\title{
Radio Galaxies as Large-Scale Cosmological Probes
}

\author{
David Nicholson
}

Presented for the Degree of Doctor of Philosophy at the University of Edinburgh 1990

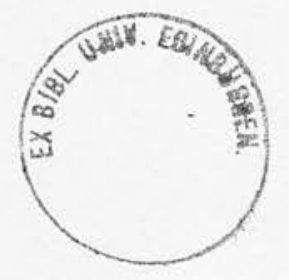


This thesis is my own composition, except where otherwise indicated in the text.

December, 1990 


\section{Acknowledgements}

I am especially grateful to John Peacock for his patient supervision of this project. I found it difficult to contend with the clinical, impersonal approach to research I encountered at ROE and this ultimately affected my ability to work well there. In spite of this I received much advice and encouragement.

I have several people to thank for contributing to some of the observations that are presented in this thesis. Simon Lilly gave me an expertly introduction to the art of CCD photometry and spectroscopy of radio galaxies. Chris Collins assisted me on the observing run in Australia. Further data was kindly provided by Lance Miller and Charles Jenkins.

Of my contemporaries in the Department of Astronomy - Karl Glazebrook, Alan Moorhouse and Phil Blanco deserve a special mention. Karl for answering most of my computing questions and providing me with several pieces of useful data reduction software. Alan \& Phil for good companionship 'after-hours' when everyone else had left.

I am especially grateful to Bernard Schutz in Cardiff for allowing me some time away from gravitational wave research to finish this dissertation. The lively, stimulating (and fun!) environment which Bernard has nurtured amongst the Relativity Group there is a far cry from the one I experienced at $\mathrm{ROE}$ and gave me the added incentive I needed to get things completed.

Of my friends in Edinburgh I wish to acknowledge Steve Proctor with whom I talked a lot, drank a lot and hurtled around a squash court with a lot. Kim Graham introduced me to the Negociant's cappucino and helped me discover the delights of the City. Finally, a special mention to Catherine who's love and support over the last two years has been a driving force.

I dedicate this thesis to my parents for their understanding and continuous support throughout the entire course of my university education.

The Royal Observatory Edinburgh is thanked for allowing me the use of its excellent research facilities during the course of my research. I acknowledge the SERC for awarding me a postgraduate studentship. 


\section{Abstract}

An all-sky sample of essentially all the radio galaxies within $30000 \mathrm{kms}^{-1}$ down to a flux-density limit of about $0.5 \mathrm{Jy}$ at $1.4 \mathrm{GHz}$ is assembled from the Parkes, Bologna, Jodrell Bank and Bonn surveys. The ease and uniformity with which this class of object can be selected render them as excellent statistical probes of the large-scale structure. This thesis is concerned with an application of the sample to study clustering and streaming motions in the universe on $10-100 h^{-1} \mathrm{Mpc}$ scales.

The observational database acquired the radio galaxies comprise spectroscopic redshifts and $I$ band $C C D$ frames, with additional $B$ frames for a, few of the galaxies. These allow a simple distance indicator relation for the radio galaxies to be derived by fitting deVaucouleurs empirical $r^{1 / 4}$ law to the azimuthally-averaged surface brightness profiles of the galaxies. This yields an effective size parameter $r_{e}$ and a distanceindependent surface-brightness $\mu$ for calibrating the 'standard rods'. In terms of rms scatter, the $\mu-r_{e}$ relation yields relative distance accurate to about $28 \%$. This compares favourably with other distance indicators which incorporate velocity dispersions, yet it has the advantage that the photometric parameters $\mu$ and $r_{e}$ are very straightforward to measure.

A useful parameter describing the richness of the environments of radio galaxies is the amplitude of the spatial cross-correlation function $B_{g r}$. Its calculation in the past has involved using full sets of galaxy counts, notably the Lick counts to do the cross-correlation. In the absence of such a set of galaxy counts, for declinations south of $-23^{\circ}$, it is shown that the Abell cluster catalogue can be used to obtain a reasonable estimate of $B_{g r}$. Considering the all-sky sample as a whole it is found that, on average, radio galaxies reside in systems of Abell richness $R \simeq 0$. A search for correlations between radio luminosity and $B_{g r}$ for the radio galaxies is performed but none are found. Similarly, no evidence is found for correlations between optical luminosity and $B_{g r}$.

The 3-d correlation function for radio galaxies is calculated here for the first time. A strong signal is detected, although not as strong as some reports in the past of the cluster-cluster correlation function would have suggested. There is no trend for the clustering signal to show a systematic increase or decrease if radio galaxies in bands of radio luminosity are considered separately. No evidence is found to support the claim by Tully that rich clusters are aligned along the supergalactic plane and exhibit correlated structure on a scale $0.1 c$.

Peculiar velocities are derived for the radio galaxies using the $\mu-r_{e}$ relation and these are analysed in terms of simple dipole and quadrupole models for the motion of the Local Group. Large error bars on the dipole solution are traced to significant offdiagonal elements in the error covariance matrix which tells us how the errors in each 
of the 6 free parameters in the non-linear least squares fit of the model to the data are coupled together. Monte-carlo simulations for an isotropised sky-distribution suggests that the large errors are a consequence of an anisotropic sky coverage - notably the sparse number of galaxies that were observed in the southern hemisphere relative to the north. A different technique for analysing peculiar velocities is to use the velocity autocorrelation tensor. Improvements are shown to exist over previous calculations principally a better weighting function can be computed from the data itself and then fed iteratively back into the calculation. These improvements are implemented here, although the overall effects they make to the solution are slight.

A simple model for biased galaxy formation is considered in which the non-linear dark matter clumps are identified as sites for galaxy formation and their luminosity is assumed to be proportional to the product of some power of their mass and a power of the collapse redshift of the system. The implications of subjecting these systems to a long-wavelength density perturbation are examined. It turns out that the FaberJackson relation for elliptical galaxies should exhibit systematic offsets which correlate with the richness of their environments. This is a testable prediction. A large sample of elliptical galaxies with photometric and kinematic data are employed. No systematic offsets are found for either the Faber-Jackson relation or the $D_{n}-\sigma_{v}$ relation. Offsets are shown to exist for systems which typically inhabit extremely poor environments, such such as spiral bulges, and extremely rich environments such as radio galaxies and Brightest Cluster Members. Offsets are found and they are in the right sense for them to be attributed to the operation of bias. However, other more likely explanations are possible. At face value the data examined here seem to suggest that the distance indicator relations are effectively unbiased. 


\section{Contents}

Acknowledgements i

Abstract

Contents iv

List of Figures xii

List of Tables $\quad$ xv

1 General Introduction 1

1.1 Introduction . . . . . . . . . . . . . . . . . 1

1.2 The Large-scale structure of the universe . . . . . . . . . . . . . . 3

1.2.1 The cosmological principle and isotropy of the universe . . . . 3

1.2 .2 Big bang cosmology . . . . . . . . . . . . 4

1.2.3 Inflation and the origin of density fluctuations $\ldots \ldots \ldots$

1.2.4 The emergence of structure ............ . 7

1.2.4.1 The radiation dominated era .......... 8

1.2.4.2 Post-recombination evolution ............ 9

1.2.5 Observational constraints on the theoretical models . . . . . . 10

1.2.5.1 Cosmic microwave background anisotropies . . . . . 10

1.2.5.2 The Large-scale distribution of galaxies and clusters . . 12

1.2.5.3 Streaming motions . . . . . . . . . . 15 
1.3 Galaxy formation . . . . . . . . . . . . . . 17

1.3.1 Radio galaxy formation ................... 18

1.4 Thesis outline . . . . . . . . . . . . . . . . . 19

2 An All-Sky Sample of Nearby/Ellipticals 20

2.1 Introduction . . . . . . . . . . . . . . 20

2.2 Sample selection criteria . . . . . . . . . . . . . . . 20

2.3 Compilation of the sample . . . . . . . . . . . . 22

2.3.1 Radio selection . . . . . . . . . . . . . . . . . 22

2.3.1.1 The Parkes survey . . . . . . . . . . . . 23

2.3.1.2 The Bologna B2 survey . . . . . . . . . . . . 24

2.3.1.3 The Jodrell Bank Survey . . . . . . . . . . . . . . . 24

2.3.1.4 The Bonn survey. . . . . . . . . . . . 25

2.3 .1 .5 Additional sources . . . . . . . . . . 25

2.3.1.6 Flux-density limits. . . . . . . . . . 26

2.3 .2 Optical selection ...................... 26

2.4 The final sample . . . . . . . . . . . . . . 28

2.5 Selected Properties of the sample . . . . . . . . . . . 28

2.5 .1 Sky coverage . . . . . . . . . . . . . . . . 29

2.5.2 Redshift distributions . . . . . . . . . . . . . 29

2.5.3 Radio power histogram .................. 33

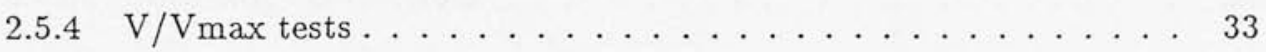

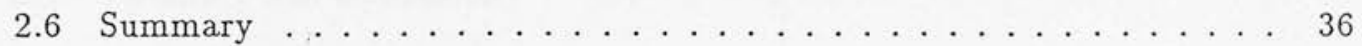




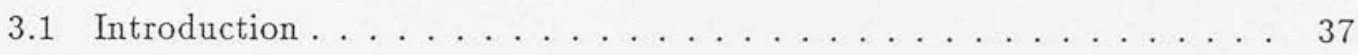

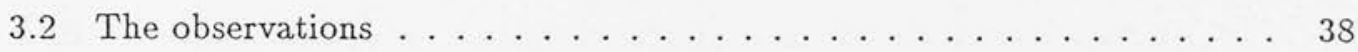

3.2 .1 General strategy . . . . . . . . . . . . . . 38

3.2.2 Instrumentation and observing conditions . . . . . . . 40

3.3 Data reduction ........................... 41

3.3.1 Bias subtraction ...................... 41

3.3.2 Flat-fielding. . . . . . . . . . . . . . 41

3.3.3 Cosmetic improvements .................... 42

3.3.4 Fringe removal . . . . . . . . . . . . . . . . 42

3.4 Photometric calibrations . . . . . . . . . . . . . . . . 42

3.4 .1 The UH88 calibrations . . . . . . . . . . . . . . . 45

3.4 .2 The INT calibrations . . . . . . . . . . . . . . . 45

3.4 .3 AAT calibrations . . . . . . . . . . . . . 47

3.5 Photometric consistency checks ... . . . . . . . . . 50

3.5.1 Measurements of aperture magnitudes for the galaxies . . . . 50

3.5.2 Comparison between UH88 \& AAT magnitudes . . . . . . . . . 51

3.5.3 The INT repeat photometry . . . . . . . . . . 53

3.6 Astrometry .......................... 53

3.7 Further corrections to galaxy magnitudes . . . . . . . . . . . 54

3.7.1 Galactic extinction corrections .............. 54

3.7 .2 Cosmology corrections . . . . . . . . . . . . . 54

3.8 Summary and conclusions . . . . . . . . . . . 55 
3.9 Introduction . . . . . . . . . . . . . 56

3.10 The observations ..................... 57

3.11 Data reduction ......................... 60

3.11 .1 Flat-fieding . . . . . . . . . . . . . 6 6 61

3.11 .2 Extracting the spectra ...................... 61

3.11 .3 Wavelength calibration . . . . . . . . . . . 61

3.11 .4 Smoothing the spectra . . . . . . . . . . . 62

3.11 .5 Removing features by interpolation . . . . . . . . . . 63

3.12 A catalogue of radio galaxy spectra . . . . . . . . . . . 63

3.12 .1 Spectral features . . . . . . . . . . . . . . 63

3.12 .2 Anomalous objects . . . . . . . . . . . . 67

3.13 Redshift results . . . . . . . . . . . . . . 67

3.13.1 The cross-correlation technique . . . . . . . . . 67

3.13.1.2 Internal errors in cross-correlation redshifts . . . . . . 69

3.13 .2 Results ......................... 71

3.13.2.1 The radio galaxy redshifts . . . . . . . . 72

3.13 .3 Comparison with published redshifts . . . . . . . 77

3.14 Summary . . . . . . . . . . . . . . . . . . . 79

4 The Optical Properties and Environments of Radio Glaxies 81

4.1 Introduction . . . . . . . . . . . . . 81

4.2 The Cluster environment of radio galaxies . . . . . . . . . . 82

4.2 .1 The cluster parameter $B_{g g} \ldots \ldots \ldots \ldots \ldots 2$ 
4.2 .2 Results ......................... 84

$4.3 B_{g r}$ vs. radio luminosity . . . . . . . . . . . . . 90

4.4 Radio galaxy surface photometry . . . . . . . . . . . . . 93

4.4 .1 The $(M, \alpha)$ diagram .......................... 93

4.4 .2 The $\mu_{e}-r_{e}$ relation ................... 95

4.4.2.1 A general fitting function . . . . . . . . . . 103

4.4.2.2 Generating light intensity profiles ... . . . . . 103

4.4.2.3 Seeing deconvolution . . . . . . . . . . 104

4.4.2.4 The optimisation algorithm . . . . . . . . . 104

4.4 .2 .5 Results .................. . . 106

4.4.3 Comparison between parameters ... . . . . . . . . . . 110

4.5 Discussion . . . . . . . . . . . . . . . . 113

4.5.1 Implications of a luminosity-size relation . . . . . . . . . . . . 114

4.5.2 The $\mu-r_{e}$ relation as a distance indicator . . . . . . . . . 114

4.6 Colour-magnitude Diagram . . . . . . . . . . . . . 114

4.7 Are optical properties related to cluster environment? . . . . . . . 116

4.8 Summary . . . . . . . . . . . . . . . . . . 116

5 The 3-d Clustering of Radio Galaxies 119

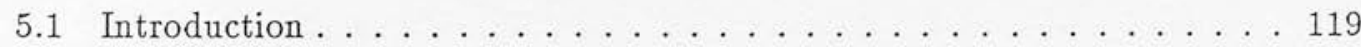

5.2 Observations of large-scale clustering in the universe . . . . . . . 120

5.2.1 The two-point correlation function . . . . . . . . 120

5.2 .2 Clustering of galaxies . . . . . . . . . . . . . . 121

5.2 .3 Clustering of clusters . . . . . . . . . . . . . . . 122 
5.2 .4 Supercluster correlations . . . . . . . . . . . . . 125

5.2.5 The clustering of radio galaxies . . . . . . . . . 125

5.3 The 3 - $\mathrm{d}$ correlation function for radio galaxies . . . . . . . . 126

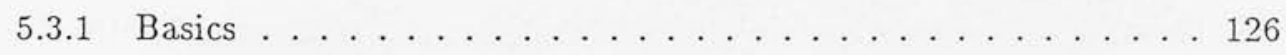

5.3.2 The monte-carlo datasets . . . . . . . . . . . . 127

5.3.3 Error estimates . . . . . . . . . . . . . . . . . 128

5.3 .4 Results . . . . . . . . . . . . . . . . 129

5.3.5 Trends with radio luminosity . . . . . . . . . . . 129

5.4 Discussion .......................... 135

5.5 The Tully effect . . . . . . . . . . . . . . 136

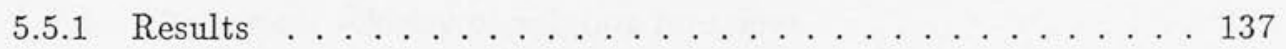

5.5 .2 Discussion . . . . . . . . . . . . . . . 141

5.5 Summary and conclusions . . . . . . . . . . . . 141

6 Large-Scale Streaming Motions $\quad 142$

6.1 Introduction . . . . . . . . . . . . . . . . . . . 142

6.2 Previous observational work . . . . . . . . . . . . . . 144

6.3 The present work . . . . . . . . . . . . . 146

6.4 Multipole solutions for the local group motion . . . . . . . . . . . 147

6.4 .1 The data ........................ 147

6.4 .2 The distance indicator relation . . . . . . . . . . . . 149

6.4 .3 The optimisation scheme ... . . . . . . . . . 150

6.4.4 Dipole solution for the local group motion . . . . . . . . . . 154

6.4 .5 Quadrupole solution ................. 156 
6.4 .6 Error analysis . . . . . . . . . . . . . . 159

6.4 .7 Monte carlo simulations . . . . . . . . . . . . 160

6.5 Streaming in the vicinity of the Great Attractor . . . . . . . . . . 160

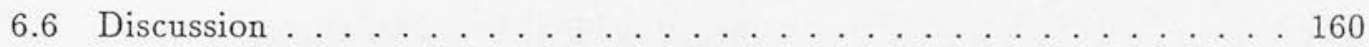

6.7 The velocity autocorrelation function . . . . . . . . . . . . 162

6.7.1 Method of calculation .................. 163

6.7 .2 An improved calculation . . . . . . . . . . 165

6.7 .2 .1 A better weighting term . . . . . . . . . 165

6.7.2.2 Reciprocal variance weighted distance . . . . . . . . . . . 167

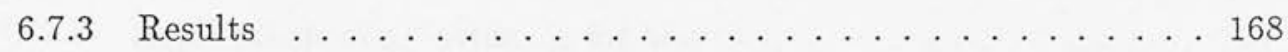

6.7.4 Theoretical velocity correlation functions . . . . . . . . . 171

6.8 Summary . . . . . . . . . . . . . . . . . . 173

7 Biased Peculiar Velocities $\quad 174$

7.1 Introduction . . . . . . . . . . . . . . . 174

7.2 Biased galaxy formation . . . . . . . . . . . 175

7.2 .1 Observations ............................ 175

7.2.2 Amplitude of biasing in galaxies and clusters . . . . . . . 176

7.2.3 Physical mechanisms for biased galaxy formation . . . . . . . 177

7.2 .4 Natural or autonomous bias . . . . . . . . . . . 178

7.3 Outline of the present work . . . . . . . . . 180

7.4 The fundamental plane for elliptical galaxies . . . . . . . . . . . . . . 181

7.4 .1 Origin of systematic offsets . . . . . . . . . . 182

7.5 Biased peculiar velocities . . . . . . . . . . . . . 183 
7.5.1 Implications of offsets for peculiar velocities . . . . . . . . . . 183

7.5 .2 Observational database . . . . . . . . . . . 185

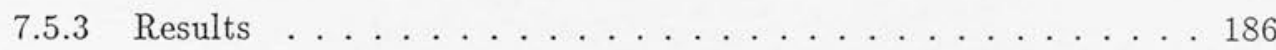

7.6 Spiral bulges, BCM's and radio galaxies . . . . . . . . . . 189

7.6 .1 Spiral bulges . . . . . . . . . . . . . . . . 189

7.6.2 Brightest cluster members . . . . . . . . . . . . . . 192

7.6.3 Radio galaxies . . . . . . . . . . . . . . . . 195

7.7 Discussion . . . . . . . . . . . . . . . 195

7.7.1 Constraining the simple bias model . . . . . . . . . . . 200

7.7.2 Implications of no evidence for bias . . . . . . . . . . . . 204

7.8 Summary . . . . . . . . . . . . . . . . 205

8 Concluding Remarks $\quad 206$

Appendices

A All-Sky Sample Database 211

B A Catalogue of Spectra 234

C Analytic Approximation to Seeing Convolution Integral 272

D Environment Parameters for G7 Ellipticals $\quad 277$ 


\section{List of Figures}

1.1 Post recombination density fluctuation spectra . . . . . . . . . 10

1.2 The distribution of galaxies ................ 14

2.1 Hubble diagram for the sample . . . . . . . . . . . 27

2.2 Sky distribution for the sample ... . . . . . . . . 30

2.3 Redshift distribution for the sample . . . . . . . . . . . . 32

2.4 Distribution of radio luminosities . . . . . . . . . . . 34

$2.5 \mathrm{~V} / \mathrm{V} \max$ against radio luminosity . . . . . . . . . . 35

3.1 KPNO $B$ \& $I$ filter profiles . . . . . . . . . . . . . . . 44

3.2 Extinction curves for UH88 observations . . . . . . . . . . . 46

3.3 Extinction curves for INT observations . . . . . . . . . . . . . . 48

3.4 Extinction curves corrected for time-varying dust extinction . . . . . . 49

3.5 Example absorption line spectrum ............... 66

3.6 Example emission line spectrum . . . . . . . . . . . 66

3.7 Cross-correlation spectrum . . . . . . . . . . . 70

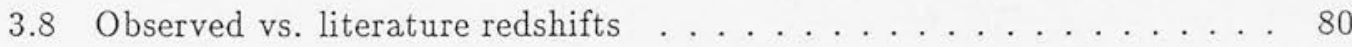

4.1 Schechter functions corresponding to the Abell criteria . . . . . . . . 87

$4.2 A_{g r}$ comparison between Lick and Abell estimates . . . . . . . . 89

$4.3 B_{g r}$ against $2.7 \mathrm{GHz}$ source luminosity $\ldots \ldots \ldots \ldots \ldots . \ldots \ldots$

4.4 The $M-\alpha$ diagram .................... 96

4.5 Relationship between growth curve and deVaucouleurs parameters . . . 102 
4.6 Convolution of an $r^{1 / 4}$ profile with a Gaussian PSF . . . . . . . . 105

4.7 A few example profile fits .................. 107

$4.8 \mu-r_{e}$ relation for the radio galaxies $\ldots \ldots \ldots \ldots \ldots 11$

4.8 Comparison between growth curve $\alpha$ and profile fit $\alpha \ldots \ldots . \ldots 112$

$4.10 B-I$ colour-magnitude diagram . . . . . . . . . . 115

4.11 Colour vs redshift . . . . . . . . . . . . . . 115

4.12 Optical luminosity against richness of environment . . . . . . . 117

5.1 The hiearchial trend of clustering in the universe . . . . . . . 123

5.23 -d correlation function for full sample . . . . . . . . . 130

5.3 3-d correlation function for radio galaxies in bands of radio luminosity . 131

5.4 Distribution of $z<0.025$ radio galaxies about the SGZ plane . . . . . 138

5.5 Distribution of $z>0.025$ radio galaxies about the SGZ plane . . . . 138

6.1 Sky distribution of subsample used in the streaming analysis . . . . . 148

6.2 The $\mu-r_{e}$ relation . . . . . . . . . . . . 151

6.3 The effect of correcting redshifts for peculiar velocities on the $\mu-r_{e}$ relation . . . . . . . . . . . . . . . 155

6.4 Showing the lack of radio galaxies in the vicinity of the Great Attractor 161

6.5 Geometry of a pair of galaxies . . . . . . . . . . . 164

6.6 Velocity correlation functions for the G7 ellipticals . . . . . . . . . 169

6.7 Pair distributions for the radio galaxies . . . . . . . 170

6.8 Velocity correlation function for the radio galaxies . . . . . . . 170

6.9 Theoretical velocity correlations . . . . . . . . . . . . 172

7.1 Schematic diagram to illustrate biased peculiar velocities . . . . . . . . 184

$7.2 D_{n}-\sigma_{v}$ relation for the $\mathrm{G} 7$ ellipticals $\ldots \ldots \ldots \ldots \ldots$ 
$7.3 \quad D_{n}$ residuals against $B_{g e} \ldots \ldots \ldots \ldots \ldots \ldots \ldots$

7.4 Affect of avoiding local-anomaly and binning in $B_{g e}$ on residuals . . . 188

7.5 Faber-Jackson relation for the G7 ellipticals . . . . . . . . . . . . 190

7.6 Faber-Jackson residuals against $B_{g e} \ldots \ldots \ldots \ldots 1$

7.7 Affect of avoiding local-anomaly and binning in $B_{g e}$ on residuals . . . . 191

7.8 Faber-Jackson relation for S0 bulges in Coma . . . . . . . . . . . . 193

$7.9 D_{n}-\sigma_{v}$ relation for So bulges in Coma . . . . . . . . . . . 193

7.10 Faber-Jackson and $\mu-R-\sigma_{v}$ relation for BCM's . . . . . . 194

7.11 Faber-Jackson relation for radio galaxies . . . . . . . . . . . 196

$7.12 D_{n}-\sigma_{v}$ slope against richness class for G7 clusters . . . . . . . . . 199

7.13 Faber-Jackson slope against richness class for G7 clusters . . . . . . 199

7.14 Expected Faber-Jackson offsets in $\beta=3$ model . . . . . . . . . . 203

B1.1 Spectra acquired at UH8s . . . . . . . . . . 235

B1.2 Spectra acquired at the AAT . . . . . . . . . . . 249

B1.3 Spectra acquired at the WHT . . . . . . . . . . . 260

B1.4 FORS spectra from the AAT . . . . . . . . . . . 262

B1.5 Spectra acquired at the INT . . . . . . . . . . 267

A1.6 Spectra acquired from ESO . . . . . . . . . . . 269

C.1 Analytic approximations to profile convolution . . . . . . . . 274 


\section{List of Tables}

1.1 Observations of the $\mathrm{CBR} \ldots \ldots \ldots \ldots \ldots \ldots$

2.1 Radio surveys from which the sample was compiled . . . . . . . . . . 22

2.2 Source-cluster angular associations . . . . . . . . . . 25

2.3 Results of $\mathrm{V} / \mathrm{V} \max$ tests $\ldots \ldots \ldots \ldots \ldots \ldots$

3.1 Summary of UHs8 \& AAT repeat photometry . . . . . . . . . . . . . . 52

3.2 Summary of INT repeat photometry . . . . . . . . . . . . 52

3.3 Summary of spectroscopic observations . . . . . . . . . . . . 58

3.4 Common features seen in radio galaxy spectra . . . . . . . . . 65

3.5 Galaxy and stellar templates . . . . . . . . . . . . 72

3.6 Redshift results . . . . . . . . . . . . . . . 73

3.7 Radio galaxies in Huchra and Fairall's catalogues . . . . . . . . . 78

4.1 Abell richness classes . . . . . . . . . . . . . . . . . . 86

$4.2 B_{g g}$ estimates for Abell clusters . . . . . . . . . . . 86

4.3 Average values of $B$ for the sample . . . . . . . . . . . . . . 92

4.4 Photometric and environment parameters for the sample . . . . . . . 97

$7.1 D_{n}-\sigma_{v}$ and Faber-Jackson slopes for G7 clusters . . . . . . . . . 197

A1.1 Definite sample members . . . . . . . . . . . . . . . 213

A1.2 Probable sample members . . . . . . . . . . . . . . . . . . . 219

A1.3 Possible sample members . . . . . . . . . . . . . . 220

A1.4 Definite sample non-members . . . . . . . . . . . . . . . . . 224 
D.1 Environment parameters for the G7 ellipticals . . . . . . . . 277

D.2 The most populous G7 clusters . . . . . . . . . . . 284 


\section{Chapter 1}

\section{General Introduction}

\subsection{Introduction}

Radio galaxies are furnished with a number of desirable properties that have established them as powerful cosmological probes. Their strong emission at radio wavelengths can be detected at large distances and acts as a convenient pointer to high-redshift galaxies. This has been exploited for the 3CR-like samples to study galaxy formation and evolution at large look-back times (e.g. Lilly \& Longair 1984; Eisenhardt \& Lebofsky 1987). Similarly, the Parkes Selected Region Survey (Dunlop \& Peacock 1990) has been used to examine the 'redshift-cutoff' for steep spectrum radio sources and the form of the Radio Luminosity Function (RLF) at high redshifts.

In this thesis the emphasis is on the nature of the present-day structure of the universe rather than that at distant epochs. Our study utilises a further important property of radio galaxies: the ease and uniformity with which this class of object can be selected render them as excellent statistical probes of the large-scale structure. Accordingly, an all-sky sample of nearby radio galaxies is assembled from the Parkes, Bologna, Jodrell Bank and Bonn surveys down to an approximate flux-density limit of $0.5 \mathrm{Jy}$ at $1.4 \mathrm{GHz}$. Redshift cutoffs are imposed on the sample selection at $z=0.01$ and $z=0.1$, so that the final sample probes the cosmologically interesting regime from $10-100 h^{-1} \mathrm{Mpc}\left(h \equiv H_{0}\right.$ in units of $\left.100 \mathrm{kms}^{-1} \mathrm{Mpc}^{-1}\right)$. This dissertation is primarily concerned with a statistical study of clustering and streaming motions on the above scale.

Vital clues to the nature of the particle content of the universe and the primordial spectrum of density fluctuations can be found by studying the present-day distribution and dynamical behaviour of matter on scales extending from just outside the Local Group 
of galaxies to redshifts of about one-tenth (Kolb \& Turner 1990 and references therein). Optically selected samples of objects have been used almost exclusively in the past to investigate this domain with some debatable results. In particular, the reality of largescale streaming motions (Davis \& Peebles 1983a; Gunn 1988) and positive correlations in the distribution of Abell clusters on $>50 h^{-1} \mathrm{Mpc}$ scales (Ling, Frenk \& Barrow 1986; Sutherland 1988) has been the subject of much controversy. The implied existence of very large-scale density inhomogeneities in the universe puts severe constraints on any model which attempts to explain the origin and growth of cosmic structure. The favoured Standard Cold Dark Matter (CDM) model, in which structure forms from the gravitational amplification of infinitessimal fluctuations laid down at very early times, is one such such scenario which apparently has problems trying to accommodate these observations (e.g. Efstathiou 1990).

One of the main motivations for this thesis is a desire to try and shed some light on this issue by providing some further observations, but from a novel perspective. Optically selected samples of normal galaxies are dispensed with in favour of an all-sky sample of radio galaxies which, we shall argue, is expected to be free from a number of systematic biases which may have influenced the outcome of previous observations of the large-scale structure to an unknown extent.

These observations take the form of optical spectroscopy and CCD photometry. Although the applications of the data are primarily geared towards studying the large-scale structure, it is important to note that they can also help to develop new insights into several aspects of the radio phenomenon itself. Attention here is focussed towards the relationships between the local cluster environments of radio galaxies with their radio and optical properties. This has already been shown by others to form a fruitful line of research (Longair \& Seldner 1979; Lilly \& Prestage 1987; Prestage \& Peacock 1988) although some important issues still stand to be resolved.

This general introduction to the thesis provides a brief but comprehensive overview of the large-scale structure from both the theoretical and observational viewpoints. An opportunity is taken to present some notation which will be required for later Chapters. Some issues, such as microwave background anisotropies and the epoch of galaxy formation 
are not directly relevant to the present work but I have included a short description here for the sake of completeness and also so that their value as probes of the large-scale structure might be weighed alongside clustering and streaming velocities, which are the principal subjects of this dissertation.

\subsection{The Large-Scale Structure of the Universe}

In order to keep pace with recent advances in theoretical and observational studies of the large-scale structure and galaxy formation, there have been a number of lucid treatments of the subject at a variety of levels. The list is somewhat exhaustive but reviews by Efstathiou \& Silk (1983) and more recently the book by Kolb \& Turner (1990) cover most of the relevant background material that is necessary for this thesis. The present overview is merely designed to introduce some key theoretical ideas which have been proposed to explain the origin of large-scale structure and examines how well these stand up to observations. We begin by tracing the origin of large-scale structure in a roughly chronological order from the generation of fluctuations in the early universe through to the current epoch.

\subsubsection{THE COSMOLOGICAL PRINCIPLE AND ISOTROPY OF THE UNIVERSE}

It is worthwhile bearing in mind that the departures from homogeneity in the distribution and dynamical behaviour of matter in the universe, such as those implied by observations of peculiar motions of galaxies and positive cluster correlations which we seek to reliably quantify in this thesis, are local phenomena only. The Cosmological Principle (CP) combines the Copernican postulate that we are not in any way privileged observers with the observation that the universe appears isotropic in all its measured properties. This isotropy is only apparent however if we envisage smoothing out all the local structure. In a universe which satisfies the CP it is easy to demonstrate that pure expansion (or indeed contraction) are the only permissable velocity fields. In the notation of Gunn (1978) the 
velocity of an observer can be expressed in Cartesian coordinates as follows

$$
v_{j}\left(x^{1}, x^{2}, x^{3}\right)=\sum_{i} \frac{\partial v_{j}}{\partial x^{i}} \delta x^{i}+O\left[\left(\delta x^{i}\right)^{2}\right]
$$

The velocity gradient $\partial v_{j} / \partial x^{i}$ is a $3 \times 3$ matrix which can be decomposed into three terms: a multiple of the identity; a traceless symmetric part; a skew part. Thus the velocity field can be written

$$
\mathbf{v}=H \delta \mathbf{x}+\mathbf{\Sigma} \cdot \delta \mathbf{x}+\Omega \times \delta \mathbf{x}
$$

where $H$ is the expansion scalar, or more familiarly the Hubble parameter, $\Sigma$ the shear tensor, and $\Omega$ the angular velocity. The isotropy constraint demands that the first two terms drop out leaving

$$
\mathbf{v}=H \mathbf{x}
$$

This simple formula for the recession velocity, expressing its proportionality to distance, is just the familiar Hubble flow. There is a great deal of activity in observational cosmology at the present time to search for systematic departures from the Hubble flow on largescales - this subject is dealt with in some detail in Chapters Six and Seven. From the theoretical point of view, large-scale coherent flows are presumably driven by gravity and thus their amplitude and direction can put severe constraints upon the size of density inhomogeneities in the universe.

\subsubsection{BIG BANG COSMOLOGY}

The CP can be combined with an assumption that Einstein's field equations provide a correct description of gravity, to model the evolution of the universe. Thus, if the universe is regarded to be an isotropically expanding fluid of matter and radiation, its expansion is governed by (Weinberg 1972)

$$
\frac{\ddot{R}}{R}=-\frac{4 \pi G}{3}\left(\rho+\frac{3 P}{c^{2}}\right)
$$

and

$$
\dot{\rho}=-\frac{3 \dot{R}}{R}\left(\rho+\frac{P}{c^{2}}\right)
$$

where $R(t)$ is the scale factor and $P$ the pressure. In the Newtonian approximation these are respectively the equations of motion and mass conservation for an expanding fluid. 
The present day universe is matter dominated and hence the above equations can be integrated to yield

$$
\frac{d}{d t}\left(\rho R^{3}\right)=0 \Rightarrow \rho R^{3}=M=\text { const }
$$

and the Friedmann equation

$$
\left(\frac{\dot{R}}{R}\right)^{2}=\frac{8 \pi G \rho}{3}-\frac{k c^{2}}{R^{2}}
$$

where $k$ is a constant of integration known as the curvature constant. Note that $k$ is only regarded as a constant of integration here in the sense that equations 1.4 and 1.5 can be derived in a quasi-Newtonian fashion. Equation 1.7 can be expressed in terms of the present day values of the Hubble and density parameters $\left(H_{0} \& \Omega_{0}\right)$, allowing the Friedmann equation to be written in its more familiar form

$$
\dot{R}^{2}-\frac{8 \pi G M}{3 R}=R_{0}^{2} H_{0}^{2}\left(\Omega_{0}^{2}-1\right)
$$

where $\Omega_{0}=8 \pi G \rho_{0} / 3 H_{0}^{2}$ and $H_{0}=(\dot{R} / R)_{0}$. The solution to eqn. 1.8 is crucially dependent on the present mass density of the universe which observations of the large-scale structure must seek to constrain.

The thermal history of the universe has been carefully traced by Yang et al. (1984) and detailed computations of cosmological nucleosynthesis require that the density parameter for baryonic matter $\Omega_{B} \lesssim 0.035 h^{-2}$. This has profound implications for the particle content of the universe since, as the next Section indicates, there are good theoretical grounds for believing that $\Omega_{0}=1$.

\subsubsection{INFLATION AND THE ORIGIN OF DENSITY FLUCTUATIONS}

The standard cosmology provides a reliable framework for describing the history of the universe as early as $10^{-2} \mathrm{sec}$ after the big-bang. However, it is not without its shortcomings and to reconcile these one must resort to some particle physics. Inflation was conceived by Alan Guth in 1981 (Guth 1981) to solve the so-called 'flatness' and 'horizon' problems. The flatness conundrum arises from the observation that the present value of $\Omega_{0}$ is close to unity and yet the Friedmann equations imply that as the universe has evolved, $\Omega$ should have been evolving away from 1 according to

$$
\Omega=\frac{1}{1-x(t)}
$$


where $x(t) \propto R(t)^{2}$ for a radiation dominated universe and $x(t) \propto R(t)$ in a matter dominated universe. This means that, at very early times, $\Omega$ was equal to 1 to an extremely high degree of precision

$$
\begin{gathered}
\left|\Omega\left(10^{-43} \mathrm{sec}\right)-1\right| \simeq O\left(10^{-60}\right), \\
|\Omega(1 \mathrm{sec})-1| \simeq O\left(10^{-16}\right)
\end{gathered}
$$

This level of fine-tuning seems particularly unpalatable. Like the flatness problem, the horizon problem can also be accommodated in a standard cosmology but again at the expense of very special initial conditions. This latter puzzle is to comprehend how a universe made out of $10^{5}$ causually distinct regions can be homogenous. Without going into detail, the inflationary model for the early universe, proposed by Guth, is able to solve both problems. The key to inflation is a that there was an epoch when the vacuum energy density dominated the energy density of the universe. During this epoch, known as the deSitter phase, $R(t)$ grew exponentially $(\propto \exp (H t))$, allowing a small, causallycoherent region to grow to a size which encompasses the region which eventually becomes our presently observable universe. It is this rapid expansion which forms the basis of a solution to the horizon and flatness puzzles. A firm prediction of the inflationary scenario is that the present day value of $\Omega_{0}$ should be exactly equal to unity. As we shall see in Chapter 7 , this value can only be accommodated if one is prepared to invoke biased galaxy formation.

A further inadequacy with the standard cosmology is its failure to shed any light upon the origin or nature of primordial density perturbations, which must have existed at a level of $\sim 10^{-3}$ at decoupling to account for the observed large-scale structure in the universe. The evolution of density fluctuations in inflation has been discussed by Bardeen et al. (1983). Quantum fluctuations left over from the smoothing influence of the rapid exponential expansion during the inflationary epoch result in a calculable spectrum of adiabatic density perturbations which turn out to be scale-invariant and are expected to obey Gaussian statistics so long as their amplitude is small. Adopting the usual convention of expanding density inhomogeneities in a Fourier expansion, then

$$
\delta \rho=(2 \pi)^{-3} \int \delta_{k} e^{-i k x} d^{3} k
$$

where $k$ is co-moving wavenumber. It is usual to refer to $\delta \rho / \rho$ on a given scale as the 
r.m.s. mass fluctuation on that scale, i.e.

$$
(\delta \rho / \rho)_{k}^{2} \equiv\left\langle(\delta M / M)^{2}\right\rangle_{k} \simeq \Delta_{k}^{2} \equiv(2 \pi)^{-3} k^{3}\left|\delta_{k}\right|^{2}
$$

The factor $\left|\delta_{k}\right|^{2}$ is called the power spectrum of the fluctuations and denoted by $P(k)$. For the sake of simplicity, a power law form for $P(k)$ is usually assumed

$$
P(k) \propto k^{n}
$$

There are physical arguments (see Efstathiou 1990) to suggest that $n \lesssim 4$, with favoured values being $n=1$ and $n=-3$, since these spectra have equal power on all scales when the relevant scale comes within the horizon, i.e. they are scale invariant. The former type of fluctuations are termed adiabatic in which the perturbation in entropy is zero; the latter are isocurvature type fluctuations or entropy perturbations. The Standard Cold Dark Matter model is based around the premise of an initial adiabatic spectrum. Isocurvature models have been considered by Peebles $(1987 \mathrm{a}, 1987 \mathrm{~b})$ and found to predict temperature fluctuations in the microwave background consistent with observations. The difficulty with these fluctuations, however, is the lack of any realistic mechanism to generate them (Bond 1988). The next section highlights some of the physics concerned with the amplification of density fluctuations and the origin of large-scale structure.

\subsubsection{THE EMERGENCE OF LARGE-SCALE STRUCTURE}

The essential ingredients in a theoretical model for the large-scale structure are a characteristic fluctuation spectrum and a given composition for the cosmic soup. If an inflationary universe appealed to earlier in this Chapter is accepted, then $\Omega_{0}=1$ and it seems inevitable that some of the mass density of the universe must be in 'dark' matter form. The dark matter candidates fall into two distinct categories, each of which give rise to markedly different evolutionary paths towards the large-scale structure that we observe today. It is towards observations of clustering and streaming motions that we must turn in order to put limits on the particle content of the universe.

The decoupling of matter from radiation at $z \sim 1000$ forms a crucial point in the evolutionary history of the universe since, at later times, density fluctuations in the dark 
matter can begin to grow. A few aspects of pre- and post-decoupling physics are now addressed in order to demonstrate the predictions of various theoretical models which the work described later in this thesis can potentially discriminate between. Attention is restricted to adiabatic fluctuations in which the local entropy is held constant with matter and radiation both perturbed together $\left(\rho_{b} \sim \rho_{\gamma} \neq 0\right)$.

\subsubsection{The radiation dominated era}

A density perturbation in a fluid will inevitably collapse once the gravitational force is able to overcome the resistance due to pressure. The critical mass for the instability to develop is termed the Jeans mass, $M_{J}$. This is the mass inside a sphere of radius $\lambda_{J}$, given by

$$
\lambda_{J}=2 \pi /\left(\frac{4 \pi G \rho}{c_{s}^{2}}\right)^{\frac{1}{2}}
$$

where $c_{s}$ is equal to the adiabatic sound speed. Note that the Jeans scale corresponds roughly to the horizon size in the radiation dominated era since $(G \rho)^{-\frac{1}{2}}$ sets the expansion timescale.

Due to the gravity-internal pressure imbalance, $M_{J}$ grows approximately as $M_{H}$ the mass enclosed within the horizon. The size of perturbations in the baryonic matter, which can continue to grow after recombination, is governed by a process called Silk damping (Silk 1968). Photon diffusion from high pressure regions leaves behind residual matter perturbations below a critical mass scale $M_{S}$ and perturbations are washed out on the scale-length of the photon random walk. This is not the case for perturbations in the dark matter which are undamped.

Prior to recombination the dark matter particles only interact with the radiation field through gravity. Neutrinos decouple from the plasma after nucleosynthesis and are relativistic with a high velocity dispersion, i.e. they are "hot" particles. Cold dark matter candidates like axions have, as their name suggests, low thermal velocities. It is only after matter-radiation decoupling that the properties of the dark matter particles become important, as we now discuss. 


\subsubsection{Post-recombination evolution}

After decoupling, fluctuations can begin to grow since the expansion timescale is of the order of the collapse timescale. Fig. 1.1 illustrates the post-recombination fluctuation spectrum as a function of mass for the hot- and cold dark matter dominated universes.

The free streaming of neutrinos has erased structure on co-moving scales which today correspond to the masses of clusters or superclusters. The suppression of substructure on these initial scales suggests that the collapse process will be highly anisotropic, to a thin sheet or pancake (Zeldovich 1970). This might conceivably fragment at later epochs into smaller structures: galaxy clusters, groups and individual galaxies (Sunyaev \& Zeldovich 1972). The whole process is often dubbed the top-down scenario for galaxy formation. Conversely, the bottom-up scenario refers to cold dark matter models in which large-scale structure develops through hierarchial clustering of smaller structures.

Theoretically, the change in shape of the initial fluctuation spectrum after recombination is characterised by a transfer function $T(k)$, usually defined so that

$$
\left|\delta_{k}\left(t_{0}\right)\right|=T\left(k, t_{i}\right)\left|\delta_{k}\left(t_{i}\right)\right|
$$

This function has been computed for a massive neutrino dominated universe by Bond \& Szalay (1983) and for an adiabatic cold dark matter universe by Bond \& Efstathiou (1984). Properties of the linear density and velocity fields can then be calculated such as the two-point correlation function, which is just the Fourier transform of the power spectrum.

\subsubsection{OBSERVATIONAL CONSTRAINTS ON THE THEORETICAL MODELS}

Theories for the large-scale structure like CDM have great predictive power. Observational cosmology today is primarily geared around designing careful tests to check whether these predictions are correct. A few such tests are reviewed here. This is by no means an exhaustive list but rather selects just a few that bear most relevance to the present work.

\subsubsection{Cosmic microwave background anisotropies}

The CBR presents us with crucial information about the physical conditions that prevailed 


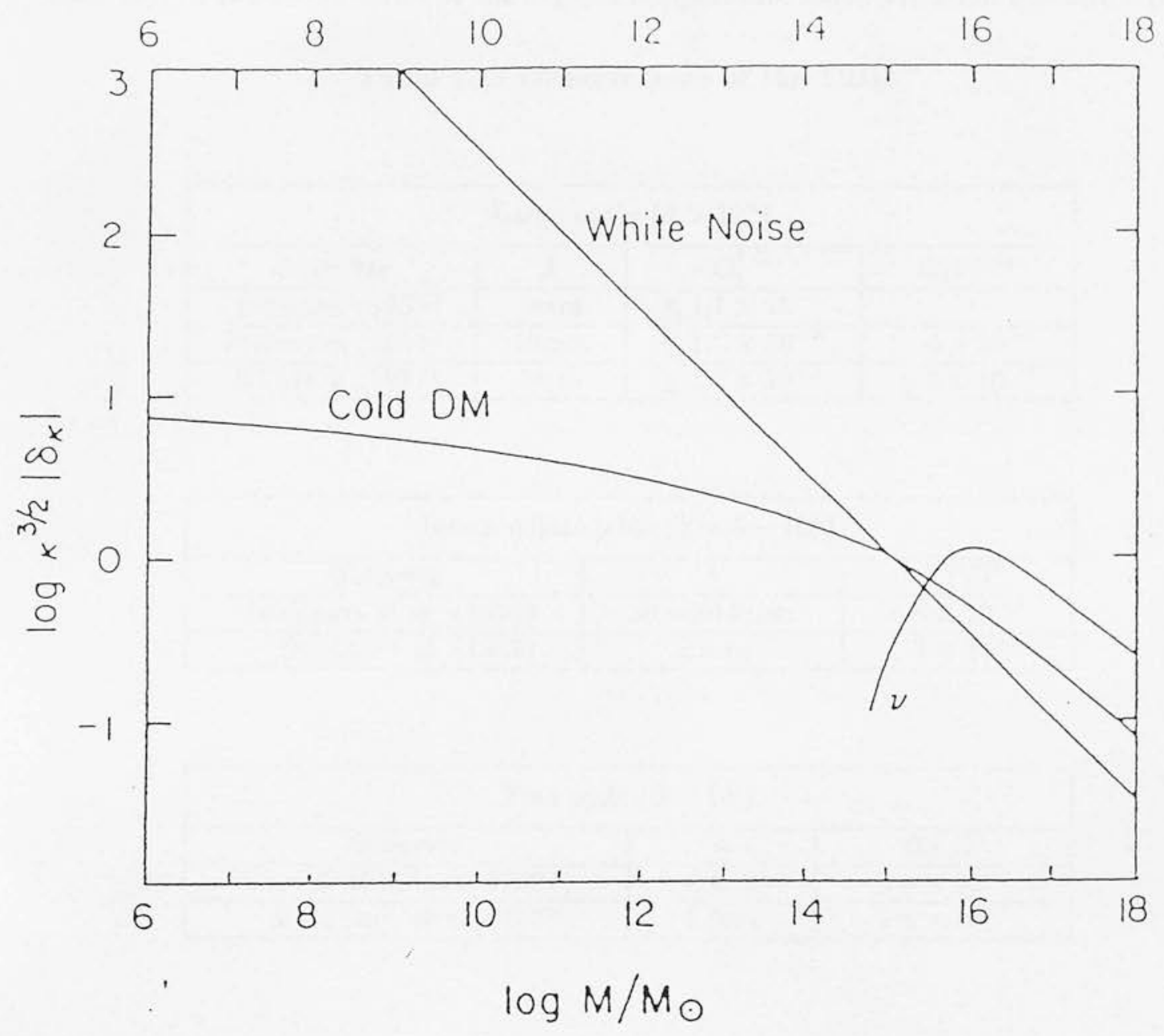

Fig. 1.1: Post recombination density fluctuation spectra for cold dark matter and neutrino $(\nu)$ dominated universes. Reproduced in part from Primack \& Blumenthal (1984). 
in the universe at the epoch $(z \sim 1000)$ when matter and radiation last interacted. Smallscale anisotropies in the CBR must be manifest at some level but so far these have proved to be elusive. Some important results from observations of the CBR for anisotropies on a variety of scales are summarised below (NB. the fluctuations in the first of these tables have been expressed in terms of the angular temperature autocorrelation function $C(\theta)$ )

Table 1.1: Observations of the CBR

\begin{tabular}{|c|c|c|c|}
\hline \multicolumn{4}{|c|}{ Large angle $\left(\theta>10^{\circ}\right)$} \\
\hline Observer & $\lambda$ & $C_{2}^{1 / 2}$ & $C(\theta)^{1 / 2}$ \\
\hline Berkeley (1985) & $3 \mathrm{~mm}$ & $\lesssim 1.1 \times 10^{-4}$ & - \\
\hline Princeton (1983) & $12 \mathrm{~mm}$ & $\lesssim 1.2 \times 10^{-4}$ & $4 \times 10^{-5}$ \\
\hline RELIKT (1987) & $8 \mathrm{~mm}$ & $\lesssim 4.8 \times 10^{-5}$ & $\lesssim 2 \times 10^{-5}$ \\
\hline
\end{tabular}

\begin{tabular}{|c|c|c|}
\hline \multicolumn{3}{|c|}{ Intermediate scale $\left(\theta \sim 5-10^{\circ}\right)$} \\
\hline Observer & $\lambda$ & $\Delta T / T$ \\
\hline Melchiorri et al. $(1981)$ & $50-3000 \mu \mathrm{m}$ & $4.8 \times 10^{-5}$ \\
\hline Davies et al. $(1987)$ & $2.9 \mathrm{~cm}$ & $3 \times 10^{-5}$ \\
\hline
\end{tabular}

\begin{tabular}{|c|c|c|}
\hline \multicolumn{3}{|c|}{ Fine scale $\left(\theta<10^{\prime}\right)$} \\
\hline Observer & $\lambda$ & $\Delta T / T$ \\
\hline Uson \& Wilkinson (1984) & $1.4 \mathrm{~cm}$ & $\lesssim 3 \times 10^{-5}$ \\
\hline Readhead et al. $(1989)$ & $1.5 \mathrm{~cm}$ & $1.5 \times 10^{-5}$ \\
\hline
\end{tabular}

On large angular scales, the experiments find a dipole anisotropy in the background. Conventional wisdom points towards a Doppler origin for this anisotropy, in which case the implied Local Group (LG) motion relative to the CBR is $614 \mathrm{kms}^{-1}$ towards $\alpha=$ $11.18 \pm 0.05^{h}$ and $\delta=-8.0 \pm 0.7^{\circ}$ (Fixsen et al. 1983). This provides an important benchmark measure and discrepancies between the LG motion relative to the CBR and samples of distant galaxies have been interpreted as being due to galaxy streaming motions as we shall explain in further detail in Chapter 6 .

In the theoretical context, CBR anisotropies on small angular scales that are predicted 
to arise in the adiabatic CDM and isocurvature baryon models have been calculated by Bond \& Efstathiou (1987). For the isocurvature model, the original entropy fluctuations show up as large temperature fluctuations. In particular, the isocurvature axion model appears to be ruled out by the observations of Readhead et al. and Uson \& Wilkinson. If the positive detection of $\mathrm{CBR}$ fluctuations on $5^{\circ}$ and $8^{\circ}$ scales that have been claimed by Davies et al. are confirmed, then the Standard CDM model may also face problems.

\subsubsection{The large-scale distribution of galaxies and clusters}

Observations of large-scale structure in the universe have accelerated rapidly over the last decade and hold great promise for the future. Much of the early work was confined to statistical studies of the angular positions of galaxies listed in the Lick and Zwicky catalogues (Peebles 1980 and references therein). Similarly the Abell catalogue of rich clusters of galaxies has been subject to extensive statistical analysis (Bahcall 1988 and references therein). Recent attention however has shifted to the redshift surveys, which provide a 3-dimensional view of the large-scale structure. The largest present survey is the Centre for Astrophysics (CfA) redshift survey (Davis et al. 1982) which includes all galaxies in the northern sky with galactic latitude $b>40^{\circ}$ and apparent magnitude $m \lesssim 14$. This survey shows a diversity of structure in the distribution of galaxies with obvious enhancements as well as large regions of low density, termed voids.

All of the surveys indicate that the distribution of galaxies on $10-100 h^{-1} \mathrm{Mpc}$ scales is highly non-uniform. The amplitude of clustering is most conveniently quantified in terms of the two-point correlation function $\xi(r)$ which is defined by the expression

$$
d P(r)=n^{2}[1+\xi(r)] d V_{1} d V_{2}
$$

where $d P(r)$ is the joint probability of finding two objects in volumes $d V_{1}$ and $d V_{2}$ separated by a distance $r$ and $n$ is the mean number density of the objects in question. Analyses of the catalogues show that, for galaxies, $\xi_{g g}(r)$ can be represented by a power law

$$
\xi_{g g}(r)=\left(\frac{r_{0}}{r}\right)^{\gamma}
$$

with $\gamma \simeq 1.8$ in the range $0.1 h^{-1} \lesssim r \lesssim 10 h^{-1} \mathrm{Mpc}$, where $r_{0}=(5 \pm 0.7) h^{-1} \mathrm{Mpc}$ is the galaxy correlation length (Davis \& Peebles 1983b). The two-point correlation function 
statistic has also been applied to the Abell cluster catalogue by Bahcall \& Soneira (1983), who find a similar power-law form for $\xi_{c c}(r)$ but in the range $7<r<150 h^{-1} \mathrm{Mpc}$ and with a correlation length of $r_{0} \simeq 25 h^{-1} \mathrm{Mpc}$. The amplitude of the cluster-cluster correlation function is thus some eighteen times larger than the galaxy correlation function. Further, there appears to be evidence in the Abell catalogue for an apparent trend of increase in clustering strength with richness of system (Bahcall 1988). This discovery is supported by the report of positive spatial correlations amongst a sample of superclusters on a scale of $\sim 100 h^{-1} \mathrm{Mpc}$ (Bahcall \& Burgett 1986). One further correlation function of interest that has been derived is the galaxy-cluster cross correlation function $\xi_{g c}(r)$ (Seldner \& Peebles 1977; Lilje \& Efstathiou 1988). This is also found to be positive on scales $\gtrsim 50 h^{-1}$ $\mathrm{Mpc}$.

The surprising clustering signals derived from a statistical analysis of the Abell cluster catalogue, led to a detailed investigation of possible systematic biases in the catalogue by Sutherland (1988) and Dekel et al. (1989). In particular, Sutherland finds large anisotropies in the redshift space correlation function which he interprets as evidence for spurious line-of-sight clustering. After correction for these anisotropies a much reduced length of $14 h^{-1} \mathrm{Mpc}$ is obtained, with no evidence for true spatial correlations beyond about $40 h^{-1} \mathrm{Mpc}$. In a few years time, reliable cluster catalogues should become available for a wide range of statistical studies. These will be compiled in an automated way from machine-based surveys of galaxies (Heydon-Dumbleton et al. 1989; Sutherland et al. 1988). Fig. 1.2 gives an example of what has already been achieved here. It is a greyscale map of the distribution of galaxies on a mosaic of Schmidt plates which have been scanned by the microdensitometer machine, COSMOS, at the Royal Observatory Edinburgh for the Edinburgh-Durham Southern Galaxy Catalogue (EDSGC). Two conspicuous largescale structures are evident as density contrasts on the bottom left half of the map. Their reality has been confirmed by redshift surveys of galaxies in these regions (Q.A. Parker, private communication). In addition to machine-based surveys, the X-ray satellite ROSAT will provide an all-sky cluster catalogue free from projection contamination. Chapter 5 describes how radio galaxies are also unbiased pointers towards rich systems, on average Abell $R \simeq 0$, in the universe and so this class of object can also be used as a check on the clustering results that have been obtained from the optically-selected rich cluster catalogues. 


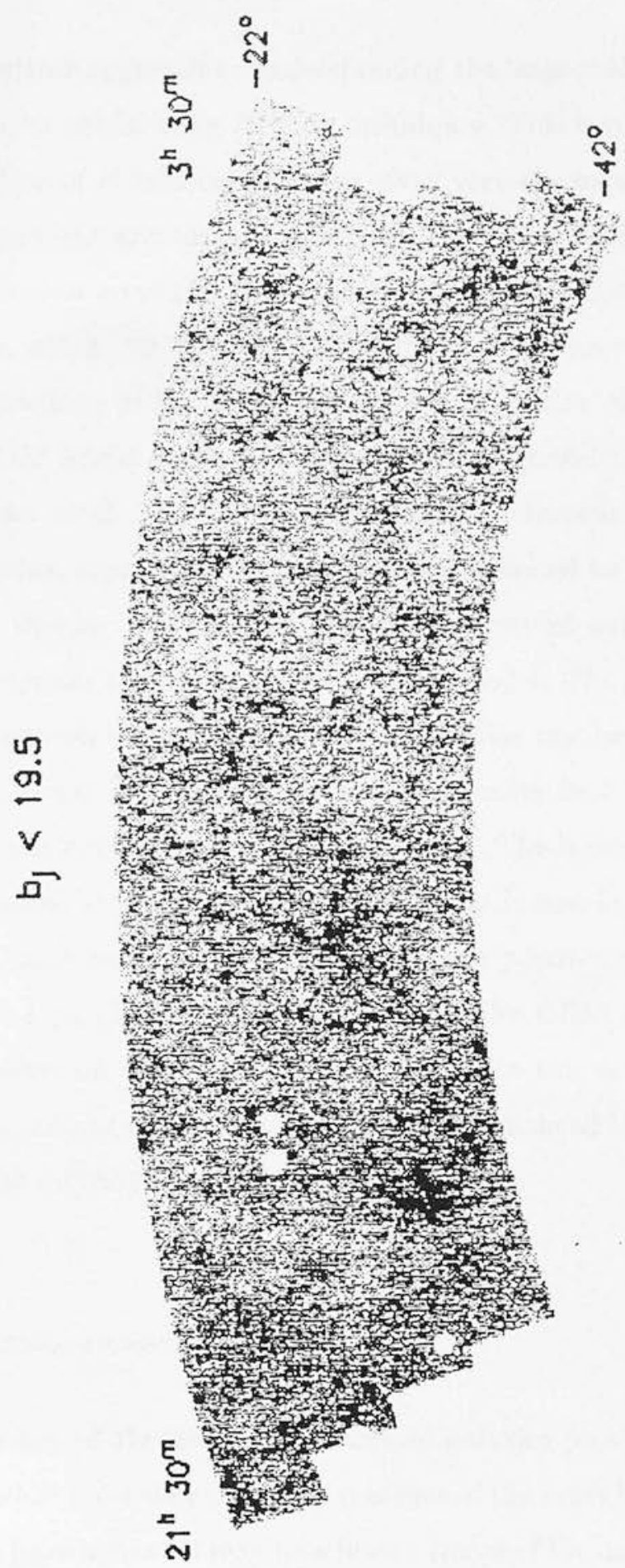

Fig. 1.2: Grey-scale map of the distribution of galaxies identified by the microdensitometer machine, COSMOS, from systematic scans of Schmidt plates. The map shown here is for a mosaic of such plates and was kindly provided by R.C Nichol. 
The theoretical approach to understanding the large-scale structure has been to analytically study its origin using $N$-body techniques. This involves numerically solving for the time evolution of $N$ massive points at given vectorial locations $\mathrm{r}(t)$ inside a sphere of radius $R(t)$ expanding according to Einstein's equations. The initial positions are chosen such that they reflect a random fluctuation field with variance $P(k)$. The main outputs of the simulations which can be used directly to confront observation are correlation functions and morphology of the large scale mass distribution as well as details of velocity fields. The HDM initial power spectrum has been considered by Klypin \& Shandarin (1983) and Frenk et al. (1983). They find that the clustering scale for galaxies exceeds the observed value, especially if the galaxies are assumed to form only in the regions of dense pancake shocks. Davis et al. (1985) have carried out simulations with a power spectrum appropriate to the the adiabatic CDM model. The observed galaxy correlation functions and pairwise peculiar velocities of galaxies can be reproduced if galaxies are assumed to form only in the peaks of the mass density field. This point is addressed in more detail in the next section and in Chapter 7. The biased CDM simulations are remarkably successful at producing the structure that is seen in redshift surveys, i.e. voids, filaments etc. Some redshift surveys however show positive galaxy correlations on large scales $\gtrsim 50 h^{-1} \mathrm{Mpc}$ (Broadhurst et al. 1990). The CDM model does apparently not have enough power on very large scales to reproduce this observation. Observations of the cluster-galaxy cross-correlation function and the Bahcall \& Soneira cluster correlation function are also difficult to accommodate in CDM.

\subsubsection{Streaming motions}

Theoretical studies of the peculiar motions of galaxies provide a much cleaner test of cosmogonic models since they provide a measure of the mass fluctuations rather than the light, which we have indicated may be a biased tracer of the underlying mass distribution. The interesting, and often rather controversial history, of streaming flow work has been reviewed by Davis \& Peebles (1983a), Gunn (1988) and Burstein (1990). Much of the recent theoretical effort has attempted to match the observational data acquired by a consortium of astronomers referred to as the 'Gang of Seven' ( e.g. Lynden-Bell et al. 1988). These comprise peculiar velocities for an all-sky sample of elliptical galaxies selected from 
a survey volume of about $6000 \mathrm{kms}^{-1}$ in radius. Initial attempts to model the peculiar velocity field as a bulk flow, roughly parallel to the CBR dipole have been superseded by a more elaborate multi-parameter model that is dominated by a spherical infall into a "Great Attractor" centred at a distance of $4200 \mathrm{kms}^{-1}$ and just north of the galactic zone of avoidance at the vicinity of the Hydra-Centaurus supercluster. The theoretical approach to modelling observations of streaming flows has been to consider a convolution of the density perturbation responsible for the peculiar velocity, with a window function reflecting the volume and geometry of the space that is surveyed (Kaiser 1988c). Thus for the simple (but unrealistic) case of a Gaussian window function, the peculiar velocities are predicted in linear theory to be distributed according to the Maxwellian law, with a dispersion

$$
\sigma_{v}^{2}\left(R_{*}\right)=H_{0}^{2} \Omega^{1.2} \int_{0}^{\infty} P(k) \exp \left(-k^{2} R_{*}^{2}\right) d k / 2 \pi^{2}
$$

Bertschinger \& Juskiewicz (1988) have tested several scenarios for the growth of cosmic structure against the observations mentioned above. In particular they find the standard CDM predictions fail by a large margin to reproduce the large-scale flows. Kaiser \& Lahav (1989) have argued that CDM may only be resurrected if the streaming velocities have been overestimated by a factor $\sim 2$ and the level of biasing is smaller than its standard value. New observations of the large-scale peculiar velocity field are presented in Chapter 6. In Chapter 7 the issue of whether the observed peculiar velocities might be partly driven by systematic offsets in the galaxy distance indicator relations is addressed.

\subsubsection{The epoch of galaxy formation}

Recent leaps in technological development have meant that the universe at redshifts $z=$ $2 \rightarrow 5$ can now be observed. The status of present observations however appears to be rather unclear (see Peebles 1989 for a review). On the one hand, there is evidence for substantial star-forming activity even at the moderate redshifts, $z \lesssim 3$. The inferred properties of gas clouds along the line-of-sight to quasars and galaxy counts to very faint magnitudes in various passbands both argue in favour of a fairly recent formation epoch. There have been conflicting observations however which suggest that at least some galaxies at high redshifts are very similar to galaxies observed today, evidence which conversely argues for late formation. In the standard CDM picture, galaxy formation is a recent 
and protracted process which continues on until the present day. If the luminosity of oldlooking objects at high redshifts (e.g. Chambers, Miley \& van Breugel 1987; Lilly 1988) is dominated by a stellar population $\sim 1 \mathrm{Gyr}$ old, it puts the formation of the stars at a redshift $z \geq 5$. The epoch of galaxy formation, if it is unequivocally observed, may thus be one of the most clear-cut discriminators between the competing theories for structure formation.

\subsection{Galaxy Formation}

Our discussions of galaxy formation in this thesis will be mainly restricted to the influence of environmental effects and the implications of spatially modulated luminosity-indicator relations on the interpretation of streaming velocities. A direct indication that environment has an important role to play in determining the global shapes and characteristics of galaxies is the observation that ellipticals generally occur in dense environments whereas their spiral counterparts are most prevalent in the field (Davis \& Geller 1976). Our present understanding of galaxy formation is only poor but it is generally thought to consist of a series of dissipative and merging processes, most of which can be modulated by the largescale environment (e.g. Silk \& Norman 1981; Silk 1987). Some of the relevant processes may include: mergers and violent relaxation; gradual infall and formation of disks; dark halo stripping; biasing of star formation; cooling flows. The local environment affects not only the supply of galaxy-building material, but also the dynamical structure of the end products (e.g. the velocity anisotropies of ellipticals are dynamical relics of past mergers), the primordial initial mass function (IMF) and thus the stellar $(M / L)$, the ratio of the dark and visible matter, etc. Large-scale variations in $(M / L)$ are of primary interest because they are naturally expected in many, and perhaps all, scenarios of large-scale structure and galaxy formation (Hoffman et al. 1982; Silk 1988). Such variations are indeed one of the cornerstones of the whole idea of biased galaxy formation (Dekel \& Rees 1987). The problem with biasing is that it is difficult to find a good physical argument that can naturally give rise to the requisite amount of mass-light segregation. A threshold model has been proposed by Davis et al. (1985) in which galaxies form at high peaks in the smoothed primordial density field. The difficulty then is understanding how galaxy formation could be impeded below the threshold. This question is discussed in greater 
detail in Chapter 7.

\subsubsection{RADIO GALAXY FORMATION}

The formation history of radio galaxies is a particularly intriguing subject since it begs the question as to what physical process is responsible for the onset of non-thermal nuclear activity. A systematic study of the cluster environment of radio galaxies might yield some clues, and this approach to the problem was first taken by Longair \& Seldner (1979) and later by Prestage \& Peacock (1988). Radio galaxies are roughly divisible into three types on the basis of their morphological structure and radio spectra. The classification scheme introduced by Fanaroff \& Riley (1974) will be used throughout this thesis. In this scheme, the diagnostic is linear separation between regions of highest radio luminosity on opposite sides of the central galaxy compared to the total extent of the source measured from the lowest contour. If the ratio is $>0.5$ then the source is denoted FRII, otherwise FRI. A compact radio source is one dominated by a $\leq 1 \operatorname{arcsec}$ core (e.g. Laing, Riley \& Longair 1983). Amongst the FRII sources there is often a sub-division into 'good-', 'doubtful-' and 'non-classical' doubles which effectively refers to a progression of dominance of compact hotspots at the outer edge of the radio structure.

As a result of their study, Longair \& Seldner concluded that FRI radio galaxies preferentially inhabit denser regions of the universe than FRIIs. They attempted to explain this in terms of the 'beam' or collimated flow of relativistic material material being disrupted in cluster radio galaxies due to motion relative to the cluster center of mass. Although a more careful analysis by Prestage \& Peacock confirmed this observation, they also noted that there appeared to be a significant scatter in properties, implying that cluster strength is not the sole factor governing structure. In addition they found no statistical evidence for environmental differences between the sub-classes of FRII radio galaxies which had originally been claimed by Longair \& Seldner.

There have also been reports that the optical properties of low-redshift radio galaxies are correlated with their cluster environment (Lilly \& Prestage 1987). This point should not be taken too lightly since, if correct, it may introduce an undesirable systematic effect into the application of the radio galaxies as standard candles. It is discussed further in Chapter 4. 


\subsection{Thesis Outline}

A guide to the content of the remaining seven Chapters is given here. Chapter 2 describes the compilation of an all-sky sample of radio elliptical galaxies, emphasising the motivations for choosing a particular set of selection criteria and describing how well these can be met in practice. The observational material and data reduction techniques are assembled in Chapter 3 - this Chapter falls into two parts dealing with the CCD photometry and spectroscopy in turn. Chapter 4 is essentially a precursor to the next two main results Chapters. It forms a study of the optical properties of low-redshift radio ellipticals and examines whether these are correlated with their local environment. Chapter 5 presents the first ever published calculation of the 3 - $\mathrm{d}$ correlation function for radio galaxies. Checks for a systematic dependence of clustering strength on radio luminosity are performed. A search for evidence of very large-scale density inhomogeneities in the universe is described. In Chapter 6, the peculiar velocities for a subsample of the radio galaxies are derived and analysed for a streaming dipole. A different analysis method, using the velocity autocorrelation tensor, is also described. Chapter 7 is concerned with models for biased galaxy formation, in particular autonomously biased gravitational collapse. A variety of observational tests are performed to search for evidence of its operation by studying the properties of nearby elliptical galaxies. Finally, Chapter 8 collates the main results and points out some promising directions for future research. 


\section{Chapter 2}

\section{An All-Sky Sample of Nearby Radio Galaxies}

\section{$2.1 \quad$ Introduction}

This Chapter describes the compilation of a powerful, new, all-sky sample of low-redshift radio galaxies. These objects are assembled from the Parkes, Bologna, Jodrell Bank and Bonn surveys and approximate a complete sample down to $0.5 \mathrm{Jy}$ at $1.4 \mathrm{GHz}$. Redshift cutoffs at $z=0.01$ and $z=0.1$ are imposed upon the object selection not only for practical reasons but for physical reasons also - in subsequent Chapters we shall employ the sample to test claims for density inhomogeneities in the universe on $10-100 h^{-1} \mathrm{Mpc}$ scales. These applications demand an optically homogeneous selection of near standard candles which are uniformly distributed across the sky. As we shall see in this and the following Chapters, the all-sky sample matches this description very well

The layout of this Chapter is as follows. Section 2.2 explains how the proposed applications of the sample set design constraints upon its assembly. In Section 2.3 the compilation of the sample is described and deals in turn with each of the radio and optical selection criteria, describing how well these can be met in practice. The all-sky sample database is included as an Appendix. Some information pertinent to the format of the data tables in this Appendix is provided in Section 2.4. Finally, Section 2.5 includes the optical data acquired in the next Chapter to examine a few properties of the sample. These include its completeness, sky coverage, redshift distribution and radio power histograms. A short summary is provided in Section 2.6.

\subsection{Sample Selection Criteria}

Firstly, an upper redshift limit needs to be imposed on the object selection. Given that the sample will be used to set limits on the large-scale clustering and dynamics of matter in the 
universe, a brief survey of existing observations is necessary. Many of these observations argue in favour of very large-scale density inhomogeneities. Bahcall \& Soneira (1983) report a two-point correlation function for Abell clusters which remains positive out to $100 h^{-1} \mathrm{Mpc}$. Chapter 5 will have more to say on this issue but for the moment let us suppose that it is correct. The most extreme claims for large-scale structure have been made by Tully (1986) who finds correlated structure in the distribution of rich clusters even on scales as large as $0.1 c$. In view of these observations, an upper redshift limit of $z=0.1$ was deemed large enough to probably contain a fair sample of the universe. In fact there are practical reasons for not wanting to push out any further than $z=0.1$. Surface photometry becomes rather difficult to interpret at larger redshifts since one is then often dealing with small angular sizes of the order of only a few times a typical seeing disk. Also, surface brightness dims very rapidly with $z$ according to $(1+z)^{-4}$.

In addition to the high redshift cutoff, a low redshift cut-off is also required and again this is dictated to some extent by the constraints imposed by CCD surface photometry. The limiting size of these detectors mean that a very low-redshift galaxy would essentially 'fill' the frame and some of its halo light would be lost at the edges. This would also pose additional problems for trying to measure a background sky estimate. Moreover at low redshift, a few galaxies are lost from the radio surveys through over-resolution. In view of these points a low-redshift cutoff of $z=0.01$ was applied. In total then the distance range of the sample spans only a factor of ten in redshift. This has the advantage of being narrow enough to ensure that a reasonably homogeneous sample of objects are selected in terms of both their optical and radio properties.

Due to the presence of large-scale gradients in dust extinction towards the galactic plane which could easily bias a photometric study of the radio galaxies, it was decided to avoid this region completely. A simple selection constraint of $|b|>15^{\circ}$ was taken rather than to consider a survey volume of complicated shape.

In summary then, the requirements are for a complete sample of radio galaxies filling the redshift shell from $0.01<z<0.1$, over the 9.3 steradians of sky which avoid the galactic plane, down to some uniform flux-density limit. The next Section describes the radio surveys at our disposal for assembling the sample and considers how well these selection criteria can be met in practice. 


\subsection{Compilation of the Sample}

There are two independent features of the sample selection to be considered. Firstly, a uniform radio selection of sources from the surveys down to a desired flux-density limit and secondly the optical selection of sources such that redshift constraints on the sample are closely matched. Each of these are discussed in turn below.

\subsubsection{RADIO SELECTION}

The whole sky away from the galactic plane is covered at radio wavelengths by one of four surveys of roughly equivalent depth. These are tabulated below together with some specific details.

Table 2.1: Radio surveys from which the all-sky sample has been compiled.

\begin{tabular}{|c|c|c|c|}
\hline Survey & Frequency & Sky coverage & Reference \\
\hline Parkes & $2.7 \mathrm{GHz}$ & $\delta \lesssim 24^{\circ}$ & Bolton et al. (1979) \\
\hline Bologna B2 & $408 \mathrm{MHz}$ & $24^{\circ} \lesssim \delta \lesssim 40^{\circ}$ & Grueff \& Vigotti $(1972,1973,1979)$ \\
\hline Jodrell Bank & $966 \mathrm{MHz}$ & $40^{\circ} \lesssim \delta \lesssim 70^{\circ}$ & Cohen et al. (1977) \\
\hline Bonn S5 & $5 \mathrm{GHz}$ & $\delta \gtrsim 70^{\circ}$ & Kühr et al $(1981,1987)$ \\
\hline
\end{tabular}

In the next Section it is shown that an upper redshift limit of 0.1 corresponds to an approximate value of apparent blue magnitude of $B \simeq 17$. As this is relatively bright it might be expected that a high percentage of the radio sources which would fall into the final sample have had an identified optical counterpart. In fact all of the surveys listed above have had extensive optical identification programmes based on radio positions, which carry a typical uncertainty of $\lesssim 10 \mathrm{arcsec}$. In addition, galaxies well above the limit of the sky survey should have been noted as candidate identifications if present. Clearly then these four surveys provide an excellent basis for selecting an all-sky sample complete to the desired distance limit of $z=0.1$. A few relevant details are given below for each of the four surveys in turn. 


\subsubsection{The Parkes Survey}

The various parts of the Parkes 2.7-GHz survey have been published in a series of papers presented in the Astrophysical supplement of the Australian Journal of Physics (e.g. Bolton, Wright \& Savage 1979; Savage \& Bolton 1979 and references therein). The Parkes radio telescope is a $64 \mathrm{~m}$ dish which yields a half power beam-width of $8 \mathrm{arcmin}$. at 2.7 GHz. A pointing model for the telescope has been described by Savage (1976) and the positional accuracies for each observed source are about 10 arcseconds in each coordinate. At galactic latitudes of $|b|>15^{\circ}$, almost all regions at $\delta<4^{\circ}$ have been surveyed to a $2.7 \mathrm{GHz}$ flux-density limit of $0.35 \mathrm{Jy}$ or deeper. The only exceptions are a few regions where the closest approach to the galactic plane is further than $15^{\circ}$, the worst affected point being at $\alpha=17^{h}, \delta=-4^{\circ},|b|=21.9^{\circ}$. These few regions however total much less than 1 percent of the survey area and thus may be safely ignored. At $\delta>4^{\circ}$ the situation is a little more complicated, the direct $2.7 \mathrm{GHz}$ survey being complete to a depth of only $0.6 \mathrm{Jy}$ in this region. The zone covering a range of declination from $4^{\circ} \rightarrow 20^{\circ}$, however, has been surveyed down to $2.5 \mathrm{Jy}$ at $408 \mathrm{MHz}$ by Day et al. (1964). All of the objects found were then subsequently remeasured at a frequency of $2.7 \mathrm{GHz}$. In addition, all of the sources listed in the $4 \mathrm{C}$ catalogue that lie in this zone down to $3 \mathrm{Jy}$ at $178 \mathrm{MHz}$ were observed at $2.7 \mathrm{GHz}$ by Wills \& Bolton (1969). For a spectral index of $\alpha=0.8$ (defined in the sense $S_{\nu} \propto \nu^{-\alpha}$ ), these searches should thus be complete to respectively $0.55 \mathrm{Jy}$ and $0.34 \mathrm{Jy}$ at $2.7 \mathrm{GHz}$. Finally, the zone $20^{\circ}<\delta<27^{\circ}$ was surveyed to $1.5 \mathrm{Jy}$ at $635 \mathrm{MHz}$ by Shimmins \& Day (1968), again with a $2.7 \mathrm{GHz}$ follow-up, at which frequency the equivalent flux-density limit is $0.47 \mathrm{Jy}$.

In summary, the Parkes survey is complete down to $0.35 \mathrm{Jy}$ at $\delta<24^{\circ}$. This is almost ensured by the rather flat flux density distribution for radio galaxies in $0.01<z<0.1$. The Parkes staff maintain a composite catalogue of the combined data from all surveys which is updated regularly as new or improved observations become available. A machine-readable version of this catalogue was kindly provided by Dr. Alan Wright. Lists of radio and optical positions for each source are given together with an optical magnitude. These are generally "photographic" magnitudes estimated from Palomar Sky Survey (PSS) prints. They are taken to be equivalent to $B$ within the accuracy of the eyeball estimates. 


\subsubsection{The Bologna B2 Survey}

The B2 survey is a compilation of 3225 radio sources observed at $408 \mathrm{MHz}$ with the Bologna Northern Cross telescope. This instrument has been described in detail by Braccesi et al. (1969) and has a resolving power of $3^{\prime}$ in right ascension and $10^{\prime}$ in declination. The survey covers a declination band $24^{\circ}<\delta<40.3^{\circ}$ down to a limiting flux-density of $0.25 \mathrm{Jy}$. Radio source positional accuracies have been quoted as $12^{\prime \prime}$ and $40^{\prime \prime}$ in right ascension and declination respectively. Careful optical identification programmes have been carried out for sources brighter than $0.9 \mathrm{Jy}$, the details of which are presented in three papers by Grueff \& Vigotti $(1972,1973,1979)$. These cover a region which again is close to but not exactly equal to $|b|>15^{\circ}$. The worst omission is from an area around $\alpha=22^{h}, \delta=30^{\circ}$ equal to about 0.2 steradians.

The optical magnitudes given by Grueff \& Vigotti (1979) approximate the Johnson $R$ system. These were converted to a nominal $B$ magnitude using a zero-redshift colour for ellipticals of $(B-R)_{J}=1.9$ (Bruzual 1983).

\subsubsection{The Jodrell Bank Survey}

The $966 \mathrm{MHz}$ Jodrell Bank survey has been constructed from observations made with the Mark $1 \mathrm{~A}$ telescope and covers $40.3^{\circ}<\delta<71^{\circ}$ to a limiting flux of $0.7 \mathrm{Jy}$. This telescope can achieve a resolution of $18 \mathrm{arcmin}$. half power beam-width. Accurate radio positions $\left( \pm 2^{\prime \prime}\right)$ were measured for the compact sources using the $\mathrm{Mk} 1 \mathrm{~A}$ and $\mathrm{MkII}$ telescopes as an interferometer with a baseline of 425.20 metres. Optical identification of these sources has been reported by Cohen et al. (1977). The more extended sources were identified by Porcas et al. (1980). Palomar Sky Survey prints were examined in order to estimate a rough $B$ magnitude.

A feature of the Jodrell Bank survey is the existence of many sources which were identified with a candidate cluster only. An attempt was made to try and identify the cluster source by cross-checking the cluster locations with all other radio survey identification data which have conveniently been collected in a catalogue published by Véron-Cetty \& Véron (1983). This left 25 cluster sources still without an optical identification. Rather than resort to the original PSS prints and probabilistic arguments, the positions of the 
radio sources were cross-checked with the positions of clusters in the Abell cluster catalogue (Abell, Corwin \& Olowin 1989) to try and pin down the parent cluster of the radio source at least. Table 2.2 below lists 10 sources for which a cluster centroid lies within $1^{\circ}$. Radio maps for the sources are really needed however before their optical counterparts can be identified with any degree of certainty.

Table 2.2: $\quad$ Source-cluster angular associations

\begin{tabular}{|cccccc|}
\hline source & $\Delta \theta\left(^{\prime}\right)$ & cluster & source & $\Delta \theta\left(^{\prime}\right)$ & cluster \\
\hline $0647+693$ & 7.75 & A562 & $0804+499$ & 43.67 & A626 \\
$0847+491$ & 55.59 & A716 & $1013+596$ & 7.06 & A959 \\
$1108+411$ & 1.56 & A1190 & $1230+486$ & 51.59 & A550 \\
$1303+684$ & 39.79 & A 1674 & $1325+553$ & 33.01 & A1734 \\
$1559+538$ & 17.77 & A2149 & $1614+473$ & 53.16 & A2180 \\
\hline
\end{tabular}

\subsubsection{The Bonn Survey}

A series of $5 \mathrm{GHz}$ surveys covering most of the Northern sky were carried out in the $1970 \mathrm{~s}$ at Greenbank and Bonn. The deepest of these was the Bonn S5 survey undertaken with a $100 \mathrm{~m}$ telescope operating at a frequency of $4.9 \mathrm{GHz}$ with a half power beam-width of 3 arcmin. (Kühr et al. 1981). This survey covered $\delta>70^{\circ}$ to a completeness limit of $0.25 \mathrm{Jy}$. Reliable optical identifications have been achieved using VLA maps (Kühr et al. 1987). Optical magnitudes were again estimated by eye from the PSS prints.

\subsubsection{Additional Sources}

Even at $z>0.01$ there are a few radio sources of such large angular size ( $\gtrsim 10 \mathrm{arcmin}$.) that they are not easily resolved as a single object. Some of these have been noted and added to the sample where known: $0503-286$ (Subrahmanya \& Hunstead 1986); $0744+559$ (Willis, Strom \& Wilson 1974); 0945 + 734 (Mayer 1979); $1029+569$ (C.R. Masson 1979); $1637+826$ (Wagget, Warner \& Baldwin 1977). There are doubtless other of these 'giant' sources, particularly in the Southern Hemisphere, but such objects must constitute a tiny incompleteness in the total sample. 


\subsubsection{Flux-Density Limits}

For an 'average' frequency of $1.4 \mathrm{GHz}$ and taking a spectral index of 0.8 to be representative, the above flux-density limits correspond to the following figures: 0.59 Jy (Parkes); 0.33 Jy (Bologna); 0.52 Jy (Jodrell Bank); 0.69 Jy (Bonn). These are all of a similar depth with the exception of Bologna. This was accordingly cut back to a limit of $1.2 \mathrm{Jy}$ at $408 \mathrm{MHz}$, which is equivalent to $0.44 \mathrm{Jy}$ at $1.4 \mathrm{GHz}$. The all-sky sample therefore approximates a complete sample down to $0.5 \mathrm{Jy}$ at $1.4 \mathrm{GHz}$.

\subsubsection{OPTICAL SELECTION}

In order to check the implied optical limits on the sample arising from the imposed redshiftcutoffs, a least squares fit of estimated $B$ magnitude against redshift was performed on 125 radio galaxies which had been extracted from the survey catalogues and which were in possession of a spectroscopic redshift. The Hubble diagram is shown in Fig. 2.1 and the following equation was derived

$$
\log _{10}(z / 0.1)=0.2(B-17)
$$

with an rms scatter of about 1 magnitude.

Thus to within a magnitude the upper redshift cut-off of $z=0.1$ corresponds to selecting objects brighter than $B=17$. In fact a more conservative optical limit of $B=19$ was finally used in order to ensure a reasonable completeness. This is important-preferentially including only the brightest galaxies in a sample as one looks out to greater distances introduces the well-known Malmquist bias (Malmquist 1920). As we shall note in Chapter 6 , presence of this bias in optically selected samples can greatly complicate the analysis of galaxy peculiar velocities. Applying this optical limit to the initial sample resulted in a further 454 candidate sample members to add to the 125 objects which were already known a priori to satisfy the redshift criteria.

In summary then, the idealised set of selection criteria on which the final sample is based are the following:

- $|b|>15^{\circ}$ 


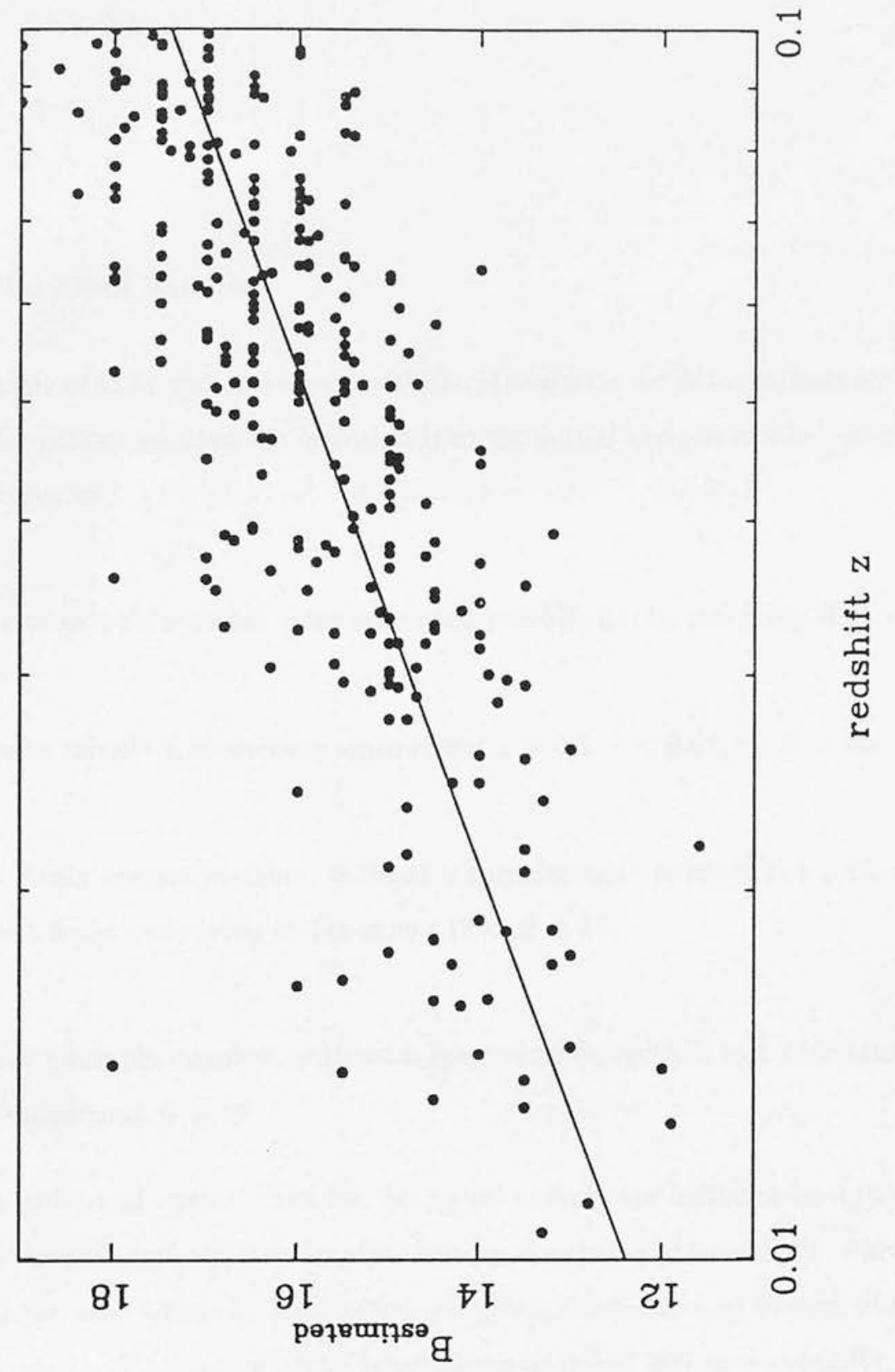

Fig. 2.1: Hubble diagram for the sample. New redshifts from Chapter 3 are included. A conservative optical limit of $B=19$ was used as the criterion for selecting galaxies from the surveys that did not have a published redshift. The line shown is a least-mean squares fit to the points. 
- $S_{\nu}>0.5 \mathrm{Jy}$ at $1.4 \mathrm{GHz}$

- $0.01<z<0.1$

- $B<19$

\subsection{The Final Sample}

On the basis of their spectroscopic redshifts, if available, or $B$ magnitude estimates if not, each radio galaxy selected for inclusion into the initial sample can be assigned to one of three categories

(i) definite sample member since measured redshift lies in the range $0.01<z<0.1$;

(ii) definite sample non-member since either $z>0.1, z<0.01$, or $B>19$;

(iii) very likely sample member, without a spectroscopic redshift but with estimated apparent magnitude lying in the range $12<B<17$;

(iv) unlikely sample member, without a spectroscopic redshift but with estimated apparent magnitude $B>17$.

The radio and optical data for the initial sample are included in Appendix A. This includes the new optical data, acquired during the course of this thesis, which will be discussed in the next Chapter. Four tables are given, corresponding to each of the categories outlined above. The spectroscopy programme acquired 300 new redshifts, boosting the redshift completeness of the sample to about $90 \%$.

\subsection{Selected Properties of the Sample}

This Section examines the sky, redshift and flux-density distributions of the sample and 
uses the $\mathrm{V} / \mathrm{V}_{\max }$ test to estimate its completeness.

\subsubsection{SKY COVERAGE}

Fig. 2.2 plots, in Aitoff projection, the sky distribution of the radio galaxies - filled circles and unfilled circles represent galaxies with and without a measured redshift respectively.

A simple measure of sky coverage anisotropy is the vector $\mathbf{R}$, which has a magnitude given by

$$
|\mathbf{R}|=\sqrt{\langle l\rangle^{2}+\langle m\rangle^{2}+\langle n\rangle^{2}}
$$

where $l, m$ and $n$ are the individual galaxy directional cosines. This is an elemental indicator of anisotropy which is sensitive to north-south distribution anomalies but not to the lack of galaxies in the zone of galactic obscuration. $|\mathbf{R}|=0$ implies a perfectly uniform sky coverage. The radio galaxy sample has an amplitude of $|\mathbf{R}|=0.18$, a small contribution of which is probably due to the natural clustering of galaxies. Although the composite sample is reasonably uniform it may well be that there is a small declinationdependent systematic incompleteness. This is shown to be a rather small effect however later in this Chapter.

\subsubsection{REDSHIFT DISTRIBUTIONS}

The local radio luminosity function (LRLF) is the comoving number of radio sources per unit volume as a function of the luminosity $P$ at a frequency $\nu$ and $\operatorname{cosmic}$ epoch $z=0$. It can be calculated from either the data compiled on a radio-optically complete sample of identified sources, or using the radio observations of an optically selected sample. Like the Schechter function for the optical luminosity function, the LRLF also has a characteristic break at some critical power $P^{*}$. The LRLF has been derived for elliptical galaxies at 1.4 $\mathrm{GHz}$ by Auriemma et al. (1977) and Windhorst et al. (1984). For an assumed parametric form

$$
\rho / h^{3} \mathrm{Gpc}^{-3}\left(\Delta \log _{10} P\right)^{-1}=\phi^{*}\left(1+\frac{P^{*}}{P}\right)^{-1}
$$

their results may be fitted by

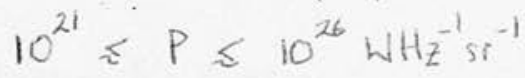

$$
\left(\phi^{*}, P^{*}\right)= \begin{cases}10^{4.3}, 22.7 & \text { Windhorst et al.; over the limiting } \\ 10^{3.8}, 23.2 & \text { Auriemma et al. range of radio power } \\ \text { indicated above }\end{cases}
$$




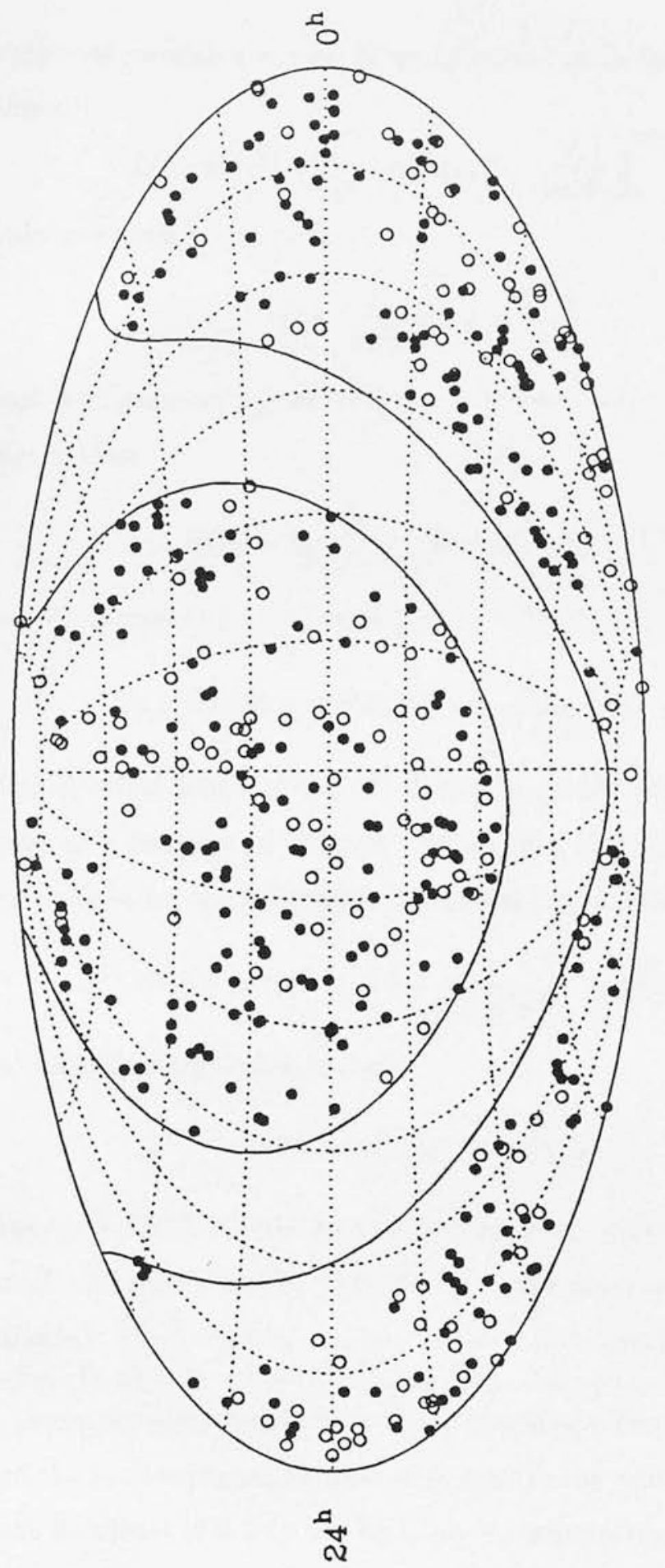

Fig. 2.2: Sky distribution of the sample. Filled circles denote objects with a spectroscopic redshift. Unfilled circles are objects without a redshift - these are very likely to have $z>0.1$ and thus to fall outside of the sample. 
The total number of comoving sources at an epoch $z$ can be found by integrating over the LRLF as follows

$$
N(>P(z))=\int_{P}^{\infty} \rho d \log _{10} P=\frac{1}{\ln 10} \int_{P}^{\infty} \rho \frac{d P}{P}
$$

which is simple to evaluate and gives

$$
N=\frac{\phi^{*}}{\ln 10} \ln \left(1+\frac{P^{*}}{P}\right)
$$

We shall adopt a Friedmann model with $H_{0}=50 \mathrm{kms}^{-1} \mathrm{Mpc}^{-1}$ and $\Omega_{0}=1$. The luminosity distance is then

$$
D(z)=\frac{2 c}{H_{0}(1+z)}[z-(\sqrt{1+z}-1)]
$$

and thus the radio power computed in $\mathrm{WHz}^{-1} \mathrm{sr}^{-1}$ by $S_{\nu} D^{2}(1+z)^{1+\alpha}$ reduces to

$$
P_{\nu}=3.4276 \times 10^{26} S_{\nu}\left(2-\frac{2}{\sqrt{1+z}}\right)^{2}(1+z)^{1.8}
$$

for an assumed spectral index of 0.8. A further quantity we require is the differential volume element as a function of redshift. Given that the all-sky sample covers a solid angle of $9.314 \mathrm{sr}$, then for the adopted world model this is evaluated by

$$
d V(z)=2012 \times\left(2-\frac{1}{\sqrt{1+z}}\right)^{2} \frac{d z}{(1+z)^{3 / 2}} \quad G_{p} c^{3}
$$

The predicted redshift distribution is then

$$
d n(z)=d V(z) \cdot N(>P(z))
$$

Theoretical curves for RLF's with the model parameters given by Auriemma et al. and Windhorst et al. are shown in Fig. 2.3 on top of the observed redshift distribution for the all-sky sample. The curves have been calculated by performing the integral in Eqn. (2.4) over limits ranging from $P=10^{21} \mathrm{wHz}^{-1} \mathrm{sr}^{-1}$ to the source luminosity, $P$ As expected, incompleteness sets in at $z \simeq 0.1$, reaching a factor of about 2 by $z=0.15$. An estimate of the total expected number of sources in the redshift shell from $z=0.01 \rightarrow$ 0.1 down to the flux limit of $0.5 \mathrm{Jy}$ can be made by integrating under these curves. Thus

$$
N=\iint \rho d V d \log P
$$

This can be solved analytically and for the Auriemma et al. and Windhorst et al. set of parameters respectively gives $N=305$ and $N=349$. These compare with a total number of expected all-sky sample members within this redshift range of 359 . 


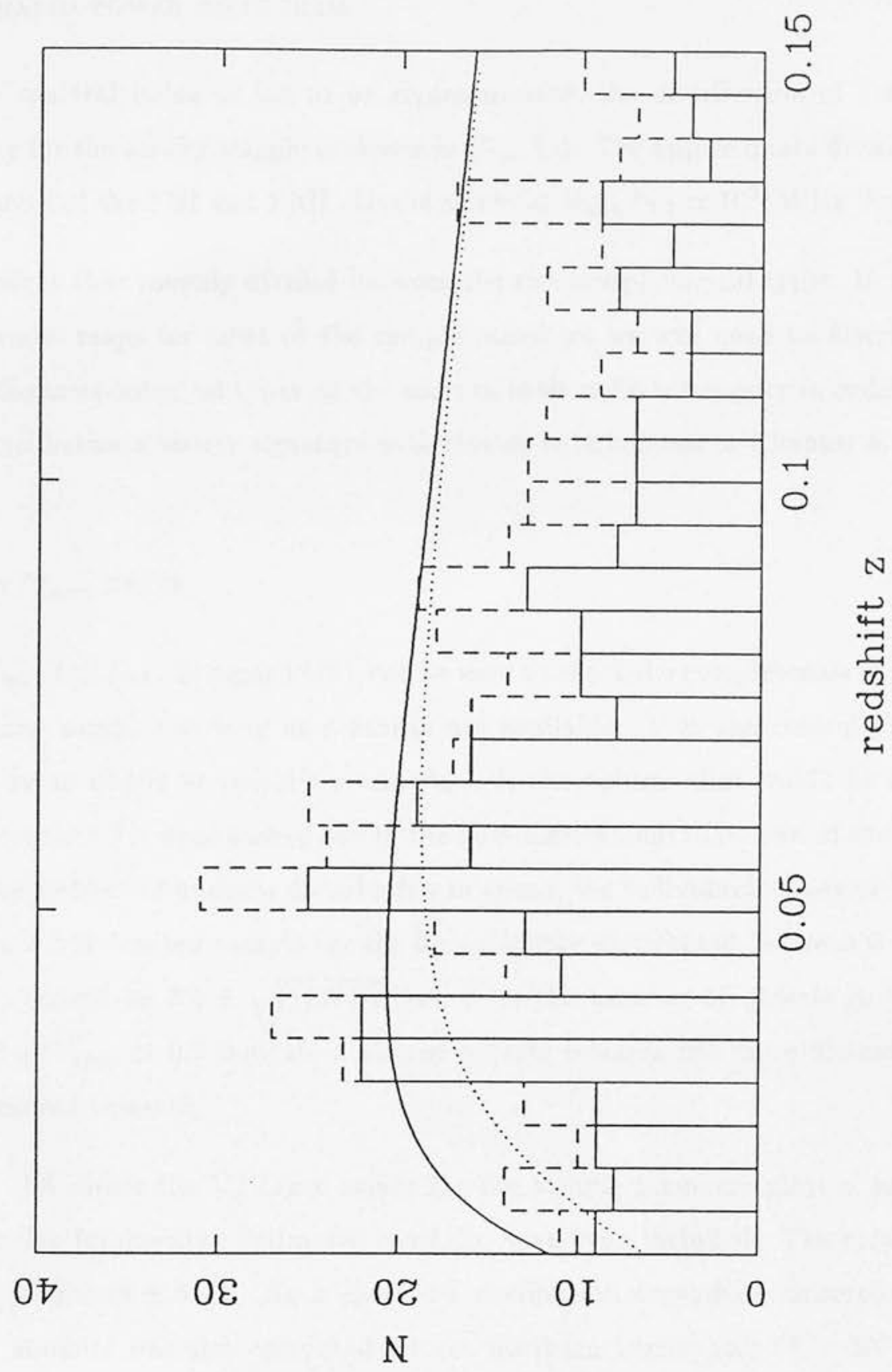

Fig. 2.3: Redshift distribution of the sample (solid histogram) with an estimate of the expected distribution of the remaining candidates (dashed line histogram). The lines mark the expected distribution for a uniform flux-density limit of $0.5 \mathrm{Jy}$ at $1.4 \mathrm{GHz}$ according to the luminosity functions of Auriemma et al. (1977) and Windhorst et al. (1984). 


\subsubsection{RADIO POWER HISTOGRAM}

Taking a spectral index of 0.8 to be representative, the distribution of $1.4 \mathrm{GHz}$ radio luminosity for the all-sky sample is shown in Fig. 2.4. The approximate division between radio sources of the FRI and FRII class is at about $\log _{10} P_{2.7} \simeq 10^{24} \mathrm{WHz}^{-1} \mathrm{sr}^{-1}$.

The sample is thus roughly divided between the two morphological types. In the absence of good radio maps for most of the sample members we will need to discriminate between radio morphological types on the basis of their radio luminosity in order to test for correlations between source structure with cluster environment in Chapter 4.

\subsection{4 $\mathrm{v} / \mathrm{v}_{\max }$ TESTS}

The $\mathrm{V} / \mathrm{V}_{\max }$ test (e.g. Longair 1978), can be used to check the completeness of flux-limited radio galaxy samples so long as redshifts are available. $\mathrm{V}$ is the cosmological volume enclosed by an object at redshift $z$ and $\mathrm{V}_{\max }$ is the volume that would be enclosed by that same object if it were pushed out to the flux-density limit of its parent sample. Under the null hypothesis of uniform distribution in space, the individual values of $\mathrm{V} / \mathrm{V}_{\max }$ for objects in a flux limited sample should be uniformly distributed between 0 and 1 , and $\left\langle\mathrm{V} / \mathrm{V}_{\max }\right\rangle$ should be $0.5 \pm \sqrt{(12 N)^{-1} \text { where }} N$ is the number of objects in the sample. Values of $\mathrm{V} / \mathrm{V}_{\max }>0.5$ indicate a bias of objects towards the more distant regions of their accessible volumes.

Fig. 2.5 shows the $\mathrm{V} / \mathrm{V} \max$ values for the sample members plotted against their $1.4 \mathrm{GHz}$ radio luminosity. Estimated redshifts have been included. The mean value for the sample is $0.49 \pm 0.02$. As a check for declination-dependent incompleteness, the $\mathrm{V} / \mathrm{V} \max$ statistic was also computed for the northern hemisphere $\left(\delta>20^{\circ}\right)$, southern hemisphere $\left(\delta<-20^{\circ}\right)$ and equatorial zone $\left(-20^{\circ}<\delta<20^{\circ}\right)$ sources. The results are given in Table 2.3. 


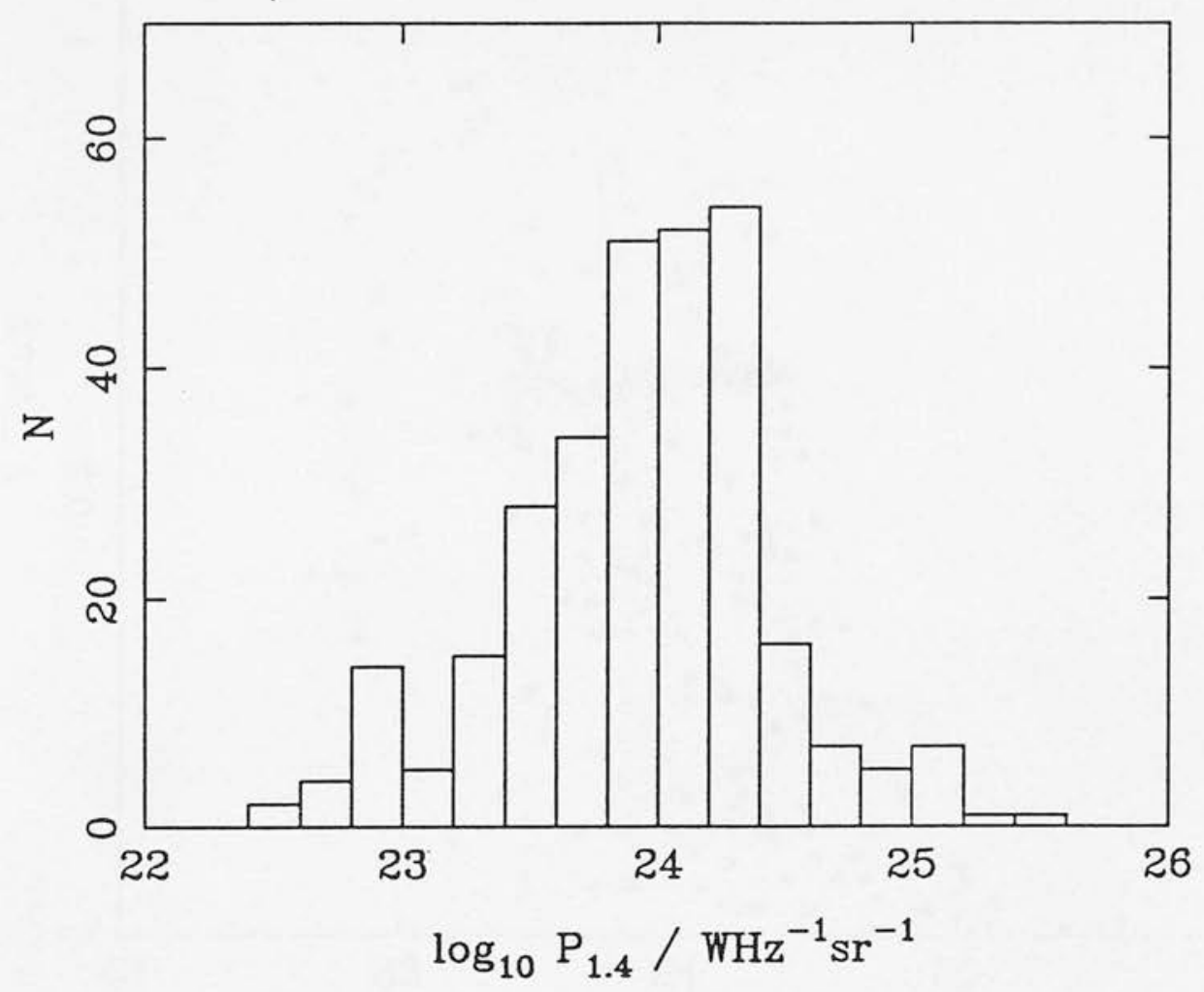

Fig. 2.4: $1.4 \mathrm{GHz}$ radio luminosity distribution for the all-sky sample. There are roughly equal numbers of radio galaxies about the Fanaroff-Riley division. 


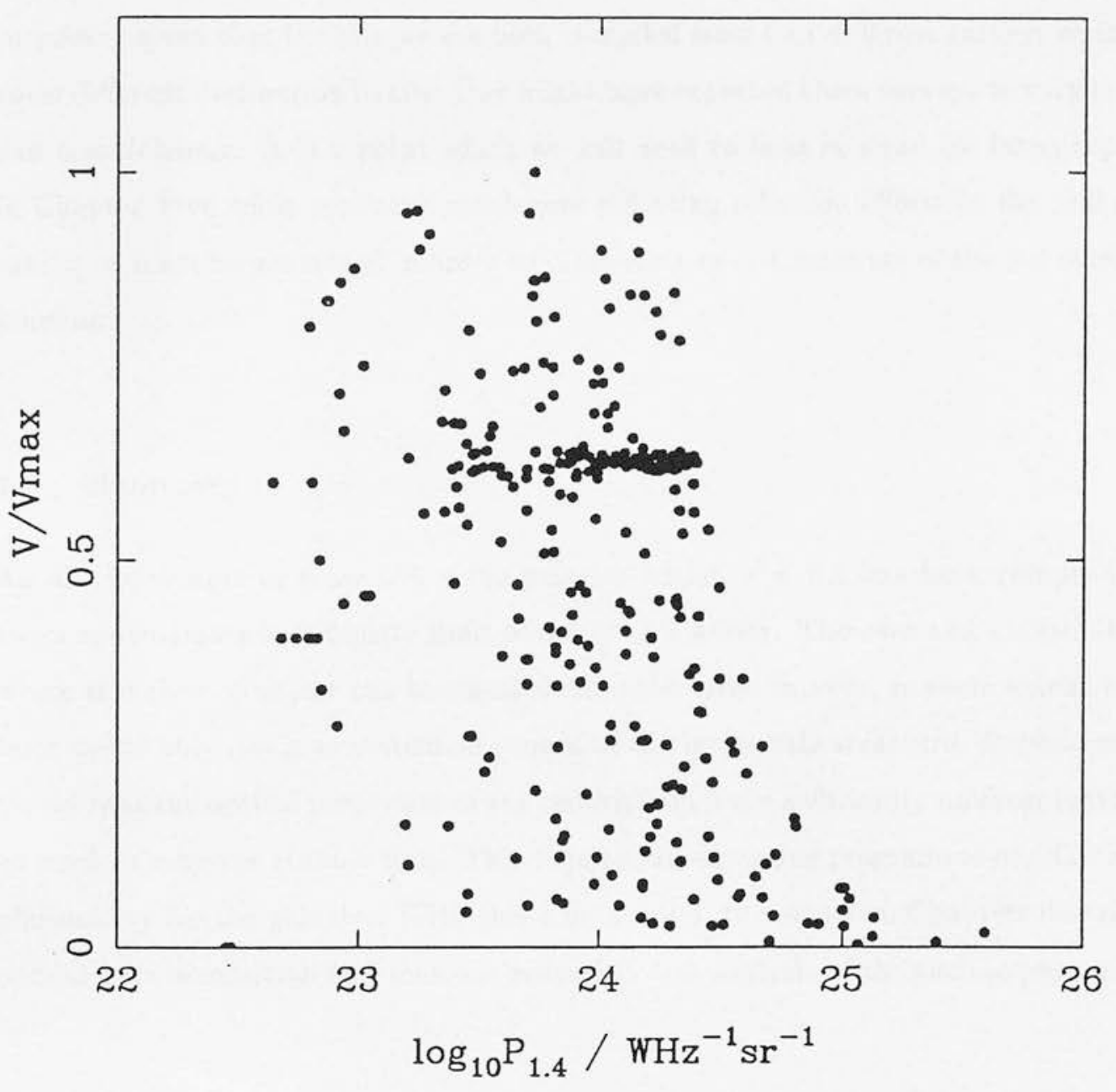

Fig. 2.5: $\mathrm{V} / \mathrm{Vmax}$ plotted against $1.4 \mathrm{GHz}$ radio luminosity $\left(\Omega_{0}=1, h=0.5, \alpha=0.8\right)$ 
Table 2.3: Results of V/Vmax Tests

all sample:

$0.49 \pm 0.02$

northern hemisphere:

$0.52 \pm 0.03$

southern hemisphere:

$0.47 \pm 0.03$

equatorial zone:

$0.45 \pm 0.03$

There is a slight declination-dependent incompleteness for the sample. This is not too surprising given that the sample has been compiled from four different surveys which each cover different declination bands. One might have expected these surveys to vary in depth and completeness. It is a point which we will need to bear in mind for later, especially in Chapter Five when synthetic catalogues reflecting selection effects in the real galaxy catalogue must be generated in order to compute a robust estimate of the 3 -d correlation function.

\subsection{Summary}

An all-sky sample of some 350 radio galaxies within $z<0.1$ has been compiled down to an approximate flux-density limit of $0.5 \mathrm{Jy}$ at $1.4 \mathrm{GHz}$. The ease and uniformity with which this class of object can be selected from the radio surveys, to some extent justifies their use in this thesis as statistical probes of the large-scale structure. It remains to be shown that the optical properties of the radio galaxies are sufficiently uniform for them to be used to map the Hubble flow. This requires an extensive programme of CCD surface photometry for the galaxies. With this aim in mind, the next two Chapters describe the optical data acquisition and then the reduction and analysis of the surface photometry. 


\section{Chapter 3}

\section{Data Acquisition and Reduction}

\section{Part I - CCD Photometry}

\subsection{Introduction}

The acquisition and photometric reduction of two-colour CCD frames for a large subsample of radio elliptical galaxies is described. Normally, CCD photometry of bright galaxies is a rather routine mode of observing and the reduction of the data poses little of a problem. However, when galaxy magnitudes are being used to predict their peculiar velocities one needs to be especially cautious. The reason for this is that an observational signature for a bulk flow of galaxies is that they appear systematically brighter in one direction of the sky than they do another. It is important then to safeguard against any adverse effects in the photometry which could potentially give rise to a spurious brightness gradient.

This Chapter begins with a discussion of the general strategy for carrying out the photometry. The instrumentation employed on each of the telescopes is listed and the observing conditions prevalent during the course of each run are mentioned. Section 3.3 describes the photometric reductions. The set of standard reduction algorithms applied to the $\mathrm{CCD}$ frames are dealt with only briefly here. A more concise account can be found in Mackay (1986) or Wall \& Laing (1986). Calibration equations for each set of observations are derived in Section 3.4. These need to be free from systematics for reliable measurements of the peculiar velocity field. Tests for the presence of systematics in the photometry are described in the next two Sections. Firstly, Section 3.5 presents the results of a cross-comparison between repeat frames for galaxies acquired at different telescopes. Section 3.6 presents the results of astrometry checks, which were performed to test whether the nominal pixel scales for the CCD frames were in error. In Section 3.7, 
further corrections to the galaxy magnitudes for the effects of galactic extinction and an expanding universe are described. Section 3.8 gives a brief summary of the observations.

\subsection{The Observations}

These were performed at three main sites: the University of Hawaii's 88 inch telescope (UH88) on Mauna Kea; the Anglo-Australian 3.9 metre telescope (AAT); the Isaac Newton 2.5 metre telescope (INT) on the Canary Island of La Palma. It was possible to identify a fairly general strategy for maximising the efficiency of the observations although this was, of course, subject to inevitable revision as the course of the project developed. Some of the key features are discussed below.

\subsubsection{GENERAL STRATEGy}

The ultimate goal of this observing programme was an ambitious one: to acquire $B$ \& $I$ CCD frames for all of the radio galaxies known to satisfy the idealised set of selection criteria listed in the previous Chapter. The choice of filters is qualified later.

A first priority at the telescopes was to observe the radio galaxies which were secured a place in the final sample on the basis of published redshifts. Next priority was given to the brightest remaining objects, specifically those with $11<B<17$. The Hubble diagram displayed in the preceding Chapter suggests that these radio galxies are almost certain to fall into the final sample.

Many of the features of the photometry were dictated by the stringent requirements of large-scale cosmological flow experiments. In particular it is essential to check that the galaxy photometry is uniform across the sky and free from any directional biases. The reason for this has been alluded to above. A magnitude dipole anisotropy of 0.1 magnitudes, for example, would translate into a peculiar velocity dipole of roughly $800 \mathrm{kms}^{-1}$ at a redshift of $z=0.05$. Clearly, then, a claim for bulk motions of galaxies could easily be discredited if photometric biases were known to exist in the data.

In order to test for the presence of any systematic bias in the radio galaxy photometry, a number of objects were observed twice from different telescopes. Tight constraints on 
the observing schedule meant that the number of repeat observations were fewer than would have been preferred. However, they still should allow for some statement to be made about the photometric consistency of the observations. We shall return to this point later.

The choice of broad-band filters was motivated by a number of points. When the project began, in 1986, infra-red astronomy with 2-d array cameras like IRCAM (Casali, Aspin \& Mclean 1987) had not yet emerged. Photometry in the $K(2.2 \mu \mathrm{m})$ window with a single-element pholtovoltaic device was not an attractive alternative. For observations in $I$, however, one has all the attractions of observing in the infra-red such as small extinction corrections and reduced sensitivity to recent bursts of star-formation, but with the added advantage that a CCD array can be used as the detector.

The importance of 2-d photometry for this work stems from the fact that observations can be made in non-photometric conditions and calibrated later by bootstrapping the standard star measurements. Further, it is straightforward to design algorithms for 2-d arrays which can interactively remove foreground stars and nearby galaxies from around the object of interest. This contrasts with single-element detectors where one would attempt to minimise the effect of nearby contaminants by making a judicious choice of aperture size.

There are several arguments in favour of complementing the near infra-red CCD observations with a further set of observations through a blue passband, such as the $B$ filter. In the context of this work, two are of particular importance. Firstly, the colour information could be used to check against cases of anomalous foreground extinction. Also, the extra colour information could conceivably be used to discriminate between multiple nuclei in galaxies and foreground stars when secondary local maxima appear in the galaxian light profiles and there is some doubt as to its origin. Secondly, the expected correlation between $B-I$ colour and $I$ magnitude (Pierce 1986) for elliptical galaxies may serve as an extra distance indicator relation for tightening up the estimates of the radio galaxy peculiar velocities.

As it turned out, only $120 \mathrm{~B}$ band CCD frames were eventually recorded. Adverse weather conditions prevaled on the INT and AAT runs and as a result it was decided to 
sacrifice the $B$ band observations in order to observe as many galaxies in the primary $I$ band as time allowed.

\subsubsection{INSTRUMENTATION AND OBSERVING CONDITIONS}

UH88. The telescope was mounted at Cassegrain focus with a Galileo/Institute for Astronomy CCD camera. This configuration normally results in a pixel scale of 0.14 arcsec. For our observations a focal reducer was used and this increased the image scale to 0.41 arcsec pixel $^{-1}$. The larger pixel size meant that nearby galaxies of large angular size could be accommodated onto the chip in addition to some background sky. One slight disadvantage of this optical set-up was an impairment to the blue response of the detector. The detector used was a Texas Instruments (TI) three-phase CCD, thinned and back-illuminated. It has $500 \times 500$ pixels, each $15 \mu \mathrm{m}$ square. The low readout noise and excellent seeing conditions prevalent on Mauna Kea compensate for the smaller aperture of this telescope.

AAT. An RGO CCD camera was used. This was equipped with a thinned, backilluminated Thompson CCD with $1024 \times 1024$ pixels each $19 \mu \mathrm{m}$ square. The nominal pixel scale was given as 0.16 arcsec. pixel $^{-1}$.

INT. An RGO CCD camera was mounted at prime focus of the 2.5 metre telescope. The detector was an RCA CCD which has $350 \times 512$ pixels, each $30 \mu m$ square. At the INT prime focus each pixel subtends a nominal angular size of 0.74 arcseconds.

Observing conditions during the UH88 run were excellent. In addition to the cloudless skies, good seeing conditions prevaled over all three nights with a typical stellar pointspread function measured at about $1^{\prime \prime}$. This is particularly crucial for galaxy surface photometry where it desired to reliably fit the light profile of the galaxies over a large radial range including well into the centre. Typical integration times on the galaxies were $180 \mathrm{~s}$ in $I$ and $300 \mathrm{~s}$ in $B$. This allowed a total of 120 radio galaxies, in addition to several sequences of standard stars, to be observed through both filters in a total of three nights.

Conditions at the INT were less favourable. A common feature of observing at the Observatori Astrofisico del Roque de los Muchachos on the Canary Island of La Palma 
is the presence of Saharan dust blown over from the east in adverse weather conditions (Whittet, Bode \& Murdin 1987; Kidger 1990). This problem afflicted two of the three nights that were allocated for the photometry. Radio galaxies were still observed on each of the dusty nights but with a fairly low duty cycle. It was essential to break off after every hour or so and observe standard stars if the data were to have any chance of being adequately calibrated later. Only radio galaxies within $30-40^{\circ}$ of the zenith were observed in order to avoid the worst of the dust clouds towards the horizon.

Finally, conditions at the AAT were acceptable for one night only. The other night suffered from dreadful seeing conditions of about $8^{\prime \prime}$. An opportunity was taken to acquire some more $B$ frames on this night although these were not used for the subsequent analyses presented in the next Chapter.

\subsection{Data Reduction}

All of the raw CCD frames were processed by the author on the Royal Observatory Edinburgh's STARLINK VAX. Use was made of the standard software packages distributed by STARLINK, namely FIGARO and ASPIC, although for some stages in the reduction it was necessary to write new software. Some of the main features of the CCD reductions are described briefly in this Section.

\subsubsection{BIAS SUBTRACTION}

This was especially simple for the UH88 data: a DC level calculated from the over readout of the chip was subtracted as a scalar from each frame. At the INT and AAT, bias frames were routinely measured at the start and end of each night's observing. A master frame was then constructed by averaging over each set of frames. An appropriately scaled version of the master frame was then subtracted from all of the remaining flat-field and galaxy frames.

\subsubsection{FLAT-FIELDING}

A set of flat-field frames were recorded each night for the purpose of removing any largescale gradients that might exist across the chip. At UH88 the flat-field frames were 
obtained by illuminating the inside of the dome with a photoflood lamp and taking a series of short exposures through each filter. After flat-fielding the galaxy frames, they were examined with a cursor for any residual trace of structure. In fact this was present at a rather high level for the $B$ frames, about $3 \%$. For the $I$ frames there was no more than a $2 \%$ gradient across the chip. The sky-flats worked very well for the AAT and INT frames. Large-scale residual gradients were of the order of $1 \%$ or less.

\subsubsection{COSMETIC IMPROVEMENTS}

The UH88 chip in particular was cosmetically rather poor and as well as the large-scale gradients across the chip had three columns of 'bad' or non-functional pixels. Rather than avoid these altogether it is better to patch over them by interpolating a value from the nearby pixels and adding random noise of appropriate amplitude. Cosmic rays could also be identified across the chip as individual pixels of high intensity. Again, their CCD count was replaced with an interpolated value from the eight contiguous pixels.

\subsubsection{FRINGE REMOVAL}

The optical set-up at the UH88 meant that these frames did not suffer from any fringing, a phenomenon attributed to interference between strong night-sky emission lines of comparable wavelength to the variable silicon substrate thickness in back-illuminated chips. They were present however on the INT frames, more so in $I$ than in $B$. To effect their removal a fringe frame was constructed by stacking up a number of long exposure galaxy frames and applying a median filter. As fringes are additive in intensity, the procedure used for their removal took the form of a global subtraction of an appropriately normalised fringe frame from each of the galaxy images. Generally this worked well, an exception being the frames recorded towards the end of each night when the intensity of the sky-lines are subject to rapid change. Fringes were evident to a lesser extent on the AAT frames and were adequately removed by the flat-fielding process.

\subsection{Photometric Calibrations}

Throughout the course of each observing run a pre-defined set of standard stars were 
routinely observed in order to monitor the stability of the observing conditions and to quantify the amount of extinction in each of the photometric passbands. The standard stars were carefully selected from Landolt's equatorial catalogue (Landolt 1983) on the basis of their $V$ magnitudes and $V-I$ colours. Only stars fainter than $V \leq 10$ were selected so as to avoid saturation. Since the stars would be used to colour correct the galaxies, only those which had a $V-I$ colour closely matched to the canonical elliptical galaxy $V-I$ colour, which is about equal to unity (Pierce 1986), were selected.

The first step in the calibration is to derive aperture magnitudes for the processed standard stars. These were measured from growth curves of sky-subtracted flux summed in apertures of increasing radii centred about the accurate stellar centroid. The curves generally exhibited a sharp rise in cumulative flux which gradually levelled off and finally broke up into noise as increasingly more of the background became enclosed by the aperture. In the one or two cases where this behaviour was not shown by the growth curve, due generally to the stars being too close to the edge of the frame or else to a nearby contaminating star, the stars were rejected from the calibration analysis.

Simple calibration equations were derived for each filter which, to a good approximation, can be treated as linear. They involve three terms:

- a constant offset term;

- an extinction term proportional to $[\sec (\chi)]$, where $\chi$ is the zenith distance of the star at the time of observation;

- a colour correction term.

The color term deserves a special mention. It arises effectively because the magnitudes listed by Landolt for his equatorial standards are given in the standard UBVRI photometric system. This is different to the KPNO system employed at the telescopes and colour equations exist between the two systems. Fig. 3.1 illustrates the broad-band KPNO $B$ and $I$ interference filter profiles with the RCA CCD spectral responses folded in.

These suggest that the colour correction term for $I$ will be small since the curve almost overlaps the filter response curve for $I$ in the Landolt system. A larger colour correction might be expected for $B$ since the KPNO $B$ filter response falls sharply longward of 4000 


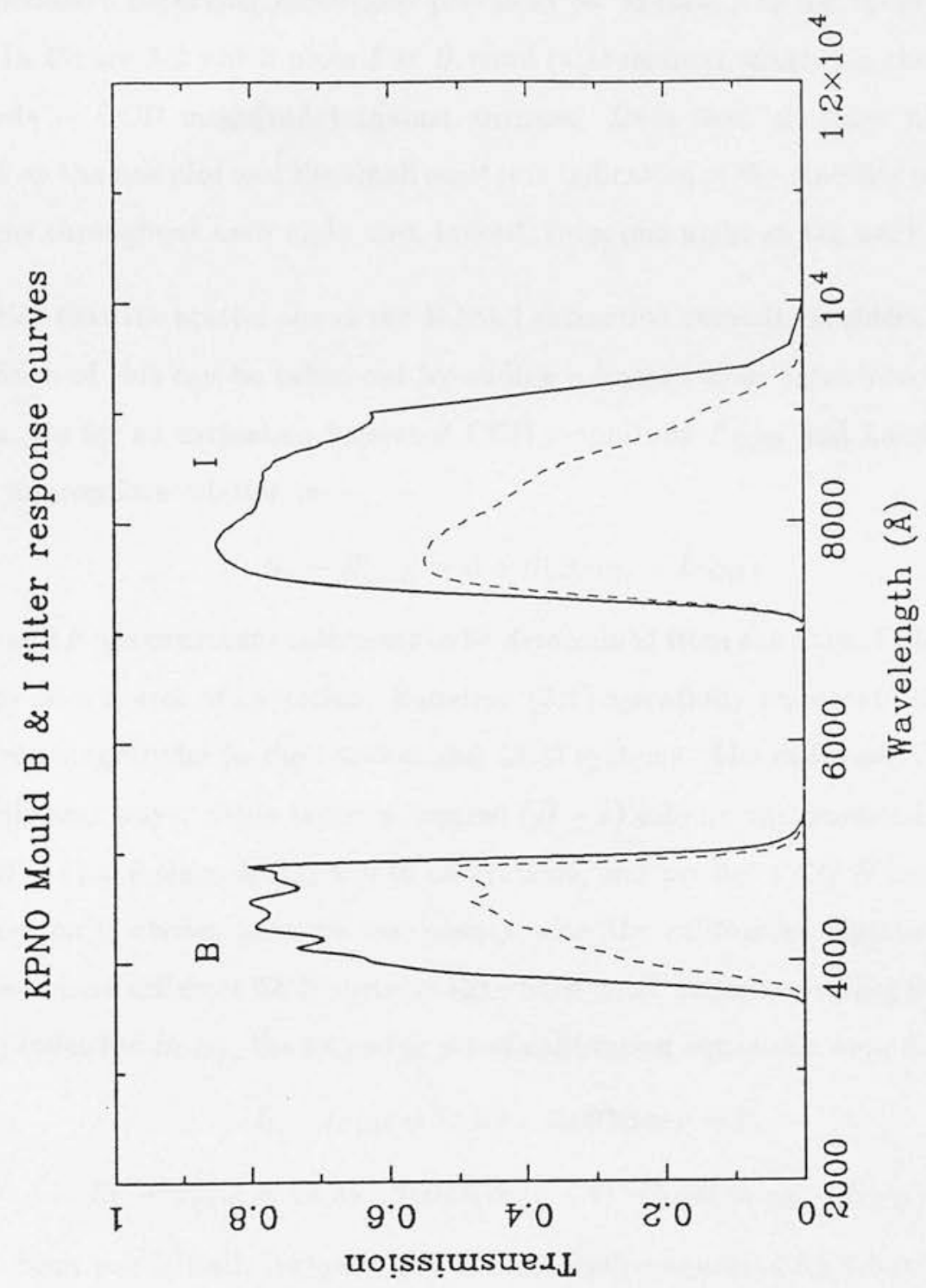

Figure 3.1: The broad-band Kitt Peak Mould B and I interference filter profiles. Dashed lines show the effect of folding in the spectral response of the RCA CCD. 
$\AA$ relative to the $B$ response curve in the Landolt system.

\subsubsection{THE UH88 CALIBRATIONS}

The superlative observing conditions prevalent on Mauna Kea for these observation is evident in Figure 3.2 which plots $I$ \& $B$ band instrumental offsets (in the sense Landolt magnitude - CCD magnitude) against airmass. Data from all three nights has been included on the one plot and the small scatter is indicative of the stability of the observing conditions throughout each night and, indeed, from one night to the next.

Notice that the scatter about the $B$ band extinction curve is considerably larger than for $I$. Much of this can be taken out by adding a linear colour term into the calibration equation. So for an extinction-corrected $\mathrm{CCD}$ magnitude $B_{\mathrm{CCD}}$ and Landolt magnitude $B_{\mathrm{L}}$, the appropriate relation is

$$
B_{\mathrm{L}}-B_{\mathrm{CCD}}^{\prime}=\alpha+\beta\left(B_{\mathrm{CCD}}-I_{\mathrm{CCD}}\right)
$$

where $\alpha$ and $\beta$ are constant coefficients to be determined from the data. Colour corrections can often be a source of confusion. Equation (3.1) essentially represents an approximate fit between magnitudes in the Landolt and CCD systems. The calibration can be viewed in two different ways: either insert measured $(B-I)$ colours and predict Landolt $B$ 's, or insert $(B-I)=0$ since $M_{\mathrm{Vega}}=0$ in all systems, and predict CCD $B$ magnitudes. The former option is chosen here for consistency with the calibration equations from other telescopes where different CCD systems have been used. After regressing for $\alpha$ and $\beta$ and iterating twice for $B_{\mathrm{CCD}}$, the following set of calibration equations were derived

$$
\begin{gathered}
I_{\mathrm{L}}-I_{\mathrm{CCD}}=23.68-0.003(\sec \chi-1) \\
B_{\mathrm{L}}-B_{\mathrm{CCD}}^{\prime}=24.66-0.005(\sec \chi-1)-0.04\left(B_{\mathrm{CCD}}-I_{\mathrm{CCD}}\right)
\end{gathered}
$$

A colour term was initially included into the calibration equation for $I$ but this was found to have a coeficcient effectively equal to zero as expected. The rms scatter about each of the above relations was less than 0.02 magnitudes.

\subsubsection{THE INT CALIBRATIONS}

The dusty conditions prevalent at the INT demanded that the object frames were complemented with a large number of standard star observations to monitor the stability of 
UH88 KPNO I Filter

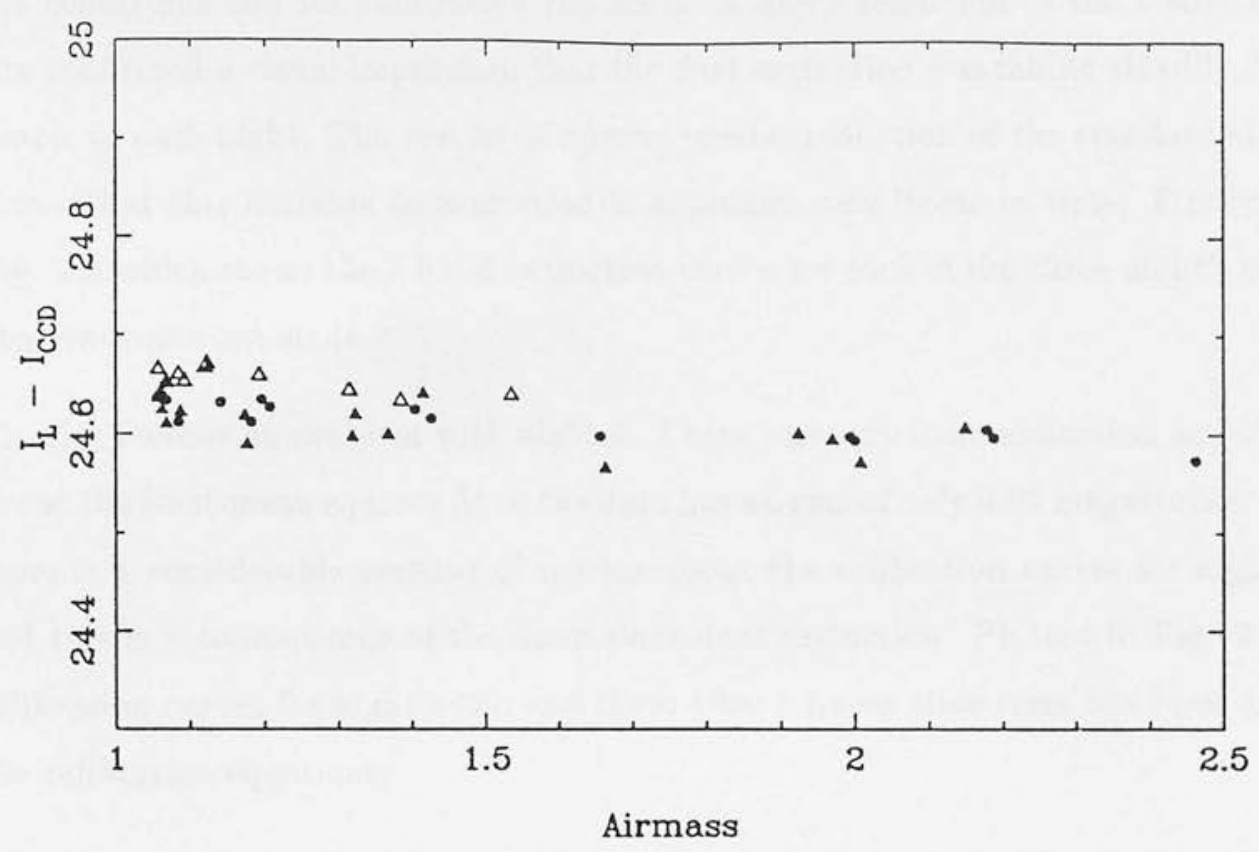

UH88 KPNO B Filter

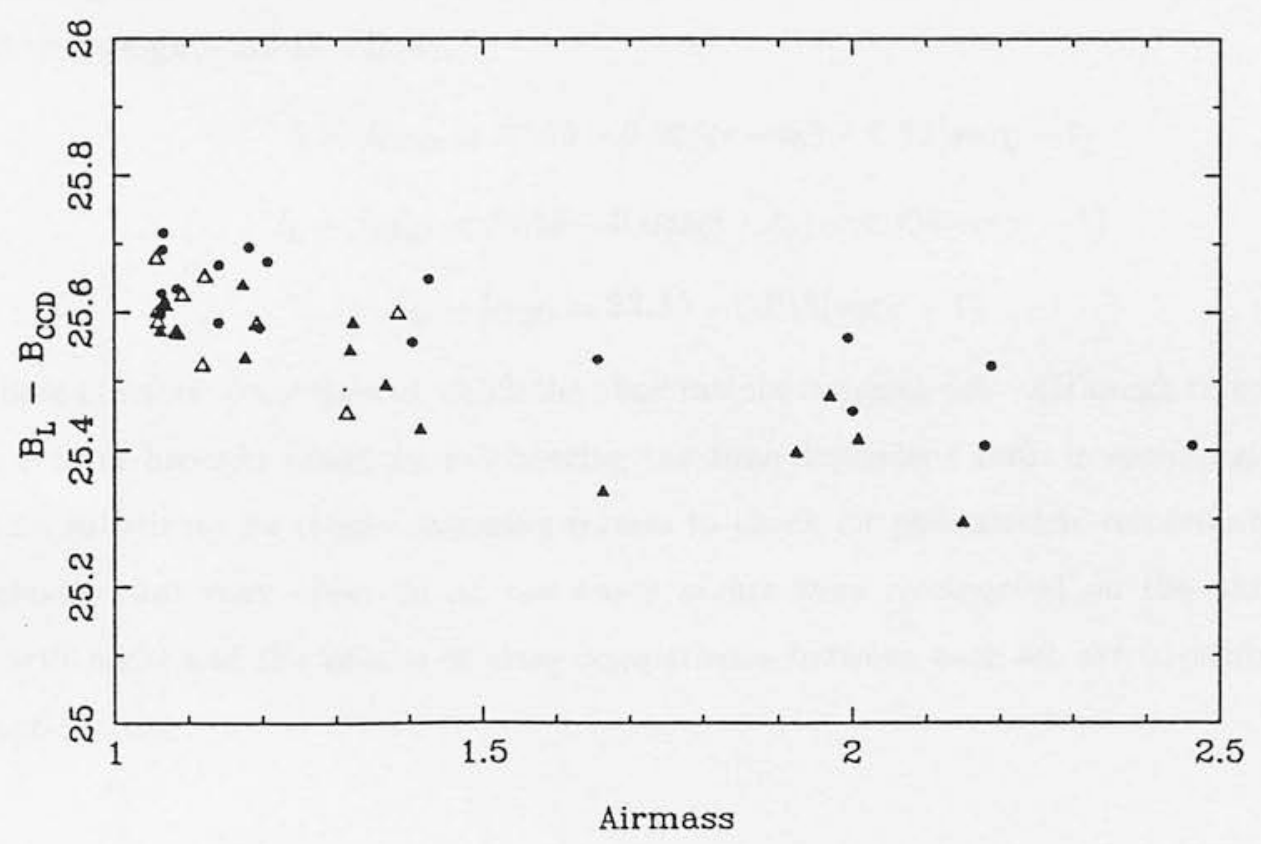

Figure 3.2: Extinction curves for the UH88 standard star data. All three nights data have been plotted (each symbol refers to a specific night). Much of the scatter about the extinction curve for $B$ is reduced when a colour correction term is added in to the calibration equation. 
the conditions and for calibrating the data. A quick reduction of the stellar images on site confirmed a visual impression that the dust extinction was falling steadily during the course of each night. The results of a more precise reduction of the standard star images shows that this decrease in extinction is approximately linear in time. Firstly, examine Fig. 3.3 which shows the $I$ band extinction curves for each of the three night's over which observations were made.

Clearly, there is no problem with night 4 . There was very little extinction and the scatter about the least-mean squares fit to the data has an rms of only 0.01 magnitudes. However, there is a considerable amount of scatter about the calibration curves for night's 2 \& 3 and this is a consequence of the anomalous dust extinction. Plotted in Fig. 3.4 are the calibration curves for night's two and three after a linear time term has been added into the calibration equations.

In each case the scatter about the extinction curves is reduced by about a factor of 3 down to the level of about $0.02-0.03$ magnitudes. The calibration equations derived for all three nights are as follows.

$$
\begin{gathered}
I_{\mathrm{L}}-I_{\mathrm{CCD}}=23.60-0.025\left(t-t_{0}\right)-0.22(\sec \chi-1) \\
I_{\mathrm{L}}-I_{\mathrm{CCD}}=23.55-0.035\left(t-t_{0}\right)-0.326(\sec \chi-1) \\
I_{\mathrm{L}}-I_{\mathrm{CCD}}=23.35-0.048(\sec \chi-1)
\end{gathered}
$$

where $t_{0}$ refers to the time at which the observations commenced. Although this reduction in scatter brought about by introducing the time-dependent term is encouraging, there is no substitute for cross-comparing frames to check for photometric consistency. A few galaxies that were observed on the dusty nights were re-observed on the photometric fourth night and the results of cross-comparisons between each set are presented in the next Section.

\subsubsection{AAT CALIBRATIONS}

These were achieved to within an rms error about the calibration curve of only 0.02 magnitudes. The following equation was derived:

$$
I_{\mathrm{L}}-I_{\mathrm{CCD}}=24.34-0.14(\sec \chi-1)
$$




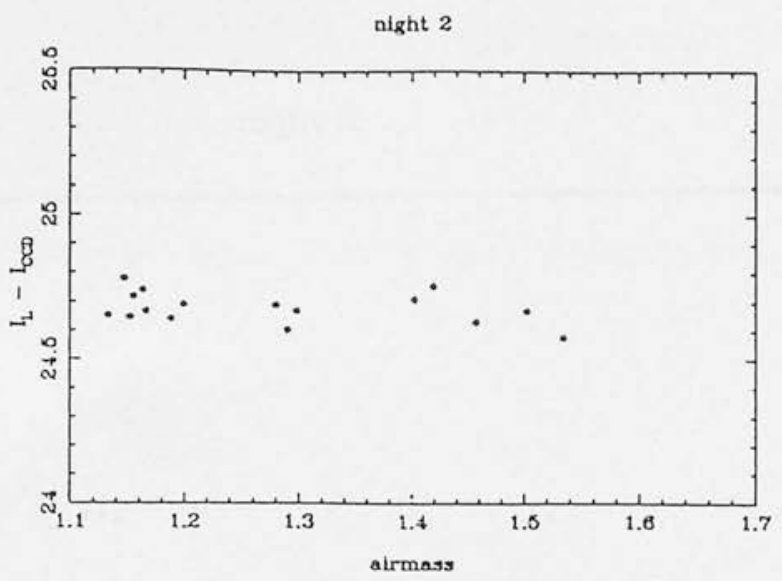

night 3
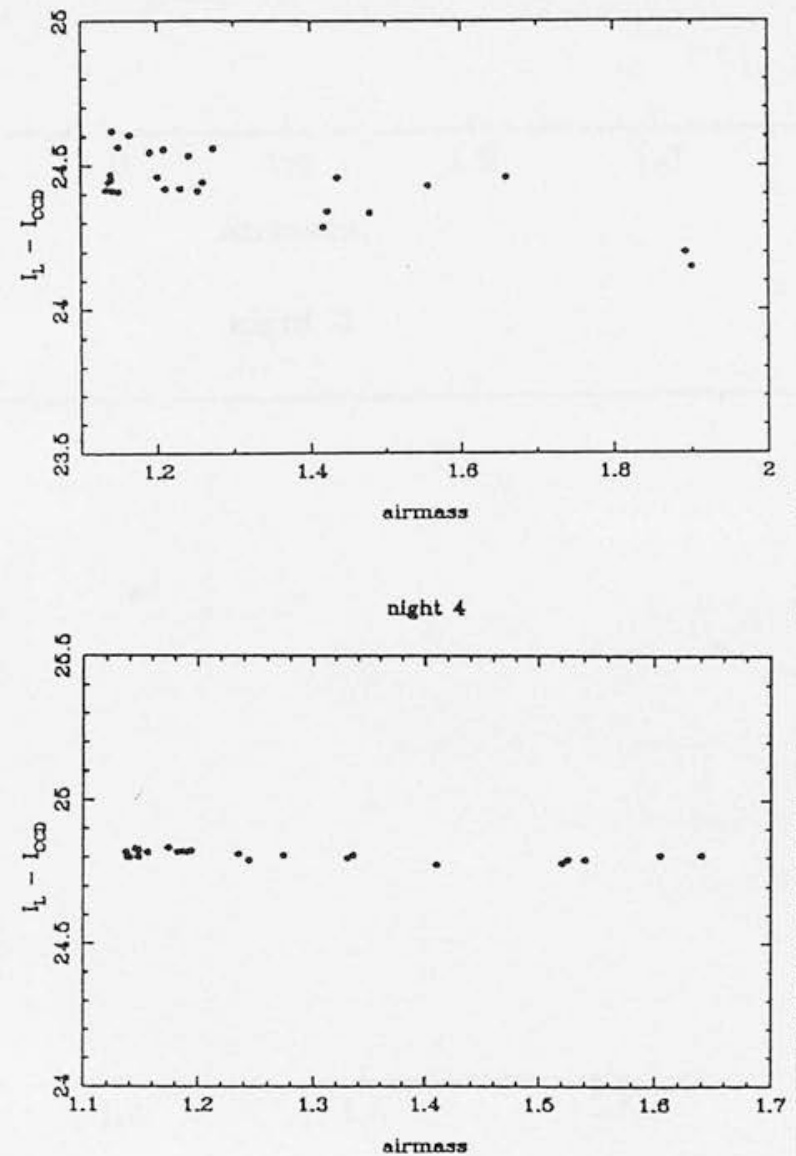

Fig. 3.3: Extinction curves in the $I$ band for the three nights at the INT spent on photometry. Night 4 was photometric. There is a large scatter about the curves for nights 2 \& 3 caused by anomalous dust extinction. 
night 2

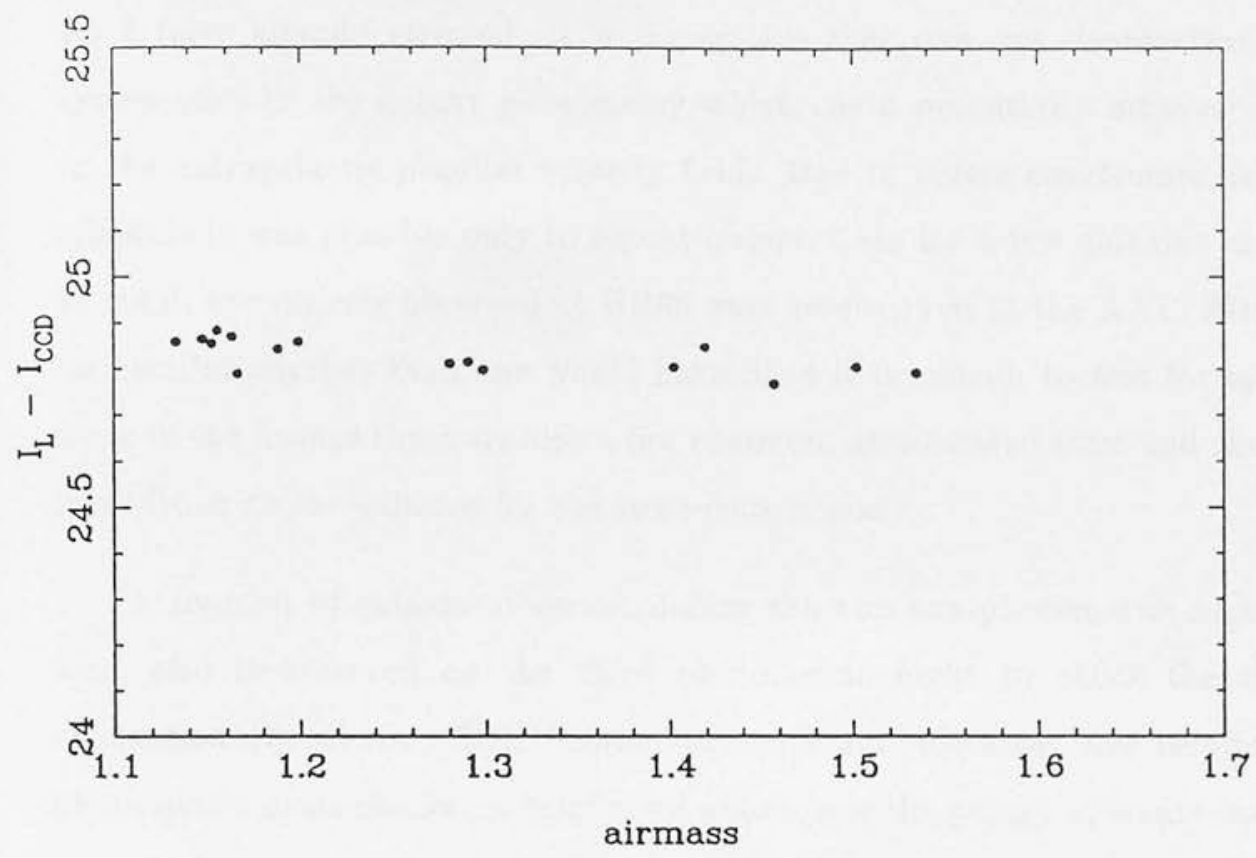

night 3

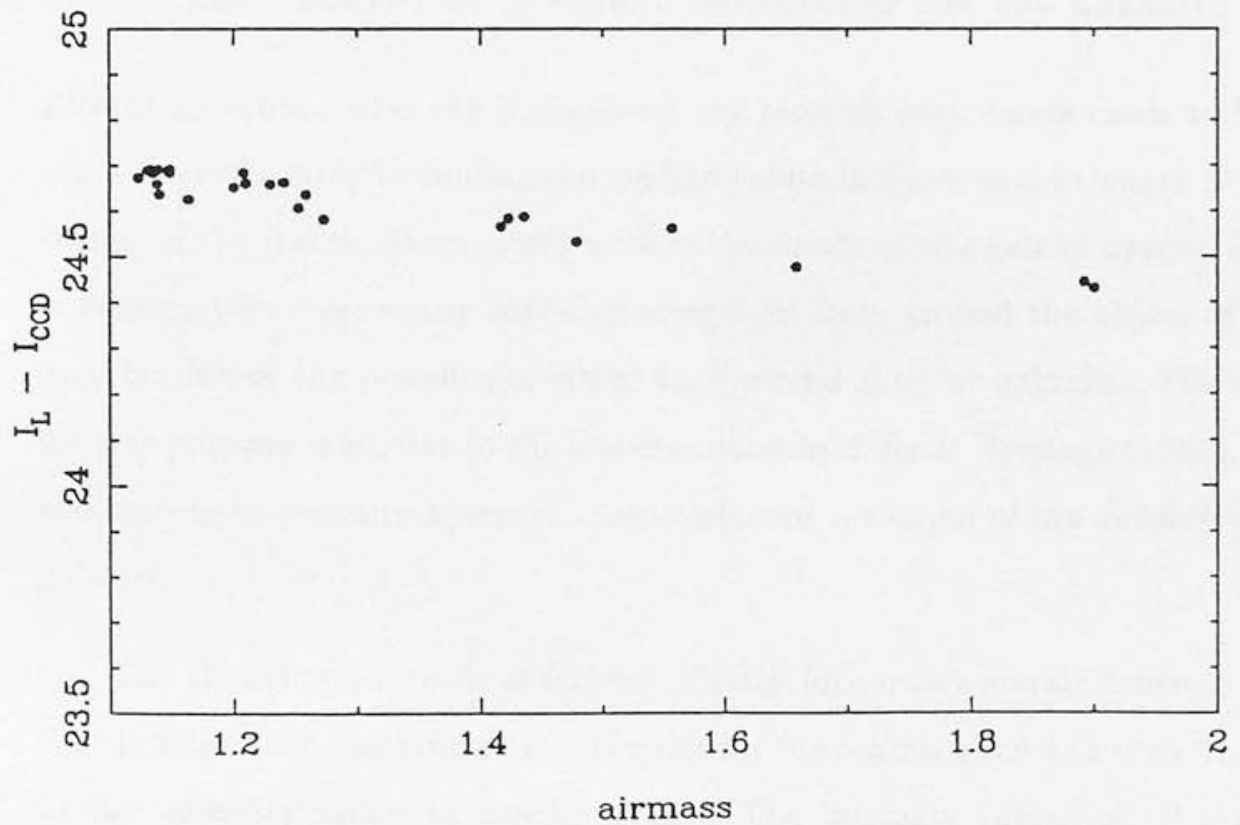

Fig. 3.4: Extinction curves in $I$ for nights $2 \& 3$ after correcting for a linear timedependent term. The rms scatter about a least-squares fit is reduced by a factor 3 to about 0.02-0.03 magnitudes. 


\subsection{Photometric Consistency Checks}

As I have already stressed, it is imperative that one can demonstrate there are no systematics in the galaxy photometry which could potentially masquerade as a dipole in the extragalactic peculiar velocity field. Due to severe constraints on the observing schedule it was possible only to repeat observations for a few galaxies at different sites. In total, five objects observed at UH88 were re-observed at the AAT. Although this is a far smaller number than one would have liked it is enough to test for systematics. On some of the frames there are also a few common, unsaturated stars and these can be used in addition to the galaxies for the cross-comparisons.

A number of galaxies observed during the two non-photometric nights on the INT were also re-observed on the third photometric night to check the time-dependent calibration equations. This Section presents and discusses the results from all the photometric cross-checks. A brief word about how the galaxy aperture magnitudes were measured is given first.

\subsubsection{MEASUREMENT OF APERTURE MAGNitudes For THE GALAXIES}

Firstly, an estimate for the background sky level on each frame needs to be found. This was achieved simply by finding the median values in boxes of side length $5^{\prime \prime}$ placed at each corner of the frame. To measure a 'clean' estimate of the galaxy aperture magnitudes it is necessary to remove any contaminating light from around the object of interest which may be due to the presence of either background stars or galaxies. The algorithm used for this purpose is similar to the one described by Lilly \& Prestage (1987), who were also attempting to measure aperture magnitudes for a sample of low redshift radio elliptical galaxies.

The algorithm proceeds as follows. Firstly, locate an accurate centroid for the galaxy. This is achieved by making an initial guess for the central pixel and then fitting a parabola to the intensity values in nearby pixels. The intensity values of all pixels which are $180^{\circ}$ reflections of each other about the galaxy centroid are then examined. If these are mismatched by an amount which exceeds some pre-set threshold then the higher value is reduced to the value of its symmetric counterpart and gaussian noise of appropriate amplitude is added. This threshold is of course rather arbitrary. Trial-and-error values 
suggested that pulling down the pixels that were too bright by about a factor of 5 worked best. Sky subtracted aperture magnitudes were then computed for each galaxy. The choice of aperture for this was dictated by the angular size of the galaxy and its location on the frame.

\subsubsection{COMPARISON BETWEEN UH8 \& \& AAT MAGNITUdES}

Only five radio galaxies observed on the UH88 run were re-observed at the AAT. In addition to comparing the galaxy magnitudes there are also typically two or three unsaturated stars on each of these frames which can be included into the crosscomparisons. The full set of magnitudes and their residuals derived from each of the repeat frames are given in Table 3.1 .

In viewing Table 3.1, the greatest weight should be given to the galaxy magnitudes since these were measured in larger apertures than the stars. This is important because errors in estimating the stellar centroids - for the AAT frames these are likely to be larger given that the pixels only subtend $0.15^{\prime \prime}$ - can lead to systematic magnitude errors. For larger apertures, the consequences of mistaking the true centroid are not so severe. Aperture sizes for computing galaxy magnitudes were dictated by the location of the galaxy on the frame and on its environment. The stellar magnitudes were total magnitudes derived from growth curves of cumulatives sky-subtracted flux. Magnitudes were obtained before and after the symmetrisation algorithm was applied to remove nearby objects. The results quoted in Table 3.1 are for the pair of magnitudes which were in closer agreement - either those before or after application of the algorithm. Error bars on the estimated magnitudes are somewhat uncertain. They were derived by simply assuming a $1 \%$ inaccuracy in the background sky value that was subtracted from the frame.

The magnitude residuals, which are what we are really interested in, are fairly evenly scattered about zero suggesting that there are no obvious systematic offsets in the photometry. A proper statistical analysis is difficult to do since the measured values from each frame are not independent. In retrospect, it would have been desirable to repeat more observations of galaxies to put the issue of systematic magnitude offsets beyond doubt. With only five repeat frames I believe it is not possible to say unequivocally that

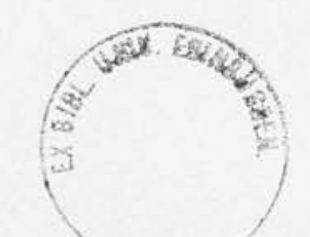


Table 3.1: Summary of UH88 \& AAT repeat photometry

$\begin{array}{ccccr} & \text { AAT } & \text { UH88 } & \text { size } & \Delta m_{I} \\ & & & & \\ 0359+193 & 13.63 \pm 0.2 & 13.79 \pm 0.1 & 15^{\prime \prime} & -0.16 \\ \text { star } & 14.72 \pm 0.24 & 14.59 \pm 0.10 & \text { total } & 0.13 \\ \text { star } & 11.43 \pm 0.03 & 11.40 \pm 0.07 & \text { total } & 0.03 \\ \text { star } & 15.73 \pm 0.40 & 15.86 \pm 0.25 & \text { total } & -0.13 \\ & & & & \\ 0431-134 & 13.25 \pm 0.09 & 13.28 \pm 0.04 & 15^{\prime \prime} & -0.03 \\ \text { star } & 15.60 \pm 0.33 & 15.51 \pm 0.15 & \text { total } & 0.09 \\ \text { star } & 16.22 \pm 0.04 & 16.19 \pm 0.04 & \text { total } & 0.03 \\ & & & & \\ 0825+247 & 15.09 \pm 0.09 & 14.86 \pm 0.1 & 12^{\prime \prime} & 0.23 \\ & & & & \\ 0945+076 & 15.14 \pm 0.06 & 15.10 \pm 0.04 & 15^{\prime \prime} & 0.04 \\ \text { star } & 17.33 \pm 0.4 & 17.36 \pm 0.25 & \text { total } & -0.03 \\ \text { star } & 16.93 \pm 0.35 & 17.31 \pm 0.6 & \text { total } & -0.38 \\ & & & & \\ 1000-043 & 14.16 \pm 0.06 & 14.10 \pm 0.09 & 13^{\prime \prime} & 0.06 \\ \text { star } & 15.97 \pm 0.08 & 15.77 \pm 0.24 & \text { total } & 0.2 \\ \text { star } & 14.77 \pm 0.2 & 15.01 \pm 0.15 & \text { total } & -0.25\end{array}$

Table 3.2: Summary of INT repeat photometry

$\begin{array}{ccccr} & \mathrm{P} & \mathrm{N} / \mathrm{P} & \text { size } & \Delta m_{I} \\ & & & & \\ 1502+262 & 13.67 \pm 0.04 & 13.78 \pm 0.10 & 21^{\prime \prime} & -0.11 \\ \text { star } & 16.41 \pm 0.21 & 16.18 \pm 0.13 & \text { total } & 0.23 \\ \text { star } & 16.03 \pm 0.15 & 15.82 \pm 0.20 & \text { total } & 0.21 \\ & & & & \\ 0206+35 & 15.06 \pm 0.09 & 15.10 \pm 0.06 & 18^{\prime \prime} & -0.04 \\ \text { star } & 14.49 \pm 0.08 & 14.49 \pm 0.10 & \text { total } & 0.0 \\ \text { star } & 15.50 \pm 0.12 & 15.58 \pm 0.09 & \text { total } & -0.08 \\ & & & & \\ 0121+429 & 14.76 \pm 0.23 & 14.59 \pm 0.14 & 24^{\prime \prime} & 0.17 \\ \text { star } & 15.54 \pm 0.07 & 15.56 \pm 0.21 & \text { total } & -0.02 \\ \text { star } & 16.94 \pm 0.10 & 16.98 \pm 0.18 & \text { total } & -0.04 \\ & & & & \\ 1713+641 & 14.93 \pm 0.09 & 15.08 \pm 0.19 & 25^{\prime \prime} & -0.15 \\ \text { star } & 15.89 \pm 0.23 & 15.82 \pm 0.24 & \text { total } & 0.07 \\ \text { star } & 16.40 \pm 0.17 & 16.25 \pm 0.21 & \text { total } & 0.15\end{array}$


there are no systematic offsets in the photometry - there appears, on the basis of the evidence we have, to be no serious inconsistencies within the data.

\subsubsection{THE INT REPEAT PHOTOMETRY}

Introducing a time-dependent extinction term into the calibration equations for the INT photometry appeared to work very well. To get a more robust idea of whether the calibrations are adequate it is important to check the galaxy magnitudes, together with a few stellar magnitudes, on repeat frames. There are four such frames, two from each of the two dusty nights, which overlap with observations that were made on the extremely photometric night. The full set of comparisons are presented in Table 3.2. The column labelled ' $\mathrm{P}$ ' refers to magnitudes for objects taken from the photometric frames and ' $\mathrm{N} / \mathrm{P}$ ' refers to the non-photometric frames. There is clearly an excellent agreement between these values, which serve to increase our confidence that the INT data can be included in the peculiar velocity analysis without introducing any bias.

\subsection{Astrometry}

The level of precision required for streaming flow work and the desire to avoid systematics in the photometry require that the nominal pixel scales quoted for each of the CCD detectors should not generally be taken on trust. A systematic difference in (true nominal) pixel size from one detector to the next can again manifest itself as a streaming dipole and consequently needs to be guarded against. A precise value for the INT pixel scale was kindly provided by Karl Glazebrook who was conducting a $B V R I$ survey for faint objects on the INT immediately prior to my own run. As part of his reductions, Glazebrook need to pair up the CCD identifications with a list of objects identified from photographic plates. This procedure provided an accurate value for the INT CCD pixel scale which turned out to be $0.741 \pm 0.004^{\prime \prime}$, i.e very close to the nominal figure of $0.74^{\prime \prime}$. Only two INT CCD frames overlapped with UH88 frames of identical galaxies. However, on these frames there were 3 and 5 unsaturated stars respectively. Comparing distances between galaxy-stellar and stellar-stellar centroids from each frame allows the UH88 pixel 
scale to be tied down to sufficient accuracy. A value of $0.410 \pm 0.008$ was estimated which is identical to the quoted value within the errors. Using the UH8s and AAT overlap frames, a similar procedure was to establish the pixel size for the chip used at the AAT. A value of $0.15 \pm 0.005$ was obtained which falls marginally outside the nominal value.

\subsection{Further Corrections to Galaxy Magnitudes}

Having derived calibrated magnitudes for the galaxies they then need to be corrected for two important effects: galactic dust extinction and cosmology. Each of these are now discussed briefly in turn below.

\subsubsection{GALACTIC EXTINCTION CORRECTIONS}

These were taken from the HI density contour maps of the galaxy presented by Burstein $\&$ Heiles (1982). The corrections are very small $(\leq 0.02)$ well away from the galactic plane, rising to $\sim 0.06$ towards $|b| \simeq 15^{\circ}$. Corrections for $I$ were taken to be about $30 \%$ of the extinction in $B$, assuming a Whitford reddening law (Pierce 1986).

\subsubsection{COSMOLOGICAL CORRECTIONS}

In an expanding universe, the (monochromatic) surface brightness of a source is given by

$$
I_{\nu}=\frac{I_{s \nu(1+z)}}{(1+z)^{3}}
$$

where $I_{s}$ is the surface brightness at the source. Integrating over bandpass frequency, the result is

$$
-2.5 \log I=-2.5 \log \frac{I_{0}}{(1+z)^{4}}+K(z)
$$

The term $K(z)$ comprises two parts: (1) the acceptance band is narrower in the rest frame of the distant object by a factor $(1+z)$, independent of wavelength or of the shape of the energy curve - the apparent magnitude of the object is thereby increased by an amount $2.5 \log (1+z)$. (2) At each wavelength $\lambda$ in the acceptance band the intensity received is not $F(\lambda)$ read from the standard energy curve in the observer's velocity frame, but $F[\lambda /(1+z)]$. The apparent magnitude will then be increased if the energy curve falls towards shorter wavelengths, or decreased if it rises. 
In order to get a handle on the magnitude of the second term above, Bruzual (1983) has considered spectral evolutionary models for ellipticals in which the galaxies are treated as a closed system for which the star-formation rate is a smooth function of time. This rate governs the spectral evolution of the galaxy. Specifically, he considers two models evolving and non-evolving. Using his tabulated values of $K$-corrections as a function of redshift and fitting these to a simple model of the form $K(z)=a z+b z^{2}$, it was found that for the particular version of the evolving model that was examined, $a=0$ and $b=0$ whereas for the non-evolving model, $\mathrm{a}=0.8, \mathrm{~b}=0$. In fact for low redshifts, where the magnitudes of the effect are small, it does not really matter which model one assumes so long as this is stated clearly in any work. The non-evolving model was selected to $K$-correct the radio galaxy magnitudes.

\subsection{Summary and Conclusions}

The CCD photometry programme resulted in about $100 \mathrm{~B}$ frames and $320 \mathrm{I}$ frames. All of the $B$ frames and about $50 \%$ of the $I$ frames were obtained in extremely photometric conditions on Mauna Kea. About $25 \%$ of the frames were acquired at the INT, where conditions were less than favourable. For studying cosmological peculiar velocities it is essential to be free from any sort of systematic offsets in the photometry which can masquerade as a dipole. No evidence was found for any such offsets in the radio galaxy photometry, although our conclusions were based on rather fewer numbers than would have been preferred. Astrometric checks were also performed as a guard against biases that can creep in if there is a systematic over/under estimation of galaxy sizes. Galaxy photometry is really only of any use when coupled with redshifts. This latter measurement allows observed photometric properties of the galaxy to be related to their intrinsic properties. It is to the redshift survey for the radio galaxy sample then that we now turn our attention. 


\section{Part II}

\section{Optical Spectroscopy}

\subsection{Introduction}

This second part of the Chapter deals with a completely different aspect of the observing programme - namely, the optical spectroscopy. Recall from Chapter 2 that, of the initial sample, only 125 radio galaxies had a published radial velocity that was sufficiently accurate not to have to be remeasured for the streaming flow work. A report is given here of a radio galaxy redshift survey which has as its main goal to acquire redshifts for the rest of the sample.

Redshift completeness, or at least as near to this ideal as possible, is usually an essential requirement for any sample being used to probe the large-scale structure. This was evident for the all-sky sample in the sense that preliminary results for the 3 - $\mathrm{d}$ correlation function (Peacock et al. 1988) led us to draw quite different conclusions about the clustering properties of radio galaxies than do a similar set of results for the full redshift survey (Chapter 5; Peacock \& Nicholson 1991).

Specific features of a spectroscopic programme for galaxies which are ultimately being used to study the Hubble flow are that the measured redshifts need to be accurate and free from systematic biases. Typical accuracies need to be of the order of $150 \mathrm{kms}^{-1}$ or less given that one is interested in detecting a dipole motion of something like $500 \mathrm{kms}^{-1}$ at $\langle z\rangle \simeq 0.03$. For identical reasons to those already alluded to in the photometry section, systematic biases in the velocity data are undesirable.

A brief summary of the remainder of this Chapter is given below. Section 3.10 presents some details regarding the data acquisition, briefly describing the instrumentation 
employed at each telescope and the observational strategy. This is followed in Section 3.11 by a discussion of the data reduction techniques. In common with the CCD photometry there is a fairly standard approach to this problem. Hence it is dealt with only briefly here. Section 3.12 describes some commonly occurring features that appear in the spectra - a catalogue of all the observed radio galaxy spectra are included as an Appendix to this thesis. The next Section derives redshifts for the galaxies using the cross-correlation technique. The chief sources of errors in these measurements are pointed out and a crosscomparison between the redshifts obtained here and those obtained by other workers for a small subset of the radio galaxies which have been observed twice is presented. A summary of this work is presented in Section 3.14.

\subsection{The Observations}

The full set of spectroscopic observations undertaken for this thesis are displayed in Table 3.3 .

The author was present on three of these runs. Further data has generously been acquired for this work by Lance Miller (INT \& Cerro-Tololo Inter-American Observatory), Charles Jenkins (using the Durham-RGO Faint Object Spectrograph (FOS) on the William Herschel 4.2 metre telescope), Simon Lilly (UH88) and John Peacock (AAT). A few details of the instrumentation and acquisition techniques are provided below.

UH88 The University of Hawaii's Faint-Object Spectrograph (FOS) was mounted at Cassegrain focus with a cryogenic CCD camera. The dispersive element in the spectrograph was a grism. This had 600 lines per mm., providing an undeviated central wavelength of $5000 \AA$ and a spectral coverage between 4500 and $6200 \AA$. The cryogenic camera employed as its detector a Texas Instruments CCD array. The optical system illuminates the central few hundred columns of pixels, with the dispersion direction parallel to the long side. A few columns in the unexposed area of the chip are read out twice to provide a direct measure of the electronic bias offset level. On-chip binning was performed, in which the summed charge from two adjacent pixels is read out as a single charge signal. 


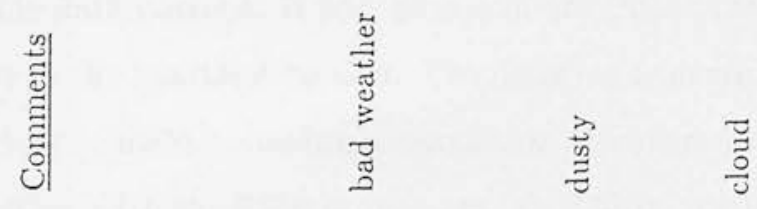

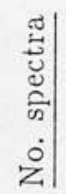

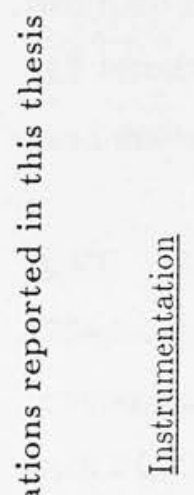

i 0 ก

m

와느ำ

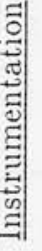

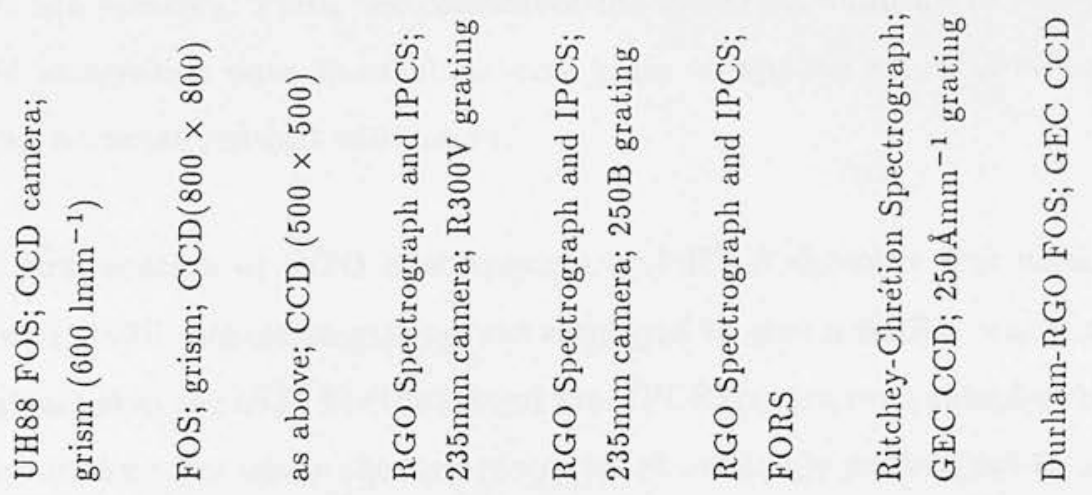

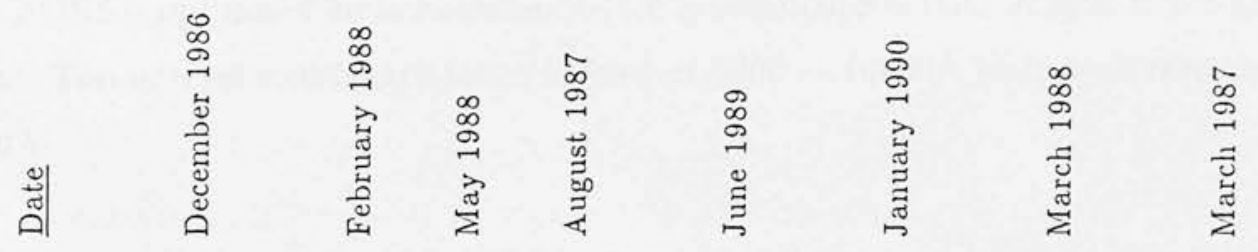

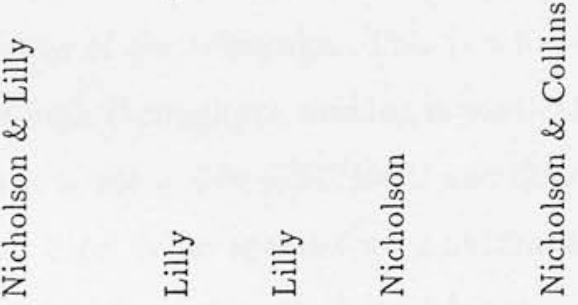

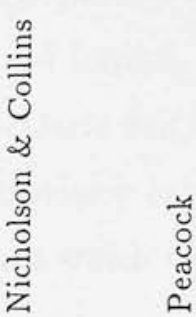

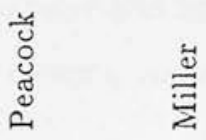

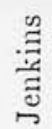

คैं

崩|

$\underset{\substack{\infty \\ \hdashline}}{\stackrel{1}{5}}$

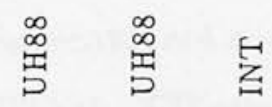

岁 委

息 
INT 2.5 metre The instrumentation employed here was an RGO Spectrograph and IPCS detector. This latter is a blue-sensitive photon-counting device which stores individual photon events in a $2 \mathrm{D}$ image of about $10^{6}$ elements, with zero readout noise and a negligible dark current. It has, at maximum, 2044 pixels available in the spectral direction and 514 in the spatial direction. The detector is particularly suited to a wavelength range from $3500 \rightarrow 6000 \AA$, having a maximum quantum efficiency of about $20 \%$ at $4000 \AA$. In conjunction with the $235 \mathrm{~mm}$. camera, an R300V grating was chosen to yield a wavelength coverage of approximately $3000 \rightarrow 7000 \AA$ at $\sim 2 \AA$ per pixel. With a CCD detector, it is not possible to examine the signal-to-noise ratio of a spectrum until the chip is read out at the end of an integration. The IPCS, however, counts individual photons and real-time readouts are possible. Thus, one can watch the spectrum build-up in real time and terminate the integration once spectral features begin to appear which are deemed good enough for an accurate redshift estimation.

AAT Again a combination of RGO Spectrograph and IPCS detector was used. A $235 \mathrm{~mm}$. camera with $250 \mathrm{~B}$ dispersive grating was employed to give a similar wavelength coverage to that attained at the INT. For the second run, IPCS spectra were complemented with FORS spectra in the cases where the former spectra showed only poor signal-to-noise ratios. FORS is optimised for low resolution, red spectroscopy of faint objects and is highly efficient. The normal wavelength range is fixed at $5200 \rightarrow 10900 \AA$ with peak response at $\sim 7000 \AA$.

WHT The Durham-RGO Faint Object Spectrograph was employed, mounted at the $\mathrm{f} / 15$ Cassegrain focus of the telescope. This is a fixed format, low resolution $(15 \rightarrow 20 \AA$ FWHM) device of high throughput making it particularly suited for faint object observations. Its detector is a $385 \times 576 \mathrm{GEC} \mathrm{CCD}$ and dispersion is provided by a transmission grating and grism. First-order spectra were obtained which cover a wavelength range of $4000 \rightarrow 10500 \AA$ with a dispersion of about $9 \AA$ per pixel.

CTIO The telescope was configured with a Ritchey-Chrétien Spectrograph in combination with pre-flashed GEC\#9 CCD and $250 \AA / \mathrm{mm}$ grating. The wavelength coverage was from about $4000 \AA$ to $7000 \AA$ at roughly $3 \AA$ per pixel. A standard exposure time of 600 s on each galaxy gave very high signal-to-noise spectra. 
Low-redshift radio galaxies are generally very bright and extended objects. This makes observing them fairly straightforward. Their high optical luminosity meant that the majority could easily be seen on the acquisition TV in the control room of the telescope. Accurate centring of the slit on the target object was then simply achieved by a manual adjustment of the telescope pointing. Accurate positional offsetting from recommended nearby guide stars was used to centre up the fainter galaxies.

One of the chief advantages of using the IPCS over a CCD is the facility to allow the spectrum to be watched being built up. This allows the observer to intercede when he feels that there is enough $\mathrm{S} / \mathrm{N}$ in the spectrum for estimating redshifts. With the CCD one has to choose an integration time and the object spectrum is 'hidden' from view until after integration. For these latter observations, integration times were based on the apparent $B$ magnitudes of the galaxy and the knowledge that cross-correlation redshifts (Section 3.13 ) accurate to $\leq 50 \mathrm{kms}^{-1}$ require an $\mathrm{S} / \mathrm{N}$ of $\geq 100$ counts per pixel. Typically, the integration times ranged between from about 5 minutes and 45 minutes.

A number of calibration spectra were acquired along with the radio galaxy observations. Arc spectra are the most important and these were measured after each slew of the telescope to guard against flexures in the optical system and to wavelength-calibrate each galaxy spectrum independently. Flat fields were needed to correct the CCD detector surfaces for large-scale gradients. At UH88 and CTIO these were obtained by replacing the arc-lamp with a tungsten filament lamp and uniformly illuminating the slit. Finally, a pair of recommended flux-standard and smooth-spectrum stars were observed to be used for taking out the instrumental wavelength responses of the detectors and correcting for atmospheric absorption effects at the red end of the spectra.

\subsection{Data Reduction}

The FIGARO 2-d spectral reductions software package, written by Keith Shortridge, was adequate for most steps in the reduction. The most important one of these is the wavelength calibration which ultimately limits how accurately redshifts can be measured. All of the reductions described here were done by the author with the exception of the second set of AAT data which were reduced by John Peacock. The IPCS and CCD spectra 
require slightly different reduction techniques which are indicated below.

\subsubsection{FLAT-FIELDING}

The CCD spectra were accompanied by flat-field images for the purpose of removing largescale intensity trends from across the surface of the detectors. Firstly, the 2-d flat field images were collapsed in the spatial direction to give a set of 1-d spectra. These represent the average spectral response of the tungsten lamps that were used to illuminate the detector and are essentially just black body curves. A spline fit was then made to each of the flat-field spectra to yield their smoothed versions. Each of the cross-sections parallel to the spatial direction in the 2-d flat-field images were divided by the smooth spectrum. This produces a corrected flat-field calibration image. All object and arc images were divided by an appropriate flat-field frame to correct for sensitivity variations across the detector. The IPCS and FORS arrays were not flat-fielded since pixel-pixel deviations for these devices are apparently $\leq 2 \%$ at most.

\subsubsection{EXTRACTING THE SPECTRA}

1-dimensional spectra were next obtained from the 2-dimensional spectral images by extracting a number of rows parallel to the dispersion direction and summing these to yield an intensity profile across the slit. The number of extracted rows in each case was determined by inspecting the images with a cursor. For emission line galaxies (such as $1127+012)$ it was often better to extract only a few rows either side of the nucleus to enhance their spectral features. A number of sky rows were extracted from each side of the object and normalised to the number of object rows to yield a composite sky spectrum. A factor of 3 or 4 times as many sky rows were extracted than object rows in order to reduce the noise level in the sky-subtracted spectrum.

\subsubsection{WAVELENGTH CALIBRATion}

This is an automatic, menu-driven procedure within the FIGARO software but requires some care given its relevance to the accuracy of the final redshifts. The first step is to identify as many arc lines as possible in an arc-lamp spectrum using a list of tabulated 
wavelengths as a guide. For all of the arc-lamp spectra measured here at least 16 lines could be readily identified over a decent range in wavelength. A polynomial is then fitted to the wavelength/channel number relation for each of the arc spectra in order to define a global relationship. After some trial-and-error a high-order (eighth) polynomial was used because it gave a good fit over a wide range in wavelength with rms residuals never exceeding $0.3 \AA$ : at a wavelength of $5000 \AA$ this corresponds to a velocity error of only $18 \mathrm{kms}^{-1}$. The accuracy of the wavelength calibration is essentially limited by the uncertainties in the gaussian fitting routine used to measure individual arc lines.

The template arc-line list measured from one arc spectrum was used to calibrate all the other arc spectra, using a comparison function within the FIGARO software. In fact the shifts between arc lines during the course of a night's observation were typically less than about $3 \AA$ with the exception of the second AAT run where shifts of up to $6 \AA$ were measured. It should also be mentioned that each arc spectrum was fitted independently. A simpler, but perhaps less safe, alternative would be to apply some constant pixel offset to each spectrum, keeping the same dispersion relation each time. The former method is easy to implement within FIGARO however and was the one used here.

The object spectra were then wavelength-calibrated by associating them with the set of wavelength coefficients obtained from the fit to their corresponding arc spectrum. In preparation for subsequent steps, the spectra were next rebinned into coordinates linear in wavelength. Any separate exposures of the same objects were coadded together at this stage to form a composite spectrum.

\subsubsection{SMOOTHING THE SPECTRA}

The global shapes of the reduced spectra reflect the instrumental response of the spectrograph and detector coupled with the quartz calibrator. Smooth spectra standard stars were observed for removing these effects. Since we shall be needing to subtract the continuum prior to doing cross-correlations this is not a crucial stage in the reductions. However, often in the absence of obvious lines in the spectrum it is necessary to resort to looking for subtle changes in the shape of the continuum (e.g. the $4000 \AA$ 'break') as an indicator of redshift. 
The spectral standards that were observed are LTT2415 (Baldwin \& Stone 1983) and HD84937 (Oke \& Gunn 1983) which are both flux-standards. Calibration of the radio galaxy spectra onto a relative flux per unit frequency scale were achieved by performing a smooth interpolation between a set of points, representing flux-density at a given wavelength, being careful to avoid known absorption features in the stellar spectrum. Division by the resultant smooth calibration spectrum left the galaxy continua acceptably flat.

\subsubsection{REMOVING FEATURES BY INTERPOLATION}

Later, when we come to discuss cross-correlations, it will be remarked that all strong nightsky lines, emission lines and spurious features in the object spectra should be removed prior to application of the cross-correlation algorithm. Cosmic rays are the main contributors to the spurious features. It is difficult to find a technique for removing them from the raw 2-d spectral images while at the same time leaving narrow emission lines unscathed. Consequently these and the night-sky lines were cautiously removed by interpolating over them. Although the sky-subtraction generally worked well, the oxygen $5875 \AA$ line often remained in the reduced spectra and had to be interpolated out. As a precaution the location within the spectrum of any excised features was stored so that any anomalous shifts found by the cross-correlation routine could be checked.

\subsection{A Catalogue of Radio Galaxy Spectra}

Some 250 radio galaxy spectra were acquired during the course of this thesis. The majority of these are presented in Appendix B where they take the form of plots of flux (arbitrary units) against wavelength. It is obviously not possible to discuss each of these in turn, nor is it possible to discuss even a fraction of these in detail. Instead I present a short summary of some of the most commonly recurring features and present two of the more extreme examples of emission and absorption line spectra.

\subsubsection{SPECTRAL FEATURES}

A large proportion of the radio galaxy spectra show discernable spectral features of some 
kind or other. Table 3.4 gives a list of common absorption and emission line features, in the wavelength range of interest, that have been observed in radio elliptical galaxies (Corwin \& Emerson 1982; Costero \& Osterbrock 1977).

Aside from these single lines one can also often discern a striking feature known as the $4000 \AA$ 'break', due to a blend of calcium $H$ and $K$ absorption lines. It is manifest as an abrupt change in continuum shape. The prevalence of features like this in elliptical galaxy spectra give credence to an earlier point that was made about flattening the continuum with a smooth standard star spectrum.

The two most common features seen in the radio galaxy spectra are due to Mgb 5174 and the sodium D doublet at $5892 \AA$. The former is relatively broad, being a blend of three lines. Further common absorption features in the spectra are calcium and iron lines at 5591.0 and $5709.9 \AA$ respectively. A particularly good example of a strong absorption line spectrum is shown in Fig. 3.5 with some prominent features marked. These lines are hardly redshifted at all and cast suspicion on the identification of this object with the radio galaxy $2053-201$ which has a $B$ magnitude of $\sim 18$. Nevertheless it is shown here for illustrative purposes. Spectra with strong emission lines are much more frequently seen in radio galaxies than normal ellipticals. Their presence is usually linked to physical conditions in the active nuclear regions of the galaxy. Hine \& Longair (1979) discovered that optical spectral type is correlated with redshift (and consequently radio luminosity) in the sense that emission line spectra are more common in the higher redshift galaxies. By far the most common emission line feature seen in the radio galaxy spectra is the OIII line which has a rest wavelength of $5007 \AA$. It is not surprising that, in the light of Hine and Longair's discovery, this line is mainly seen in the higher redshift radio galaxies $(z \geq 0.09)$ in the sample such as $0124+117,0145+000,0213+025,0254+054,1127+012$. A particularly striking example of a narrow emission line radio galaxy is $1127+012$. The spectrum is shown in Fig. 3.6 with five prominent lines indicated. This galaxy has been identified as being of $\mathrm{N}$ type in the literature (Véron-Cetty \& Véron 1983). These objects are partly stellar in appearance and display low-level nuclear activity. 
Table 3.4: Some common features seen in the spectra of radio galaxies.

\begin{tabular}{|c|c|c|c|}
\hline \multicolumn{2}{|r|}{ absorption } & \multicolumn{2}{|c|}{ emission } \\
\hline$\lambda_{0}$ & feature & $\lambda_{0}$ & feature \\
\hline 3742.5 & FeI (+TiII ?) & 3346 & $\mathrm{Ne} \mathrm{V}$ \\
\hline 3769.0 & $\mathrm{H} \lambda+\mathrm{FeI}$ & 3426 & $\mathrm{Ne} \mathrm{V}$ \\
\hline 3794.5 & FeI & 3727 & O II \\
\hline 3798.6 & $\mathrm{H} \theta$ & 3869 & $\mathrm{Ne} I I I$ \\
\hline 3827.0 & FeI & 3889 & $\mathrm{He} \mathrm{I}$ \\
\hline 3834.7 & $\mathrm{H} \eta(+\mathrm{MgI}+\mathrm{FeI})$ & 3967 & $\mathrm{Ne}$ III \\
\hline 3879.1 & $\mathrm{FeI}$ & 3970 & $\mathrm{H} \epsilon$ \\
\hline 3888.7 & $\mathrm{H} \zeta$ & 4071 & S II \\
\hline 3933.4 & CaII & 4102 & $\mathrm{H} \delta$ \\
\hline 3969.2 & $\mathrm{CaII}+\mathrm{H} \epsilon$ & 4340 & $\mathrm{H} \gamma$ \\
\hline 4071.1 & $\mathrm{FeI}$ & 4363 & O III \\
\hline 4102.8 & $\mathrm{H} \delta$ & 4686 & $\mathrm{He}$ II \\
\hline 4227.8 & $\mathrm{CaI}$ & 4861 & $\mathrm{H} \beta$ \\
\hline 4272.1 & $\mathrm{FeI}+\mathrm{CrI}$ & 4959 & O III \\
\hline 4298.1 & $\mathrm{CH}$ etc. & 5007 & O III \\
\hline 4304.4 & $\mathrm{CH}$ etc. & 5199 & N I \\
\hline 4310.4 & $\mathrm{CH}$ etc. & 5309 & $\mathrm{Ca} \mathrm{V}$ \\
\hline 4340.5 & $\mathrm{H} \gamma$ & $5 \pi 21$ & Fe VII \\
\hline 4376.7 & $\mathrm{FeI}+\mathrm{CH}$ & 5876 & $\mathrm{He} \mathrm{I}$ \\
\hline 4384.1 & $\mathrm{FeI}$ & 6087 & $\mathrm{Fe}$ VII \\
\hline 4864.5 & $\mathrm{H} \beta$ & 6300 & O I \\
\hline 5166.6 & $\mathrm{MgI}$ & 6364 & O I \\
\hline 5174.0 & MgI & 6374 & $\mathrm{Fe} \mathrm{X}$ \\
\hline 5183.2 & $\mathrm{MgI}$ & 6548 & N II \\
\hline 5268.6 & $\mathrm{FeI}+\mathrm{CaI}($ or $\mathrm{MgH})$ & 6563 & $\mathrm{H} \alpha$ \\
\hline 5591.0 & $\mathrm{CaI}+\mathrm{NiI}$ & 6583 & N II \\
\hline 5709.9 & $\mathrm{FeI}+\mathrm{MgI}(+\mathrm{NiI} ?)$ & 6716 & S II \\
\hline 5847.6 & $\mathrm{FeI}(+\mathrm{NiI} ?)$ & 6731 & S II \\
\hline 5856.3 & $\mathrm{CaI}+\mathrm{FeI}$ (or $\mathrm{TiO})$ & & \\
\hline 5892.4 & $\mathrm{NaI}$ & & \\
\hline 5917.6 & FeI? & & \\
\hline 5948.2 & SiI ? & & \\
\hline 5955.3 & $\mathrm{FeI}+\mathrm{TiI}$ & & \\
\hline
\end{tabular}




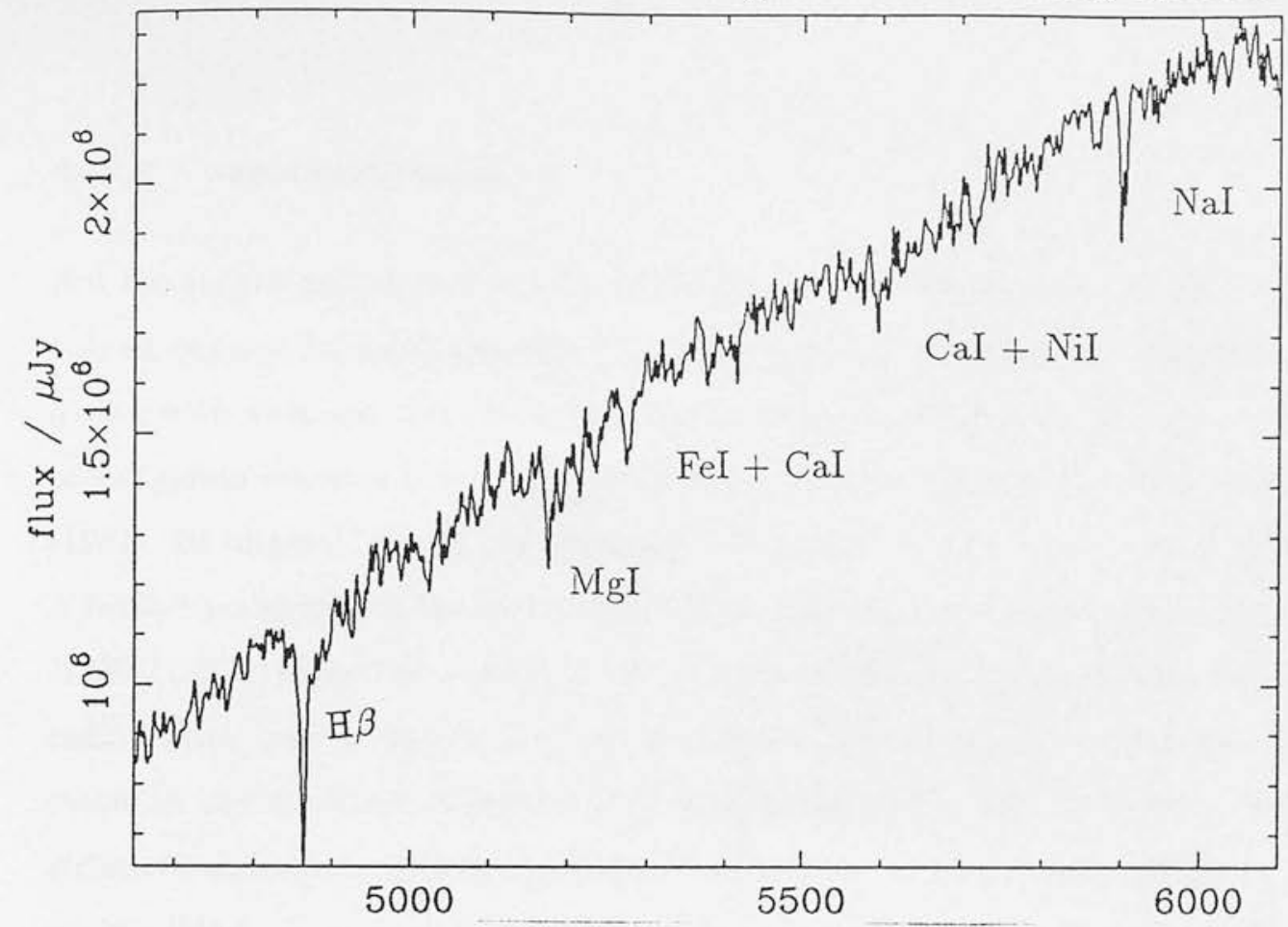

Fig. 3.5: Spectrum for 2053 - 201. Most of the absorption features which are evident in the other radio galaxy spectra are present here.

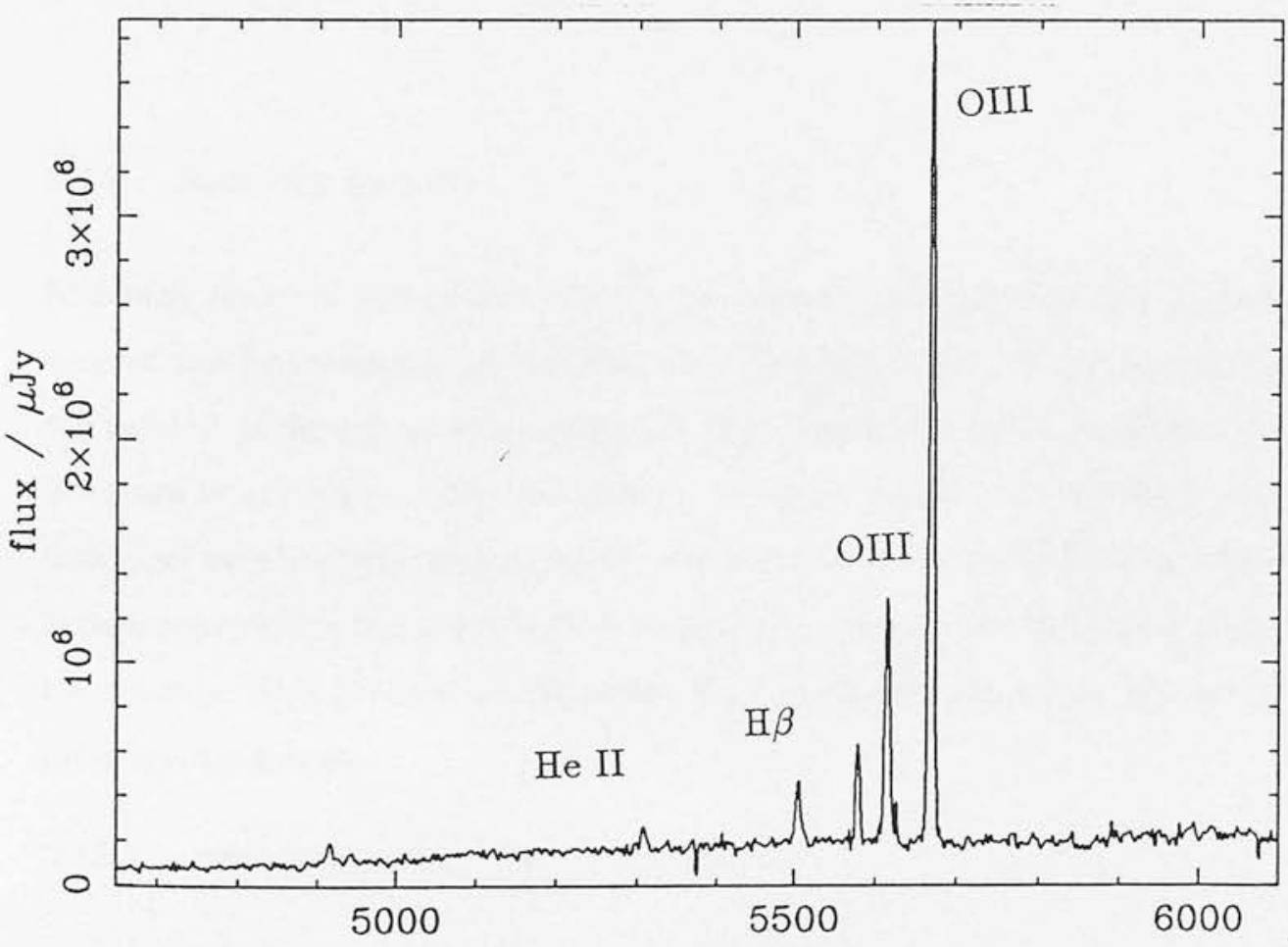

Fig. 3.6: Spectrum for $1127+012$. This galaxy is noted as being of $N$-type in the iterature. Its spectrum shows a variety of strong emission lines and is fairly atypical with egard to the rest of the radio galaxy spectra. 


\subsubsection{ANOMALOUS OBJECTS}

Not too surprisingly a very small percentage of the objects included in the initial sample turned out not be radio galaxies. A WHT spectrum of $0850+51 \mathrm{~b}$ revealed it to be a quasar with its broad MgII emission line and continuum shape suggesting $z \sim 1.3$. Further investigation revealed that this object had in fact been identified as such by Kühr et al. (1981). Its original inclusion in the sample has arisen via an error in Cohen et al. (1977). A further peculiarity is the spectrum for $2005-489$ which is clearly stellar. In fact Savage \& Wall (1976) expressed a suspicion that the most likely optical candidate for this Parkes radio source may in fact be a planetary nebula. The spectrum we have for this object seems to confirm their suspicion. The spectra for $0424-268$ and $0955+035$ are also stellar. It also seems that the optical counterpart to 2053-201, discussed above, has been misidentified. It is unclear whether these objects were originally misidentified as the optical components of radio sources or whether the wrong objects have been observed erroneously at the telescope. They have been retained in the sample pending further observation.

\subsection{Redshift Results}

Although many of the galaxy spectra show discernable features whose observed wavelengths can be measured and ratioed with their rest values to put a rough estimate on the redshift of the galaxy, this simple but crude approach obviously needs to be surpassed if we are to calculate radial velocities to the sort of accuracies needed for studying cosmological peculiar velocities. Cross-correlation techniques are able to achieve the desired accuracy as well as being much more reliable than the eye for identifying weak features in the spectra. This Section describes the cross-correlation algorithm and applies it to the radio galaxy spectra.

\subsubsection{THE CROSS-CORRELATION TECHNIQUE}

Prior to the cross correlation, a subset of each spectrum ranging from $4650 \AA$ to $6100 \AA$ was mapped to 1872 bins with coordinates linear in $\ln \lambda$. This wavelength range includes most of the common spectral features and avoids the atmospheric absorption contaminated red 
more suspect. This scale was such that a relative velocity of $\sim 44 \mathrm{kms}^{-1}$ shifts a feature by one bin. A further essential part of the pre-processing stage prior to cross-correlation was to excise sharp emission-line features in the spectra which can give rise to spurious velocity shifts.

The 'absorption-line only' spectra were next continuum subtracted by removing an eighth-order polynomial-fit to the continuum and apodizing with a cosine bell to remove any discontinuity between the beginning and end of the spectra. Experiments with continuum division rather than subtraction were found to make negligible difference to the derived redshifts. Finally, the spectra were bandpass-filtered to remove any residual curvature that remains in the continuum at low frequency as well as to attenuate the highest spatial frequency components due to noise.

Cross-correlation analysis is performed on absorption-line only spectra which have been linearly binned in $\ln \lambda$ such that a uniform linear shift in bins gives a measure of velocity redshift. In terms of observed $\left(\lambda^{\prime}\right)$ and emitted $(\lambda)$ wavelengths of a given spectral feature, the redshift is defined to be

$$
1+z=\lambda^{\prime} / \lambda
$$

and thus the velocity shift corresponding to the shift in bins between a given feature in the observed object spectrum and a template spectrum is

$$
\Delta z_{\text {shift }}=\mathrm{e}^{\Delta \ln \lambda}-1
$$

It is now straightforward to compute the absolute redshift of the object rather than its shift relative to the template. Denoting object and template with the suffices ' $O$ ' and ' $T$ ' respectively allows us to write that

$$
1+z_{0}=\lambda_{0}^{\prime} / \lambda_{0}=\left(\lambda_{0}^{\prime} / \lambda_{T}^{\prime}\right)\left(\lambda_{T}^{\prime}\right)\left(\lambda_{T}^{\prime} / \lambda_{0}\right)
$$

and

$$
1+z_{T}=\lambda_{T}^{\prime} / \lambda_{T}
$$

Dividing these and carrying the denominator over gives the following

$$
(1+z)_{0}=(1+z)_{T}(1+z)_{0 \rightarrow T}
$$


The cross-correlations were performed using a modified version of Dr. Philip Hill's SPICA code. As part of its output, this program provides an error estimate, $\delta$, in the cross-correlation function (CCF) peak according to the formulae derived by Tonry \& Davis (1979). Along with $\delta$, the CCF peak position, height and width are also returned. These are all useful parameters for assessing the reliability of the computed velocity shift.

An example cross-correlation function derived from correlating two galaxy spectra is shown in Fig. 3.7

This has been normalised so that a perfect correlation would give a peak height equal to unity. The CCF peak is quite unambiguous in this example although this is not always the case. A series of cross-correlations were performed with spectra consisting purely of random noise. This test indicated that no feature arising by chance has a peak height exceeding 0.2 .

\subsubsection{Internal errors in cross-correlation redshifts}

A thorough description of the many sources of error which can arise in slit spectroscopy has been given by Parker et al. (1986). A fundamental limit on the accuracy of the derived redshifts is set by the accuracy to which the spectra can be wavelength calibrated. The gaussian fitting to arc lines in the wavelength range from about $4500 \AA$ to $6000 \AA$ is sufficiently precise that any induced velocity errors should be no more than $30 \mathrm{kms}^{-1}$. Repeated measures of the OI and NaI sky lines at $5577 \AA$ and $5891 \AA$ lend support to this claim.

Tonry \& Davis (1979) describe how to evaluate internal errors arising from inaccuracies in the CCF peak position. The error is estimated according to

$$
E=\frac{N}{8 B(1+r)}
$$

where $N=2048$, the number of bins in the CCF, and $B$ is the half-maximum point in the CCF. The latter can be expressed simply in terms of the FWHM of the CCF peak,W

$$
B=2 N \times \frac{\ln 2}{\pi W}
$$




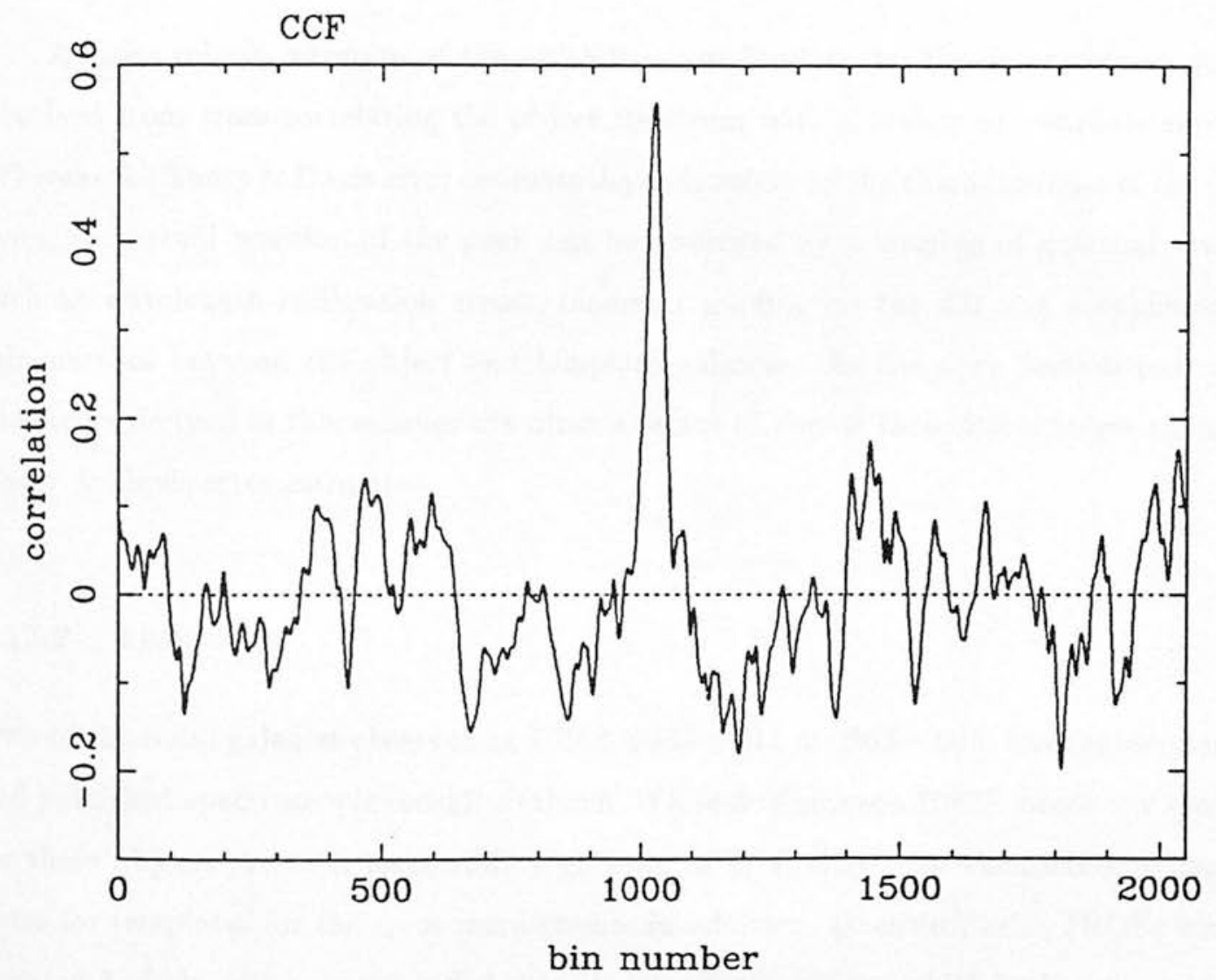

Fig. 3.7: Typical cross-correlation function of radio galaxy spectrum with template spectrum. The peak has been normalised to unity for an autocorrelation. 
The remaining parameter, $\mathrm{r}$, describes the significance of the peak which is greater the more symmetry it has. This is expressed as

$$
r=h / \sigma_{0} 2^{\frac{1}{2}}
$$

where $\mathrm{h}$ is the peak height (normalised to unity for an autocorrelation) and $\sigma_{0}$ is the rms of the antisymmetric component of the CCF. Combining these, gives as a final expression for the error

$$
E=0.283 W /(1+r)
$$

A more robust estimate of the redshift errors is given by the dispersion in values obtained from cross correlating the object spectrum with a variety of template spectra. Whereas the Tonry \& Davis error estimate depends solely on the characteristics of the CCF peak, the actual position of the peak can be governed by a number of external sources such as wavelength calibration errors, incorrect guiding on the slit and morphological mis-matches between the object and template galaxies. As the next Section indicates, the errors derived in this manner are often a factor of two or three times larger than the Tonry \& Davis error estimates.

\subsubsection{RESULTS}

Two of the radio galaxies observed at UH88, 0039 + $211 \& 0803-008$, have subsequently had published spectroscopic redshifts (Owen, White \& Thronson 1988). Since our spectra for these objects are at a reasonably high level of $\mathrm{S} / \mathrm{N}$, they offer themselves as candidates for templates for the cross-correlations. In addition, Quentin Parker (ROE) kindly donated 3 of his fully-reduced radial-velocity 'standard' spectra which he has successfully used as templates in the past (Parker et al. 1986). A full list of all the templates is given overleaf. 
Table 3.5: Galaxy and Stellar Templates

name

HD35410

HD203638

IC5175

$0039+211$

$0803-008$
$\mathrm{V}\left(\mathrm{kms}^{-1}\right)$

Ref.

Fehrenbach \& Duflot 1981

Evans, Menzies \& Stoy 1959

West \& Barbier 1982

Owen, White \& Thronson 1988

Owen, White \& Thronson 1988

These templates were used initially to estimate redshifts for a few of the radio galaxies with very strong absorption-line features which gave a sharp CCF peak and hence an accurate redshift. This increased the number of templates for then trying to extract a redshift from the poorer $\mathrm{S} / \mathrm{N}$ spectra. It is important to include a few radio galaxy spectra as templates since one is then cross-correlating like objects and the chances of any gross inaccuracies in the derived redshifts arising purely from morphological mis-matches is reduced. The reliability of the cross-correlation redshifts is discussed later.

\subsubsection{The radio galaxy redshifts}

A full list of results for the redshifts and their error estimates is given in Table 3.6

The CCF redshift, $\mathrm{V}_{\mathrm{ccf}}$, is a mean velocity derived from cross-correlations with several template galaxy spectra. The quoted standard deviation in this value has been carefully assessed from all the cross-correlations performed for each galaxy. It has not been divided by the square root of the number of templates used, and thus assumes that the scatter represents real differences in template spectral shapes. In addition to this more robust error estimate, the Tonry \& Davis error resulting from an inherent imprecision in the CCF peak position, $\sigma_{\mathrm{TD}}$, has also been tabulated. Noted that the latter estimates of the error are often much smaller than the error derived from comparing results of cross-correlations with several templates. 
Table 3.6: Redshift results for the all-sky sample members observed during the course of this thesis

\begin{tabular}{|c|c|c|c|c|c|c|c|}
\hline IAU & B & $\mathrm{v}_{\mathrm{ccf}}$ & $\sigma_{\mathrm{v}}$ & $\sigma_{\mathrm{TD}}$ & Telescope & $t_{\exp }$ & comments \\
\hline $0000-550$ & 14.0 & 9683 & 150 & 112 & $\operatorname{AAT}(1)$ & 3 & \\
\hline $0001-531$ & 14.5 & 9803 & 120 & 87 & $\operatorname{AAT}(1)$ & 3 & \\
\hline $0007+124$ & 17.8 & - & - & - & UH 88 R 1 & 20 & \\
\hline $0038+086$ & 17.5 & - & - & - & UH 88 R1 & 20 & $\left({ }^{*}\right)$ \\
\hline $0039+211$ & 16.5 & 30489 & 90 & 61 & UH $88 \mathrm{R} 1$ & 15 & \\
\hline $0040-065$ & 17.0 & - & - & 70 & UH 88 R1 & 15 & $\left(^{*}\right)$ \\
\hline $0043-424$ & 17.0 & 22904 & 300 & 225 & AAT (1) & 7 & \\
\hline $0043-638$ & 16.0 & 22215 & 420 & 120 & AAT (2) & & \\
\hline $0057-180$ & 18.0 & 29979 & 300 & 91 & UH 88 R 1 & 30 & $(*)$ \\
\hline $0058-507$ & 17.0 & 18407 & 270 & 104 & $\operatorname{AAT}(1)$ & 5 & \\
\hline $0115-261$ & 16.7 & 15529 & 40 & 120 & AAT (2) & & \\
\hline $0116-190$ & 18.0 & - & - & - & UH 88 R 1 & 30 & \\
\hline $0116+310$ & 14.9 & 17868 & 60 & 83 & INT & 22 & \\
\hline $0121+429$ & 16.8 & 9833 & 120 & 43 & UH $88 \mathrm{R} 1$ & 15 & \\
\hline $0124-117$ & 17.5 & 37054 & 180 & 43 & UH 88 R 1 & 15 & \\
\hline $0128+002$ & 16.1 & 23923 & 90 & 61 & UH 88 R1 & 5 & \\
\hline $0131-449$ & 17.5 & 27011 & 180 & 240 & AAT (2) & & \\
\hline $0137-177$ & 18.0 & - & - & - & UH 88 R 1 & 77 & \\
\hline $0144-552$ & 17.5 & 29200 & 240 & 120 & AAT (2) & & \\
\hline $0145+000$ & 16.5 & 27551 & 210 & 52 & $\mathrm{UH} 88 \mathrm{R} 1$ & 15 & \\
\hline $0157+405$ & 17.5 & 24433 & 180 & 91 & UH 88 R 1 & 10 & \\
\hline $0207+095$ & 17.0 & 26472 & 120 & 52 & UH 88 R 1 & 15 & \\
\hline $0208-067$ & 15.0 & 12591 & 150 & 48 & UH 88 R1 & 10 & \\
\hline $0213+025$ & 18.0 & 43410 & 240 & 43 & UH 88 R1 & 30 & \\
\hline $0213-132$ & 17.5 & 44789 & 180 & - & UH 88 (2) & & emission lines \\
\hline $0226-284$ & 18.0 & 62777 & 210 & - & AAT $(2)$ & & emission lines \\
\hline $0245-044$ & 17.5 & 40771 & 300 & 89 & UH 88 R1 & 15 & $(*)$ \\
\hline $0254+064$ & 17.5 & 39723 & 210 & 52 & UH 88 R1 & 20 & \\
\hline $0301-123$ & 17.0 & 30279 & 150 & 57 & UH $88 \mathrm{R} 1$ & 15 & \\
\hline $0305-131$ & 18.5 & - & - & - & UH 88 R3 & 50 & \\
\hline $0305-226$ & 18.5 & 80914 & - & 一 & AAT (2) & & $\left({ }^{*}\right)$ \\
\hline $0309-316$ & 18.5 & 76687 & 150 & - & AAT (2) & & emission lines \\
\hline $0312-400$ & 16.5 & 22724 & 570 & 150 & AAT (2) & & \\
\hline $0326-288$ & 17.5 & 32917 & 150 & - & AAT (2) & & emission lines \\
\hline $0326-461$ & 16.0 & 20686 & 270 & 150 & AAT (2) & & \\
\hline $0357-247$ & 18.5 & 30759 & 600 & 120 & AAT (2) & & \\
\hline $0359+193$ & 16.8 & 16279 & 60 & 69 & UH $88 \mathrm{R} 1$ & 15 & \\
\hline $0402+179$ & 18.0 & 33397 & 240 & 65 & UH 88 R 1 & 30 & \\
\hline $0419+140$ & 18.0 & 19278 & 180 & 87 & UH $88 \mathrm{R} 1$ & 30 & \\
\hline $0420-263$ & 18.5 & 39603 & 130 & - & AAT (2) & & emission lines \\
\hline $0424-268$ & 17.0 & 809 & 600 & - & AAT (2) & & $4000 \AA$ feature \\
\hline $0442-282$ & 18.5 & 44369 & 150 & - & AAT (2) & & emission lines \\
\hline $0454+066$ & 19.0 & - & - & - & UH $88 \mathrm{R} 3$ & 60 & \\
\hline $0456-301$ & 18.0 & 18887 & 180 & 120 & AAT (2) & & \\
\hline $0502-103$ & 15.4 & 11812 & 150 & 70 & UH 88 R 3 & 10 & \\
\hline $0509+011$ & 18.0 & 36574 & 300 & 70 & UH 88 R 1 & 60 & $\left(?^{*}\right)$ \\
\hline $0517-562$ & 17.0 & 28450 & 90 & 96 & CTIO & 10 & \\
\hline $0521-328$ & 18.0 & 63766 & - & - & AAT (2) & & $(*)$ \\
\hline $0522-483$ & 18.5 & 57170 & 180 & - & AAT (2) & & emission lines \\
\hline $0530+040$ & 19.0 & 44519 & 300 & 81 & UH88 R3 & 45 & $\left({ }^{*}\right)$ \\
\hline $0533-120$ & 17.8 & - & - & - & UH 88 R1 & 20 & \\
\hline
\end{tabular}




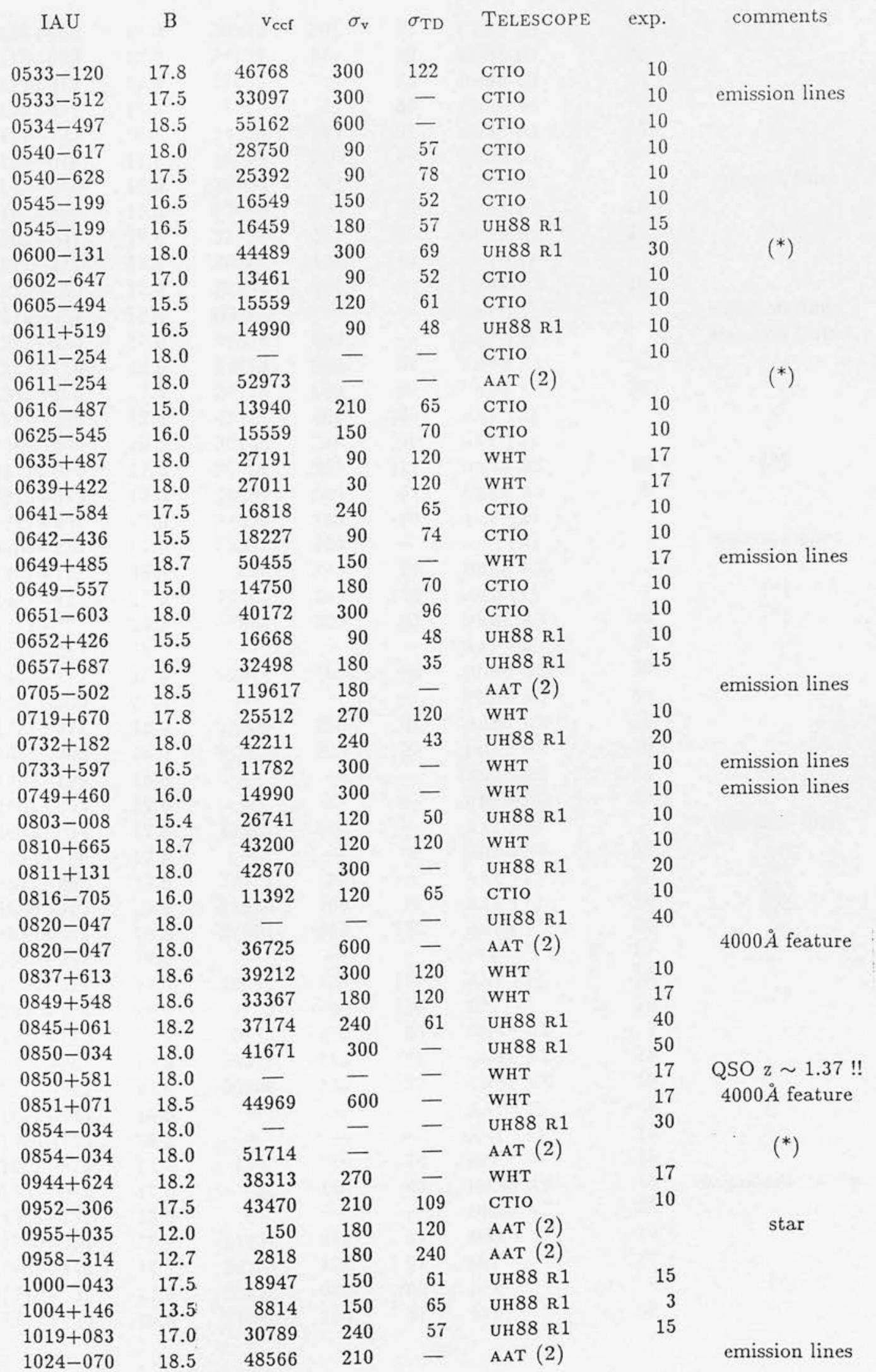


Table 3.6: cont.........

\begin{tabular}{|c|c|c|c|c|c|c|c|}
\hline IAU & $\mathrm{B}$ & $\mathrm{v}_{\mathrm{ccf}}$ & $\sigma_{\mathrm{v}}$ & $\sigma_{\mathrm{TD}}$ & Telescope & exp. & comments \\
\hline $1036+058$ & 18.0 & 36874 & 300 & 39 & UH $88 \mathrm{R} 1$ & 45 & $(*)$ \\
\hline $1118+000$ & 17.0 & 29739 & 180 & 52 & UH 88 R 1 & 25 & \\
\hline $1127+012$ & 17.8 & 39633 & 240 & 43 & UH 88 R 1 & 20 & \\
\hline $1130-037$ & 15.3 & 15469 & 90 & 39 & UH 88 R 1 & 10 & \\
\hline $1137+123$ & 16.5 & 24223 & 120 & 61 & UH 88 R 1 & 15 & \\
\hline $1139-078$ & 17.0 & 19516 & 210 & 48 & UH 88 R 1 & 20 & \\
\hline $1148-353$ & 18.0 & 37804 & 90 & - & $\mathrm{AAT}(2)$ & & emission lines \\
\hline $1151+099$ & 16.5 & 30459 & 210 & 52 & UH 88 R 1 & 15 & \\
\hline $1201-041$ & 18.0 & 31778 & 300 & 81 & UH 88 R2 & 20 & $(*)$ \\
\hline $1211-417$ & 17.5 & 20566 & 400 & 180 & $\operatorname{AAT}(2)$ & & \\
\hline $1215+039$ & 17.0 & 23534 & 300 & 87 & UH 88 R2 & 15 & \\
\hline $1215-457$ & 18.0 & 158021 & 150 & - & $\operatorname{AAT}(2)$ & & emission lines \\
\hline $1221-423$ & 17.0 & 51354 & 300 & - & $\operatorname{AAT}(2)$ & & emission lines \\
\hline $1227+119$ & 16.0 & 24613 & 210 & 57 & UH 88 R 1 & 15 & \\
\hline $1233+168$ & 17.5 & 20776 & 180 & 87 & UH 88 R 2 & 30 & \\
\hline $1254-286$ & 17.5 & 40232 & 400 & 270 & $\mathrm{AAT}(2)$ & & \\
\hline $1301-086$ & 16.5 & 30249 & 270 & 120 & AAT (2) & & \\
\hline $1312+089$ & 17.5 & 27101 & 219 & 111 & $\mathrm{UH} 88 \mathrm{R} 2$ & 25 & $(*)$ \\
\hline $1313+073$ & 15.5 & 15499 & 300 & 83 & UH 88 R 2 & 5 & \\
\hline $1317-407$ & 17.0 & 14930 & 180 & 90 & $\mathrm{AAT}(2)$ & & \\
\hline $1406-230$ & 17.5 & 25692 & 300 & - & AAT (2) & & emission lines \\
\hline $1407+177$ & 15.5 & 5066 & 240 & 87 & $\mathrm{UH} 88 \mathrm{R} 2$ & 5 & \\
\hline $1407-425$ & 15.5 & 15259 & 240 & 100 & $\operatorname{AAT}(1)$ & 7 & $(*)$ \\
\hline $1411+094$ & 18.3 & 47667 & 300 & 81 & UH 88 R 3 & 45 & $(*)$ \\
\hline $1414-212$ & 18.0 & - & - & - & $\operatorname{AAT}(1)$ & 17 & \\
\hline $1423-177$ & 18.0 & 32078 & 300 & 83 & UH $88 \mathrm{R} 2$ & 30 & \\
\hline $1426+030$ & 18.5 & - & - & - & UH 88 R3 & 50 & \\
\hline $1427+074$ & 18.0 & 15859 & 210 & 87 & $\mathrm{UH} 88 \mathrm{R} 2$ & 10 & \\
\hline $1443-085$ & 16.0 & 21825 & 300 & 91 & $\mathrm{UH} 88 \mathrm{R} 2$ & 10 & \\
\hline $1449-129$ & 18.0 & - & - & - & UH 88 R 2 & 30 & \\
\hline $1452-054$ & 17.0 & - & - & - & UH88 R2 & 15 & \\
\hline $1452-054$ & 17.0 & 11182 & 180 & - & $\mathrm{AAT}(2)$ & & emission lines \\
\hline $1453+166$ & 17.5 & - & - & - & $\mathrm{UH} 88 \mathrm{R} 2$ & 25 & \\
\hline $1517-283$ & 17.5 & 36725 & 300 & - & $\operatorname{AAT}(1)$ & 10 & $(*)$ \\
\hline $1520-329$ & 18.0 & 22335 & 700 & 89 & AAT (1) & 14 & $\left(^{*}\right)$ \\
\hline $1538+082$ & 16.5 & 37924 & 210 & 103 & $\mathrm{UH} 88 \mathrm{R} 2$ & 20 & \\
\hline $1549+202$ & 15.0 & - & - & - & INT & 90 & \\
\hline $1553-328$ & 17.5 & 19666 & 180 & 101 & $\operatorname{AAT}(1)$ & 16 & \\
\hline $1557+708$ & 14.0 & 7735 & 90 & 130 & INT & 14 & \\
\hline $1601+173$ & 17.5 & 10613 & 270 & 83 & UH 88 R 2 & 5 & \\
\hline $1603+001$ & 16.5 & 18317 & 210 & 91 & UH88 R2 & 15 & \\
\hline $1616-029$ & 16.6 & 20536 & 240 & 122 & UH $88 \mathrm{R} 2$ & 10 & \\
\hline $1617-235$ & 16.5 & - & - & - & AAT (1) & 6 & $(?)$ \\
\hline $1625-750$ & 18.0 & - & - & - & AAT (1) & 15 & . \\
\hline $1637+820$ & 14.0 & 7345 & 210 & 78 & INT & 15 & \\
\hline $1643+022$ & 17.0 & 450 & 120 & 63 & UH 88 R 2 & 15 & magnitude wrong? \\
\hline $1654-137$ & 18.0 & - & - & - & UH $88 \mathrm{R} 2$ & 30 & \\
\hline $1706+094$ & 15.7 & 11452 & 210 & 87 & UH $88 \mathrm{R} 2$ & 10 & \\
\hline $1707+340$ & 17.2 & 24133 & 180 & 91 & INT & 28 & \\
\hline $1719+242$ & 17.5 & 26532 & 600 & 100 & INT & 40 & $(*)$ \\
\hline $1733+710$ & 16.0 & 17808 & 150 & 87 & INT & 30 & \\
\hline
\end{tabular}




\begin{tabular}{|c|c|c|c|c|c|c|c|}
\hline IAU & B & $\mathrm{v}_{\mathrm{ccf}}$ & $\sigma_{\mathrm{v}}$ & $\sigma_{\mathrm{TD}}$ & Telescope & exp. & comments \\
\hline $1739+173$ & 15.9 & 19397 & 180 & 78 & UH 88 R 2 & 10 & \\
\hline $1740+162$ & 14.5 & 10373 & 270 & 74 & UH 88 R 2 & 6 & \\
\hline $1754+626$ & 15.0 & 8274 & 210 & 96 & INT & 30 & \\
\hline $1801-702$ & 16.5 & 11842 & 240 & 89 & $\operatorname{AAT}(1)$ & 4 & \\
\hline $1814-766$ & 14.0 & 5666 & 210 & 74 & $\operatorname{AAT}(1)$ & 4 & \\
\hline $1819+689$ & 16.4 & 26442 & 180 & 91 & INT & 35 & \\
\hline $1833-772$ & 15.0 & 5336 & 150 & 190 & $\operatorname{AAT}(1)$ & 2 & \\
\hline $1839-486$ & 17.5 & 32677 & 240 & 62 & $\operatorname{AAT}(1)$ & 7 & $(*)$ \\
\hline $1847-796$ & 17.0 & 22934 & 270 & 109 & $\operatorname{AAT}(1)$ & 10 & \\
\hline $1921-577$ & 16.0 & 17838 & 180 & 182 & $\operatorname{AAT}(1)$ & 15 & \\
\hline $1922-430$ & 18.0 & 24433 & 210 & 100 & $\operatorname{AAT}(1)$ & 10 & \\
\hline $1950+671$ & 18.4 & 22065 & 150 & 87 & INT & 30 & \\
\hline $2005-489$ & 16.5 & - & - & - & AAT (1) & 2 & planetary nebula !! \\
\hline $2049+175$ & 18.0 & 33705 & 400 & 93 & UH 88 R 2 & 30 & $\left(^{*}\right)$ \\
\hline $2053-201$ & 17.8 & 540 & 120 & 87 & UH88 R1 & 15 & id?? \\
\hline $2054-581$ & 16.0 & 15559 & 240 & 109 & $\operatorname{AAT}(1)$ & 5 & \\
\hline $2056-369$ & 18.0 & 32305 & 240 & 193 & AAT (1) & 12 & $\left({ }^{*}\right)$ \\
\hline $2101-715$ & 16.5 & 22215 & 120 & 100 & $\operatorname{AAT}(1)$ & 10 & \\
\hline $2117-269$ & 18.0 & 27731 & 300 & - & AAT (1) & 13 & $4000 \AA$ 'break' feature \\
\hline $2123+007$ & 17.5 & 40322 & 300 & 78 & $\mathrm{UH} 88 \mathrm{R} 1$ & 15 & \\
\hline $2134-281$ & 16.0 & 21435 & 300 & 101 & AAT (1) & 10 & \\
\hline $2148-555$ & 16.0 & 11542 & 300 & 176 & $\operatorname{AAT}(1)$ & 3 & \\
\hline $2146-016$ & 18.0 & - & - & - & INT & 115 & \\
\hline $2148-427$ & 16.3 & 19067 & 270 & 145 & AAT (1) & 3 & \\
\hline $2156-564$ & 16.0 & 22694 & 120 & 122 & AAT (1) & 3 & \\
\hline $2206-251$ & 18.0 & - & - & - & $\operatorname{AAT}(1)$ & 15 & \\
\hline $2212+135$ & 14.3 & 7345 & 180 & 78 & UH 88 R2 & 5 & \\
\hline $2212+135$ & 14.3 & 7345 & 180 & 69 & UH 88 R 2 & 3 & \\
\hline $2225+100$ & 17.5 & 40322 & 150 & 74 & UH 88 R 1 & 20 & \\
\hline $2229-086$ & 15.5 & 24763 & 180 & 52 & UH 88 R 1 & 10 & \\
\hline $2235+408$ & 17.0 & 17088 & 150 & 43 & UH 88 R 1 & 15 & \\
\hline $2236-176$ & 16.0 & 15289 & 210 & 89 & UH 88 R 2 & 10 & \\
\hline $2253-465$ & 17.5 & 25482 & 240 & 49 & AAT (1) & 10 & \\
\hline $2309-416$ & 17.0 & 28480 & 240 & 62 & AAT (1) & 10 & $(*)$ \\
\hline $2316-423$ & 16.0 & 16279 & 150 & 82 & AAT (1) & 7 & \\
\hline $2317-277$ & 17.5 & 29110 & 240 & 130 & AAT (1) & 15 & $\left({ }^{*}\right)$ \\
\hline $2320+203$ & 14.5 & 11512 & 180 & 52 & UH 88 R 1 & 5 & \\
\hline $2320+417$ & 18.0 & 44519 & 240 & 87 & UH 88 R1 & 15 & \\
\hline $2327-215$ & 18.0 & 23234 & 270 & 123 & AAT (1) & 15 & \\
\hline $2338-001$ & 17.2 & - & - & - & UH 88 R 1 & 20 & \\
\hline $2353-184$ & 15.5 & 21675 & 150 & 109 & INT & 40 & \\
\hline
\end{tabular}

$\left({ }^{*}\right)$ treat redshift with caution. Only reproducible with a few templates but seem reasonable either on the basis of its magnitude or else associating very weak absorption features in the spectrum with $\mathrm{Mgb}$ or $\mathrm{NaD}$.

(?) spectrum is rather :odd. Foreground contaminating object perhaps. 
The rate of success for producing a consistent redshift via the cross-correlation technique is good. The main failures were usually for galaxies with very weak $S / N$. Crosscorrelation has been able to produce redshifts for many of the spectra which appear to the eye to be completely featureless. A few of the galaxies in Table 3.6 have a tentative redshift only in the sense that a sensible and consistent value emerged upon cross-correlation with only a few of the template spectra and varied wildly when others were used. These galaxies have been flagged with an asterisk. A number of objects failed to give an absorption line redshift but the present of a few emission lines meant that a fairly accurate redshift could still be measured.

Although the cross-correlation routine failed on only a few occasions to give a sensible redshift, the rms errors were often rather large. On average these were about $150 \mathrm{kms}^{-1}$ but worsened for the poorer $\mathrm{S} / \mathrm{N}$ objects. It is important to test whether there are any systematics in the derived velocities. The galaxy redshifts have effectively been zeropointed to the system of templates. We now discuss a test to check for this.

\subsubsection{COMPARISON WITH PUBLISHED REDSHIFTS}

The Huchra (unpublished at time of writing) and Fairall \& Jones (1988) galaxy redshift catalogues contain entries for every galaxy which has been observed spectroscopically and a radial velocity measured. They are invaluable source of reference not only for this work but for other projects also. These catalogues were acquired in machine-readable form and scanned for objects which coincided, to within the optical position error bars, with our radio galaxies. Fourteen such candidates were found. These are listed in Table 3.7 together with their positions, heliocentric redshifts and redshift errors.

The catalogue objects selected for 0043 - 424 and 0816 - 705 stand out as probable mis-identifications. For 0816 - 705 this is not unreasonable since a glance at the finding chart of the radio galaxy alerts one to the fact that this galaxy resides in a rich field with many nearby neighbours. However, $0043-424$ is a bit more of a puzzle. There are no nearby neighbours to this radio galaxy making mis-identification a less likely explanation. The CCF redshift is secure, to within $300 \mathrm{kms}^{-1}$, from cross-correlation with several templates. Also the spectrum reveals a $4000 \AA$ break at a wavelength that the puts the 


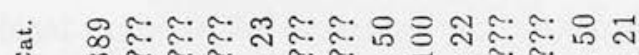
施 H

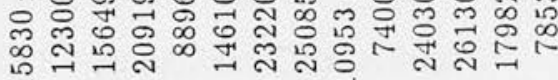

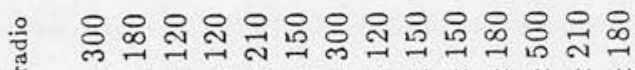
$\mathrm{H} H \mathrm{H} H \mathrm{H} H \mathrm{HH} H \mathrm{H} H \mathrm{H}$

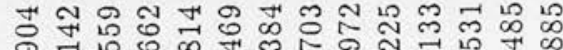

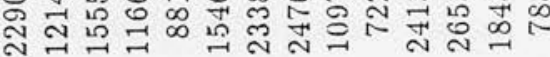

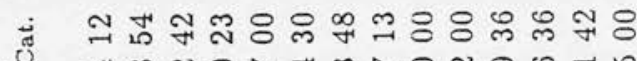

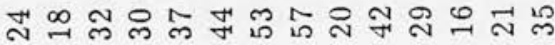

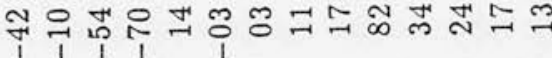

ธึ่

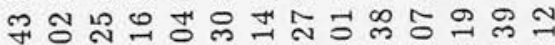

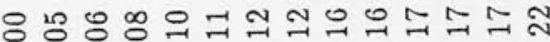

ๆ

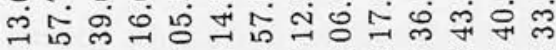
స్

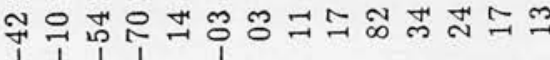

으의 ம்

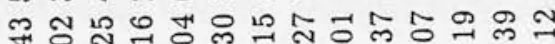

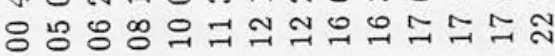

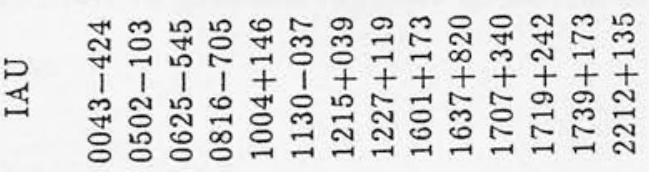


radio galaxy at its CCF redshift. It may mean then that the catalogue entry for the object that coincides with $0043-424$ is erroneous.

Unfortunately, only eight of the catalogue entries which duplicate the radio galaxies have a redshift error. Like the photometry, these numbers are rather too few to put beyond doubt the issue as to whether there are any systematics in the data. However, the results do at least seem to be encouraging. Given that it is a ratio or product of $(1+z)$ 's rather than $z$ itself that is used for the CCF redshift estimate, Fig. 3.8 plots the logarithmic residuals of $(1+z)$ against CCF redshift.

The filled in points are the most important ones from the point of view of a statistical analysis since both measurements have an estimate of the redshift error. According to Fig. 3.8, the points are reasonably well scattered about zero indicative of no systematics in the radio galaxy redshift data. For the 8 galaxies with redshift errors, the chi-squared statistic turns out to be $\chi^{2} \simeq 2$. In summary then the radio galaxy redshifts compare favourably with literature redshifts with no evidence for any systematic differences. More points on Fig. 3.8 would have been desirable to increase the confidence of this assertion.

\subsection{Summary}

A radio galaxy redshift survey which had as its aim to complete the redshift data for the all-sky sample has been undertaken. Some 250 redshifts have been measured boosting the redshift completeness of the sample to about $90 \%$. The cross-correlation technique was found to yield a redshift for all except the very weakest $\mathrm{S} / \mathrm{N}$ spectra. In many cases the error bars were rather large though, ranging from $100 \mathrm{kms}^{-1}$ through to $600 \mathrm{kms}^{-1}$ with a median value of about $150 \mathrm{kms}^{-1}$. Comparing measured redshifts with literature values for a small number of galaxies suggests good agreement with no evidence for any systematic differences. 


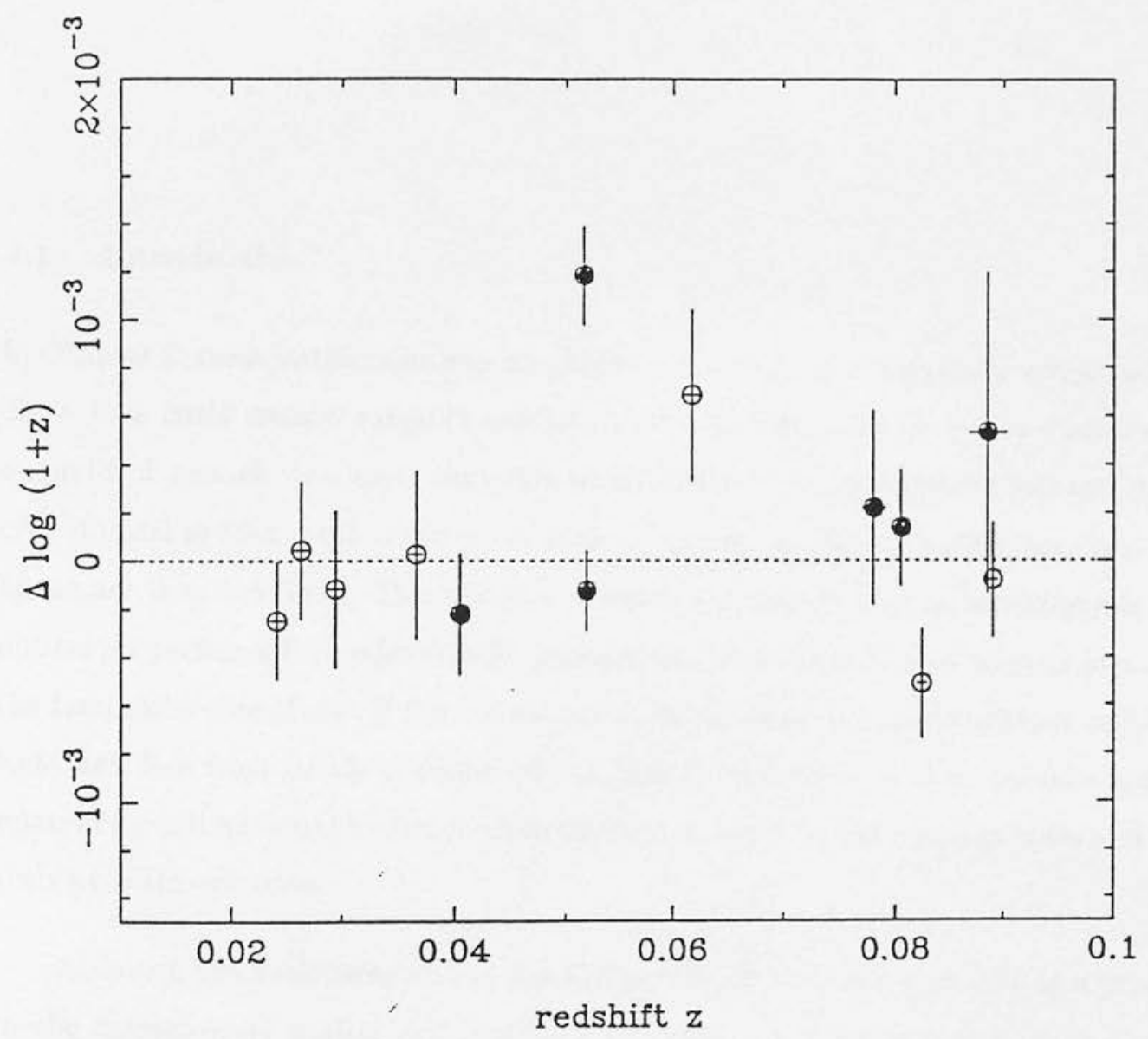

ig. 3.8: Results of comparison between measured redshifts and literature values given the Huchra and Fairall catalogues. The filled in points are for galaxies whose catalogue dshifts have a quoted rms error. 


\section{Chapter 4}

\section{The Cluster Environment and Optical Prop- erties of Low-Redshift Radio Galaxies}

\subsection{Introduction}

In Chapter 2, some justification was sought for restricting the radio galaxy sample selection down to a fairly narrow range in redshift, spanning only a factor ten in distance. The unqualified remark was made that this would likely yield an optically homogeneous set of statistical probes. Such a sample is desirable for cosmological studies since systematic biases are then less likely. This Chapter is concerned mainly with an investigation of the optical properties of low-redshift radio galaxies and, in particular, derives their location on the luminosity-size plane. If the correlation between these two observables is sufficiently tight and free from subtle selection effects, then it can be used as a distance indicator relation for estimating the relative photometric distances for the radio galaxies and hence their peculiar velocities.

Although the work presented in this Chapter should be viewed mainly as a precursor to the cosmological studies undertaken in the next two Chapters, it is clear that the all-sky sample database can potentially offer many new insights into the radio galaxy phenomenon. I have chosen here to focus attention onto testing the purported relations between the radio and optical properties of radio galaxies with their cluster environment.

The layout of this Chapter is as follows. Section 4.2 introduces $B_{g r}$, the amplitude of the spatial cross-correlation function, which is used to quantify the richness of environment for the radio galaxies. It is shown that, in the absence of a full set of galaxy counts for the southern hemisphere, the Abell cluster catalogue can be used to get a reasonable estimate of $B_{g r}$. Although good quality radio maps are scarce for the all-sky sample members, it is still possible to differentiate between the FRI and FRII classes roughly 
on the basis of radio luminosity. This is done in Section 4.3 and the results of a test for correlations between FR class and $B_{g r}$ are presented. There has been some evidence in the past to suggest that the intermediate power radio sources prefer markedly richer environments than their high- and low- power counterparts. Section 4.4 considers the optical properties of low-redshift radio galaxies - this is a far more relevant question as far as the remaining few Chapters in this thesis are concerned. In particular, we wish to establish the location of low-redshift radio galaxies on the luminosity-size plane. This has generally been discussed in the past in terms of an $M-\alpha$ relation, but for the present applications it is more convenient to re-cast this relation in terms of an intrinsic surface brightness - effective size $\left(\mu_{e}-r_{e}\right)$ relation. Optical profile fits are presented and used to derive an empirical set of structural parameters for each galaxy. The implications of a luminosity-size relation for radio galaxies are discussed in Section 4.5. In Section 4.6 the colour-magnitude diagram is derived. Section 4.7 examines whether there is any evidence for correlations between the optical properties of radio galaxies with their cluster environment. Finally, a short summary is presented in Section 4.8.

\subsection{The Cluster Environment of Radio Galaxies}

The amplitude of the spatial cross-correlation function $B_{g g}$ is well-known in the context of measuring the local cluster environment of radio galaxies (Longair \& Seldner 1979; Prestage \& Peacock 1988) and quasars (Yee, \& Green 1987). In order to measure this parameter effectively, a full set of galaxy counts is ususally required. Such a catalogue exists for the northern declination zone bounded by $\delta=-23^{\circ}$. This is the Lick Galaxy Catalogue compiled by Shane \& Wirtanen from painstaking eyeball-scans of photographic plates and published in 1967 (Shane \& Wirtanen 1967). There is, at present, no corresponding set of counts for the southern hemisphere. The main purpose of this Section is to explain how to implement the Abell Cluster Catalogue (Abell, Corwin \& Olowin 1989) for estimating $B_{g g}$ and to show that these estimates are reasonable.

\subsubsection{The Cluster parameter $B_{g g}$}

The correlation function $\xi(r)$ is undoubtedly the most ubiquitous tool for studying clus- 
tering of galaxies. According to definition

$$
n(r) d V=\rho[1+\xi(r)] d V
$$

where $n(r)$ is the number of galaxies in a volume element $d V$ at distance $r$ from the radio source and $\rho$ is the mean number density of galaxies. Taking $\xi(r)$ to have power-law form with an index of -1.77 (Groth \& Peebles 1977) gives

$$
\xi(r)=B_{g r}\left(\frac{h \mathrm{r}}{\mathrm{Mpc}}\right)^{-1.77}
$$

where the amplitude $B_{g r}$ is directly related to the number of excess galaxies around the source. Prestage \& Peacock (1988; hereafter PP) have shown that the exact choice of power-law index is unimportant providing it is close to 2 for all sources. Instead of a spatial correlation function one usually measures an angular correlation function defined as

$$
w_{z}(\theta)=A_{g r}(z)\left(\frac{\theta}{\text { deg. }}\right)^{-0.77}
$$

where

$$
A_{g r}(z)=H(z) B_{g r}
$$

$A_{g r}$ can be measured directly from the source positions and galaxy catalogue and $H(z)$ is a conversion function which allows for the effect of different magnitude limits on the observability of clustering at different redshifts (Longair \& Seldner 1979; hereafter LS).

In practice, the way to compute $A_{g r}$ is by noting that the 2-d analogue of equation (41) is simply

$$
N(\theta) d \Omega=N_{g}[1+w(\theta)] d \Omega
$$

where $N(\theta) d \Omega$ is the number of galaxies in solid angle $d \Omega$ at angular distance $\theta$ from the radio source and $N_{g}$ is the average surface density of galaxies. Then from equations (4.4) and (4.5)

$$
\int N(\theta) d \Omega=\int N_{g} d \Omega+N_{g} A_{g r} \int \theta^{-(1.77-1)} d \Omega
$$

which, following PP, we choose to write as

$$
N_{\mathrm{obs}}=N_{\mathrm{bc}}+N_{g} A_{g r} J
$$

where $N_{o b s}$ is the number of galaxies observed to lie within $\theta$ of the source position and $N_{b c}$ is the expected number of background objects in an identical area. $J$ is an integral of the 
correlation function over this area, i.e. $J=\int \theta^{-(1.77-1)} d \Omega$. There are $N_{t}=\left(N_{\mathrm{obs}}-N_{\mathrm{bc}}\right)$ objects physically associated with the radio galaxy and thus

$$
A_{g r}=\frac{N_{t}}{N_{g} J}
$$

Clearly $A_{g r}$ can be simply regarded as a measure of the ratio of the surface number density of galaxies associated with the source to the field number density, with the integral $J$ accounting for the effects of the area used to count galaxies and the radial profile of the nearby galaxy distribution.

The conversion function $H(z)$ is written as

$$
H(z)=\frac{I}{N_{g}}\left(\frac{D}{1+z}\right)^{3-1.77} \phi\left(m_{\lim }, z\right)
$$

where $I$ is a definite integral and $D$ is the comoving distance to the source. $\phi\left(m_{\lim }, z\right)$ is the integral number of galaxies per unit volume which at redshift $z$ are observed to be brighter than the apparent magnitude limit of the galaxy count survey: for the Lick counts $m_{\text {lim }} \sim 18.7$. Also absorbed into $H(z)$ are the Hubble constant, density parameter, galaxy $K$-correction and the characteristic magnitude, slope and normalisation of the luminosity function for galaxies. The choice of these parameters is limited however by observations of galaxy number-magnitude relations (e.g. Shanks 1984 et al.). In any case PP have shown that varying the parameters changes $H(z)$ by generally less than $20 \%$, increasing somewhat at low and high values of $z$. For these calculations an identical set of parameter values to those chosen by PP are adopted. Note that they use $h=0.5$ whereas LS work with $\mathrm{h}=1$.

\subsubsection{RESULTS}

Firstly, the Lick catalogue was used to derive $A_{g r}$ for the sources north of $-23^{\circ}$. The Lick counts of galaxies (Shane \& Wirtanen 1967) are binned number counts and the counts from all bins with centres lying within $1 \mathrm{Mpc}$ of the source position were included in a summation to find $N_{\text {obs }}$. The choice of a $1 \mathrm{Mpc}$ rather than a $1^{\circ}$ counting radius (used by LS) is preferable for the fairly obvious reasons cited by PP. Similarly, $N_{g}$ can be estimated from the mean count within all bins between $3^{\circ}-5^{\circ}$ away from the source. Note that the resulting estimates of Agr are not very sensitive to the width of 
of the background annulus nor its distance from the source position. The integral $J$ is performed numerically over an identical area to that used for $N_{\text {obs }}$. Errors on $A_{g r}$ were also computed in an identical manner to that described by PP, essentially by just placing down search apertures at random locations in the catalogue and calculating the scatter in $A_{g r}$.

Now consider the radio galaxies occupying the region south of $\delta=-23^{\circ}$ which is not covered by the Lick counts. As indicated earlier, the Abell Cluster Catalogue can be used to derive a reasonable estimate of $B_{g r}$ for these radio galaxies. This can be seen as follows. Firstly, recall that $B_{g r}$ measures a luminosity overdensity or number excess of galaxies at a given location. For an observed excess $\Delta N$ of galaxies around a source, the correlation function may be integrated over a cone defined by the 2 -d data

$$
\Delta N=\int d \Omega \phi\left(m_{\lim }, z\right) \theta^{1-\gamma} D^{3-\gamma}(1+z)^{\gamma-3} B I_{\gamma}
$$

For a fixed metric distance $x=\theta D /(1+z)$ this reduces to

$$
\Delta N=\frac{2 \pi}{3-\gamma} \phi B x^{3-\gamma} I_{\gamma}
$$

For a power-law index of 1.77 and noting that the search radius used by Abell to count galaxies, based on the distance estimated from the magnitude of the tenth brightest cluster member, is equal to $3 \mathrm{Mpc}(h=0.5)$ we obtain

$$
\Delta N=70 B_{g r} \phi
$$

The idea then is to compute $\phi$ and hence $B_{g r}$ using some information about the criteria used by Abell to assign galaxies to clusters. To do this we will parameterise $\phi$ with a Schechter function of slope -1.25 . Accordingly, the number of galaxies in the range of luminosity from $L$ to $L+d L$ is given by

$$
N(L)=\phi_{*} \int_{L}^{L+d L} \frac{L^{-1.25}}{L_{*}} \mathrm{e}^{-L / L *} d \frac{L}{L_{*}}
$$

The strategy employed by Abell in his original work (Abell 1958) was to count galaxies that fell within the magnitude range from $m_{3}$ to $m_{3}+2$, where $m_{3}$ is the magnitude of the third brightest cluster member, and lay inside the pre-defined metric search radius. He then defined six classes of cluster richness according to population, the details of which are tabulated below: 
Table 4.1: Abell Richness Classes

\begin{tabular}{|c|c|}
\hline \multicolumn{2}{|c|}{ Abell Richness Classes } \\
\hline $\mathrm{N}$ & $\mathrm{R}$ \\
\hline $30-49$ & 0 \\
$50-79$ & 1 \\
$80-129$ & 2 \\
$130-199$ & 3 \\
$200-299$ & 4 \\
$300+$ & 5 \\
\hline
\end{tabular}

Knowing the Abell magnitude limit and the excess number of galaxies for a given richness class it should be possible to compute a corresponding $\left(\mathrm{L} / \mathrm{L}_{*}\right)_{\lim }$ by using the Abell criterion, which, in our previous notation is $N\left(m_{3}+2\right) / N\left(m_{3}\right)=\Delta N / 3$. Schechter functions corresponding to the appropriate limiting magnitudes have been plotted in Fig. 4.1 , where we have taken $\phi_{*}=2.2 \times 10^{-3} \mathrm{Mpc}^{-3}$. If a mean number excess for each Abell cluster richness class is assumed then a limiting value of $L / L_{*}$ can be read off from the curves.

The expected number of galaxies down to these limiting luminosities can be computed by integrating equation (4.14) between $\left(L / L_{*}\right)_{l i m} \rightarrow \infty$. The results are tabulated below together with the estimates of $B_{g r}$ which these values yield upon substitution into equation (4.13). The values should be compared with mean estimates of $B_{g g}$ derived by PP for each of the Abell richness classes (NB these values were originally in error and accordingly have have been corrected upward by the numerical factor 2.537, Prestage \& Peacock 1989).

Table 4.2: $\quad B_{g g}$ estimates for Abell clusters

\begin{tabular}{|ccccc|}
\hline $\mathrm{R}$ & $\overline{\Delta N}$ & $\left(\mathrm{~L} / \mathrm{L}_{*}\right)_{\lim }$ & $\overline{B_{g g^{*}}}$ & $\overline{B_{g g^{*}}}(\mathrm{PP})$ \\
\hline 0 & 40 & 0.22 & 191 & 289 \\
1 & 60 & 0.30 & 410 & 690 \\
2 & 105 & 0.37 & 852 & 984 \\
\hline
\end{tabular}

The agreement is reasonable given the uncertainties in $\phi_{*}$ and limiting Lick magni- 
tude, in addition to the considerable differences between search radii that have been used for each calculation. A crude average of these tabulated results suggests that the Abell $B_{g r}$ should be computed from the cluster overdensity as roughly $9 \Delta N$.

The procedure for calculating $A_{g r}$ for a galaxy using the Abell catalogue was the following. Firstly, a comoving separation was computed between each radio source and all of the Abell clusters. If spectroscopic redshifts were not available for the clusters then a redshift was predicted from an empirical cluster magnitude-redshift relation constructed for the tenth brightest cluster members. If the source-cluster comoving separation exceeded $10 \mathrm{Mpc}$, the cluster was skipped and the next one read. Beyond this distance there is negligible power in the angular cross-correlation function $\left(w(\theta) \propto \theta^{-0.77}\right)$. If the radio source was found to sit in the centre of the cluster then the contribution to $A_{g r}$ from that cluster is given simply by calculating 9 times the cluster number overdensity, according to our earlier result. Otherwise, a two-dimensional de-projection is required. Thus for a source located at $(x, \phi)$ and a source-cluster centroid separation of $\theta$, assuming circular symmetry the contribution from the cluster to $A_{g r}$ is given by the integral

$$
A_{g r}^{\text {deproj. }}=\frac{A_{g r} \times 1.23}{2 \pi} \int_{0}^{1} \int_{0}^{2 \pi}\left(x^{2}+\theta^{2}-2 x \theta \cos \phi\right)^{\frac{-0.77}{2}} \cos \theta d \theta d x
$$

where the upper limit of $1^{\circ}$ on the integral over $x$ corresponds to about $10 \mathrm{Mpc}$ at a redshift of $z=0.1(h=0.5)$. These values require two small corrections: (i) to account for clusters that are not near to the source in redshift space, and (ii) to eliminate contributions from background galaxies. The first was achieved by rejecting clusters with redshift $z_{c}$ relative to the source redshift $z_{s}$ such that $\log \left|\frac{z_{c}}{z_{s}}\right|>0.5$. The reference annulus chosen for the background correction was enclosed by arcs at $2^{\circ}$ and $7^{\circ}$ from the source and the correction term computed by solving a similar integral to that given by equation 4.14 . The most appropriate Abell estimate of $A_{g r}$ to compare with the Lick values however should only have had the background correction made.

In order to assess how reasonable the Abell estimates of $A_{g r}$ really are, the recently published Northern Abell Cluster Catalogue (Abell, Corwin \& Olowin 1989) was employed to estimate $A_{g r}$ for the radio galaxies north of $\delta=-23^{\circ}$ for which a Lick estimate had already been computed. These two estimates are shown plotted against each other in Fig. 4.2 . 


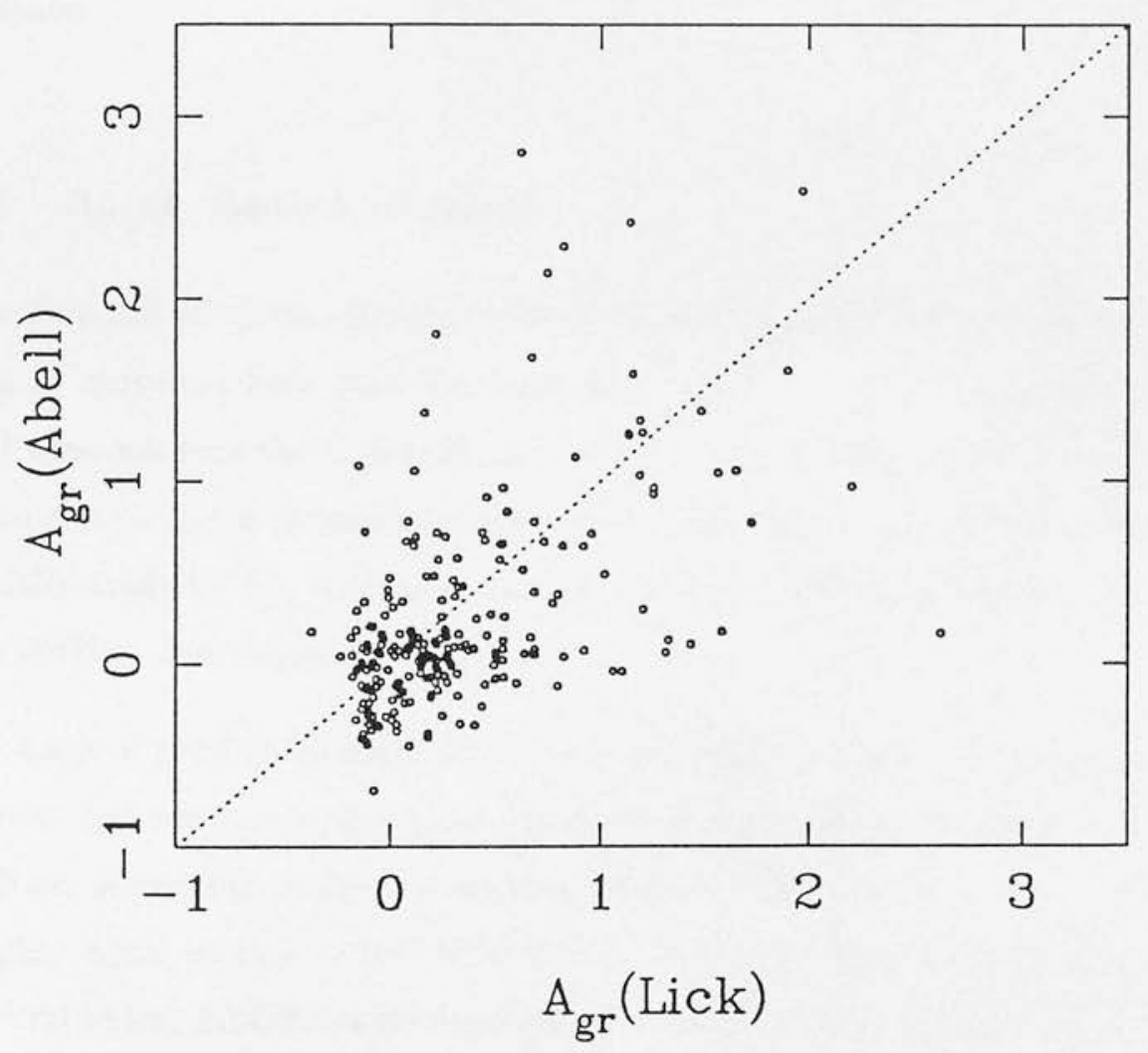

ig. 4.2: A comparison between estimates of $A_{g r}$ for the radio galaxies north of $=-23^{\circ}$ using the Lick galaxy counts and the Abell cluster catalogue. 
Clearly, the Abell estimates are not too bad given the crudity of the approximations that have been made. Certainly, they are at least good enough to include the southern radio sources in statistical tests for correlations between cluster environment with properties of the radio galaxies. The line of points around zero along the Abell $A_{g r}$ axis corresponds to radio galaxies for which there are no nearby clusters, according to Abell's definition of a cluster.

\section{3 $B_{g r}$ vs. Radio Luminosity}

The Fanaroff-Riley classification scheme for radio galaxies was defined in Chapter 1. We remind ourselves here that low luminosity radio sources are generally found to be of FRI type whereas the high-luminosity sources are typically FRIIs. The claim that FRI radio galaxies seem to prefer a more richer environment than their FRII counterparts was initially made by LS. Although confirmed later by PP, the effect was found to be a lot less striking than originally thought.

Lack of good radio maps for most of the all-sky sample sources meant that only an indirect and rough morphological classification is possible on the basis of their radio luminosities. According to LS, the division between FRIs and FRIIs is at a radio luminosity roughly equal to $P_{2.7} \sim 10^{24} \mathrm{WHz}^{-1} \mathrm{sr}^{-1}$. Taking a representative value of 0.8 for the spectral index, $2.7 \mathrm{GHz}$ radio luminosities were calculated for each of the all-sky sample members and these are shown plotted against their values of $B_{g r}$ in Fig. 4.3.

There is clearly no obvious fall in $B_{g r}$ towards higher luminosity and the general impression is similar to the one found by PP - namely, that individual objects either side of the FR division show similar extremes in $B_{g r}$ and that there is a large scatter (greater than the measurement error) in $B_{g r}$ at all luminosities. The mean values of $B_{g r}$ for the radio galaxies either side of $P_{2.7}=10^{24} \mathrm{WHz}^{-1} \mathrm{sr}^{-1}$ are shown in Table 4.3 together with mean values for Abell clusters of richness $R=0,1 \& 2$ taken from PP. 


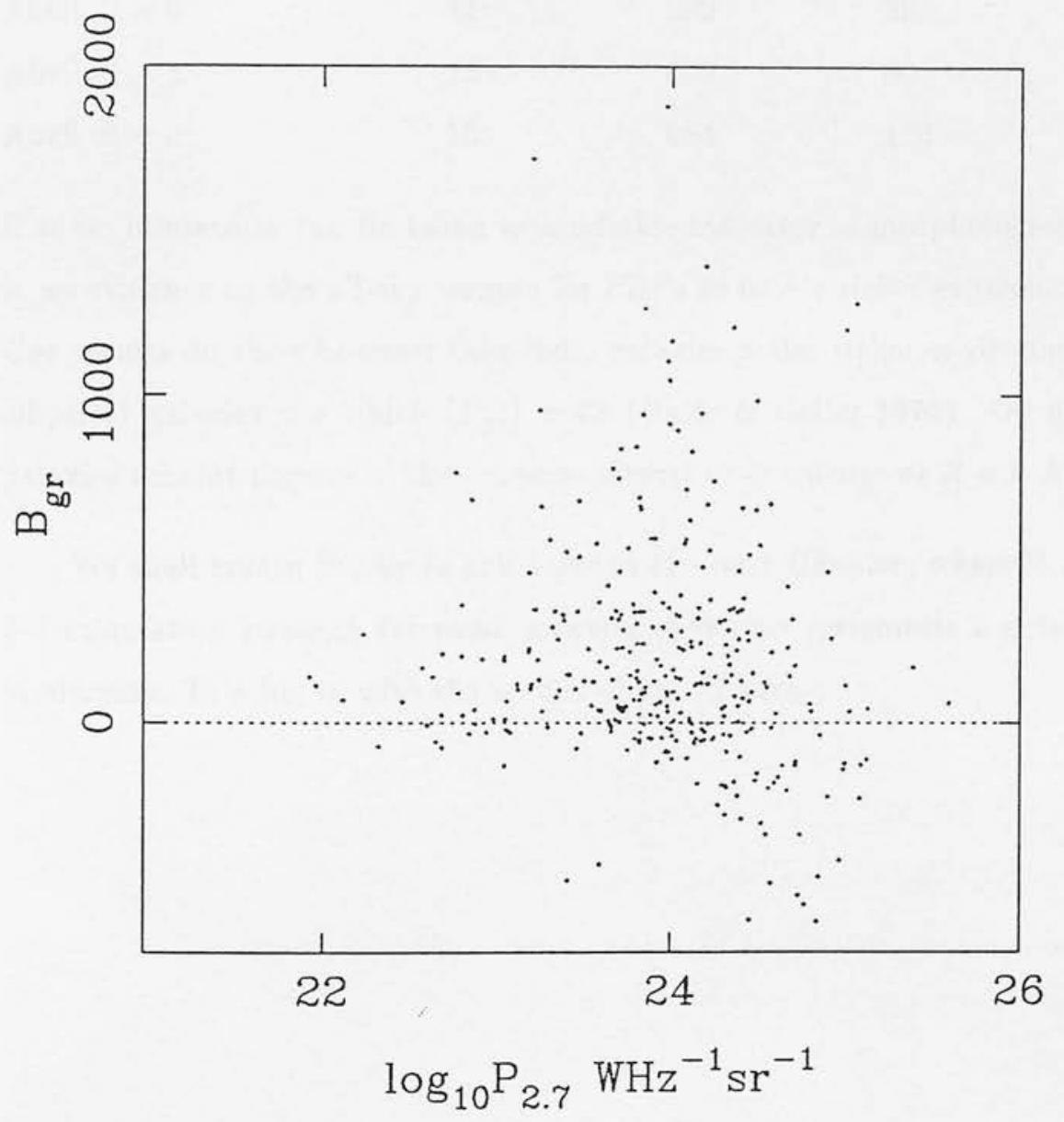

Fig. 4.3: $2.7 \mathrm{GHz}$ radio luminosity plotted against $B_{g r}$ for the all-sky sample. The transition point between FRI and FRII radio sources is roughly at $\log _{10} P=24 \mathrm{WHz}^{-1} \mathrm{sr}^{-1}$. 
Table 4.3: Average Values of $\mathrm{B}$

$\begin{array}{llll} & \mathrm{N} & \left\langle B_{g g}\right\rangle & \sigma \\ \text { all sample } & 333 & 168 & 20 \\ \log _{10} P_{2.7}<24 & 169 & 111 & 28 \\ \log _{10} P_{2.7}>24 & 164 & 108 & 28 \\ \text { Abell } R=0 & 11 & 289 & 29 \\ \text { Abell } R=1 & 15 & 690 & 60 \\ \text { Abell } R=2 & 13 & 984 & 120\end{array}$

If radio luminosity can be taken as a reliable indicator of morphological type then there is no evidence in the all-sky sample for FRI's to locate richer environments than FRIIs. Our results do show however that radio galaxies prefer richer environments than normal elliptical galaxies, for which $\left\langle B_{g e}\right\rangle \simeq 68$ (Davis \& Geller 1976). On average, the radio galaxies inhabit regions of the universe almost as overdense as $R=0$ Abell clusters.

We shall return briefly to this issue in the next Chapter, where it is shown that the 3-d correlation strength for radio galaxies shows no systematic trends with their radio luminosity. This fits in with the results obtained here. 


\subsection{Radio Galaxy Surface Photometry}

Optical studies of large samples of radio galaxies are something of a rarity in the astronomical literature. Important in the context of this work is the study by Lilly \& Longair (1984) of a subsample of $3 \mathrm{CR}$ radio galaxies. They present a remarkably tight $(K-z)$ Hubble diagram for these objects which has an rms scatter of about 0.45 magnitudes only, rendering radio galaxies as excellent standard candles. The main goal of this Section is to demonstrate that with a little extra work one can establish an improved distance indicator relation for radio galaxies. This is just the intrinsic surface brightness $\left(\mu_{e}\right)$ versus effective size $\left(r_{e}\right)$ relation which is well-known for normal ellipticals (Kormendy 1977). Although simple, it turns out that this luminosity indicator fares almost as well in terms of percentage distance accuracy with the more standard distance indicator relations for elliptical galaxies which incorporate correlations between photometric variables with internal velocity dispersion.

\subsubsection{ThE $\left(M_{\gamma}, \alpha\right)$ DIAGRAM}

Previous studies of the location of radio galaxies on the luminosity-size plane have concentrated on the $M_{\gamma}-\alpha$ relation (Lilly, Mclean \& Longair 1984; Lilly \& Prestage 1987). Gunn $\&$ Oke (1975) defined $\alpha$ to be a dimensionless parameter which measures the logarithmic slope of the growth curve of enclosed galactic flux density at a fiducial metric sampling radius $(\gamma)$, which they chose to be $19.2 \mathrm{kpc}$ (for $h=0.5$ ). If $L(r)$ is the luminosity enclosed within a radius $r$, then

$$
\alpha=\left[\frac{\mathrm{d} \log L(r)}{\mathrm{d} r}\right]_{\gamma}
$$

For the all-sky sample, which fills the redshift shell from $0.01<z<0.1, \gamma$ ranges from between $8^{\prime \prime}$ up to 1 arcminute. In practice this means that $\gamma$ is not usually large enough to enclose all of the galactic halo light, although it probably falls well outside the radius of a typical seeing disk.

Despite our main interest here, which is the $\mu_{e}-r_{e}$ relation for radio galaxies, deriving an $M_{\gamma}-\alpha$ relation is also desirable for comparative purposes. As indicated above, 
much of the early CCD photometry for radio galaxies and brightest cluster members has concentrated on the form of the $M_{\gamma}-\alpha$ relation. Since these two quantities are measured directly from growth curves, without having to fit a model to the surface brightness distribution of the galaxies, they are a useful pair of parameters which other astronomers can quickly compare with should they wish to repeat our photometry at a later date.

In view of this, an $M-\alpha$ diagram was constructed for a subsample of the radio galaxies which had good $I$-band CCD photometry. This was done in the following way. Firstly, nearby stars and galaxies were removed from the vicinity of the radio galaxy using the symmetrisation algorithm described in the last Chapter. Radio galaxies with multiple nuclei, defined loosely as secondary maxima in the light profiles that have an integrated luminosity considerably less than that of the main body of the galaxy, also had their nuclei removed. The justification for this is partly physical in that there is some doubt anyway as to whether these nuclei are actually associated with the galaxy or else whether they are simply chance superpositions (e.g. Merrit 1984). On the practical side, multiple nuclei can add scatter to the $M-\alpha$ relation, the sign of which depends on whether the secondary component is enclosed or bisected by the $19.2 h^{-1} \mathrm{kpc}$ sampling radius. Given that only a small fraction $(\leq 8 \%)$ of the sample appeared to possess multiple nuclei it seemed reasonable to remove these nuclei on the premise that doing so was not going to seriously bias our study.

A sky background was estimated for each frame by stepping around its periphery with a box of dimension $5^{\prime \prime}$ and finding the median pixel value. Growth curves were then computed for the radio galaxies by summing up sky-subtracted flux in apertures of increasing radii. Digitisation effects were allowed for in the smaller apertures by actually calculating the fractional area of pixels that were cut by the aperture arc. $M_{\gamma}$ and $\alpha$ were measured by fitting a third-order polynomial to the growth curves in the vicinity of $\gamma$. The chief source of error in these estimates arise from uncertainties in the background sky level. This was judged to produce roughly 6-7\% rms errors in $\alpha$ and about $0.04^{\mathrm{m}}$ in $M_{\gamma}$. Finally, a small correction was made to $\alpha$ for the effects of atmospheric seeing. The corrections were read off directly from the graph presented by Lilly, Mclean \& Longair (1984) which is the result of simulations that they performed on a family of synthetic galaxy models. Corrections for airmass, colour, galactic extinction and cosmology were 
made to $M_{\gamma}$ as discussed in the last Chapter. The final values for the parameters have been listed in Table 4.4 and are shown plotted in Fig. 4.4.

There is an obvious correlation between $M_{\gamma}$ and $\alpha$. A discussion of the implications of this are reserved till Section 4.5 , until after the $\mu_{e}-r_{e}$ relation has been examined.

\subsubsection{THE $\mu_{e}-r_{e}$ RELATION}

It is a simple matter to verify that the $(M, \alpha)$ parameters are related to the more familiar de Vaucouleurs half-light radius $r_{e}$ and effective surface brightness $\mu_{e}$. From the Oke \& Gunn definition of $\alpha$ we have

$$
\alpha_{\gamma}=\left[\frac{2 \pi r^{2} I(r)}{L(r)}\right]_{\gamma}
$$

into which any empirical form for $I(r)$, such as deVaucouleurs $r^{1 / 4}$ law (deVaucouleurs 1948) can be substituted. One then has an expression relating $\alpha$ to $r_{e}$ and this is shown in Fig. 4.5.

Also marked is a line showing the difference between 'total' absolute magnitude and absolute magnitude derived from within an aperture of metric size $\gamma$.

Converting between $\alpha$ and $M_{\gamma}$ to deVaucouleur's $I_{e}$ and $r_{e}$ in this way assumes that the galaxies surface brightness profiles are exactly fit with a empirical function of the form (deVaucouleurs 1948)

$$
I(r)=I_{e} \exp \left[-7.76\left\{\left(r / r_{e}\right)^{1 / 4}-1\right\}\right]
$$

In fact this is a often a rather gross assumption and for elliptical galaxies with peaked central profiles or extended halos the simple $r^{1 / 4}$ law turns out to be rather a poor fit. The $r^{1 / 4}$ profile has no shape parameter and yet it is known that ellipticals have a wide variety of profile shapes (e.g. Schombert 1986). Thus one might expect an $r^{1 / 4}$ fit to deviate at some level from the true galaxian light profile. This begs the question - do better alternatives exist? 


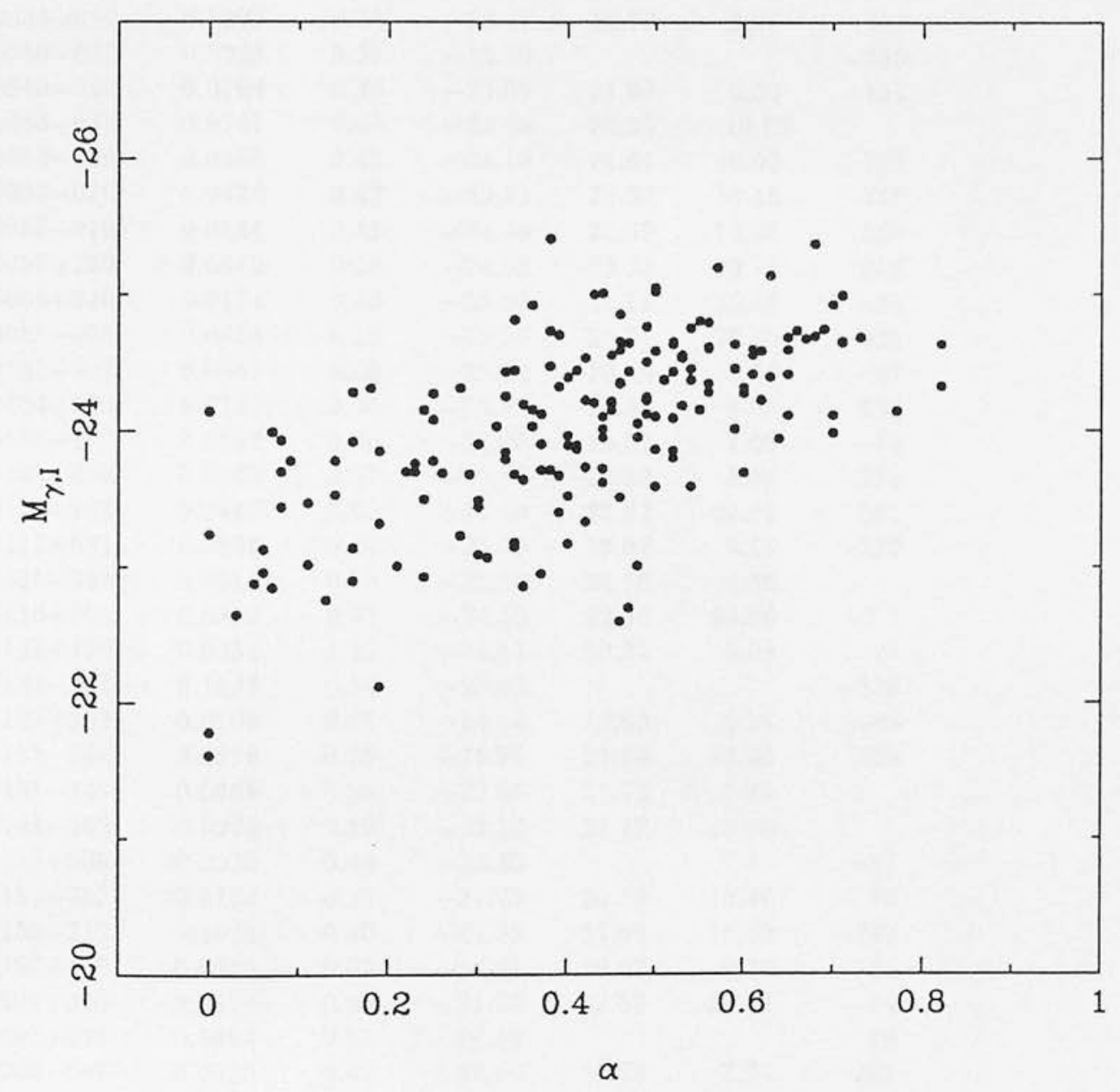

Fig. 4.4: $M-\alpha$ diagram for a subsample of the radio galaxies with adequate $I$ band CCD photometry. 
Table 4.4: The photometric and environment parameters discussed in this Chapter

\begin{tabular}{|c|c|c|c|c|c|c|}
\hline $\mathrm{IAU}$ & z & $\alpha_{I}$ & $M_{I, \gamma}$ & $\mu_{e}^{I}$ & $r_{e}$ & $B_{g r}$ \\
\hline $0000-550$ & 0.0323 & 0.22 & -23.70 & 20.10 & 7.70 & 137 \\
\hline $0001-531$ & 0.0327 & 0.00 & -24.14 & 20.43 & 8.80 & -18 \\
\hline $0005-199$ & 0.1175 & 0.62 & -24.22 & 21.96 & 20.80 & \\
\hline $0007-325$ & 0.0256 & 0.09 & -23.78 & 20.22 & 7.96 & 12 \\
\hline $0023-333$ & 0.0515 & 0.60 & -23.69 & 22.60 & 29.60 & 188 \\
\hline $0032+101$ & 0.0570 & 0.53 & -24.18 & 19.94 & 5.96 & 105 \\
\hline $0034-014$ & 0.0730 & 0.36 & -24.15 & 18.02 & 2.40 & 146 \\
\hline $0036+030$ & 0.0140 & 0.23 & -23.70 & 20.47 & 6.58 & 181 \\
\hline $0038-210$ & 0.0900 & 0.60 & -24.27 & 19.30 & 4.30 & -305 \\
\hline $0038+086$ & 0.1290 & 0.38 & -24.47 & 18.70 & 3.96 & -652 \\
\hline $0040-065$ & 0.1228 & 0.37 & -23.70 & & & -280 \\
\hline $0043-424$ & 0.0764 & 0.30 & -23.09 & 21.90 & 6.56 & -151 \\
\hline $0043-638$ & 0.0741 & 0.40 & -23.89 & 20.96 & 10.22 & \\
\hline $0053-015$ & 0.0385 & 0.45 & -24.19 & 21.61 & 16.02 & 79 \\
\hline $0053-016$ & 0.0436 & 0.42 & -23.73 & 22.32 & 18.18 & 758 \\
\hline $0055-010$ & 0.0444 & 0.41 & -24.44 & 21.69 & 14.98 & 286 \\
\hline $0055+260$ & 0.0479 & 0.36 & -24.92 & 23.33 & 50.76 & 206 \\
\hline $0055+030$ & 0.0174 & 0.43 & -25.00 & 21.11 & 15.58 & -31 \\
\hline $0058-508$ & 0.0614 & 0.50 & -23.59 & 22.31 & 20.02 & 621 \\
\hline $0101-802$ & 0.0567 & 0.00 & -23.24 & 19.49 & 3.66 & -67 \\
\hline $0104+320$ & 0.0163 & 0.38 & -25.41 & 20.88 & 9.50 & 256 \\
\hline $0108-142$ & 0.0517 & 0.46 & -24.85 & 19.52 & 7.00 & -72 \\
\hline $0109+490$ & 0.0662 & 0.37 & -23.90 & 18.82 & 3.04 & -273 \\
\hline $0110+152$ & 0.0445 & 0.60 & -24.30 & 22.02 & 22.62 & 381 \\
\hline $0111+021$ & 0.0466 & 0.44 & -23.72 & 19.07 & 3.14 & -122 \\
\hline $0115-261$ & 0.0518 & 0.13 & -22.76 & 20.58 & 4.86 & \\
\hline $0116+310$ & 0.0599 & 0.61 & -24.55 & 22.30 & 24.96 & -7.3 \\
\hline $0121+429$ & 0.0336 & 1.11 & -24.17 & 20.31 & 3.08 & 21 \\
\hline $0124-117$ & 0.1235 & 0.50 & -23.63 & & & -326 \\
\hline $0128+002$ & 0.0789 & 0.71 & -24.64 & 19.60 & 6.38 & -48 \\
\hline $0131-367$ & 0.0295 & 0.25 & -23.78 & 21.14 & 13.96 & 229 \\
\hline $0131-449$ & 0.0901 & 0.35 & -22.86 & 21.74 & 8.20 & \\
\hline $0144-552$ & 0.0974 & 0.19 & -22.12 & 21.17 & 13.60 & \\
\hline $0145+000$ & 0.0925 & 0.46 & -23.89 & & & -11 \\
\hline $0153+053$ & 0.0186 & 0.38 & -24.73 & 20.33 & 10.46 & 79 \\
\hline $0155-212$ & 0.1621 & 0.40 & -24.39 & 21.66 & 16.58 & -743 \\
\hline $0157+405$ & 0.0818 & 0.60 & -24.31 & 19.08 & 3.84 & -64 \\
\hline $0206+350$ & 0.0374 & 0.50 & -23.86 & 22.55 & 21.70 & -64 \\
\hline $0207+095$ & 0.0884 & 0.57 & -24.30 & & & 20 \\
\hline $0208-067$ & 0.0420 & 0.47 & -22.70 & 18.72 & 2.74 & 258 \\
\hline $0214-480$ & 0.0640 & 0.49 & -24.22 & 21.48 & 18.44 & 1178 \\
\hline $0217+017$ & 0.0409 & 0.35 & -24.19 & 20.34 & 7.86 & 27 \\
\hline $0219+427$ & 0.0209 & 0.52 & -24.43 & 21.43 & 11.86 & 268 \\
\hline $0229-208$ & 0.0900 & 0.61 & -24.55 & 20.66 & 10.98 & 146 \\
\hline $0238+085$ & 0.0214 & 0.38 & -23.71 & 20.89 & 8.12 & -27 \\
\hline $0247-207$ & 0.0870 & 0.63 & -25.14 & 19.45 & 7.68 & 270 \\
\hline $0255+058$ & dumbell & & & & & \\
\hline $0257-398$ & 0.0676 & 0.16 & -23.92 & 19.69 & 5.38 & -106 \\
\hline $0300+162$ & 0.0326 & 0.37 & -22.95 & 19.98 & 3.28 & -59 \\
\hline $0301-123$ & 0.1010 & 0.51 & -24.61 & & & 1149 \\
\hline $0305+039$ & 0.0288 & 0.39 & -24.70 & 20.67 & 12.18 & 75 \\
\hline $0307-305$ & 0.0675 & 0.30 & -23.44 & 21.11 & 8.96 & -106 \\
\hline
\end{tabular}


Table 4.4: cont....

\begin{tabular}{|c|c|c|c|c|c|c|}
\hline IAU & z & $\alpha_{I}$ & $M_{I, \gamma}$ & $\mu_{e}^{I}$ & $r_{e}$ & $B_{g r}$ \\
\hline $0312-400$ & 0.0758 & 0.22 & -23.63 & & & \\
\hline $0314-440$ & 0.0628 & 0.42 & -24.22 & 21.75 & 22.58 & 1104 \\
\hline $0316-444$ & 0.0758 & 0.63 & -24.39 & 22.52 & 41.00 & 1381 \\
\hline $0319-454$ & 0.0633 & 0.52 & -23.85 & 22.54 & 28.62 & 815 \\
\hline $0325+023$ & 0.0302 & 0.52 & -23.80 & 21.76 & 14.08 & -28 \\
\hline $0326-461$ & 0.0690 & 0.37 & -23.87 & & & \\
\hline $0332-391$ & 0.0626 & 0.14 & -23.78 & 20.63 & 8.54 & 906 \\
\hline $0344-345$ & 0.0538 & 0.21 & -23.01 & 21.10 & 7.36 & -58 \\
\hline $0356+102$ & 0.0306 & 0.34 & -23.18 & 20.95 & 5.98 & -13 \\
\hline $0359+193$ & 0.0543 & 0.48 & -23.95 & 20.35 & 6.12 & 185 \\
\hline $0402+179$ & 0.1114 & 0.24 & -22.93 & 19.16 & 2.50 & -525 \\
\hline $0404+035$ & 0.0891 & 0.66 & -22.35 & & & -265 \\
\hline $0419+140$ & 0.0643 & 0.46 & -22.60 & 21.64 & 6.82 & -172 \\
\hline $0427-539$ & 0.0390 & 0.45 & -24.15 & 21.38 & 18.18 & 208 \\
\hline $0431-134$ & 0.0364 & 0.44 & -23.62 & 20.08 & 4.92 & 488 \\
\hline $0434-225$ & 0.0682 & 0.51 & -24.37 & 21.63 & 22.22 & 106 \\
\hline $0446-206$ & 0.0724 & 0.44 & -23.95 & 18.80 & 3.12 & 1202 \\
\hline $0449-175$ & 0.0318 & 0.43 & -24.20 & 20.33 & 7.72 & 64 \\
\hline $0453-206$ & 0.0343 & 0.54 & -24.37 & 21.23 & 14.38 & 351 \\
\hline $0456-301$ & 0.0630 & 0.00 & -21.61 & 20.89 & 4.80 & \\
\hline $0502-103$ & 0.0405 & 0.29 & -23.68 & 20.15 & 5.28 & -32 \\
\hline $0503-286$ & 0.0381 & 0.08 & -23.70 & 20.97 & 11.46 & -25 \\
\hline $0511-305$ & 0.0583 & 0.31 & -23.07 & 21.19 & 7.84 & -72 \\
\hline $0518-458$ & 0.0358 & 0.00 & -21.78 & 21.76 & 6.12 & -22 \\
\hline $0521-365$ & 0.0554 & 0.11 & -23.47 & 19.35 & 3.54 & -63 \\
\hline $0523-327$ & 0.0758 & 0.56 & -24.28 & 20.65 & 4.32 & -123 \\
\hline $0546-329$ & 0.0369 & 0.35 & -24.19 & 20.96 & 14.00 & -429 \\
\hline $0545-199$ & 0.0556 & 0.59 & -24.01 & 18.87 & 3.14 & 116 \\
\hline $0548-317$ & 0.0326 & 0.16 & -23.14 & 20.56 & 6.50 & -460 \\
\hline $0602-647$ & 0.0449 & 0.07 & -22.85 & 20.52 & 5.48 & -38 \\
\hline $0605-494$ & 0.0519 & 0.39 & -23.67 & 21.50 & 14.22 & 237 \\
\hline $0616-487$ & 0.0465 & 0.41 & -23.86 & 21.46 & 15.70 & -91 \\
\hline $0618-371$ & 0.0324 & 0.28 & -23.23 & 21.05 & 9.90 & 344 \\
\hline $0620-526$ & 0.0511 & 0.49 & -24.50 & 22.52 & 56.86 & 348 \\
\hline $0625-354$ & 0.0553 & 0.82 & -24.32 & 21.11 & 14.34 & 818 \\
\hline $0625-536$ & 0.0539 & 0.59 & -24.45 & 22.32 & 42.06 & 764 \\
\hline $0641-584$ & 0.0561 & 1.73 & -22.11 & & & -65 \\
\hline $0642-436$ & 0.0608 & 0.48 & -24.05 & 21.69 & 19.46 & -80 \\
\hline $0649-557$ & 0.0492 & 0.49 & -24.12 & 22.17 & 33.64 & -550 \\
\hline $0704+351$ & 0.0789 & 0.65 & -24.68 & 19.02 & 4.78 & 512 \\
\hline $0755+370$ & 0.0413 & 0.56 & -24.79 & 20.25 & 10.06 & 20 \\
\hline $0802+240$ & 0.0601 & 0.35 & -23.64 & 18.18 & 2.00 & 62 \\
\hline $0803-008$ & 0.0892 & 0.68 & -25.37 & 20.53 & 16.14 & 114 \\
\hline $0816-705$ & 0.0380 & 0.42 & -23.33 & 21.75 & 7.96 & -25 \\
\hline $0819+061$ & 0.0815 & 0.48 & -23.01 & 18.07 & 1.56 & -37 \\
\hline $0825+240$ & 0.0833 & 0.40 & -23.52 & 18.77 & 2.64 & 456 \\
\hline $0828+320$ & 0.0513 & 0.37 & -23.71 & 19.17 & 3.32 & -85 \\
\hline $0836+290$ & 0.064 & 0.45 & -24.21 & 21.20 & 12.88 & -28 \\
\hline $0838+325$ & 0.0697 & 0.52 & -24.63 & 20.18 & 9.90 & 314 \\
\hline $0844+310$ & 0.0675 & 0.50 & -25.02 & 20.01 & 10.04 & 287 \\
\hline $0915-118$ & 0.0548 & 0.55 & -24.15 & 23.36 & 78.60 & 114 \\
\hline $0921-213$ & 0.0519 & 0.03 & -22.65 & 20.43 & 4.98 & -83 \\
\hline
\end{tabular}


Table 4.4: cont....

\begin{tabular}{|c|c|c|c|c|c|c|}
\hline IAU & $\mathrm{z}$ & $\alpha_{I}$ & $M_{I, \gamma}$ & $\mu_{e}^{I}$ & $r_{e}$ & $B_{g r}$ \\
\hline $0945+076$ & 0.0861 & 0.30 & -23.49 & 15.71 & 0.74 & -81 \\
\hline $1000-043$ & 0.0636 & 0.33 & -23.79 & 20.43 & 6.48 & -18 \\
\hline $1003+350$ & 0.0989 & 0.49 & -24.24 & 20.38 & 7.82 & -366 \\
\hline $1004+146$ & 0.0305 & 0.45 & -24.55 & 20.24 & 8.58 & 30.8 \\
\hline $1019+083$ & 0.1022 & 0.62 & -24.58 & 19.66 & 6.42 & 337 \\
\hline $1037+300$ & 0.0911 & 0.32 & -24.03 & 18.66 & 3.12 & -206 \\
\hline $1043-290$ & 0.0574 & 0.05 & -22.88 & 21.15 & 11.54 & -69 \\
\hline $1040+310$ & 0.0351 & 0.46 & -23.51 & 21.04 & 7.90 & 76 \\
\hline $1053-282$ & 0.0594 & 0.06 & -22.96 & 22.17 & 18.12 & -75 \\
\hline $1056-360$ & 0.0700 & 0.11 & -23.02 & 21.15 & 9.82 & -117 \\
\hline $1113+290$ & 0.0491 & 0.33 & -23.84 & 18.30 & 2.32 & 549 \\
\hline $1118+000$ & 0.0989 & 0.65 & -24.59 & 17.77 & 2.62 & 291 \\
\hline $1123-351$ & 0.0329 & 0.19 & -23.85 & 21.15 & 13.08 & 92 \\
\hline $1130-037$ & 0.0521 & 0.59 & -24.63 & 21.10 & 15.10 & 268 \\
\hline $1131+493$ & 0.0338 & 0.39 & -24.32 & 20.35 & 9.14 & 386 \\
\hline $1132+492$ & 0.0324 & 0.14 & -23.53 & 19.12 & 3.20 & 483 \\
\hline $1137+180$ & 0.0105 & 0.06 & -23.13 & 20.67 & 5.78 & -545 \\
\hline $1137+123$ & 0.0811 & 0.55 & -24.80 & 21.59 & 21.22 & 12 \\
\hline $1139-078$ & 0.0654 & 0.43 & -22.84 & & & 44 \\
\hline $1200+519$ & 0.0631 & 0.47 & -24.43 & 22.33 & 24.24 & 378 \\
\hline $1201-041$ & 0.1050 & 1.37 & -23.49 & 22.05 & 11.90 & -159 \\
\hline $1204+225$ & 0.0650 & 0.50 & -24.58 & 21.90 & 17.84 & 73 \\
\hline $1215+039$ & 0.0785 & 0.71 & -24.65 & 21.28 & 16.44 & 603 \\
\hline $1216-100$ & 0.0874 & 0.30 & -22.99 & & & -78 \\
\hline $1227+119$ & 0.0824 & 0.50 & -25.05 & 20.18 & 9.30 & 398 \\
\hline $1250-102$ & 0.0143 & 0.24 & -24.15 & 20.32 & 7.66 & -147 \\
\hline $1251+270$ & 0.0857 & 0.50 & -23.80 & & & -1124 \\
\hline $1251-289$ & 0.0578 & 0.44 & -25.01 & 21.72 & 40.14 & 700 \\
\hline $1254-300$ & 0.0540 & 0.70 & -24.06 & & & 426 \\
\hline $1257-253$ & 0.0645 & 0.32 & -23.79 & & & -94 \\
\hline $1258-321$ & 0.0149 & 0.46 & -21.95 & & & 749 \\
\hline $1309+210$ & 0.0300 & 0.24 & -23.50 & 20.89 & 6.04 & -90 \\
\hline $1312+089$ & 0.0904 & 0.39 & -23.40 & 17.93 & 2.06 & 96 \\
\hline $1313+073$ & 0.0524 & 0.71 & -24.99 & 22.32 & 33.36 & 250 \\
\hline $1316+290$ & 0.0728 & 0.50 & -24.10 & 18.96 & 3.76 & 32 \\
\hline $1319+428$ & 0.0794 & 0.34 & -23.68 & 21.14 & 7.78 & 313 \\
\hline $1321+310$ & 0.0163 & 0.26 & -23.69 & 21.28 & 9.52 & 141 \\
\hline $1322+360$ & 0.0174 & 0.19 & -23.32 & 20.34 & 4.42 & -271 \\
\hline $1331-090$ & 0.0814 & 1.07 & -23.65 & & & -4 \\
\hline $1339+260$ & 0.0720 & 0.73 & -24.68 & 23.20 & 40.64 & 835 \\
\hline $1346+260$ & 0.0633 & 0.82 & -24.63 & 23.10 & 34.14 & 914 \\
\hline $1350+310$ & 0.0450 & 0.25 & -24.08 & 21.71 & 14.58 & -138 \\
\hline $1358-113$ & 0.0366 & 0.53 & -23.93 & & & 241 \\
\hline $1407+177$ & 0.0189 & 0.16 & -24.28 & 19.71 & 5.14 & -23 \\
\hline $1414+110$ & 0.0237 & 0.34 & -24.44 & 21.05 & 11.30 & -7.0 \\
\hline $1422+268$ & 0.0370 & 0.54 & -23.59 & 21.88 & 9.84 & 52 \\
\hline $1427+074$ & 0.0534 & 0.47 & -24.64 & 21.13 & 13.30 & 25 \\
\hline $1443+178$ & 0.0653 & 0.41 & -23.96 & & & -62 \\
\hline $1448+634$ & 0.0410 & 0.18 & -24.31 & 19.43 & 5.38 & 9 \\
\hline $1452+165$ & 0.0456 & 0.46 & -24.65 & 21.40 & 14.32 & 94 \\
\hline $1502+262$ & 0.0538 & 0.65 & -24.11 & 23.16 & 27.22 & 296 \\
\hline $1514+072$ & 0.0350 & 0.67 & -24.67 & 23.03 & 44.94 & 132 \\
\hline
\end{tabular}


Table 4.4: cont.....

\begin{tabular}{|c|c|c|c|c|c|c|}
\hline IAU & $z$ & $\alpha_{I}$ & $u_{I, \gamma}$ & $\mu_{e}^{I}$ & $r_{e}$ & $B_{g r}$ \\
\hline $1519+070$ & 0.0455 & 0.65 & -24.41 & 22.81 & 33.14 & 292 \\
\hline $1519+045$ & 0.0526 & 0.54 & -24.45 & 20.96 & 11.40 & 297 \\
\hline $1521+280$ & 0.0825 & 0.53 & -24.53 & 20.19 & 8.44 & 140 \\
\hline $1529+240$ & 0.0956 & 0.33 & -24.43 & 19.05 & 4.96 & 164 \\
\hline $1538+082$ & 0.1240 & 0.34 & -24.81 & 18.74 & 5.16 & 822 \\
\hline $1549+202$ & 0.0318 & 0.98 & -21.70 & & & 160 \\
\hline $1555-140$ & 0.0971 & 1.02 & -24.64 & 23.88 & 73.32 & -473 \\
\hline $1557+708$ & 0.0267 & 0.53 & -24.55 & 21.54 & 16.06 & -47 \\
\hline $1601+173$ & 0.0366 & 0.33 & -24.25 & 20.20 & 6.60 & 208 \\
\hline $1602+240$ & 0.0318 & 0.66 & -24.73 & 22.19 & 27.02 & 120 \\
\hline $1602+178$ & 0.0315 & 0.41 & -23.89 & 21.49 & 11.54 & 870 \\
\hline $1603+001$ & 0.0614 & 0.30 & -23.90 & 19.46 & 4.34 & -146 \\
\hline $1615+350$ & 0.0296 & 0.36 & -24.03 & 21.60 & 13.28 & 525 \\
\hline $1616-029$ & 0.0689 & 0.56 & -24.61 & 19.64 & 6.42 & 207 \\
\hline $1626+390$ & 0.0297 & 0.49 & -24.76 & 23.09 & 47.94 & 520 \\
\hline $1636+370$ & 0.1603 & 0.69 & -24.74 & 20.17 & 8.56 & -3221 \\
\hline $1657+320$ & 0.0630 & 0.23 & -23.76 & 17.84 & 2.00 & 117 \\
\hline $1658+300$ & 0.0351 & 0.08 & -23.44 & 19.62 & 3.58 & -43 \\
\hline $1706+094$ & 0.0396 & 0.37 & -24.12 & 19.89 & 5.52 & 218 \\
\hline $1707+340$ & 0.0801 & 0.25 & -24.27 & 19.66 & 5.32 & 950 \\
\hline $1709+390$ & 0.0621 & 0.44 & -24.10 & 18.32 & 2.68 & 477 \\
\hline $1712+640$ & 0.0798 & 0.44 & -24.01 & 17.82 & 2.10 & 1754 \\
\hline $1713+641$ & 0.0810 & 0.56 & -24.60 & 21.31 & 6.08 & 975 \\
\hline $1717-009$ & 0.0304 & 0.40 & -23.17 & 21.50 & 6.30 & 1182 \\
\hline $1719+242$ & 0.0885 & 0.16 & -22.90 & 17.85 & 1.66 & -470 \\
\hline $1733+710$ & 0.0599 & 0.46 & -24.35 & 20.10 & 6.14 & 99 \\
\hline $1739+173$ & 0.0653 & 0.56 & -24.34 & 21.62 & 15.18 & 704 \\
\hline $1740+162$ & 0.0345 & 0.08 & -23.93 & 20.61 & 6.72 & 122 \\
\hline $1744+557$ & 0.0304 & 0.28 & -24.31 & 20.57 & 8.68 & -5 \\
\hline $1754+626$ & 0.0295 & 0.40 & -23.96 & 22.63 & 32.78 & 145 \\
\hline $1754+626$ & 0.0295 & 0.52 & -24.65 & 21.38 & 10.28 & 133 \\
\hline $1807+698$ & 0.0501 & 0.22 & -24.31 & & & 19 \\
\hline $1819+689$ & 0.0877 & 0.46 & -24.59 & 17.79 & 2.86 & -239 \\
\hline $1842+455$ & 0.0908 & 0.70 & -24.92 & 22.00 & 23.72 & 984 \\
\hline $1845+790$ & 0.0561 & 0.35 & -23.71 & & & -27 \\
\hline $1950+671$ & 0.0738 & 0.40 & -23.67 & & & -221 \\
\hline $2058-135$ & 0.0291 & 0.70 & -23.98 & 20.79 & 8.16 & 582 \\
\hline $2123+007$ & 0.1330 & 0.68 & -24.69 & 23.15 & 36.60 & -366 \\
\hline $2206+237$ & 0.0862 & 0.31 & -23.73 & & & -220 \\
\hline $2212+135$ & 0.0262 & 0.64 & -23.94 & 20.64 & 6.72 & 454 \\
\hline $2221-023$ & 0.0562 & 0.54 & -22.91 & & & -80 \\
\hline $2229+390$ & 0.0161 & 0.34 & -23.15 & 19.41 & 9.30 & 37 \\
\hline $2229-086$ & 0.0827 & 0.57 & -25.20 & 20.92 & 18.82 & 390 \\
\hline $2235+408$ & 0.0575 & 0.52 & -24.09 & 18.88 & 3.06 & -61 \\
\hline $2236-176$ & 0.0525 & 0.77 & -24.14 & 22.10 & 19.98 & -129 \\
\hline $2243+390$ & 0.0806 & 0.70 & -24.11 & 19.17 & 3.64 & -120 \\
\hline $2247+113$ & 0.0261 & 0.54 & -24.75 & 20.67 & 13.02 & 71 \\
\hline $2308+073$ & 0.0430 & 0.61 & -24.59 & 20.89 & 13.60 & 604 \\
\hline $2318+079$ & 0.0111 & 0.07 & -23.99 & 19.80 & 5.56 & 183 \\
\hline $2320+203$ & 0.0392 & 0.46 & -24.34 & 20.28 & 8.02 & 165 \\
\hline $2322-123$ & 0.0821 & 0.61 & -24.41 & 21.91 & 22.18 & 257 \\
\hline $2335+264$ & 0.0293 & 0.42 & -24.53 & 21.71 & 16.72 & 707 \\
\hline
\end{tabular}


Table 4.4: cont.....

$\begin{array}{ccccccc}\text { IAU } & \mathrm{z} & \alpha_{I} & \boldsymbol{M}_{I, \gamma} & \mu_{e}^{I} & r_{e} & B_{g r} \\ 2353-184 & 0.0725 & 0.51 & -24.37 & 18.31 & 3.02 & 354\end{array}$




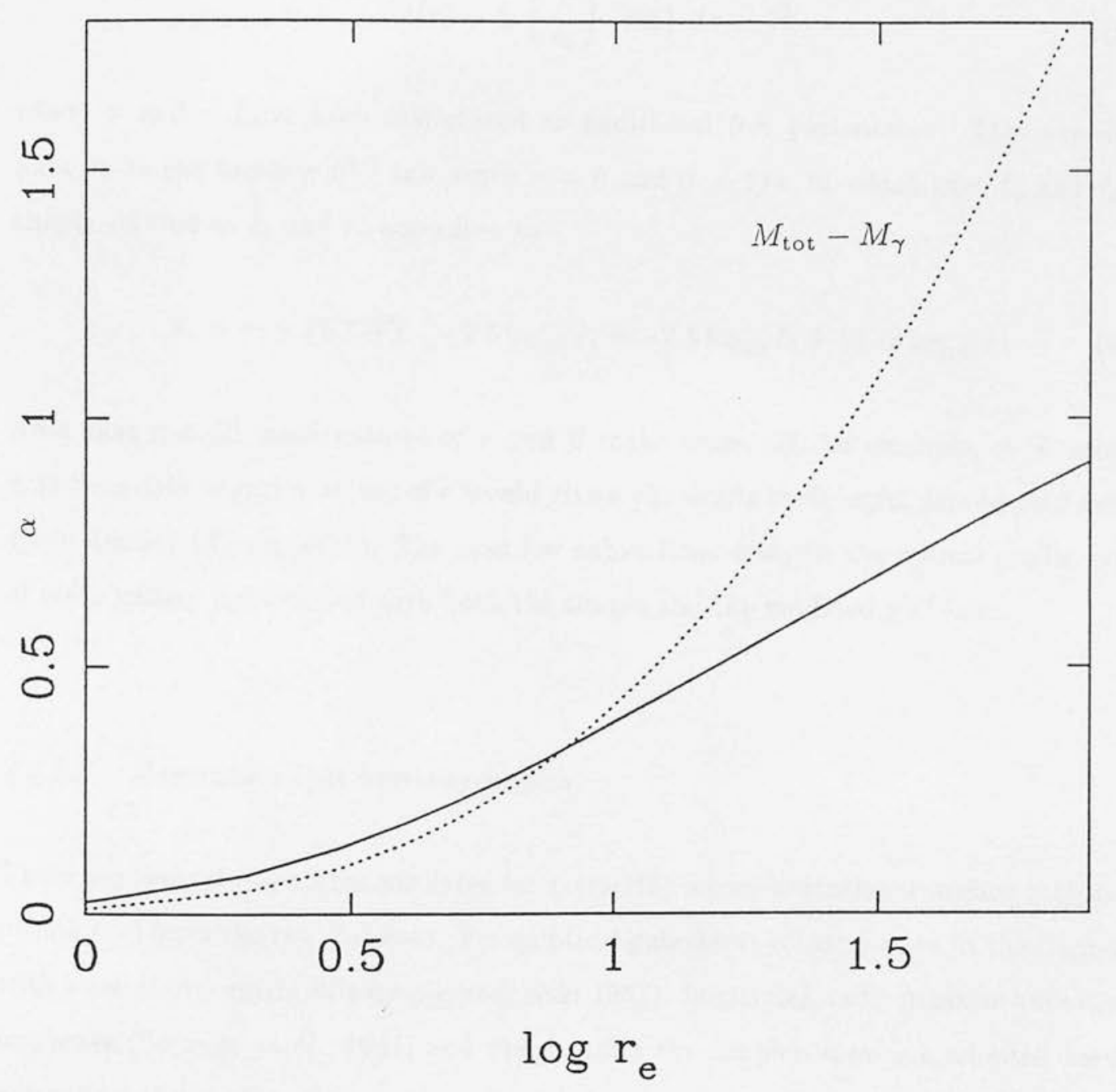

Fig. 4.5: Relationship between the alpha parameter and deVaucouleurs half-light radius $r_{e}$. Also marked is the difference between a 'total' absolute magnitude and the metric magnitude with aperture size equal to $\gamma$. 


\subsubsection{A general fitting function}

Consider a more general fitting function of the form

$$
I(r)=I_{0}\left(\frac{r}{r_{0}}\right)^{\nu} \exp \left[-\left(r / r_{0}\right)^{\beta}\right]
$$

where $\nu$ and $\beta$ have been introduced as additional free parameters. This expression reduces to the familiar $r^{1 / 4}$ law when $\nu=0$ and $\beta=1 / 4$, in which case $I_{0}$ and $r_{0}$ are simply related to $I_{e}$ and $r_{e}$ according to

$$
r_{e}=r_{0} \times(7.67)^{4} ; \quad-2.5 \log _{10} I_{e}=-2.5 \log _{10} I_{0}+19.75 \log _{10}(e)
$$

Note that not all combinations of $\nu$ and $\beta$ make sense. If, for example, $\beta$ is equal to 0.25 then only negative values of $\nu$ would give a physically meaningful deprojected central space density (Young 1976). The next few subsections describe the optical profile fitting of radio galaxy light curves with both the simple and the modified $r^{1 / 4}$ law.

\subsubsection{Generating light intensity profiles}

There are several techniques available for extracting a one-dimensional surface brightness profile $I(r)$ from the raw 2-d data. For elliptical galaxies it is common to fit the isophotes with a set of concentric ellipses (Jedrzejewski 1987). In general, radio galaxies have round isophotes (Sansom et al. 1987) and this justifies the simpler approach adopted here of extracting the profiles from concentric circular apertures of increasing radii about the galaxy centroid. An accurate centroid was found for each galaxy by fitting a parabola to intensity values in pixels located near to an estimated central pixel. In most cases, it was possible to generate data points from the galaxy centroid right out to the edge of the CCD frame. For the higher redshift galaxies, or galaxies in crowded fields, profiles were generated up until the point when they became excessively noisy. The main contribution to the error bars on each of the points is sky-subtraction inaccuracies. Errors were obtained by assuming that the final data point in each profile may be out by a factor of 2 either way and adding this up in quadrature with a flat-fielding error of $2 \%$. 


\subsubsection{Seeing deconvolution}

Usually optical profile fits to the galaxian light distribution avoid the central regions of the galaxy where atmospheric seeing creates uncertainties. Schweizer (1979) has remarked that many of the elliptical galaxy 'cores' that had been claimed at the time were probably an artifact due to the adverse effects of seeing on the central parts of the light profile. In order to model the observed brightness, one needs to convolve some assumed intrinsic intensity distribution $i(x, y)$ with a point-spread function $s(x, y)$ reflecting the seeing conditions at the time of the observation. The seeing-convolved intensity distribution is given by

$$
I(x, y)=\iint i(\xi, \eta) s(x-\xi, y-\eta) d \xi d \eta
$$

where $x, y, \xi$ and $\eta$ are apparent Cartesian coordinates in the plane of the sky with origin at the galaxy centroid. The results of convolving a pure $r^{1 / 4}$ law with a gaussian pointspread function (PSF) of dispersion $5 r_{0}$ are shown in Fig. 4.6.

Its main effect is to give the $r^{1 / 4}$ law a flat-topped appearance. The light removed from the flat-topped area reappears at larger radii as a small excess which is only just discernable in the diagram.

The 2-d integral in eqn. 4.20 is computationally rather intensive to solve numerically for each choice of free parameters in the fitting function. Monte Carlo techniques were therefore employed to gain a little bit more speed. Later, an analytic approximation to the full 2-d integral came to light (Bailey \& Sparks 1983; Sparks 1988) and these formulae are explored further in Appendix C.

\subsubsection{The optimisation algorithm}

Given the extracted 1-d profile for a galaxy, the problem is to optimise the four free parameters in the fitting function. Since the errors on $I(r)$ are fixed, a minimum $\chi^{2}$ optimisation scheme is most appropriate. The algorithm requires an initial guess for each of the parameters. A fit to the data with this parameter choice yields a goodness-of-fit equal to $\chi_{0}^{2}$. The parameters are then iteratively varied in such a way that $\chi^{2}$ is lowered. 
deconvolved $r^{1 / 4}$

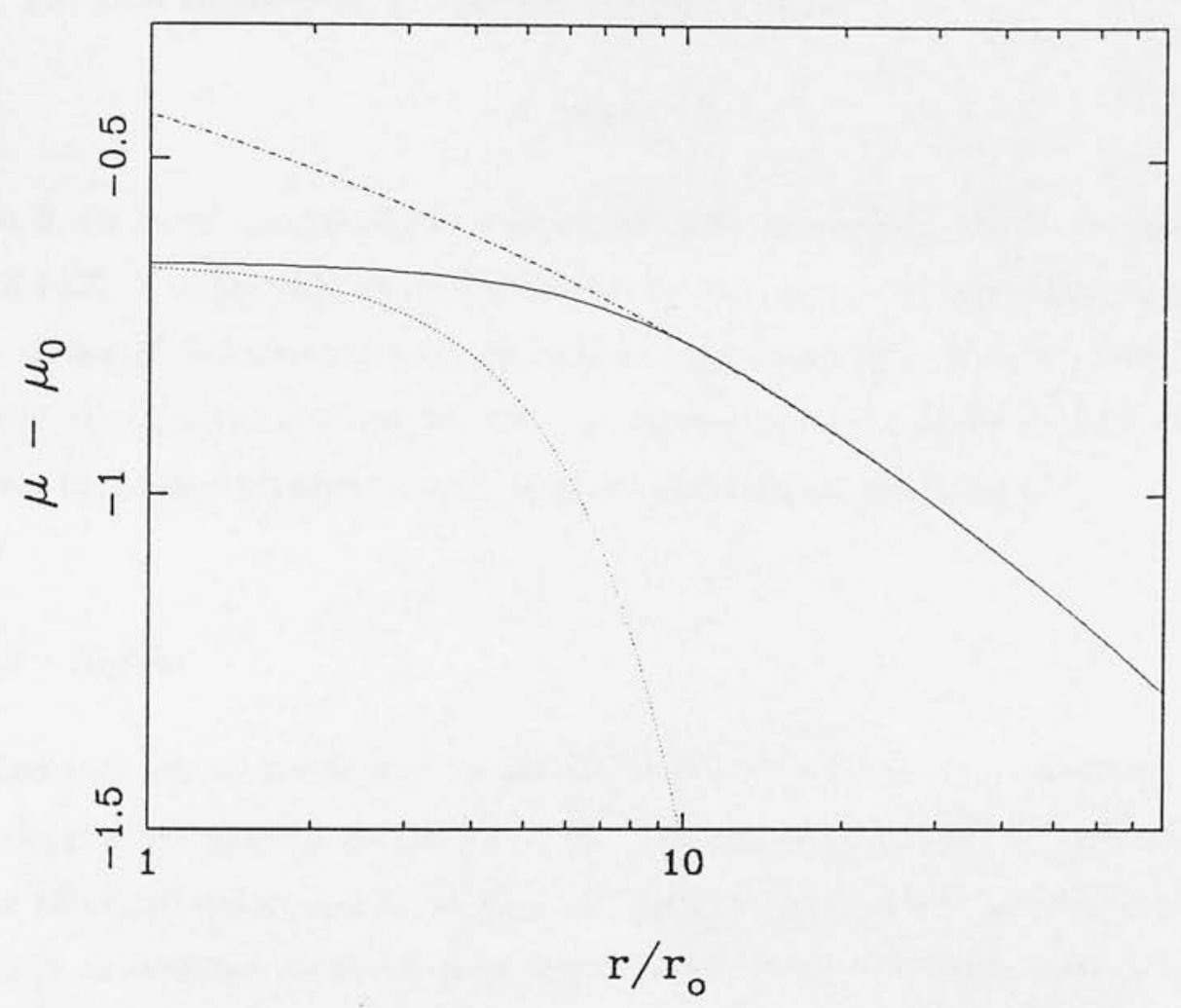

Fig. 4.6: The effect of convolving an $r^{1 / 4}$ light profile with a gaussian point-spread function. The dot-dashed line is the unconvolved profile. The bold line is the resultant profile after convolving with the point-spread function indicated by the dotted line. 
This yields a new value for $\chi^{2}$ found from a simple Taylor expansion as follows

$$
\chi^{2} \simeq \chi_{0}^{2}+\mathrm{Y} \cdot d \mathrm{X}+\frac{1}{2} d \mathrm{X} \cdot H \cdot d \mathrm{X}
$$

where $\mathrm{X}$ is a vector whose elements are the free parameters in the fitting function, $\mathrm{Y}$ is the vector whose elements are the derivatives of $\chi^{2}$ with respect to the parameters in $\mathrm{X}$ and $H$ is the Hessian matrix of second derivatives of $\chi^{2}$ with respect to the parameters in $\mathbf{X}$. The local minimum in $\chi^{2}$ can then be found by solving

$$
H \cdot d \mathbf{X}=-\mathbf{Y}
$$

$\mathrm{Y}$ and $H$ are found analytically and the optimisation proceeds iteratively by replacing $\mathrm{X}$ with $\mathbf{X}+d \mathbf{X}$. Two precautions were taken: firstly, large jumps in $\mathbf{X}$ due to the complicated shape of the $\chi^{2}$ hypersurface from the assumed quadratic approximation were avoided; secondly, the eigenvalues of the Hessian were checked whenever a minimum was reported to check that this was indeed a true minimum and not just a saddle point.

\subsubsection{Results}

The first task was to decide whether the modified $r^{1 / 4}$ law really was providing a better fit to the profiles than the standard version. To begin with, the pure $r^{1 / 4}$ law, convolved for the effects of seeing, was fit to all of the profiles. In general it proved to work well especially, as expected, over a range of radius outside the central region of the galaxy and excluding the faint halo. A fairly representative set of example fits are shown in Fig. 4.7.

In particular note the excellent fit for $1754+626 \mathrm{~b}$ over $80^{\prime \prime}$ in radius. For the radio galaxies $2058-135$ and $0229-208$ the $r^{1 / 4}$ fit, marked by the bold line, is not very good. Since fitting the modified $r^{1 / 4}$ law for four free-parameters was computationally expensive only a subset of the profiles were fit as a trial. In all cases it was found that the parameter $\beta$ remained essentially fixed at $1 / 4$ and that either $\nu$ remained at zero or else took a small negative value in the range $-0.4\langle\nu<-0.2$. Instances where a negative $\nu$ profile fit proved to be generally better than a pure $r^{1 / 4}$ fit were when the galaxy light profile rose slowly above an $r^{1 / 4}$ at large radii. Examples are the two galaxies, 2058 - 135 and $0229-208$ already noted above. The dashed lines shown fitted to their profiles in Fig. 

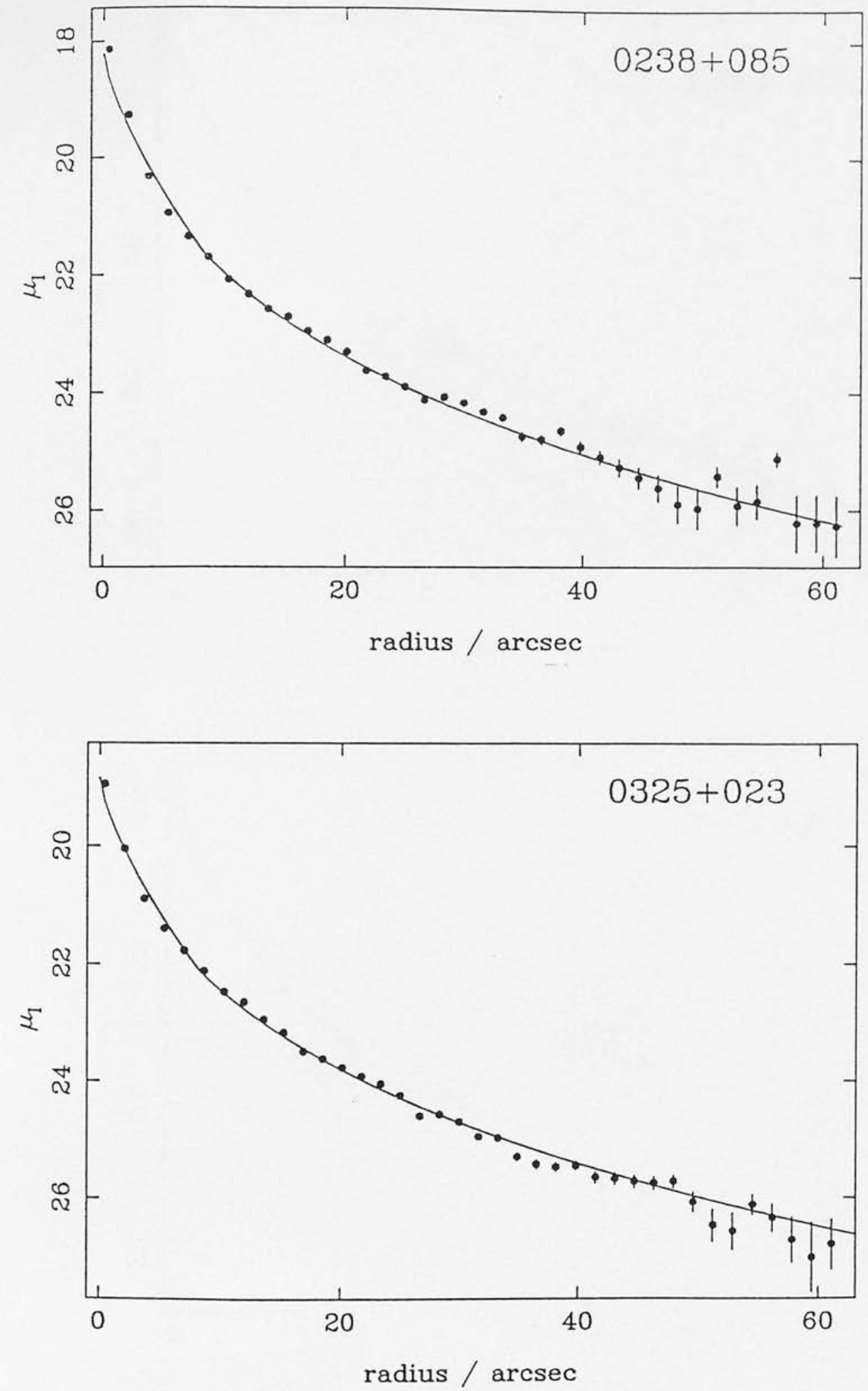

Fig. 4.7: A few representative examples of empirical profile fits to the radio galaxy $I$ band surface brightness distributions. 
4.4 Radio Galaxy Surface Photometry
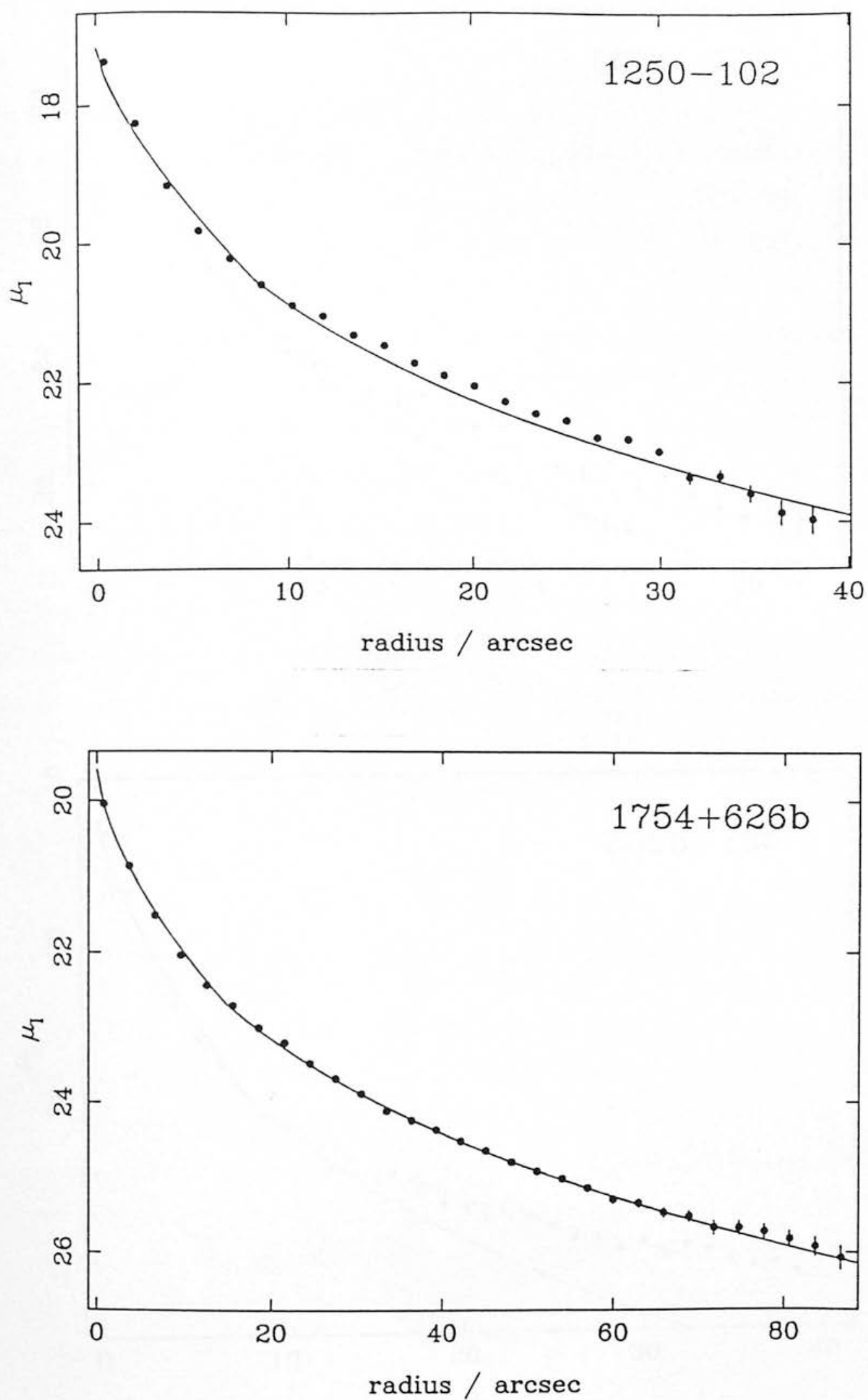

Fig. 4.7: cont.... 

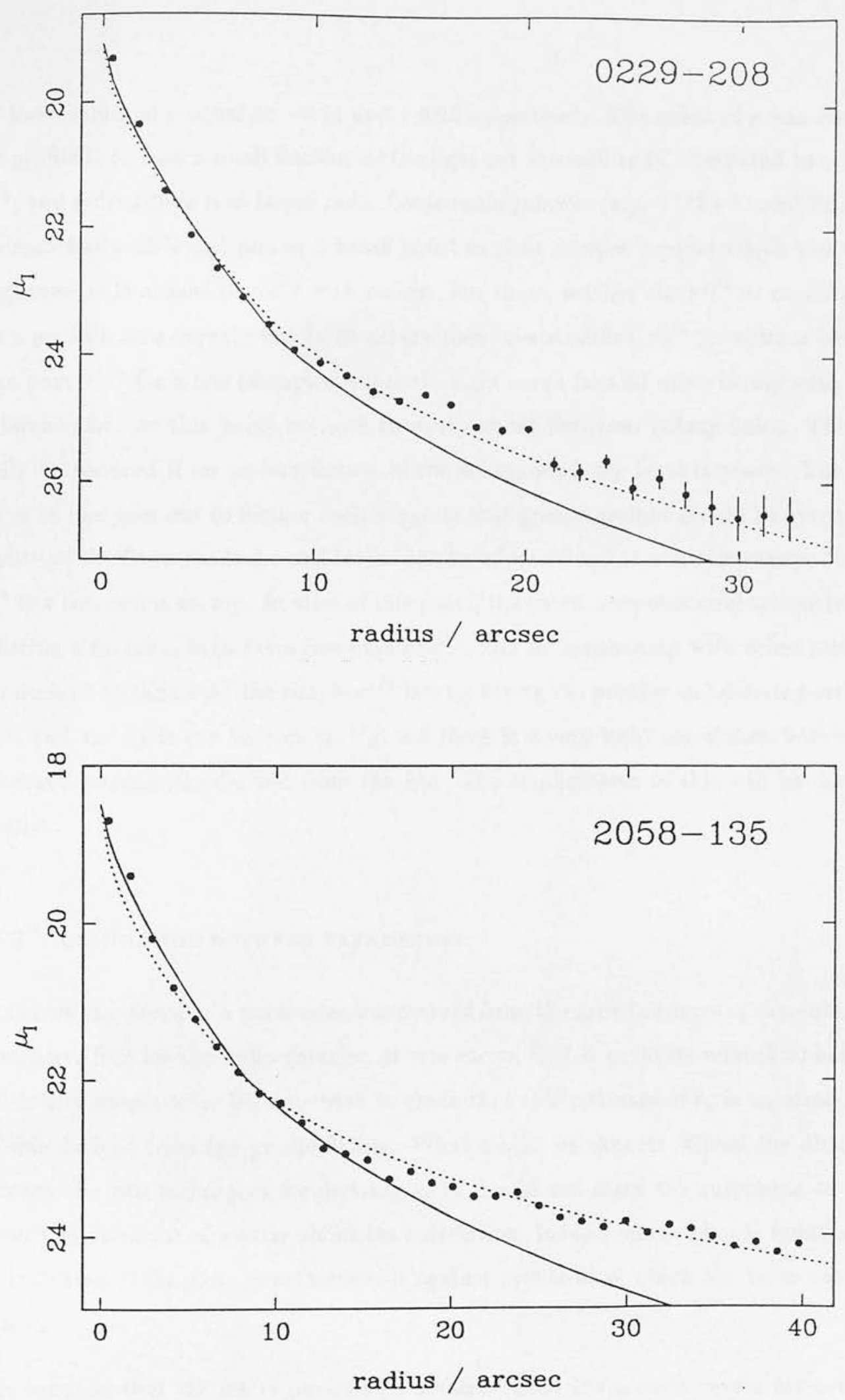

Fig. 4.7: cont.... 
4.7 have values of $\nu$ equal to -0.21 and -0.23 respectively. The effect of a non-zero $\nu$ on the profile is to take a small fraction of the light out at small radii, compared to standard $r^{1 / 4}$, and redistribute it at larger radii. Some radio galaxies (e.g. $1733+71$ and $\left.2229+39\right)$ are manifestly $c D$ 's and posses a break point in their profiles beyond which the surface brightness falls almost linearly with radius. For these, neither the $r^{1 / 4}$ or modified $r^{1 / 4}$ law's provide an adequate fit. In summary then, the modified $r^{1 / 4}$ provides a better fit than pure $r^{1 / 4}$ for a few examples where the light curve falls off more slowly with radius at large radii. At this point we need to worry about ficticious galaxy halos. These can easily be induced if an underestimate of the background sky level is made. The larger errors as one goes out to farther radii suggests that greater weight should be given to the quality of the fit in the inner and intermediate values of radius - in this regime the pure $r^{1 / 4}$ law comes out on top. In view of this point, the extra computational labour involved in fitting a function with extra free parameters, and for consistency with other workers it was decided to stick with the simple $r^{1 / 4}$ law for fitting the profiles and deriving estimates of $\mu_{e}$ and $r_{e}$. As it can be seen in Fig. 4.8 there is a very tight correlation between the structural parameters derived from the fits. The implications of this will be discussed shortly.

\subsubsection{COMPARISON BETWEEN PARAMETERS}

Earlier we saw how the $\alpha$ parameter was derived from the growth curves of sky-subtracted cumulative flux for the radio galaxies. It was shown that $\alpha$ could be related to half-light radius in a simple way. We now wish to check that this estimate of $r_{e}$ is consistent with the one derived from the profile fitting. What might we expect? Given the differences between the two techniques for deriving $r_{e}$ it should not seem too surprising to find a rather large amount of scatter about the correlation. Indeed this is what is found as Fig. 4.9 indicates. This plots growth-curve $\alpha$ against profile-fit $\alpha$ which has been converted from $r_{e}$.

This suggests that the set of parameters obtained from the growth curves are probably too simplistic to be used for the streaming analysis. Better is to use $r_{e}$ (and $\mu_{e}$ ) obtained from the profile fitting, which includes a better calculation to account for the effects of seeing and does not restrict attention to only that portion of the light within $19.2 \mathrm{kpc}$ 


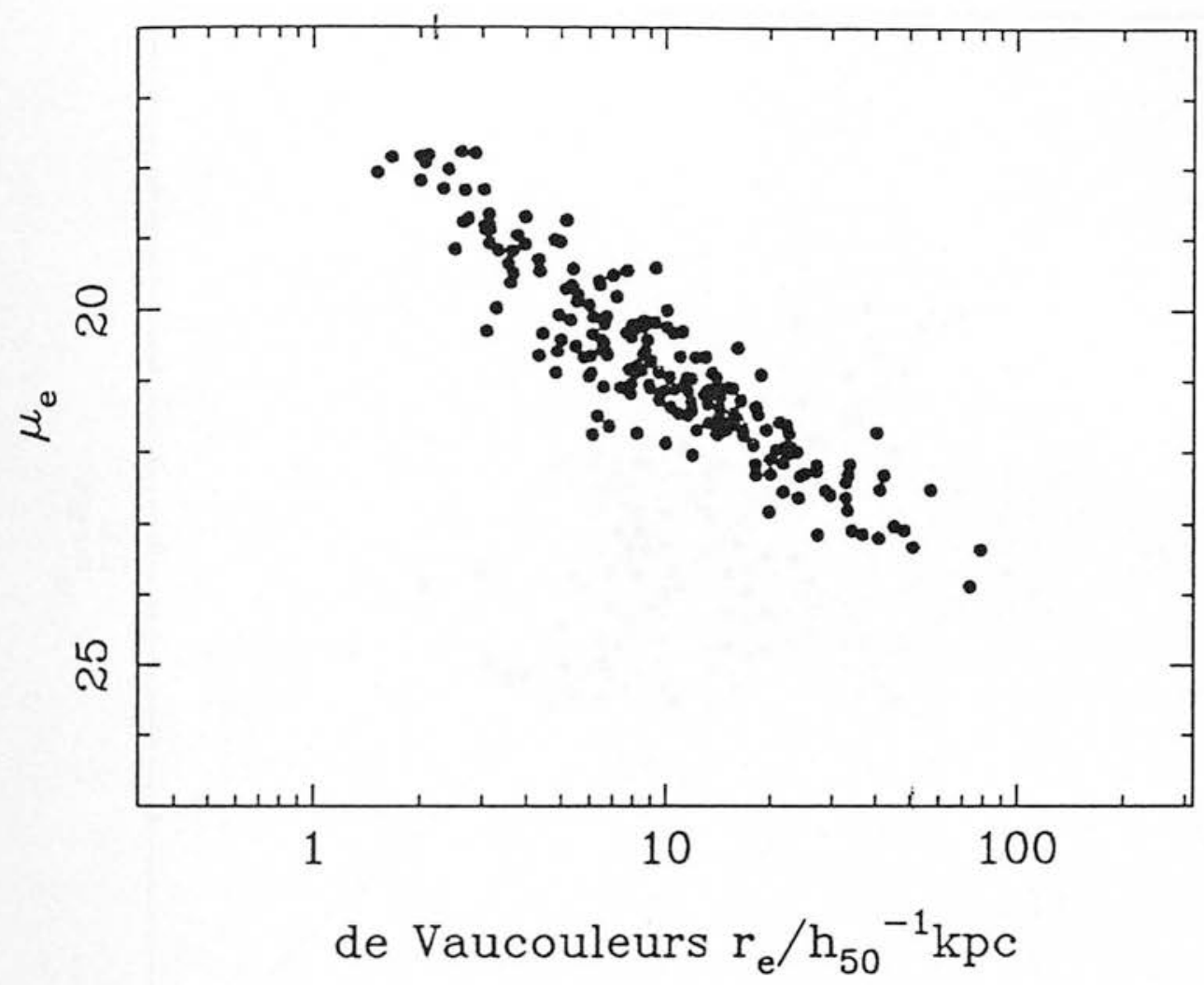

Fig. 4.8: The $\mu-r_{e}$ relation for the radio galaxies, where the parameters have been derived from empirical profile fits to the surface brightness distribution of each galaxy. 


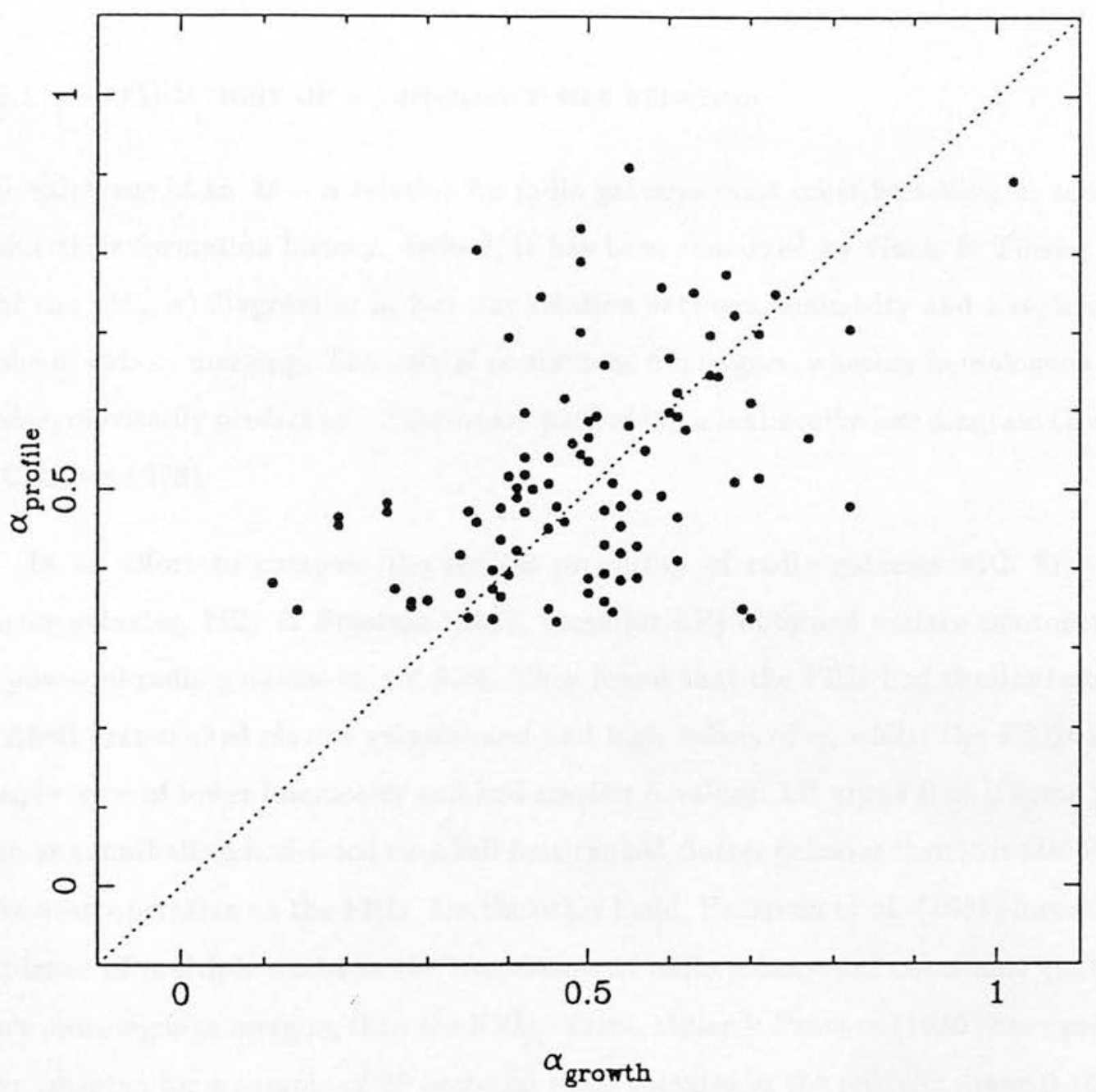

Fig. 4.9: Comparison between $\alpha$ values obtained directly from growth curves and from optical profile fits converting from $r_{e}$. The radio galaxies worst affected by seeing (i.e those with effective angular size less than $4^{\prime \prime}$ are not included on the diagram). 
which the growth curve analysis does.

\subsection{Discussion}

\subsubsection{IMPLICATIONS OF A LUMINOSITY-SIZE RELATION}

The existence of an $M-\alpha$ relation for radio galaxies must must be telling us something about their formation history. Indeed, it has been remarked by Gunn \& Tinsley (1976) that the $\left(M_{\gamma}, \alpha\right)$ diagram or in fact any relation between luminosity and size, is a direct probe of galaxy merging. Theoretical predictions of mergers, whether homologous or otherwise, inevitably predict an evolutionary path along a luminosity-size diagram (Hausman \& Ostriker 1978).

In an effort to compare the optical properties of radio galaxies with first-ranked cluster galaxies, Lilly \& Prestage (1987, hereafter LP) obtained surface photometry for 31 powerful radio galaxies at $z<0.25$. They found that the FRIs had similar luminosity to Abell first-ranked cluster galaxies and had high values of $\alpha$, whilst the FRIIs in their sample were of lower luminosity and had smaller $\alpha$ values. LP argue that if some process such as cannibalism had acted on Abell first-ranked cluster galaxies then it is also likely to have been operative on the FRIs. On the other hand, Heckman et al. (1986) looked at the incidence of multiple nuclei in the two classes of radio galaxy and concluded that FRIIs show more signs of merging than the FRIs. Yates, Miller \& Peacock (1989) have presented deep imaging for a sample of 25 powerful radio galaxies in the redshift range $0.15<z<$ 0.82. Only 2 FRIIs in their sample show any low-surface brightness features and a further 4 of the FRIIs have multiple nuclei. These observation support the conclusions of LP, i.e. that FRIIs appear not to have had a particularly violent history. However, as Yates, Miller \& Peacock note, there is an alternative explanation: the FRI radio galaxies may be old mergers and the FRII galaxies young mergers.

The observations of LP can be tested with the photometry presented here if we roughly discriminate between the two FR types, as we did earlier, according to their radio luminosity. Recall that the Fanaroff-Riley division is at a radio power of about $\log _{10} P_{2.7}=$ 
$24 \mathrm{WHz}^{-1} \mathrm{Sr}^{-1}$. The results for the mean $(M, \alpha)$ pair for the FRIs are $(-24.06,0.41)$ and for the FRIIs these are $(-24.14,0.47)$. Thus, there is only very marginal evidence for any differentiation of sources on the $M-\alpha$ plane for the all-sky sample radio galaxies. A more reliable test of this could be made if radio maps were forthcoming for the sources allowing for a more precise morphological identification.

\subsubsection{THE $\mu_{e}-r_{e}$ RELATION AS A Distance INDicAtor}

The implications of the tight correlation between $\mu_{e}$ and $r_{e}$ for measuring galaxy peculiar velocities will be discussed in some detail in Chapter 6 . The basic idea is that we have measured a rest-frame surface brightness $\mu_{e}$ which is distant-independent and allows the 'standard rods' to be calibrated. Other distance indicator relations for elliptical galaxies, the best of which is the $D_{n}-\sigma_{v}$ relation (Dressler et al. 1987), involve measuring velocity dispersions which are a lot more difficult to acquire at the telescope than the simple 'snapshot' CCD frames used to measure $\mu_{e}$ and $r_{e}$. Given the simplicity of the $\mu_{e}-r_{e}$ relation, it fares almost as well as the $D_{n}-\sigma_{v}$ relation in terms of relative percentage distance measurement accuracy as we shall see in Chapter 6.

\subsection{Colour-Magnitude Diagram}

Only a few $B$ frames were eventually acquired for the radio galaxies but these allow us take a preliminary look at the $B-I$ vs. $I$ colour-magnitude relation. Our main interest is to examine how well such a relation might be able to be used to tighten up our estimates of peculiar velocities based on the $\mu_{e}-r_{e}$ relation alone.

Metric colours were derived for 79 of the radio galaxies, where the aperture size was kept at $19.2 \mathrm{kpc}$ to save having to duplicate measurements. Figs. 4.10 and 4.11 plot $B-I$ colour against $I$ magnitude and redshift respectively.

The scatter about the colour-magnitude relation is clearly far too large for it to tighten up distances estimates by very much. It is also, unfortunately, a very flat relationship. The solid line marked on the colour-redshift diagram is a guide to the expected colours for elliptical galaxies. It thus represents the colour of redshifted starlight. The line has a 


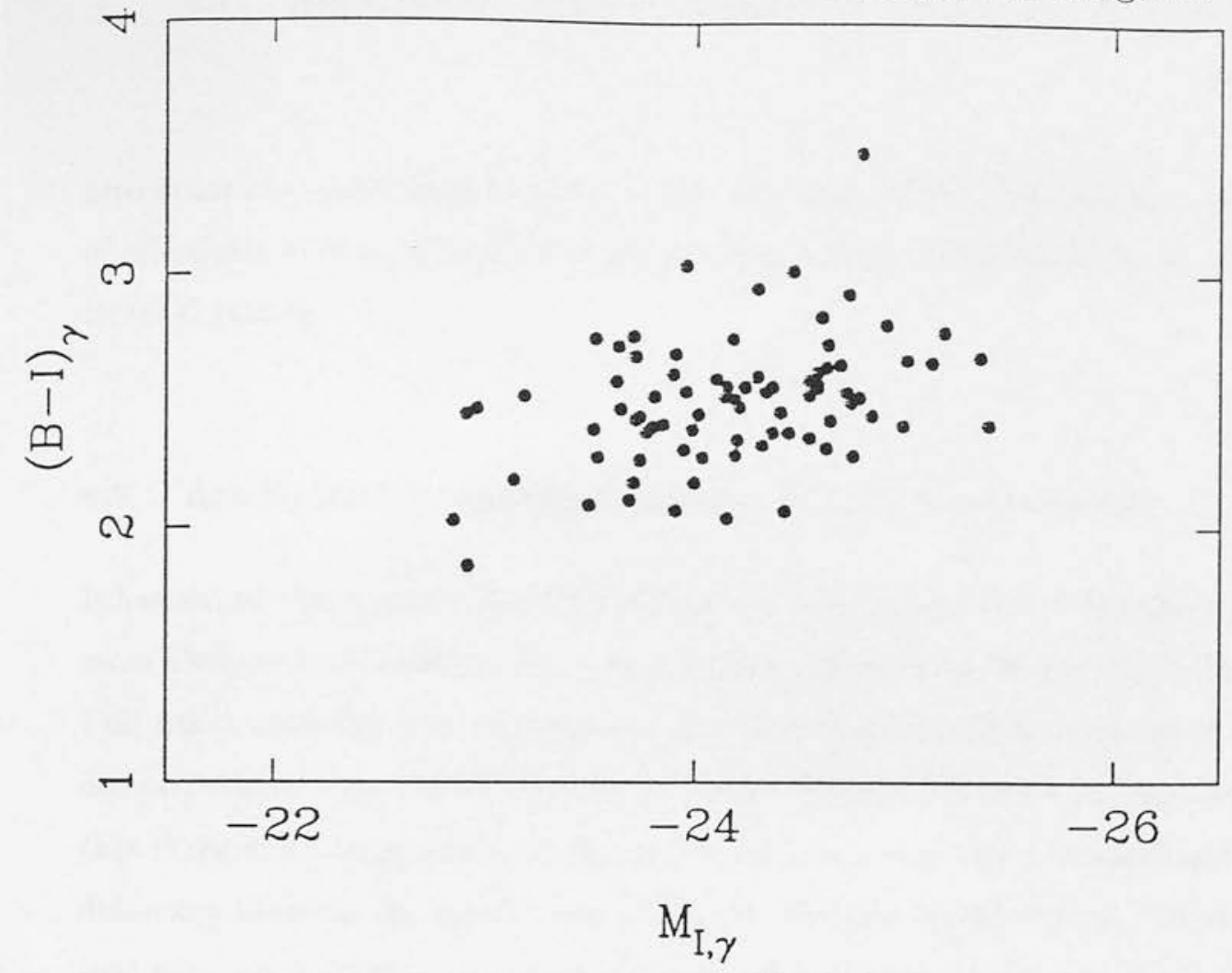

Fig. 4.10: Colour-magnitude diagram for a small subsample of radio galaxies which have both $B$ and $I$ frames.

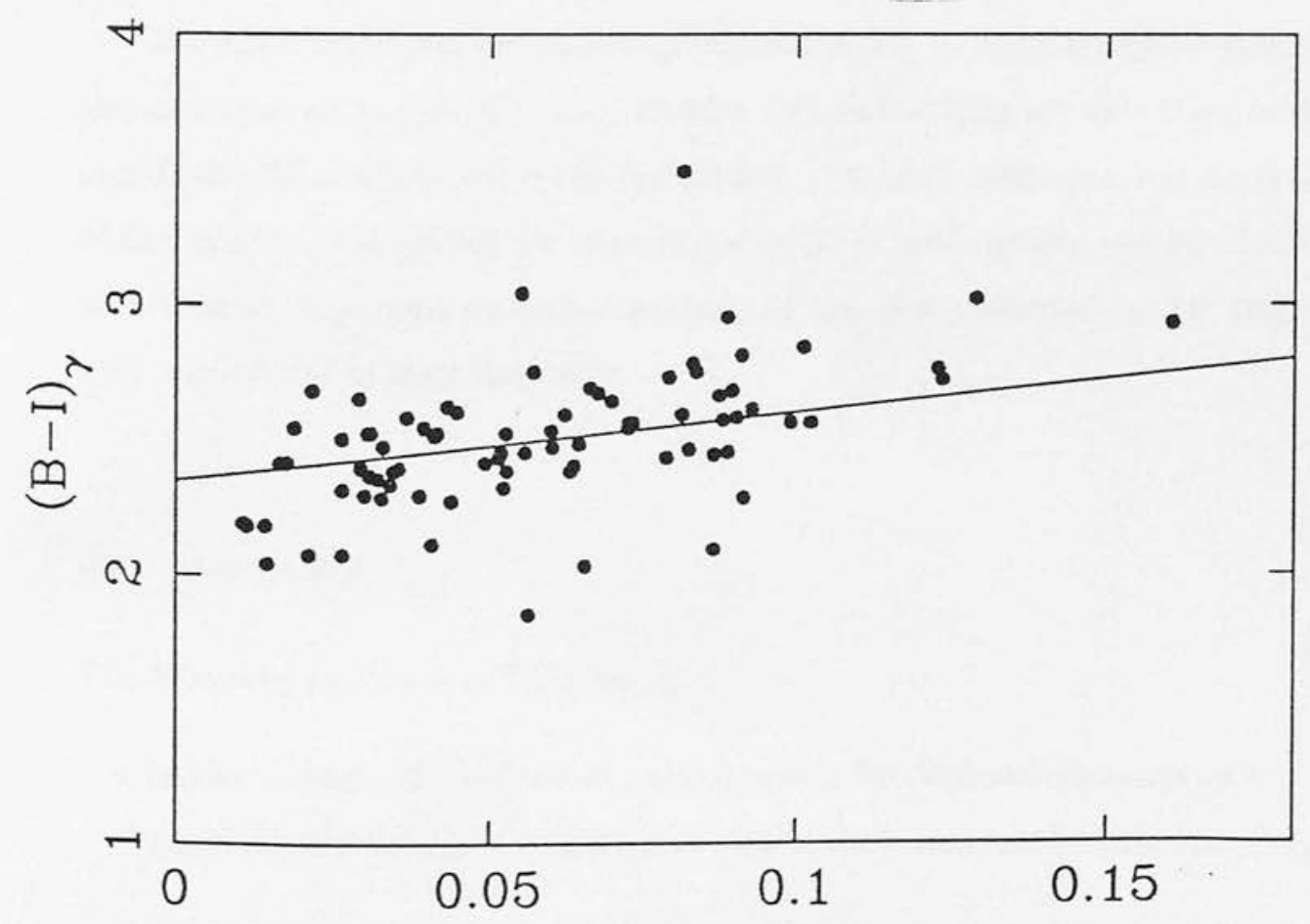

\section{redshift $\mathrm{z}$}

Fig. 4.11: $\quad B-I$ colour plotted against redshift. The line has a slope of $2.5 z$, taken from the $K$-corrections of Bruzual (1983). 
zero-point of $\sim 2.35$ taken from the results of a study of $B-I$ colours for a small sample of ellipticals in Virgo (Pierce 1986) and a slope of $2.5 z$ taken from the $K$-corrections of Bruzual (1983).

\subsection{Are Optical Properties Related to Cluster Environment?}

It has already been noted that Lilly \& Prestage (1987) found that FRI radio galaxies were more likely to have multiple nuclei than FRII's and hence to be more optically luminous. This fits in with the observed tendency that they found for FRIs to reside, on average, in denser systems than the FRIIs. Earlier in this Chapter we found for the all-sky sample that there was a large scatter in $B_{g r}$ over a range in power and no statistically significant difference between the mean value of $B_{g r}$ for the two morphological classes, assuming that they can be differentiated on the basis of their radio luminosity. Similarly, we find no evidence for any trend in optical luminosity with richness of environment. The data are shown in Fig. 4.12 which plots $M_{\gamma}$ in $I$ against $B_{g r}$.

If we divide the sample into two groups about $B_{g r}=250$, then either side of this value the mean metric magnitudes are $-23.92 \pm 0.69$ and $-23.93 \pm 0.64$ - there is therefore no significant difference between the two classes. A similar conclusion was reached by Yates, Miller and Peacock (1989) for their higher redshift radio galaxy sample. Moreover, they note that an improved statistical analysis of the data presented by LP suggests only a very weak trend in their data also.

\subsection{Summary}

The following results have been found:

- in the absence of a full set of galaxy counts for declinations south of $\delta=-23^{\circ}$, the Abell cluster catalogue can be used to a make a reasonable estimate of $B_{g g}$;

- radio galaxies either side of the Fanaroff-Riley transition show similar extremes in $B_{g r}$, with no tendency for FRI's to occupy, on average, richer environments than FRIIs; 


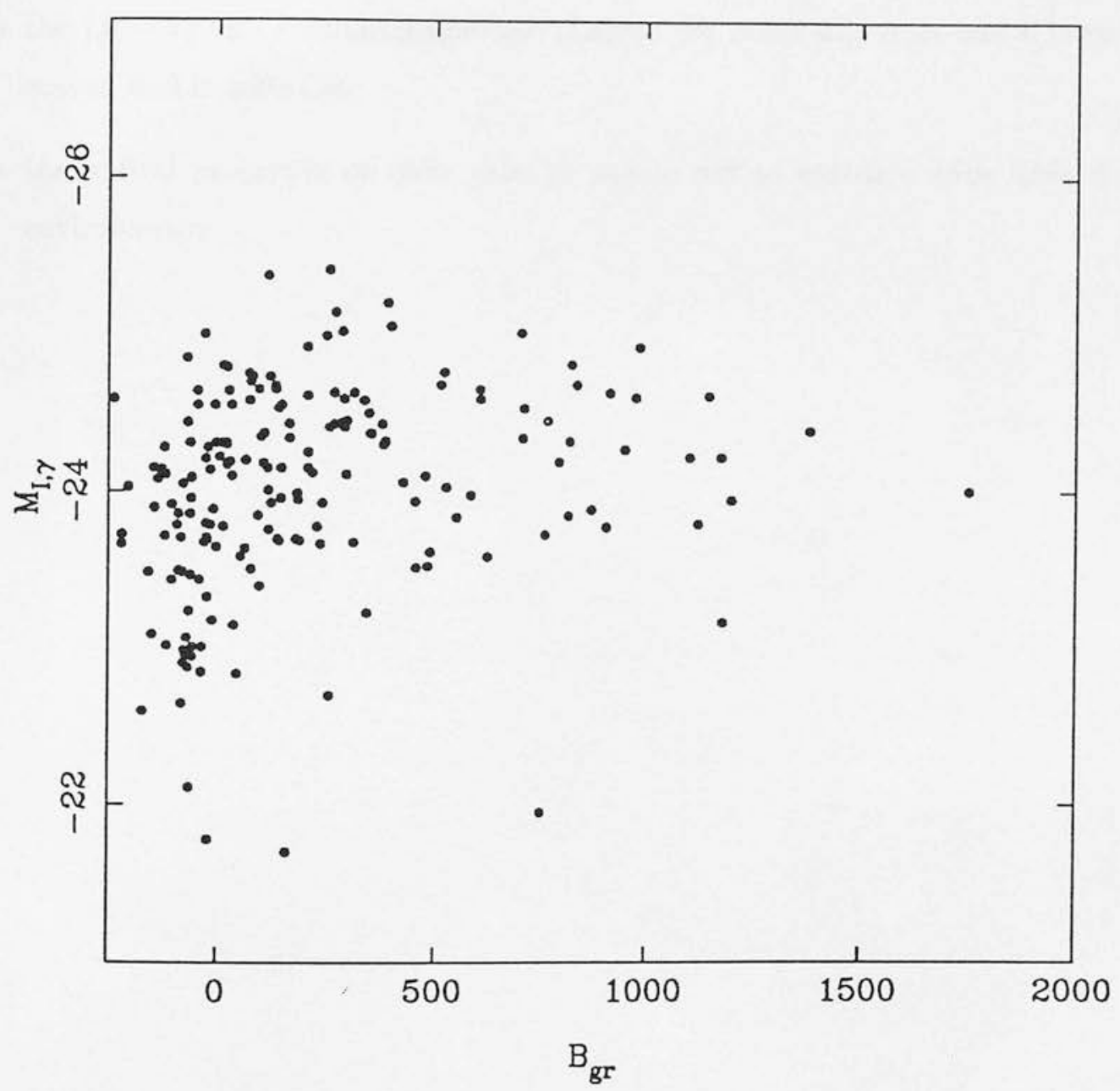

4.12: Optical luminosity against richness of environment for the all-sky sample radio ellipticals. No trend is apparent in the data. 
- on average, radio galaxies inhabit regions of the universe which are as overdense as Abell clusters of richness $R=0$;

- the $\mu_{e}-r_{e}$ relation for radio ellipticals is extremely well defined with a scatter that corresponds to a relative photometric percentage distance accuracy of about $28 \%$;

- the $(B-I)$ vs. $I$ colour-magnitude diagram for radio ellipticals has a large rms scatter and is quite flat;

- the optical properties of radio galaxies appear not to correlate with their cluster environments. 


\section{Chapter 5}

\section{The 3-d Clustering of Radio Galaxies}

\subsection{Introduction}

Observations of large-scale clustering in the universe arguably rank alongside microwave background fluctuations as a discriminator between the cosmological models for structure formation. Some recent observations of clustering seem to suggest the existence of very large-scale density inhomogeneities in the universe. These pose something of a challenge for most theoretical models and, in particular, appear to exclude the Standard Cold Dark Matter cosmology. Naturally, before revising a theoretical model it is necessary to convince oneself that the observations are reliable. To date, these have presently been based mostly around optically selected samples of rich clusters, especially the Abell cluster catalogue. It is well-known that this catalogue suffers from pathological selection effects, but the effect that these might have on the derived cluster correlations is not entirely understood. In any case these doubts make it rather premature to rule out any cosmology simply on the basis of statistics derived from optically-selected cluster catalogues alone.

In a few years time the situation will have improved considerably and less subjective cluster catalogues are likely to be at our disposal. The X-ray satellite, ROSAT, for example is currently conducting an all-sky search for clusters. This X-ray selected cluster catalogue will be ideal for an unbiased statistical study of the large-scale structure. In addition, automated cluster surveys are under way at the Royal Observatory Edinburgh and at Cambridge.

The main result of this Chapter is the 3-d correlation function for radio galaxies. It is unique in the sense that such a quantity has never been calculated before for radio galaxies. Previous investigations of the distribution of radio galaxies have used 2-d data only to compute the angular correlation function. Since radio galaxies can be consid- 
ered to be abnormally bright ellipticals which reside in rich environments, the theoretical expectation is that these objects should show an enhanced correlation over that for normal galaxies. The radio galaxy redshift survey can test this point, which has important implications for galaxy formation.

The radio galaxy survey volume is also deep enough for constraints to be put on the existence of very large-scale structure in the universe. The chief pioneer of such structure has been Tully (1986). His claim is that the distribution of rich clusters shows a marked flattening throughout the volume $z<0.1$ towards the supergalactic plane.

The Chapter is divided into the following Sections. Firstly, Section 5.2 presents a brief overview of some of the key observations of large-scale clustering in the universe. A description of the calculation of the radio galaxy spatial correlation function is given in Section 5.3 and the main results are presented. A check for any radio power-dependence of the observed clustering is made. The Tully effect is examined in Section 5.5. Finally, the Chapter is concluded in Section 5.6 with a short summary.

\subsection{Observations of Large-Scale Clustering in the Universe}

Simple visual scans of 2-d galaxy maps suggest a wealth of large-scale structure. The nearby galaxies show a marked concentration into groups and clusters. In 3 - $d$ it becomes clear that the hierarchy of structure extends to even larger scales, and terms such as voids, filaments, sheets, walls and superclusters have been coined by cosmographers to describe the clustering patterns in the universe.

Although useful from a simple phenomological point of view, a simple but reliable statistical tool is really needed in order to quantify this structure. The two-point correlation function $\xi(r)$ is the most widely utilised diagnostic and we shall use it here. For a more general description of n-point correlation functions the reader is referred to Layzer (1975) and Peebles (1980).

\subsubsection{THE TWO-POINT CORRELATION FUNCTION}

This is defined by the joint probability $d P(r)$ of finding two objects separated by a distance 
$r$ and within volume elements $d V_{1}$ and $d V_{2}$ such that

$$
d P(r)=n^{2}[1+\xi(r)] d V_{1} d V_{2}
$$

where $n$ is the space density of objects in the sample. The correlations are therefore zero for a random distribution of points and are positive for a clumped distribution on the relevant clumping scale, i.e. $\xi(r)$ essentially measures deviations in the clustering pattern from a random distribution. In practice there are only a few statistically complete redshift surveys of galaxies and angular correlation functions $w(\theta)$ are more common. This latter quantity is the projected analogue in 2-d of $\xi(r)$ and it can be used to infer the spatial twopoint correlation function from Limber's equation, which is an integral equation relating $\xi(r)$ to $w(\theta)$ (Peebles 1980$)$.

In the theoretical context, $\xi(r)$ is a useful statistic since it is simply related (at least in the regime $\xi \ll 1$ ), through a Fourier transform, to the power spectrum of the density field. Thus, observations of $\xi(r)$ can be used directly to set constraints on the shape of the primordial fluctuation spectrum.

\subsubsection{CLUSTERING OF GALAXIES}

Groth and Peebles (1977) have applied the two-point angular correlation function to study the clustering of galaxies in the Zwicky and Lick catalogues. They find that the data can be most simply fit by a power-law form for $w_{g g}(\theta)$ on scales $\lesssim 2.0^{\circ}$. More specifically

$$
w_{g g}(\theta)=A \theta^{-\delta} \quad \text { with } \quad A=0.0684 \pm 0.0057 \text { and } \delta=0.734 \pm 0.035
$$

On scales $\gtrsim 2.5^{\circ}$ there is a sharp break from the power-law and this may yield important important clues to the nature of the primordial density fluctuation spectrum (e.g. Maddox et al. 1990).

The observed power-law form for $w_{g g}(\theta)$ suggests that the spatial two-point correlation function can be fit by a power-law but with an index of $\gamma=-(1+\delta)$, such that

$$
\xi_{g g}(r)=B r^{-\gamma} \equiv\left(\frac{r}{r_{0}}\right)^{\gamma}
$$


where $r_{0}$ is the characteristic scale length of clustering. This statistic has been employed to analyse clustering in the CfA redshift survey (Davis et al. 1982) by Davis \& Peebles (1983b) who find that $r_{0}=5.3 \pm 0.3 h^{-1} \mathrm{Mpc}$ for an assumed slope of $\gamma=1.77$. Likewise, de Lapparent, Geller \& Huchra (1986) estimate that $5 h^{-1} \mathrm{Mpc} \lesssim r_{0} \lesssim 12 h^{-1} \mathrm{Mpc}$ with $1.3 \lesssim \gamma \lesssim 1.9$ from the extended CfA survey. Both these results should be treated with some caution on scales $\gtrsim 10 h^{-1} \mathrm{Mpc}$ (corresponding roughly to the break scale from $w_{g g}$ ) because of errors involved in determining a background count of galaxies and with the luminosity function. Evidence for correlated structure in the CfA survey is seen even at the limits of the sample depth.

For galaxies, there is strong evidence to suggest a trend in clustering strength with the local environments of the systems. Most weakly clustered are the HII galaxies, a class of gas-rich dwarf galaxies. These objects have been found primarily in underdense regions such as voids (Thuan, Gott \& Schneider 1987). The correlation function for these systems has been measured by Iovino, Melnick \& Shaver (1988), who find a clustering scale-length of about $3 h^{-1} \mathrm{Mpc}$.

At the next level in the hierarchy are the spiral galaxies. These are generally found in less rich environments than their elliptical counterparts (Davis \& Geller 1976). Fig. 5.1 plots the clustering signals that have been observed for systems of different richness. The hierarchy extends beyond galaxies to incorporate clusters and perhaps even superclusters as we shall now discuss.

\subsubsection{CLUSTERING OF Clusters}

Optically selected cluster catalogues (Abell 1958; Zwicky et al. 1961-68; Schectman 1985) have been used extensively to compute the cluster-cluster correlation function. An enhancement is found over the galaxy correlation function which is strikingly large. In particular, Bahcall \& Soneira (1983, hereafter BS) have performed statistical analysis of clustering in the Abell catalogue and report a value of $\xi_{c c}(r)$ for clusters of richness $\mathrm{R} \geq 1$ which is unity at approximately $25 h^{-1} \mathrm{Mpc}$ and remains positive out to $\sim 100 h^{-1} \mathrm{Mpc}$. This corresponds to an enhancement over galaxies by about a factor of 18 , but it is the scale-length over which the structure is found to persist that is the most worrying feature. 


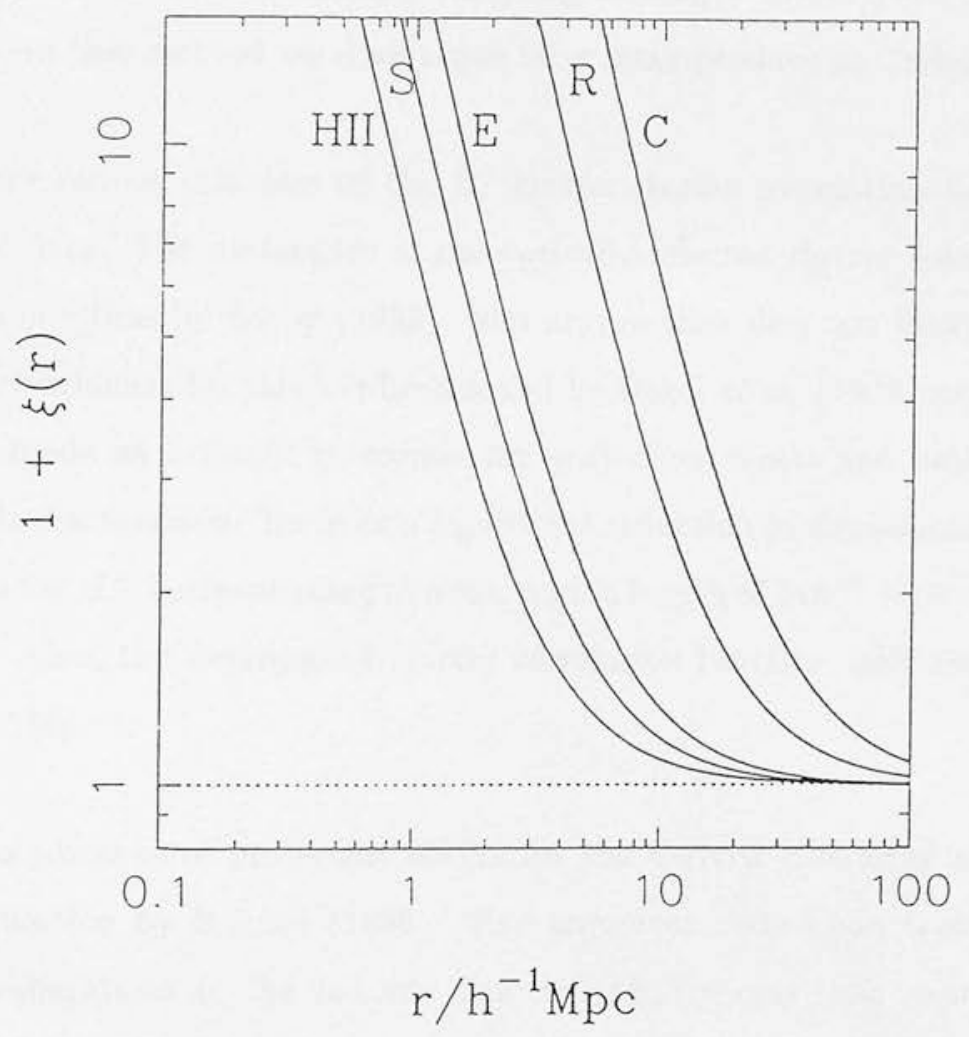

Fig. 5.1: The observed hierarchial trend for systems of increasing richness to show enhanced correlations. The key is: HII - HII dwarf galaxies; S - spirals; E-ellipticals; R - radio ellipticals (this thesis); C - clusters. The references for these are in the main text. 
The conclusions drawn by Bahcall \& Soneira have been challenged on two main grounds - for statistical reasons and for reasons concerned with the reliability of the observational data. Statistically, one is dealing with only a relatively small number of clusters. The analysis presented by BS was based on only 104 clusters. The Poissonian error bars on $\xi(r)$ are therefore likely to be large. Moreover, it has been argued by Ling, Frenk \& Barrow (1986) that the Poisson error bars are an underestimate of the true errors on $\xi(r)$. They advocate a bootstrap resampling technique to get a better handle on the errors, but even this method we shall argue later, may produce an underestimate.

A more serious criticism of the BS cluster-cluster correlation function refers to the source of data. The uniformity of the optically-selected cluster catalogues has been brought into question by Lucey (1983), who argues that they are biased by projection effects. Direct evidence for this has been found by Dekel et al. (1989) and by Sutherland (1988), who made an attempt to correct for projection effects and derived a corrected cluster correlation function. He finds a significant reduction in the enhancement of $\xi_{c c}(r)$ by about a factor of 6 , corresponding to a correlation length of $14 h^{-1} \mathrm{Mpc}$ with no richnessdependence. Also, the deprojected cluster correlation function falls essentially to zero beyond $50 h^{-1} \mathrm{Mpc}$.

The importance of projection effects for the derived clustering results has been called into question by Bahcall (1988). Her argument rests upon scatter-diagrams of cluster pair separations in the redshift direction $\left(R_{z}\right)$ versus their separation in right ascension $\left(R_{\alpha}\right)$ or declination $\left(R_{\delta}\right)$. A strong and systematic elongation in the $z$-direction was seen by Bahcall and deemed unlikely to be due to projection effects since this would have produced an excess of pairs at any $R_{z}$, as well as any $R_{\alpha}$ and $R_{\delta}$, rather than the excess observed specifically at small separations $(\Delta z \leq 0.015)$.

It is evident that in order to compute $\xi_{c c}(r)$ with any degree of confidence, an alternative and more objective technique for selecting clusters must be sought. Future prospects for doing just this look very good with redshift surveys of machine-based cluster catalogues currently underway (Collins, private communication). Also, with the launch of the ROSAT satellite in May 1990, there is the prospect of an X-ray selected sample of clusters to look forward to in a year or two hence. 


\subsubsection{SUPERCLUSTER CORRELATIONS}

Remarkably, there is also some evidence to suggest that the clustering hierarchy extends beyond rich clusters of galaxies to include superclusters. Their spatial distribution has been studied by Bahcall \& Burgett (1986). Although the analysis performed by Bahcall $\&$ Burgett was based on only a small sample of superclusters, they claimed a $3 \sigma$ effect which implies the existence of very large-scale structure in the universe on a scale of $100-150 h^{-1} \mathrm{Mpc}$. In addition, the supercluster correlation amplitude fits in well with the increased correlation-richness trend defined by galaxies and clusters (Bahcall 1988).

\subsubsection{THE CLUSTERING OF RADIO GALAXIES}

Searches for angular clustering have also been carried out in samples of radio galaxies. Seldner \& Peebles (1978) analysed the 4C survey and detected weak angular correlations at the $2-3 \sigma$ level on a scale of $1-3^{\circ}$. These correlations however were later shown by C. Masson (1979) to be artifacts. Recently, Langston (1988) has reported clustering on scales of up to $24^{\prime}$ amongst the weaker sources $\left(60 \lesssim S_{5} \lesssim 89 \mathrm{mJy}\right)$ in the MG $5 \mathrm{GHz}$ survey.

One of the earliest and best attempts to detect clustering of radio galaxies was that of Webster (1977), who used a power spectrum analysis method to show that fluctuations in radio galaxy number density between randomly-place cubes of side $1 \mathrm{Gpc}$ were $\leq 3 \%$. The degree of isotropy of the universe that this implies, $\leq 10^{-4}$ scaling density perturbations back to last scattering at $z \simeq 1000$, provides a comparable constraint to present observations of microwave background anisotropies on $\sim 10^{\circ}$ scales. Webster found no angular clustering because the cm-wavelength surveys he used for his study comprised of radio galaxies with a typical redshift of $z \simeq 1$, so that projection effects dilute clustering on smaller scales very severely.

Recently, Shaver \& Pierre (1989) have performed various statistical tests on the distribution of strong radio sources in the Molonglo catalogue. They find an anisotropy, which is manifest in the $\log (N)-\log (S)$ relation as a slightly different slope from the -1.5 prediction of a uniformly-filled and static Euclidean universe. A possible explanation for their results is advanced later. 


\subsection{The 3-d Correlation Function for Radio Galaxies}

The all-sky sample redshift survey presents us with a unique opportunity to carry out a full 3-d search for radio galaxy clustering. This Section describes the details of the calculation and collects together the main results.

\subsubsection{BASICS}

The computation of $\xi(r)$ is relatively straightforward given a sample of galaxies with accurate spectroscopic redshifts and angular position on the sky. A pair of galaxies at distance $r_{1}$ and $r_{2}$ respectively, separated by an angle $\theta_{12}$ on the sky, have a spatial separation given by:

$$
r_{12}^{2}=r_{1}^{2}+r_{2}^{2}-2 r_{1} r_{2} \cos \theta_{12}
$$

This is valid for an $\Omega_{0}=1$ cosmology only, which we shall assume. A more complicated expression can be found for the case of an open universe and this leads to a negligible increase in $\xi(r)$ by a few percent. One generally assumes that the maximum scale of appreciable galaxy clustering is small compared to the typical distances of the galaxies in the sample. Peebles (1980) shows that there are only appreciable contributions to the relevant integrals if the objects are nearly at the same distance, $\left|r_{1}-r_{2}\right| \ll r_{1}$, and the angular separation $\theta$ is much less than one radian. For $\theta \ll 1$, the separation given by equation (5.2) reduces to

$$
r_{12}^{2}=\left(r_{1}-r_{2}\right)^{2}+r_{1} r_{2} \theta^{2}
$$

and defining

$$
u=r_{2}-r_{1}, \quad r=\left(r_{1}+r_{2}\right) / 2
$$

this becomes

$$
r_{12}^{2}=u^{2}+(r \theta)^{2}
$$

Co-moving separations were computed for all pairs of radio galaxies, adopting a value for $H_{0}$ of $100 \mathrm{kms}^{-1} \mathrm{Mpc}^{-1}$. Using proper separations decreases the co-moving separation by a factor of only $(1+z)^{-1} \lesssim 10 \%$, hence slightly decreasing the amplitude of $\xi$.

The statistic we wish to compute, $\xi(r)$, is usually found by creating a random catalogue, with many more members than the true catalogue, and by counting pairs 
either within the two catalogues or between catalogues. These give the two estimates

$$
\begin{aligned}
& 1+\xi_{1}=\langle D D\rangle /\langle R R\rangle \\
& 1+\xi_{2}=\langle D D\rangle /\langle D R\rangle
\end{aligned}
$$

where $D D$ and $D R$ are the number of actual galaxy pairs and galaxy-random point pairs with redshift separations in the range $(r-d r / 2, r+d r / 2)$. There is little difference between these estimators, although the second is generally held to be more robust and it is the one that we shall use here.

\subsubsection{THE MONTE-CARLO DATASETS}

The various methods for creating synthetic catalogues in order to compute $\xi(r)$ must be weighed up quite carefully. For the radio galaxy sample it is essential that we take due account of the fact that the galaxies have been assembled from four different surveys, each of which have covers different declination bands. Since the surveys are undoubtedly incomplete (although to only a small extent as we noted in Chapter 2) there is a corresponding declination-dependent incompleteness in the sample which must be allowed for. It is important that one has a clear understanding of the sample selection effects so that they might be disentangled from any real clustering that is present in the sample.

The most simple and least realistic synthetic catalogue can be constructed by simply shuffling observed redshifts and generating a random right ascension and declination $(\alpha, \delta)$ pair, ensuring that these are consistent with $|b|>15^{\circ}$. Strictly speaking one should perturb the observed redshifts with a small velocity drawn from a normal distribution with a dispersion of, say, $150 \mathrm{kms}^{-1}$. This safeguards against reproducing any redshift clumping that might exist in the real survey into the simulated dataset - in practice the effect that this had on $\xi(r)$ was minimal.

An improved synthetic catalogue again shuffles observed redshifts, but this time assumes declination-dependent depth and correspondingly selects declinations randomly from the list of observed declinations. As before, a random right ascension is generated and the $(\alpha, \delta)$ pair are checked to ensure that $|b|>15^{\circ}$. 
Finally, a further improvement one could make is to allow both the completeness and redshift distribution of the sample to vary over the sky. Simulated catalogues are generated in this way by selecting randomly from the list of observed galaxy coordinates and redshifts. This last method was used for computing the estimates of $\xi$ presented in the next Section.

\subsubsection{ERROR ESTIMATES}

Having described a method for calculating $\xi(r)$, we now need to consider how to estimate realistic error bars. Suppose $N_{p}$ is the number of independent pairs that are counted in a given bin. Then a simple Poissonion estimate of the error on $\xi$ in that bin is given by

$$
\frac{\Delta \xi}{1+\xi}=\frac{1}{\sqrt{N_{p}}}
$$

Peebles (1973) has argued that this gives an underestimate of the true uncertainty in $\xi$. The right-hand side needs to be increased by a factor of roughly $1+4 \pi J_{3}$, where $4 \pi J_{3}$ is the volume integral of $\xi$ out to the radius of interest. Since $J_{3}$ is generally difficult to measure, an alternative scheme for producing correct errors - the bootstrap resampling method - has been suggested by Ling, Frenk \& Barrow (1986). We shall not waste time describing the details of this method here, but note that it too is likely to produce an underestimate of the true errors. The key point is that one is always concerned with sampling errors in the sense that large-scale variations in the properties of the density field cause the area under study not to be a totally fair sample. One straightforward way to deal with this is to subdivide the sample and look at fluctuations between sub-samples located in independent regions of space. Although rather inefficient, this method is at least a safer option than either of the other two for estimating reasonably representative values of the error bars on $\xi(r)$.

In order to put this method into effect, the radio galaxy sample was sub-divided up into four declination bands, each of which covered an approximately equal area of the celestial sphere. $\xi(r)$ was calculated for each subsample of the radio galaxies and it is the scatter about these four estimates then which gives an indication of the true uncertainty in $\xi(r)$. A mean value of $\xi(r)$ can be found for each bin if desired by weighting the individual estimates of $\xi(r)$ with the square-root of the number of galaxies in each declination band. Similarly, a weighted error can be calculated on the mean. 
The correlation function for the composite sample of 354 radio galaxies is shown plotted in Fig. 5.2.

Four points are plotted per bin, each of which represents the value of $\xi(r)$ for galaxies subdivided up into different declination zones. The scatter in these points gives a rough guide of the true errors on $\xi(r)$. In fact these agree quite well with the Poisson error bar estimates, which are omitted so as not to unduly clutter up the diagrams.

Due to the uncertainties inherent in fitting a model to the various points, which are not guaranteed to be independent, this was not attempted here. Instead, a dashed line corresponding to a power-law for $\xi(r)$ with index of -1.8 and lag of $14 h^{-1} \mathrm{Mpc}$ has been marked. The data clearly follow this line very closely - the characteristic scale length for clustering in the all-sky sample is very close to $14 h^{-1} \mathrm{Mpc}$ and $\xi$ falls approximately as a power-law with slope -1.8 .

\subsubsection{TRENDS WITH RADIO LUMINOSITY}

In view of the points that we discussed in Chapter 4, regarding the environmental differences between FRI (low radio power) and FRII (high radio power) radio galaxies it is interesting to check for any trends in the clustering signal with radio luminosity. Fig. 5.3 plots $\xi(r)$ for radio galaxies in different bands of $1.4 \mathrm{GHz}$ radio power which have been indicated on the diagrams.

The errors on $\xi(r)$ are obviously larger than those for the composite sample but it is still clear that the strength of clustering is virtually identical for the low- $P_{1.4}$ radio sources as it is for the most powerful sources in the sample. These results fit in neatly with the conclusions of Chapter 4, in which it was found that there was no significant fall-off in $B_{g r}$, the amplitude of the spatial cross-correlation function, going from low values of radio luminosity through to higher values. 


\section{all galaxies}

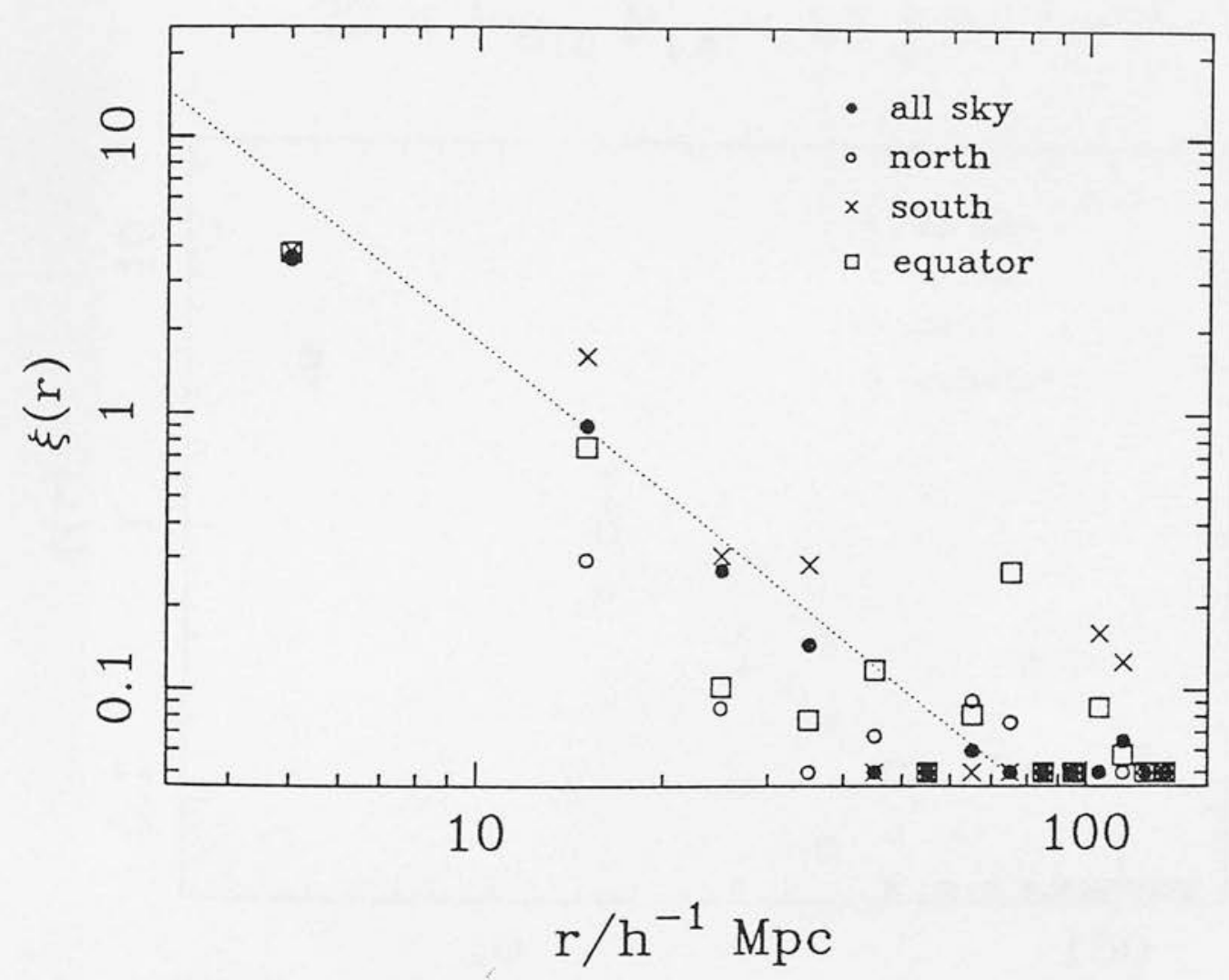

Fig. 5.2: The 3-d correlation function for all of the radio galaxies in the $0.01<z<0.1$ sample. Different symbols refer to the different declination bands as indicated in the key. The scatter about the points gives an indication of the true errors on $\xi$. The dashed line marked is for a power-law model for $\xi$ with slope equal to -1.8 and characteristic length equal to $14 h^{-1} \mathrm{Mpc}$. 


$$
23<\log _{10} \mathrm{P}_{1.4}<25 \mathrm{WHz}^{-1} \mathrm{sr}^{-1}
$$

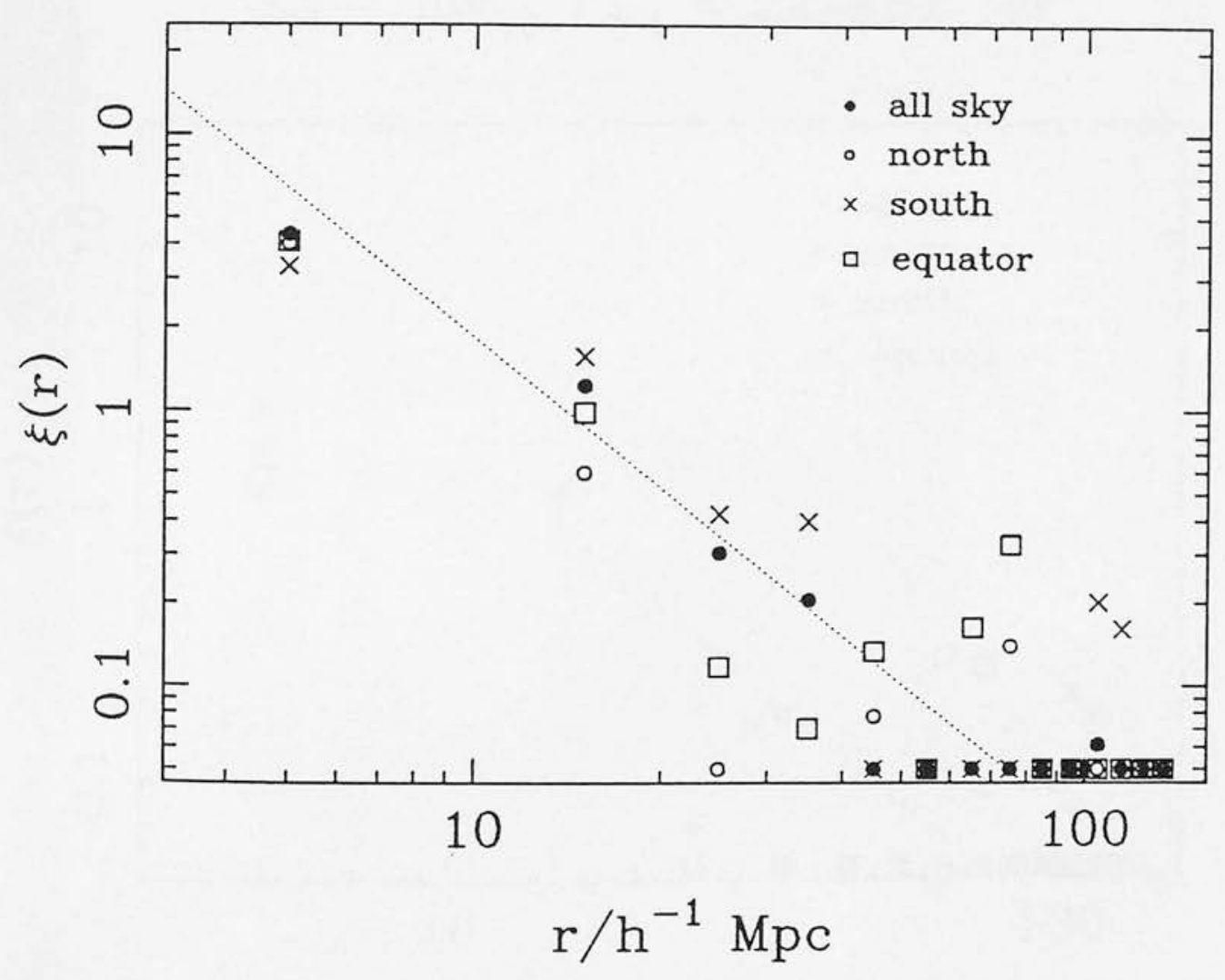

Fig. 5.3: 3-d correlation function for radio galaxies in different bands of $1.4 \mathrm{GHz}$ radio luminosity. The error bars are larger due to the poorer numbers in each declination band. 
$23<\log _{10} \mathrm{P}_{1.4}<24 \mathrm{WHz}^{-1} \mathrm{sr}^{-1}$

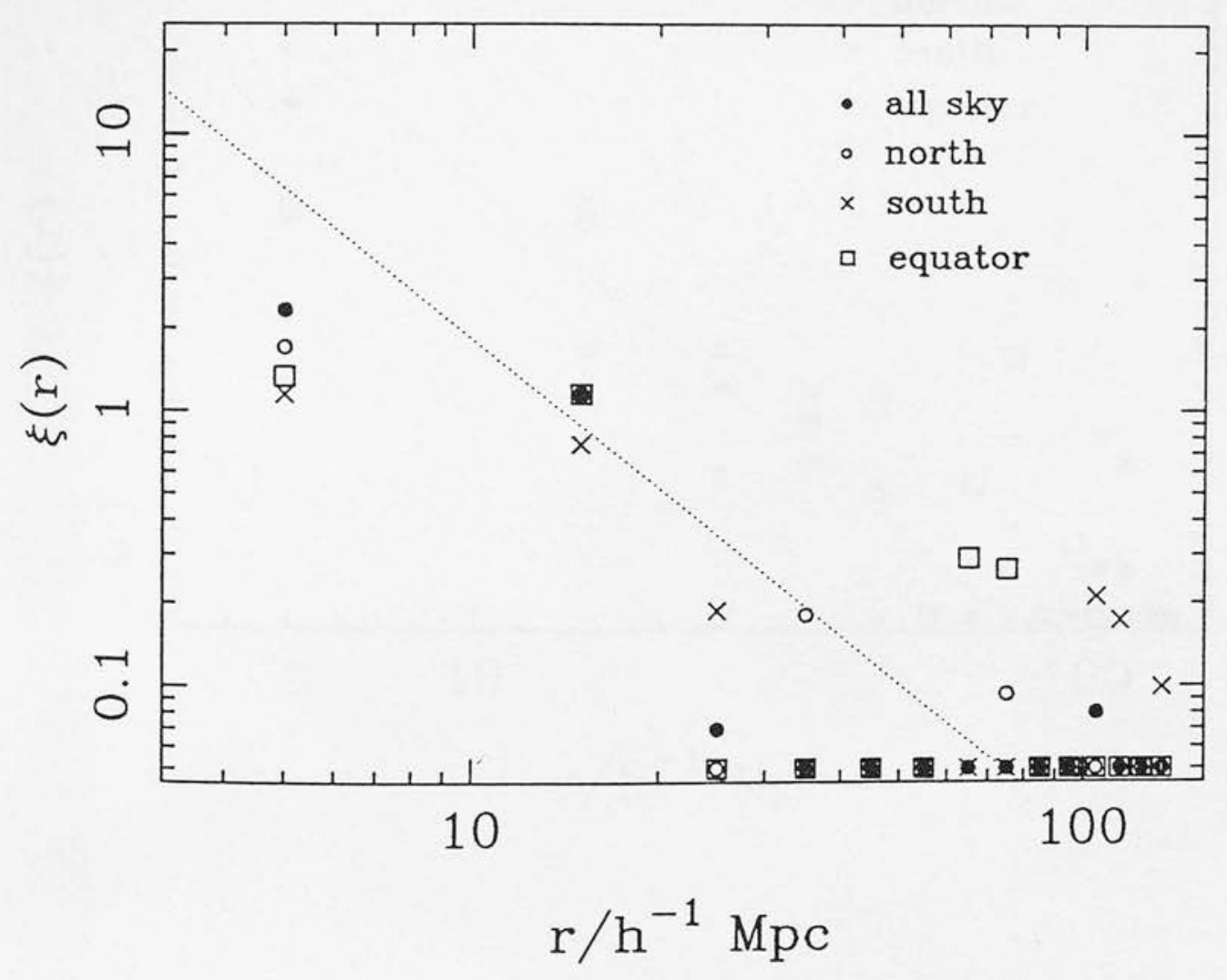

Fig. 5.3: cont.... 
$23.5<\log _{10} \mathrm{P}_{1.4}<24.5 \mathrm{WHz}^{-1} \mathrm{sr}^{-1}$

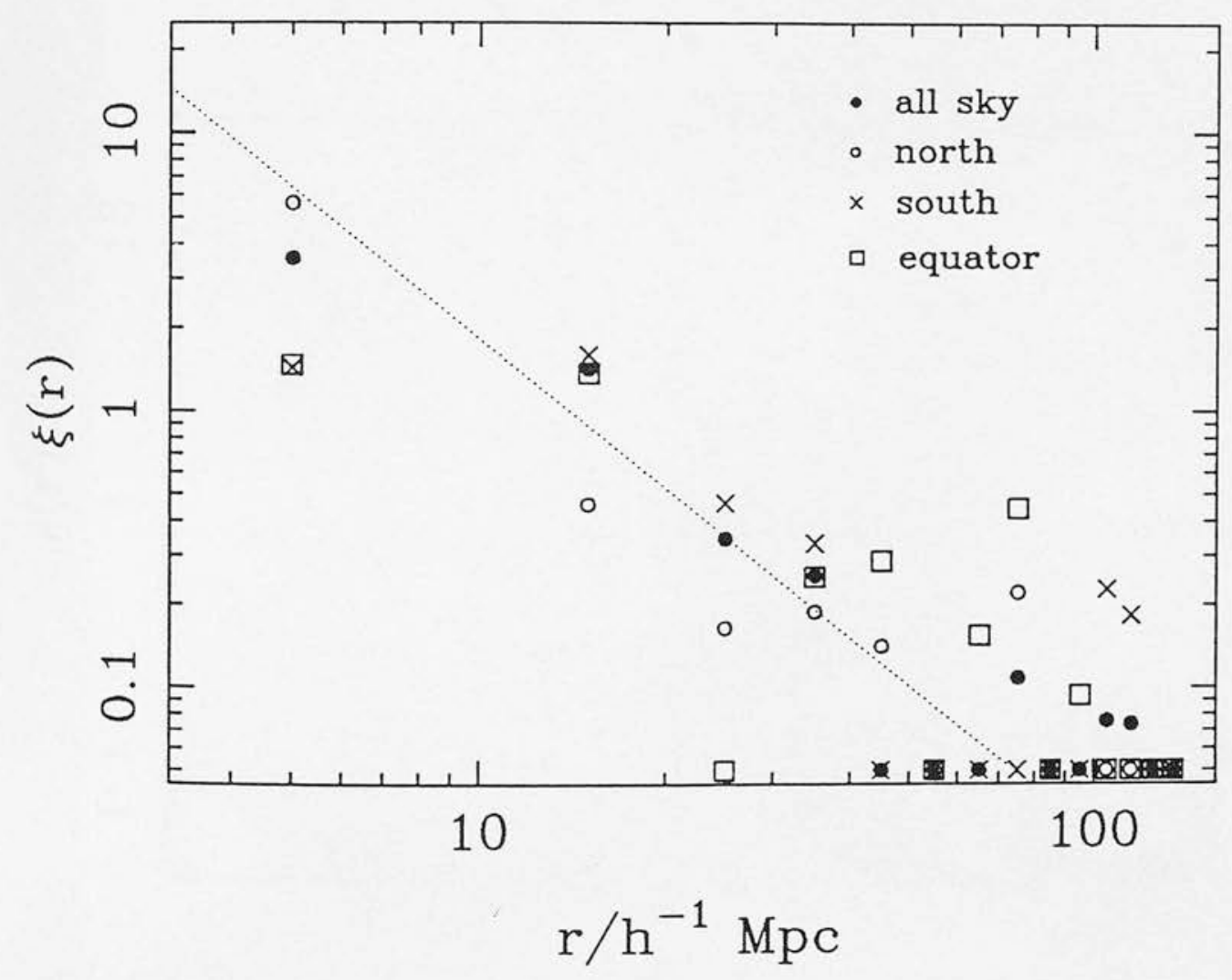

Fig. 5.3: cont.... 
$24<\log _{10} \mathrm{P}_{1.4}<25 \mathrm{WHz}^{-1} \mathrm{sr}^{-1}$

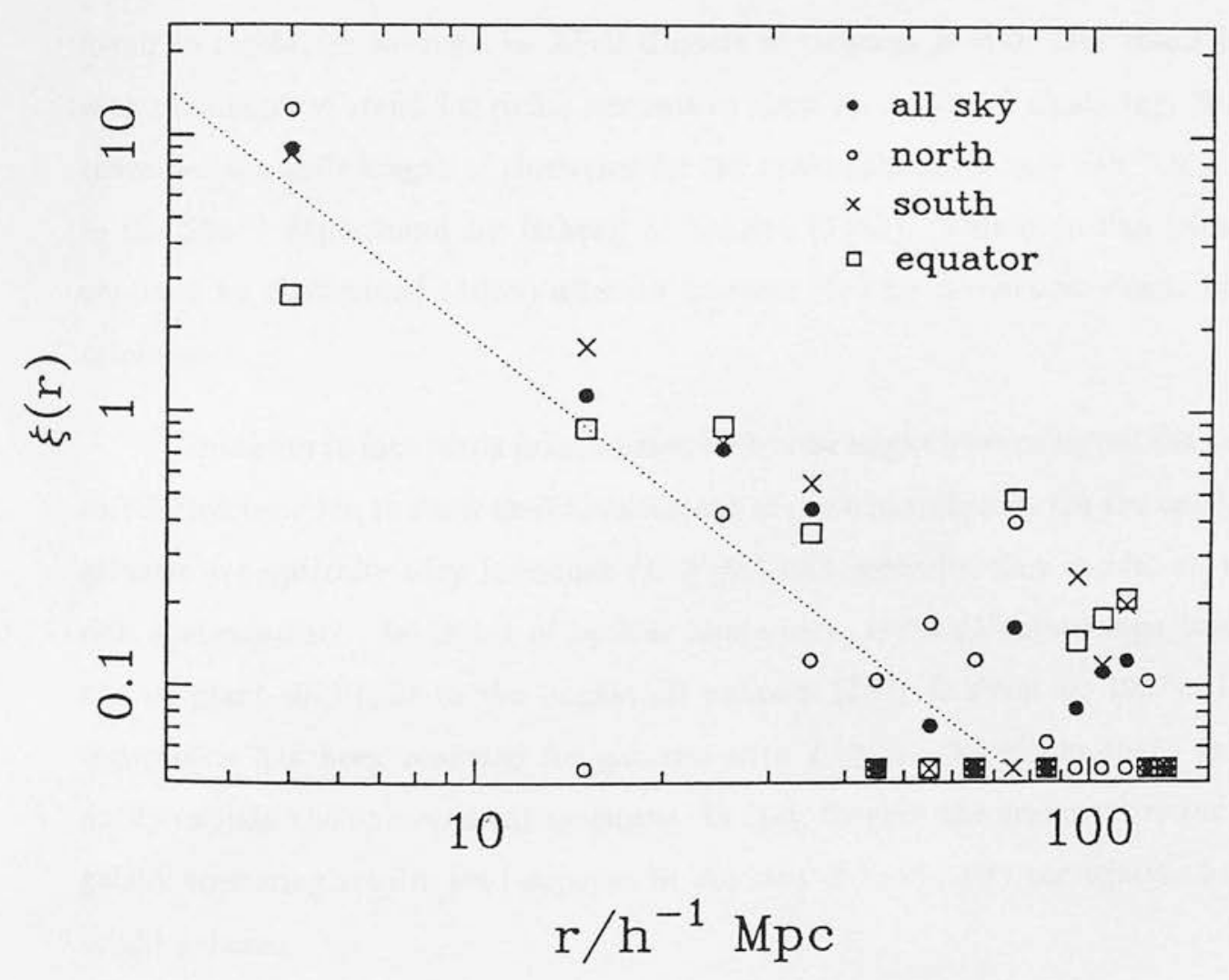

Fig. 5.3: cont.... 


\subsection{Discussion}

The first point to note is that the conclusions drawn from the above results are quite different from those inferred from the results presented in Peacock et al. (1988). These latter were based on the partially complete redshift survey and it was found then that the strong clustering signal was restricted to a band in radio power at close to the FanaroffRiley division. The new results presented here are much more robust.

The radio galaxy correlation function shows an enhancement over that for normal elliptical galaxies by about a factor of six. Recall from Chapter 4 that radio galaxies were found to reside, on average, in Abell clusters of richness $R=0$. Our result fits in then with the observed trend for richer systems to show an enhanced clustering. However, the characteristic scale length of clustering for the radio galaxies is only $14 h^{-1} \mathrm{Mpc}$ compared to the $25 h^{-1} \mathrm{Mpc}$ found by Bahcall \& Soneira (1983). This is in line with the value obtained by Sutherland (1988) after he corrects $\xi(r)$ for projection effects in the Abell catalogue.

There are in fact two a priori reasons why one might have expected the radio galaxy correlation function to show the enhancement that we have found. On the one hand, radio galaxies are optically very luminous $\left(L \geq L_{*}\right)$ and secondly they reside, on average, in rich environments. In terms of optical luminosity, radio galaxies range from that for normal giant ellipticals to the largest $c D$ galaxies (Lilly \& Prestage 1987). Luminosity segregation has been reported for galaxies with $L \geq L_{*}$ (Hamilton 1988) and this can partly explain the observed enhancement. In fact, turning the argument round, the radio galaxy clustering results lend support to the idea of luminosity segregation for optically bright galaxies.

The picture regarding the cluster environments of low-redshift radio galaxies that appears to have emerged in this thesis, from the present Chapter and also Chapter 4 , is that they occupy a range of environments from poor groups to richest clusters, on average residing in Abell clusters of richness $R=0$. There are no significant correlations between source luminosity with their local environments or 3-d clustering signal.

At higher redshifts $\langle z\rangle=0.2$, Yates, Miller \& Peacock (1989) have studied a small sample of powerful radio galaxies and find that they occupy environments on average as 
rich as Abell class 0 clusters. They see a trend in clustering properties with both redshift and radio power but due to limited statistics were unable to say which was the primary effect. The results here seem to favour the epoch-dependence of clustering properties since it would be rather difficult to envisage how power-dependency could be dominant at high redshifts but not for the low-redshift radio galaxies.

\subsection{The Tully Effect}

An important issue in cosmography, or the mapping of large-scale structure in the universe, is the precise coherence length of structure in the distribution of galaxies and clusters. The 'Great Attractor', which we shall explore in more detail in the next Chapter, is a name which has been coined to describe a huge mass concentration thought to be responsible for some of the local large-scale streaming motions (Faber \& Burstein 1988). This purported structure lies near the southern galactic plane in the vicinity of HydraCentaurus, at an estimated redshift of $z \sim 0.01-0.015$. Other evidence for large-scale density homogeneities in the universe has been claimed by Lonsdale \& Hacking (1989), who find evidence for structure in the distribution of faint IRAS galaxies and, in particular, a possible background extension of the Pavo-Indus supercluster at $z \sim 0.02-0.05$.

The most extreme claims, however, have been made by Tully (1986) who believes that we reside in an overdense region of the universe that involves $10^{18} M_{\odot}$ and extends across a dimension corresponding to $10 \%$ of the distance to the event horizon. This belief is founded in the observation that there is a significant enhancement of rich clusters of galaxies coincident with the super-galactic equator, i.e. coincident with the plane of the Local Supercluster as defined by Shapley-Ames galaxies (see deVaucouleurs, deVaucouleurs \& Corwin (1976) for a description of the supergalactic coordinate system).

The discovery of this surprisingly coherent distribution of light in the universe, hereafter referred to as the Tully effect, challenges most cosmological models for largescale structure formation. It is based on a study of an all-sky sample of 214 rich Abell clusters and has received corroborative evidence from the apparent distribution of the poorer clusters studied by Schectman (1985). Specifically, Tully reports a $4.6 \sigma$ excess of rich clusters in an $80 h_{75}^{-1} \mathrm{Mpc}$ interval perpendicular to the super-galactic equator centred 
$10 h_{75}^{-1} \mathrm{Mpc}$ below the plane of the Local Supercluster. The most obvious features of this excess stretch along a long dimension of $500 h_{75}^{-1} \mathrm{Mpc}$.

Tully's analysis involved taking the observed SGZ distribution and attempting to renormalise it to allow for sample selection (zone of avoidance and radial selection) in order to produce a plot of SGZ-dependent density. His method is a little dangerous in that it amplifies the statistical noise in the distribution at large distances from the plane.

The volume of space that is surveyed by the radio galaxy sample, is deep enough for a test to be made of the reality of Tully's claims. A simple approach is adopted. The technique described earlier for constructing synthetic galaxy catalogues is used to estimate the expected SGZ distribution on the null hypothesis that the density field is isotropic. These are compared directly with the histogrammed data for the radio galaxy sample and from these one can draw inferences about whether there is a concentration of structure towards the SGZ plane.

\subsubsection{RESULTS}

The binned data for the most nearby, $z<0.025$, radio galaxies is shown first in Fig. 5.4. The histogram presented for the synthetic catalogues has been normalised to the number of radio galaxies.

It is clear that for the nearby galaxies there is a concentration towards the supergalactic plane. This is simply a reflection of the local large-scale structure in the universe. Such a concentration can also be seen for the Wall \& Peacock radio galaxy sample (1985) as Shaver \& Pierre (1989) have remarked.

Now consider the histogrammed data for higher redshift shells of radio galaxies, shown in Fig. 5.5.

These figures indicate that there is no significant evidence at all for any concentration of radio galaxies towards the SGZ plane on these scales. The radio galaxy redshift survey fails to confirm the Tully effect. 


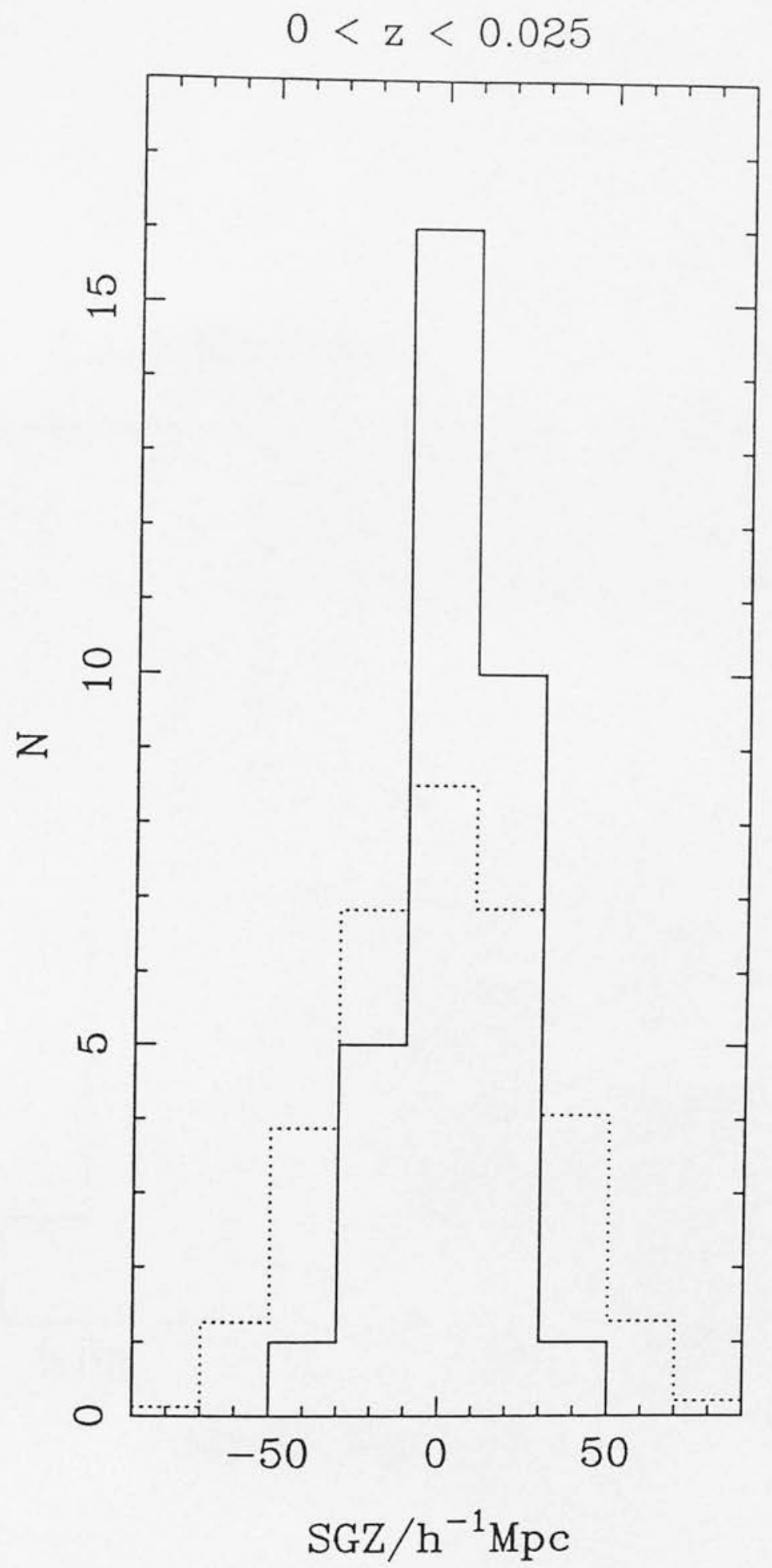

Fig. 5.4: Histograms showing the distribution of radio galaxies (full line) within $z<0.025$ and galaxies drawn from a synthetic catalogue (dashed line) above and below the supergalactic (SGZ) plane. The obvious concentration along the SGZ plane is due to the local large-scale structure. 
$0.025<\mathrm{z}<0.05$

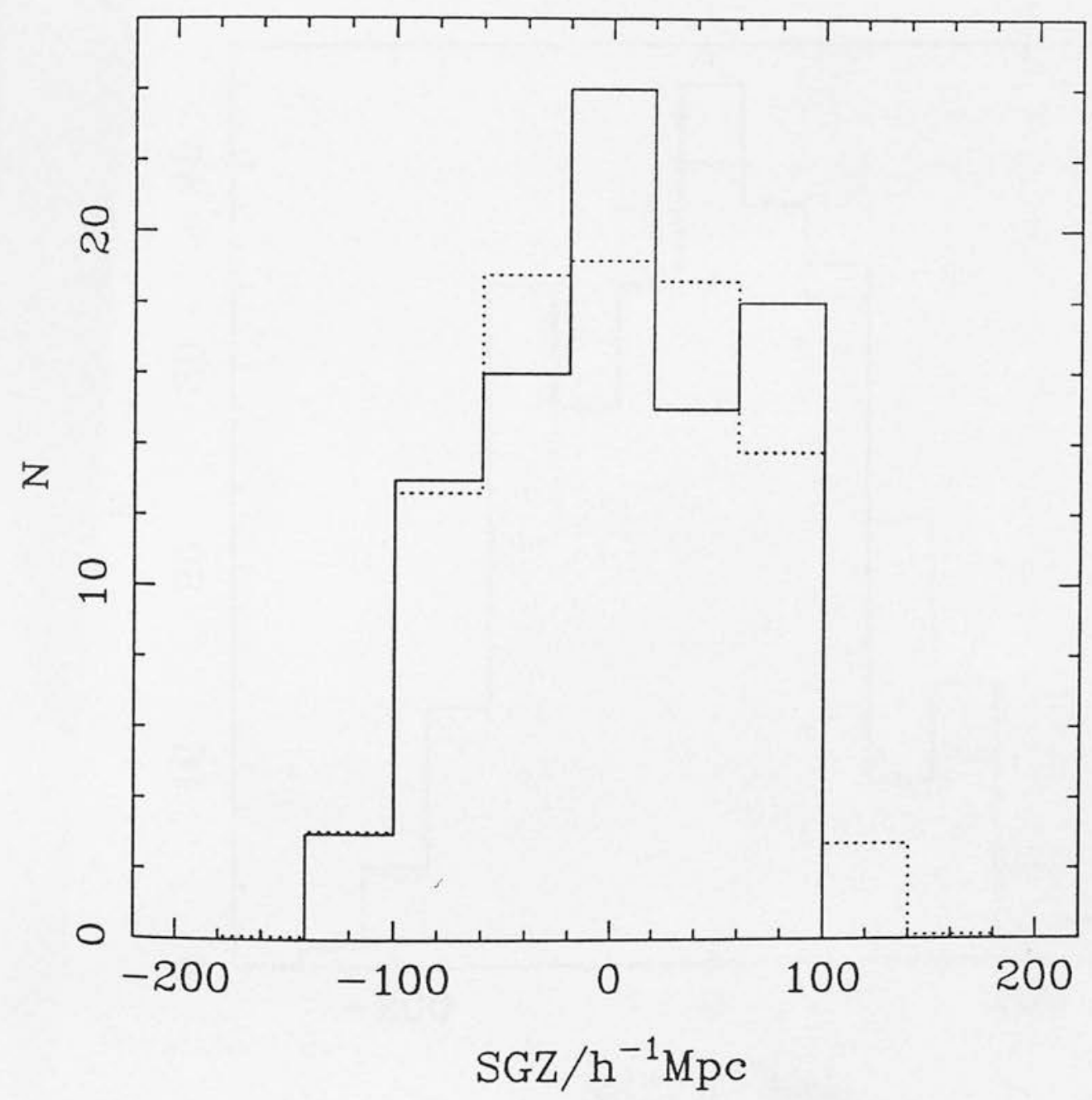

Fig. 5.5: Histograms for the radio galaxies beyond $z>0.025$ and galaxies drawn from synthetic catalogues. There is no evidence for any significant concentration of radio galaxies towards the SGZ plane. 


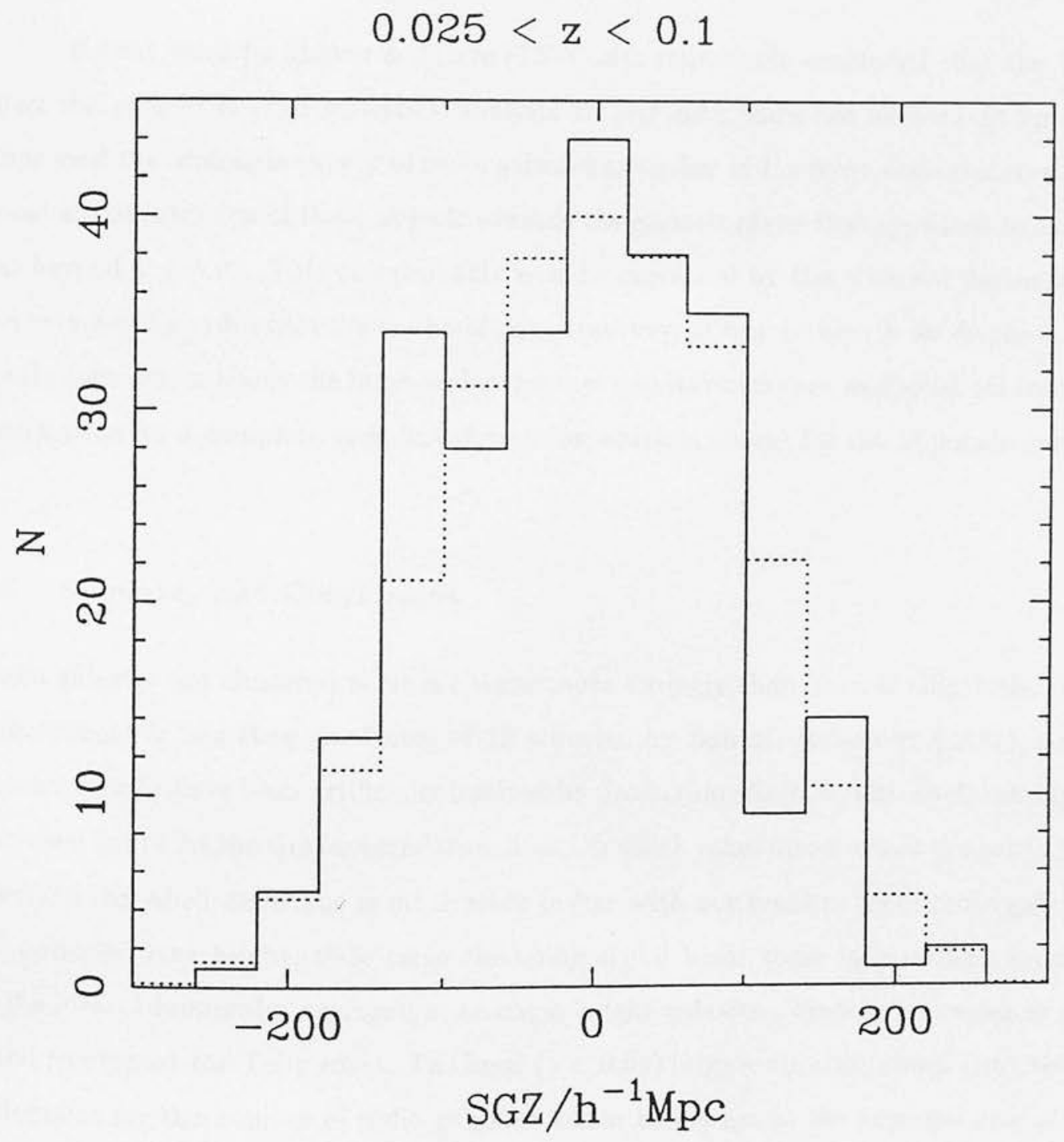

Fig. 5.5: cont.... 


\subsubsection{DISCUSSION}

It seems likely that the very large-scale structure seen by Tully in the distribution of rich clusters is a result of projection effects in the Abell catalogue - the catalogue is simply far too unreliable to make any satisfactory statements about large-scale clustering in the universe.

Recent work by Shaver \& Pierre (1989) also tentatively concluded that the Tully effect was evident in their statistical analysis of their data. How can we account for this? They used the Molonglo survey of radio galaxies as probes of the large-scale structure and found a concentration of these objects towards the galactic plane that appeared to extend out beyond $z \leq 0.02$. This can probably now be explained by the observed flattening of the very nearby radio galaxies in the Molonglo survey, of which there is no dispute. Any conclusions drawn about the large-scale structure and its extent are weakened without 3-d information for a complete sample, information which is absent for the Molonglo survey.

\subsection{Summary and Conclusions}

Radio galaxies are clustered some six times more strongly than normal ellipticals. This enhancement is less than the factor of 18 reported by Bahcall \& Soneira (1983), which appears now to have been artificially boosted by projection effects in the Abell catalogue. A revised figure for the cluster correlation function which takes into account the projection effects in the Abell catalogue is much more in line with our results. Since radio galaxies are optically very bright, their large clustering signal lends some independent support to the idea of luminosity segregation amongst bright galaxies. Finally, no evidence was found to support the Tully effect. The local $(z<0.02)$ large scale structure is detected in histograms for the number of radio galaxies within bands about the supergalactic plane compared to identical histograms for simulated catalogues. No significant evidence is found for any structure beyond this scale. We therefore reject Tully's claims as arising mainly because of contaminations in the Abell cluster catalogue. 


\section{Chapter 6}

\section{Large-Scale Streaming Motions}

\subsection{Introduction}

The radio galaxy spectroscopy and photometry database presents us with an exciting opportunity to explore the topical issue of large-scale streaming motions in the universe. This subject has recently returned to the forefront of cosmological research after a latent period, borne out of a basic mistrust in the reality of earlier results, which claimed significant $(\sim 10 \%)$ deviations from the Hubble flow on surprisingly large scales. In fact these claims have stood the test of time and still present one of the most severe challenges for theoretical models of large-scale structure formation.

The key ingredients required for mapping the extragalactic peculiar velocity field are spectroscopic redshifts and a set of redshift-independent variables which can be combined in some way to predict galaxy distances. Line-of-sight velocities, measurable as redshifts, assume that the galaxies are essentially at rest in the frame of reference defined by the cosmic microwave background radiation (CBR). Redshift-independent measures of distance, however, require no such prior assumptions about the isotropy of the universal expansion. Together, the two independent distance estimates yield galaxy peculiar velocities, or deviations from Hubble flow. The usual practice then is to examine the variation of these perturbations across the sky and check whether they can be fit by a simple dipole, or perhaps some higher multipole moment model for the extragalactic peculiar velocity field. A multi-parameter regression analysis of the data yields an estimate for the Local Group (LG) motion relative to the reference frame defined by the survey volume occupied by the galaxies. The amplitude and direction of this motion can be compared directly with theoretical predictions to set constraints on the particle content of the universe and 
on the spectral shape of the initial density fluctuations from which galaxies formed.

In principle, then, observations of streaming flows are a very powerful discriminator between models for galaxy formation, on an almost equal footing to observation of microwave background anisotropies and galaxy clustering. From a theoretical viewpoint, large-scale velocity fields are endowed with a number of useful features. They provide a measure of the mass fluctuations rather than of the light (we shall see why this is important in the next Chapter); they can be analysed using linear perturbation theory; they give direct information about the statistical properties of the initial density field which should have been preserved; they measure density fluctuations on a scale well beyond that at which the normalisation of the arbitrary initial fluctuation amplitude is usually performed. Unfortunately, from the observational side, peculiar velocities take a considerable amount of time and effort to measure and their analysis is not straightforward, especially if systematic effects in the data need to be entangled from any real peculiar velocities that may be present.

One of the chief motivations for using a sample of radio galaxies to probe the velocity field is that, for this class of object, a remarkably tight yet simple-to-measure distanceindicator relation can be found and, in addition, the ease and uniformity with which radio galaxies can be selected means that sample selection effects are much easier to avoid.

This Chapter begins with a short overview of some previous observations of the Hubble flow. Section 6.3 emphasises why the all-sky sample is particularly suited to study the velocity field. The data is presented in Section 6.4 and peculiar velocities are derived using the $\mu_{e}-r_{e}$ relation for the radio galaxies that was established in Chapter 4. Section 6.5 presents the data analysis. This takes the form of fitting dipole and quadrupole models for the peculiar velocity field to the data. A careful error analysis is performed to test the robustness of the solutions. Monte Carlo simulations are carried out in order to test whether inhomogeneous sky coverage of the sample galaxies can have an adverse effect on the error bars associated with the Cartesian components of the Local Group motion. Section 6.5 discusses the main results and compares them with those obtained by other workers. In Section 6.7 a rather different approach to analysing the peculiar velocities is described. This uses the velocity autocorrelation tensor and a number of improvements over existing calculations are found. A short summary is given in Section 6.8 


\subsection{Previous Observational Work}

The early background to observations and theoretical interpretation of deviations from universal expansion has been reviewed by Davis \& Peebles (1983a). More up-to-date reviews have been presented by Gunn (1988) and Burstein (1990), to which the reader is referred for a more comprehensive discussion than the one given here. In fact this overview is deliberately rather biased towards a description of the pioneering study by Rubin et al. (1976; hereafter RF) and the more recent investigation undertaken by a large consortium of astronomers (Lynden-Bell et al. 1988) dubbed the 'Gang of Seven' (hereafter, G7). The RF results provide a useful set of yardstick measures for comparing with the radio galaxy dipole solutions since the two samples are of comparable depth and attain a similar percentage distance accuracy. The importance of the G7 work lies in the fact that a very good percentage distance accuracy $(\sim 21 \%)$ was achieved by using a variant of the Faber-Jackson relation, known as the $D_{n}-\sigma_{v}$ relation (Dressler et al. 1987; Djorgovski \& Davis 1987), to estimate galaxy distances. Also their sample of ellipticals was large, with good sky coverage, allowing the peculiar velocity field to be mapped in some detail.

One of the first key observations of the Hubble flow was the survey by RF of a sample of spiral galaxies out to a depth of about $13000 \mathrm{kms}^{-1}$. Their analysis, however, was restricted to a redshift shell between 3500 and $6000 \mathrm{kms}^{-1}$ where, they argued, the effects of Malmquist bias were likely to be less severe. In fact this was an unnecessary precaution as we shall see later. RF concluded from their analysis of the spiral's peculiar velocities that the LG motion was about $600 \mathrm{kms}^{-1}$ relative to the frame defined by the shell of galaxies. The true implications of this result, however, were not fully appreciated until Smoot et al. (1977) reported the discovery of a dipole anisotropy in the CBR at a level of $\delta T / T \sim 10^{-3}$. The preferred interpretation of this is a Doppler-induced effect arising from a combination of the Earth's rotational and orbital motions. The measurement of the CBR dipole implied a LG motion of $614 \mathrm{kms}^{-1}$ relative to the $\mathrm{CBR}$ and in a direction towards $l=270^{\circ}, b=30^{\circ}$. Although the two independent measures of the amplitude of the LG motion are almost identical in each case, the vectors are misaligned by about $90^{\circ}$. If the CBR really is a universal rest frame, and the failure to find a quadrupole anisotropy of comparable magnitude to the dipole suggests that it is, then the next most 
likely explanation of the RF dipole seems to be that the spiral galaxy sample are partaking of a coherent bulk flow.

The Rubin-Ford effect has been criticised on various grounds, notably by Fall \& Jones (1976) and by Schechter (1977). As a result, it took a further ten years for new results to begin to emerge. A catalyst for this renewed activity was the work of Collins et al. (1986), who were able to answer at least one of the criticisms levelled at the RF study. They re-observed a selection of the RF spirals in the infra-red, thus avoiding the large corrections for interstellar dust extinction required for optical observations of spiral galaxies. Their analysis confirmed the Rubin-Ford effect, raising speculation that there were indeed large, coherent flows of galaxies in the universe.

Interest in streaming flows again began to wane following the publication of two null results. Firstly, Aaronson et al. (1986) observed a small sample of clusters and employed the infra-red Tully-Fisher relation for estimating relative distances. Their data were consistent with the clusters being at rest in the Hubble flow. This sample of clusters was not ideal for testing the reality of the Rubin-Ford effect however, since it was observed from Arecibo which surveys a region of space almost orthogonal to the direction of the RF dipole. The other null result was obtained by Hart \& Davies (1982) for a nearby sample of spirals, using the Tully-Fisher relation to estimate their relative distances. Staveley-Smith (1985) has since called into question their regression analysis which casts some doubt on the validity of their result.

Recent attention has focussed on the results of G7. These have set the pace for observers and theoreticians alike. Their sample comprised 385 elliptical galaxies out to about $6000 \mathrm{kms}^{-1}$. The key feature of their observations has been the application of a new luminosity indicator for elliptical galaxies, the $D_{n}-\sigma_{v}$ relation, which achieves impressive accuracy (Dressler et al. 1987; Djorgovski \& Davis 1987). Here, $D_{n}$ is the angular size within which the galaxy mean surface brightness attains some fiducial value. Note that all three variables which arise in the virial equation are consequently represented in the $D_{n}-\sigma_{v}$ relation - size, surface brightness and velocity dispersion. The picture that has emerged from the data analysis performed by G7 (Lynden-Bell et al. 1988) is again that of a large-scale flow of galaxies, but one which is dominated by the gravitational action of a 'Great Attractor', a mass concentration of about $5 \times 10^{16} M_{\odot}$ lying at a distance of 
about $4500 \mathrm{kms}^{-1}$ just beyond the Hydra-Centaurus complex. Although a full discussion of these results is not necessary here, we shall be returning to several aspects of their analysis throughout the course of this Chapter.

Finally, an interesting recent development has been the attempts to reconstruct the density field from redshift surveys, principally of the IRAS galaxies (Yahil 1988; Strauss \& Davis 1988), to see whether this is consistent with the G7 picture of the velocity field. In short, the peculiar motions predicted by the IRAS survey succeed in matching the observed motions within regions of $\sim 1500 \mathrm{kms}^{-1}$ in size, but fail to predict the largescale coherent flow that exists in the observations over a distance of $7000 \mathrm{kms}^{-1}$. The IRAS peculiar velocity predictions do not appear to support a Great Attractor model.

\subsection{The Present Work}

The radio galaxy sample offers a number of potential advantages over other samples which have been used in the past to study the Hubble flow. One of the key features of the sample is that we expect it to be free from systematic biases of the kind that generally plague optically selected samples. The Malmquist bias (Malmquist 1920) is perhaps the most obvious of these. This can arise quite simply because as one observes out to greater distances in the universe, an increasing number of only the brightest galaxies get included into the sample at the expense of their fainter counterparts. The radio galaxy sample was initially selected according to radio flux-density criteria and although there is undoubtedly a radio Malmquist bias in the sample, there should be no optical analogue because the optical properties of radio galaxies are effectively uncorrelated with their radio properties at low redshifts. Also worth recalling here is that the optical criterion used to select galaxies without a redshift was a cautious one, allowing for the 1 magnitude scatter about the Hubble diagram, so that is is unlikely that galaxies have been 'missed' at the higher redshifts.

The reason why a Malmquist bias can severely complicate the analysis for peculiar velocities is that the systematic magnitude error it introduces (in $\mathrm{kms}^{-1}$ ) is proportional to distance (errors in magnitude are logarithmic errors). G7 have argued that for cluster galaxies the effect is small since for these the combined error in distance is reduced by 
a factor $n^{1 / 2}$ where $n$ is the number of observed galaxies in the cluster. The Malmquist error is consequently reduced by a factor $n$. Ironically, it is the presence of clusters and underdense regions in the universe that make the Malmquist bias difficult to correct. If one was dealing with a universe in which there was a smooth and homogeneous distribution of galaxies then the size of the effect could be computed and the appropriate corrections made. G7 have described in some detail their method for dealing with Malmquist bias. It requires some judicious assumptions to be made about the distribution of galaxies. Although it is probably unlikely that the surprising results obtained by G7 can be wholly attributed to a lack of understanding about how to correct for Malmquist bias, it is not impossible.

A further advantage offered by the all-sky sample is its depth. Deviations from Hubble flow have been found on all scales that have so far been observed - the breakover point to universal expansion has not been found. The all-sky sample offers the first opportunity to test whether streaming flows persist beyond the Rubin-Ford scale.

\subsection{Multipole Solutions for the Local Group Motion}

A variety of solutions are presented in this Section for the Local Group motion, based on a careful analysis of the radio galaxy peculiar velocities. These are derived by minimising the scatter about the $\mu-r_{e}$ relation and a justification for this choice of regression scheme forms an important part of the discussion. Having obtained the solutions, a combination of Monte-Carlo tests and statistical arguments are presented to check whether they are reasonable given the sample depth, percentage accuracy of the distance indicator and inhomogeneous sky distribution of the galaxies.

\subsubsection{THE DATA}

The data comprise accurate spectroscopic redshifts, effective angular size $\theta_{e}$ and intrinsic surface brightness $\mu_{e}$, for some 200 radio galaxies within $z<0.1$. Surface brightness has been corrected for $(1+z)^{4}$ and $k$-dimming according to the formulae given in Chapter 3 .

Fig. 6.1 plots the sky distribution of the radio galaxies, differentiating between the low redshift $(z<0.04)$ and distant objects with a filled and unfilled circle respectively. There 


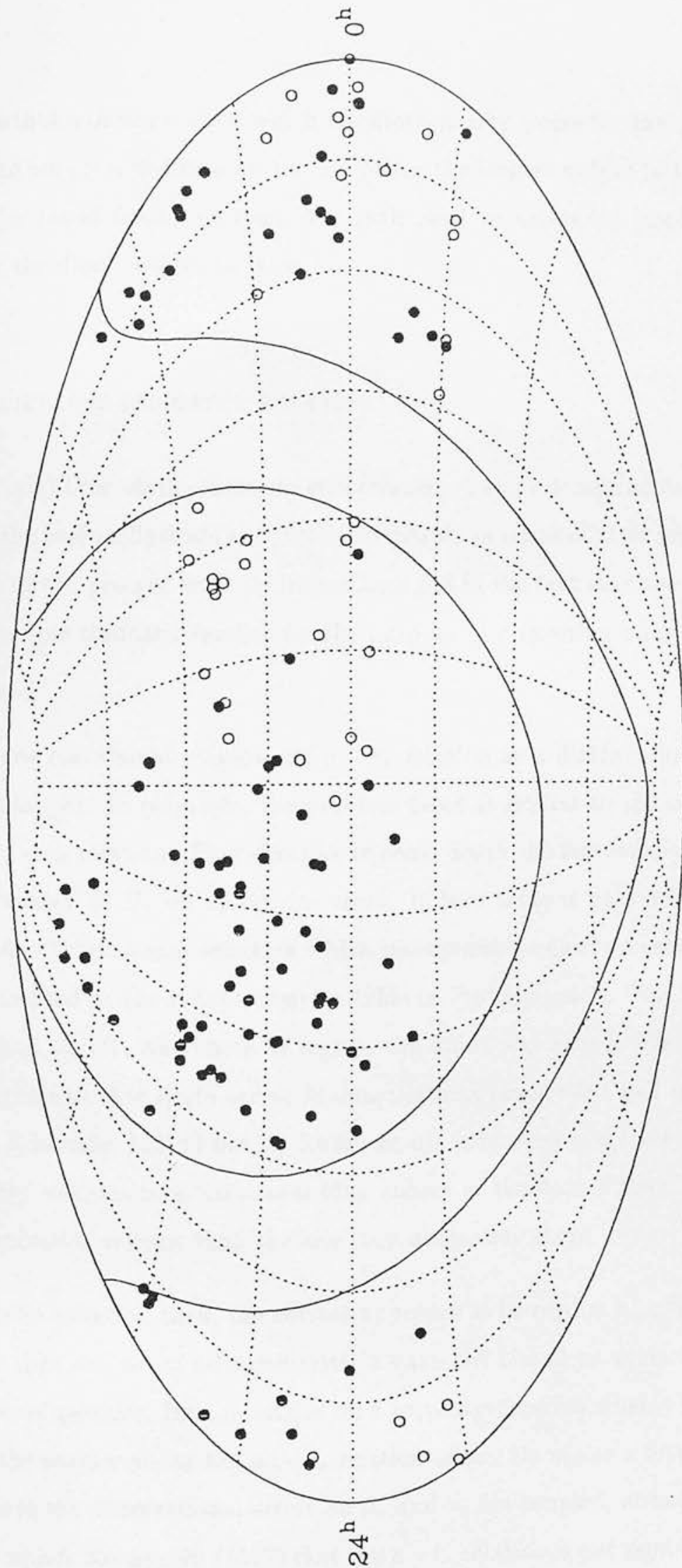

Fig. 6.1: Sky distribution of the all-sky sample members with adequate $I$ band $C C D$ photometry. The filled circles are for radio galaxies within $12000 \mathrm{kms}^{-1}$. 
is a strong north-south anisotropy which is unfortunately worse for the nearby galaxies, which carry the smallest distance errors and hence the largest weight in the optimisation schemes for the Local Group motion. We shall need to assess the implications of this anisotropy for the dipole solutions later.

\subsubsection{THE DISTANCE INDICATOR RELATION}

In Chapter 4, a tight correlation between effective size $r_{e}$ and intrinsic surface brightness $\mu_{e}$ was found for the radio ellipticals and discussed mainly in terms of their formation history. In the context of the present work its importance lies in the fact that the galaxies can be used as calibratable standard candles for the purpose of measuring relative extragalactic distances.

Exactly how one should employ the $\mu-r_{e}$ relation as a distance indicator requires some careful thought. In principle, the problem faced is similar to the one discussed by G7 for their $D_{n}-\sigma_{v}$ relation. They describe in some depth the factors affecting the choice between a regression of $D_{n}$ on $\sigma_{v}$ or vice versa. In fact there is only one correct choice: $D_{n}$ contains the observational selection which incorporates unknown errors and therfore $\sigma_{v}$ should be treated as the independent variable in the regression. This fact appears to have been missed by G7, who chose to regress the other way round. The penalty to pay for this oversight was that quite severe Malmquist bias corrections had to be applied to the data. As Schechter (1977) noted, Rubin et al. also need not have restricted their peculiar velocity analysis to a 'minimum bias' subset of the data if they had opted for a different minimisation scheme than the one they ultimately chose.

For the radio galaxies, then, the correct approach is to regress $\mu_{e}$ upon $r_{e}$ since it is almost certain that our set of selection criteria have not biased us against selecting lowsurface brightness galaxies. Having settled on a correct regression scheme we now need to consider how the scatter about the $\mu_{e}-r_{e}$ relation affects its use as a distance indicator. Firstly, note that the observational errors on $\mu_{e}$ and $r_{e}$ are coupled, although it has been shown by Hamabe \& Kormendy (1987) that the $\mu-r_{e}$ relation is not significantly affected by this coupling. In any case the experimental errors in the observables are swamped by cosmic scatter. Still, one is faced with an essentially unknown cosmic covariance matrix 
to determine. This was done by Staveley-Smith \& Davies (1990) for their sample of spirals using multivariate statistical techniques to separate a total intrinsic error into contributions from each of the variables.

A more transparent solution to the problem for the radio galaxies is to truncate the data in $r_{e}$ and assume that all the scatter is in the $\mu_{e}$ direction. This is reasonable given that any large differences between horizontal and vertical scatter would show up most at the edges of the data, so that by truncating a consistent picture is being made. The reason for truncating in $r_{e}$ rather than $\mu_{e}$ again relates to the fact that the distribution in $\mu_{e}$ at a given size is expected to be unbiased by observational selection whereas the converse is not true.

The $\mu_{e}-r_{e}$ relation was therefore truncated at values of $r_{e}$ equal to $2.5 h_{50}^{-1} \mathrm{kpc}$ and $50 h_{50}^{-1} \mathrm{kpc}$ which meant that only 11 galaxies were lost. Although this is a somewhat arbitrary choice, it is important to note that none of the solutions presented later in this Section are severely affected by removing a few galaxies at the edge of the data. Any galaxies lying further than $3 \sigma$ away from the least-mean squares line through the truncated $\mu-r_{e}$ relation were also rejected. The $\mu-r_{e}$ relation for the remaining galaxies (prior to truncation) is shown in Fig. 6.2. There is an rms scatter about the $\mu-r_{e}$ relation in the $r_{e}$ direction which corresponds to a relative distance accuracy of about $28 \%$. Although this is not as good as $D_{n}-\sigma_{v}$ it is still acceptable for measuring peculiar velocities. Certainly, the photometric parameters are a lot less trouble to acquire than velocity dispersions.

\subsubsection{THE OPTIMISATION SCHEME}

Let us begin by modelling the observed velocity field as a simple Local Group dipole motion superimposed on the uniform expanding background. The model can be extended to include higher order multipole moments of the velocity field if desired. For the dipoleonly case

$$
H_{0} r=V_{c}+\mathbf{u} \cdot \hat{\mathbf{n}}+\Delta_{v}=V_{H}
$$

where $V_{c}$ is the observed velocity reduced to the Local Group barycentre (to correct heliocentric velocities the IAU convention of a solar motion in the LG frame of $300 \mathrm{kms}^{-1}$ 


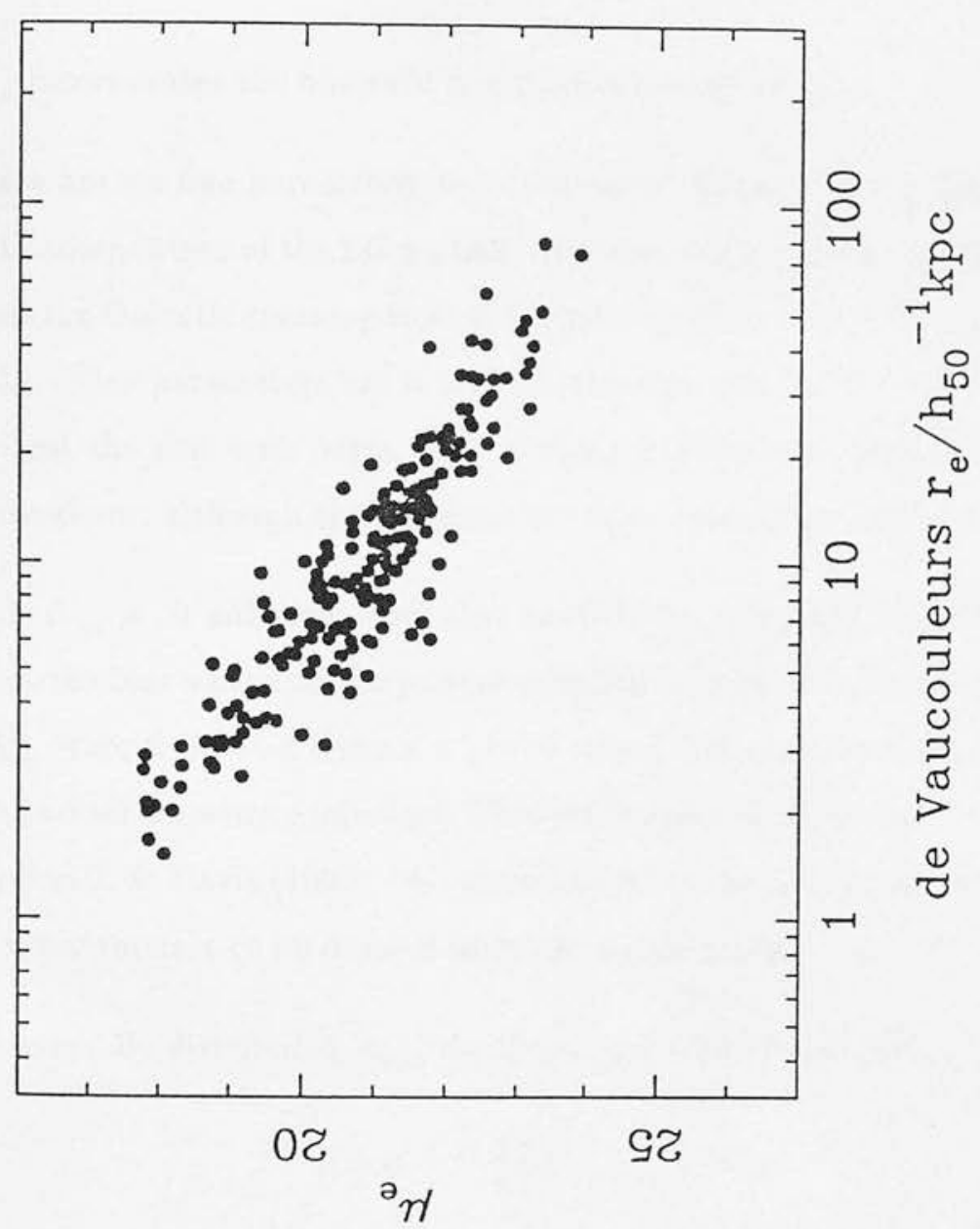

Fig. 6.2: The $\mu_{e}-r_{e}$ relation for 172 radio galaxies. Five $3 \sigma$ outliers have been rejected and truncation in $r_{e}$ at values of $2.5 h_{50}^{-1} \mathrm{kpc}$ and $50 h_{50}^{-1} \mathrm{kpc}$ will remove a further eleven galaxies. 
towards $l=90^{\circ}, b=0^{\circ}$ was adopted), $\mathrm{u}$ is the $\mathrm{LG}$ motion vector, $\hat{\mathrm{n}}$ is a unit vector in the direction of the galaxy and $\Delta_{v}$ is the 'thermal' noise in the Hubble flow. The perturbed velocities can be used to compute a 'true' physical size for the galaxies and the corresponding scatter about the $\mu_{e}-r_{e}$ relation is minimised, where

$$
\mu_{e}+\Delta_{\mu_{e}}=\alpha+\beta \log _{10} r_{e}
$$

and $\Delta_{\mu_{e}}$ incorporates the observed and intrinsic errors in $\mu_{e}$.

There are six free parameters to be estimated from the data. Firstly, the $x, y$ and $z$ Cartesian components of the LG motion. We shali work in Galactic Cartesian coordinates $x$ towards the Galactic centre, $y$ toward Galactic rotation, and $z$ toward the north Galactic pole. The other parameters are $\alpha$ and $\beta$, intercept and slope respectively in the $\mu-r_{e}$ relation and the rms error term $\Delta_{\mu_{e}}$. A fixed Hubble flow noise term of $250 \mathrm{kms}^{-1}$ is used throughout, although the solutions are fairly insensitive to this choice.

Since $\Delta_{\mu_{e}}$ is an unknown error that needs to be estimated from the data, the optimisation scheme best suited to the present problem is maximum likelihood. If, on the other hand, $\Delta_{\mu_{e}}$ were fixed at a certain a priori value, then minimum $\chi^{2}$ could be used since then the two schemes are equivalent. This point appears not to have been appreciated by Staveley-Smith \& Davis (1989) who choose to minimise $\chi^{2}$ despite treating the errors as extra free parameters to be derived from the optimisation.

For normally distributed $\Delta_{\mu_{e}}$, the likelihood $L$ can be written

$$
L=\mathrm{e}^{-\chi^{2} / 2} \prod\left[2 \pi\left(\Delta_{\mu_{e}}^{2}+\Delta_{v}^{2}\right)\right]^{-1 / 2}
$$

As we have argued, it is the scatter about $\mu-r_{e}$ that should be minimised and hence $\chi^{2}$ is given by

$$
\chi^{2}=\sum_{1}^{N} \frac{\left(\mu_{e}-\alpha-\beta \log _{10} r_{e}\right)^{2}}{\Delta_{\mu_{e}^{k}}^{2}+\Delta_{v}^{2}}
$$

Inserting this into equation 6.3 and noting that maximising $L$ and $\ln L$ are equivalent gives a final expression

$$
\ln L=-\frac{1}{2} \sum_{1}^{N} \ln \left[2 \pi\left(\Delta_{\mu_{e}}^{2}+\Delta_{v}^{2}\right)\right] \approx \frac{1}{2} \sum_{1}^{N} \frac{\left(\mu_{e}-\alpha-\beta \log _{10} r_{e}\right)^{2}}{\Delta_{\mu_{e}}^{2}+\Delta_{v}^{2}}
$$


This is quite difficult to solve. It is easier to use the fact that, for fixed $\Delta_{\mu_{e}}^{2}$, maximum likelihood is equivalent to least-mean squares. The best approach, then, seems to be to solve least-mean squares for a range of trial values for $\Delta_{\mu_{e}}$ and to use the resulting values of $\chi^{2}$ to examine the one-dimensional variation in maximum likelihood with $\Delta_{\mu_{e}}$ and hence find the best value for $\Delta_{\mu_{e}}$.

The optimization problem one faces is essentially a weighted, non-linear least squares. There is a NAG routine to deal with such problems and briefly this works in the following way. Suppose the function we wish to minimise is $F(x)$ which is a function of the $n$ variables, $x_{1}, x_{2}, \ldots, x_{n}$. Then the vector of partial derivatives of $F(x)$ is called the gradient vector and is denoted by

$$
g(x)=\left[\frac{\partial F(x)}{\partial x_{1}}, \frac{\partial F(x)}{\partial x_{2}}, \ldots, \frac{\partial F(x)}{\partial x_{n}}\right]^{T}
$$

The gradient vector is an important diagnostic in optimisation problems since it must be zero at an unconstrained minimum of any function with continuous first derivatives. Similarly, the matrix of second derivatives termed the Hessian matrix must be positive semi-definite at any unconstrained minimum of $F(x)$. Thus for the point $x^{*}$ to be an unconstrained local minimum of $F(x)$ :

(i) $\left\|\bar{g}\left(x^{*}\right)\right\|=0$, and

(ii) $G\left(x^{*}\right)$ is positive-definite;

where $\|\bar{g}\|$ denotes the Euclidean length of $\bar{g}$.

The elements in the variance-covariance matrix of the estimated regression coefficients are derived from the Jacobian of the function $f(x)$ at the solution. Thus suppose that

$$
F(x)=f^{\mathrm{T}}(x) f(x)
$$

represents the goodness-of-fit of our non-linear model to the observed data. We shall assume that the Hessian of $f(x)$, at the solution, can be adequately represented by $2 J^{\mathrm{T}} J$, where $J$ is the Jacobian of $f(x)$ at the solution. The estimated variance-covariance matrix $C$ is then given by

$$
C=\sigma^{2}\left(J^{\mathrm{T}} J\right)^{-1} \quad J^{\mathrm{T}} J \quad \text { non - singular }
$$

where $\sigma^{2}$ is the estimated variance of the residual at the solution $\bar{x}$, given by

$$
\sigma^{2}=\frac{F(\bar{x})}{m-n}
$$


$m$ being the number of data points and $n$ the number of variables. The diagonal elements of $C$ are estimates of the variances of the regression coefficients.

As a test of the algorithm, it was ran on a mock dataset into which a dipole had been added. A minimum value of $\chi^{2}$ was found at the correct values of the 6 free parameters.

\subsubsection{DiPOLE SOLUTION FOR THE LOCAL GROUP MOTION}

The algorithm was next ran on 72 radio galaxies lying within $12000 \mathrm{kms}^{-1}$. The observables, $\theta_{e}$, redshift etc., were fed into the computer programme and a trial value of 0.35 mag. $\operatorname{arcsec}^{-2}$ was supplied for the rms scatter on $\mu_{e}$. Again the algorithm converged to a solution quite quickly and it was found that this was fairly insensitive to choices of $\Delta_{\mu_{e}}$ around the initial trial value. The resulting components of the Local Group motion together with their rms errors, derived from the diagonal elements of the error covariance matrix, are given below

$$
V_{x}=-1484 \pm 644 \mathrm{kms}^{-1} ; \quad V_{y}=776 \pm 425 \mathrm{kms}^{-1} ; \quad V_{z}=1066 \pm 424 \mathrm{kms}^{-1}
$$

The constant coefficients $\alpha$ and $\beta$ were calculated to be $23.2 \pm 0.14$ and $2.94 \pm 0.17$ respectively. Minimum $\chi^{2}$ turned out to be very close to unity for the six degrees of freedom in the problem.

It is interesting now to check whether using the dipole-corrected redshifts for calculating $r_{e}$ brings down the scatter in the $\mu-r_{e}$ relation. The corrected distance indicator relation is shown in Fig. 6.3. Also shown is the uncorrected $\mu-r_{e}$ relation for the 72 galaxies used in the streaming analysis. Inspection of these shows that there is a small reduction in scatter - in terms of percentage distance accuracy, the change is from $28 \%$ to $25 \%$.

It is also illuminating to express the LG motion dipole components in terms of equatorial coordinates $\alpha$ and $\delta$. In this case, they turn out to be:

$$
V_{x}=-292 \pm 344 \mathrm{kms}^{-1} ; \quad V_{y}=304 \pm 605 \mathrm{kms}^{-1} ; \quad V_{z}=1988 \pm 548 \mathrm{kms}^{-1}
$$

Note the large amplitude of the motion in the $z$-component which, in the equatorial system, points towards the north pole. This reflects the conspicuous north-south anisotropy in the distribution of the radio galaxies about the sky. 

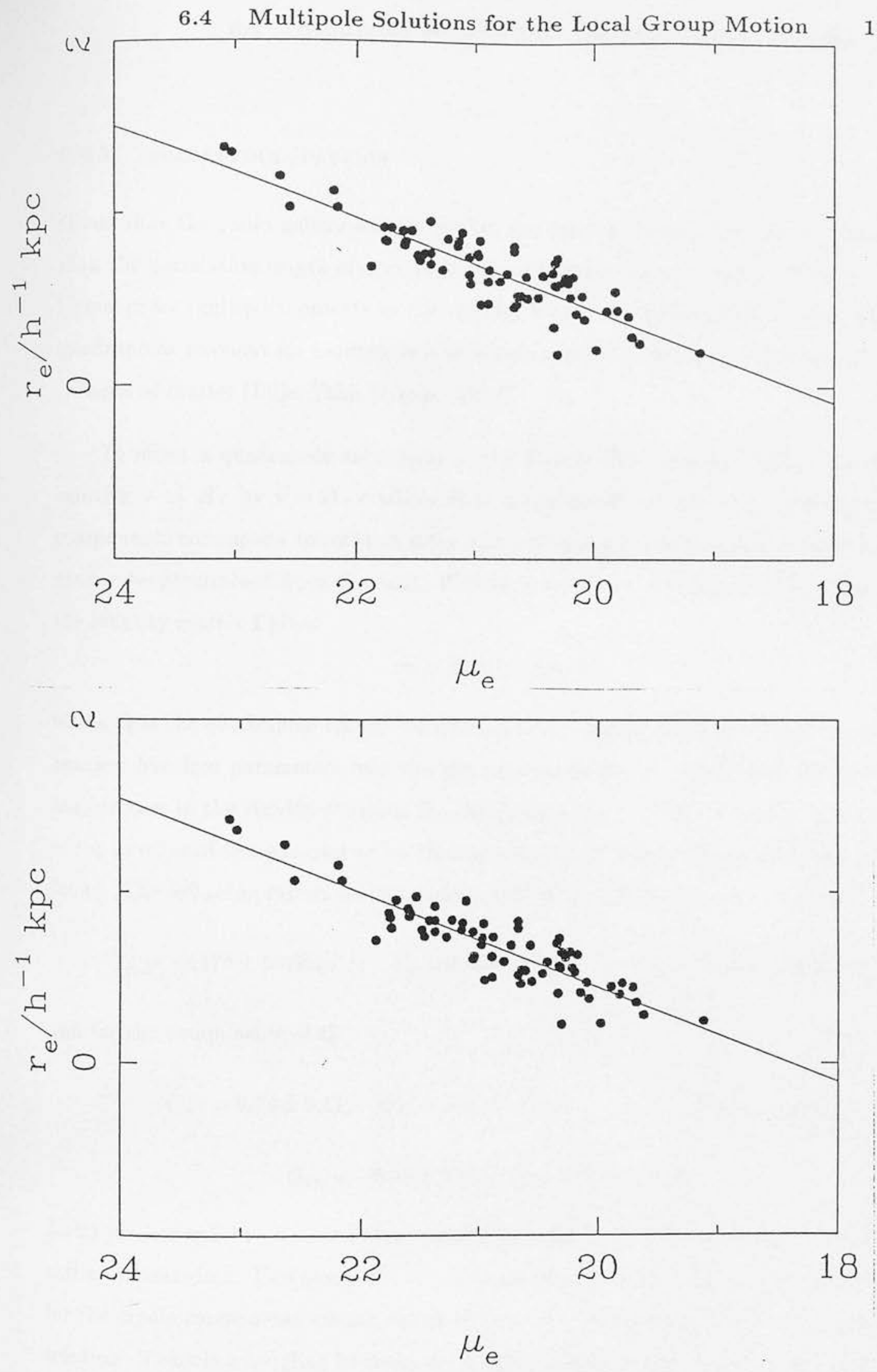

Fig. 6.3: The $\mu_{e}-r_{e}$ relation for 72 galaxies which have a measured peculiar velocity. The upper diagram used observed redshifts to compute $r_{e}$. Observed redshifts were corrected for peculiar velocities to produce the bottom diagram. 


\subsubsection{QUADRUPOLE SOLUTION}

Given that the radio galaxy sample probes the large-scale structure on a greater scale than the correlation length of gravitationally attracting masses one might expect to find higher order multiple moments in the velocity field (Clutton-Brock \& Peebles 1981). A quadrupolar moment for example is a signature of tidal fields set up by massive concentrations of matter (Lilje, Yahil \& Jones 1986).

To effect a quadrupole anisotropy in the Hubble flow we must replace the Hubble velocity $\mathbf{v}=H \mathbf{r}$ by $\mathbf{v}=\mathbf{H} \cdot \mathbf{r}$ where $\mathbf{H}$ is a symmetric matrix - the antisymmetrical components correspond to rotation rates which do not give rise to radial velocities and so cannot be determined from the data. Writing $\mathrm{r}$ as $r \hat{\mathrm{r}}$ and expressing $\mathbf{H}$ as a multiple of the identity matrix I gives

$$
\mathbf{v}=H_{0} r(\mathbf{I}+\mathbf{Q}) \cdot \hat{\mathbf{r}}
$$

where $\mathbf{Q}$ is the quadrupole tensor. Solving for both a dipole and a quadrupole introduces another five free parameters into the the expression for $\chi^{2}$ - these are effectively the anisotropies in the Hubble constant $Q_{x x}, Q_{y y}, Q_{z z}, Q_{x y}$ and $Q_{x z}$. Despite this doubling of the number of free parameters for the regression code to solve for, a minimum was still found. The following results for the components of the LG motion were obtained:

$$
V_{x}=-1174 \pm 687 \mathrm{kms}^{-1} ; \quad V_{y}=883 \pm 453 \mathrm{kms}^{-1} ; \quad V_{y}=1092 \pm 454 \mathrm{kms}^{-1}
$$

and for the components of $\mathrm{Q}$

$$
\begin{gathered}
Q_{x x}=0.08 \pm 0.11 ; \quad Q_{x y}=-0.07 \pm 0.06 ; \quad Q_{x z}=-0.062 \pm 0.05 \\
Q_{y y}=-0.03 \pm 0.04 ; \quad Q_{z z}=0.04 \pm 0.03
\end{gathered}
$$

Little further will be set about these results here since the large error bars render them rather meaningless. Two points are worth mentioning however. Firstly, the above values for the dipole components are somewhat different from those obtained for the dipole-only solution. There is a coupling between the dipole and quadrupole moments due to the sky anisotropy of the sample. They are effectively independent of each other for a perfectly uniform sky coverage. Secondly, there is a much neater way to treat the quadrupole. Like every symmetric tensor, $\mathbf{Q}$ can be brought to principle axes. From the basic definition of a 
quadrupole moment (e.g. Landau \& Lifschitz 1975) it follows that the diagonal elements of $Q$ sum to zero. In general, only two of its three principal values will be independent. The axis of symmetry in the present problem lies along the galactic plane so this must be one of the principle axes of the tensor $\mathbf{Q}$. The location of the other two axes in the $x, y$ plane are arbitrary. The diagonalised form of $Q$ is written

$$
Q_{D}=\left(\begin{array}{ccc}
-Q_{x^{\prime}} & 0 & 0 \\
0 & -Q_{y^{\prime}} & 0 \\
0 & 0 & Q_{z^{\prime}}
\end{array}\right)
$$

where $Q_{z^{\prime}}=Q_{x^{\prime}}+Q_{y^{\prime}}$. The $Q_{z^{\prime}}$ axis of the quadrupole is defined as the direction of maximum expansion and, in a hypothetical situation where motions are dominated by a single mass concentration, would represent the direction of infall.

\subsubsection{ERROR ANALYSIS}

The errors on the dipole solution are strikingly large especially when compared with the results obtained by Rubin et al. for a sample of similar size which probed a comparable depth. In an effort to understand these, first consider the error covariance matrix for the free parameters in the regression analysis for the Local Group motion. In order to avoid this looking disproportionately messy due to the large differences in magnitude between redshifts and the other observables, recession velocities have been scaled by a factor of $10^{3} \mathrm{kms}^{-1}$. The covariance matrix, $\mathrm{C}$, is then

$\begin{array}{lrrrr}\sigma_{\alpha} & \sigma_{\beta} & \sigma_{z_{x}} & \sigma_{z_{y}} & \sigma_{z_{z}} \\ 0.021 & - & - & - & - \\ 0.024 & 0.032 & - & - & - \\ 0.020 & 0.009 & 4.66 & - & - \\ -0.039 & -0.048 & 0.47 & 2.06 & - \\ -0.029 & -0.027 & -1.33 & 0.63 & 2.02\end{array}$

There are significant off-diagonal terms in $\mathrm{C}$ (especially the $\sigma_{z_{x}} \sigma_{z_{z}}$ term) and the hypothesis we wish to test here is that they are due to the radio galaxy sample having an anisotropic sky distribution. Monte-carlo simulations are clearly in order, but before these consider the following heuristic argument. 
Suppose that the distribution of gravitating matter in the universe induces a dipole $v_{r}=\mathbf{v} \cdot \hat{\mathbf{r}}$, where $\hat{\mathbf{r}}$ describes the position of a galaxy relative to the observer. A least mean-squares optimisation scheme can be used to compute $\mathrm{v}$ since the observational errors $\sigma_{i}$ are independent of $\mathbf{v}$. Thus

$$
\chi^{2}=\sum_{i} \frac{\left(V_{i}-\mathbf{V} \cdot \hat{\mathbf{r}}_{\mathrm{i}}\right)^{2}}{\sigma_{i}^{2}}
$$

Solving for $\partial / \partial \mathbf{V}=0$ yields a matrix equation for $\mathbf{V}$.

The errors on the dipole are found from the matrix of second derivatives, or the Hessian. If this is approximately diagonal it can be expressed as

$$
\frac{\partial \chi^{2}}{\partial V_{j}^{2}}=2 \sum_{i} \frac{\left(\hat{\mathrm{r}}_{\mathrm{i}}\right)_{\mathrm{j}}^{2}}{\sigma_{i}^{2}}
$$

Suppose the matrix equation for $\mathbf{V}$ admitted a solution $V_{\min }$, then an approximate expression can be written for $\chi^{2}$ as

$$
\chi^{2}=\frac{\left(V-V_{\min }\right)^{2}}{\sigma_{V}^{2}}
$$

and hence from equation (6.12)

$$
\sigma_{V_{j}}^{2}=\left[\sum \frac{\left(\hat{\mathrm{r}}_{\mathrm{i}}\right)_{j}^{2}}{\sigma^{2}}\right]^{-1}
$$

where the subscript $j$ runs from 1 to 3 and labels each of the Cartesian components of the Local Group motion.

The variance of the errors on the dipole solution, which we shall denote $\Delta_{|V|^{2}}^{2}$, is given as usual by

$$
\Delta_{|V|^{2}}^{2}=\left\langle|V|^{4}\right\rangle-\left\langle|V|^{2}\right\rangle^{2}
$$

In terms of the $\sigma_{V_{i}}^{2}$ 's we have that

$$
\left\langle|V|^{2}\right\rangle=\sum \sigma_{V_{i}}^{2}
$$

and

$$
\left\langle|V|^{4}\right\rangle=\sum \sigma_{V_{i}}^{4}+\sum_{i \neq j} \sigma_{V_{i}}^{2} \sigma_{V_{j}}^{2}
$$


An analytic expression for $\left\langle V^{4}\right\rangle$ can be found if $V$ is assumed to have been drawn from a gaussian distribution. In this case we have

$$
\left\langle|V|^{4}\right\rangle=2 \sum \sigma_{V_{i}}^{4}+\left(\sum \sigma_{V_{i}}^{2}\right)^{2}
$$

and thus

$$
\left\langle|V|^{4}\right\rangle-\left\langle|V|^{2}\right\rangle^{2}=2 \sum \sigma_{V_{i}}^{4}
$$

from which

$$
\begin{aligned}
\Delta_{|V|^{2}} & =\left(2 \sum \sigma_{V_{i}}^{4}\right)^{\frac{1}{2}} \\
& =2|V| \Delta_{|V|}
\end{aligned}
$$

and so finally we have

$$
\Delta_{|V|} \simeq \frac{\left(\sum \sigma_{V_{i}}^{4}\right)^{\frac{1}{2}}}{\sqrt{2}\left(\sum \sigma_{V_{i}}^{2}\right)^{\frac{1}{2}}}
$$

This result is used below to predict a theoretical estimate for what the errors on the dipole for the radio galaxy sub-sample should be in the case of a uniform sky distribution. It assumes there is no signal - i.e. deviations of $|V|$ from zero are due to random noise only.

\subsubsection{MONTE CARLO SIMULATIONS}

In the case of a uniform sky distribution the expected variance on the LG motion can be computed by noting that

$$
\left\langle\sum \frac{(\hat{\mathrm{r}})_{j}^{2}}{\sigma^{2}}\right\rangle=\left\langle\cos ^{2} \theta\right\rangle \sum \frac{1}{\sigma^{2}}=\frac{1}{3} \sum \frac{1}{\sigma^{2}}
$$

from which it follows that

$$
\Delta_{|V|}=\sqrt{\frac{3}{2}} \times\left[\sum \frac{1}{\sigma^{2}}\right]^{1 / 2}
$$

For 71 galaxies in the redshift range $3000-12000 \mathrm{kms}^{-1}$ with distance measurement errors of $28 \%$ per galaxy one therefore expects approximately $\sigma_{V_{j}} \simeq 400 \mathrm{kms}^{-1}$ and hence, $\Delta_{|V|} \simeq 285 \mathrm{kms}^{-1}$.

To check this, a number of simulations were ran for different realisations of an isotropic sky distribution, i.e the observables $\theta_{e}$ and $\mu_{e}$ for the radio galaxies were kept unchanged but their sky distribution was isotropised. Average rms errors on the three components of the Local Group motion after 20 realisations were as follows

$$
\Delta V_{x}=520 \mathrm{kms}^{-1} ; \Delta V_{y}=447 \mathrm{kms}^{-1} ; \Delta V_{z}=474 \mathrm{kms}^{-1}
$$


These errors are closer to the expected values for an isotropic distribution than the actual values for the real sample. In particular the error bar on the $x$-component of the LG motion has been reduced considerably. The main reason for this is that all of the offdiagonal elements in the covariance matrix are now small whereas before the $\sigma_{z_{x}} \sigma_{z_{z}}$ element was large. There is still some remaining discrepancy between the errors derived from the Monte-Carlo simulations with the expected errors. This is partially explained by the fact that although the galaxies were given a random pair of coordinates, these were such that $|b|>15^{\circ}$, so the sky distribution was not completely isotropised. Also, the off-diagonal elements in the covariance matrix are not exactly equal to zero.

\subsection{Streaming in the Vicinity of the Great Attractor}

The frustrating lack of southern hemisphere objects within $12000 \mathrm{kms}^{-1}$ that have a CCD frame from which $\mu_{e}$ and $r_{e}$ can be measured does not allow any statement to be made at this stage about streaming in the vicinity of the Great Attractor. This is demonstrated in Fig. 6.4 which is a vector diagram of the kind used by G7 to dislpay their peculiar velocity data. Points have been plotted for galaxies which lie within $30^{\circ}$ of the supergalactic plane. The Great Attractor region lies in the top-right part of the diagram. It is only very poorly sampled.

\subsection{Discussion}

How well does our dipole solution compare with other results on the RF scale? To check this we would ideally like to know the six independent components of the observed velocity error covariance matrices. Unfortunately, other workers usually just quote the errors on the three cartesian components of the dipole. Progress can be made however if we assume that the matrices are of the form $\left.\operatorname{diag}\left[\left(\delta v_{x}\right)^{2},\left(\delta v_{y}\right)^{2}\right),\left(\delta v_{z}\right)^{2}\right]$, i.e. orthogonal error components are uncorrelated to first order, then

$$
\left[\begin{array}{c}
v^{4}(\delta v)^{2} \\
v^{6} \cos ^{2} b(\delta b)^{2} \\
\left(v_{x}^{2}+v_{y}^{2}\right)^{2} \sin ^{2} l(\delta l)^{2}
\end{array}\right]=\left[\begin{array}{ccc}
v^{2} v_{x}^{2} & v^{2} v_{y}^{2} & v^{2} v_{z}^{2} \\
v_{x}^{2} v_{z}^{2} & v_{y}^{2} v_{z}^{2} & \left(v^{2}-v_{z}^{2}\right)^{2} \\
v_{y}^{4} & v_{x}^{2} v_{y}^{2} & 0
\end{array}\right]\left[\begin{array}{c}
\left(\delta v_{x}\right)^{2} \\
\left(\delta v_{y}\right)^{2} \\
\left(\delta v_{z}\right)^{2}
\end{array}\right]
$$




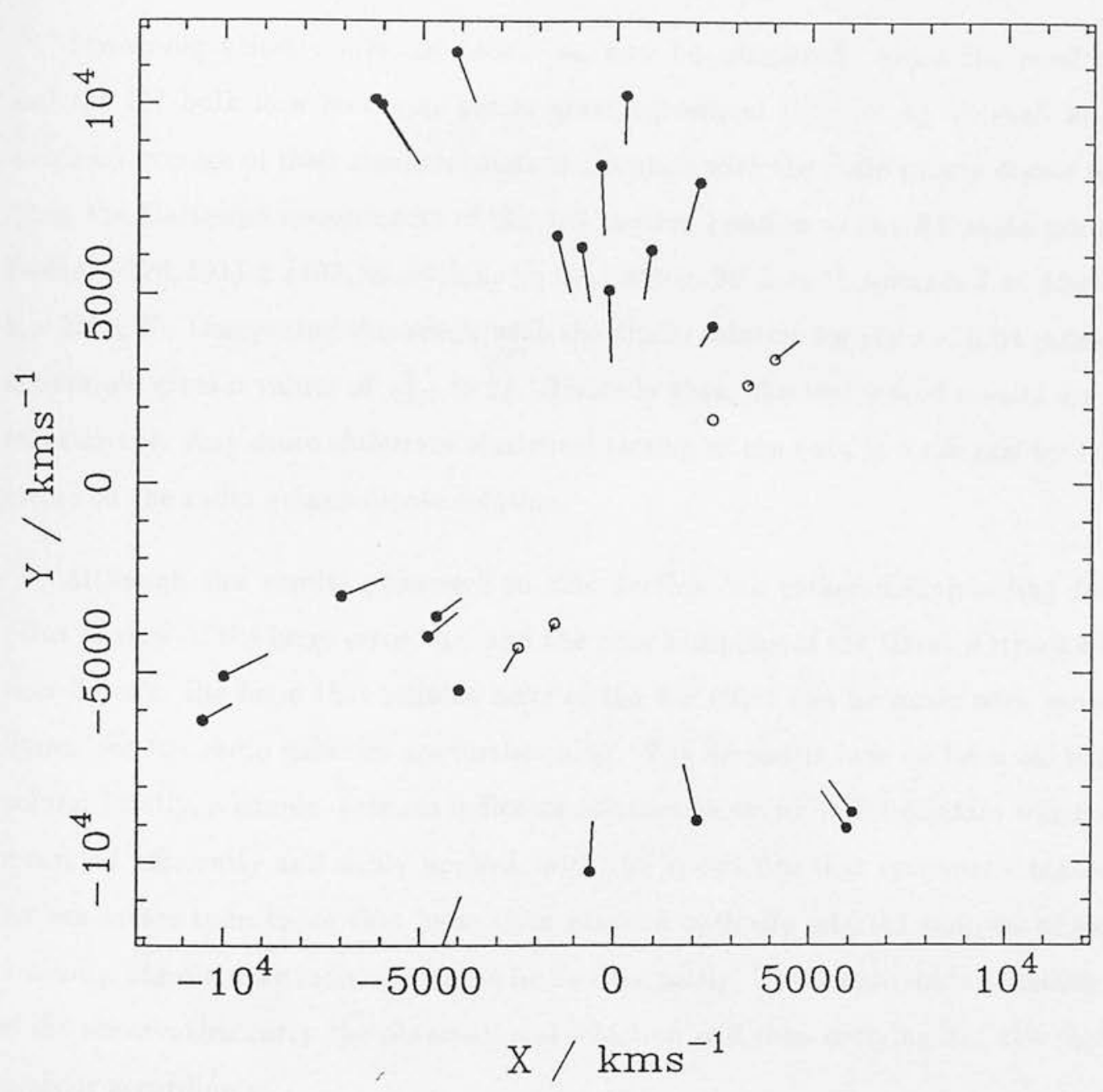

Fig. 6.4: Vector diagram indicating the lack of galaxies in the vicinity of the Great Attractor (top right). Radio galaxies lying within $30^{\circ}$ of the supergalactic plane only are shown. 
If one or more of the $\left(\delta v_{i}\right)^{2}$ is negative then equation (6.24) may not have a sensible solution. This can arise in practice when the errors on $l$ and $b$ are small given the error on $v$. A conservative procedure then is to assume isotropic errors with $\delta\left(v_{i}\right)^{2}=(\delta v)^{2}$.

Streaming velocity measurements can now be compared. Since the results of RF and the G7 bulk flow solutions are in good agreement $\left(\chi_{3 \mathrm{df}}^{2} \simeq 4\right)$ we shall adopt the weighted average of their measurements to compare with the radio galaxy dipole solution. Thus the Cartesian components of the LG motion relative to the RF scale are close to $(-443,-126,191) \pm(102,85,56) \mathrm{kms}^{-1}$, i.e. $499 \pm 96 \mathrm{kms}^{-1}$ towards $l=196^{\circ} \pm 11^{\circ}$, $b=23^{\circ} \pm 7^{\circ}$. Comparing this result with the dipole solution for the $z<0.04$ radio galaxy subsample gives a values of $\chi_{3 \mathrm{dr}}^{2} \simeq 11$. Basically then, the two sets of results are totally inconsistent. Any more elaborate statistical testing of the data is weakened by the large errors on the radio galaxy dipole solution.

Although the results presented in this Section are rather disappointing from the point of view of the large error bars and the poor sampling of the Great Attractor region, they do offer the hope that reliable tests of the RF effect can be made once more CCD frames for the radio galaxies are forthcoming. The emphasis here as been on two main points. Firstly, a simple distance indicator relation exists for radio galaxies which can be measured efficiently and easily applied, with the knowledge that systematic biases are a lot less severe than those that have often plagued optically selected samples of galaxies. Secondly, the distance indicator has to be used correctly. This means understanding which of the observables carry the observational selection and then carrying out the regression analysis accordingly.

Observed dipoles can be compared with theory in a straightforward way. The prescription for doing this has been given by Kaiser (1988c). In many respects the velocity autocorrelation tensor discussed in the next Section is a better statistic for comparing against theory however and we postpone such tests until then.

\subsection{The Velocity Autocorrelation Function}

There are two approaches to analysing cosmological peculiar velocity data with a view towards testing models for structure formation. The first involves comparing the observers 
models for their data (e.g. a simple dipole or streaming towards a Great Attractor) with theory. This approach was used by Bertschinger \& Juskiewicz (1988) to test the G7 observations. Here we shall consider a different method - using the data to compute a velocity autocorrelation tensor. The coherence length of the deviations from Hubble flow can be measured and this provides a useful statistic with which the predictions of galaxy formation models can be compared.

The velocity autocorrelation tensor $\xi_{v v}$ has recently been computed for the G7 elliptical data by Kaiser (1988b) and Groth et al. (1989). Their methods of calculating $\xi_{v v}(r)$ are slightly different and we shall argue that here neither is strictly ideal and that improvements can be found.

\subsubsection{METHOD OF CALCULATION}

In order to calculate $\xi_{v v}(r)$, two simplifying assumptions need to be made about the large-scale velocity field. Firstly, that the field is curl-free as predicted in the gravitational instability picture. Secondly, that it is statistically isotropic, i.e. that the power spectrum $P_{v}(k)$ is a function only of the modulus of the wave vector. If one could observe galaxies and access information about their tangential motion in addition to their line-of-sight velocity then the calculation would be straightforward: sum the dot product $\hat{v} \cdot \hat{v}$ for all pairs of galaxies with a given separation and divide by the number of pairs. Constructing a correlation function from line-of-sight velocity information alone $(u=\hat{\mathrm{r}} \cdot \mathrm{v})$ introduces geometrical functions of the three variables $r, \theta, \phi$ which describe the location of a pair of galaxies relative to the observer. The geometry of a pair of galaxies in the Cartesian reference frame is shown in Fig. 6.5.

An expression for the correlation function can be written in terms of the unit vectors describing the positions of the galaxies. This is

$$
\left\langle u_{1} u_{2}\right\rangle=P_{12} \Pi(r)+S_{12} \Sigma(r)
$$

where

$$
P_{12}=\hat{\mathbf{r}}_{1} \cdot \hat{\mathrm{r}}, \quad S_{12}=\hat{\mathrm{r}}_{1} \cdot \hat{\mathrm{r}}_{2}-P_{12}, \quad \mathrm{r}=\mathrm{r}_{1}-\mathrm{r}_{2}
$$

Here $\Pi$ and $\Sigma$ refer to the "parallel" and "perpendicular" components of the velocity correlation tensor which are related to the familiar dot product correlation function in 


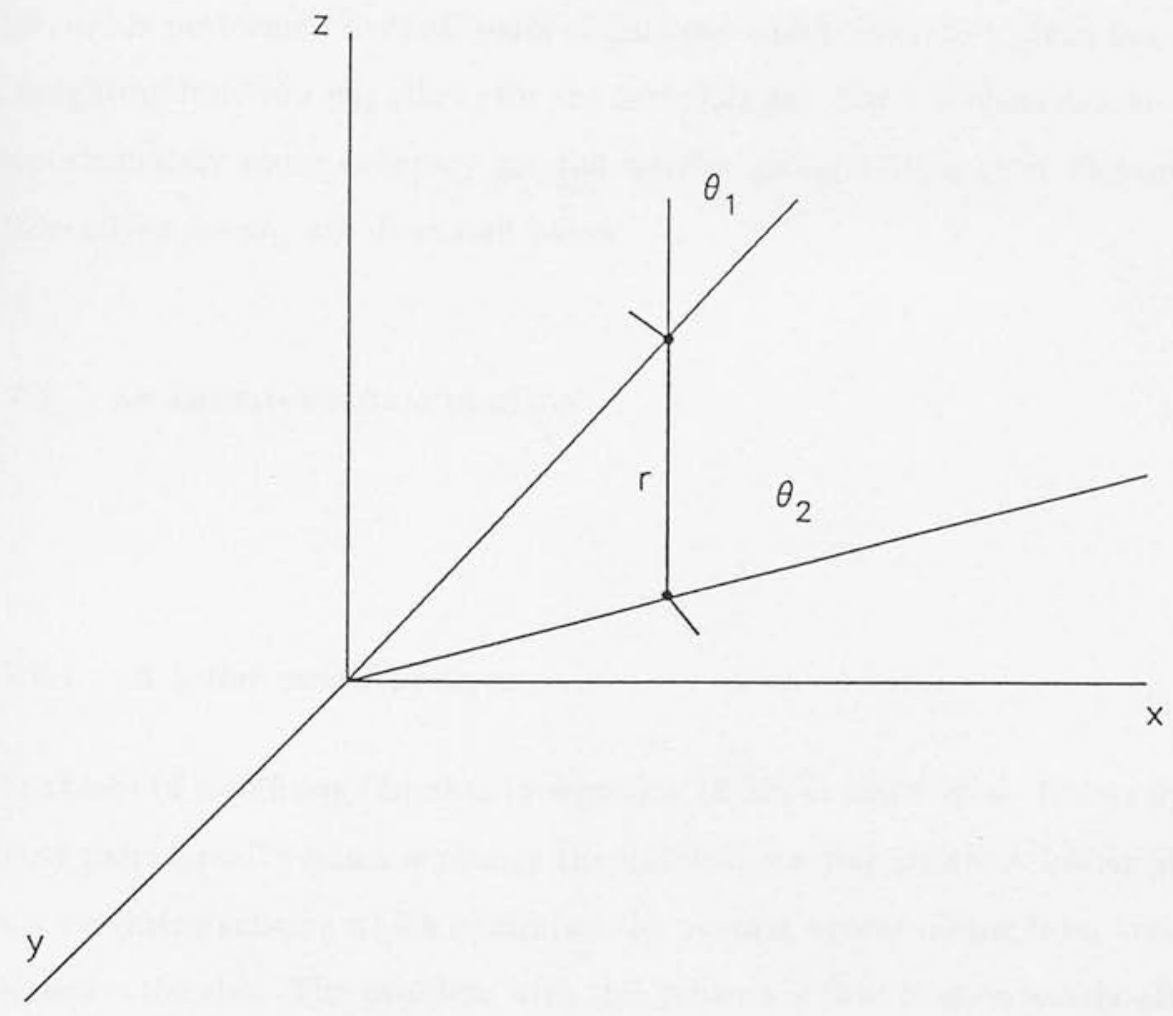

Fig. 6.5: The geometry of a pair of galaxies used for the computation of the velocity autocorrelation tensor $\xi_{v v}$. 
the following way

$$
\xi_{v v}(r)=\Pi+2 \Sigma
$$

The scheme that has then been used by both Kaiser and Groth et al. to estimate II and $\Sigma$ for a given data set is to minimise a 'chi-squared' statistic, where

$$
\chi^{2}(r)=\sum_{i, j} w_{i j}\left[u_{i} u_{j}-P_{i j} \Pi(r)-S_{i j} \Sigma(r)\right]^{2}
$$

The sum is performed over all pairs of galaxies which fall into a given bin in $r, \theta, \phi$ space. A weighting function $w_{i j}$ allows for the fact that peculiar velocities can be estimated with proportionately more accuracy for the nearby galaxies than their distant counterparts. Alternatives for $w_{i j}$ are discussed below.

\subsubsection{AN IMPROVED CALCULATION}

\subsubsection{A better weighting term}

The choice of weighting function in equation (6.28) is non-trivial. Kaiser opted to weight galaxy pairs equally which is clearly the simplest starting point. A better alternative is to use a weighting scheme which minimises the 'formal' errors arising from noisy photometric distance estimates. The problem with this scheme is that it gives nearly all weight to the very nearby galaxies and thus results in huge errors, since one is then predominantly sampling only a small region.

The weighting function used by Groth $e t$ al. was the reciprocal of a total variance which comprised of the noisy distance estimate errors added up in quadrature to a term which can best be though of as a 'thermal' noise in the Hubble flow. The total variance was therefore written as

$$
\sigma_{v}^{2}=\sigma_{f}^{2}+r^{2} \Delta_{r}^{2} / N
$$

where $\sigma_{f}$ is the Hubble flow noise, $r$ is the distance to the group or cluster and $N$ is its number of component galaxies. $\Delta_{r}$ represents the fractional error in estimated distance, which G7 quote as being about $21 \%$. The weighting function is then, $w_{i j}=1 /\left(\sigma_{i}^{2} \sigma_{j}^{2}\right)$, 
which follows from the fact that if $u_{i}$ and $u_{j}$ are normally distributed with zero mean and have independent errors with variances $\sigma_{i}^{2}$ and $\sigma_{j}^{2}$, then the variance of their product is just $\sigma_{i}^{2} \sigma_{j}^{2}$.

For their calculations, Groth et al. kept the value of $\sigma_{f}$ fixed at a nominal value of $250 \mathrm{kms}^{-1}$. However, the Hubble flow noise has cosmic origin and is properly a part of the correlation tensor itself. An expression for $\sigma_{f}$ in terms $\Pi(r)$ and $\Sigma(r)$ can be calculated from first principles and added to the observational variance to get a more realistic weighting function.

In order to derive such an expression for the Hubble flow noise we first note that it can be written as

$$
\sigma_{f}^{2}=\left\langle\left(u_{1}^{2} u_{2}^{2}\right)\right\rangle-\left\langle u_{1} u_{2}\right\rangle^{2}
$$

and that we have already written down an equation for the second term as a function of $\Pi(r)$ and $\Sigma(r)$. Thus we are left with the problem of how to calculate the correlation function of $u^{2}(r)$. This has been done by Rice (1954) and we summarise his calculation here.

In fact it is a rather simple calculation if we make the realistic approximation that peculiar velocities are normally distributed. Consider a random vector $r$ in $K$ dimensions which has values $x_{1}, x_{2}, \ldots x_{K}$. Then $r$ is normally distributed when the probability density of the $x$ 's is of the form

$$
(2 \pi)^{-K / 2}|M|^{-1 / 2} \exp \left[-\frac{1}{2} x^{\prime} M^{-1} x\right]
$$

where the exponent is a quadratic form in the $x$ 's. The square matrix $M$ is composed of the second moments of the $x$ 's.

$$
M=\left(\begin{array}{cccc}
\mu_{11} & \mu_{12} & \ldots & \mu_{1, K} \\
\ldots & & & \ldots \\
\mu_{1 K} & \ldots & \ldots & \ldots
\end{array}\right)
$$

where the second moments are defined by

$$
\mu_{11}=\left\langle x_{1}^{2}\right\rangle ; \quad \mu_{12}=\left\langle x_{1} x_{2}\right\rangle, \quad \text { etc }
$$

We now require the two dimensional distribution in which the first variable is the peculiar velocity $u\left(r_{0}\right)$ and the second variable is its value $u\left(r+r_{0}\right)$ at some distance $r$. This 
distribution is normal, and has second moments

$$
\begin{aligned}
& \mu_{11}=\left\langle u^{2}\left(r_{0}\right)\right\rangle=\Pi(0) \\
& \mu_{22}=\Pi(0) \\
& \mu_{12}=\left\langle u\left(r_{0}\right) u\left(r+r_{0}\right)\right\rangle=\phi(r)
\end{aligned}
$$

Thus an expression for the correlation function of $u^{2}(r)$ is the following

$$
\begin{aligned}
\left\langle u_{1}^{2} u_{2}^{2}\right\rangle=\frac{1}{2 \pi A} \int_{-\infty}^{+\infty} d u_{1} \int_{-\infty}^{+\infty} d u_{1} u_{1}^{2} u_{2}^{2} \exp & \\
& \left(-\frac{1}{2 A^{2}}\left(\Pi_{0} u_{1}^{2}+\Pi_{0} u_{2}^{2}-2 \xi_{v} u_{1} u_{2}\right)\right)
\end{aligned}
$$

where

$$
A^{2}=\Pi^{2}(0)-\phi^{2}(r), \quad u_{1}=u\left(r_{0}\right), \quad u_{2}=u\left(r_{0}+r\right)
$$

This integral can be solved analytically, in terms of the hypergeometric function, by changing variables. The solution is

$$
\left\langle u_{1}^{2} u_{2}^{2}\right\rangle=\Pi^{2}(0)+\phi\left(r, \theta_{1} \theta_{2}\right)^{2}
$$

giving us finally for the theoretical part of the weighting term

$$
\sigma_{\mathrm{th}}^{2}=\Pi(0)^{2}
$$

The calculation of $\xi_{v v}$ proceeds as follows. A starting value of $\sigma_{f}$, say $250 \mathrm{kms}^{-1}$ is assumed. This is used to obtain a value of $\Pi(0)$ which is then fed back into the calculation in an iterative way until the values of $\xi_{v v}$ have converged. In practice it was found that only 3 iterations were necessary.

\subsubsection{Reciprocal variance weighted distance}

The next question concerns the choice of galaxy distance to be used for estimating pair separations. The options are to use recession velocity or else the noisy photometric distance. The former was adopted by Kaiser, whereas Groth et al. chose the latter. Clearly neither are ideal. A better alternative weights these options in the sense that photometric distances are more appropriate for the nearby galaxies since they are less noisy 
whereas redshifts are probably best to use at larger distance for the converse reason. A reciprocal-variance weighted distance, $D^{\prime}$, should therefore be employed. This is written as follows:

$$
D^{\prime}=\frac{\frac{V_{H}}{\sigma_{f}^{2}}+\frac{V_{R}}{\sigma_{R}^{2}}}{\frac{1}{\sigma_{f}^{2}}+\frac{1}{\sigma_{R}^{2}}}
$$

where $\sigma_{f}^{2}$ and $\sigma_{R}^{2}$ are respectively the Hubble flow noise and the variance on the photometric distance estimates (which, recall, proportionately increases with distance) and $V_{z}$ and $V_{H}$ are redshift-distance and photometric distance respectively.

\subsubsection{RESULTS}

Firstly, we want to check how the implementation of these two improvements to the calculation affect the results. This is shown in Fig. 6.6.

The top diagram presents $\xi_{v v}$ for the G7 elliptical dataset which was computed according to the prescription given by Groth et al.. The bin-size for counting pairs is $500 \mathrm{kms}^{-1}$ and the points have been plotted at the bins centres. The bottom diagram shows $\xi_{v v}$, again for the G7 dataset, but incorporating the improved weighting term and a reciprocalvariance weighted distance. An initial Hubble flow noise of $250 \mathrm{kms}^{-1}$ was fed into the computer program and this value was iterated three times after which the values of $\xi_{v v}$ had converged.

The differences between these new results and the old set are minimal, which is not too surprising. There is a small reduction in $\xi_{v v}$ for the first 3 bins. Also the error bars are slightly increased because the value of $\sigma_{f}$ obtained from the data is more like $350 \mathrm{kms}^{-1}$ rather than the smaller input value.

Although there are significantly fewer radio galaxies than in the elliptical sample, it is still possible to compute $\xi_{v v}$ for them by increasing the bin size for counting pairs. A bin size of $2000 \mathrm{kms}^{-1}$ was therefore used. First consider the distribution of pairs shown in Fig. 6.7. This suggests that greatest weight should be given to the values of $\xi_{v v}$ between separations of $5000 \mathrm{kms}^{-1}$ and $12000 \mathrm{kms}^{-1}$. The velocity autocorrelation function is plotted for the $0.01<z<0.04$ subsample of radio galaxies in Fig. 6.8. The points are consistent with zero correlation at all separations up to $13000 \mathrm{kms}^{-1}$. 

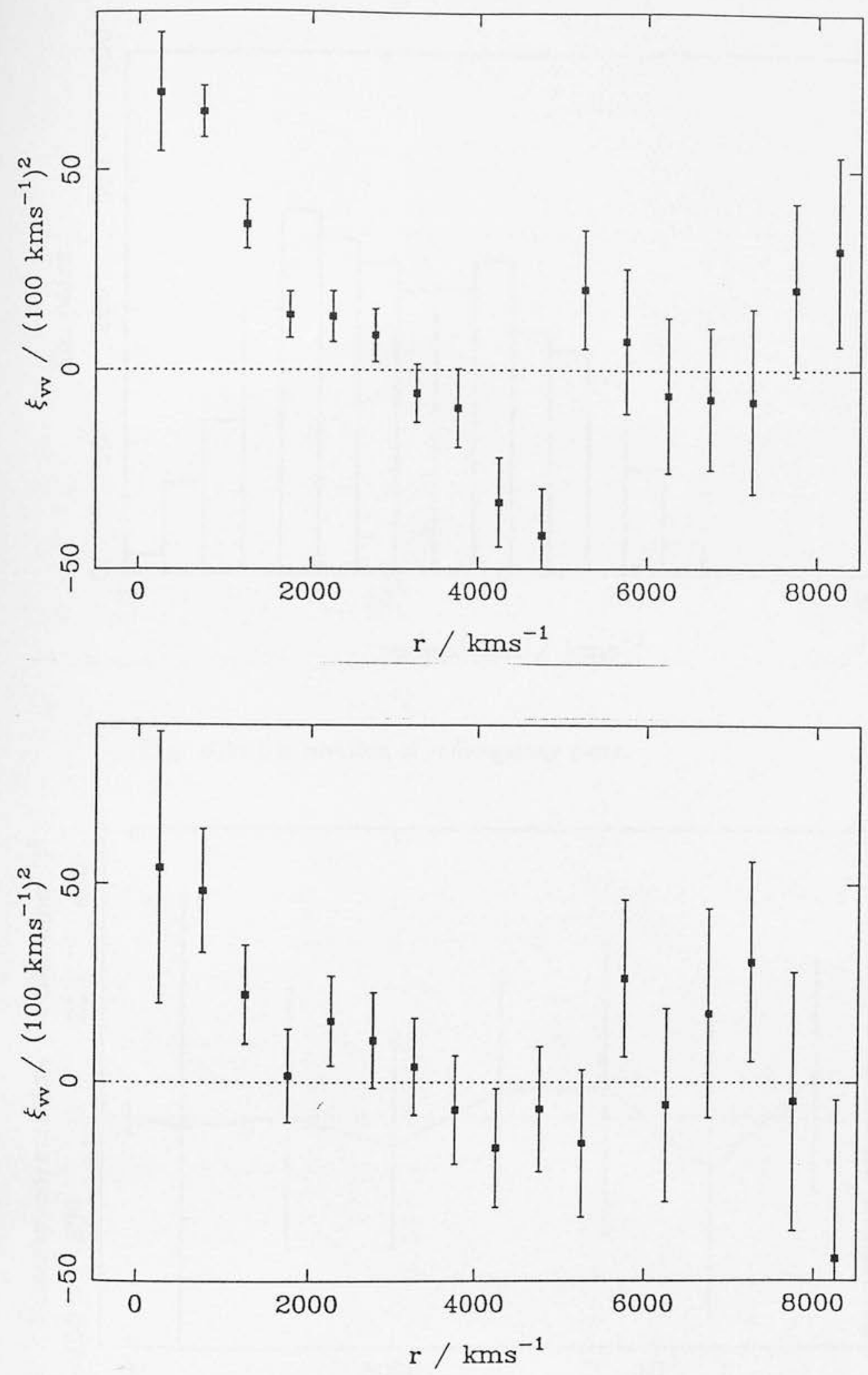

Fig. 6.6: Velocity autocorrelation functions for the G7 ellipticals. The top diagram used the Groth et al. method. The bottom diagram is for an improved weighting function and using reciprocal-variance weighted distance. 


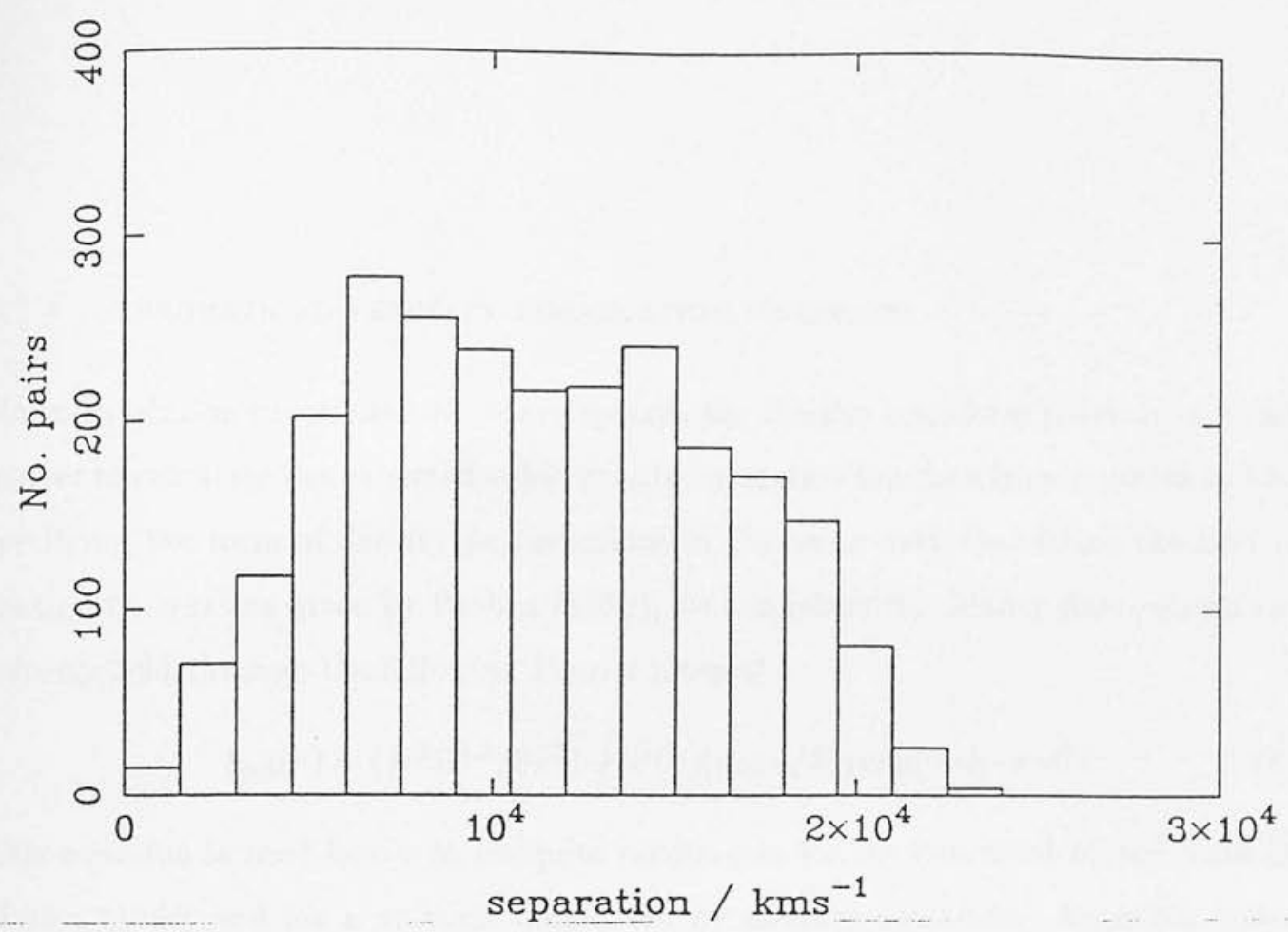

Fig. 6.7: Distribution of radio-galaxy pairs.

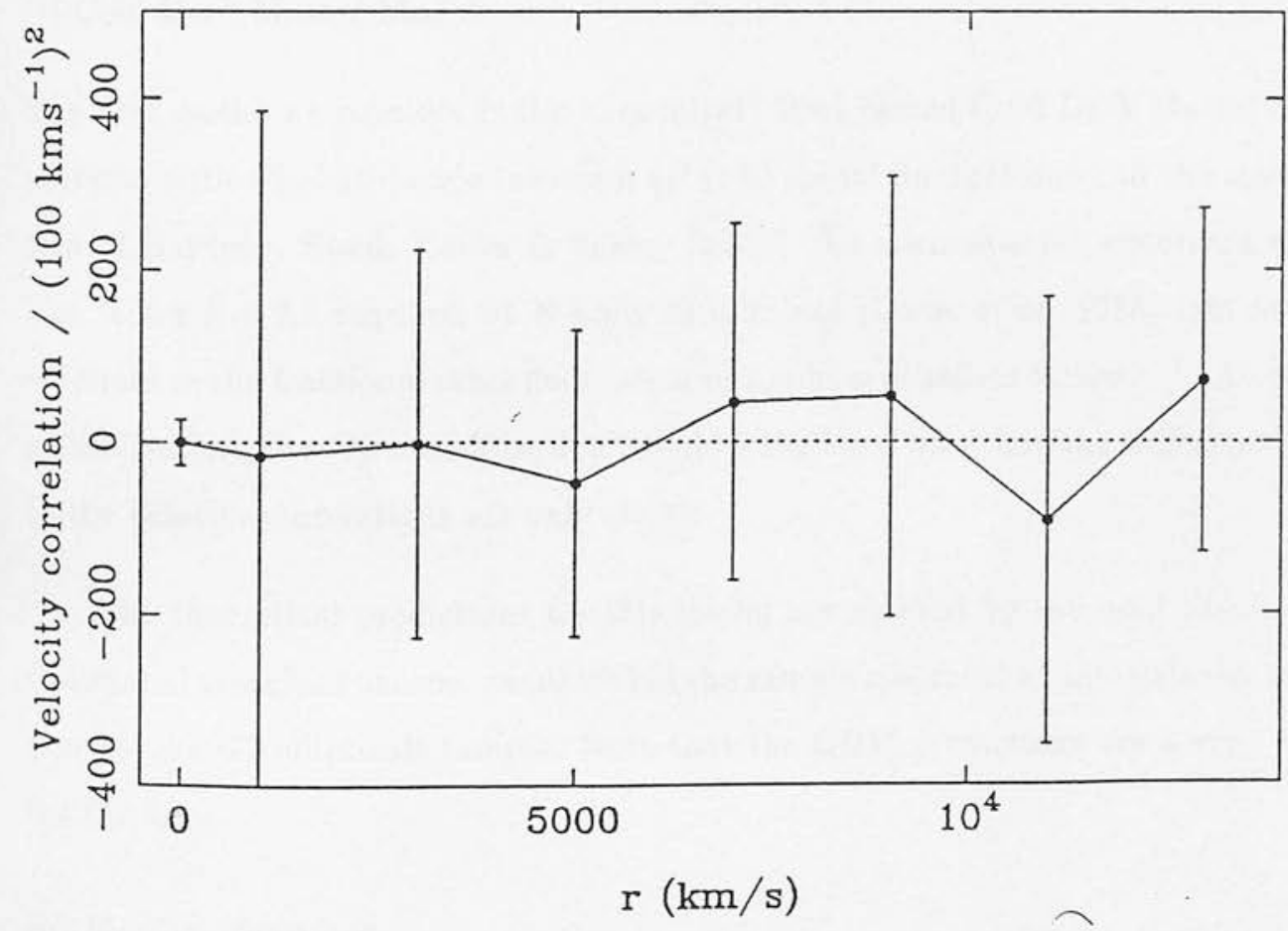

Fig. 6.8: Velocity correlation function for the $0.01<z<0.04$ subsample of radio galaxies. 


\subsubsection{THEORETICAL VELOCITY CORRELATION FUNCTIONS}

Since correlation functions and power spectra are Fourier transform pairs, it is a simple matter to calculate the expected velocity autocorrelation function from a power spectrum specifying the form of density perturbations in the early universe. Using the linearised continuity equation given by Peebles (1980), we can relate the density fluctuations to the velocity field through the following Fourier integral

$$
\xi_{\alpha \beta}(r)=\left(H^{2} \Omega^{1.2} / 8 \pi^{3}\right) \int P(k)\left(k_{\alpha} k_{\beta} / k^{4}\right) \exp (-i \mathrm{k} \cdot \mathrm{r}) d^{3} k
$$

This equation is used below to compute predictions for the canonical biased Cold Dark Matter model and for a universe dominated by massive neutrinos. An extra factor of $\exp \left(-k^{2} r_{f}^{2}\right)$, with $r_{f}$ equal to $400 \mathrm{kms}^{-1}$ is introduced into equation (6.38), to smooth out any small-scale, non-linear structure.

\section{(i) Cold Dark Matter Model}

The first model we consider is the "canonical" $\Omega=1$ biased Cold Dark Matter dominated universe with adiabatic scale invariant $(P \propto k)$ initial fluctuations and the transfer function of Bardeen, Bond, Kaiser \& Szalay (1986). To normalise the spectrum we use the bias factor $b=2.5$ required by $N$-body simulations (Davis et al. 1985). Its reciprocal is set equal to the fractional mass fluctuation in a sphere of radius $800 \mathrm{kms}^{-1}$. An alternative normalisation, the ' $J_{3}$ normalisation', could have been used but the differences it makes to the velocity correlations are only slight.

The theoretical predictions for this model are marked by the bold line in Fig. 6.9. Correlated velocities become small within the sample size for the radio galaxies and indeed also for the G7 ellipticals sample. Note that the CDM predictions are a very poor fit to the G7 data.

\section{(ii) Massive Neutrinos}

The second model is an $\Omega=1$ universe dominated by one species of massive neutrino. The initial spectrum goes as $k$ and we use the transfer function of Bond \& Szalay (1983), 


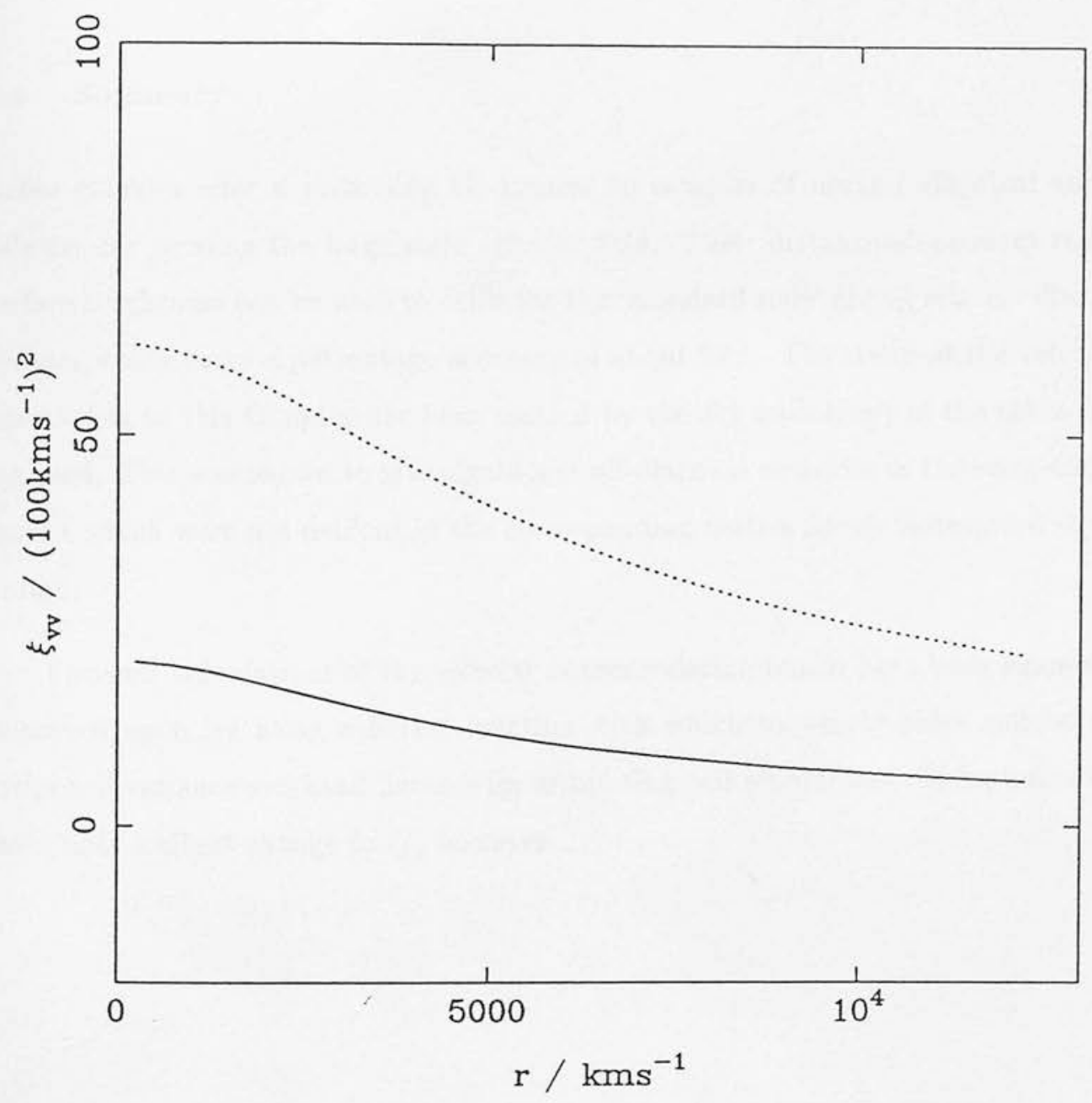

Fig. 6.9: Theoretical velocity correlations. The bold line is for standard CDM. The dashed line is for a massive neutrino universe. 
normalising the spectrum this time to satisfy the $J_{3}$ integral constraint (Peebles 1980). The dashed line on Fig. 6.9 marks the theoretical predictions for this model. Recall from the introductory Chapter that neutrinos are free-streaming dark matter particles. This explains the large correlations predicted by this model.

\subsection{Summary}

Radio galaxies offer a promising alternative to samples of normal elliptical and spiral galaxies for probing the large-scale velocity field. Their distant-independent rest-frame surface brightness can be used to calibrate the 'standard rods' giving relative distance estimates which carry a percentage accuracy of about $28 \%$. The study of the velocity field undertaken in this Chapter has been marred by the sky anisotropy of the subsample being used. This was shown to give significant off-diagonal elements in the error-covariance matrix which were not evident in the corresponding matrix for an isotropised sky distribution.

Previous calculations of the velocity autocorrelation tensor have been examined and improved upon by using a better function with which to weight pairs and by using a reciprocal variance weighted distance for estimating pair separations. These improvements make only a slight change to $\xi_{v v}$ however. 


\section{Chapter 7}

\section{Biased Peculiar Velocities}

\subsection{Introduction}

In our introductory survey of galaxy formation and cosmology, we traced back the possible origin of large-scale structure to quantum fluctuations laid down at very early times. Inflation was deemed a desirable aspect of the evolution of the early universe, not only because it resolved some standard problems with the Big Bang theory (e.g. flatness, horizon), but also predicted the desired scale-invariant Harrison-Zeldovich power spectrum, $\left|\delta_{k}\right|^{2}=A k$, for the initial fluctuations. Recall that in an inflationary universe with vanishing cosmological constant $(\Lambda=0)$, a density parameter of $\Omega_{0}=1$ is implied. If we demand a lower value, say $\Omega_{0}=0.2$ to match observational evidence from the dynamics of clusters, this requires a remarkably fine tuning of the initial exponential growth factor to yield a present radius of curvature of the order of the Hubble radius $\left(R_{0} H_{0} \sim 1\right)$. Thus, there is much aesthetic appeal to retain an $\Omega_{0}=1$ model, despite the conflicting evidence, and considerable effort has been devoted over the last decade to try and reconcile this discrepancy. The present Chapter is concerned with perhaps the most convincing explanation: biased galaxy formation.

By means of introduction, a short review outlining the basics of the threshold model for biased galaxy formation is given first. The flaws in this model are made apparent and these lead us to consider alternatives, of which one of the most promising candidates is 'natural' bias or autonomously biased gravitational collapse. The present Chapter is mainly concerned with a variety of tests to check whether there is any evidence for the operation of this type of bias in the photometric and kinematic properties of galaxies. 


\subsection{Biased Galaxy Formation}

The value of $\Omega_{0}$ is still very much open to debate, more than fifty years on from the 'missing mass' problem discovered by Zwicky (1933). This refers to the factor of 10 discrepancy between the luminous mass in clusters of galaxies and that inferred dynamically from the virial theorem. Luminous matter in the universe contributes a value of $\Omega_{0} \sim 0.03$ (Faber \& Gallagher 1979). Dark matter within galaxies, whose presence is indicated by flat rotation curves, may increase this value by a factor $\sim 3$ (Rubin et al. 1982). Additional dark matter between galaxies, suggested by the virial masses of clusters and groups of galaxies, could increase the contribution to $\Omega_{0}$ by a further factor $\sim 2$. The resulting value of $\Omega_{0} \sim 0.1-0.2$ is still short, by about a factor of 5 , from closing the universe. This value is also very close to the maximum baryon density consistent with the observed hydrogen/deuterium ratio in standard models of cosmological nucleosynthesis (Yang et al. 1984). If the surplus matter needed to bring the observed value of $\Omega_{0}$ up to the theoretically preferred value of unity exists, it is therefore likely to be non-baryonic and distributed more smoothly than galaxies so as not to conflict with the virial cluster estimates. It was this latter presumption, in particular, that gave rise to the notion of biased galaxy formation in which luminous objects may form preferentially in regions of greater large-scale density enhancement (Kaiser 1984; Schaeffer \& Silk 1985; Umemura \& Ikeuchi 1985).

\subsubsection{OBSERVATIONS}

There are several observations which lend support to the idea that luminous matter may not be a faithful tracer of the underlying mass distribution. For example, the range of clustering strengths displayed by various classes of object. It was remarked in Chapter 5 that there seems to be a hierarchy of structure in the universe with dwarf galaxies occupying the bottom rung of the ladder and continuing upwards through normal galaxies, radio galaxies, rich clusters and perhaps even superclusters. Other observations which argue against a light-traces-mass universe are the morphology-density effect (Dressler 1980; Postman \& Geller 1984) for galaxies, an observed relation between the morphology and topology of large-scale structure (Giovanelli et al. 1986) and the surface brightnessclustering relation reported by Djorgovski \& Davis (1985). 


\subsubsection{AMPLITUDE OF BIASING IN GALAXIES AND CLUSTERS}

Dekel \& Rees (1987) have presented a simple argument to illustrate the need for bias if an $\Omega_{0}=1$ universe is to be tenable. They consider how mass estimates are made on scales $10 h^{-1} \mathrm{Mpc}$, where the linear approximation to the density field is assumed valid. These estimates are based on either modelling the infall of the Local Group into the Local Supercluster (LSC) (Davis \& Peebles 1983a) or else applying a cosmic virial theorem to pairs of galaxies or to cluster-galaxy pairs (Peebles 1986). Adopting the former approach, a linear, spherical model for the LSC gives an estimate

$$
\Omega \simeq \delta^{-1.7}\left(3 v_{c} / H r\right)^{1.7}
$$

where $\delta$ is the density enhancement within the Local Group radius $r$, and $v_{c}$ is the infall velocity at $r$ (a value of $250 \pm 50 \mathrm{kms}^{-1}$ was adopted by Tammann \& Sandage 1985). A further dependence is supplied by the cosmic virial theorem defined by Peebles (1980). This is

$$
\Omega \propto \xi(r)^{-1}(v / r)^{2}
$$

where $v$ is a mean pair-velocity and $\xi(r)$ is the 3-point spatial correlation function. Applying these simple expressions yields a value for $\Omega_{0}=0.1-0.3$. The key point is, however, that the number overdensity $\delta_{g}$ and correlation function $\xi_{g}(r)$ both refer to galaxies. If these luminous objects are biased tracers of the mass distribution in the LSC, then the rms fluctuations in galaxies will in general be different from those of the underlying mass. It is conventional to characterise this difference by the parameter

$$
b \equiv \frac{\sigma_{g}\left(8 h^{-1} \mathrm{Mpc}\right)}{\sigma_{\rho}\left(8 h^{-1} \mathrm{Mpc}\right)}=\frac{1}{\sigma_{\rho}\left(8 h^{-1} \mathrm{Mpc}\right)}
$$

i.e. the ratio of galaxy to mass fluctuations in a sphere of $8 h^{-1} \mathrm{Mpc}$ radius, corresponding to a mass scale $M=6 \times 10^{14} \Omega h^{-1} M_{\odot}$, roughly that of rich clusters. Since the level of fluctuations in galaxy counts on this scale is observed to be unity (Davis \& Peebles 1983b), $b$ can be interpreted as the inverse of the spectrum normalisation on the mass scale of rich clusters. Suppose then that, by virtue of the linear approximation, galaxies are more strongly clustered than the underlying matter such that $\xi_{g}(r)=b^{2} \xi_{\rho}(r)$. The true value of $\Omega_{0}$, obtained from equations (7.1) and (7.2), is then larger by a factor $b^{1.7}$ or $b^{2}$. This would comply with $\Omega_{0}$ if the bias parameter had the value $b \sim 1.5-3$. 
For rich clusters, the degree of biasing is measured by comparing the mean cosmic mass-to-light ratio to the cluster mass-to-light ratio. This has been quite difficult to pin down observationally. Efstathiou et al. (1988) have analysed an extensive redshift survey for cluster galaxies and report a critical value for $(M / L)$ to close the universe of $1500 M_{\odot} / L_{\odot}$, which implies a bias $B=5$ (note the difference in notation between the biasing factors for galaxies and clusters).

A further indicator of the need for bias comes from the numerical simulations of largescale structure formation in the CDM cosmology by Davis et al. (1985). They find that the predicted current-epoch correlation length for galaxies is smaller than the observed $5 h^{-1} \mathrm{Mpc}$, unless $\Omega h \lesssim 0.2$. The amount of biasing required to solve this dilemma is consistent with the value deduced above from the Virgo infall argument.

\subsubsection{PHYSICAL MECHANISMS FOR BIASED GALAXY FORMATION}

The general idea is that high peaks in a Gaussian random field display enhanced clustering (Kaiser 1984; Peacock \& Heavens 1985; Bardeen et al. 1986). This enhancement is a function of scale: on large scales there is a linear amplification of the correlation function which is independent of scale. On smaller scales, but larger than the mass scale of the peaks themselves, a non-linear (exponential) enhancement of the number-density of high peaks in overdense regions arises.

Kaiser (1984) has explored the observational consequences of identifying high peaks in the smoothed primordial density field as sites for galaxy and cluster formation. In particular the observation that different classes of object exhibit different clustering properties can be understood if these arguments are followed through properly.

A basic flaw with the threshold model is that the peak height (relative to the rms variation of $\delta \rho / \rho$ ) has to be fixed externally from numerical experiments. In numerical Nbody simulations it is hypothesised that galaxies form only from regions which are $\gtrsim 2.5 \sigma$ peaks of the initial mass distribution smoothed over a galactic scale. This a priori choice results in simulations which give good fits to the observed galaxy covariance function, cluster $M / L$ ratio and the Virgo infall. 
The mass-light segregation invoked by the threshold model makes the value of $b$ dependent on the details of the procedure used to identify sites of galaxy formation. Using a peak formalism to reproduce the observed abundance and two-point correlation function of galaxies, Bardeen et al. (1986) find a preferred value of $b \simeq 1$.7. This is close to the value demanded by $\mathrm{N}$-body simulations although any small difference is still crucial since there are observable quantities (e.g. pairwise peculiar velocities of galaxies and the epoch of galaxy formation) which depend exponentially on $b$.

So far nothing has been said about the physical meaning of a threshold for galaxy formation. If the threshold model is to remain attractive, a plausible astrophysical process which could impede the formation of galaxies below the peak threshold in the density field must be found. To shut off galaxy formation completely requires heating the intergalactic medium to a temperature above $10^{6} \mathrm{~K}$. Although this may eventually happen as a result of galactic winds, explosions and virialisation in clusters of galaxies, the heating would be most effective in regions of high galaxy density. The inhomogeneity of the heating would seem unlikely to counteract strongly any statistical enhancement of galaxy clustering. More promising is to look for mechanisms of suppressing star formation. For example, one might envisage the initial formation of massive stars whose explosions would then make further star formation difficult and might expel much of the gas from the galaxy (Larson 1974). Gas would still fall into a potential well produced by the dark matter, but would not yield a high surface brightness galaxy which would be counted in redshift surveys. There is some doubt as to whether this mechanism alone can set a sufficiently sharp threshold for galaxy formation and moreover, our theories of star formation are not well enough developed to predict with confidence what might happen at high redshift. Other feedback mechanisms to inhibit galaxy formation in the lower peaks have been reviewed by Dekel \& Rees (1987). All of these are equally uncertain.

\subsubsection{NATURAL OR AUTONOMOUS BIAS}

A promising alternative to the 'sharp' threshold model has been proposed by Kaiser (1988a) and discussed in some detail by Cole \& Kaiser (1989). The general idea has also been advocated by White et al. (1987) although in a rather different context. Kaiser argues that we observe only a 'snapshot' of galaxy and cluster formation and that low 
amplitude perturbations are not in fact destined to become 'failed' galaxies, but will eventually make luminous galaxies but at a later time. The need to suppress star formation in some objects altogether is obviated by postulating a simple variation in star forming efficiency with epoch. If the non-linear dark matter concentrations (halos) are identified as potential sites for galaxy formation, then a 'theory for galaxy formation' tells us how to assign luminosities to these sites. The epoch-dependency of star forming efficiency can be expressed in terms of the collapsed mass $(M)$ and redshift of collapse $\left(z_{c}\right)$ as follows

$$
L\left(M, z_{c}\right)=M^{\alpha}\left(1+z_{c}\right)^{\beta}
$$

Although simple, there is some hope at least that this basic characterisation of the stellar luminosity upon halo properties might still embody some of the ill-defined physics governing star formation.

Kaiser (1988a) has shown that the model results in a positive bias for all objects. This is quite distinct from the threshold model, where a positive bias for the most massive objects was at the expense of a negative bias for the least massive. At the site of a protocluster the abundance of galaxies per unit mass is enhanced by a factor $B$ given by

$$
B=\left(1+\frac{\Delta_{C}}{\Delta_{G}}\right)^{\frac{6 \beta}{6 \alpha-(n+3) \beta}}
$$

where $n$ is the effective power-law index of the density fluctuations, $\Delta_{C}$ and $\Delta_{G}$ are the linear values of $\delta \rho / \rho$ on cluster and galaxy scales respectively. Notice that the implied bias here is local. In the threshold model this was not the case and collapsing peaks were required to propagate malign effects over a distance to shut off galaxy formation from the lower amplitude perturbations.

The model is capable of reconciling the cluster $M / L$ ratio with $\Omega=1$, provided $B=3-5$. Kaiser argues that such a level of bias is expected if there is a quite rapid variation of star-forming efficiency with epoch, $\beta=2 \rightarrow 3$. In addition a negative powerlaw index is needed. Thus, for $n=-2$ (see next section) $\delta \rho / \rho \propto M^{-\frac{1}{6}}$. For bright galaxies with $M$ typically $10^{12} M_{\odot}$ and clusters of $M \sim 10^{15} M_{\odot}$ one finds that $\Delta_{C} / \Delta_{G} \sim 1 / 3$ or it may even be as large as unity for rare, rich clusters. It would be surprising if large values of $\beta$ were not to leave some mark on the properties of present-day galaxies. The remainder of this Chapter is devoted to a search for such relics. 


\subsection{Outline of the Present Work}

This Chapter forges a link between much of the work that was presented in earlier Chapters. As we shall note in the next Section, Kaiser's biasing model predicts environmental offsets in luminosity-internal velocity type distance indicator relations (except for the special case when $\beta=2, \alpha=4 / 3)$. From Chapter 4 we have already discussed how to calculate a parameter for measuring the local environment of a galaxy. Chapter 6 described how distance indicator relations are used to measure peculiar velocities. This information equips us well-enough to be able to perform a number of tests in order to search for evidence of autonomous bias by examining the properties of present-day galaxies.

The layout of the rest of this Chapter is as follows. Section 7.4 describes how the fundamental plane for galaxies can be explored for clues to the operation of bias. In Section 7.5 the implications of spatially-modulated distance indicator relations for the interpretation of peculiar velocities is discussed. A large sample of elliptical galaxies which have had photometric and kinematical observations made is then used to check for systematic offsets. Section 7.6 considers other observational data - notably for spiral bulges and brightest cluster members which are generally located in vastly disparate environments. Section 7.7 discusses the results and places them in their theoretical context. A short summary is given in Section 7.8. 


\subsection{The Fundamental Plane for Elliptical Galaxies}

Elliptical galaxies exhibit a tight correlation between luminosity $L$ and central velocity dispersion $\sigma_{v}$ (Faber \& Jackson 1976), which must reflect a remarkable uniformity in their formation history. Moreover, by incorporating surface brightness $I$, so that all three quantities appearing in the virial theorem are represented, an even tighter fundamental relation can be found. This is the $D_{n}-\sigma_{v}$ relation encountered in the last Chapter, where $D_{n}$ is a scale-length corresponding to the diameter within which the mean surface brightness of the galaxy attains some fiducial value.

In terms of autonomous bias it is worth noting that in an unbiased, light traces mass, universe the observed correlation between $L$ and $\sigma_{v}$ can be simply attributed to a small, negative power law index in the density fluctuation spectrum (Blumenthal et al. 1984). For example, in CDM, the spectral index is close to -2 on galaxy scales. Thus, the rms variation of $\delta \rho / \rho$ when smoothed with a box containing a galactic mass $M$ will vary as $\delta \rho / \rho \propto M^{-1 / 6}$. The final density of the system at collapse varies as the cube of the initial perturbation amplitude, i.e. as $M^{-1 / 2}$. Invoking the virial theorem then gives $M \propto \sigma_{v}^{4}$. This corresponds to values of $\alpha=4 / 3$ and $\beta=2$ in equation 7.4. The theoretical prediction bears a strong resemblance to the observed Faber-Jackson relation. It is also possible to derive the form of the $D_{n}-\sigma_{v}$ relation from first principles but no assumptions about the initial hierarchy are required. It follows directly from the fact that all bright elliptical galaxies have surface brightness distributions which approximate de Vaucouleurs law when projected on the sky and that the mass-to-light ratio of their inner parts are very nearly constant (Gunn 1988).

What clues, if any, might the fundamental plane yield to aid us in our search for evidence of autonomous bias? There are essentially two aspects of the plane that are of interest. These are (i) the distribution of points on the fundamental plane, and (ii) offsets in the equation of the fundamental plane and whether these correlate with environment. The first of these has been addressed by Peacock (1990) and his results will be discussed briefly later. The present work is concerned mainly with the second, which we now discuss 
in some detail.

\subsubsection{ORIGIN OF SYSTEMATIC OFFSETS}

The abundance of galaxies per unit mass inside a cluster is enhanced by a factor $B$ in response to long wavelength density perturbations. This enhancement is proportional to $\beta$, which governs the rate of star formation, through the following relation which is valid in the linear regime only

$$
B=\frac{\beta}{(\alpha-(n+3) \beta / 6)} \frac{\Delta_{B}}{\Delta_{O B J}}
$$

where $n$ is the index of the power-law density fluctuation spectrum and $\triangle_{O B J}$ is the initial density perturbation for an object subject to the modulating influence of the longwavelength perturbation.

As a specific example, consider the unbiased case of $\beta=0$. A proto-galactic object subject to a positive long-wavelength swell will have its collapse redshift, and therefore its internal velocity, enhanced. The luminosity, however, by definition remains unchanged. Thus in regions of the universe which are now overdense one would expect to find less luminous galaxies at a given velocity dispersion.

Systematic offsets are also a feature of models with a high degree of bias, i.e. $\beta>2$. The prediction here though is that galaxies of a given internal velocity dispersion will be brighter in overdense regions of the universe. This begs the question why an earlier collapse should give rise to a brighter galaxy? Some of the options have been discussed by Cole \& Kaiser (1989). Most compelling is the idea that one can form more stars per unit mass of gas in deeper potential wells (Larson 1974; Dekel \& Silk 1986).

A further interesting case is the one for which $\alpha=4 / 3, \beta=2$ whereupon all galaxies lie along a universal $L-\sigma_{v}^{4}$ relation with no intrinsic scatter and no systematic offsets. This choice has been explored in numerical simulations (White et al. 1987; Carlberg \& Couchman 1989) in which galaxies above a luminosity limit are identified with the dark matter halos with rotation velocities above a corresponding threshold. Although appealing, Kaiser (1988a) has remarked that this particular choice of parameters seems to imply too weak a bias.

The arguments presented here suggest that for reasonable values of the parameters 
one might expect to find systematic offsets in the Faber-Jackson relation but not for the $D_{n}-\sigma_{v}$ relation. However it is still important to test for offsets in the latter, since the biasing model under study is only rather simple and offsets in $D_{n}-\sigma_{v}$ may well be an inherent feature of some more realistic model.

\subsection{Biased Peculiar Velocities}

\subsubsection{IMPLICATIONS OF OFFSETS FOR PECULIAR VELOCITIES}

If systematic offsets were present in the Faber-Jackson and $D_{n}-\sigma_{v}$ relations then their application as galaxy distance indicators would lead us to question the reality of largescale streaming motions. The tacit assumption that is made when these relations are used as galaxy distance indicators is that they are universally valid and apply as equally to poor groups of galaxies as they do to the richest of clusters. Clearly, this assumption must break down at some level especially if we are to hold on to the general belief that galaxy formation consists of a series of dissipative merging and infall processes, most of which can be conditioned by the large-scale environment (e.g. Silk \& Norman 1981; Silk 1987).

The implications of systematic offsets for an interpretation of the large-scale velocity field have been remarked upon by several authors (Kaiser 1988b; Djorgovski, de Carvalho \& Han 1988; Silk 1989). In terms of the simple biasing model discussed earlier, offsets in the $L-\sigma_{v}$ relation are a feature of all but the special case for which $\alpha=4 / 3, \beta=2$. In the unbiased $\beta=0$ model, one would infer a smaller Hubble constant in regions of dense environment. The converse would be true if a high degree of autonomous bias $(\beta \simeq 3)$ were operating. The latter case has a particularly intriguing consequences for an interpretation of peculiar velocities and this is illustrated schematically in Fig. 7.1.

The sketch depicts a hypothetical situation in which an observer's peculiar-motion is driven by the overdensity shown. A boundary has also been drawn within which the observer can reliably estimate photometric distances. In a super-naturally (high- $\beta$ ) biased universe, galaxies in the dense region are brighter (or bigger) than the application of a universal 


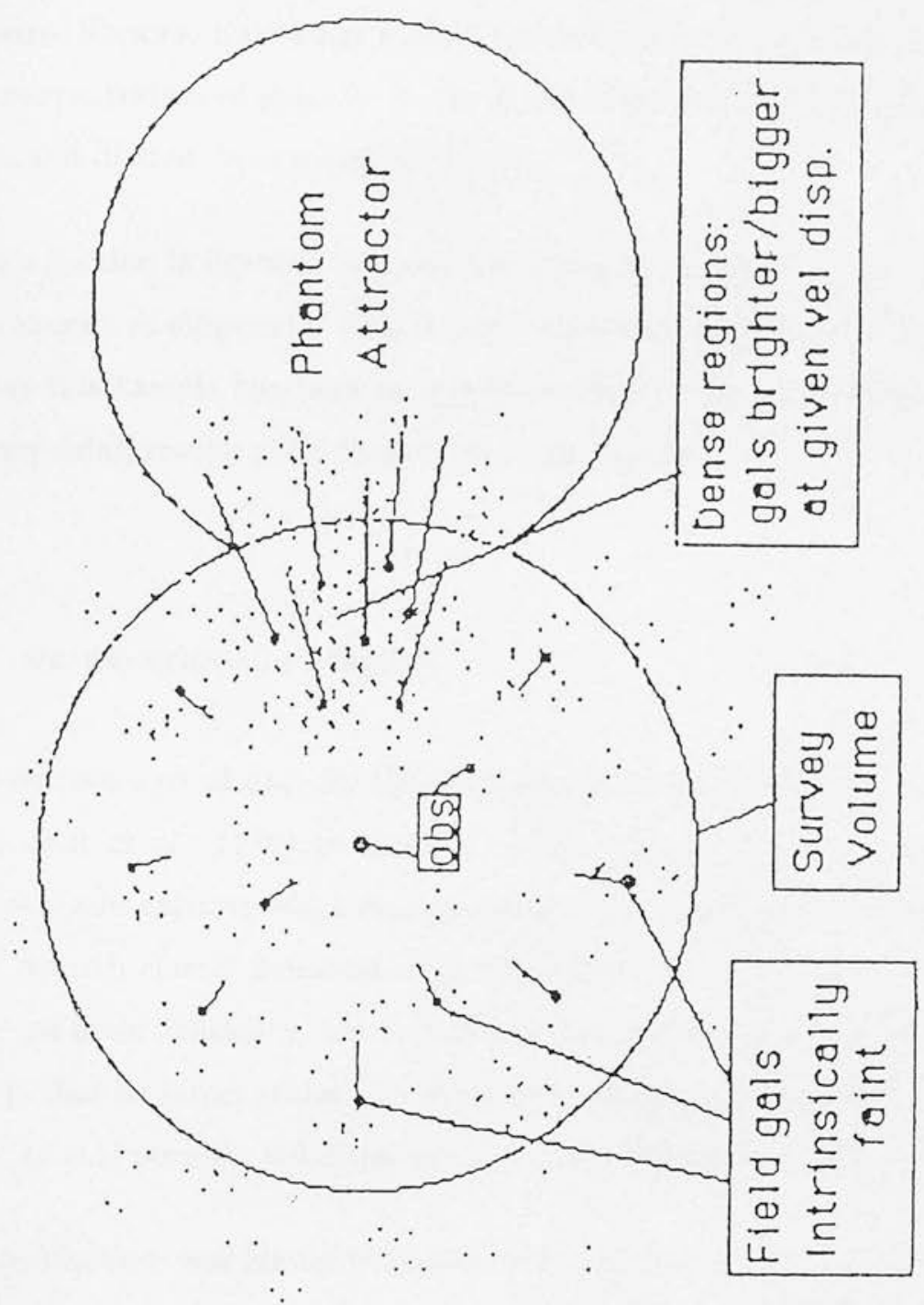

Fig. 7.1: A schematic diagram (reproduced from Kaiser 1988b) showing how an observer in a 'super-naturally' biased universe might conclude that the overdensity responsible for his own peculiar motion is itself being pulled in a direction away from the observer by a more distant 'phantom' attractor. 
Faber-Jackson or $D_{n}-\sigma_{v}$ relation would predict. Thus one might be led to infer that the peculiar velocity of the clump, in the same sense as that of the observer, was being induced by a mass concentration at greater depth. Such an implied mass concentration is of course illusory. Kaiser has likened the situation to that which we appear to observe: a rich concentration of galaxies in the Hydra Centaurus region being pulled by a more massive and distant "attractor."

This Section is devoted to a test for offsets in the $D_{n}-\sigma_{v}$ and $L-\sigma_{v}$ relations for a large sample of ellipticals. This is none other than the 'Gang of Seven' database. The fact that this sample has been used extensively to map the peculiar velocity field with some surprising results gives these tests an added impetus.

\subsubsection{OBSERVAtional Database}

This comprises optical data for the large sample of ellipticals used by the Gang of Seven (Lynden-Bell et al. 1988) to study the local velocity field. In the sense that the G7 sample contains galaxies which occupy a range of environments from isolated field galaxies through to rich cluster members, it is well-suited to test the predictions of natural bias models. Its main deficiency, however, lies in its shallow depth. The advantage of a deeper sample is that on larger scales, any systematic effects present in the data would probably swamp the real peculiar velocities and thus have been more easier to detect.

The database was kindly provided by David Burstein in machine-readable form. It lists heliocentric radial velocities, peculiar velocities relative to the $\mathrm{CBR}$, galaxy coordinates and $D_{n} \& \sigma_{v}$ for some 385 elliptical galaxies. In addition to this set of parameters the total blue magnitude $B_{T}$ for each galaxy was extracted from Burstein et al. (1987) and added to the list. A value of $A_{g e}, B_{g e}$ and associated errors were computed for each of the ellipticals using the Lick catalogue for objects north of $\delta=-23^{\circ}$ and the Abell cluster catalogue to get a rough estimate for galaxies south of this declination. The method used was identical to that described in Chapter 4 using the same set of values for the parameters $H_{0}, \phi^{*}$ etc. The resulting values of $A_{g e} \& B_{g e}$ are tabulated in Appendix D, together with some other details about the sample. 


\subsubsection{RESULTS}

Firstly, consider the $D_{n}-\sigma_{v}$ relation. The observed angular sizes for each galaxy were converted into true physical sizes by correcting observed redshifts for peculiar motions. In view of the points made in the last Chapter about which way round to do a regression, $\sigma_{v}$ was regressed on $D_{n}$ since it is the latter quantity that contains the observational selection. The data are shown in Fig. 7.2 together with a least-mean squares fit.

The equation of this best-fitting line is

$$
\log _{10} \sigma_{v}=1.89 \pm 0.01+(0.64 \pm 0.02) \log _{10} D_{n}
$$

where the units of $D_{n}$ are kiloparsecs $\left(H_{0}=50 \mathrm{kms}^{-1} \mathrm{Mpc}\right)$.

It is the scatter about $D_{n}-\sigma_{v}$ and in particular whether it correlates with $B_{g e}$, rather than the equation of the line that is of most interest however. Fig. 7.3 plots $D_{n}$ residuals against $B_{g e}$ for all 385 elliptical galaxies. There is little evidence for any systematic trend in the data. This is confirmed by an insignificant value $(r=0.04)$ for the Spearman Rank Correlation Coefficient.

One can conceive of two reasons for why any potentially weak signal in the data as it has been presented in Fig. 7.3 might be masked. One, is the inclusion in the elliptical sample of nearby galaxies inhabiting the so-called 'local anomaly' - this is a region within $2500 \mathrm{kms}^{-1}$, discussed by Faber \& Burstein (1988), in which the motions of galaxies are confused by irregular gravitational attractions induced by nearby visible matter. Also, the low values of $B_{g e}$ introduce noise and this may also be partially responsible for masking any weak signal. It is better to bin the data in $B_{g e}$ to help reduce this noise. The effects of avoiding the local anomaly region and binning in $B_{g e}$ with a bin size of ten units and plotting points at the bin centres is shown in Fig. 7.4

Still no significant correlation is revealed. One must conclude then that, on the basis of this evidence alone, the $D_{n}-\sigma_{v}$ relation is an effectively unbiased distance indicator relation as the simple bias model has led us to expect.

Next, consider the $L-\sigma_{v}$ relation. From the $k$-corrected and $(1+z)^{4}$ corrected values of $B_{T}$, absolute magnitudes were computed for each galaxy, again perturbing redshifts 


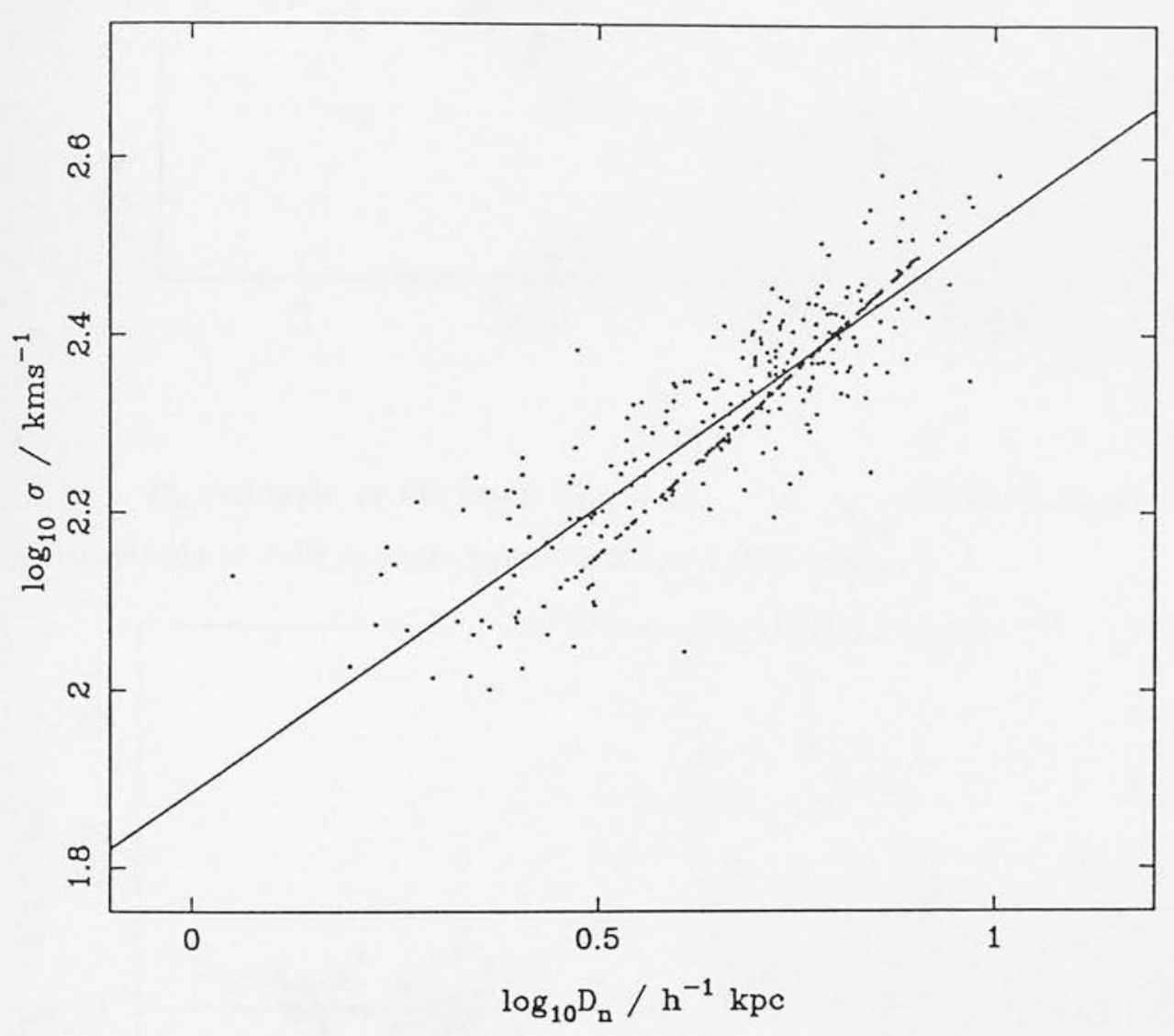

Fig. 7.2: The $D_{n}-\sigma_{v}$ relation for the G7 elliptical galaxies. $D_{n}$ represents the diameter within which the mean blue surface brightness of the galaxy is 20.75 mag.arcsec ${ }^{2}$. Redshifts have been perturbed with peculiar velocities appropriate to each galaxy. A least-mean squares fit to the data has been shown. 
for a measured peculiar velocity. The data are show in Fig. 7.5 fitted with a least-mean squares straight line.

The equation of this line is

$$
\log _{10} \sigma_{v}=0.316 \pm 0.007-(0.1 \pm 0.004) M_{B}
$$

As before, it is the offsets about this line that are of interest. Magnitude residuals (in the sense $\left.M_{\text {obs }}-M_{\text {pred }}\right)$ are plotted against $B_{g e}$ in Fig. 7.6.

Surprisingly, there is still no evidence for a correlation. The data were 'cleaned' in an identical manner to that described above for the $D_{n}-\sigma_{v}$ relation and the effect of doing this is illustrated in Fig. 7.7. Again, no signal is unmasked. A discussion of these results is reserved till Section 7.7 until after an opportunity has been taken to examine some further data.

\subsection{Spiral Bulges, BCM's and Radio Galaxies}

The results from the last Section appear to argue against any systematic, environmentdependent offsets in the distance indicator relations for elliptical galaxies. Any signal we might have expected to find would of course have been weak, especially for relatively nearby galaxies where offsets due to real, gravitationally-induced peculiar motions might be expected to dominate over any spurious motions driven by offsets in the distance indicators. In this Section we will focus our attention onto classes of astrophysical objects which occupy extreme environments. For example, S0 galaxies which are generally found in isolation or often well away from the centre of clusters. Also, radio galaxies and Brightest Cluster Members (BCM's) which typically inhabit some of the richest of environments in the universe.

\subsubsection{SPIRAL BULGES}

It is well-known that there is a zero-point shift in the Faber-Jackson relation for S0 bulges relative to elliptical galaxies (Whitmore \& Kirshner 1981, Dressler \& Sandage 1983). Originally it was thought that this effect may be related to the presence of bars or the inclination of these systems (Kormendy \& Mlingworth 1983). However, the $D_{n}-\sigma_{v}$ 


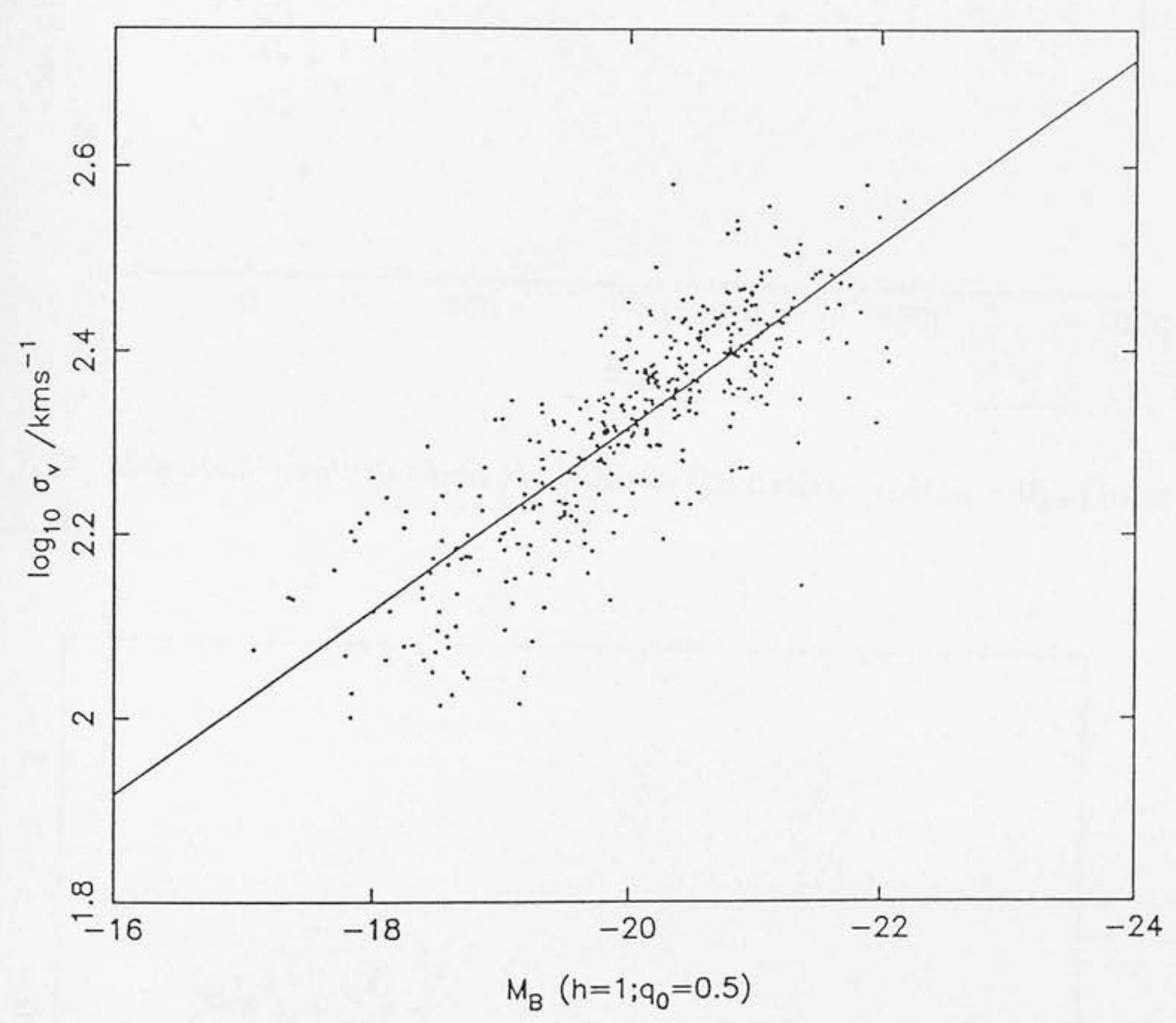

Fig. 7.5: Faber-Jackson relation for the G7 ellipticals. Absolute magnitudes have been determined from the tabulated values of cosmology-corrected apparent blue magnitudes with redshifts perturbed for the observed galaxy peculiar velocities. A least-mean squares fit to the data is shown. 


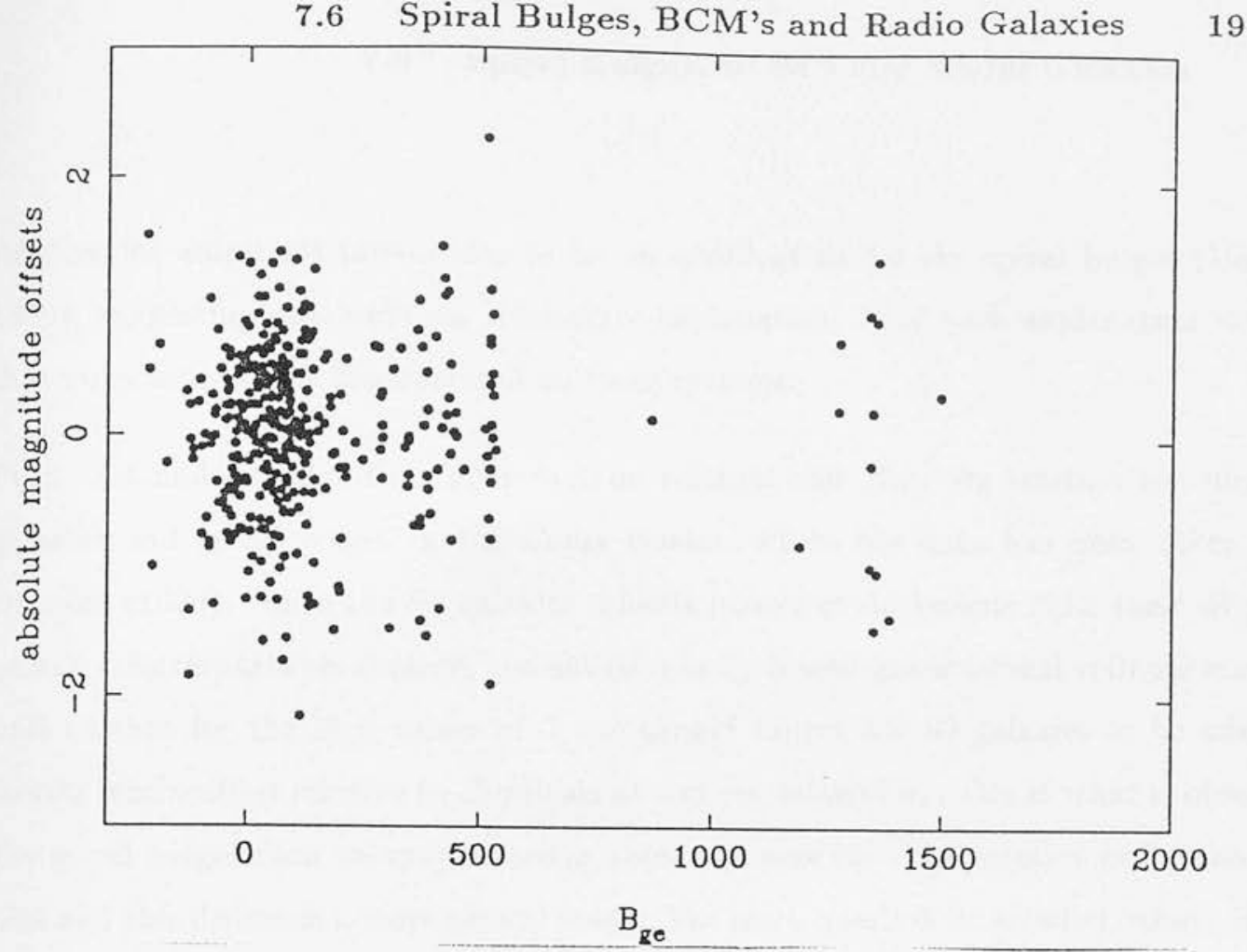

Fig. 7.6: Magnitude residuals about the Faber-Jackson relation $\left(M_{\mathrm{obs}}-M_{\text {pred }}\right)$ plotted against $B_{g e}$.

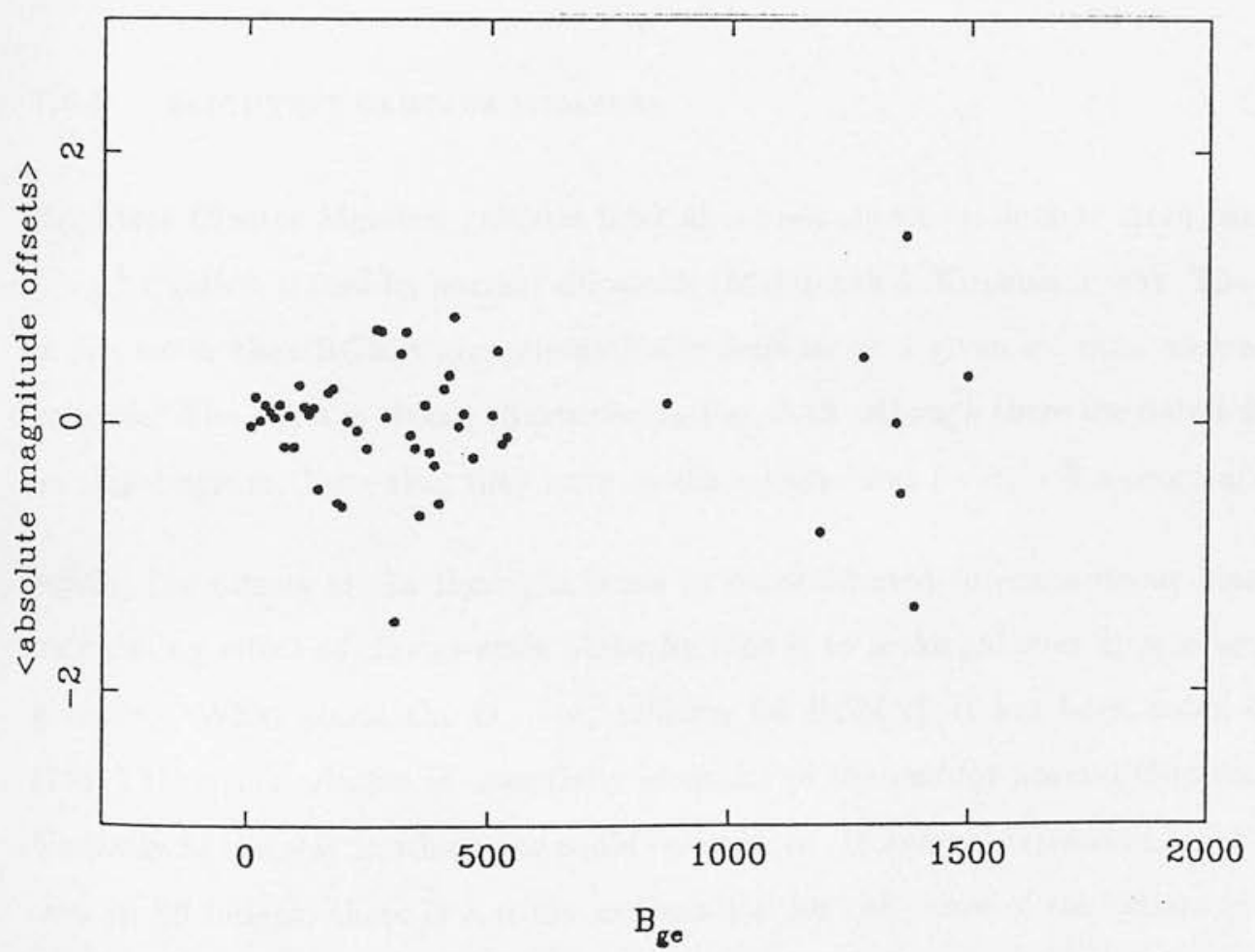

Fig. 7.7: As for Fig. 7.6 but with galaxies lying in the Local Anomaly removed and the data has been binned in $B_{g e}$. 
relation for ellipticals proves also to be an excellent fit for the spiral bulges (Dressler 1986), suggesting one seeks an alternative explanation. One such explanation may be that autonomous bias has operated on these systems.

Figs. 7.8 and 7.9 plot the Faber-Jackson relation and $D_{n}-\sigma_{v}$ relation for elliptical galaxies and spiral bulges in the Coma cluster, where the data has been taken from Dressler (1986). Since the S0 galaxies inhabit poorer environments than their elliptical galaxy counterparts on average, the autonomously biased gravitational collapse scenario tells us that for the high values of $\beta$ one should expect the S0 galaxies to be offset to fainter luminosities relative to ellipticals at a given value of $\sigma_{v}$ : this is what is observed. For spiral bulges then we may be seeing some evidence for the operation of autonomous bias and this demands a more careful study. The main question is: to what extent, if any, do the contrasting disk and elliptical galaxy internal dynamics dictate the position of the galaxies on the $L-\sigma_{v}$ plane? This issue needs to be considered in further detail before the offsets can be attributed to the operation of natural bias.

\subsubsection{BRIGHTEST CLUSTER MEMBERS}

Brightest Cluster Member galaxies have also been shown to deviate from the canonical $L-\sigma_{v}^{4}$ relation traced by normal ellipticals (Malumuth \& Kirshner 1985). The offsets are in the sense that BCM's are sytematically brighter at a given $\sigma_{v}$ than normal elliptical galaxies. The effect is clearly illustrated in Fig. 7.10 although there are only a few objects on this diagram. Note that they have an almost identical $I-\sigma_{v}-R$ to normal ellipticals.

Again, the offsets are in the right sense to be attributed to autonomous bias since the modulating effect of cluster-scale perturbations is to make galaxies appear brighter at a given $\sigma_{v}$. What about the $D_{n}-\sigma_{v}$ relation for BCM's? It has been noted by Hoessel (1987) that this relation is essentially identical to the one for normal elliptical galaxies. Similarly to the way in which one could conceive of alternative explanations for the offsets seen in S0 bulges, there is a ready explanation for the cause of the offsets in the BCM $L-\sigma_{v}$ relation. It may be that we are simply seeing the evidence for a past merging history for BCM's in which tidal accretion was probably important. The idea is that when one galaxy merges with another then the internal velocity of the system is unchanged but 


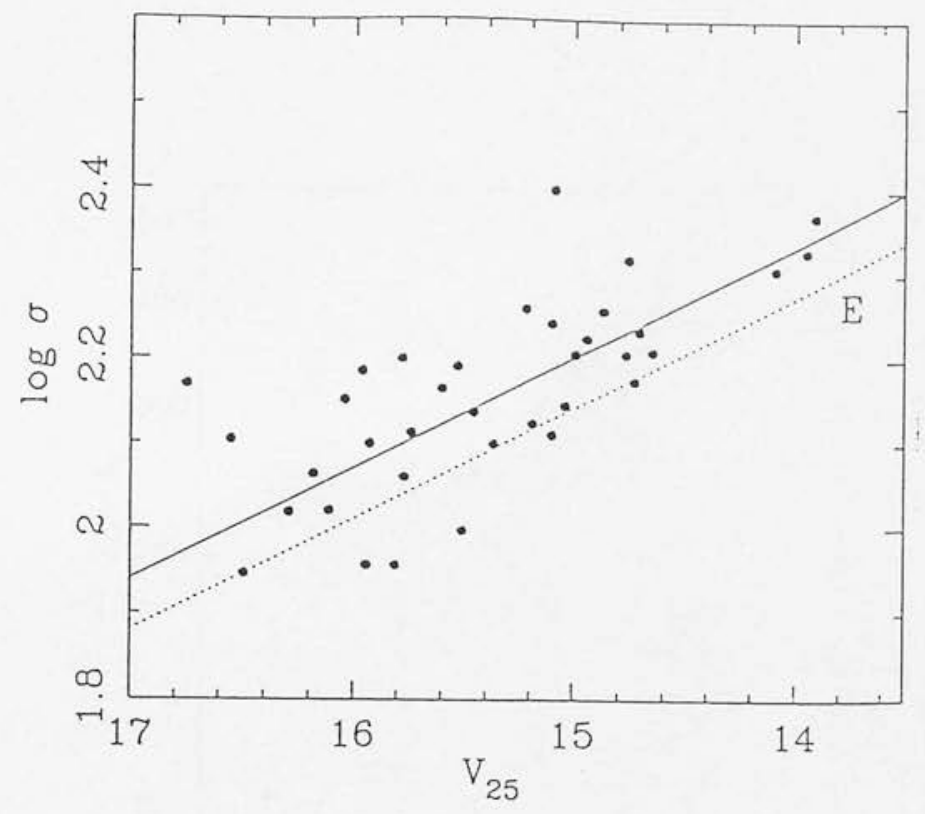

Fig. 7.8: Faber-Jackson relation for S0 galaxies in Coma. The dashed curve represents the relation for a sample of elliptical galaxies in the cluster.

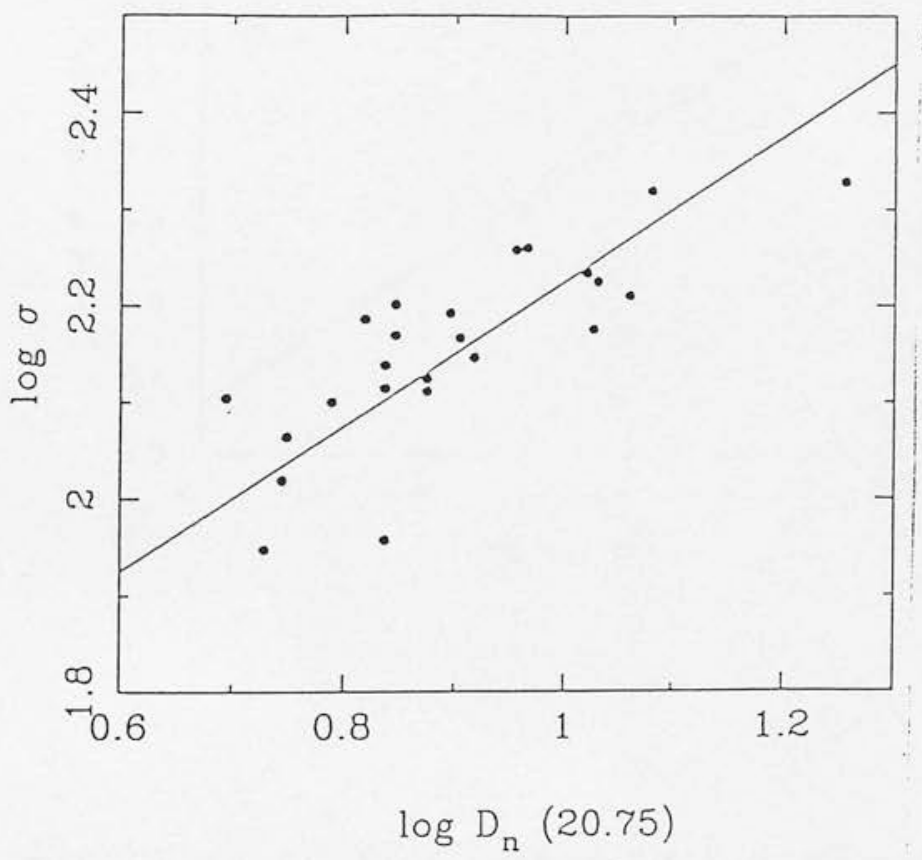

Fig. 7.9: $D_{n}-\sigma_{v}$ relation for spiral bulge galaxies in Coma (data taken from Dressler 1986). The line marked is the least-mean squares fit to the $D_{n}-\sigma_{v}$ relation for a sample of elliptical galaxies in Coma. 

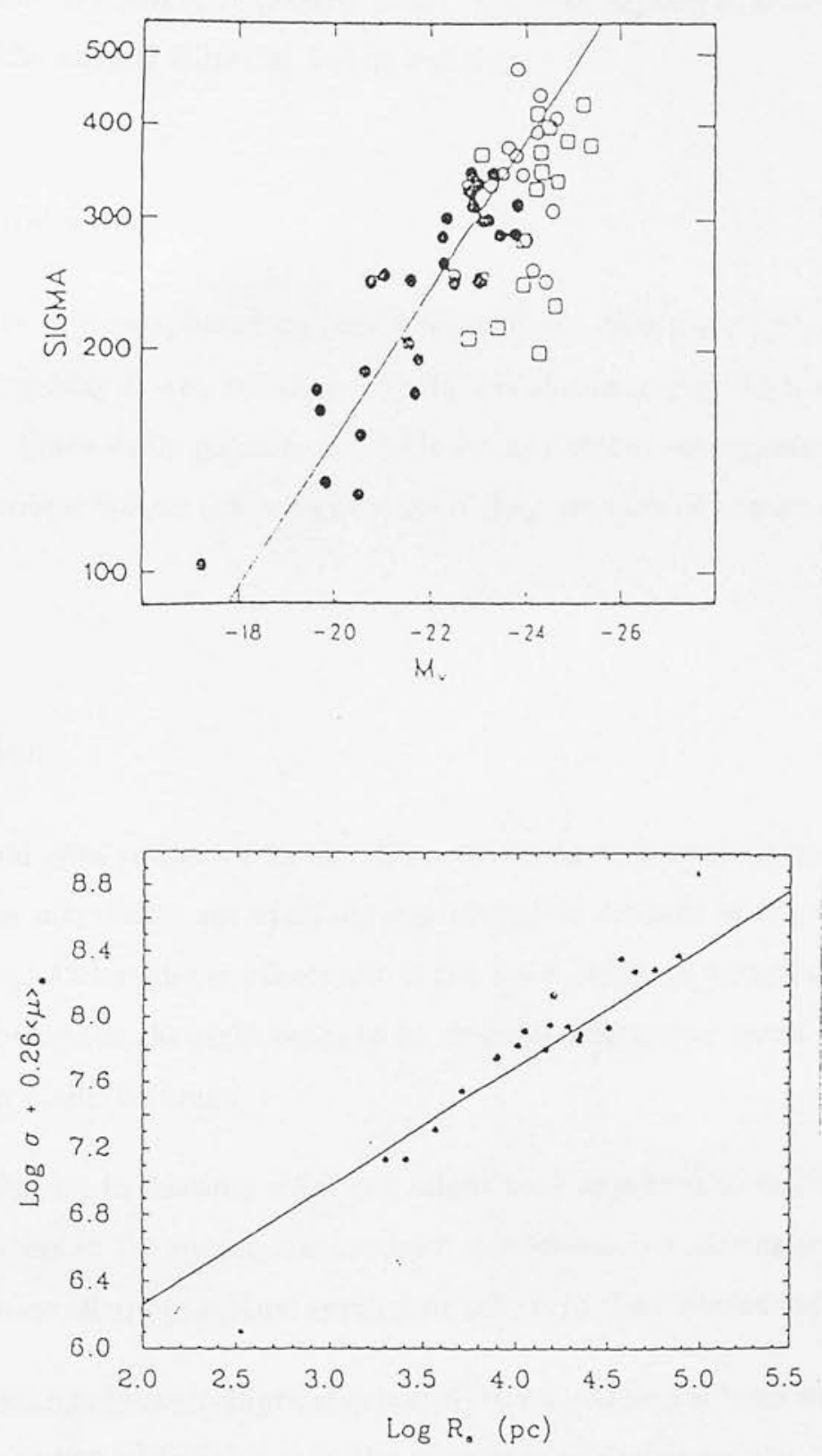

Fig. 7.10: Faber-Jackson relation for Brightest Cluster Member galaxies (unfilled circles) compared to the canonical relation for normal elliptical galaxies data taken from Malumuth \& Kirshner 1985). They have an $I-\sigma_{v}-R$ relation which is almost identical to that for normal ellipticals however. 
there is an increase in luminosity (Merrit 1984). This can explain the observed deviations of BCM's from the normal elliptical $L-\sigma_{v}$ relation.

\subsubsection{RADIO GALAXIES}

There is tentative evidence, based on only a few objects, that radio galaxies also deviate from the normal galaxy $L-\sigma_{v}$ relation. The data is shown in Fig. 7.11, reproduced from Samson (1987). Since radio galaxies typically occupy richer environments than normal ellipticals, these observations again make sense if they are viewed in terms of autonomous bias.

\subsection{Discussion}

The observational data presented in this Chapter seems to favour distance indicator relations which are manifestly not spatially modulated as a result of the operation of autonomous biasing. Although the offsets about the $L-\sigma_{v}$ relation for spiral bulges, BCM's and radio galaxies are in the right sense to be explained by autonomous bias, alternative explanations can easily be found.

Before moving on to consider what one might have expected to find for a reasonable choice of parameters in the simple bias model it is worthwhile collating some of the other observations. These all argue against systematic offsets in the distance indicator relations.

Firstly, the Gang of Seven elliptical galaxy data was also considered by Djorgovski, de Carvalho \& Han (1988) although not in the context of autonomous bias. Their approach was to examine the six most populous clusters in the sample (these are noted in the Appendix D) for correlations between their $D_{n}-\sigma_{v}$ slope and various parent cluster properties such as richness and velocity dispersion. The data are listed in Table 7.1. and slopes have been plotted against cluster richness class in Fig. 7.12. Note that a 'universal' slope of 1.2 was adopted by G7 to derive peculiar velocities.

Although six clusters present only poor statistics, there is evidence for a dependency of slope on richness. Djorgovski, de Carvalho \& Han derive a Spearman Rank Correlation 


\section{radio galaxies}

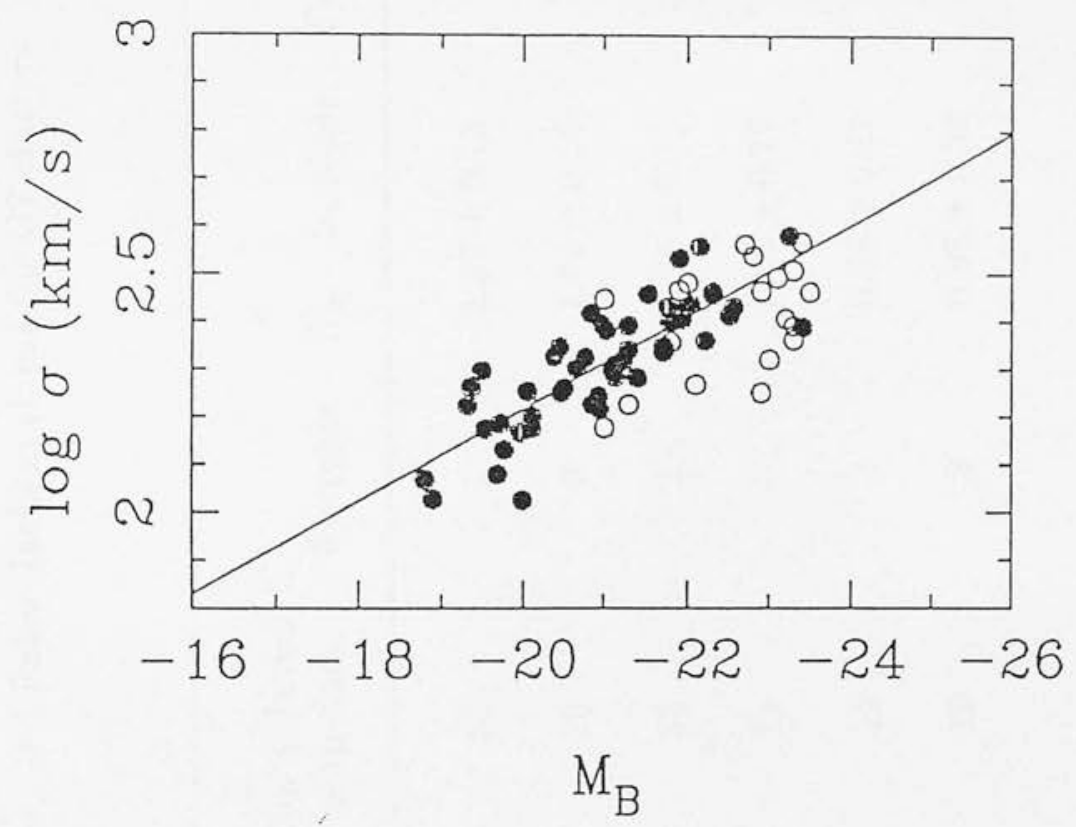

Fig. 7.11: Faber-Jackson relation for radio elliptical galaxies (unfilled symbols) compared to that for normal ellipticals (data taken from Sansom 1987). 

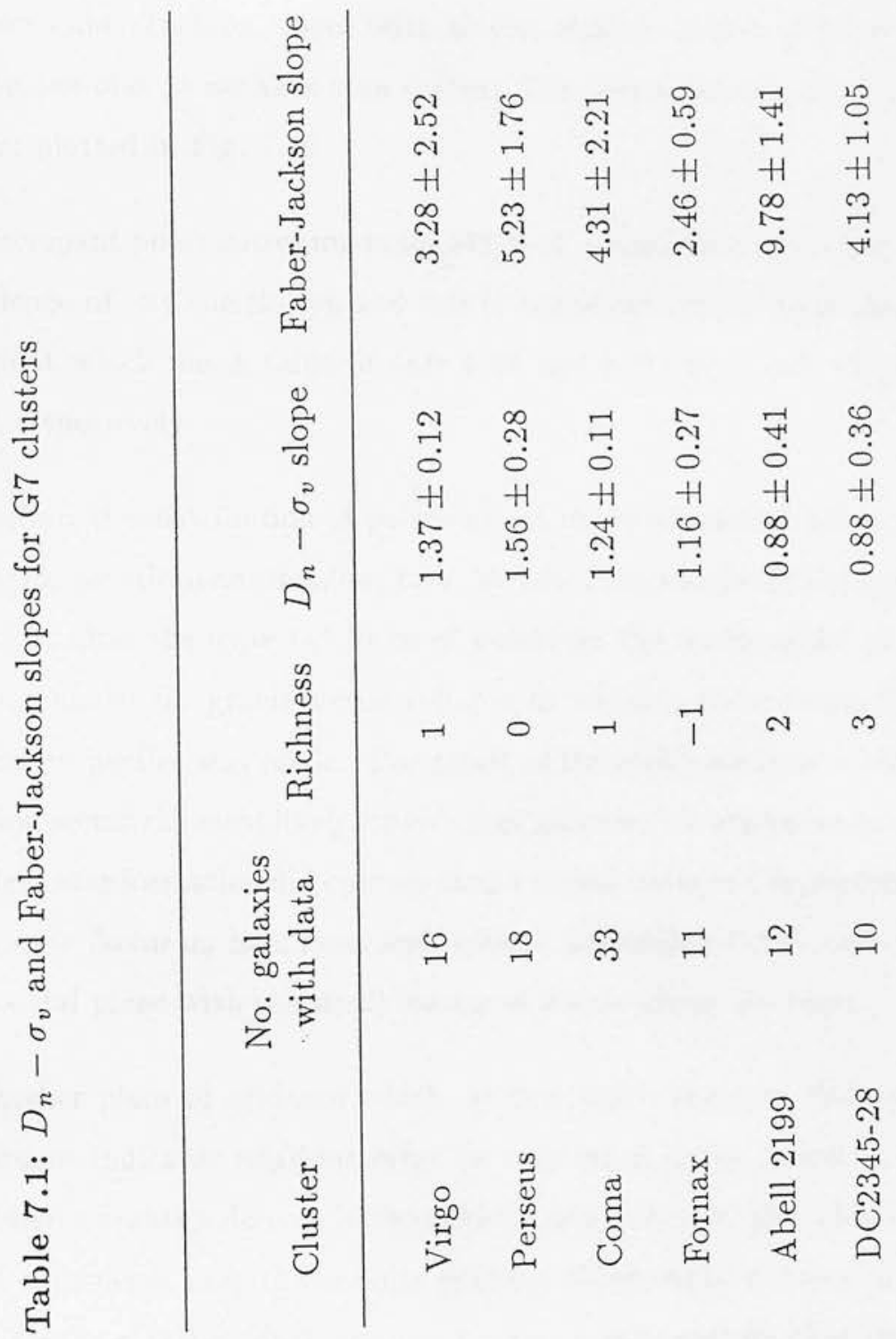
Coefficient of -0.62 . Furthermore, one can divide the galaxies in each cluster into inner and outer sub-sample and look for correlations between the difference in slopes and intercepts with the parent cluster properties. The systematic trend with richness is stronger for galaxies in the cluster cores, where the interactions dominate, and is weak or absent in the cluster envelopes. Djorgovski, Carvalho \& Han did not, however, consider the variation Faber-Jackson slopes with cluster richness which is more relevant for testing autonomous bias as we have seen earlier. The slopes are tabulated in Table 7.1 and the data are plotted in Fig. 7.13

The discrepant point corresponds to $A 2199$ at a distance of $9150 \mathrm{kms}^{-1}$. There is clearly no evidence of any correlation and this is borne out by the Spearman Rank Correlation Coefficient which has a value of only 0.38 and 0.21 , with and without the inclusion of A2199, respectively.

Earlier, the distribution of points about the fundamental plane was mentioned as a possible diagnostic of autonomous bias. In fact, Peacock (1990) has suggested that natural bias might alter the expected locus of points on the fundamental plane. He considered a general model for gravitational collapse in which a clear distinction between baryon and halo properties was made. The result of Peacock's analysis is that an unbiased star formation seems the most likely for elliptical galaxies, his argument being that if an epochdependent star formation did operate then a rather contrived dependence of baryonic mass and collapse factor on halo mass and redshift are needed to reconcile the equation of the fundamental plane with the small scatter of points about the plane.

Another piece of evidence which, at first sight, suggests that systematic offsets in the distance indicator relations must be very small is the following. The Coma/Virgo cluster distance ratios derived independently using $D_{n}-\sigma_{v}$ and Tully-Fisher relations are in good agreement, despite the quite extreme difference in richness of these two systems. In fact this is a rather weak argument since it may well be that the two relations are based on a very similar physics, perhaps with an identical dependency on environment. Burstein (personal communication) has also examined the spiral galaxy database compiled by Aaronson et al. (1982) to look for systematic trends in their derived peculiar motions with $21 \mathrm{~cm}$ HI deficiency. This latter may be thought of as a diagnostic of local environment since it is known to be correlated with distance from the centre of a cluster (Kraan- 


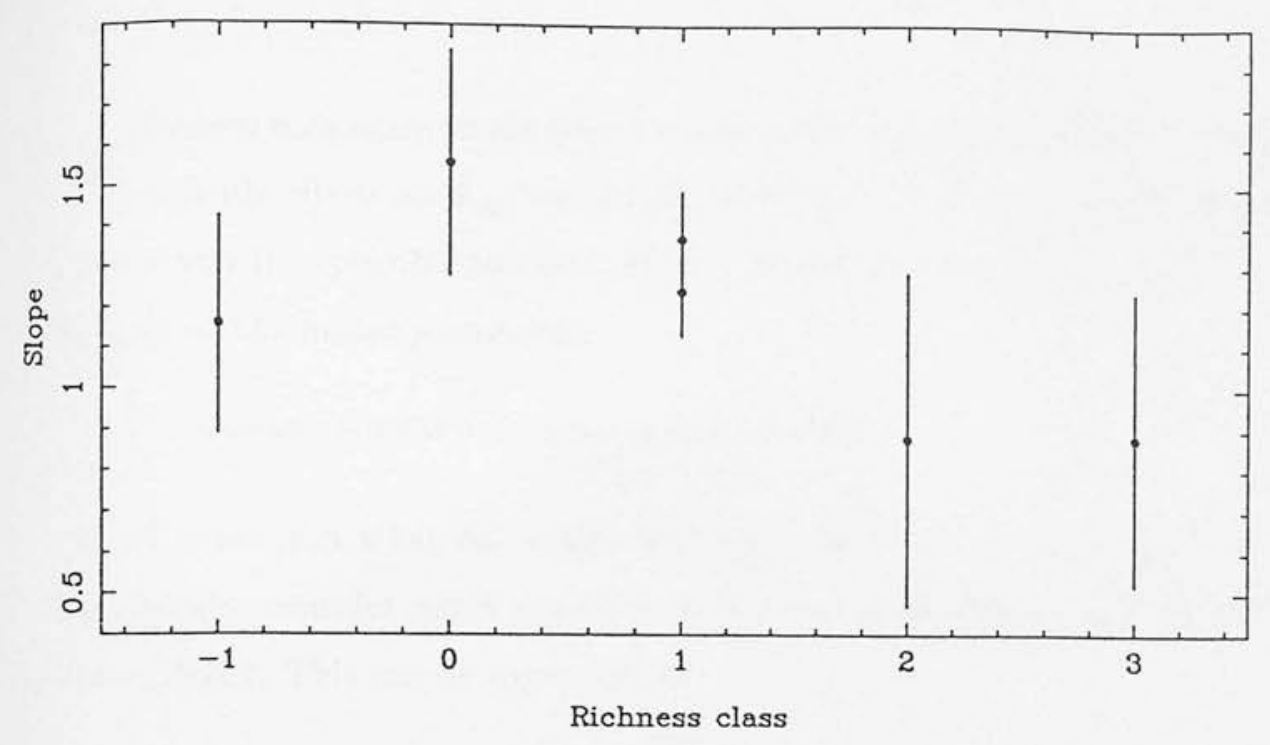

Fig. 7.12: $D_{n}-\sigma_{v}$ slopes for the six most populous clusters in the G7 sample plotted against their Abell richness class.

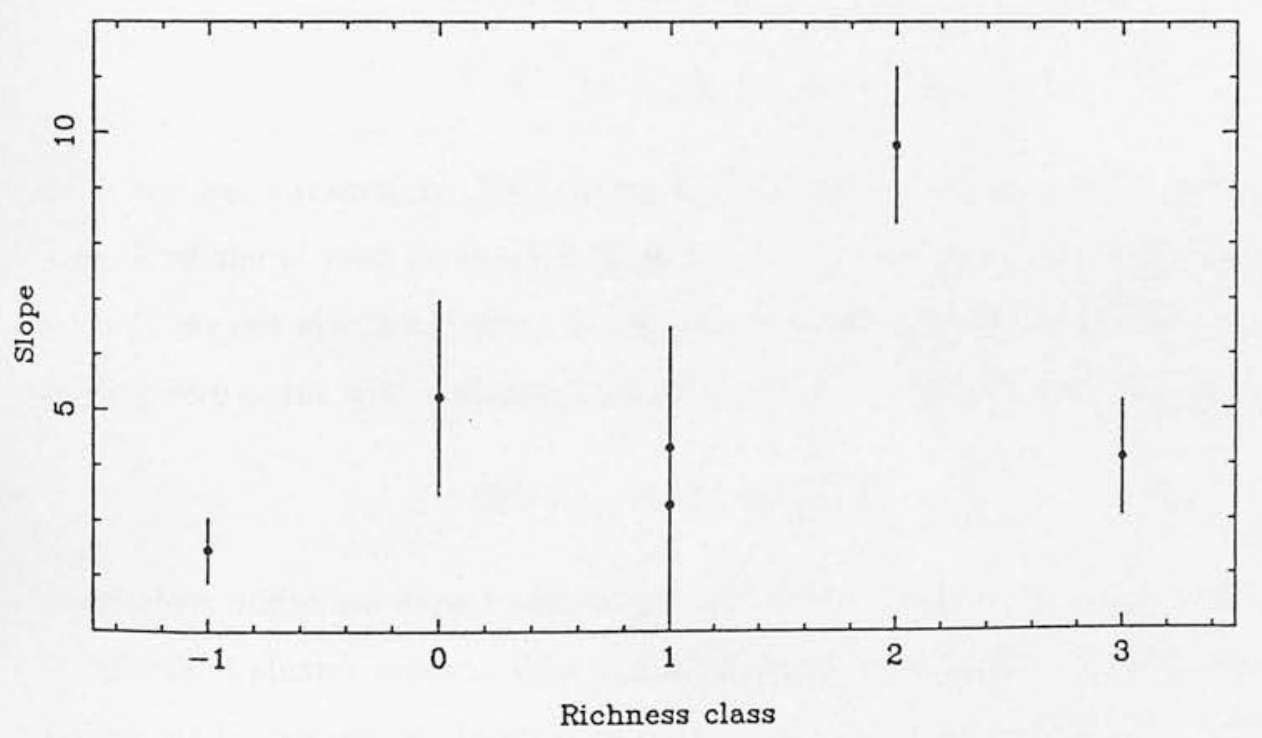

Fig. 7.13: Faber-Jackson slopes for the six most populous clusters in the G7 sample plotted against their Abell richness class. The most discrepant point is for the cluster A2199. 
We now turn again to the simple model and examine more clearly how the dependency of systematic offsets on $B_{g g}$, the amplitude of the spatial cross correlation function, arise. In this way it is possible to check what limits the observations presented in this Chapter impose on the model parameters.

\section{7 .1}

\section{CONSTRAINING THE SIMPLE BIAS MODEL}

To get some idea what one might have expected to detect for reasonable values of the parameters, consider again the effect of a long-wavelength density perturbation upon a proto-object. This can be expressed as

$$
L / L^{\prime}=\left(1+\frac{\Delta_{C}}{\Delta_{G}}\right)^{\beta}
$$

whereby the long-wavelength perturbation $\Delta_{C}$ has been added to the initial Gaussian random field. It was remarked earlier that the primary effect is to enhance or retard the collapse time of objects and hence modify their luminosity according to the above equation. If we now invoke the requirement that the objects satisfy a luminosity - velocity dispersion relation $L \propto \sigma_{v}^{\gamma}$, then this produces the following constraint

$$
\alpha-(n+3) \beta / 6=\gamma(1-n) / 12
$$

upon the free parameters. Demanding that $\alpha$ satisfies equation (7.9) and that $\beta=\gamma / 2$, then all of the objects lie exactly on on the $L-\sigma_{v}$ relation. It is clear that models with $\beta \neq \gamma / 2$ do not give a universal $L-\sigma_{v}$ relation, but one which exhibits a systematically varying zero-point with correlates with richness of environment according to

$$
(\Delta \ln L)_{\sigma_{v}}=(\beta-\gamma / 2) \ln \left(1+\frac{\Delta_{c}}{\Delta_{g}}\right)
$$

What then might we expect the magnitude of these offsets to be in view of recent observations of cluster mass-to-light ratios? Consider the earlier expression for $B$, the enhancement in the specific luminosity density of a virialised system

$$
B=\left(1+\frac{\Delta_{c}}{\Delta_{g}}\right)^{\frac{6 \beta}{6 \alpha-(n+3) \beta}}
$$

Substituting from equation (7.9) yields

$$
B=\left(1+\frac{\Delta_{c}}{\Delta_{g}}\right)^{\frac{12 \beta}{\gamma(1-n)}}
$$


Substituting from equation (7.9) yields

$$
B=\left(1+\frac{\Delta_{c}}{\Delta_{g}}\right)^{\frac{12 \beta}{\gamma(1-n)}}
$$

For an assumed $\gamma=4$ and $n=-2$ one thus finds that

$$
\begin{gathered}
1+\frac{\Delta_{c}}{\Delta_{g}}=3.2 \quad(B=10, \beta=2) \\
1+\frac{\Delta_{c}}{\Delta_{g}}=1.3 \quad(B=5, \beta=4)
\end{gathered}
$$

It is therefore evident that for reasonable values of the parameters, $\ln \left(1+\Delta_{c} / \Delta_{g}\right)$ should be of the order of unity and one would expect to see large offsets in $\Delta L / L$ for $\beta \neq \gamma / 2$.

To quantify the effect further it is again possible to appeal to observations and get estimates for $\Delta_{c}$ and $\Delta_{g}$. Since overdensities grow inversely as $(1+z)^{-1}$ and freeze out at $\Delta \sim 1$ one can write

$$
\frac{\Delta_{c}}{\Delta_{g}}=\frac{1+z_{f}(c)}{1+z_{f}(g)}
$$

where $z_{f}(c)$ and $z_{f}(g)$ are the formation redshifts for clusters and galaxies respectively. For clusters, the observed quantity is a number excess of galaxies within some fiducial radius. An expression can be derived for this in terms of the amplitude of the spatial crosscorrelation function $B_{g g^{*}}$, which has been calculated in this thesis by counting galaxies within a $1 \mathrm{Mpc}$ radius of the source location. For the simple case in which the correlation function can be integrated over a sphere in three-dimensional space

$$
\Delta N=\int \rho B_{g g^{*}} r^{-1.77} d V
$$

which for a counting radius of $1 \mathrm{Mpc}$ reduces to

$$
\Delta N=\frac{4 \pi}{1.23} \rho B_{g g^{*}}
$$

Of course, by counting galaxies one is looking at the light which we have argued in this Chapter is probably not a faithful tracer of the underlying mass distribution. Thus the density contrast is given by

$$
\Delta \rho / \rho=\frac{4 \pi}{1.23} \frac{B_{g g^{*}}}{B}
$$


For rich clusters, e.g. Coma for which $B_{g g^{*}} \simeq 1000$, the formation redshift is quite recent. For $B=5$ one finds $z_{f}=1.3$ and for $B=10$ then $z_{f}=0.8$. The formation redshift for galaxies however can only be estimated from indirect means, making using of the observed values of $B$. Consider the ratio $\Delta_{c} / \Delta_{g}$. The numerator depends on the richness of the cluster whereas the denominator is assumed fixed. Thus let us suppose that

$$
\frac{\Delta_{c}}{\Delta_{g}} \propto B_{g g^{*}}^{1 / 3}
$$

Then from equation (7.12) we can write

$$
B=\left(1+x B_{g g^{*}}^{1 / 3}\right)^{\frac{12 \beta}{\gamma(1-n)}}
$$

For an assumed $\gamma=4$ and $n=-2$ one can calculate the following values of $x$ for the two preferred values of $B=5$ and $B=10$,

$$
\begin{aligned}
& x=0.22 \quad(B=10, \beta=2) \\
& x=0.03 \quad(B=5, \beta=4)
\end{aligned}
$$

Defining $B_{\text {crit }}$, a critical value for $B$, equation (7.10) can be written

$$
(\Delta \ln L)_{\sigma_{v}}=(\beta-\gamma / 2) \ln \left(1+\left(\frac{B_{g g^{*}}}{B_{\text {crit }}}\right)^{1 / 3}\right)
$$

where $B_{\text {crit }}$ can range roughly between $100-30000$. Clearly the observations of $M / L$ ratios of clusters does not provide a very sensitive constraint on the formation redshift of galaxies. The amplitude of the predicted offsets are shown plotted in Fig. 7.14 for a specific case of $\beta=4$, for the two extreme values for $B_{\text {crit }}$.

A number of remarks concerning equation (7.20) are pertinent. Firstly, note that large values of $\beta$ reduce the value of $x$. This has the effect of making the offsets in $\Delta L / L$ more similar whatever the value of $\beta$. Secondly, it is interesting to examine the behaviour of equation (7.20) in the limits of small and large values for $x$. For $x$ small one can write

$$
B-1 \simeq 1000^{1 / 3} x \frac{12 \beta}{\gamma(1-n)}
$$

and hence

$$
\Delta L / L \simeq x B_{g g^{*}}^{1 / 3}(\beta-\gamma / 2) \quad B_{g g^{*}}^{1 / 3}(\beta-\gamma / 2) \frac{\gamma(1-n)(B-1)}{120 \beta}
$$


$\beta=4$

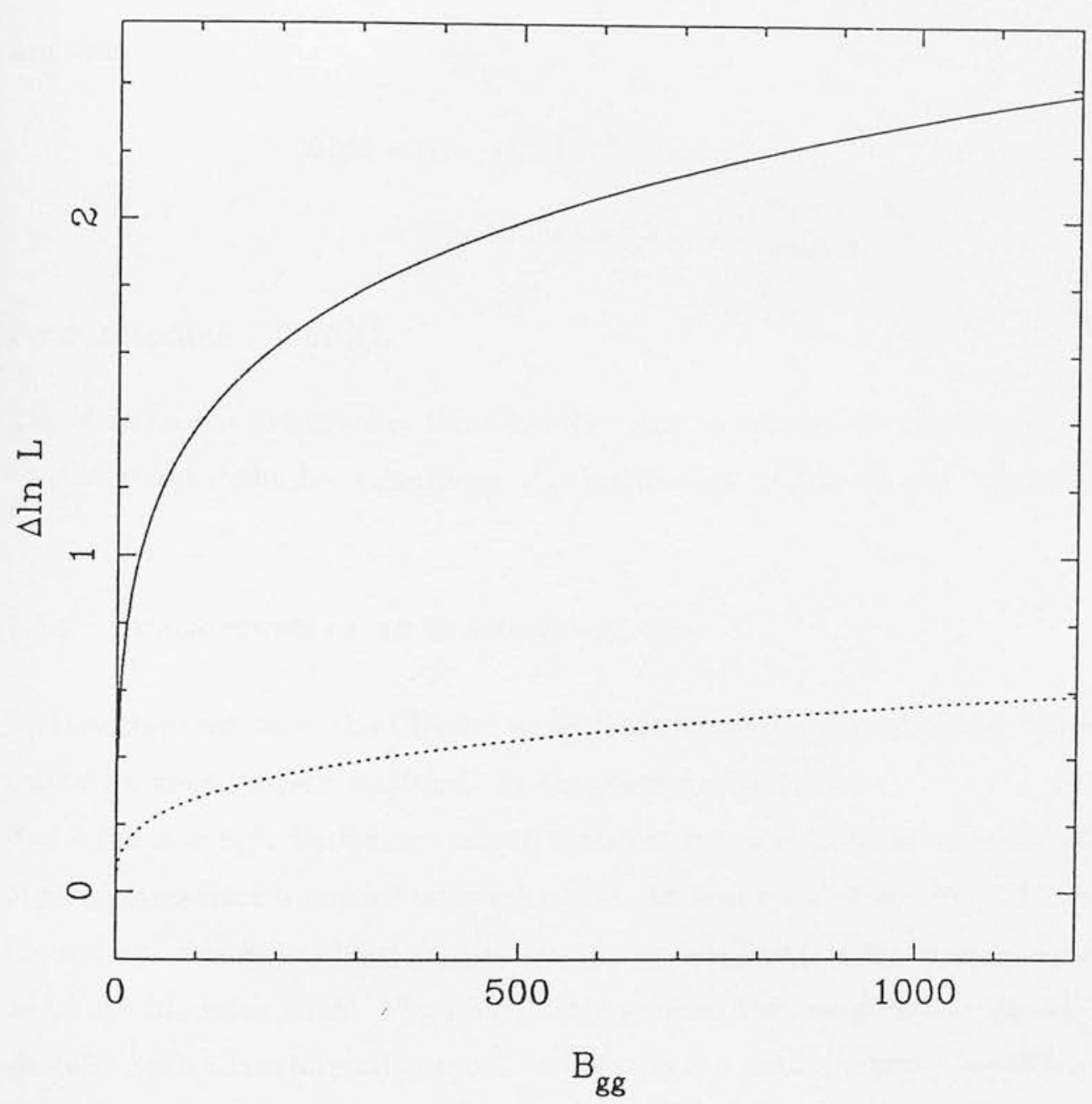

Fig. 7.14: Amplitude of offsets about the $L-\sigma_{v}$ relation of slope $\gamma=4$ as a function of $B_{g g^{*}}$ for the extreme values of $B_{\text {crit }}, 100$ and 30000 indicated by the bold and dashed lines respectively, and a value of $\beta=4$ 
For $\beta \gg 1$, one finds that $\Delta L / L \simeq 0.4 B_{g g^{*}}^{1 / 3}$

In the converse case of large $x$,

$$
B \simeq\left(x 1000^{1 / 3}\right)^{\frac{12 \beta}{\gamma(1-n)}}
$$

and thus

$$
\begin{aligned}
\Delta \ln L & =(\beta-\gamma / 2)\left(\ln B_{g g^{*}}^{1 / 3}+\ln x\right) \\
& =(\beta-\gamma / 2)\left(\ln B_{g g^{*}}^{1 / 3}+\ln \frac{B^{\gamma(1-n) / 12 \beta}}{1000^{1 / 3}}\right)
\end{aligned}
$$

For $\beta \gg 1, \Delta \ln L \simeq \beta \ln B_{g g^{*}}^{1 / 3}$.

The observations presented in this Chapter suggest a value of $\beta$ about equal to two, i.e. no scatter about the $L-\sigma_{v}$ relation. The implications of this are now briefly assessed.

\subsubsection{IMPLICATIONS OF NO EVIDENCE FOR BIAS}

The results presented in this Chapter imply that luminosity-internal velocity type distance indicators are effectively unbiased. In the context of autonomous bias this arises when $\beta=2$ and $\alpha=4 / 3$. Earlier, we argued that this appeared to be an unreasonable choice of parameters since it implied too weak a bias. An inspection of equation 7.12 shows that the ratio of cluster to object density contrasts needs to be of the order of unity to get an acceptable value for $B$. This implies that galaxies form only slightly less earlier than clusters. Such a late formation epoch for galaxies is a critical feature of CDM numerical simulations. Unfortunately though this picture is at odds with the one that emerges from the statistics of the initial perturbations. Here, the rms fluctuations on galactic and cluster scales respectively are discrepant by a factor 6 . This means that perturbations of similar amplitude to those that made clusters would form objects at $1+z \simeq 6$. Working fully through this with the Press-Schechter machinery yields the result that these objects have about the right abundance and velocity dispersions as ordinary bright galaxies, although the number of objects at any $\sigma_{v}$ remains constant to much later times. The implied bias of $\Delta_{C L} / \Delta_{O B J} \simeq 1 / 6$ is too small to reconcile the cluster mass-to-light ratios with closure density. If natural bias fails to work, what are the most physically realistic options for 
solving the cluster $M / L$ problem? The most promising candidate appears to be dynamical friction which may be efficient at producing a segregation between light and mass in the non-linear environment provided by the cluster and its subunits. This is discussed further by Hoffman et al. 1982, Barnes 1983, Evrard 1987 and especially West \& Richstone 1988.

\subsection{Summary}

A simple model for biased galaxy formation has been examined, in which the non-linear dark matter clumps are identified as sites for galaxy formation and a luminosity is assigned to these sites which depends upon some power of their collapse redshift. For reasonable choices of the parameters, this model predicts that objects lying along the $L-\sigma_{v}$ plane should be offset from the mean line and that the magnitudes of these offsets should correlate with their environment. The observations amassed in this Chapter argue against such offsets. It remains to be seen whether these can be found for a deeper sample where one has a better chance of seeing them because, if present, they would swamp any real peculiar velocities which would likely be very small on large scales. This might be a promising, albeit observationally very demanding, area for future research. 


\section{Chapter 8}

\section{Concluding Remarks}

This final Chapter summarises a few of the main results that have been obtained and suggests, what seem to the author, to be a few promising directions for future research.

To begin with let us recall the principal motivation for this thesis. It was basically that, at the time of its conception, most existing observations of large-scale clustering and streaming motions in the universe were in conflict with each other at some level. This unsatisfactory state-of affairs was probably almost entirely due to the complicating effects of systematic biases in the optically-selected samples of galaxies that had been used exclusively for probing the cosmic structure. It was therefore judged to be too premature just yet to reject theoretical models for galaxy formation, which observations of clustering and streaming can potentially discriminate between, simply on the basis of these observations alone.

The prospects for achieving a more consistent picture look good. Specifically, a reliable estimate of the cluster-cluster correlation function should be forthcoming within a year or two's time. In fact three independent measures seem likely. Two groups are presently working on a redshift survey of clusters which have been selected in an automated way by applying special cluster-finding algorithms to machine-based galaxy surveys (Collins, personal communication). In addition, the X-ray satellite, ROSAT, will conduct an unbiased all-sky search for clusters and this database will form an excellent basis for a statistical study of clustering.

In the intermittent time, it was argued that a sample of radio elliptical galaxies might be able to shed some light on the issue of clustering and streaming motions. An all-sky sample comprising effectively all the radio galaxies within a redshift of one-tenth was therefore compiled from the various radio surveys and this approximated a complete sample down to a flux-density limit of $0.5 \mathrm{Jy}$ at $1.4 \mathrm{GHz}$. A considerable observational 
effort was then made to acquire CCD frames for the sample and spectroscopic redshifts.

The main applications of the all-sky sample in this thesis were towards the large-scale structure of the universe. As a consequence, Chapter 4 was really only able to touch the surface of what can be potentially learned about radio galaxies from the observational database that had been assembled. In particular, one of the most intriguing questions that one could wish to ask about radio galaxies - namely, why they are active - was not addressed in any detail at all. This presents one of the key areas for future applications of the all-sky sample. It is likely that an answer to the above question may hinge around the importance of interactions and/or mergers during the evolution of radio galaxies and whether these can trigger non-thermal nuclear activity (Smith \& Heckman 1989). To answer the first of these questions, the CCD images of the radio galaxies need to be examined in greater detail than that described in Chapter 4. A study of the prevalence of isophote twisting or distortions and the presence of close companion galaxies are important in this context. The optical properties of the radio galaxies could then be compared with a similar set of properties for a control sample of radio-quiet ellipticals spanning an identical redshift-range to the radio galaxies. Perhaps the 'Gang of Seven' elliptical galaxy sample might have a rôle to play here.

The $I$ band photometry should also be complemented with photometry in $B$, for some of the reasons outlined in Chapter 3, but also to enable a study of colour gradients in radio ellipticals which were not examined in this thesis. Providing that the corrections for the blurring effects of seeing on the galaxy light profiles can be done carefully enough, then it would be interesting to check how the radio galaxy $B-I$ colour differentials behaved on moving inwards from the outer envelope of the galaxy towards the nucleus. This could yield further important clues as to the nature and predominance of non-thermal nuclear activity in radio galaxies.

A radio observational programme for the all-sky sample is also worth pursuing. This would help to clear up the radio morphologies of the sources and allow for better testing of correlations between radio structure with source environment and the location of FRI and FRII radio galaxies on the $M-\alpha$ diagram than the fairly rough guides that were given in Chapter 4. 
The central result of this thesis was presented in Chapter 5. Here it was demonstrated for the first time that radio galaxies are clustered and, moreover, that the strength of their clustering exceeds that for normal galaxies by about a factor of six. It had already been reported in Chapter 4 that radio galaxies inhabit, on average, clusters of average Abell richness $R=0$. Radio galaxies are therefore an unbiased pointer to rich systems in the universe. In addition to their typically rich environments, radio galaxies ar also generally very bright. In this respect, our clustering results give some independent support in favour of luminosity-segregation in optically luminous galaxies. The results presented in Chapter 5 further underlined the unsuitability of optically-selected cluster catalogues, notably the Abell catalogue, for studies of large-scale clustering in the universe. One of the most extreme claims for the existence of very large-scale structure had been made by Tully. No evidence was found in the radio galaxy redshift data to support his claims - namely that rich clusters are compressed along the supergalactic equator and correlated structure exists on a scale of $0.1 c$. The conclusion seems to be one that has already been suspected by other groups: namely that there are severe projection effect in the Abell catalogue and these can conspire to give a spurious, strong clustering signal.

The analysis presented in Chapter 5 to test the Tully effect was only simple. Montecarlo datasets were constructed and histograms for the number of galaxies within fixed distance bins above and below the supergalactic plane were compared with a similar set of histograms for the real data. One can conceive of a much more precise way for putting limits on the existence of very large-scale density inhomogeneities in the universe. Such a test has received an added impetus recently following the reports by Broadhurst et al. (1990) of strong clustering of galaxies on scales $\geq 50 h^{-1} \mathrm{Mpc}$. The technique best-suited for this improved study of the radio galaxy redshift survey is power spectrum analysis (PSA) discussed originally in this context by Webster (1976). Preliminary results that have been obtained from applying of a slightly modified version of his PSA algorithm, suggest a variance in $\delta \rho / \rho$ of $\sigma^{2} \leq 0.3$ for wavelengths $100<\lambda<200 h^{-1} \mathrm{Mpc}$ (Peacock $\&$ Nicholson 1991). The 3-d distribution of the radio galaxies does therefore not support either of the claims of Tully or Broadhust et al.

The other exciting opportunity which beckoned at the outset of this work was to test the reality of the Rubin-Ford effect, i.e. the existence of $\sim 10 \%$ perturbations in 
the Hubble flow at $\langle z\rangle \simeq 0.03$. In fact the depth of the radio galaxy sample was such that searches for streaming flows could be pushed well beyond the Rubin-Ford scale in search of a transition point from noisy to smooth expansion of the universe. The initial excitement waned when it became clear that not enough galaxies had been observed, during the course of the thesis, in the southern hemisphere and that the sky distribution of the low-redshift sample was therefore severely biased. A clear target for future research should therefore be to acquire more CCD frames for southern hemisphere galaxies and consequently reduce the sky anisotropy of the sample. Given that the $\mu-r_{e}$ distance indicator was shown to yield distances accurate to about $28 \%$, there is then a realistic hope that interesting limits can begin to be put on the magnitude of deviations from Hubble flow. The key point regarding the $\mu-r_{e}$ relation for estimating peculiar velocities is that it yields an acceptable photometric distance accuracy yet, at the same time, $\mu$ and $r_{e}$ are two very simple quantities to measure. This contrasts with having to measure velocity dispersions which are required for the $D_{n}-\sigma_{v}$ relation.

It is still not clear whether streaming motions are dominated by the gravitational attraction of a massive "attractor". An interesting future prospect for the radio galaxy sample, once more observations have been added, is to check for a "backflow" of galaxies lying just beyond the position of the Great Attractor. This would provide conclusive evidence for the reality of such an intriguing structure. Although the Gang of Seven do have some data from behind the Great Attractor which lends support to its reality, this region is one of the worst affected by Malmquist bias. A sample which is effectively biasfree, such as the radio galaxy sample, should therefore be able to address this question more confidently.

In order to link some of the earlier work on measuring the environments of galaxies and their peculiar velocities, Chapter Seven examined the properties of elliptical galaxies for evidence of environment-dependent systematic offsets in the $D_{n}-\sigma_{v}$ and $L-\sigma_{v}$ distance indicator relations. According to a simple model for autonomously biased gravitational collapse, these should be present for the Faber-Jackson relation at a level which is proportional to the rate of star-forming efficiency for these systems. No offsets were found. Only for spiral bulges, Brightest Cluster Members and radio galaxies were offsets seen to be present. These were in the right sense for them to be attributed to autonomous bias 
although alternative explanations can be found which are a lot more likely. The problem with a sample of nearby galaxies for checking bias models is that real peculiar velocities are expected to be large. For a deeper sample it is likely that they would be swamped by any systematic offsets that are present in the data and hence the latter could be much more readily detected. This is easier said than done of course. For distance samples of galaxies the problems associated with measuring accurate photometric differences are considerable. A further study of biasing models and a closer inspection of other existing data is therefore desirable before embarking on such a project.

In conclusion, then, this thesis has demonstrated that radio galaxies are excellent candidates for statistically probing the large-scale structure of the universe. As a result of the work undertake here, the largest optical database for a nearby sample of radio galaxies that has ever been published is available. This should pave the way for both an extension of the work described in this thesis and for equally exciting new projects, such as trying to determine an answer to the question - just why are radio galaxies active? 


\section{Appendix A}

\section{The All-Sky Sample Database}

The all-sky sample is presented in this Appendix in tabular form. There are four tables representing (i) radio galaxies which are known to lie within the final sample; (ii) radio galaxies which are more likely than not to lie in the final sample on the basis of their $B$ magnitudes; (iii) radio galaxies which may lie in the final sample but are unlikely to do so on the basis of their $B$ magnitudes; (iv) radio galaxies which definitely do not lie in the final sample.

A key to the information contained in each of the columns of the table is given below

Column 1: $\quad$ IAU name of galaxy;

Column 2: Right ascension of optical component of radio source;

Column 3: Declination of optical component;

Column 4: $\quad$ Inaccuracy in optical position in arcseconds;

Column 5: $\quad 1.4 \mathrm{GHz}$ radio flux-density in Jansky;

Column 6: $\quad$ Estimated $B$ magnitude;

Column 7: $\quad$ Heliocentric radial velocity in $\mathrm{kms}^{-1}$;

Column 8: $\quad$ Error in radial velocity;

Column 9: $\quad B \& I$ CCD frame indicator;

Column 10: $\quad$ Numbered reference to optical position;

Column 11: Numbered reference to magnitude;

Column 12: Numbered reference to redshift.

A few comments are relevant. Firstly, it was not possible to track down each numbered reference for all of the objects. Thus there are some blanks or zeroes in column's 10, 11 
$\&$ 12. Secondly, for a few objects a radio source position only could be found. These have still been entered into the table and can be distinguished by the large error boxes $\left(\geq 10^{\prime \prime}\right)$ in column 4.

The * flags in column 9 indicate whether CCD frames have been acquired for the object. A single * means that only an $I$ frame has been recorded. A double * means that $B$ \& $I$ frames exist. Note that a $*$ sometimes precedes the IAU name in of the object in column 1. Absence of $\mathrm{a} *$ indicates that either the published redshift of the object has too large an error $\left(\geq 600 \mathrm{kms}^{-1}\right.$ to be of any use for peculiar velocity studies or else that a spectrum has been recorded during the course of this thesis, but of too poor a signal-to-noise to yield a redshift.

At the bottom of each table some information is given about the status of observations, namely the percentage of objects with spectroscopic redshifts and $B \& I$ CCD frames. 
Table A1.1: Definite Sample Members

\begin{tabular}{|c|c|c|c|c|c|c|c|c|c|c|c|}
\hline & $\alpha_{o p t}(1950)$ & $\delta_{o p t}(1950)$ & $\pm^{\prime \prime}$ & $S_{1.4}$ & $m$ & $c z$ & \pm & $\mathrm{ccd}$ & & Refs & \\
\hline $000-550$ & $\begin{array}{llll}00 & 00 & 37.64\end{array}$ & $\begin{array}{l}-550138.0 \\
0.0\end{array}$ & 1.8 & 1.20 & 14.0 & 9683 & 150 & * & 190 & 1 & \\
\hline $001-531$ & 000141.36 & -531140.7 & 1.8 & 0.88 & 14.5 & 9803 & 120 & $*$ & 190 & 2 & \\
\hline $07-325$ & $\begin{array}{llll}00 & 07 & 03.20\end{array}$ & -323319.7 & 1.2 & 0.81 & 13.5 & 7687 & 18 & * & 0 & 6 & \\
\hline $23-333$ & $\begin{array}{lll}00 & 23 & 02.25\end{array}$ & -331922.2 & 0.5 & 1.23 & 16.0 & 15439 & 150 & $*$ & 19 & 20 & \\
\hline $2+101$ & 003220.40 & 101123.0 & 2.0 & 0.95 & 15.5 & 17100 & 0 & ** & 23 & 4 & \\
\hline $34-014$ & 003430.59 & -012538.0 & 0.7 & 4.40 & 18.0 & 21885 & 60 & ** & 25 & 26 & \\
\hline $36+030$ & 003644.15 & $\begin{array}{lll}03 & 0322.0\end{array}$ & 1.1 & 2.05 & 13.5 & 4197 & 90 & $* *$ & 0 & 29 & \\
\hline $38-210$ & $0038 \quad 18.90$ & $\begin{array}{llll}-21 & 00 & 06.0\end{array}$ & 0.1 & 0.76 & 17.0 & 26981 & 150 & ** & 32 & 32 & \\
\hline $43-638$ & 004357.64 & -634942.3 & 1.8 & 1.05 & 16.0 & 22215 & 420 & * & 190 & 17 & \\
\hline 43 & 55.98 & -422413.6 & 0.5 & 8.29 & 17.0 & 22904 & 300 & * & 19 & 20 & \\
\hline $53-016$ & 005329.31 & $\begin{array}{lll}-0136 & 16.9\end{array}$ & 0.6 & 1.25 & 16.4 & 13071 & 750 & ** & 37 & 51 & \\
\hline 53 & 005352.08 & -013157.1 & 0.6 & 1.49 & 16.7 & 11542 & 240 & $* *$ & 37 & 37 & \\
\hline 6 & $\begin{array}{l}00 \\
55 \quad 01.57\end{array}$ & -013939.4 & 0.3 & 6.09 & 15.6 & 13311 & 0 & ** & & & \\
\hline & 005505.60 & 300456.8 & 2.0 & 0.78 & 13.2 & 5216 & 150 & $* *$ & 40 & 40 & \\
\hline $5+265$ & 005540.70 & 263544.0 & 2.0 & 1.34 & 14.9 & 14360 & 150 & ** & 40 & 40 & \\
\hline $57-180$ & 005742.00 & -180354.0 & 12.0 & 1.20 & 18.0 & 29979 & 300 & ** & 41 & 41 & \\
\hline & 00 & -50 & 1.8 & 1.03 & 17.0 & 18 & 270 & 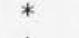 & 190 & 17 & \\
\hline $10-2>$ & 010157.94 & $\begin{array}{llll}-80 & 12 & 09.7\end{array}$ & 1.8 & 0.64 & 17.0 & 169 & 300 & * & 190 & 44 & 70 \\
\hline & 010439.20 & 320846.0 & 2.0 & 2.99 & 14.5 & 4875 & 60 & $* *$ & 46 & 46 & \\
\hline 130 & $0106 \quad 13.80$ & 130344.0 & $? ? . ?$ & 11.33 & 15.6 & 17538 & 60 & ** & 0 & 0 & \\
\hline 142 & 01 & $-1413 \quad 35.5$ & 1.6 & 1.49 & 14.7 & 15499 & 180 & ** & 0 & 41 & 22 \\
\hline 11 & 011020.36 & $15 \quad 13 \quad 35.2$ & 0.9 & 1.05 & 14.0 & 133 & 210 & ** & 0 & 53 & DJ \\
\hline 111 & 011108.80 & 020624.0 & & 1.07 & 16.3 & 139 & 1500 & ** & 51 & 51 & 24 \\
\hline$[7$ & 011551.54 & -260745.0 & 2.1 & 0.96 & 16.7 & 15529 & 120 & * & 0 & 4 & \\
\hline $16-19$ & 0116 & -19 & 0.5 & 1.13 & 18.0 & 10763 & 300 & ** & 32 & 32 & \\
\hline & 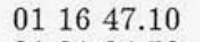 & 3 & 2.0 & 1. & 14 & 17 & 6 & ** & 40 & 40 & 5 \\
\hline & 01 & 4 & 0.5 & 0.59 & 16.8 & 98 & 120 & ** & 50 & 50 & \\
\hline & 012 & -01 & & 3.67 & 13.0 & 53 & 30 & ** & 59 & 59 & 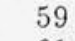 \\
\hline & 01241 & 18 & 0.7 & 1.69 & 15.5 & 12951 & 120 & ${ }^{* *}$ & 60 & 62 & 01 \\
\hline 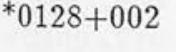 & 012 & 00 & $?$ & 0.59 & 16.1 & 23923 & 90 & ** & 0 & 51 & \\
\hline & 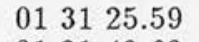 & 44 & & 1.84 & 17 & & 18 & & 64 & 64 & \\
\hline & 0 & 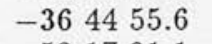 & & & 15 & & 27 & ** & & & 02 \\
\hline & 014 & -52 & & 0.73 & 17.5 & 292 & 240 & * & 190 & 13 & \\
\hline & 014 & 00 & 0 & 0.71 & 16.5 & 27551 & 210 & $* *$ & 66 & 66 & \\
\hline 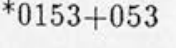 & 015344.31 & $\begin{array}{lll}05 & 23 & 05.4\end{array}$ & $\cdot 7$ & 0.85 & 13.2 & 5564 & 51 & ** & 68 & 68 & \\
\hline & & $a^{2}+2$ & & & 17 & & 18 & * & 50 & 50 & \\
\hline & 0 & 3 & & 1. & 14 & & 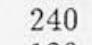 & * & 40 & 40 & 40 \\
\hline & & & & 1 & 17 & & 12 & ${ }^{* *}$ & 55 & 0 & \\
\hline & 02 & -06 & .8 & 0.61 & 15.0 & 1 & 150 & $* *$ & 0 & 35 & \\
\hline & 021 & -48 & 1.0 & 2.25 & 14.0 & 19187 & 180 & ** & 75 & 75 & \\
\hline & & & & & 15 & 12 & 15 & $*$ & 0 & 4 & \\
\hline & & & 0 & 0. & 15 & & ) & 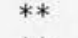 & 58 & 78 & \\
\hline & 0 & & & 0 . & 16 & & 21 & $* *$ & 0 & 54 & \\
\hline & & -20 & 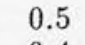 & 1.2 & 16 & 26981 & -1 & ${ }^{* *}$ & 55 & 4 & \\
\hline 年 & 023825.85 & 083129.0 & 0.4 & 1.18 & 14.8 & 6416 & 120 & ** & 68 & 68 & \\
\hline & & & & & 11 & & & & & & \\
\hline & & & & & & & 1. & $*$ & 87 & 4 & \\
\hline & 02 & & $? ?$ & & 1 & & & * & 0 & 0 & \\
\hline & & -3 & 2 & . & 16 & 202 & 15 & ${ }^{* *}$ & 0 & 16 & \\
\hline & $\begin{array}{lll}03 & 00 & 27.27\end{array}$ & 161436.6 & 0.9 & 3.21 & 16.0 & 9773 & 180 & ** & 0 & 9 & \\
\hline & & & & 8. & 14 & & & $*$ & 58 & 78 & \\
\hline & 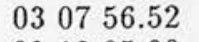 & & & & 16 & 20 & 15 & 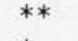 & 0 & 6 & \\
\hline & & 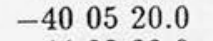 & & 0. & 16 & 22 & 57 & * & 16 & & \\
\hline & 0 & No & & 1. & 15 & 188 & 13 & ** & 1 & 16 & \\
\hline & $\begin{array}{lll}03 & 16 & 14.00\end{array}$ & -442523.0 & 6.0 & 1.49 & 16.0 & 22710 & 70 & ** & 1 & 16 & 203 \\
\hline
\end{tabular}




\begin{tabular}{|c|c|c|c|c|c|c|c|c|c|c|c|}
\hline${ }^{*} 0319-454$ & $\begin{array}{lll}03 & 19 & 15.54\end{array}$ & -452554.3 & 0.4 & 3.42 & 15.0 & 18977 & 300 & ** & 203 & 203 & 203 \\
\hline${ }^{*} 0325+023$ & $\begin{array}{lll}03 & 25 & 18.19\end{array}$ & $\begin{array}{lll}02 & 23 & 20.3\end{array}$ & 0.4 & 5.11 & 15.0 & 9054 & 90 & ** & 160 & 89 & 154 \\
\hline${ }^{*} 0326-461$ & 032654.20 & -460635.2 & 1.8 & 0.61 & 16.0 & 20686 & 270 & & 190 & 1 & 0 \\
\hline${ }^{*} 0332-391$ & $\begin{array}{lll}3 & 32 & 17.20\end{array}$ & -390924.0 & 6.0 & 1.59 & 16.5 & 18767 & 150 & ** & 1 & 1 & 7 \\
\hline${ }^{*} 0344-345$ & 34434.95 & -343202.0 & 1.0 & 3.52 & 16.5 & 16129 & 150 & ** & 142 & 1 & 7 \\
\hline${ }^{*} 0349-278$ & 034932.00 & -275324.0 & 6.0 & 4.89 & 16.8 & 19786 & 60 & ** & 20 & 161 & 161 \\
\hline${ }^{*} 0356+102$ & 035610.21 & 101731.7 & 0.3 & 10.32 & 15.6 & 9174 & 30 & ** & 58 & 78 & 154 \\
\hline * $0359+193$ & $\begin{array}{lll}03 & 59 & 19.50\end{array}$ & 192100.0 & 6.0 & 0.86 & 16.8 & 16279 & 60 & ** & 9 & 9 & \\
\hline *0404+035 & 040438.54 & 033427.2 & & 5.95 & 17.5 & 26712 & 0 & * & & & \\
\hline${ }^{*} 0419+140$ & 041940.89 & 44.9 & 1.0 & 1.10 & 18.0 & 19278 & 180 & ** & 0 & 130 & \\
\hline $0427-539$ & 42757.60 & -535605.4 & 0.5 & 4.90 & 13.2 & 11692 & 600 & ** & 19 & 87 & 95 \\
\hline *0431-134 & 43151.49 & -132823.1 & 0.9 & 0.73 & 16.3 & 10912 & 270 & ** & 0 & 22 & \\
\hline${ }^{*} 0434-225$ & 043427.50 & -223243.4 & 0.8 & 1.12 & 15.8 & 20446 & 150 & * & 0 & 4 & \\
\hline *0446-206 & 044620.69 & -203725.2 & 0.5 & 0.64 & 16.5 & 21705 & 150 & ** & 87 & 4 & \\
\hline *0449-175 & 044907.01 & -173511.8 & 1.6 & 1.08 & 14.6 & 9533 & 60 & ** & 0 & 22 & 22 \\
\hline${ }^{*} 0453-206$ & $453 \quad 14.03$ & -203900.8 & 1.8 & 4.72 & 14.0 & 10283 & 150 & ** & 0 & 163 & \\
\hline *0456 & 045630.20 & -301154.0 & ???? & 2.67 & 18.0 & 18887 & 100 & * & 0 & 0 & 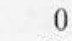 \\
\hline${ }^{*} 0502-103$ & 050231.07 & -101857.7 & 1.1 & 1.18 & 15.4 & 11812 & 150 & ** & 0 & 26 & 52 \\
\hline${ }^{*} 0503-286$ & 050351.00 & -283919.0 & $? ? . ?$ & 2.23 & 15.0 & 11422 & 28 & ** & 0 & 0 & 202 \\
\hline${ }^{*} 0511-305$ & $\begin{array}{llll}05 & 1137.20\end{array}$ & -303212.0 & 6.0 & 2.15 & 16.5 & 17478 & 150 & ** & 20 & 20 & 7 \\
\hline${ }^{*} 0513$ & 51321.15 & -490650.0 & 1.8 & 0.66 & 17.0 & 27300 & 0 & & 190 & & 204 \\
\hline${ }^{*} 051^{\prime}$ & $\begin{array}{lll}05 & 1731.06\end{array}$ & $-56 \quad 17 \quad 16.2$ & 1.8 & 1.30 & 17.0 & 28450 & 90 & & 190 & 17 & \\
\hline${ }^{*} 0518-458$ & $\begin{array}{lll}05 & 18 & 23.00\end{array}$ & -454944.0 & & 11.16 & 17.0 & 10733 & 0 & ** & & & \\
\hline${ }^{*} 0521-365$ & $\begin{array}{lll}05 & 21 & 12.95\end{array}$ & -363016.0 & 0.6 & 21.14 & 16.8 & 16609 & 30 & ** & 96 & 20 & 165 \\
\hline${ }^{*} 0523-327$ & 052335.70 & -324454.0 & 6.0 & 1.15 & 16.0 & 22724 & 150 & $*$ & 20 & 20 & 7 \\
\hline *054 & 3 & -61 & 1.8 & 0. & 18.0 & 28750 & 90 & & 190 & 13 & \\
\hline${ }^{*} 054$ & 5402 & $\begin{array}{lll}-62 & 52 & 13.2\end{array}$ & 1.8 & 0.64 & 17.5 & 25392 & 90 & * & 190 & 13 & \\
\hline *054 & 054546.00 & -195906.0 & 10.0 & 1.00 & 16.5 & 16549 & 150 & ** & 4 & 4 & 0 \\
\hline *0546 & 054636.30 & -325832.0 & $? ? . ?$ & 1.37 & 14.0 & 11062 & 150 & ** & 0 & 0 & \\
\hline *0548-317 & 054857.38 & -31 & 1.2 & 1.15 & 14.5 & 9773 & 150 & $* *$ & 0 & 6 & 8 \\
\hline${ }^{*} 0602-647$ & 6 & -64 & 1. & 1. & 17.0 & 13461 & 90 & ** & 190 & 2 & 0 \\
\hline${ }^{*} 060$ & 06 & -49 & 1.8 & 1.0 & 15.5 & 15559 & 120 & ** & 190 & 18 & 0 \\
\hline${ }^{*} 0609+710$ & $06 \quad 0948.37$ & $\begin{array}{llll}71 & 03 & 09.1\end{array}$ & 0.5 & 1.19 & 14.5 & 4047 & 30 & & 168 & 168 & 169 \\
\hline${ }^{*} 0611+519$ & $06 \quad 1134.32$ & 515945 & 0.5 & 0.82 & 16.5 & 14990 & 90 & & 168 & 168 & 0 \\
\hline${ }^{*} 0616-487$ & 061655.17 & -484341.6 & 1.8 & 1.67 & 15.0 & 13940 & 210 & $* *$ & 190 & 171 & 0 \\
\hline${ }^{*} 061$ & 9.00 & -37 & 6. & 3. & 15.6 & 97 & 150 & & 20 & 20 & 7 \\
\hline${ }^{*} 062$ & 0620 & -524001.0 & 12.0 & 4. & 15.5 & 9 & 210 & $* *$ & 0 & 0 & 0 \\
\hline${ }^{*} 062$ & 06 & -53394 & 1. & 6.2 & 15.4 & 16159 & 300 & ** & 190 & 64 & 95 \\
\hline${ }^{*} 0625-354$ & 062521.00 & $-3527 \quad 12.0$ & 6.0 & 4.90 & 16.5 & 16 & 150 & $* *$ & 20 & 20 & 7 \\
\hline${ }^{*} 0625-545$ & 062548.96 & -543039.6 & 1.8 & 2.93 & 16.0 & 15559 & 150 & & 190 & 2 & 205 \\
\hline & & 10 & 0 & 0 & 18.0 & 27 & 120 & & 50 & 50 & \\
\hline${ }^{*} 063$ & 06 & 42 & 0. & 0. & 18.0 & & 120 & & & 50 & . \\
\hline 84 & 064131.42 & -58 & 1. & 0. & 17.5 & 16 & 240 & ** & 190 & 17 & 0 \\
\hline *064 & 064254.1 & $\begin{array}{lll}-43 & 40 & 39\end{array}$ & 1.0 & 1.6 & 15.5 & 18227 & 90 & ** & 172 & 172 & 0 \\
\hline *0649- & 064923.60 & -554549.0 & 10.0 & 1.12 & 15.0 & 14750 & 180 & ** & 2 & 2 & 0 \\
\hline & & & & & 15.5 & $16 t$ & 90 & & 16 & 94 & \\
\hline & & 4 & 2. & 2 & 16.0 & 17922 & 90 & & 46 & 46 & 105 \\
\hline *070 & 07 & ? & ??. & 0 . & 17.2 & 236 & 60 & ** & 0 & 0 & 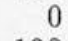 \\
\hline *0705 & 070 & 4 & 1. & 0.82 & 13.7 & 5546 & 120 & & 138 & 136 & 138 \\
\hline 0712 & 071242.14 & 532830.9 & 0.4 & 1.56 & 15.4 & 19307 & 900 & & 115 & 46 & 42 \\
\hline${ }^{*} 07$ & 071 & 6 & 0 & & 17 & 25 & 270 & & 1 & 168 & 0 \\
\hline & 073 & 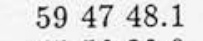 & 0 & & 16.5 & 11782 & 300 & & 68 & 168 & 0 \\
\hline 0744 & 07443 & 555629.0 & ??.? & 0.00 & 15.2 & 10583 & 0 & & 0 & 0 & 0 \\
\hline & $\begin{array}{lll}07 & 49 & 09.1\end{array}$ & 460443.6 & 0.5 & 0.67 & 16.0 & 14990 & 300 & & 50 & 50 & 0 \\
\hline${ }^{*} 0755$ & 075509.20 & 375522.0 & 2.0 & 1.87 & 14.6 & 12381 & 240 & ** & 40 & 40 & 77 \\
\hline & & & 7. & 3. & 17 & 18 & 120 & $* *$ & 89 & 89 & 00 \\
\hline $03-008$ & 080304.90 & 41.3 & 0.7 & 1.23 & 15.4 & 26741 & 90 & $* *$ & 0 & 73 & \\
\hline
\end{tabular}


*0816-705

*0819+061

$0825+247$

*0828+324

*0833-016

$* 0836+299$

* $0838+325$

${ }^{*} 0844+540$

*0844+319

*0915-118

*0921-213

*0945+076

*0945+734

*1000-043

*1002-320

$1003+351$

* $1004+146$

$* 1037+302$

* $1040+317$

*1043-290

*1053-282

*1056-360

* $1108+411$

$1113+295$

$* 1113+295$

* $1118+000$

*1123-351

*1130-037

$1131+493$

$1132+492$

* $1137+180$

* $1137+123$

*1138-078

* $1142+198$

* $1200+519$

* $1204+225$

*1211-417

* $1215+039$

*1216-100

* $1227+119$

* $1233+168$

*1250-102

* $1251+278$

*1251-289

*1251-122

*1254-300

*1257-253

*1258-321

*1308-441

$1309+210$

* $1312+089$

* $1313+073$

*1316+299

*1317-407

* $1319+428$

* $1321+318$

* $1322+366$

*1323-271
$08 \quad 16 \quad 26.28$ $\begin{array}{llll}08 & 19 & 52.10\end{array}$ $08 \quad 2541.86$

$0828 \quad 20.60$ 083302.06 083659.10 083806.75 084406.00

084454.20 $\begin{array}{llll}09 & 15 & 41.18\end{array}$ 092121.82 094506.00 094509.90

$1000 \quad 57.28$ 100226.68 100306.00 $\begin{array}{llll}10 & 04 & 09.93\end{array}$ 103742.70

104031.00

104347.90

105309.83

$1056 \quad 34.14$

110852.50

111353.59

111354.30

111846.10

112326.43

113031.85

113116.13

113203.83

113741.20

113754.00

113900.00

114229.55

120034.12

120400.10

121141.80

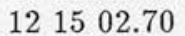

121600.60

122720.35

123358.90

125030.63

125146.29

125158.00

125158.56

125438.80

125707.00

$1258 \quad 16.22$

$1308 \quad 30.44$

$\begin{array}{lll}13 & 09 & 33.20\end{array}$

$13 \quad 12 \quad 39.49$

$\begin{array}{lll}13 & 13 & 45.97\end{array}$

$13 \quad 16 \quad 43.17$

$\begin{array}{llll}13 & 17 & 40.20\end{array}$

131905.22

132126.00

$1322 \quad 35.40$

132324.24

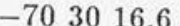
060648.0

244709.8

$\begin{array}{lll}32 & 29 & 37.0\end{array}$

-01 4034.2

295945.0

323543.7

540300.0

$\begin{array}{lll}31 & 58 & 12.0\end{array}$

$-115304.4$

$-212252.4$

$\begin{array}{lll}07 & 39 & 24.0\end{array}$

732822.2

-042337.3
-32

$-320208.9$

$\begin{array}{lll}35 & 08 & 49.0\end{array}$

143705.5

$\begin{array}{llll}30 & 13 & 38.0\end{array}$

314645.0

$-290516.0$

$-281532.1$

$-360315.6$

410620.4

$\begin{array}{lll}29 & 31 & 40.3\end{array}$

293144.0

000306.0

$-350712.5$

$-034414.3$

$\begin{array}{lll}49 & 20 & 16.8\end{array}$

$4913 \quad 56.2$

180022.0

122000.0

$-075115.0$

195302.6

$\begin{array}{lll}5157 & 13.0\end{array}$

223221.6

$-414313.2$

035457.0

$\begin{array}{lll}-10 & 02 & 50.0\end{array}$

$1157 \quad 12.2$

$1648 \quad 49.0$

$-101321.0$

275349.5

$-285734.0$

$-121751.8$

$\begin{array}{lll}-30 & 05 & 37.0\end{array}$

$-252328.0$

$\begin{array}{lll}-32 & 1021.0\end{array}$

$\begin{array}{llll}-4406 & 45.1\end{array}$

$\begin{array}{lll}21 & 03 & 57.0\end{array}$

085641.7

071835.6

295420.0

$-404600.0$

$4250 \quad 55.7$

$\begin{array}{llll}31 & 49 & 33.0\end{array}$

$\begin{array}{llll}36 & 38 & 19.0\end{array}$

$-271047.0$
1.8

$\begin{array}{lll}1.67 & 16.0 & 11392\end{array}$

$\begin{array}{llll}6.0 & 1.52 & 17.5 & 24433\end{array}$

$\begin{array}{llll}2.0 & 0.83 & 17.9 & 24973\end{array}$

$\begin{array}{llll}5.0 & 1.64 & 16.9 & 15379\end{array}$

$\begin{array}{llll}1.0 & 0.93 & 13.9 & 8994\end{array}$

$\begin{array}{llll}2.0 & 0.60 & 15.9 & 19307\end{array}$

$\begin{array}{llll}0.4 & 0.60 & 16.9 & 20896\end{array}$

$\begin{array}{llll}0.4 & 1.63 & 15.0 & 13551\end{array}$

$\begin{array}{llll}2.0 & 1.14 & 15.9 & 20236\end{array}$

$\begin{array}{llll}0.3 & 39.74 & 14.8 & 16429\end{array}$

$\begin{array}{llll}1.0 & 0.90 & 16.5 & 15559\end{array}$

$\begin{array}{llll}6.0 & 6.93 & 17.3 & 25812\end{array}$

$\begin{array}{llll}0.6 & 2.06 & 15.9 & 17268\end{array}$

$\begin{array}{llll}0.4 & 0.74 & 17.5 & 18947\end{array}$

$\begin{array}{llll}1.0 & 0.68 & 17.0 & 26292\end{array}$

$\begin{array}{llll}2.0 & 2.29 & 17.6 & 29649\end{array}$

$\begin{array}{llll}1.3 & 0.88 & 13.5 & 8814\end{array}$

$\begin{array}{llll}5.0 & 0.45 & 17.9 & 27311\end{array}$

$\begin{array}{llll}2.0 & 0.60 & 16.9 & 10523\end{array}$

$\begin{array}{llll}6.0 & 0.66 & 16.0 & 17208\end{array}$

$\begin{array}{llll}0.8 & 2.16 & 16.5 & 17808\end{array}$

$\begin{array}{llll}2.1 & 1.62 & 16.5 & 20985\end{array}$

$\begin{array}{llll}\text { ??? } & 0.97 & 0.0 & 22095\end{array}$

120

$\begin{array}{rr}30 & \\ 120 & 9\end{array}$

17

9

204

$180 \quad * * \quad 109 \quad 117 \quad 111$

$120 \quad * * \quad 0 \quad 41 \quad 95$

$90 * * \quad 40 \quad 94 \quad 111$

$\begin{array}{llll}90 & 68 & 68 & 111\end{array}$

$113 \quad 113$

$180 \quad * * \quad 40 \quad 57 \quad 111$

$90 \quad * * \quad 58 \quad 29 \quad 95$

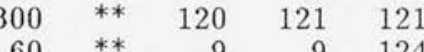

$\begin{array}{lrrr}90 & 97 & 125 & 126\end{array}$

$\begin{array}{rlrrr}150 & * * & 127 & 4 & 0 \\ 150 & & 0 & 6 & 7 \\ 0 & * * & 89 & 89 & 124 \\ 150 & * * & 0 & 43 & 0 \\ 120 & * * & 109 & 117 & 111\end{array}$

120

150

150

150

210

$\begin{array}{llll}\text { ?? ? } & 1.56 & 16.7 & 14630\end{array}$

$\begin{array}{llll}6.0 & 0.73 & 17.0 & 29739\end{array}$

$\begin{array}{llll}1.0 & 2.42 & 14.5 & 9863\end{array}$

120

180

120

$\begin{array}{lllll}0.5 & 0.95 & 15.3 & 15469 & 150\end{array}$

$\begin{array}{llll}0.4 & 0.00 & 14.2 & 10133\end{array}$

$\begin{array}{llll}1.1 & 0.00 & 15.2 & 9713\end{array}$

$\begin{array}{llll}6.0 & 1.27 & 13.3 & 3148\end{array}$

$\begin{array}{llll}6.0 & 1.52 & 16.5 & 24223\end{array}$

$\begin{array}{llll}6.0 & 0.74 & 17.0 & 19516\end{array}$

9
12
210

\section{$\begin{array}{llll}0.6 & 5.24 & 13.5 & 6476\end{array}$}

$\begin{array}{llll}1.0 & 0.74 & 15.7 & 18917\end{array}$

$\begin{array}{llll}2.0 & 1.05 & 15.5 & 19654\end{array}$

$\begin{array}{llll}1.0 & 1.32 & 17.5 & 20566\end{array}$

$\begin{array}{llll}\text { ??? } & 2.01 & 17.0 & 23534\end{array}$

21

210

400

300

$\begin{array}{llll}\text { ??.? } & 2.54 & 19.0 & 26202\end{array}$

$\begin{array}{llll}0.9 & 1.76 & 16.0 & 24613\end{array}$

$\begin{array}{llll}? ? . ? & 1.69 & 17.5 & 20776\end{array}$

$\begin{array}{llll}1.6 & 1.15 & 12.0 & 4287\end{array}$

$\begin{array}{llll}0.4 & 2.35 & 18.4 & 25692\end{array}$

$\begin{array}{llll}6.0 & 0.81 & 14.5 & 17328\end{array}$

$\begin{array}{llll}0.4 & 7.83 & 13.5 & 3987\end{array}$

$\begin{array}{llll}1.1 & 1.05 & 16.0 & 16189\end{array}$

$\begin{array}{llll}6.0 & 0.76 & 16.0 & 19337\end{array}$

$\begin{array}{llll}1.0 & 1.56 & 13.0 & 4467\end{array}$

$90 * 0 \quad 12$

210

180

150

90

90

30

150

150

150

$\begin{array}{llll}0.4 & 0.96 & 15.0 & 15439\end{array}$

$\begin{array}{llll}6.0 & 0.61 & 15.0 & 8994\end{array}$

$\begin{array}{llll}0.6 & 0.68 & 17.5 & 27101\end{array}$

$\begin{array}{llll}0.4 & 2.03 & 15.5 & 15499\end{array}$

$\begin{array}{llll}0.3 & 0.95 & 15.9 & 21825\end{array}$

240

210

$\begin{array}{lllll}14.0 & 0.79 & 17.0 & 14930 & 180\end{array}$

$\begin{array}{llll}0.3 & 2.01 & 16.7 & 23804\end{array}$

$\begin{array}{llll}2.0 & 0.78 & 13.9 & 4887\end{array}$

$\begin{array}{llll}2.0 & 0.64 & 14.3 & 5216\end{array}$

$\begin{array}{rrrr}10.0 & 1.59 & 15.0 & 12951\end{array}$

$\begin{array}{lr}* * & 40 \\ * * & 129 \\ * * & 133 \\ * & \end{array}$

$40 \quad 11$

129

133

16

0

102

138

$\begin{array}{lrrr} & 0 & 0 & 0 \\ * * & 51 & 51 & 0 \\ * & 142 & 20 & 61 \\ * * & 83 & 51 & 0\end{array}$

$\begin{array}{llll}* * & 127 & 148 & 148\end{array}$

** $\quad 0 \quad 41 \quad 150$

** 131

** 9

131 


\begin{tabular}{|c|c|c|c|c|c|c|c|c|c|c|c|}
\hline${ }^{*} 1329-328$ & 132934.69 & -325252.6 & 1.0 & 1.30 & 15.5 & 14450 & 150 & ** & 182 & 6 & \\
\hline $1331-099$ & 133140.08 & -095407.8 & ??.? & 1.86 & 17.5 & 24403 & 0 & 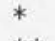 & 87 & 87 & \\
\hline${ }^{*} 1333-337$ & 133347.30 & -334240.0 & 2.0 & 4.62 & 11.9 & 3867 & 90 & ** & 185 & 0 & \\
\hline * $1339+266$ & 3933.50 & 263731.2 & ??.? & 0.47 & 16.5 & 21585 & 90 & * & 92 & 141 & \\
\hline $346+268$ & 4633.98 & 265027.8 & 0.4 & 1.17 & 16.4 & 18977 & 240 & * & 56 & 40 & \\
\hline $350+316$ & $13 \quad 5003.20$ & $\begin{array}{llll}31 & 413.8\end{array}$ & 0.6 & 3.77 & 14.9 & 13491 & 60 & ** & 56 & 40 & \\
\hline $354-251$ & 135427.00 & -250849.0 & 6.0 & 0.73 & 15.0 & 11272 & 150 & $*$ & 129 & 129 & \\
\hline${ }^{*} 1359-113$ & 135901.40 & -112158.0 & 1.0 & 1.79 & 15.0 & 10972 & 120 & * & 8 & 73 & \\
\hline${ }^{*} 1404-267$ & 140438.30 & -264647.0 & 6.0 & 0.85 & 13.5 & 6206 & 150 & ** & 129 & 129 & \\
\hline *1406-230 & 140623.20 & -230145.0 & 6.0 & 0.74 & 17.5 & 25692 & 300 & & 129 & 129 & \\
\hline $407-425$ & 140722.48 & -423249.0 & 1.0 & 0.96 & 15.5 & 15259 & 240 & & 188 & 16 & \\
\hline $407+177$ & 40734.82 & 174652.5 & 1.0 & 1.18 & 15.5 & 5066 & 240 & * & 25 & 25 & \\
\hline *1413 & $1413 \quad 31.82$ & -362700.4 & 1.0 & 2.33 & 17.0 & 22604 & 150 & & 142 & 20 & \\
\hline *1414 & 141425.80 & $\begin{array}{lll}11 & 02 & 22.0\end{array}$ & 7.0 & 3.89 & 13.3 & 7105 & 150 & * & 89 & 89 & \\
\hline *1422 & 142226.50 & 265126.0 & 2.0 & 0.60 & 15.8 & 11092 & 240 & * & 40 & 40 & \\
\hline 74 & 142726.74 & $07 \quad 28 \quad 34.4$ & ???? & 1.79 & 18.0 & 15859 & 240 & * & 0 & 192 & \\
\hline 44 & 144337.80 & $\begin{array}{lll}75107.0 & 07\end{array}$ & 6.0 & 0.74 & 15.5 & 19576 & 90 & ** & 131 & 131 & \\
\hline *144: & 144355.70 & -083319.0 & 10.0 & 0.91 & 16.0 & 21825 & 210 & ** & 4 & 4 & \\
\hline *1448 & 144816.98 & $\begin{array}{lll}63 & 28 & 35.2\end{array}$ & 0.5 & 3.20 & 15.0 & 12291 & 42 & ** & 168 & 89 & \\
\hline *1452 & 145203.32 & 163327.9 & 0.5 & 1.35 & 14.0 & 13671 & 210 & * & 87 & 194 & \\
\hline 45 & 145 & -052724.0 & 12.0 & 0.9 & 17.0 & 11182 & 180 & * & 41 & 41 & \\
\hline${ }^{*} 150$ & 150248.50 & 261125.1 & ???? & 8.7 & 16.8 & 16141 & 90 & $*$ & 0 & 46 & \\
\hline *151 & 151414 & $\begin{array}{llll}00 & 26 & 01.0\end{array}$ & 1.7 & 3.1 & 16.5 & 155 & 90 & * & 195 & 195 & \\
\hline *1514 & $1514 \quad 17.00$ & 71216.7 & 0.4 & 3.89 & 14.5 & 10493 & 90 & ** & 58 & 196 & \\
\hline 1514 & 151445.28 & -241122.9 & 0.4 & 3.38 & 15.0 & 14570 & 600 & & 197 & 20 & \\
\hline 1518 & $1518 \quad 52.73$ & 3.7 & 0.7 & 0. & 16. & 5 & 0 & ** & 87 & 87 & \\
\hline$* 152$ & $1:$ & -3 & 12.0 & 0 & 18.0 & 22 & 700 & & 6 & 6 & \\
\hline * 152 & 152121.30 & $2848 \quad 10.8$ & 1.0 & 0.5 & 17.5 & 24733 & 240 & * & 108 & 199 & \\
\hline 152 & 152933.42 & 241426 . & 0.8 & 3. & 16.0 & 28660 & 0 & * & & & \\
\hline${ }^{*} 1540-077$ & 154020.30 & -074738.0 & 10.0 & 2.05 & 18.0 & 4317 & 90 & * & 4 & 4 & \\
\hline 549 & 56 & $\begin{array}{llll}20 & 14 & 17.3\end{array}$ & ?.? & & 15. & & 240 & * & 0 & 0 & \\
\hline *155 & 1. & -3 & 1.0 & & 5 & & 180 & & 182 & 6 & \\
\hline 155 & 155 & -140126 & $2 .($ & 1. & 0 & 291 & 0 & 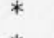 & 121 & 121 & \\
\hline${ }^{*} 155$ & 155 & 704 & 0.4 & 2.5 & 14 & & 90 & * & 193 & 193 & \\
\hline${ }^{*} 1601-$ & 160116.39 & 06.8 & 1.0 & 0.79 & 13.5 & 10613 & 270 & * & 92 & 130 & \\
\hline 602 & & & $2 .($ & 0.6 & 4 & & 60 & & 192 & 158 & \\
\hline${ }^{*} 160$ & 5 & 17 & 0. & 0. & ) & & 60 & * & 56 & 130 & \\
\hline 60 & 10 & & 6. & 2. & 16 & 183 & 210 & * & 9 & 9 & \\
\hline *161 & 16 & 35 & 2.0 & 1.3 & 15.5 & 88 & 240 & * & 40 & 40 & \\
\hline *1616 & 31.40 & -025712.0 & 6.0 & 0.71 & 16.6 & 20536 & 240 & * & 51 & 51 & \\
\hline *162 & & & 2.0 & & 13.7 & & 90 & ** & 40 & 40 & \\
\hline & & -77 & 1. & 5.9 & 16 & 72 & 600 & & 190 & 166 & \\
\hline * 163 & 16 & 82 & - & & & & & ** $*$ & 201 & 201 & \\
\hline$* 165$ & 1 & & ???? & 0. & 15 & 101 & 240 & ** & 0 & 0 & \\
\hline${ }^{*} 1655$ & 165512.49 & -77 & 0.5 & 2.42 & 17.0 & 20086 & 210 & & 19 & 19 & \\
\hline 65 & & & & & & & 60 & * & 15 & & \\
\hline & & & & & & & 2 & & 5 & & \\
\hline *170 & 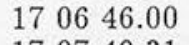 & 0 & 10 & & & & & * & 130 & 130 & \\
\hline * 170 & 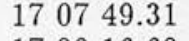 & & 1. & 0 . & 17. & & 10 & 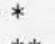 & 83 & 83 & \\
\hline${ }^{*} 1709+397$ & 170916.69 & $\begin{array}{llll}39 & 45 & 07.9\end{array}$ & 1.0 & 0.46 & 17.1 & 18617 & 210 & ** & 92 & 34 & \\
\hline 7 & & & & & & & & $* *$ & 92 & 34 & \\
\hline & & & & & & & 15 & $*$ & 136 & 143 & \\
\hline $\begin{array}{l}17 \\
*^{*} 17\end{array}$ & 5. & & 0. & 61. & 16 & 91 & 90 & * & 58 & 73 & \\
\hline $\begin{array}{l}* 1718- \\
* 1719\end{array}$ & $=0$ & . & 1. & 6. & 15 & & 21 & $* *$ & 190 & 122 & \\
\hline *1719+ & 171958.05 & 241643.8 & 0.6 & 0.88 & 17.5 & 26532 & 600 & ** & 33 & 33 & \\
\hline & & & & & 15 & & & & 201 & 201 & \\
\hline & 73 & & ?.? & 1.52 & 15. & 19397 & 30 & & 0 & 0 & \\
\hline
\end{tabular}


* $1740+162$ * $1744+557$

*1754+626

* $1755+626$

*1801-702

* $1807+698$

*1814-766

*1814-637

* $1820+689$

$1833+326$

*1833-772

*1842+455

* $1845+797$

*1847-796

*1921-577

*1921-430

*1928-340

*1929-397

*1941-554

* $1950+672$

*1954-552

*2013-308

*2014-558

*2031-359

*2040-267

*2054-581

*2058-282

*2058-135

*2101-715

*2104-256

*2117-269

*2128-388

2134-281

*2148-555

*2148-427

*2152-699

*2156-564

*2158-380

2159-335

2206-237

* $2212+135$

*2221-023

*2225-308

*2229-086 $2229+391$

* $2235+408$

*2236-176

*2236-364

$2243+394$

* $2247+113$

*2253-465

* $2308+073$

*2309-416

*2316-423

*2317-277

* $2318+079$

* $2320+203$

* $2322-123$
174036.23 174400.58 175424.00

175521.20 180135.45 180719.00 181411.46 181446.34

$18 \quad 2001.22$

$1833 \quad 11.97$

183315.08

184235.44

$1845 \quad 37.64$

184754.52 192153.33

192158.80

192823.10

192959.70

194123.30

195005.64

195419.46

$\begin{array}{llll}20 & 13 & 07.74\end{array}$

$2014 \quad 06.12$

203132.20

204046.00

205446.97

205838.00

205859.38

210109.64

$\begin{array}{lll}21 & 04 & 29.70\end{array}$

211749.00

212826.00

213418.73

214803.93

214814.40

215257.81

215659.76

215817.21

215900.48

220632.71

221213.30

222114.76

222501.11

222905.60

222907.67

223548.07

$2236 \quad 30.20$

223640.00

$2243 \quad 32.79$

224724.93

225348.51

$\begin{array}{lll}23 & 08 & 10.20\end{array}$

230921.36

231622.50

$\begin{array}{lll}23 & 17 & 16.00\end{array}$

231804.00

232050.62

$2322 \quad 43.72$
$16 \quad 14 \quad 10.5$ $\begin{array}{llll}55 & 43 & 25.0\end{array}$ 623900.0

623705.0 $-701257.6$

$\begin{array}{llll}69 & 49 & 03.0\end{array}$

$-763615.9$

$-634705.0$

$\begin{array}{lll}68 & 5522.6\end{array}$

$\begin{array}{lll}32 & 39 & 18.2\end{array}$

$\begin{array}{llll}-77 & 1209.1\end{array}$

$\begin{array}{llll}45 & 30 & 21.7\end{array}$

$\begin{array}{llll}79 & 43 & 06.1\end{array}$

$-793627.4$

$-574615.7$

$\begin{array}{lll}-43 & 0345.0\end{array}$

$-340108.0$

$-394654.0$

$\begin{array}{lll}-55 & 28 & 16.0\end{array}$

$67 \quad 1204.2$

$-551752.1$

$-305047.6$

$-554852.0$

$-355916.0$

$-264400.0$

$-580814.3$

$\begin{array}{llll}-28 & 13 & 30.0\end{array}$

$-133038.2$

$-713418.6$

$-253751.0$

$-265733.0$

$-385012.0$

$-280828.1$

$-553418.2$

$-424742.0$

$-695540.2$

$-562507.2$

$-380050.8$

$-333533.5$

$-234638.1$

$\begin{array}{llll}13 & 35 & 33.0\end{array}$

$-022126.1$

$-304905.0$

$-083959.0$

$\begin{array}{lll}39 & 06 & 03.9\end{array}$

405158.6

$-173606.0$

$-362500.0$

$\begin{array}{rrrrrrrrr}0.6 & 0.61 & 14.5 & 10373 & 240 & * * & 33 & 33 & 0 \\ 0.4 & 0.59 & 14.7 & 9114 & 210 & * * & 56 & 168 & 61 \\ 30.0 & 0.00 & 14.8 & 8274 & 210 & * * & 54 & 54 & 54 \\ & & & & & & & & \\ ? ? . ? & 0.00 & 15.0 & 8274 & 210 & * * & 50 & 50 & 0 \\ 1.8 & 1.10 & 16.5 & 11842 & 240 & & 190 & 2 & 0 \\ 7.0 & 2.16 & 15.6 & 15020 & 30 & * * & 89 & 89 & 112 \\ 1.8 & 0.66 & 14.0 & 5666 & 210 & & 190 & 107 & 0 \\ 0.4 & 12.51 & 18.0 & 18797 & 150 & * * & 76 & 0 & 98\end{array}$

$\begin{array}{llll}\text { ??.? } & 0.82 & 16.4 & 26442\end{array}$

$\begin{array}{llll}0.1 & 0.00 & 15.9 & 17088\end{array}$

$\begin{array}{llll}1.8 & 0.96 & 15.0 & 5336\end{array}$

$\begin{array}{llll}0.4 & 5.95 & 17.2 & 27221\end{array}$

$\begin{array}{llll}0.4 & 11.18 & 15.0 & 16818\end{array}$

$180 * * \quad 0$

$150 \quad 190$

$\begin{array}{lllll}1.8 & 0.66 & 17.0 & 22934 & 280\end{array}$

$\begin{array}{lllll}1.8 & 1.15 & 16.0 & 17838 & 180\end{array}$

$\begin{array}{lllll}12.0 & 0.96 & 18.0 & 24433 & 210\end{array}$

$\begin{array}{llll}6.0 & 0.61 & 17.0 & 28930\end{array}$

$\begin{array}{llll}6.0 & 2.60 & 16.0 & 22095\end{array}$

$\begin{array}{llll}\text { ??.? } & 1.44 & 14.0 & 4407\end{array}$

$\begin{array}{llll}0.5 & 0.74 & 18.4 & 22065\end{array}$

$\begin{array}{llll}1.0 & 6.32 & 16.5 & 18000\end{array}$

$\begin{array}{llll}1.0 & 0.85 & 16.5 & 26622\end{array}$

$\begin{array}{llll}0.5 & 1.18 & 15.5 & 18227\end{array}$

$\begin{array}{llll}14.0 & 1.57 & 15.5 & 26500\end{array}$

$\begin{array}{rrrr}6.0 & 2.64 & 15.4 & 12082\end{array}$

$\begin{array}{llll}1.8 & 0.78 & 16.0 & 15559\end{array}$

$\begin{array}{llll}6.0 & 5.24 & 15.6 & 11302\end{array}$

$\begin{array}{llll}1.0 & 1.01 & 15.2 & 8724\end{array}$

$\begin{array}{lllll}1.8 & 1.35 & 16.5 & 22215 & 120\end{array}$

$\begin{array}{llll}1.0 & 10.49 & 16.8 & 11662\end{array}$

$\begin{array}{llll}10.0 & 1.00 & 18.0 & 27731\end{array}$

$\begin{array}{lllr}14.0 & 1.10 & 14.5 & 5464\end{array}$

$\begin{array}{llll}1.2 & 1.08 & 16.0 & 21435\end{array}$

$\begin{array}{lllll}1.8 & 1.47 & 16.0 & 11542 & 300\end{array}$

$\begin{array}{lllll}14.0 & 0.61 & 16.3 & 19067 & 270\end{array}$

$\begin{array}{lllll}0.5 & 30.27 & 13.8 & 8544 & 90\end{array}$

$\begin{array}{lllll}1.8 & 0.79 & 16.0 & 22694 & 120\end{array}$

$\begin{array}{lllll}0.2 & 1.71 & 15.0 & 9989 & 9\end{array}$

$\begin{array}{llll}1.0 & 1.45 & 17.5 & 16159\end{array}$

$\begin{array}{llll}1.1 & 2.25 & 17.0 & 25842\end{array}$

$\begin{array}{lllr}\text { ??.? } & 2.37 & 14.3 & 7345\end{array}$

$\begin{array}{llll}0.4 & 5.85 & 17.0 & 16848\end{array}$

$\begin{array}{llll}1.0 & 0.93 & 16.5 & 16459\end{array}$

$\begin{array}{llll}1.0 & 0.76 & 15.5 & 24763\end{array}$

$\begin{array}{llll}0.4 & 0.00 & 14.2 & 4827\end{array}$

$\begin{array}{llll}0.5 & 0.82 & 17.0 & 17088\end{array}$

$\begin{array}{llll}1.0 & 1.79 & 16.0 & 15289\end{array}$

$\begin{array}{llll}14.0 & 0.71 & 15.5 & 17058\end{array}$

$\begin{array}{lll}39 & 25 & 27.3\end{array}$

$\begin{array}{lll}11 & 20 & 36.8\end{array}$

$-463337.4$

071852.0

$\begin{array}{llll}0.5 & 10.05 & 17.0 & 24163\end{array}$

$\begin{array}{lrrr}0.5 & 2.37 & 13.0 & 7825\end{array}$

$\begin{array}{llll}1.8 & 1.20 & 17.5 & 25482\end{array}$

$\begin{array}{llll}\text { ??? } & 1.62 & 14.9 & 13221\end{array}$

$\begin{array}{llll}0.5 & 0.68 & 17.0 & 28480\end{array}$

$-413917.3$

$\begin{array}{llll}6.0 & 1.25 & 16.0 & 16279\end{array}$

$\begin{array}{llll}6.0 & 3.23 & 17.5 & 29110\end{array}$

$\begin{array}{rrrr}6.0 & 0.68 & 12.8 & 3328\end{array}$

$\begin{array}{llll}0.8 & 1.03 & 14.5 & 11512\end{array}$

$\begin{array}{llll}0.5 & 1.49 & 15.4 & 24613\end{array}$
$-422323$.

075712.0

$2018 \quad 52.8$

$-122357.4$
$90 \quad * * \quad 58$

60

60

180

30
150

180

180
150
150

$\begin{array}{lrr}* * & 56 & 20 \\ & 190 & 1 \\ & 190 & \\ & 1 & 1 \\ * * & 1 & \\ * * & 1 & 3 \\ & & \\ * * & 1 & \\ * * & 50 & 5 \\ & 172 & 17 \\ * * & 182 & \\ * * & 19 & 1 \\ & & \\ * * & 16 & 1 \\ & 20 & 2 \\ & 190 & \\ * * & 20 & 2 \\ & 72 & 2\end{array}$

18
58

201

154

90

$\begin{array}{rr}18 & 0 \\ 1 & 0 \\ 16 & 0 \\ 1 & 7 \\ 30 & 7\end{array}$

1
50

172

204

627

$16 \quad 203$

$20 \quad 61$

$20 \quad 61$

$\begin{array}{rrr}2 & 2 & 0 \\ 77 & 20 & 7 \\ 4 & 4 & 0 \\ 16 & 16 & 202 \\ 0 & 4 & 0\end{array}$

$\begin{array}{lll}190 & 17 & 0\end{array}$

$\begin{array}{lll}19 & 71 & 95\end{array}$

$\begin{array}{rrr}17 & 17 & 0 \\ 69 & 16 & 69\end{array}$

182

$6 \quad 182$

$58 \quad 154$

$\begin{array}{lll}182 & 6 & 7\end{array}$

$\begin{array}{rrr}8 & 4 & 0 \\ 50 & 50 & 0 \\ 8 & 35 & 7 \\ 16 & 16 & 7\end{array}$

$\begin{array}{rlrrr}0 & * * & & & \\ 60 & * * & 87 & 87 & 47 \\ 240 & & 190 & 17 & 0 \\ 60 & * * & 0 & 0 & 29 \\ 240 & & 87 & 16 & 0\end{array}$

150

240

60

180

30

7
0
7
2
3
1
0
1 $\begin{array}{lll}16 & 16 & 0\end{array}$

$\begin{array}{ll}4 & 65 \\ 0 & 29\end{array}$

\section{,}

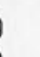

0

8

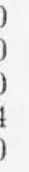

7
7

2




\begin{tabular}{|c|c|c|c|c|c|c|c|c|c|c|c|}
\hline$* 2327-215$ & 232702.82 & -213018.3 & 1.0 & 0.74 & 18.0 & 23234 & 270 & & 0 & 4 & ( \\
\hline $2331-240$ & 233117.90 & -240016.0 & ???? & 1.76 & 16.5 & 14330 & 1500 & ** & 0 & 0 & ( \\
\hline * $2335+267$ & 233558.94 & 264516.4 & 0.4 & 3.87 & 14.9 & 8784 & 90 & ** & 56 & 40 & 15 \\
\hline${ }^{*} 2353-184$ & 235321.70 & -182634.0 & 6.0 & 0.83 & 15.5 & 21675 & 150 & ** & 32 & 32 & ? \\
\hline$* 2354-350$ & 235426.50 & -350218.0 & 6.0 & 0.59 & 15.0 & 14600 & 30 & * & 20 & 20 & $2:$ \\
\hline$* 2356-611$ & 235629.37 & -611140.6 & 0.5 & 20.29 & 16.0 & 28870 & 30 & * & 19 & 61 & \\
\hline
\end{tabular}

Number of adequate spectra: 267 ( $91 \%)$

Number of I frames: $229(78 \%)$

Number of B frames: 167 ( $57 \%$ ) 
Table A1.2: Probable Sample Members

\begin{tabular}{|c|c|c|c|c|c|c|c|c|c|c|}
\hline & $\alpha_{o p t}(1950)$ & $\delta_{\text {opt }}(1950)$ & $\pm^{\prime \prime}$ & $S_{1.4}$ & $m$ & $c z$ & \pm & ccd & & \\
\hline $0114-211$ & 011422.00 & $\begin{array}{llll}-21 & 07 & 18.0\end{array}$ & 0.1 & 4.06 & 16.5 & 0 & 0 & & 20 & 20 \\
\hline $0207-224$ & 020750.60 & -222749.0 & 10.0 & 1.45 & 15.5 & 0 & 0 & & 4 & \\
\hline $0411-647$ & 041131.30 & -644357.0 & 10.0 & 1.20 & 16.5 & 0 & 0 & & 17 & 17 \\
\hline $0610+783$ & 061041.64 & $7822 \quad 28.6$ & 0.6 & 0.64 & 12.9 & 0 & 0 & & 201 & 01 \\
\hline $0955+035$ & 095548.10 & 033448.0 & ??.? & 0.68 & 17.0 & 0 & 0 & ** & 0 & \\
\hline * $1027+749$ & $\begin{array}{llll}0 & 27 & 13.38\end{array}$ & 745722.5 & 0.6 & 0.61 & 16.8 & 0 & 0 & & 201 & \\
\hline${ }^{*} 1029+569$ & 102947.10 & 565958.0 & ??.? & 0.74 & 15.0 & 0 & 0 & & 0 & 0 \\
\hline $1042+736$ & 104223.66 & $73 \quad 36 \quad 58.4$ & 0.6 & 0.76 & 14.7 & 0 & 0 & & 201 & \\
\hline $1048+556$ & 104846.20 & $\begin{array}{llll}55 & 38 & 35.0\end{array}$ & ??.? & 0.59 & 15.7 & 0 & 0 & & 0 & \\
\hline${ }^{*} 1127+553$ & 112744.70 & 552028.0 & ??.? & 0.74 & 15.5 & 0 & 0 & & 0 & \\
\hline 1141 & 114 & 463800.0 & 1.2 & 0.97 & 16.7 & 0 & 0 & & 114 & \\
\hline 1152 & 115255.56 & $\begin{array}{llll}55 & 10 & 38.3\end{array}$ & 1.0 & 2.82 & 15.7 & 0 & 0 & & 156 & 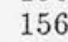 \\
\hline 1249 & 124 & $\begin{array}{llll}03 & 32 & 12.0\end{array}$ & 6.0 & 0.98 & 16.8 & 0 & 0 & * & 51 & \\
\hline $1252+533$ & 125224.68 & $\begin{array}{lll}53 & 2137.8\end{array}$ & 0.5 & 0.00 & 15.0 & 0 & 0 & * & 50 & 50 \\
\hline $1323+370$ & 132346.81 & $\begin{array}{lll}37 & 03 & 33.6\end{array}$ & 0.5 & 0.78 & 16.3 & 0 & 0 & * & 55 & 84 \\
\hline$*_{1} 1551+239$ & 15 & 23 & 6.0 & 0.90 & 17.0 & 0 & 0 & * & 158 & \\
\hline & 161759.90 & -233523.0 & 10.0 & 1.15 & 16.5 & 0 & 0 & & 4 & \\
\hline *1824+468 & 182426.45 & $46 \quad 52 \quad 19.8$ & 0.5 & 0.52 & 16.0 & 0 & 0 & $* *$ & 50 & \\
\hline $2005-489$ & 200546.80 & -485842.0 & ??.? & 1.79 & 16.5 & 0 & 0 & & 18 & \\
\hline $2116+818$ & $21 \quad 16 \quad 39.56$ & 815213.0 & 0.6 & 0.73 & 15.9 & 0 & 0 & ** & 201 & 20 \\
\hline
\end{tabular}

Number of adequate spectra: 5 ( $25 \%)$

Number of I frames: 7 ( $35 \%)$

Number of B frames: 3 (15\%) 
Table A1.3: Possible Sample Members

\begin{tabular}{|c|c|c|c|c|c|c|c|c|c|c|}
\hline & $\alpha_{o p t}(1950)$ & $\delta_{o p t}(1950)$ & $\pm^{\prime \prime}$ & $S_{1.4}$ & $m$ & $c z$ & \pm & $\mathrm{ccd}$ & & \\
\hline $002+125$ & $\begin{array}{llll}00 & 02 & 17.50\end{array}$ & 123206.0 & 6.0 & 1.35 & 18.7 & 0 & 0 & * & & \\
\hline $0003-833$ & $\begin{array}{lll}00 & 03 & 55.39\end{array}$ & -832249.9 & 1.8 & 1.89 & 19.0 & 0 & 0 & & 190 & \\
\hline $0005-062$ & 000556.00 & -061530.0 & 12.0 & 1.15 & 19.0 & 0 & 0 & ** & 5 & \\
\hline $007+124$ & $\begin{array}{llll}00 & 07 & 18.20\end{array}$ & 122722.3 & 0.6 & 1.69 & 17.8 & 0 & 0 & ** & 0 & \\
\hline $0010+775$ & $\begin{array}{lll}00 & 10 & 22.15\end{array}$ & 773206.3 & 0.6 & 2.23 & 18.6 & 0 & 0 & $* *$ & 201 & \\
\hline $0010-564$ & $\begin{array}{llll}00 & 10 & 50.73\end{array}$ & -562705.8 & 1.8 & 0.69 & 19.0 & 0 & 0 & & 190 & \\
\hline $0012+319$ & $\begin{array}{llll}00 & 12 & 29.88\end{array}$ & 315933.3 & 1.0 & 1.34 & 18.7 & 0 & 0 & * & 14 & \\
\hline $014-387$ & $\begin{array}{lll}00 & 14 & 07.00\end{array}$ & -384326.0 & 14.0 & 0.91 & 18.0 & 0 & 0 & & 16 & \\
\hline $16-515$ & 001621.66 & -513135.7 & 1.8 & 0.93 & 19.0 & 0 & 0 & & 190 & \\
\hline $20-747$ & 002 & -74 & 1.8 & 0.90 & 18.0 & 0 & 0 & & 190 & \\
\hline $028-505$ & $00 \quad 28 \quad 14.60$ & -503503.0 & 6.0 & 1.01 & 19.0 & 0 & 0 & & 13 & \\
\hline 035 & 00355 & -21364 & & 0.00 & 19.0 & 0 & 0 & & 4 & \\
\hline $036-216$ & $\begin{array}{llll}00 & 36 & 00.47\end{array}$ & -213634.6 & 0.4 & 0.00 & 19.0 & 0 & 0 & & 120 & \\
\hline $36-627$ & 003631.76 & -624740.5 & 1.8 & 1.35 & 18.5 & 0 & 0 & & 190 & \\
\hline 448 & 004 & -44 & 16.0 & 1.15 & 18.5 & 0 & 0 & & 16 & \\
\hline 504 & 5 & -502 & 1.8 & 0.61 & 18. & 0 & 0 & & 190 & \\
\hline 0054 & 005 & -45 & 1.8 & 0.7 & 18 & 0 & 0 & & 190 & \\
\hline $105-122$ & 01054 & -121702.0 & 10.0 & 0.88 & 19.0 & 0 & 0 & ** & 4 & \\
\hline 656 & 010 & -65 & 1.8 & 0.59 & 18.5 & 0 & 0 & & 190 & \\
\hline $109+415$ & $\begin{array}{lll}01 & 09 & 10.80\end{array}$ & 413106.8 & 0.5 & 1.11 & 18.2 & 0 & 0 & * & 50 & \\
\hline $14+074$ & 011450.45 & 072600.1 & 0.7 & 1.52 & 19 & 0 & 0 & & 25 & \\
\hline 22 & 01 & -25 & 10.0 & 1.23 & 18 & 0 & 0 & & 4 & \\
\hline 30 & 013 & $-62 c$ & 1.8 & 1.00 & 18. & 0 & 0 & & 190 & \\
\hline 177 & 013 & -17 & 1.2 & 0.71 & 18.0 & 0 & 0 & ** & 0 & \\
\hline $0149-394$ & 014935.15 & -39 & 0.7 & 0.91 & 19.0 & 0 & 0 & & 67 & \\
\hline $5 \cdot$ & 0 & 41 & 14.0 & 2 & 18 & 0 & 0 & & 16 & \\
\hline 08 & 02 & -24 & 0.5 & 0.91 & 17. & 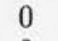 & 0 & & 32 & \\
\hline $0 s$ & 02 & -62 & 1.8 & 0.91 & 17. & 0 & 0 & & 190 & \\
\hline 75 & 021 & -473150.7 & 1.8 & 0.85 & 18.0 & 0 & 0 & & 190 & \\
\hline $22+$ & 022 & 263916.0 & 10.0 & 0.59 & 19.0 & 0 & 0 & ** & 80 & \\
\hline 25 & & -30 & & & & & & & 5 & \\
\hline 2 & & & ??.? & 0.6 & 18 & 0 & 0 & ** & 0 & \\
\hline 52 & 02 & -71 & 0.7 & 5.2 & 19 & 0 & 0 & & 18 & \\
\hline $50-2>$ & & -31 & & 0.6 & 18 & 0 & 0 & & 131 & \\
\hline 30 & 030 & -130947.0 & 6.2 & 0.79 & 18.5 & 0 & 0 & ** & 159 & \\
\hline 20 & & 46 & & & & & & & & \\
\hline & & -42 & 10.0 & 0 & & 0 & 0 & & 0 & \\
\hline 28 & 0 & -26 & 6.0 & 0 & 18 & 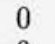 & 0 & & 32 & \\
\hline & 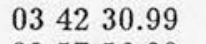 & -52 & 1.8 & 0.6 & 18 & 0 & 0 & & 190 & \\
\hline 57 & 035 & -37 & 6.0 & 1.37 & 18.0 & 0 & 0 & & 1 & \\
\hline & & & 6. & & & 0 & 0 & & 32 & \\
\hline & & -17 & 6.0 & & & 0 & 0 & ** & 32 & \\
\hline & & 30 & ??? & & 18 & 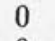 & 0 & & 0 & \\
\hline & & & 1.0 & 0.8 & 10 & 0 & 0 & $* *$ & 164 & 16 \\
\hline $588-$ & 0458 & 01 & 6.0 & 0.95 & 18 & 0 & 0 & ** & 51 & \\
\hline & & & & & & 0 & $\mathrm{c}$ & & & \\
\hline & & -22 & & & & 0 & 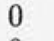 & & 162 & 162 \\
\hline & 0 & -24 & $? ?$ & & $18,+2$ & 0 & 0 & & 0 & \\
\hline & & -45 & 0.6 & $1.2^{2}+x$ & 19 & 0 & 0 & & 33 & J. \\
\hline $03+$ & 070306.95 & 465243.9 & 1.0 & 1.49 & 0.0 & 0 & 0 & & 174 & \\
\hline & & & ???? & & & & 0 & & & \\
\hline & & & ???? & & & 0 & 0 & & 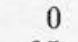 & \\
\hline & & & 20.0 & & 19. & 0 & 0 & $* *$ & 35 & \\
\hline & & & 1.3 & & & 0 & 0 & & 11 & \\
\hline 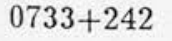 & 073316.90 & $24 \quad 1724.3$ & 1.0 & 0.45 & 19.0 & 0 & 0 & & 108 & \\
\hline
\end{tabular}


$0740+578$

$0756+383$

$0804+499$

0814-779

$0818+179$

$0823+379$

$0847+491$

$0909+165$

$0919+532$

0935-289

0941-080

0943-761

0945-321

*0955+320

1003-137

1006-214

$1012+410$

$1013+596$

$1019+648$

$1043+552$

$\begin{array}{llll}1102-185 & 1102 & 56.00 \\ 1103-244 & 11 & 03 & 45.03 \\ 1106+023 & 1106 & 11.10 \\ 1108+201 & 1108 & 41.04 \\ 1111+111 & 11 & 11 & 56.50 \\ & & & \\ 1111-037 & 11 & 11 & 58.90 \\ 1118+237 & 11 & 18 & 02.91 \\ 1119+216 & 11 & 19 & 51.78 \\ 1127-130 & 11 & 27 & 44.60 \\ 1130+339 & 11 & 30 & 30.16 \\ & & & \\ 1134+616 & 1134 & 34.72 \\ 1140+217 & 1140 & 21.25 \\ 1140+303 & 1140 & 51.30 \\ 1143-331 & 11 & 43 & 57.20 \\ 1151+384 & 1151 & 17.38\end{array}$

$1153-231$

$1155+266$

1156-402

1207-013

$1210+208$

1222-252

$1227+181$

$1230+486$

$1233-416$

$1236+612$

1243-849

1247-194

$1249+092$

$1258-229$

$1303+684$

$1307+000$ 1324-300

1325-017

$1325+553$

1329-257

$1338+044$ $1343-416$

074051.07 075632.71 $\begin{array}{lll}08 & 04 & 12.75\end{array}$ $08 \quad 1411.63$ $08 \quad 1853.00$

$\begin{array}{lll}08 & 23 & 49.81\end{array}$ 084752.80 090915.00 $\begin{array}{llll}09 & 19 & 19.21\end{array}$ 093548.70

094109.50 094323.44 094557.80 095501.80 100300.80

$\begin{array}{lll}10 & 06 & 12.70\end{array}$ $10 \quad 1257.90$ 101357.00 101924.58 104316.40

110256.00

115335.90

115545.50

$1156 \quad 15.50$

120757.60

121023.60

122214.90

122701.50

$\begin{array}{lll}12 & 30 & 11.70\end{array}$

123300.84

123607.20

$1243 \quad 11.47$

124741.00

124911.00

$1258 \quad 17.34$

130320.20

$1307 \quad 15.97$

132455.00

132504.00

$1325 \quad 38.94$

132945.00

133850.10 134305.40
$-183533.0$ $-242831.0$ $\begin{array}{llll}02 & 19 & 00.0\end{array}$

201154.8 110648.0

$-034454.0$ 234419.5

214049.9

$-130340.0$

335947.0

$\begin{array}{lll}61 & 36 & 30.2\end{array}$

$\begin{array}{lll}21 & 45 & 50.9\end{array}$

302230.0

$\begin{array}{llll}-33 & 1153.0\end{array}$

$\begin{array}{lll}38 & 28 & 29.0\end{array}$
$-231029.0$

263750.0

$-401316.0$

$-012006.0$

204918.0

$\begin{array}{lll}-25 & 12 & 07.0\end{array}$ 180652.0 483755.0

$-413607.0$

$\begin{array}{lll}61 & 15 & 47.5\end{array}$

$-845607.5$

-1929 42.0

$\begin{array}{lll}09 & 1248.0\end{array}$

$-225606.8$

682443.0

000321.4

$-300228.0$

$-014736.0$

$\begin{array}{llll}55 & 19 & 37.4\end{array}$

$-254430.0$

042907.0

$-414109.0$

$\begin{array}{rrrrr}? ? . ? & 0.59 & 0.0 & 0 & 0 \\ ? ? . ? & 0.53 & 18.4 & 0 & 0 \\ 0.5 & 0.67 & 0.0 & 0 & 0 \\ 1.8 & 0.61 & 19.0 & 0 & 0 \\ 6.0 & 1.88 & 19.0 & 0 & 0\end{array}$

$\begin{array}{ll}0 & 0 \\ 0 & 0\end{array}$

50

190

50
18

9

$\begin{array}{rrrrrrrr}1.0 & 0.49 & 19.0 & 0 & 0 & & 14 & 14 \\ ? ? . ? & 0.97 & 0.0 & 0 & 0 & & 0 & 0 \\ 12.0 & 1.01 & 18.5 & 0 & 0 & * * & 33 & 3 \\ ? ? . ? & 1.86 & 0.0 & 0 & 0 & & 0 & 0 \\ ? ? . ? & 1.69 & 18.5 & 0 & 0 & & 0 & 0\end{array}$

$\begin{array}{llllllll}12.0 & 2.93 & 19.0 & 0 & 0 & * * & 123 & 123\end{array}$

$\begin{array}{lllllll}0.5 & 1.86 & 19.0 & 0 & 0 & 96 & 96\end{array}$

$\begin{array}{llllll}0.98 & 19.0 & 0 & 0 & 17 & 17\end{array}$

$\begin{array}{lllllll}18.0 & 0.65 & 17.9 & 0 & 0 & 117 & 117\end{array}$

$\begin{array}{llllllll}6.0 & 0.59 & 19.0 & 0 & 0 & * * & 128 & 128\end{array}$

$\begin{array}{lllllll}6.0 & 0.78 & 18.5 & 0 & 0 & 129 & 129\end{array}$

$\begin{array}{lllllll}5.0 & 1.04 & 18.3 & 0 & 0 & 99 & 99\end{array}$

$\begin{array}{rrrrrrr}? ? . ? & 0.52 & 0.0 & 0 & 0 & 0 & 0\end{array}$

$\begin{array}{rrrrrrr}0.5 & 0.59 & 18.8 & 0 & 0 & 50 & 50 \\ ? ? ? & 1.19 & 0.0 & 0 & 0 & 0 & 0\end{array}$

$\begin{array}{llllllll}6.0 & 0.59 & 18.0 & 0 & 0 & * * & 129 & 129\end{array}$

$\begin{array}{lllllll}0.5 & 1.05 & 17.5 & 0 & 0 & 55 & 55\end{array}$

$\begin{array}{llllllll}6.0 & 1.13 & 18.9 & 0 & 0 & * * & 51 & 51\end{array}$

$\begin{array}{llllllll}0.5 & 1.51 & 18.5 & 0 & 0 & * * & 101 & 101\end{array}$

$\begin{array}{llllllll}6.0 & 0.88 & 18.2 & 0 & 0 & * * & 9 & 9\end{array}$

$\begin{array}{llllllll}6.0 & 0.66 & 18.0 & 0 & 0 & * * & 0 & 0\end{array}$

$\begin{array}{lllllll}0.5 & 1.18 & 18.0 & 0 & 0 & 139 & 140\end{array}$

$\begin{array}{lllllll}2.0 & 0.64 & 18.5 & 0 & 0 & 141 & 81\end{array}$

$\begin{array}{rrrrrrrr}10.0 & 0.86 & 18.5 & 0 & 0 & * * & 4 & 4\end{array}$

$\begin{array}{lllllll}1.0 & 0.82 & 18.9 & 0 & 0 & 83 & 117\end{array}$

$\begin{array}{lllllll}0.5 & 0.74 & 18.2 & 0 & 0 & 50 & 50\end{array}$

$\begin{array}{lllllll}0.6 & 0.71 & 18.0 & 0 & 0 & 33 & 33\end{array}$

$\begin{array}{llllllr}\text { ??.? } & 0.71 & 18.9 & 0 & 0 & 0 & 0\end{array}$

$\begin{array}{rrrrrrr}12.0 & 1.79 & 18.5 & 0 & 0 & 6 & 6 \\ 1.2 & 1.09 & 18.7 & 0 & 0 & 114 & 114\end{array}$

$\begin{array}{lllllll}1.2 & 1.09 & 18.7 & 0 & 0 & 114 & 114\end{array}$

$\begin{array}{lllllll}6.0 & 0.66 & 19.0 & 0 & 0 & 129 & 129\end{array}$

$\begin{array}{lllllll}6.0 & 0.79 & 19.0 & 0 & 0 & 158 & 158\end{array}$

$\begin{array}{lllllll}14.0 & 0.79 & 18.5 & 0 & 0 & 16 & 16\end{array}$

$\begin{array}{llllllll}6.0 & 0.68 & 19.0 & 0 & 0 & & 51 & 51\end{array}$

$\begin{array}{llllllll}\text { ??? } & 1.35 & 18.5 & 0 & 0 & * & 0 & 0\end{array}$

$\begin{array}{lllllll}6.0 & 0.78 & 18.0 & 0 & 0 & 129 & 129\end{array}$

$\begin{array}{llllllll}10.0 & 1.01 & 19.0 & 0 & 0 & * & 130 & 130\end{array}$

$\begin{array}{lllll}? ? . ? & 0.67 & 0.0 & 0 & 0\end{array}$

$\begin{array}{lllll}1.0 & 1.54 & 17.5 & 0 & 0\end{array}$

$\begin{array}{lllll}\text { ??? } & 0.67 & 0.0 & 0 & 0\end{array}$

$\begin{array}{rr}0 & 0 \\ 79 & 2\end{array}$

$0 \quad 0$

$\begin{array}{llllll}1.8 & 1.13 & 19.0 & 0 & 0 & 190\end{array}$

$\begin{array}{llllllll}12.0 & 1.17 & 19.0 & 0 & 0 & * & 41 & 41\end{array}$

$\begin{array}{llllllll}6.0 & 1.69 & 19.0 & 0 & 0 & * & 9 & 9\end{array}$

$\begin{array}{lllllll}0.5 & 1.01 & 17.5 & 0 & 0 & 87 & 43\end{array}$

$\begin{array}{lllllll}? ? . ? & 0.82 & 0.0 & 0 & 0 & 0 & 0\end{array}$

$\begin{array}{llllllll}3.0 & 1.45 & 19.0 & 0 & 0 & * & 74 & 51\end{array}$

$\begin{array}{rrrrrrr}12.0 & 0.98 & 18.0 & 0 & 0 & 6 & 6\end{array}$

$\begin{array}{llllllll}12.0 & 1.34 & 18.5 & 0 & 0 & * & 41 & 41\end{array}$

$\begin{array}{lllll}? ? . ? & 0.67 & 0.0 & 0 & 0\end{array}$

$\begin{array}{lllll}12.0 & 1.57 & 18.0 & 0 & 0\end{array}$

$\begin{array}{ll}0 & 0 \\ 5 & 5\end{array}$

$\begin{array}{lllll}6.0 & 0.64 & 19.0 & 0 & 0\end{array}$

$131 \quad 131$

$18 \quad 18$ 


\begin{tabular}{|c|c|c|c|c|c|c|c|c|c|c|}
\hline $1353-341$ & 135310.10 & -340629.0 & ??.? & 1.08 & 18.5 & 0 & 0 & & 0 & 0 \\
\hline $1405-298$ & 140535.80 & -295011.5 & 1.0 & 0.98 & 18.0 & 0 & 0 & & 182 & 182 \\
\hline $1410-069$ & 141057.72 & -065607.6 & 0.5 & 1.13 & 19.0 & 0 & 0 & & 127 & 127 \\
\hline $1414-212$ & 141438.60 & -211259.0 & 12.0 & 1.10 & 18.0 & 0 & 0 & & 53 & 53 \\
\hline $1416-374$ & 141658.80 & -372953.0 & 14.0 & 1.42 & 18.0 & 0 & 0 & & 16 & 16 \\
\hline $1426+030$ & 142632.67 & 030510.0 & 1.0 & 0.71 & 18.5 & 0 & 0 & * & 191 & 74 \\
\hline $1436-167$ & 143642.00 & $-1646 \quad 12.0$ & 12.0 & 1.76 & 18.7 & 0 & 0 & & 73 & 73 \\
\hline $1447+402$ & 144704.71 & 401302.6 & ??.? & 0.45 & 18.4 & 0 & 0 & & 0 & 0 \\
\hline $449-129$ & 144951.50 & -125900.0 & 12.0 & 1.34 & 18.0 & 0 & 0 & & 5 & 5 \\
\hline $453+166$ & 145345.70 & 163858.0 & 3.0 & 0.95 & 17.5 & 0 & 0 & & 194 & 194 \\
\hline $1514+186$ & 151439.90 & 184120.3 & 0.5 & 1.22 & 19.0 & 0 & 0 & & 115 & 115 \\
\hline $1518+046$ & 151845.70 & 044048.0 & 6.0 & 3.89 & 19.0 & 0 & 0 & & 9 & 9 \\
\hline $1521+111$ & 152133.50 & 110617.0 & 3.0 & 0.95 & 18.5 & 0 & 0 & * & 194 & 131 \\
\hline $526-082$ & 152601.40 & -081607.0 & 10.0 & 0.78 & 19.0 & 0 & 0 & & 4 & 4 \\
\hline $529+700$ & 152905.30 & $\begin{array}{llll}70 & 05 & 07.0\end{array}$ & ??.? & 0.00 & 0.0 & 0 & 0 & & 0 & 0 \\
\hline $1543+019$ & 154303.70 & 015912.0 & 12.0 & 0.95 & 18.5 & 0 & 0 & * & 3 & 3 \\
\hline $1555+455$ & 155543.70 & 453100.0 & 10.0 & 0.00 & 0.0 & 0 & 0 & & 200 & 200 \\
\hline $1559+538$ & 155901.39 & 534804.3 & ??.? & 0.82 & 0.0 & 0 & 0 & & 0 & 0 \\
\hline $601-003$ & 160119.10 & -002142.0 & 6.0 & 0.76 & 17.5 & 0 & 0 & * & 51 & 51 \\
\hline $601+$ & 160129.19 & 525211.0 & 0.5 & 0.00 & 18.7 & 0 & 0 & * & 50 & 50 \\
\hline $1602+495$ & 160244.16 & 493537.3 & 0.5 & 0.59 & 0.0 & 0 & 0 & & 55 & 55 \\
\hline$* 1610+407$ & 161005.92 & 404759.4 & 0.5 & 0.59 & 18.7 & 0 & 0 & * & 168 & 168 \\
\hline $1614+473$ & 161414.85 & 471850.9 & ??.? & 0.67 & 0.0 & 0 & 0 & & 0 & 0 \\
\hline $1625-750$ & 162510.26 & -750235.6 & 1.8 & 0.81 & 18.0 & 0 & 0 & & 190 & 18 \\
\hline * $1654-137$ & 165421.70 & -134423.0 & 10.0 & 1.61 & 18.0 & 0 & 0 & * & 4 & 4 \\
\hline $1711-670$ & 171113.58 & -670304.1 & 1.8 & 0.74 & 18.5 & 0 & 0 & & 190 & 18 \\
\hline $1746+712$ & 174626.21 & $71 \quad 1649.6$ & 0.6 & 0.69 & 18.9 & 0 & 0 & * & 201 & 201 \\
\hline $1756-663$ & 175612.17 & -662246.2 & 1.8 & 1.78 & 19.0 & 0 & 0 & & 190 & 2 \\
\hline$* 1759+211$ & 175940.50 & 210925.1 & 0.7 & 0.88 & 17.5 & 0 & 0 & ** & 193 & 158 \\
\hline $1807+707$ & 180724.36 & 704413.5 & 0.6 & 0.00 & 17.2 & 0 & 0 & & 201 & 201 \\
\hline$* 1853+505$ & 185352.17 & 503139.8 & 0.5 & 0.67 & 18.6 & 0 & 0 & ** & 168 & 168 \\
\hline $1908+484$ & 190824.80 & $48 \quad 2938.0$ & ??.? & 0.52 & 0.0 & 0 & 0 & * & 50 & 50 \\
\hline $1913-$ & 191319.06 & $6206 \quad 13.4$ & ??.? & 0.82 & 0.0 & 0 & 0 & * & 0 & 0 \\
\hline $1918-355$ & 191835.80 & -353229.0 & 14.0 & 1.62 & 18.5 & 0 & 0 & & 16 & 16 \\
\hline$* 1922+690$ & 192224.39 & 690519.6 & 0.5 & 0.82 & 18.2 & 0 & 0 & ** & 168 & 168 \\
\hline $1939-406$ & 193948.17 & -403643.1 & 0.5 & 0.83 & 19.0 & 0 & 0 & & 87 & 87 \\
\hline $1943+546$ & 194322.28 & 544048.6 & ??.? & 1.49 & 0.0 & 0 & 0 & * & 0 & 0 \\
\hline $2016-438$ & 201659.70 & -435333.0 & 10.0 & 0.63 & 19.0 & 0 & 0 & & 18 & 18 \\
\hline $2041-551$ & $2041 \quad 09.49$ & -550654.5 & 1.8 & 1.12 & 18.5 & 0 & 0 & & 190 & 18 \\
\hline $2048-147$ & 204822.00 & -144624.0 & 12.0 & 1.86 & 19.0 & 0 & 0 & * & 26 & 26 \\
\hline$* 2053-201$ & $2053 \quad 12.50$ & -200806.0 & 6.0 & 2.71 & 17.8 & 0 & 0 & & 20 & 20 \\
\hline $2121+028$ & 212111.54 & 025152.2 & 0.4 & 0.61 & 19.0 & 0 & 0 & * & 66 & 66 \\
\hline $2130-425$ & 213050.80 & -423201.0 & ??.? & 0.63 & 18.5 & 0 & 0 & & 0 & 0 \\
\hline $2135-189$ & 213519.14 & $-1857 \quad 12.3$ & 1.2 & 2.50 & 19.0 & 0 & 0 & * & 0 & 26 \\
\hline *2146-016 & 214608.63 & -013636.3 & 0.9 & 0.66 & 18.0 & 0 & 0 & ** & 66 & 66 \\
\hline $2206-251$ & $2206 \quad 34.52$ & -250817.5 & 0.5 & 0.81 & 18.0 & 0 & 0 & & 32 & 32 \\
\hline $2213-456$ & 221352.36 & -453642.1 & 0.5 & 1.83 & 19.0 & 0 & 0 & & 17 & 17 \\
\hline $2222-405$ & 222257.20 & -403543.0 & 14.0 & 0.95 & 18.5 & 0 & 0 & & 16 & 16 \\
\hline $2234-253$ & 433.90 & -252221.0 & 6.0 & 1.01 & 19.0 & 0 & 0 & & 32 & 32 \\
\hline $2235-143$ & 223534.80 & -142138.0 & ??.? & 0.79 & 19.0 & 0 & 0 & * & 0 & 0 \\
\hline $2248+067$ & $2248 \quad 15.43$ & 064610.0 & 0.5 & 1.69 & 19.0 & 0 & 0 & ** & 127 & 127 \\
\hline $2251-324$ & 225148.64 & -322526.9 & 1.0 & 0.68 & 18.0 & 0 & 0 & & 182 & 6 \\
\hline $2303-008$ & $\begin{array}{llll}23 & 03 & 11.73\end{array}$ & -005222.2 & 0.7 & 0.63 & 18.5 & 0 & 0 & ** & 25 & 48 \\
\hline $2305+183$ & 230528.10 & $18 \quad 1846.0$ & 6.0 & 0.73 & 18.0 & 0 & 0 & ** & 131 & 131 \\
\hline $2308-048$ & 230817.00 & -045354.0 & 6.0 & 0.63 & 18.5 & 0 & 0 & ** & 32 & 32 \\
\hline $2313-182$ & 231308.57 & -181653.9 & 1.0 & 1.40 & 19.0 & 0 & 0 & ** & 0 & 26 \\
\hline $2313+124$ & 231309.00 & 122628.0 & 12.0 & 0.88 & 19.0 & 0 & 0 & ** & 43 & 43 \\
\hline $2313+012$ & $23 \quad 1343.79$ & 011229.8 & 0.6 & 1.05 & 19.0 & 0 & 0 & ** & 0 & 43 \\
\hline
\end{tabular}




\begin{tabular}{|c|c|c|c|c|c|c|c|c|c|c|}
\hline $2313-122$ & 231346.00 & -121346.0 & 10.0 & 1.94 & 19.0 & 0 & 0 & $* *$ & 4 & * \\
\hline $2318-195$ & $2318 \quad 11.50$ & -193545.0 & 6.0 & 0.88 & 19.0 & 0 & 0 & ** & 32 & 32 \\
\hline $2322-052$ & 232245.00 & -051424.0 & 12.0 & 1.22 & 18.5 & 0 & 0 & ** & 123 & 123 \\
\hline $2324-259$ & 232400.20 & -255822.0 & 6.0 & 0.71 & 18.0 & 0 & 0 & & 32 & 32 \\
\hline $2324-023$ & 232419.48 & $-02 \quad 1844.6$ & 0.8 & 2.66 & 18.0 & 0 & 0 & $* *$ & 0 & 26 \\
\hline $2326-196$ & 40 & -193929.0 & 6.0 & 1.22 & 18.5 & 0 & 0 & $* *$ & 32 & 2 \\
\hline $2334+085$ & 233407.40 & 083318.0 & 2.0 & 1.10 & 18.5 & 0 & 0 & $* *$ & 23 & 43 \\
\hline $2338+000$ & 233832.70 & 000154.0 & 6.0 & 0.63 & 19.0 & 0 & 0 & $* *$ & 51 & 51 \\
\hline $2354-320$ & 235406.10 & -320548.0 & 10.0 & 0.61 & 19.0 & 0 & 0 & & 18 & \\
\hline
\end{tabular}

Number of adequate spectra: $16(8 \%)$

Number of I frames: $64(35 \%)$

Number of B frames: 41 ( $22 \%)$ 
Table A1.4: Definite Sample Non-Members

*0005-199

*0038+086

*0039+211

*0040-065

${ }^{*} 0059+144$

*0124-117

*0155-212

*0213-132

* $0213+025$

*0226-284

0245-044

*0246-135

*0254+064

*0301-122

*0305-226

*0309-316

*0326-288

0344-291

*0357-247

*0402+179

*0420-263

*0424-268

*0442-282

*0509+011

*0521-328

*0522-483

$* 0530+040$

*0533-120

*0533-512

*0534-497

*0600-131

*0611-254

*0647+693

*0649+485

*0651-603

*0657+687

*0705-502

${ }^{*} 0732+182$

*0810+665

$0811+398$

*0811+131

*0814+547

*0820-047

*0829+187

* $0837+613$

${ }^{*} 0845+061$

${ }^{*} 0849+548$

*0850-034

*0851+071

*0854-034

$0858+299$

*0936+361

*0944+624

*0952-306

*0958-314

$$
\alpha_{o p t}(1950)
$$

$\begin{array}{ll}00 & 0542.90\end{array}$

$\begin{array}{lll}0 & 38 & 17.00\end{array}$

$\begin{array}{llll}00 & 39 & 02.88\end{array}$

$00 \quad 4014.32$

005927.42

012407.08

015532.15

$\begin{array}{llll}02 & 13 & 12.00\end{array}$

$\begin{array}{llll}02 & 13 & 59.77\end{array}$

$02 \quad 2617.40$

024538.60

024616.00

025431.71

030136.15

030518.10

$\begin{array}{llll}03 & 09 & 49.40\end{array}$

032631.50

034458.00

035749.70

040227.60

042030.00

042437.00

044238.00

050921.90

052141.70

$05 \quad 2200.92$

053025.50

053313.00

$\begin{array}{lll}05 & 33 & 15.27\end{array}$

053458.22

060049.50

061131.50

064749.00

$06 \quad 4900.41$

065115.93

065735.02

070543.82

073213.78

$\begin{array}{lll}08 & 10 & 10.13\end{array}$

081153.88

081155.50

081412.02

082006.60

082924.51

$08 \quad 3714.99$

$0845 \quad 57.00$

084922.24

085057.36

085108.54

085440.90

$\begin{array}{llll}08 & 58 & 10.97\end{array}$

093650.50

094453.32

095219.30

095827.50

$$
\delta_{o p t}(1950)
$$

$-195626.0$

083730.0

210740.6

$-063019.4$

142718.8

$$
\pm^{\prime \prime} \quad S_{1.4}
$$

m

$c z$

$\pm \quad \operatorname{ccd}$

Refs

$-114656.1$

$\begin{array}{lll}1.0 & 0.76 & 17.0\end{array}$

$\begin{array}{lll}0.2 & 1.35 & 17.5\end{array}$

$\begin{array}{lll}0.4 & 0.69 & 16.5\end{array}$

$\begin{array}{lll}0.8 & 1.05 & 17.0\end{array}$

$\begin{array}{lll}0.5 & 1.56 & 18.5\end{array}$

35226

38673

30489

36815

56361

$\begin{array}{rl}150 & * \\ 300 & * * \\ 90 & * \\ 240 & * * \\ 150 & \end{array}$

8
5
33
0
42

$\begin{array}{lll}0.8 & 0.79 & 17.5\end{array}$

$-211653.7$

$-131330.0$

$\begin{array}{lll}1.4 & 0.68 & 17.0\end{array}$

$\begin{array}{lll}12.0 & 4.90 & 18.5\end{array}$

023022.0

$\begin{array}{lll}1.7 & 0.64 & 18.0\end{array}$

37054

48596

44789

43410

180 **

150

180

240

$\begin{array}{lll}6.0 & 0.64 & 18.0\end{array}$

62777

210

$\begin{array}{lllll}6.0 & 0.59 & 17.5 & 40771 & 300\end{array}$

$300 \quad 32 \quad 32$

-04 2743.0

$12.0-1.05$

40172

062602.4

$-121739.6$

$1.51 \quad 17.5$

39723

$\begin{array}{llll}0.7 & 0.74 & 17.0 & 30279\end{array}$

$\begin{array}{lll}10.0 & 1.76 \quad 18.5\end{array}$

80914

400

210

150

300

$\begin{array}{lll}12.0 & 1.05 & 18.5\end{array}$

76687

$\begin{array}{lll}10.0 & 1.40 & 17.5\end{array}$

??? $0.83 \quad 17.0$

$\begin{array}{lll}10.0 & 0.74 & 18.5\end{array}$

32917

39722

150

$-290938.0$

$-244225.0$

175812.0

$\begin{array}{lll}6.0 & 0.79 & 18.0\end{array}$

30759

33397

$-262330.0$

$\begin{array}{lll}18.0 & 1.40 & 18.5\end{array}$

39603

$-265042.0$

$-281518.0$

010705.1

$\begin{array}{lll}6.0 & 1.01 & 17.0\end{array}$

$\begin{array}{lll}6.0 & 6.26 & 18.5\end{array}$

809

$\begin{array}{lll}0.6 & 0.66 & 18.0\end{array}$

44369

36574

$-325359.0$

$\begin{array}{lll}12.0 & 0.98 & 18.0\end{array}$

63766

$\begin{array}{lllll}1.8 & 1.03 & 18.5 & 57170 & 180\end{array}$

$-481913.3$

040348.0

$-120430.0$

$-511507.0$

$\begin{array}{lll}12.0 & 2.01 & 19.0\end{array}$

$\begin{array}{lll}12.0 & 1.35 & 17.8\end{array}$

44519

46768

33097

$\begin{array}{lll}0.8 & 1.69 & 18.5\end{array}$

55162

150

1200

600

240

130

600

150

300

300

180
300

300

300

600

$\begin{array}{lllll}12.0 & 1.27 & 18.0 & 44489 & 300\end{array}$

$\begin{array}{lllll}18.0 & 1.07 & 18.0 & 52973 & 300\end{array}$

$\begin{array}{lllll}\text { ?? ? } & 1.78 & 0.0 & 32827 & 60\end{array}$

$-252948.0$

692325.0

483509.3

$\begin{array}{lll}0.5 & 0.52 & 18.7\end{array}$

$50455 \quad 150$

$-601829.6$

$\begin{array}{lll}1.8 & 1.39 & 18.0\end{array}$

40172

300

$6845 \quad 36.6$

$\begin{array}{llll}0.5 & 1.49 & 16.9 & 32498\end{array}$

$\begin{array}{llll}0.6 & 1.01 & 18.5 & 119617\end{array}$

$\begin{array}{llll}0.6 & 0.85 & 18.0 & 42211\end{array}$

181458.7

$\begin{array}{lll}66 & 35 & 47.9\end{array}$

$\begin{array}{ll}1.0 & 0.52\end{array}$

$0.4 \quad 0.56$

18.7

43200

39573

180

180

240

120

130724.0

130724.0

544628.8

$-044712.0$

184224.5

612316.8

$\begin{array}{lll}6.0 & 1.86 & 18.0\end{array}$

$\begin{array}{lll}0.5 & 0.59 & 17.7\end{array}$

42870

$\begin{array}{llll}10.0 & 0.85 & 18.0 & 36725\end{array}$

$\begin{array}{lll}0.5 & 1.44 & 18.0\end{array}$

45868

$\begin{array}{lll}0.5 & 1.19 & 18.6\end{array}$

39212

300

90

600

150

300

060612.0

544825.5

$-032943.2$

070620.0

$-032803.0$

$\begin{array}{lllll}6.0 & 1.18 & 18.2 & 37174 & 24\end{array}$

$\begin{array}{lllll}0.5 & 0.59 & 18.6 & 33367 & 180\end{array}$

$\begin{array}{lll}0.4 & 1.34 & 18.0\end{array}$

41671

$? ? ? \quad 1.18 \quad 18.5$

44969

300

600

$? ? . ? \quad 1.13 \quad 18.0$

51714

300

$\begin{array}{lllll}1.1 & 0.47 & 17.9 & 58220 & 0\end{array}$ 


\begin{tabular}{|c|c|c|c|c|c|c|c|c|c|c|}
\hline$* 1019+083$ & 101912.57 & 082340.7 & 1.0 & 0.66 & 17.0 & 30789 & 240 & $* *$ & 0 & 130 \\
\hline * $1024-070$ & 102459.30 & -070328.0 & 10.0 & 0.64 & 18.5 & 48566 & 210 & $* *$ & 4 & 4 \\
\hline$* 1036+058$ & 103652.00 & 055156.0 & 6.0 & 0.59 & 18.0 & 36874 & 300 & $* *$ & 131 & 35 \\
\hline$* 1107-372$ & 110735.50 & -371548.0 & 6.0 & 0.69 & 12.1 & 2878 & 150 & $*$ & 134 & \\
\hline $1125+260$ & 112526.70 & 260124.0 & ??? & 0.51 & 17.3 & 2698 & 0 & $* *$ & 0 & 0 \\
\hline$* 1125+588$ & 112541.94 & 585020.6 & 1.3 & 1.04 & 11.8 & 2971 & 45 & $* *$ & 0 & 50 \\
\hline$* 1127+012$ & 112747.51 & 011456.6 & 0.4 & 0.76 & 17.8 & 39633 & 270 & & 68 & 51 \\
\hline $1141+374$ & 114143.02 & 372406.3 & ??.? & 0.88 & 18.1 & 34476 & 0 & & 0 & 0 \\
\hline *1146-117 & 114633.64 & -114752.7 & 0.5 & 1.45 & 18.3 & 35065 & 300 & & 87 & 41 \\
\hline *1148-353 & 114844.90 & -352010.0 & 14.0 & 1.30 & 18.0 & 37804 & 90 & & 16 & 16 \\
\hline $1151+099$ & 115114.24 & $0958 \quad 10.9$ & 0.8 & 0.61 & 16.5 & 30459 & 210 & & 0 & 9 \\
\hline$* 1159+583$ & 115930.50 & $58 \quad 1848.9$ & $? ? . ?$ & 1.11 & 17.4 & 30459 & 120 & & 0 & 0 \\
\hline *1201-041 & 120129.00 & -040606.0 & 12.0 & 2.23 & 18.0 & 31778 & 300 & * & 26 & 26 \\
\hline *1215-457 & 121527.37 & -454349.3 & 1.0 & 5.58 & 18.0 & 158021 & 150 & & 142 & 142 \\
\hline$* 1221-423$ & 122103.84 & -421855.3 & 1.0 & 2.74 & 17.0 & 51354 & 300 & & 142 & 20 \\
\hline * $1231+674$ & 123103.69 & 672415.4 & ??.? & 0.97 & 17.7 & 32228 & 180 & & 0 & 0 \\
\hline *1254-268 & 125447.10 & -265132.0 & 6.0 & 0.61 & 17.5 & 40232 & 400 & & 129 & 129 \\
\hline * $1301-086$ & 130141.80 & -083817.0 & $? ? . ?$ & 0.74 & 16.5 & 30249 & 270 & ** & 0 & 0 \\
\hline $1304-215$ & 130401.70 & -213142.0 & 10.0 & 0.78 & 17.5 & 37800 & 0 & & 4 & 4 \\
\hline *1411+094 & 141133.00 & 092918.0 & 6.0 & 1.01 & 18.3 & 46468 & 700 & * & 9 & 9 \\
\hline$* 1423-177$ & 142311.60 & -174316.0 & 10.0 & 1.18 & 18.0 & 32078 & 300 & * & 4 & 4 \\
\hline$* 1517-283$ & 151706.00 & -282337.0 & 10.0 & 1.03 & 17.5 & 36725 & 300 & & 4 & 4 \\
\hline$* 1538+082$ & 153803.54 & 081442.6 & 0.5 & 0.93 & 16.5 & 37924 & 210 & * & 55 & 130 \\
\hline $1636+$ & 163615.90 & 375848.0 & 4.0 & 0.51 & 18.3 & 48057 & 1500 & $* *$ & 102 & 102 \\
\hline$* 1643+022$ & 164311.40 & $02 \quad 17 \quad 12.0$ & ??.? & 1.94 & 17.0 & 450 & 120 & * & 0 & 0 \\
\hline$* 1839-486$ & 183926.18 & -483923.5 & 1.0 & 3.38 & 17.5 & 32677 & 240 & & 64 & 64 \\
\hline$* 2049+175$ & 204917.30 & 173210.0 & $? ? . ?$ & 0.74 & 18.0 & 33705 & 300 & $* *$ & 130 & 130 \\
\hline${ }^{*} 2056-369$ & 205632.70 & -365735.0 & 14.0 & 0.86 & 18.0 & 32305 & 240 & & 16 & 16 \\
\hline$* 2123+007$ & 212313.00 & 004248.0 & 6.0 & 0.64 & 17.5 & 40322 & 270 & $* *$ & 9 & 9 \\
\hline$* 2225+100$ & 222547.60 & 100457.9 & 0.8 & 0.83 & 17.5 & 40322 & 150 & * & 0 & 130 \\
\hline$* 2254-367$ & 225422.90 & -364352.0 & 14.0 & 1.39 & 11.3 & 1709 & 150 & & 16 & \\
\hline$* 2320+417$ & 232020.40 & 414205.0 & $? ? . ?$ & 0.59 & 0.0 & 44519 & 240 & & 0 & 0 \\
\hline *2338- & 233826.20 & -001148.0 & 6.0 & 0.63 & 17.2 & 31029 & 0 & ** & 51 & 51 \\
\hline
\end{tabular}

Number of adequate spectra: 80 ( $90 \%)$

Number of I frames: 40 ( $45 \%)$

Number of B frames: 28 ( $31 \%)$ 


\section{A1.2 Numbered References}

1. Wall, J.V. \& Cannon, R.D., 1973. Austr. J. Phys. Astrophys. Suppl., 31.

2. Savage, A., 1975. Mon. Not. astr. Soc., 174, 259.

3. Bolton, J.G. \& Ekers, J., 1966. Austr. J. Phys., 19, 471.

4. Bolton, J.G., Shimmins, A.J. \& Wall, J.V., 1975. Austr. J. Phys., 19, 471.

5. Bolton, J.G., Shimmins, A.J. \& Merkelijn, J., 1968. Austr. J. Phys., 21, 81.

6. Shimmins, A.J. \& Bolton, J.G., 1974. Austr. J. Phys., Astrophys. Suppl., 32.

7. Shaver,P. private communcication.

8. Mills, B.Y. \& Hoskins, D.G., 1977. Austr. J. Phys., 30, 9.

9. Clarke, M.E., Bolton, J.G. \& Shimmins, A.J., 1966. Austr. J. Phys., 19, 375.

10. Schaffer, D.B., 1978. Astron. J. 83, 209.

11. Véron, M.P. \& Véron, P., 1975. Astron. Astrophys., 42, 1.

12. Pauliny-toth, I.I.K., Kellerman, K.I., Davis, M.M., Fomalont, E.B., Schaffer, D., 1972. Astron. J. 77, 265.

13. Bolton, J.G. \& Savage, A., 1977. Austr. J. Phys., Astrophys. Suppl. 41.

14. Rudnick, L. \& Adams, M.T., 1979. Astron. J., 84, 437.

15. Callahan, P.S., 1977. Astron. Astrophys., 55, 73.

16. Bolton, J.G. \& Shimmins, A.J., 1973. Austr. J. Phys., Astrophys. Suppl., 30.

17. Savage, A., Bolton, J.G. \& Wright, A.E., 1976. Mon. Not. astr. Soc., 175, 517.

18. Savage, A., Bolton, J.G. \& Wright, A.E., 1977. Mon. Not. astr. Soc., 179, 135.

19. Westurland, B.E. \& Smith, L.F., 1965. Austr. J. Phys., 19, 181.

20. Bolton, J.G., Clarke, M.E. \& Ekers, J.,1965. Austr. J. Phys., 18, 627.

21. Day, G.A., Shimmins, A.J., Ekers, R.D. \& Cole, D.J., 1966. Austr. J. Phys., 19, 35.

22. Whiteoak, J.B., 1972. Austr. J. Phys., 25, 233.

23. Hunstead, R.W. \& Jauncey, D.L., 1970. Mon. Not. astr. Soc., 149, 91.

24. Wills, D. \& Wills, B.J., 1976. Astrophys. J., Suppl., 31, 143.

25. Ghigo, F.D., 1977. Astrophys. J., 35, 359.

26. Bolton, J.G. \& Ekers, J., Austr. J. Phys., 19, 559.

27. Burbridge, E.M., 1967. Astrophys. J., 149, L51.

28. Lang, K.R., Sutton, J., Hazard, C. \& Gulgis, S., 1970. Astrophys. J., 160, 17.

29. Westurland, B.E. \& Wall, J.V., 1969. Astron. J., 74, 335. 
30. Wills, D., 1967. Astrophys. J., 148, L57.

31. Wills, B.J., 1965. Austr. J. Phys., Astrophys. Suppl., 38.

32. Wall, J.V., Bolton, J.G., Wright, A.E., Savage, A. \& Hagen, J.V., 1976. Austr. J. Phys., Astrophys. Suppl., 39.

33. Bolton, J.G., Trett, J., Carignan, C. \& Binette, L., 1981. Austr. J. Phys., 34, 445.

34. Rudnick, L. \& Owen, F.N., 1976. Astrophys. J., 203, L107.

35. Bolton, J.G., Wall, J.V. \& Shimmins, A.J., 1971. Austr. J. Phys., 24, 889.

36. de Vaucouleurs, G., de Vaucouleurs, A. \& Corwin, H. 1976, Second Reference Catalogue of Bright Galaxies (Austin: University of Texas Press)

37. Downes, A.J.B., Peacock, J.A., Savage, A. \& Carrie, D.R., 1986. Mon. Not. astr. Soc., 218,31 .

38. Melnick, J. \& Quintana, H., 1981. Astron. J., 86, 1567.

39. Andernach, H., Waldthausen, H. \& Wielebinski, R., 1980. Astron. Astrophys., Suppl. , 41, 339 .

40. Colla, G., Fanti, C., Giola, I., Lari, C., Lequeux, J., Lucas, R., Ulrich, M.H., 1975. Astron. Astrophys., Suppl., 20, 1.

41. Bolton, J.G. \& Ekers, J., 1967. Austr. J. Phys., 20, 109.

42. Wills, B.J. \& Wills, D., 1979. Astrophys. J., Suppl. , 41, 689.

43. Merkelijn, J.K., 1969. Austr. J. Phys., 22, 237.

44. Anguita, C. \& Pedreros, M., 1977, Astron. J., 82, 102.

45. Jauncey, D.L., Wright, A.E., Peterson, B.A. \& Condon, J.J., 1978. Astrophys. J., 219, L1.

46. Burns, J.A. \& Owen, F.N., 1979. Astron. J., 84,1478.

47. Humason, M.L., Mayall, N.U. \& Sandage, A.R., 1956. Astron. J., 61, 97.

48. Wall, J.V., 1971. Austr. J. Phys., Astrophys. Suppl., 20.

49. Simkin, S.M. \& Ekers, R.D., 1979. Astron. J., 84, 56.

50. Porcas, R.W., Urry, C.M., Browne, I.W.A., Cohen, A.M., Daintree, E.J., Walsh, D., 1980 Mon. Not. astr. Soc., 191, 607.

51. Merkelijn, J.K. \& Wall, J.V., 1970. Austr. J. Phys., 23, 575.

52. Wills, D. \& Wills, B.J., 1971. Astrophys. J., Suppl., 31, 143.

53. Merkelijn, J.K., 1972. Austr. J. Phys., 25, 451.

54. Ulrich, M., 1976. Astrophys. J., 206, 364. 
55. Bozyan, E.P., 1979. Astron. J., 84, 910.

56. Goodson, R.E., Palimaka, J.J. \& Briddle, A.H., 1979. Astron. j., 84, 1111.

57. Wallace, L.W. Sargent, 1973. Astrophys. J., 182, L13.

58. Griffin, R.F., 1963. Astron. J., 68, 421.

59. Zwicky, F. \& Humason, M.L., 1964. Astrophys. J., 139, 269.

60. McHardy, I.M., 1978. Mon. Not. astr. Soc., 185, 927.

61. Tritton, K.P., 1972. Mon. Not. astr. Soc., 158, 277.

62. Shaffer, D.B., 1978. Astron. J., 83, 215.

63. Mills, B.Y. \& Hoskins, D.G., 1977. Austr. J. Phys., 30, 509.

64. Tritton, K.P. \& Whitworth, D.P.D., 1973. Mon. Not. astr. Soc., 165, 253.

65. Wright, A.E., Ables, J.G. \& Allan, D.A., 1983. Mon. Not. astr. Soc., 84, 793.

66. Savage,A., Bolton, J.G. \& Trett, J., 1982. Austr. J. Phys., 35, 207.

67. Savage, A. \& Wright, A.E., 1981. Mon. Not. astr. Soc., 196, 927.

68. Palimaka, J.J., Bridle, A.H. \& Folomont, E.B., 1980. Astron. J., 85, 995.

69. Fosbury, R.A.E., Boksenberg, A., Danziger, I.J., Disney, M.J., Goss, W.M., Penston, M.V., Wamsteker, W., Wellington, K.J. \& Wilson, A.S. 1982. Mon. Not. astr. Soc., 201, 991.

70. Fanti, C., Fanti, R., Giola, I.M., Lari, C., Parma, P., Ulrich, M.H., 1977. Astron. Astrophys., Suppl., 29, 279.

71. Marenbach, G. \& Appenzeller, I., 1982. Astron. Astrophys., 108, 95.

72. Fomalont, F.B. \& Bridle, A.H., 1978. Astron. J., 83, 725.

73. Bolton, J.G. \& Ekers, J., 1966. Austr. J. Phys., 19, 559.

74. McEwan, N.J., Browne, I.W.A. \& Crowther, J.H., 1975. Mem R. astr. Soc., 80, 1.

75. Tritton, K.P. \& Schilizzi, R.T., 1973. Mon. Not. astr. Soc., 165, 245.

76. Hunstead,R.W., Lasker, B.M., Mintz, B. \& Smith, M.G., 1971. Austr. J. Phys., 24, 601.

77. Christiansen, W.N., Frater, R.H., Watkinson, A., O'Sullavan, J.D., Lockhart,I.A., Goss, W.M., 1977. Mon. Not. astr. Soc., 181, 183.

78. Maltby, P., Matthews, T.A. \& Moffet, A.T., 1963. Astrophys. J., 137, 153.

79. Northover, K.J.E., 1973. Mon. Not. astr. Soc., 165, 369.

80. Olsen, E.T., 1970. Astron. J., 75, 764.

81. Merkrlijn, J.K., 1968. Austr. J. Phys., 21, 903. 
82. Padrielli, L. \& Conway, R.G., 1977. Astron. Astrophys., suppl. , 27, 171.

83. Katgert-Merkelijn, J., Lari, C., Pandrielli,L., 1980. Astron. Astrophys., Suppl. , 40, 91.

84. Grueff, G. \& Vigotti, M., 1973. Astron. Astrophys., Suppl., 11, 41.

85. Burbridge, M.E. \& Strittmatter, P.A., 1972. Astrophys. J.. 172, L37.

86. Schilizzi, R.T. \& McAdam, W.B., 1975. Mem R. astr. Soc., 79, 1.

87. Schilizzi, R.T., 1975. Mem R. astr. Soc., 79, 75.

88. Peterson, B.A., 1970. Astron. J., 75, 695.

89. Wyndham, J.D., 1966. Astrophys. J., 144, 459.

90. Sandage, A., 1966. Astrophys. J., 145, 1.

91. Harris, A., 1973. Mon. Not. astr. Soc., 163, 19P.

92. Simon, A.J.B., 1979. Mon. Not. astr. Soc., 188, 637.

93. Wilkinson, A., Hine, R.G. \& Sargent, W.L.W., 1981. Mon. Not. astr. Soc., 196, 669.

94. Wilson, M.A.G., 1972. Mon. Not. astr. Soc., 156, 7.

95. Danziger, I.J. \& Goss, W.M., 1983. Mon. Not. astr. Soc.. 202, 703.

96. Hunstead, R.W., 1971. Mon. Not. astr. Soc., 152, 277.

97. Mayer, C.J., 1979. Mon. Not. astr. Soc., 186, 99.

98. Danziger, I.J. \& Goss, W.M., 1979. Mon. Not. astr. Soc., 186, 93.

99. Widram, M.D. \& Kenderdine, S., 1969. Mon. Not. astr. Soc., 146, 265.

100. Masson, C.R., 1979. Mon. Not. astr. Soc., 187, 253.

101. Hoskins, D.G., Murdoch, H.S., Adgie, R.L., Crowther, J.H. \& Gent, H., 1974. Mon. Not. astr. Soc., 166, 235.

102. Riley, J.M., 1975. Mon. Not. astr. Soc., 170, 53.

103. Caswell, J.L. \& Wills, D., 1967. Mon. Not. astr. Soc., 135, 231.

104. Dickens, R.J. \& Moss, C., 1967. Mon. Not. astr. Soc., 174, 47.

105. Gregory, S.A. \& Burns, J.O., 1982. Astrophys. J., 255, 373.

106. Schmidt, M. \& Greenstein, J.L., 1964. Astrophys. J., 140, 1.

107. Anguita, C., Campusano, L.E., Torres, C. \& Pedreros, M., 1979. Astron. J., 84, 718.

108. Grueff, G., Kotanyi, C., Schiavo-campo, P., Tanzella-Nitti, G. \& Vigotti, M., 1981. Astron. Astrophys, Suppl. , 44, 241.

109. Fanti, R., Gioia, I., Lari, C. \& Ulrich, M.H., 1978. Astron. Astrophys., Suppl. , 34, 341. 
110. Fricke, K.J., Kollatschny, W. \& Witzel, A., 1983. Astron. Astrophys., 117, 60.

111. Sandage, A., 1978. Astron. J., 83, 904.

112. Arp, H. \& Visvanathan, N., 1970. Ast. Lett., 5, 73.

113. Wills, D. \& Wills, B.J., 1974. Astrophys. J., 190, 271.

114. Machalski, J., Maslowski, J., Condon, J.J. \& Condon, M.A., 1982. Astron. J., 87, 1150.

115. Wills, B.J., Wills, D. \& Douglas, J.N., 1973. Astron. J., 78, 521.

116. Kühr, H., 1977. Astron. Astrophys., Suppl., 29, 139.

117. Grueff, G. \& Vigotti, M., 1972. Astron. Astrophys., Suppl. , 6, 1.

118. Gregorini et. al. Bologna preprint 1988.

119. Kühr, H., 1980. PhD. Thesis, Bonn.

120. Condon, J.J., Hicks, P.D. \& Jauncey, D.L., 1977. Astron. J., 82, 692.

121. Peterson, B.A., Wright, A.E., Jauncey, D.L. \& Condon, J.J., 1979. Astrophys. J., 232,400 .

122. Fosbury, R.A.E., 1977. Mon. Not. astr. Soc., 179, 89.

123. Bolton, J.G. \& Ekers, J., 1966. Astrophys. J., 19, 713.

124. Sandage, A., 1967. Astrophys. J., 150, L145.

125. Véron, P., 1977. Astron. Astrophys., Suppl., 30, 131.

126. Demoulin, M., 1970. Astrophys. J., 160, L79.

127. Wills, B.J., 1976. Astron. J., 81, 1031.

128. Savage, A. \& Bolton, J.G., 1979. Austr. J. Phys., Astrophys. Suppl., 46, 19.

129. Bolton, J.G. \& Savage, A., 1977. Austr. J. Phys., Astrophys. Suppl., 44.

130. Shimmins, A.J., Bolton, J.G. \& Wall, J.V., 1975. Austr. J. Phys., 22, 775.

131. Wills, D. \& Bolton, J.G., 1969. Austr. J. Phys., 22, 775.

132. De Ruiter, H.R., Willis, A.G. \& Arp, H.C., 1977. Astron. Astrophys., Suppl., 28, 211.

133. Véron, M.P., Véron, P., Adgie, R.L. \& Gent, H., 1976. Astron. Astrophys., 47, 401.

134. Whiteoak, J.B., 1970. Astrophys. Lett., 5, 29.

135. Valentijn, E.A., 1979. Astron. Astrophys., Suppl., 38, 319.

136. Rudnick, L. \& Owen, F.N., 1977. Astron. J., 82, 1.

137. Bolton, J.G. \& Wall, F.N., 1970. Austr. J. Phys., 23, 789.

138. Fanti, C., Fanti, R., Feretti, L., Ficarra, A., Giola, I.M., Giovannini, G., Gregorini, L., Mantovini, F., Marano, B., Padrielli, L., Parma, P., Thomassi, P. \& Vettolani, G., 
1982. Astron. Astrophys., 105, 200.

139. Kristian, J. \& Sandage, A., 1977. Astrophys. J., 162, 391.

140. Kristian, J., Sandage, A. \& Katem, B., 1978. Astrophys. J., 219, 803.

141. Owen,F.N., Rudnick, L. \& Peterson, B.M., 1977. Astron. J., 82, 677.

142. Lu, P.K., 1970. Astron. J., 75, 1167.

143. Slingo, A., Mebold, M., Goss, W.M. \& van Woerden, H., 1974. Mon. Not. astr. Soc., $166,101$.

144. Tarenchi, M. \& Scott, J.S., 1976. Astrophys. J., 207, L9.

145. Peterson, S.D., 1973. Astron. J., 78, 811.

146. Sargent, W.L.W., 1972. Astrophys. J., 173, 7.

147. Ulrich, M.J., 1971. Astrophys. J., 163, 441.

148. Owen, N. \& Rudnick, L., 1976. Astrophys. J., 205, L1.

149. Webber, J.C., 1974. Pub.A.S.P., 86, 223.

150. Coleman, G.D., Hintzen, P., Scott, J.S. \& Tarenghi, M. 1976. Nature, 262, 476.

151. Bijleveld, W. \& Valentijn, E.A., 1982. Astron. Astrophys., 111, 50.

152. Giovanni et. al. 1988. Bologna preprint.

153. Gavazzi, G., 1979. Astron. Astrophys., 72, 1.

154. Schmidt, M., 1965. Astrophys. J., 141, 1.

155. Gedehus, D.H., 1976. Astrophys. J., 208, 267.

156. Kapahi, V.K. 1981. Astron. Astrophys., Suppl., 43, 381.

157. Véron, M.P., 1971. Astron. Astrophys., Suppl., 11, 1.

158. Merkelijn, J.K., Shimmins, A.J. \& Bolton, J.G., 1968. Austr. J. Phys., 21, 523.

159. Condon, J.J., Jauncey, D.L. \& Wright, A.E., 1978. Astron. J., 83, 1036.

160. Fomalont, E.B., Palimaka, J.J. \& Bridle, A.H., 1980. Astron. J., 85, 981.

161. Searle, L. \& Bolton, J.G., 1968. Astrophys. J., 154, L101.

162. Bolton, J.G. \& Ekers, J., 1966. Austr. J. Phys., 19, 275.

163. Burbridge, E.M. \& Burbridge, G.R., 1972. Astrophys. J., 172, 37.

164. Johnson, K.H., 1974. Astron. J., 79, 1006.

165. Danziger, I.J., Fosbury, R.A.E., Goss, W.M. \& Ekers, R.D., Mon. Not. astr. Soc., 174. 259.

166. Ekers, R.D., 1970. Austr. J. Phys., 23, 217.

167. Minskowski, R., 1961. Astron. J., 66, 558. 
168. Cohen, A.M., Porcas, R.W., Browne, I.W.A., Daintree, E.J. \& Walsh, D., 1977. Mem. R. Ast. Soc., 84, 1 .

169. Weedman, D.W. \& Khachikyan, E.E., 1968. Astrophysics, 4, 243.

170. Kronberg, P.P. \& Biermann, P., 1981. Astrophys. J., 243, 89.

171. Bajaja, E., 1970. Astron. J., 75, 667.

172. Lu, P.K., 1974. Astron. J., 79, 453.

173. Bailey, J.A. \& Pooley, G.G., 1968. Mon. Not. astr. Soc., 138, 51.

174. Edwards, T., Kronberg, P.P. \& Menard, G., 1975. Astron. J., 80, 1005.

175. Peterson, B.M., Craine, E.R. \& Strittmatter, P.A. 1978. PASP, 90, 386.

176. Burbridge, M.E., Smith, M.E. \& Burbridge, G.R., 1975. Astron. J., 199, L137.

177. Burbridge, E.M., 1970. Astrophys. J., 160, L33.

178. Ulrich, M., 1978. Astrophys. J., 221, 422.

179. Michalec, A. \& Roman, G., 1976. ACTA Cosm., 4, 19.

180. Vidal, N.V., 1975. Astron. Astrophys., 42, 145.

181. Greenstein, J.L., 1961. Astrophys. J., 133, 335.

182. Jauncey, D.L., Batty, M.J., Gulkis, S. \& Savage, A., 1982. Astron. J., 87, 763.

183. Strittmatter, P.A., Carswell, R.F., Gilbert, G. \& Burbridge, E.M., 1974. Astrophys. J., 190, 509.

184. Hazard, C., Jauncey, D.L. \& Barker, D.C., 1970. Astron. J., 75, 1039.

185. Goss, W.M., Wellington, K.J., Christiansen, W.N., Lockhart, I.A., Watkinson, A., Frater, R.H. \& Little, A., 1977. Mon. Not. astr. Soc., 178, 525.

186. Evans, D.S., 1963. MNASSA, 22, 140.

187. Chicarni, G., Rood, H.J., Sastry, G.N. \& Welch, G.A., 1971. Astrophys. J., 168, 11.

188. Vernon, M.P., 1977.Astron. J., 82, 937.

189. Jenkins, C.R., 1982. Mon. Not. astr. Soc., 200, 705.

190. Freeman, M.A., A radio study of galaxy streaming motions : Physics 1V Report, 1987. Astrophysics Department, University of Sydney.

191. Hoskins, D.G., Murdoch, H.S., Hazard, C. \& Jauncey, D.L., 1972. Austr. J. Phys., 25,559 .

192. Burns, J.O., White, R.A. \& Hough, D.H., 1981. Astron. J., 86, 1.

193. Bridle, A.H. \& Fomalont, E.B., 1978. Astron. J., 83, 704.

194. Hazard, C. \& Murdoch, H.S., 1977. Austr. J. Phys., Astrophys. Suppl., 42. 
195. Bolton, J.G. \& Kinman, T.D., 1966. Astrophys. J., 145, 951.

196. Mathews, T.A., Morgan, W.W. \& Schmidt, M., 1964. Astrophys. J., 140, 35.

197. Hunstead, R.W., 1971. Nature, 233, 401.

198. Disney, M.J., Peterson, B.A. \& Rodgers, A.W., 1974. Astrophys. J., 194, L79.

199. Hazard, C. \& Jauncey, D.L., 1972. Astron. J., 77, 621.

200. Slingo, A., 1974. Mon. Not. astr. Soc., 168, 307.

201. Kühr, H., Johnston, J., Odenwald, S. \& Adlhoch, J., 1987. Astron. Astrophys. Supp. Ser., 71, 493.

202. Jones, P.A., 1987. Proc. Astr. Soc. Aus., 7, 208.

203. Jones, P.A., 1989. Proc. Astr. Soc. Aus., 8, 1. 


\section{Appendix B}

\section{Catalogue of Radio Galaxy Spectra}

Herein are plotted the spectra for all the radio galaxies observed during the course of this thesis. Only six spectra recorded in the May 1988 run on Mauna Kea however were considered useable and these are only of poor S/N so have been omitted.

The presentation is such that observations from each telescope have been individually grouped. Objects are arranged in order of increasing right ascension.

The CCD spectra from UH88 and CTIO have been fluxed so that the scale along the ordinate of the plots is a relative flux measure. For the WHT, AAT and INT spectra the units are photons/second. The quantity plotted along the abscissa in all cases is wavelength in Angstoms. The spectra have all been scrunched to linear wavelength bins and for the CTIO spectra in particular the wavelength range for this was specifically chosen so as to reject the long wavelength end of the spectrum where atmospheric $A$ and B absorption bands are a problem. For further details see Chapter 3: Part II. 

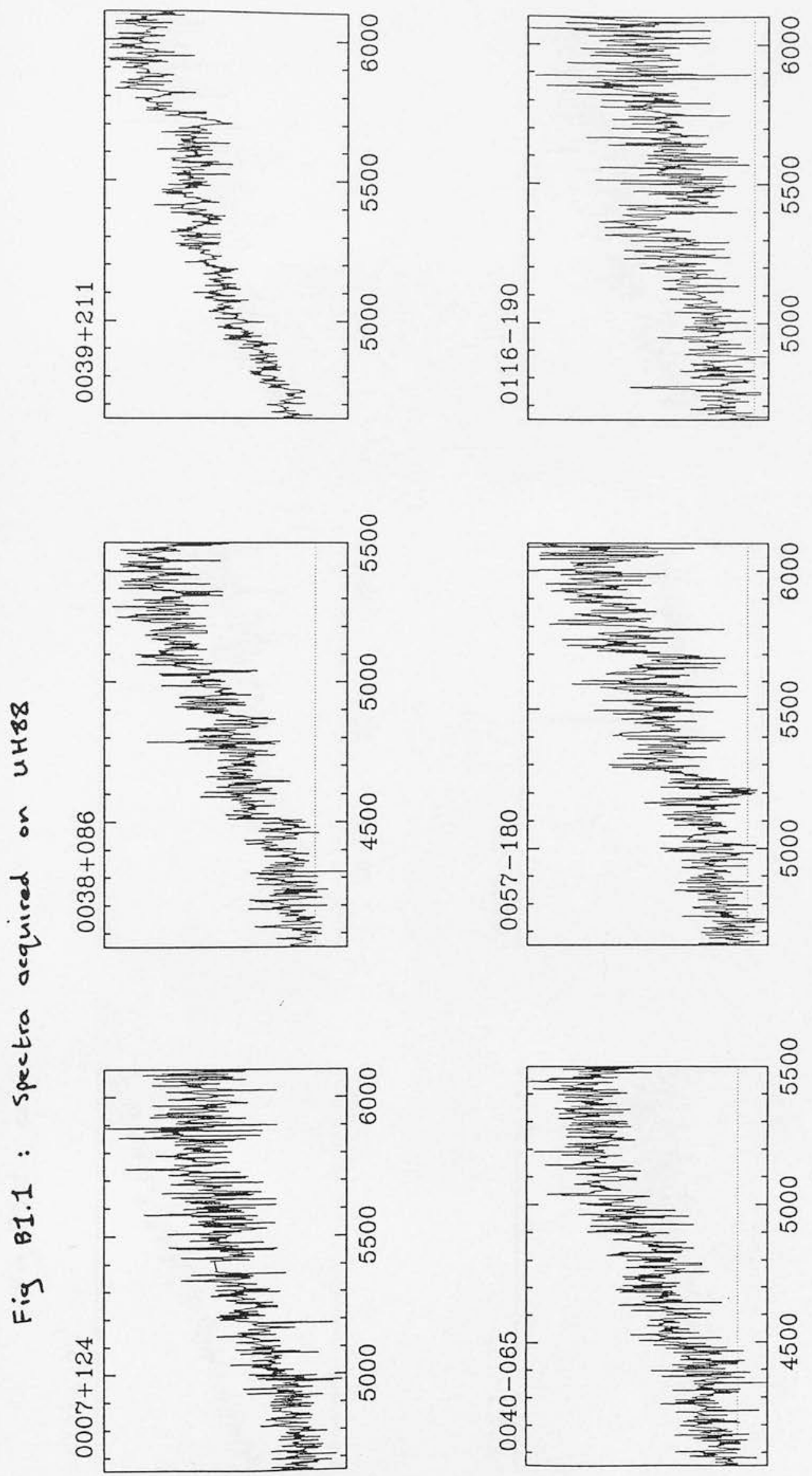

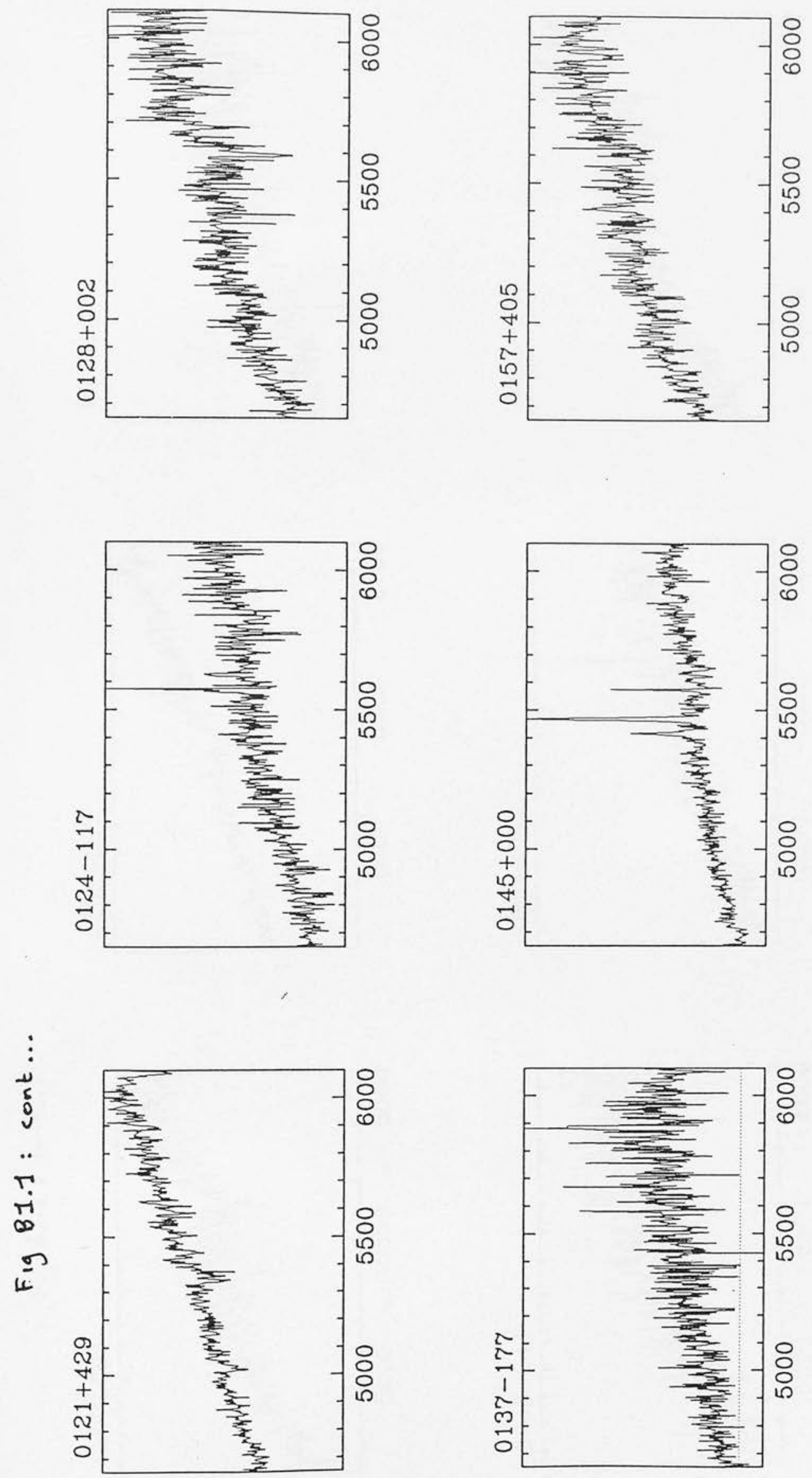

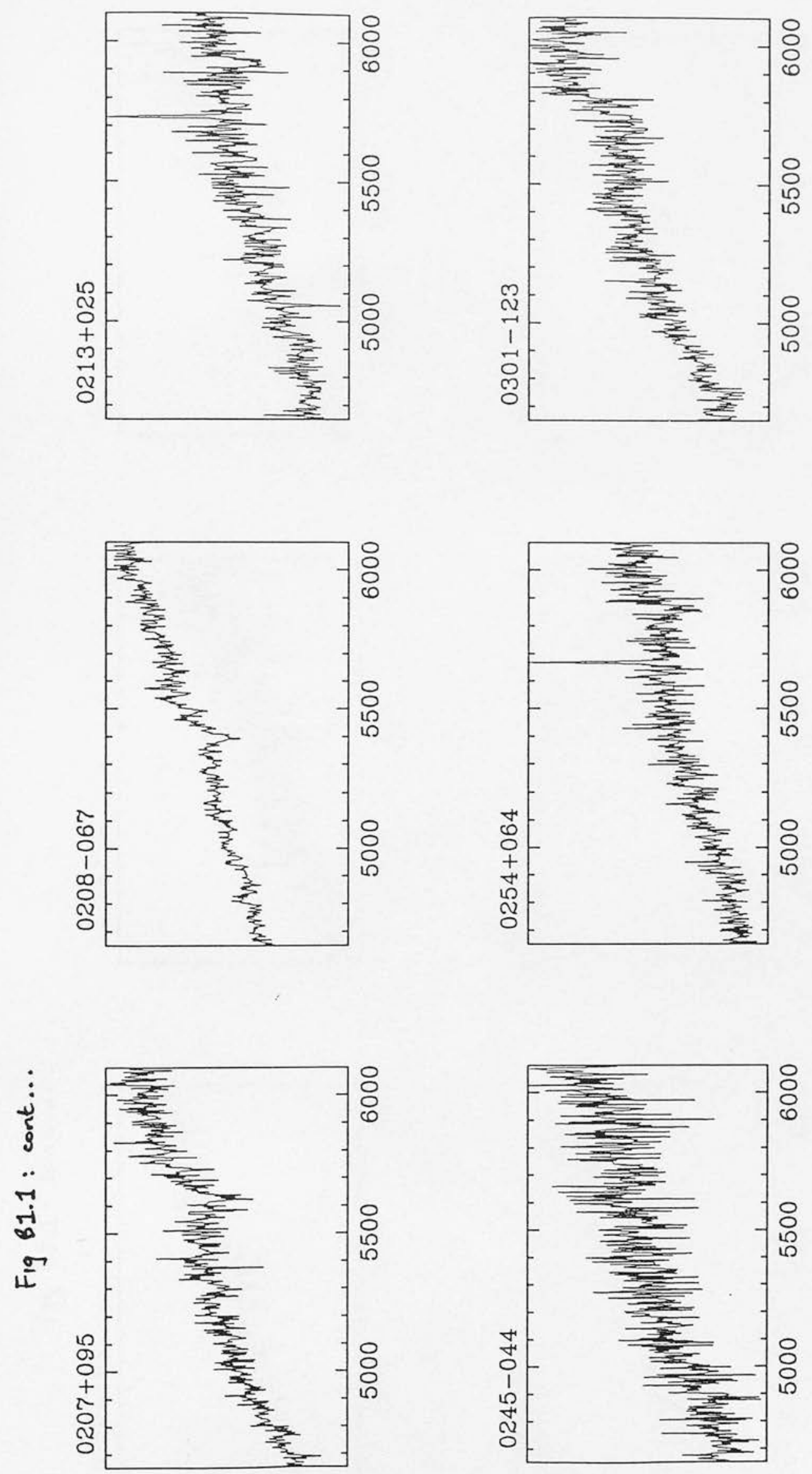

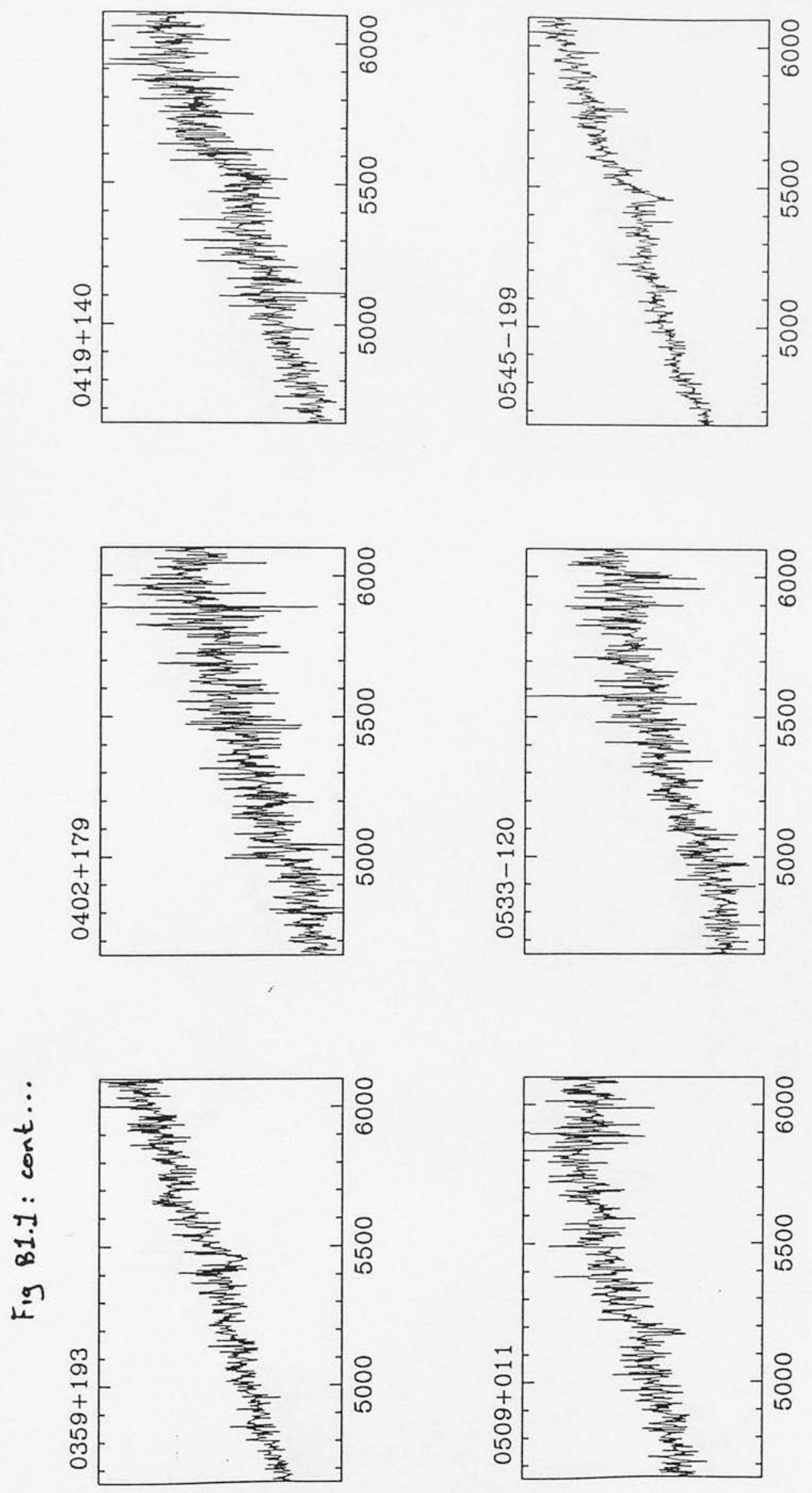

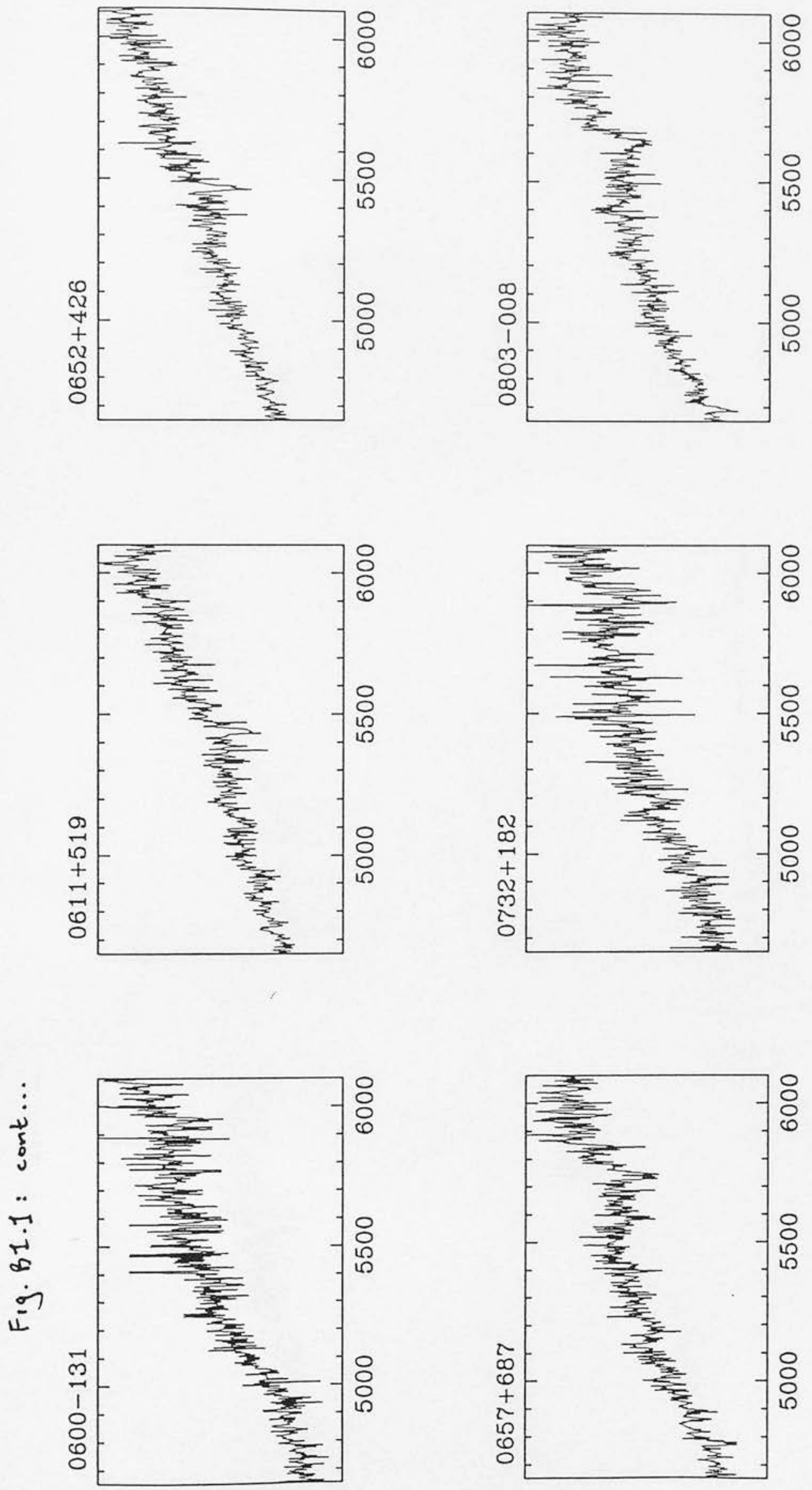

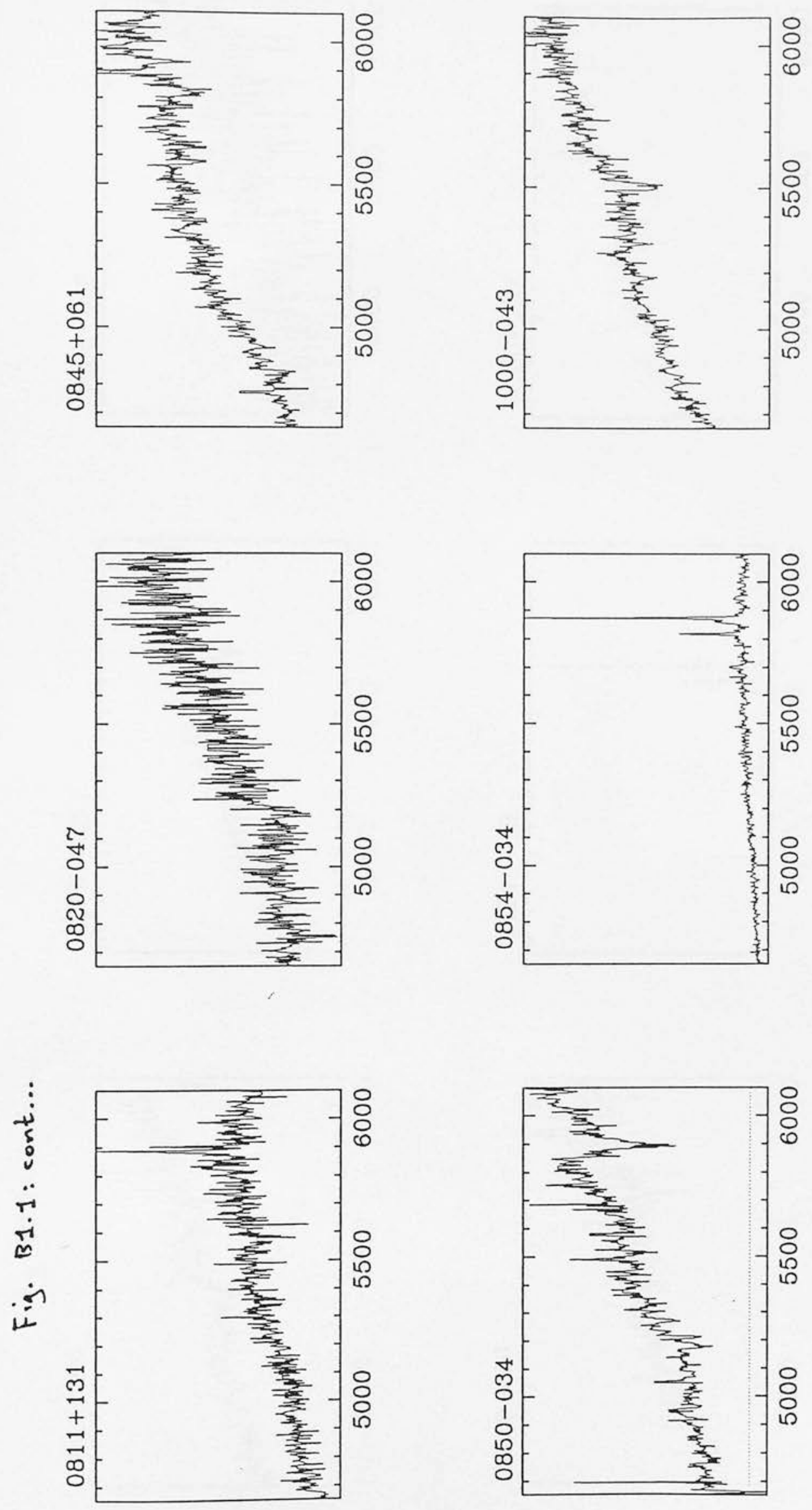

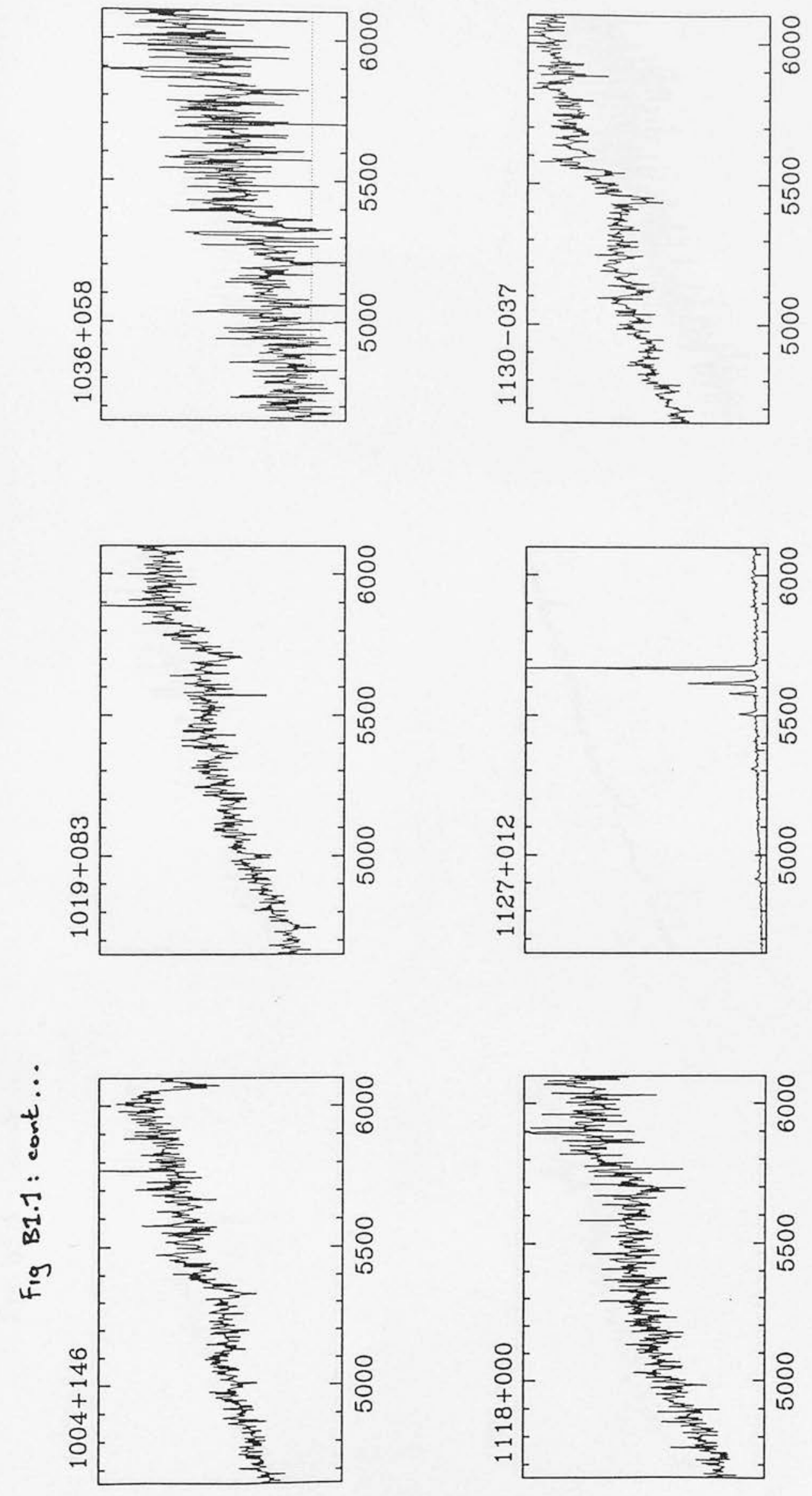

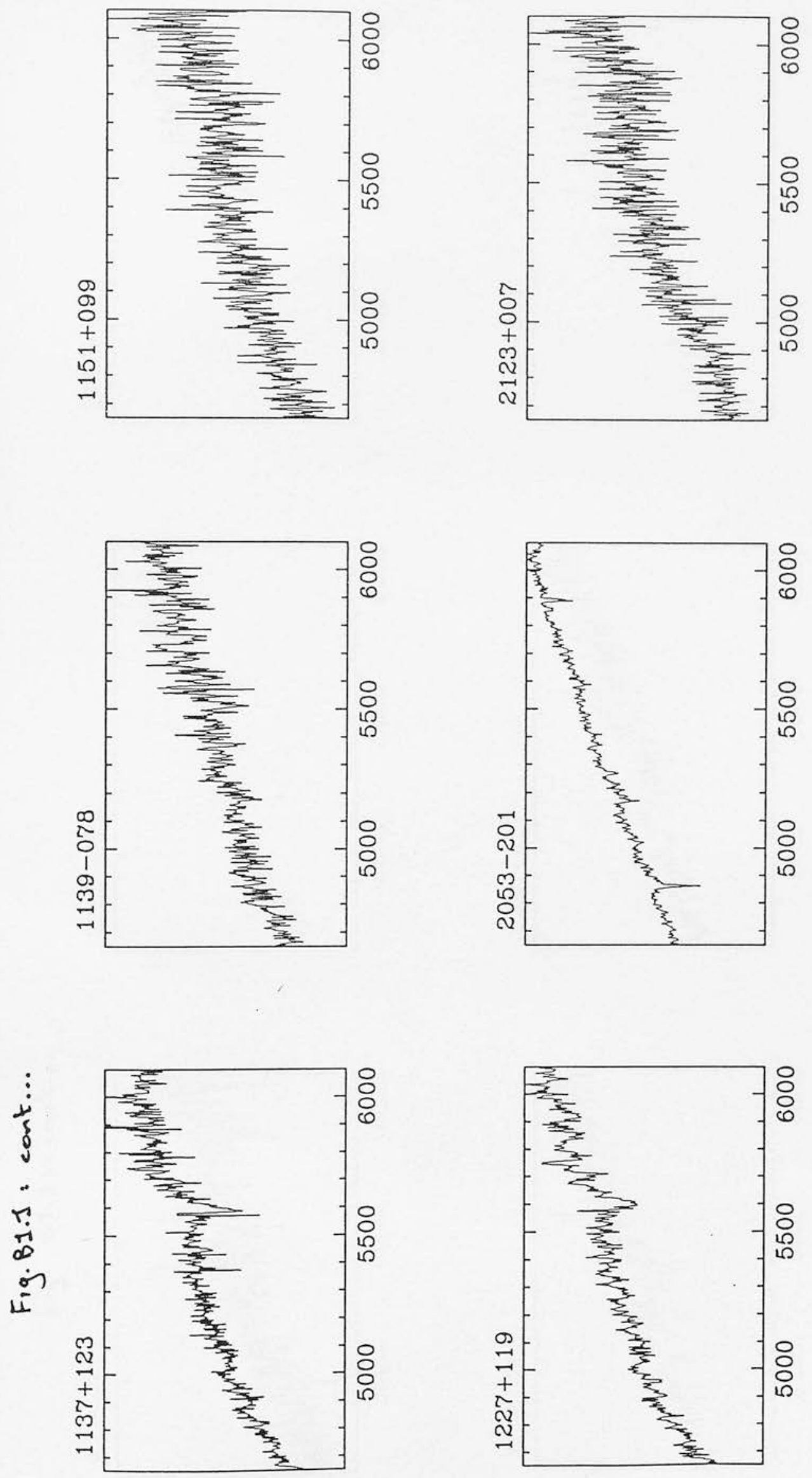

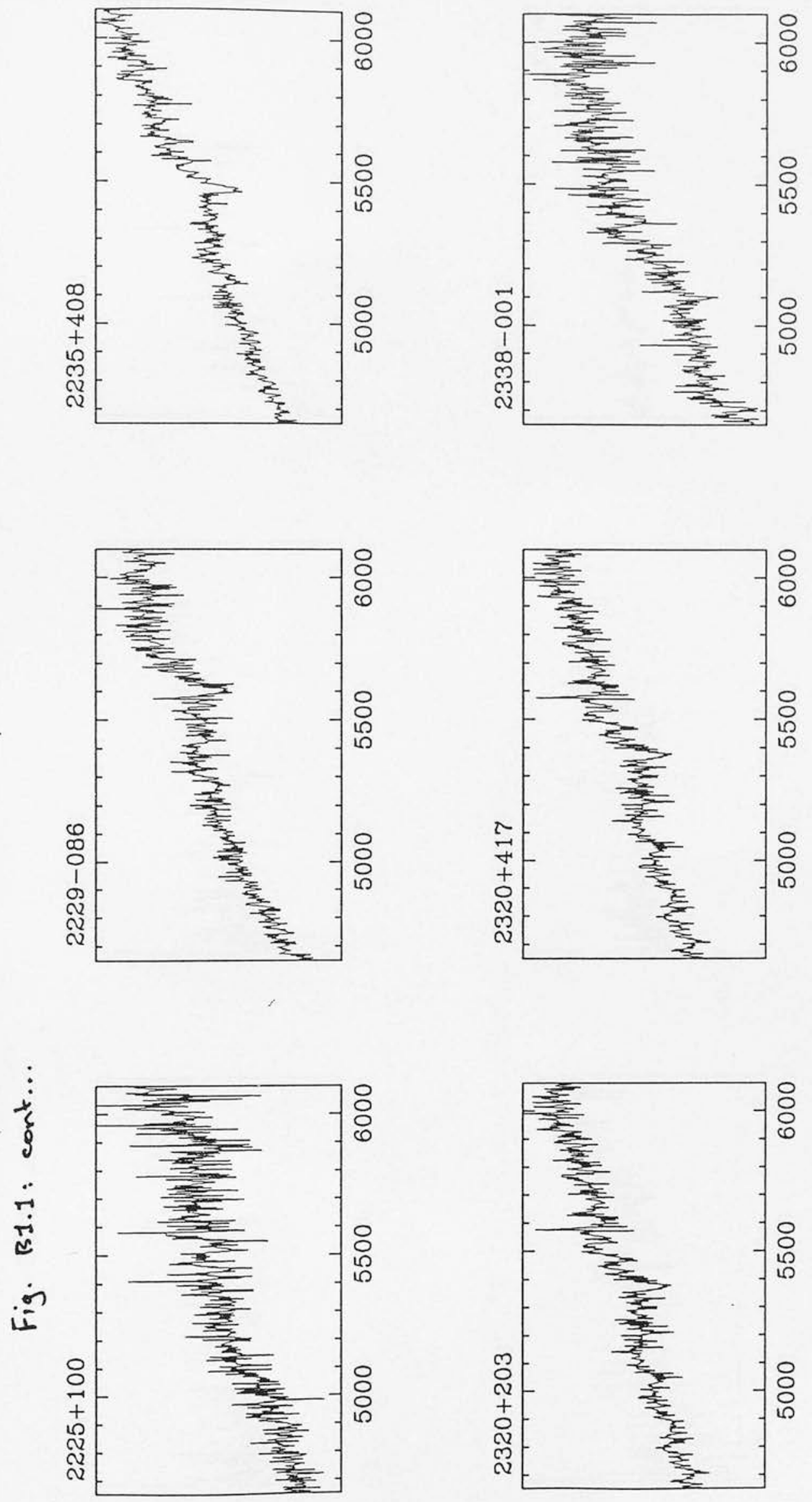

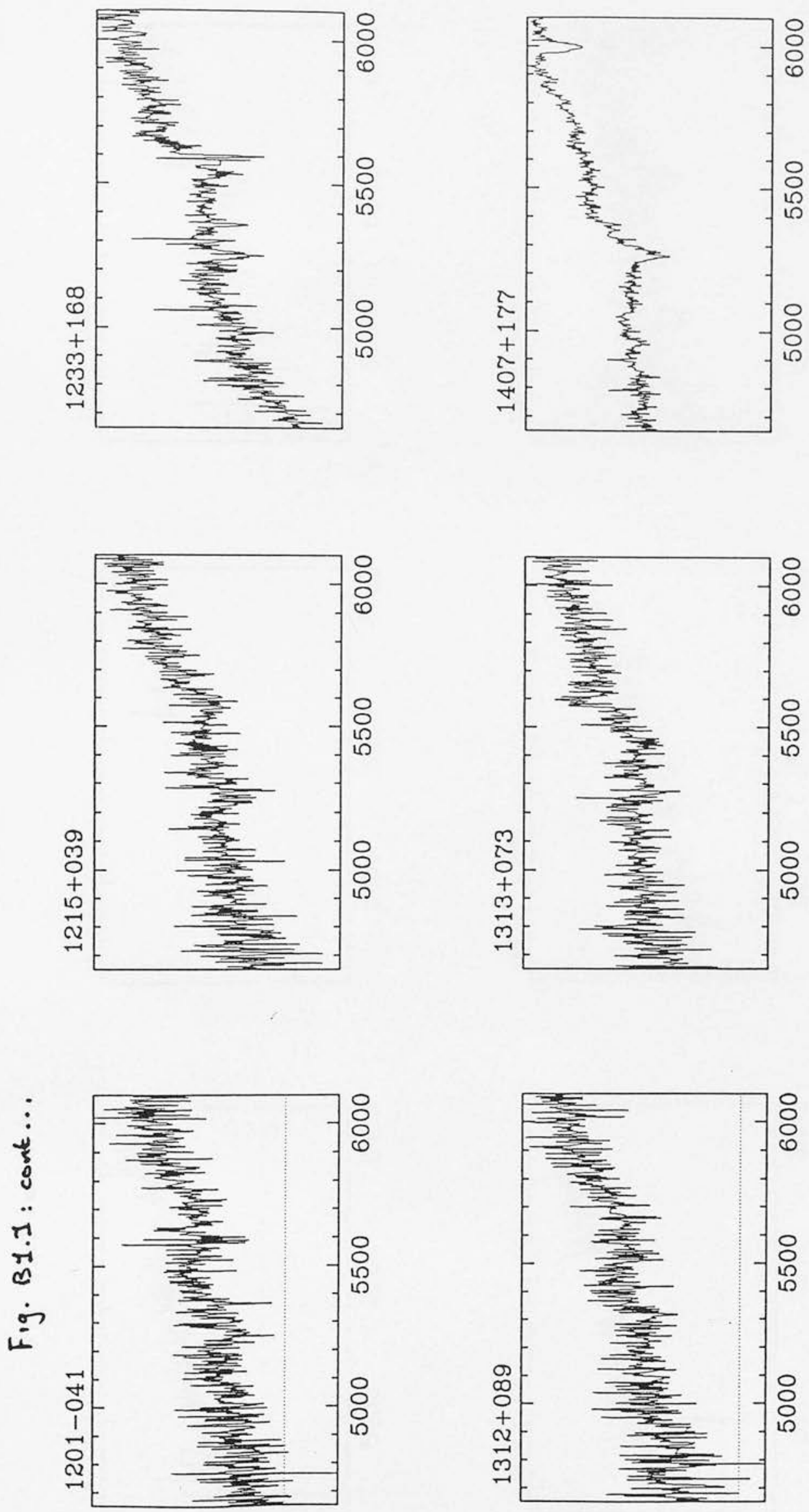

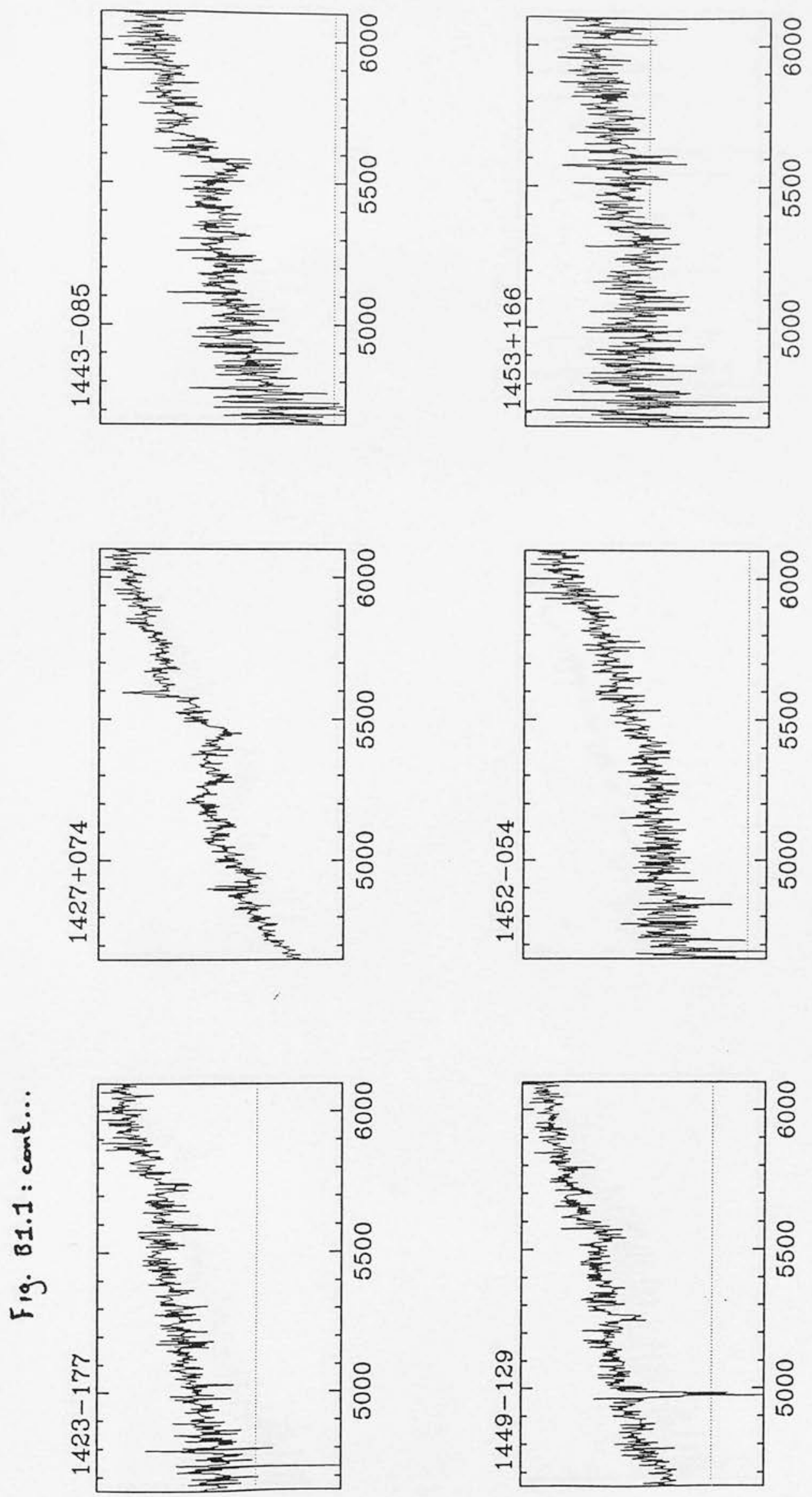

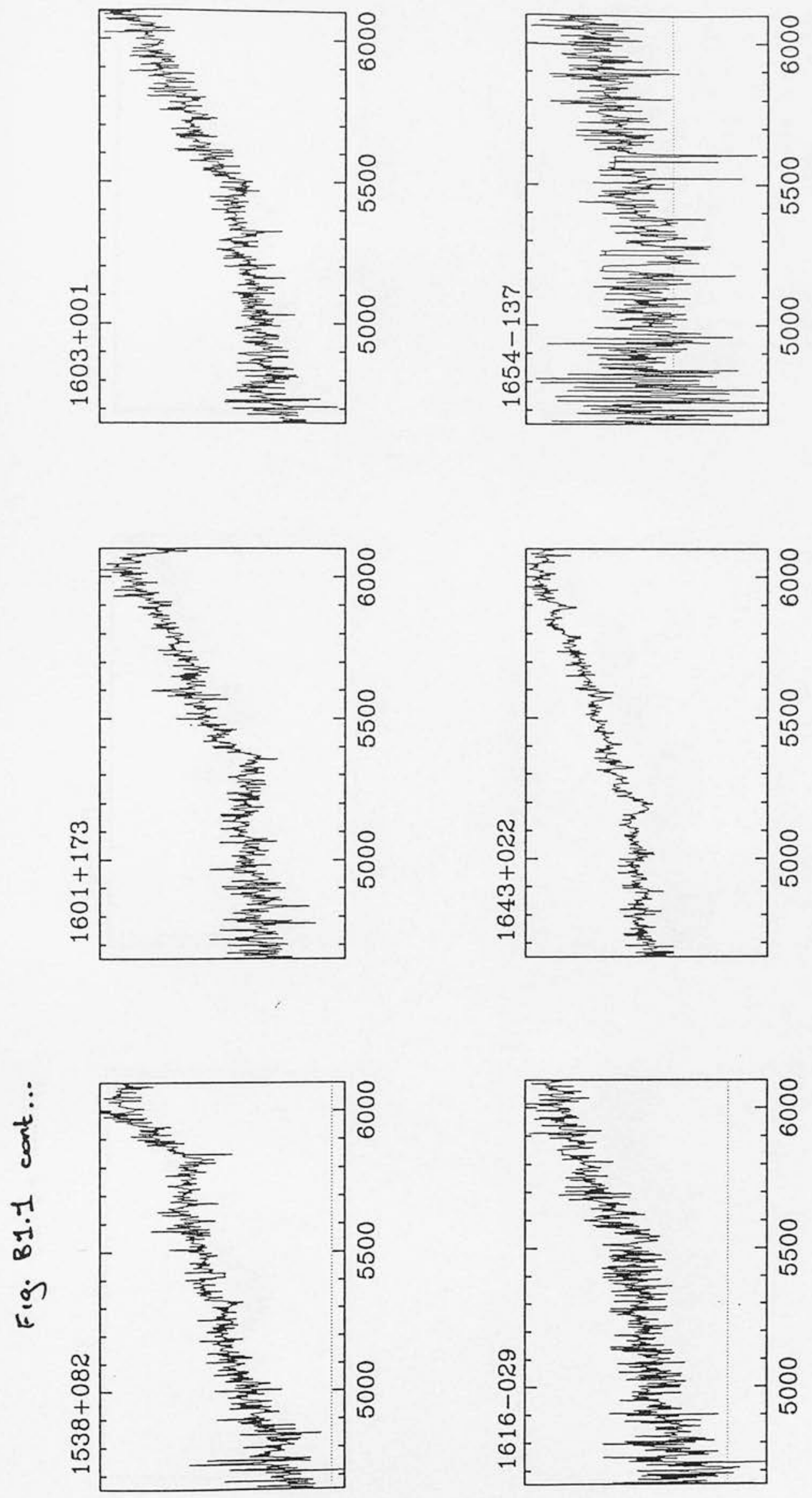

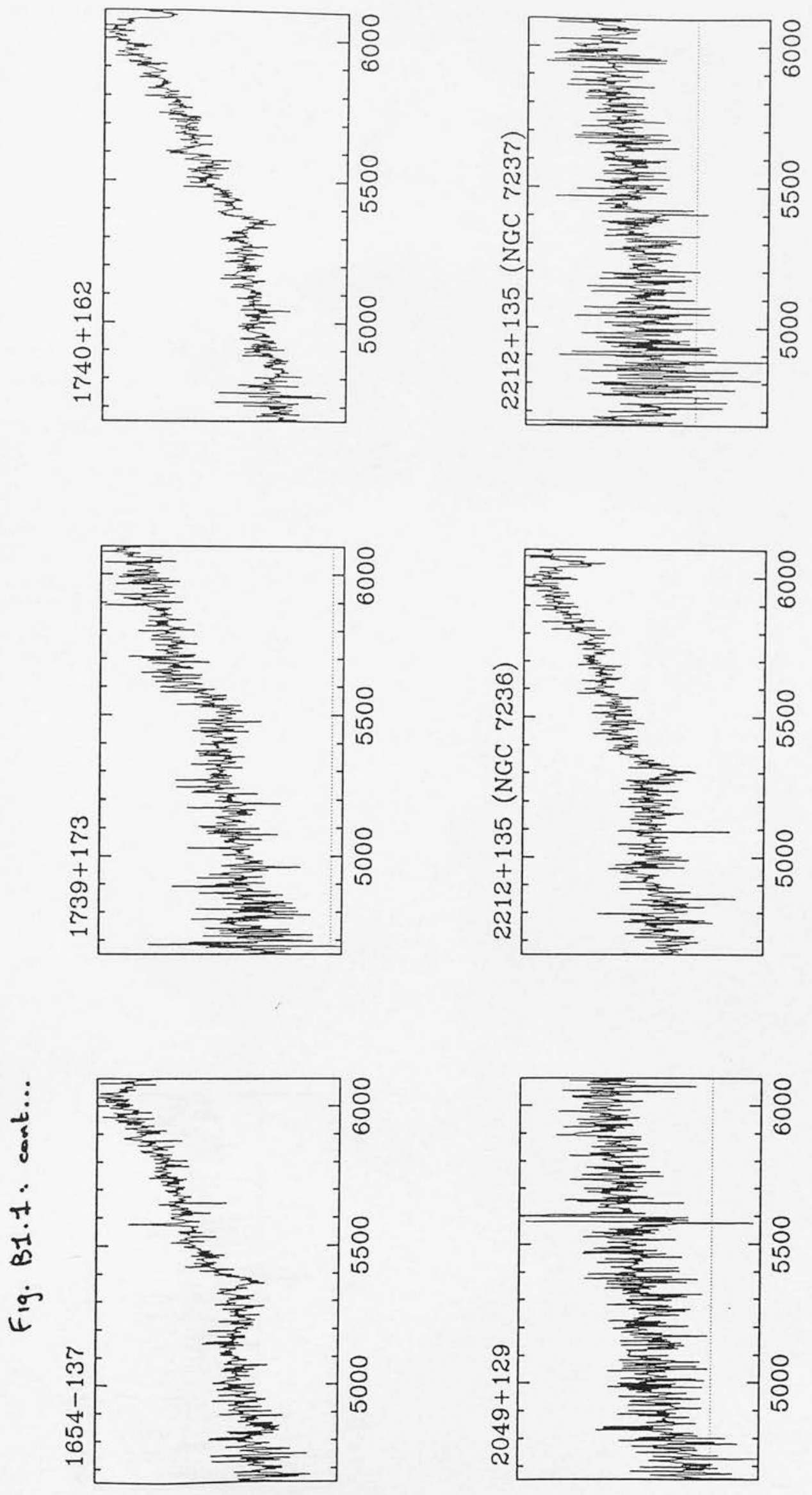


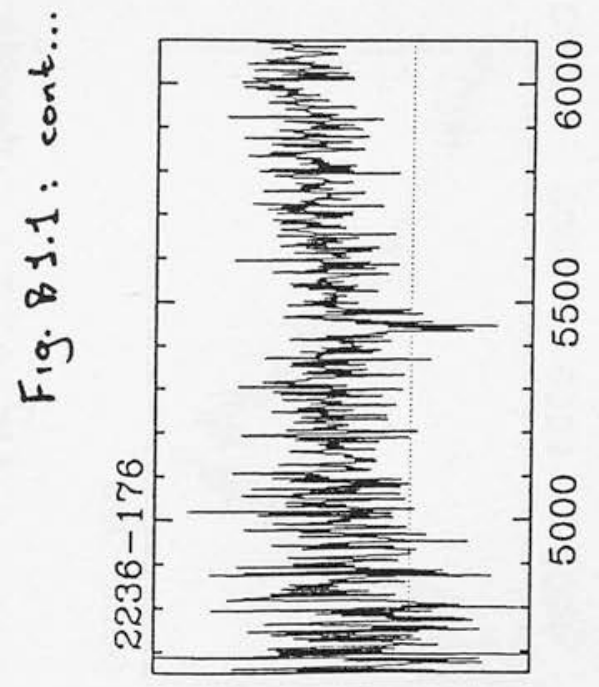



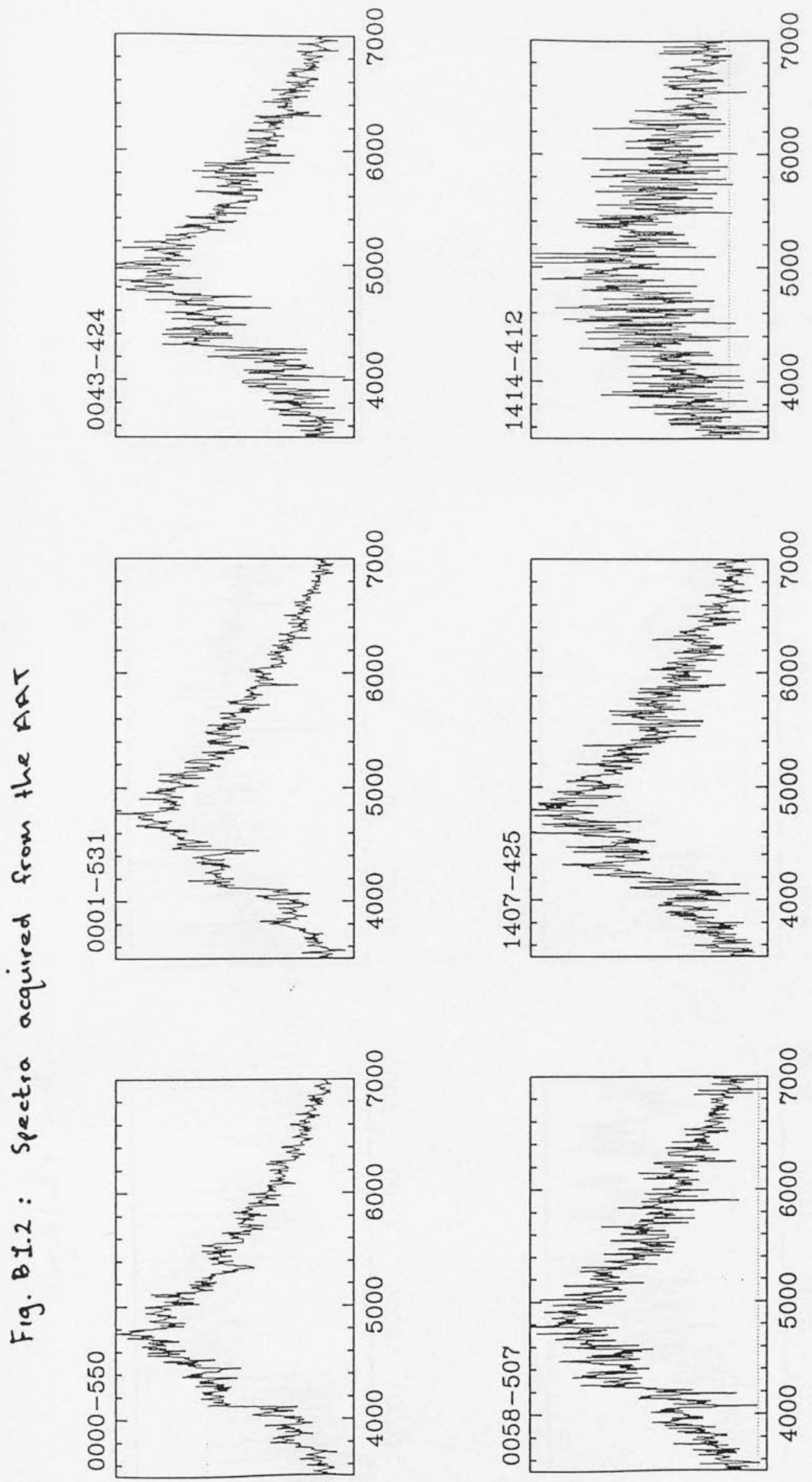

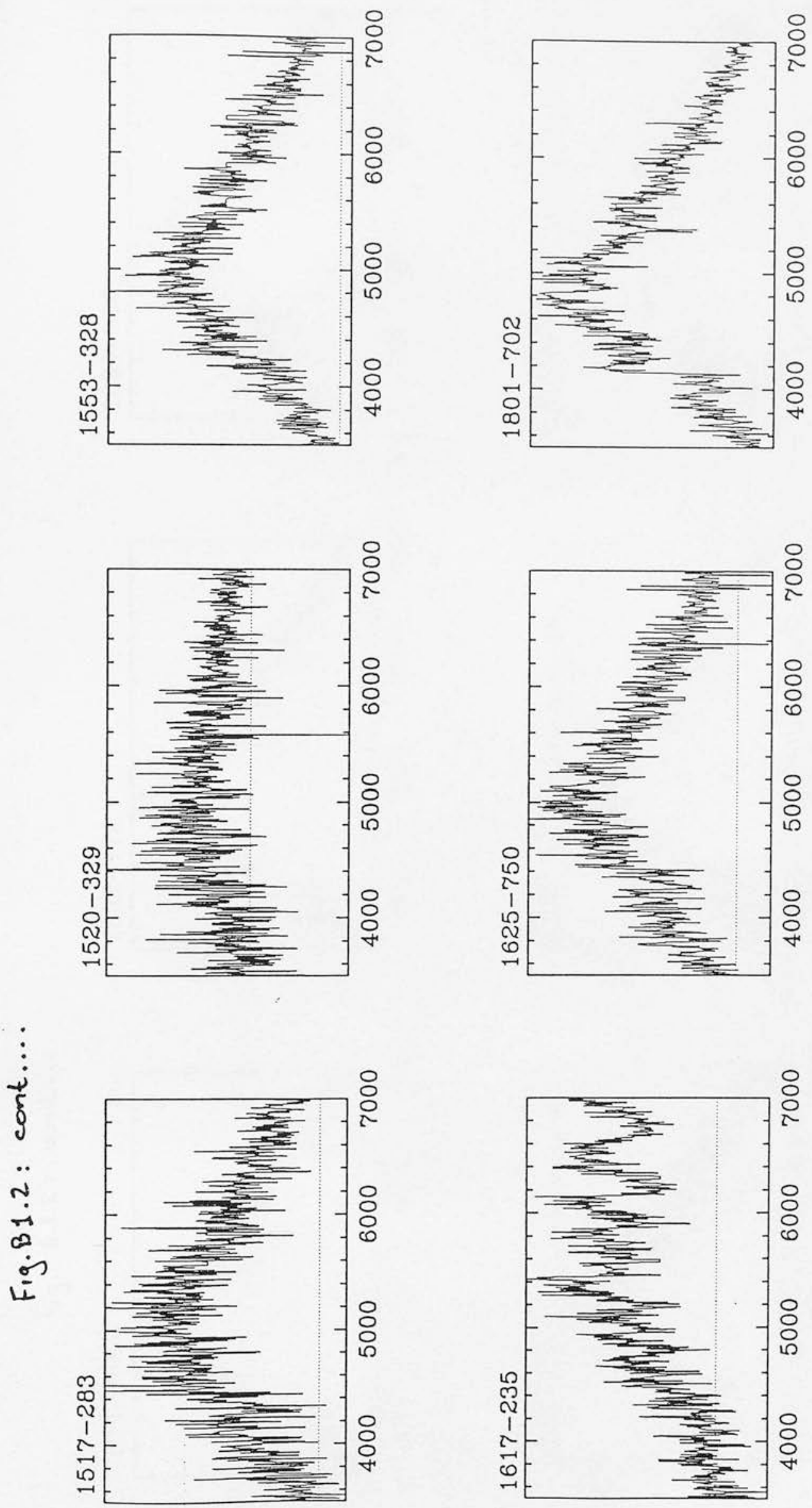

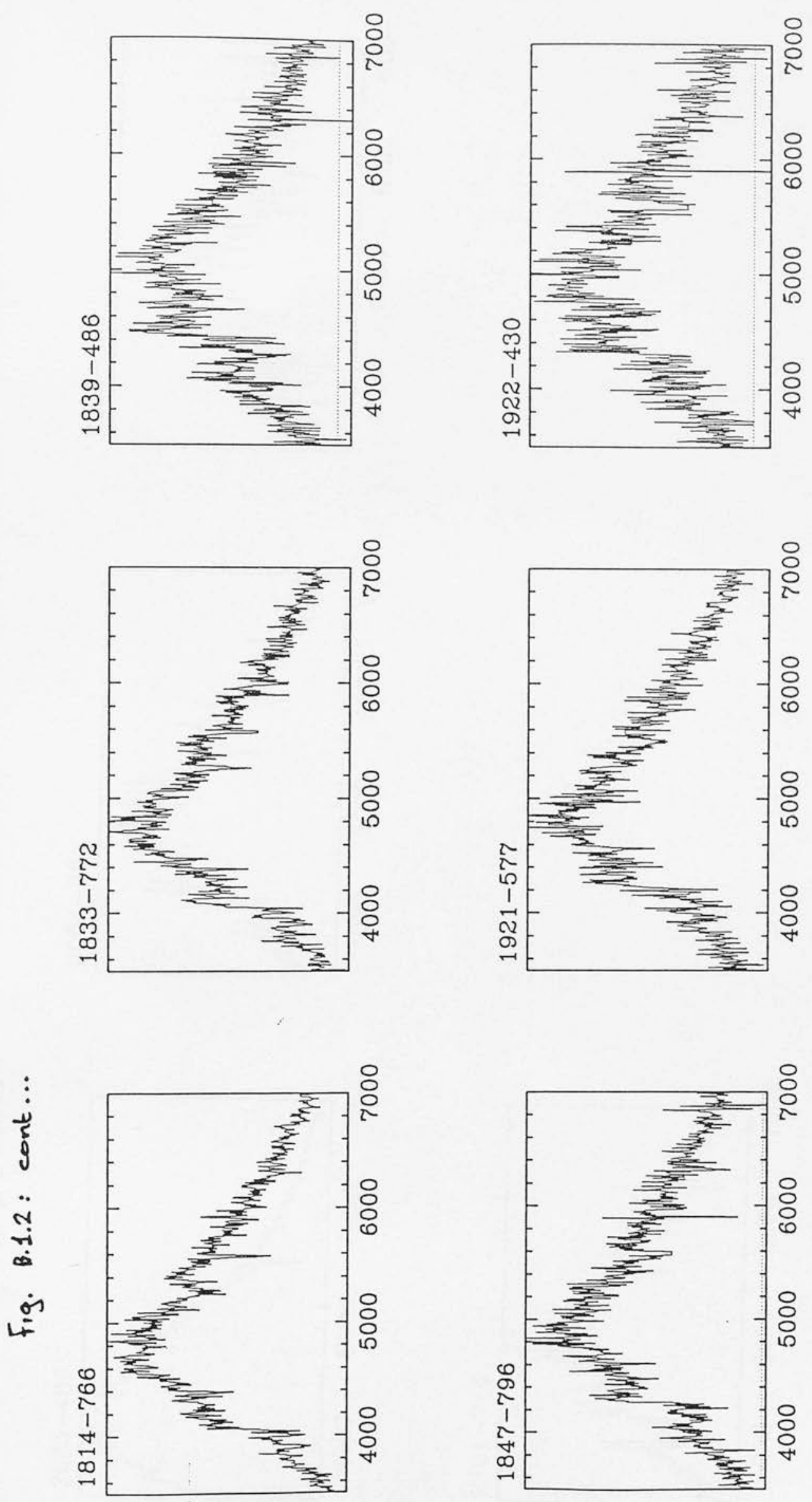

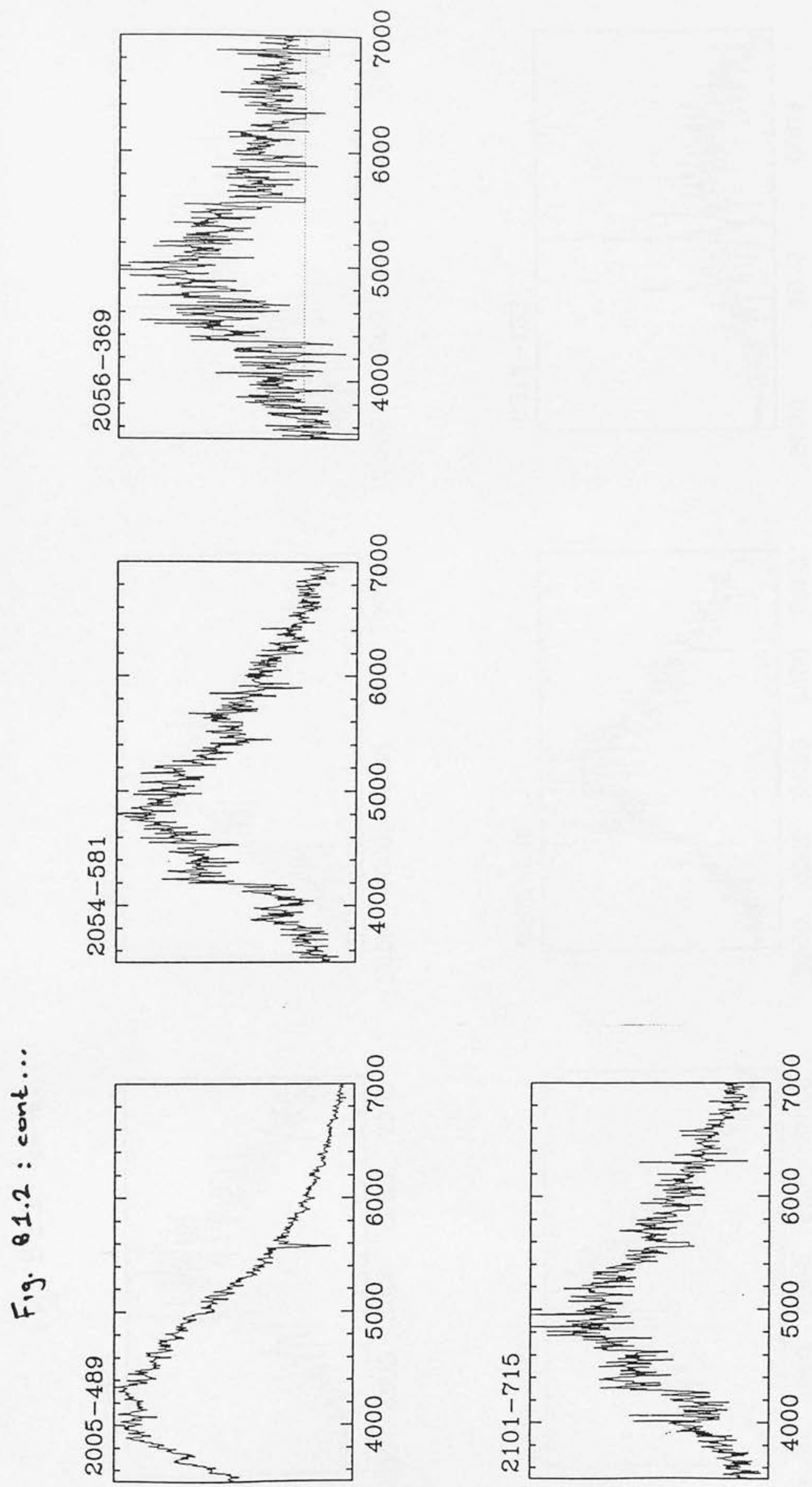

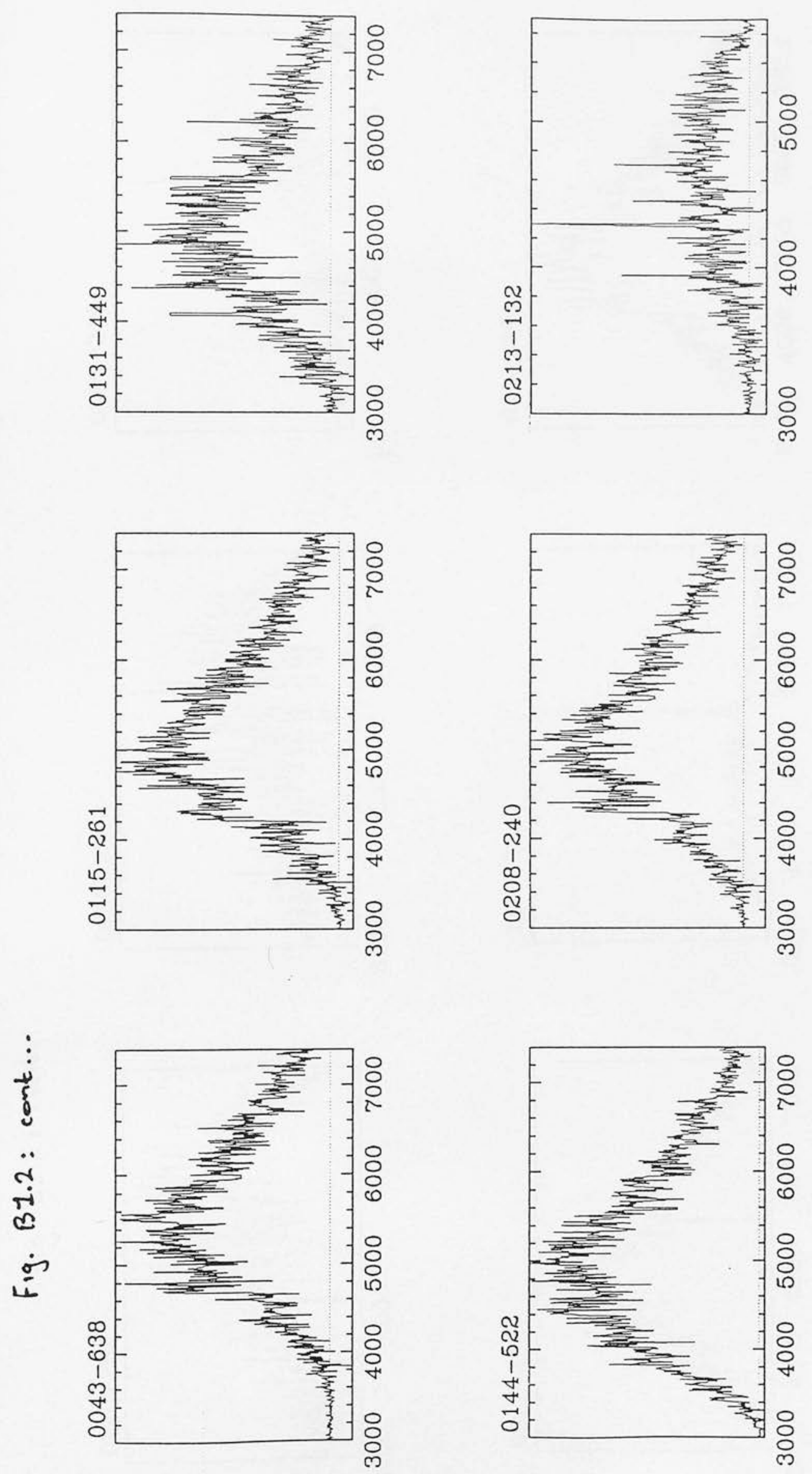

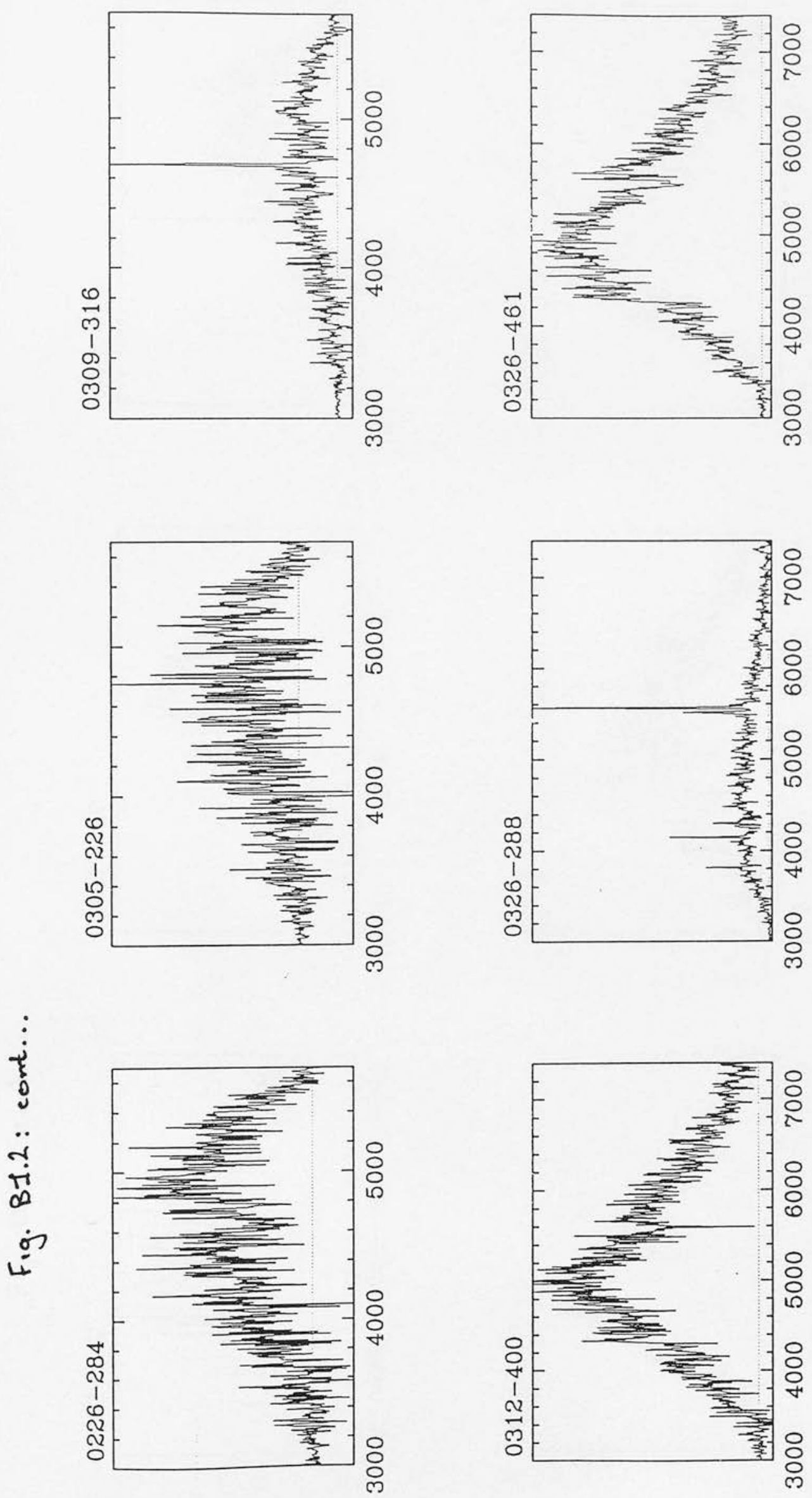

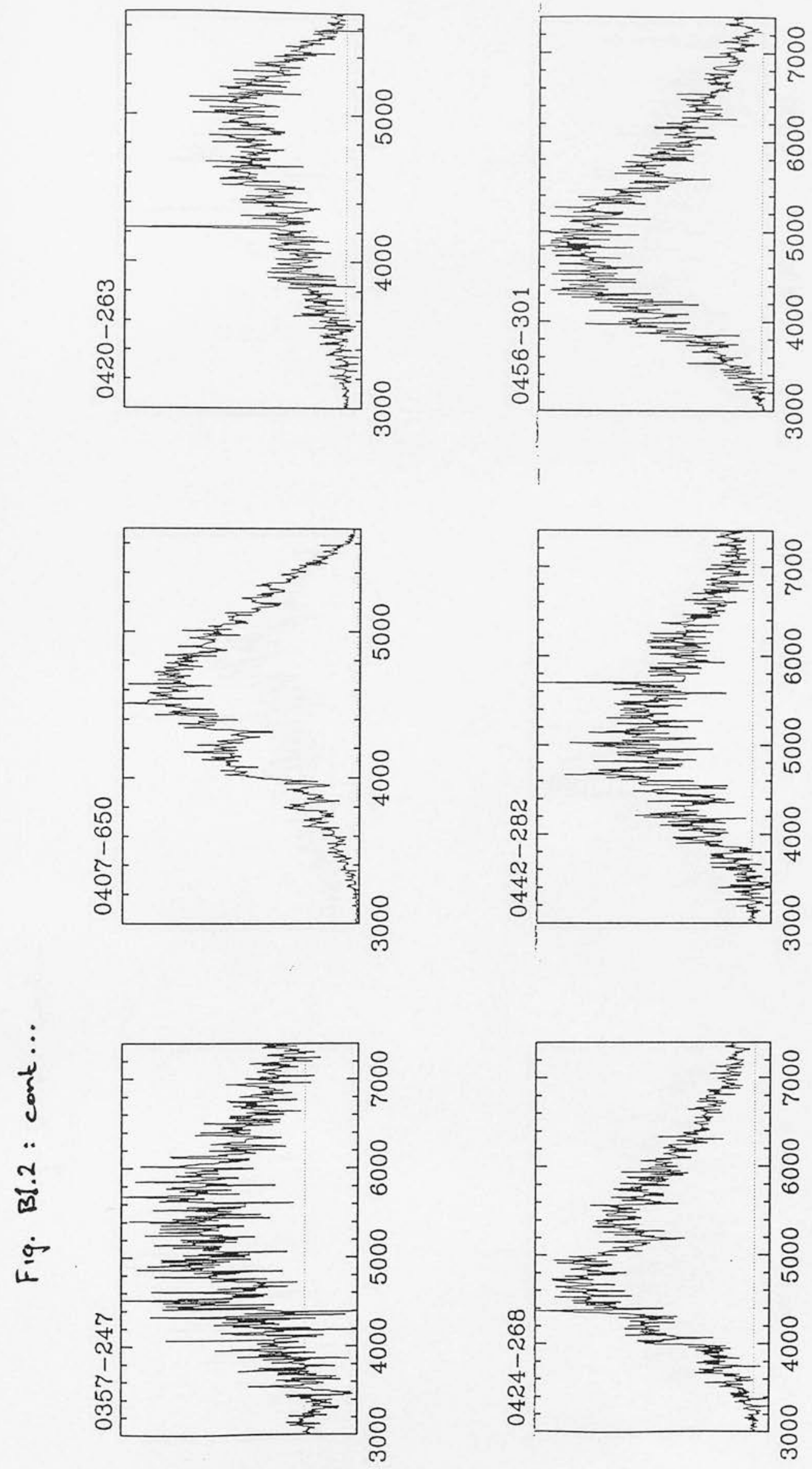

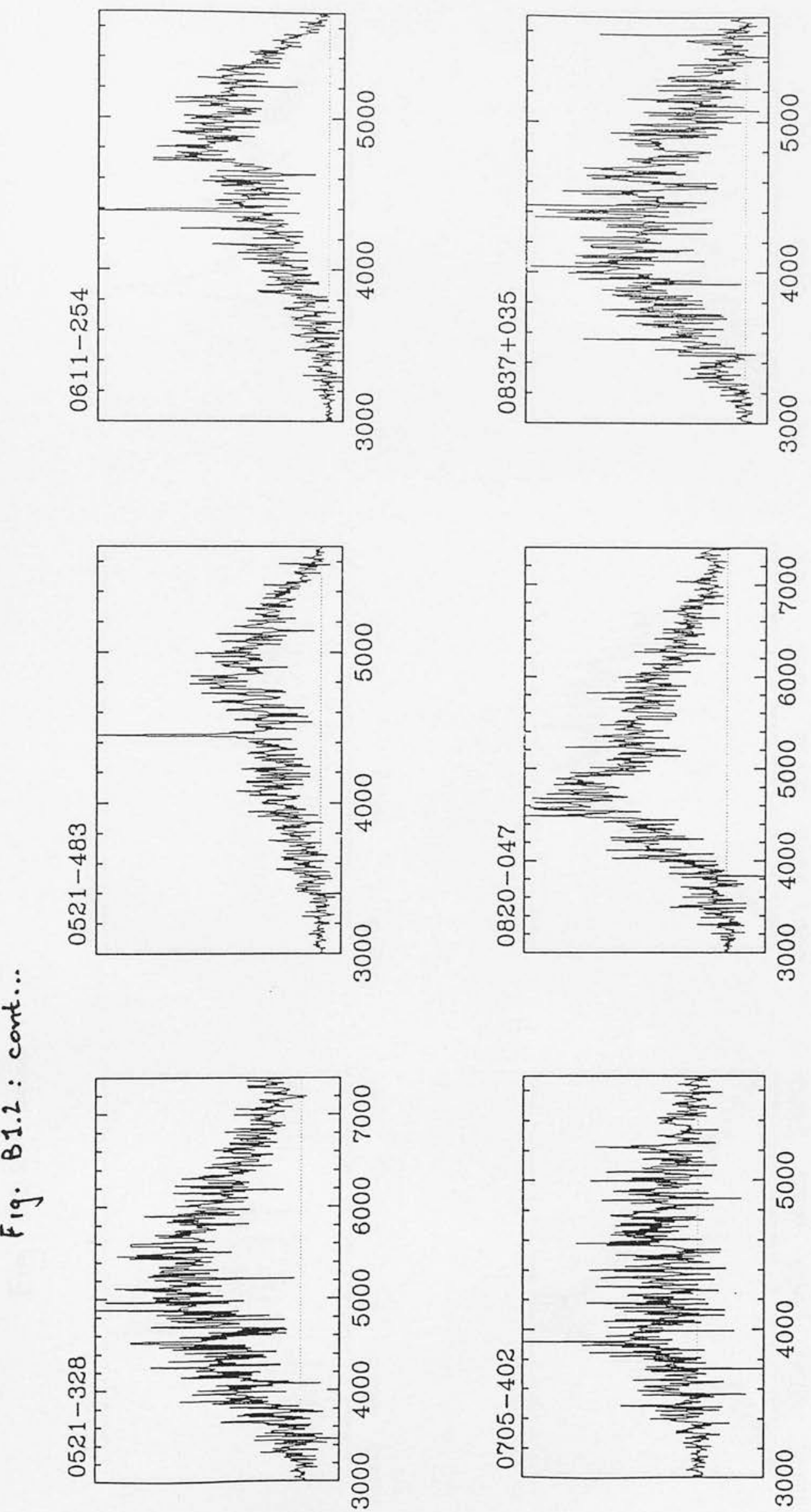

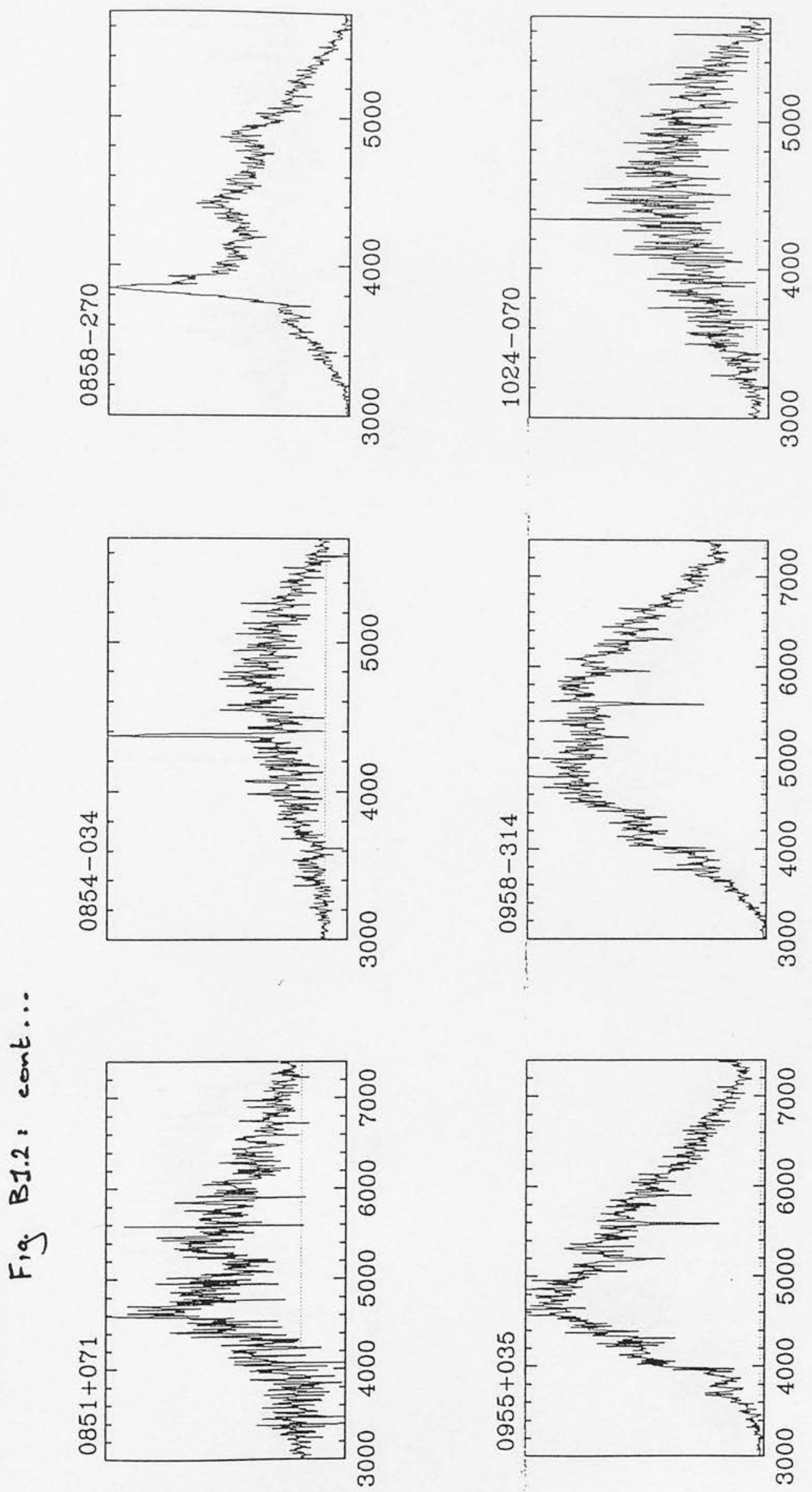

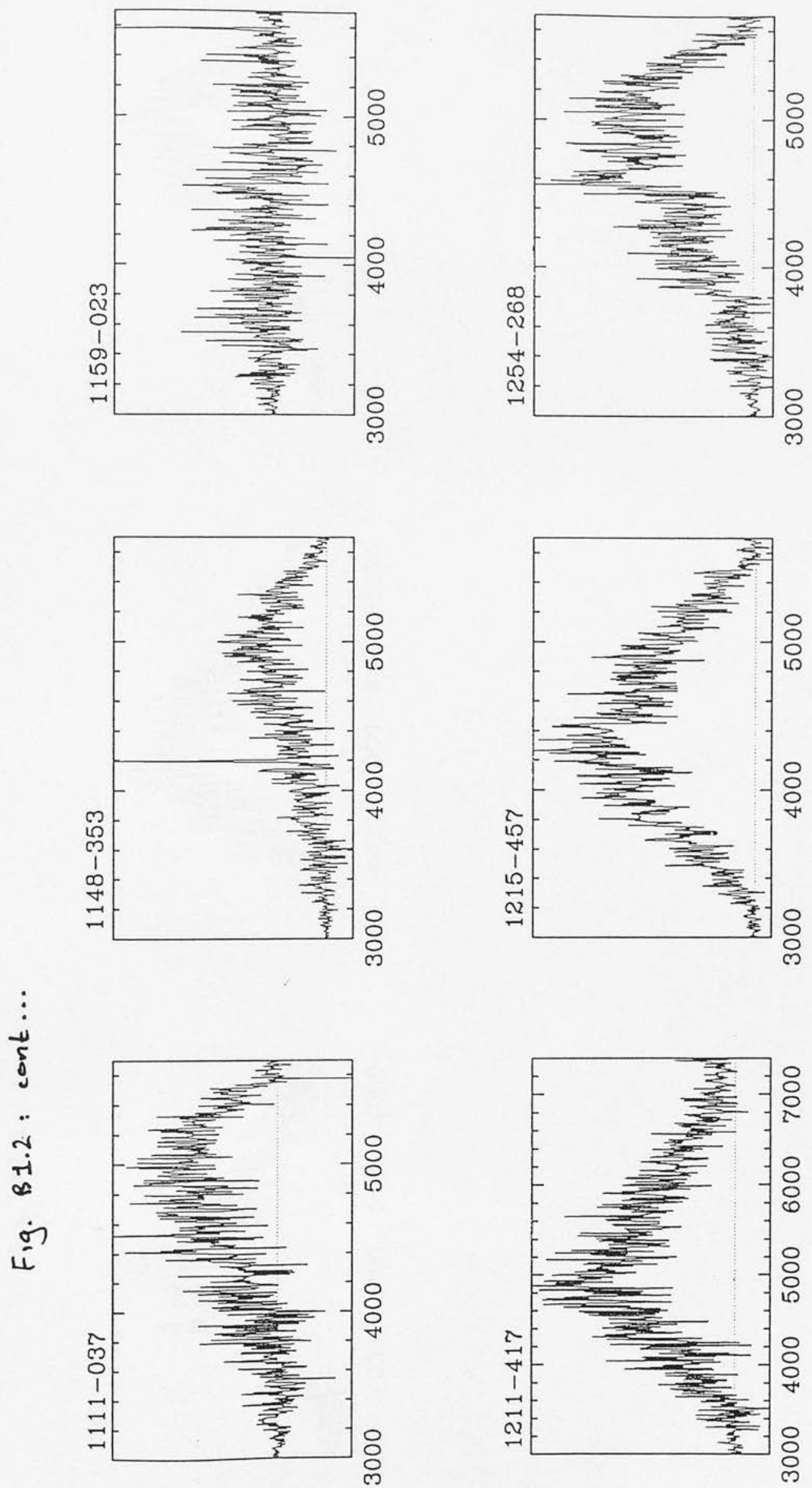

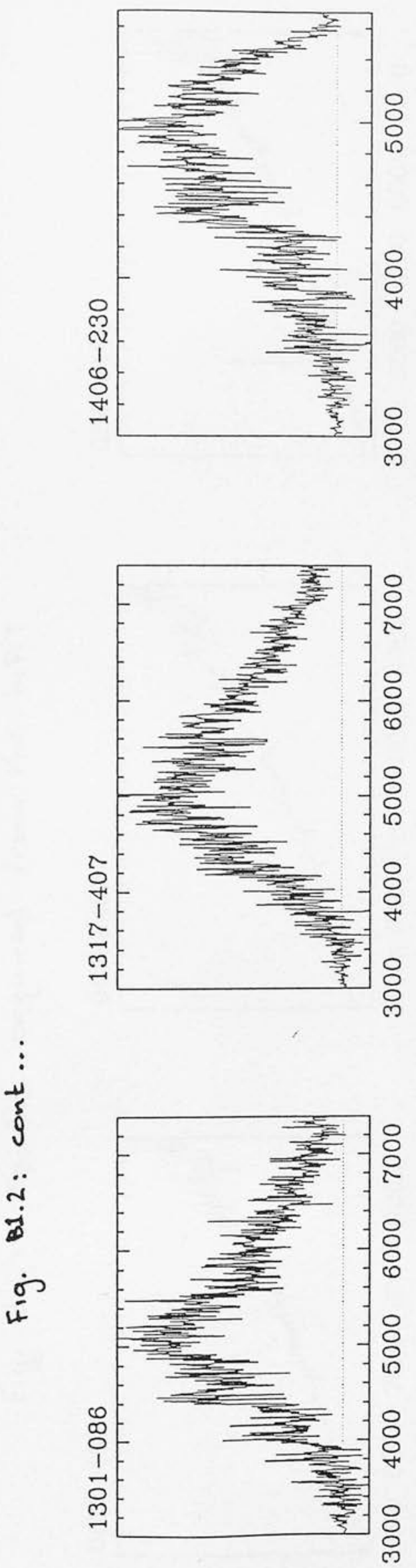

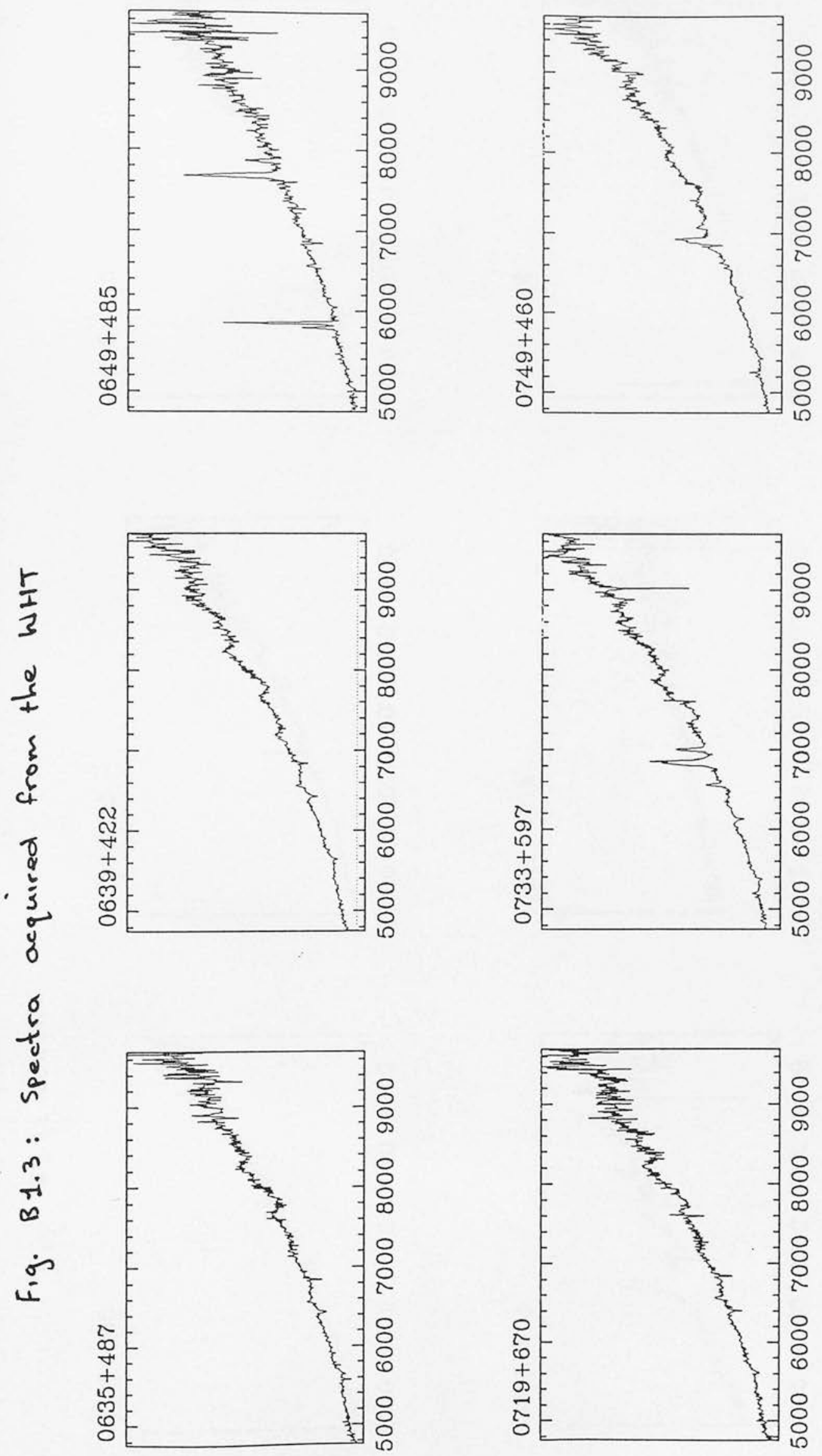

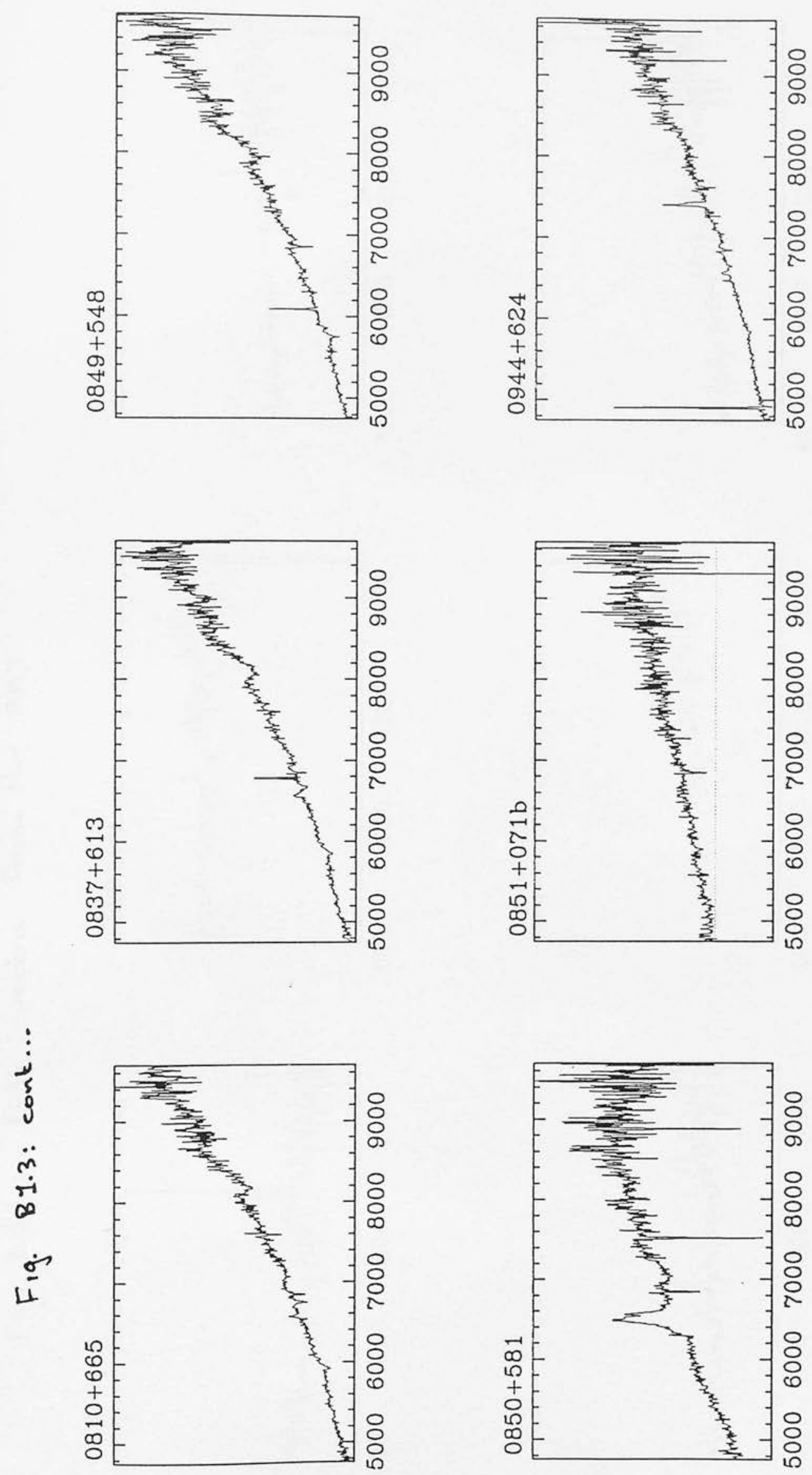

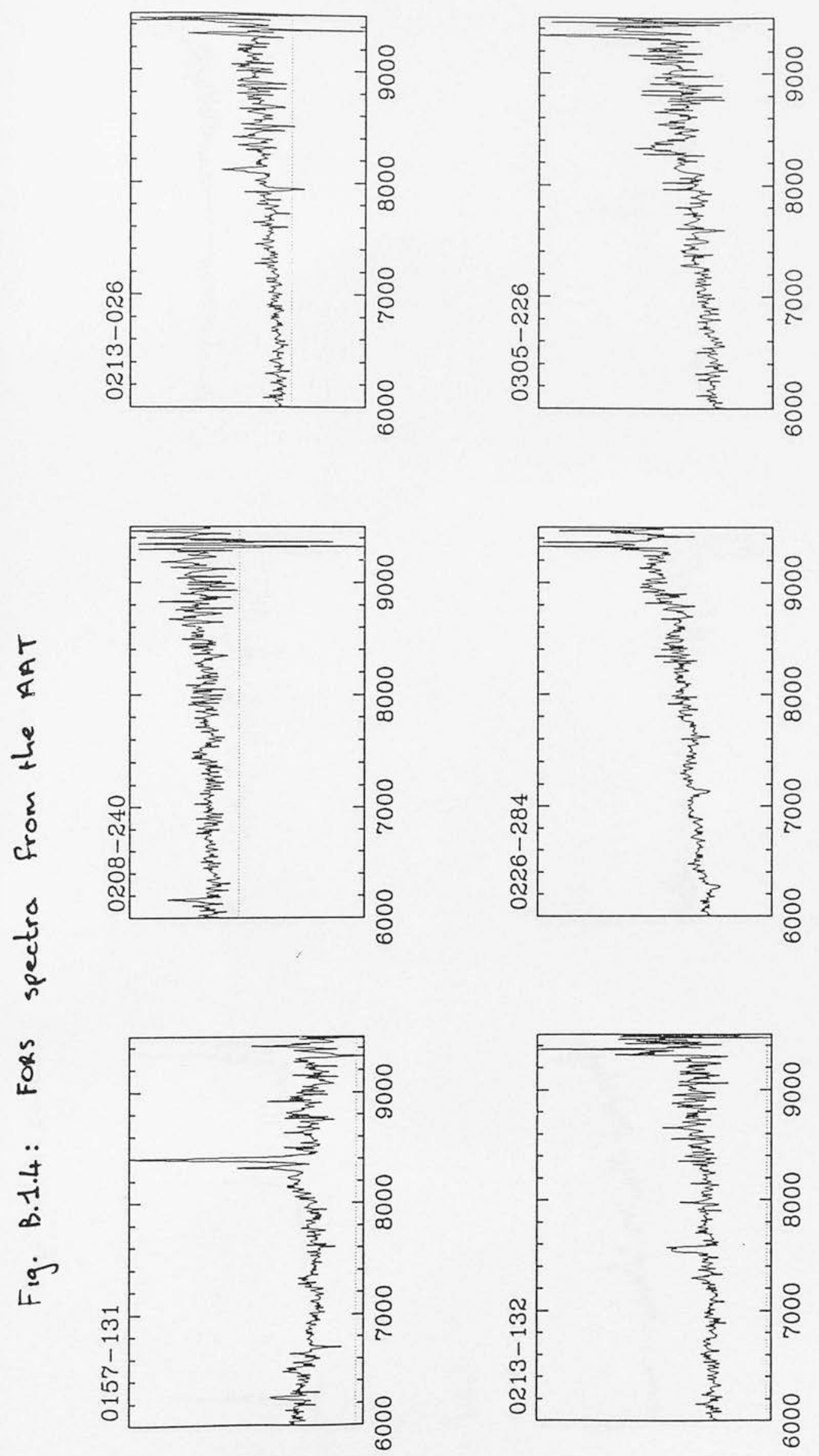

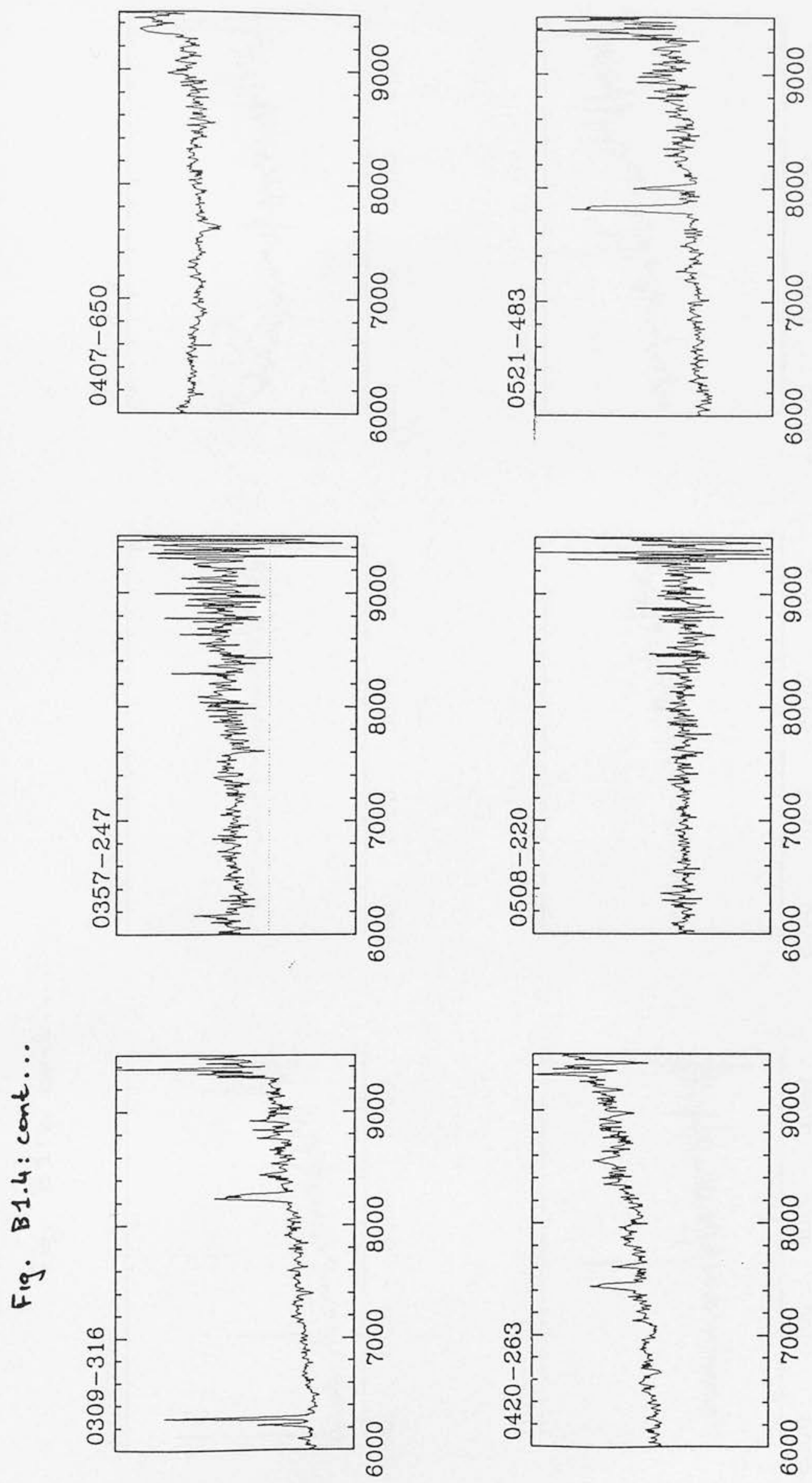

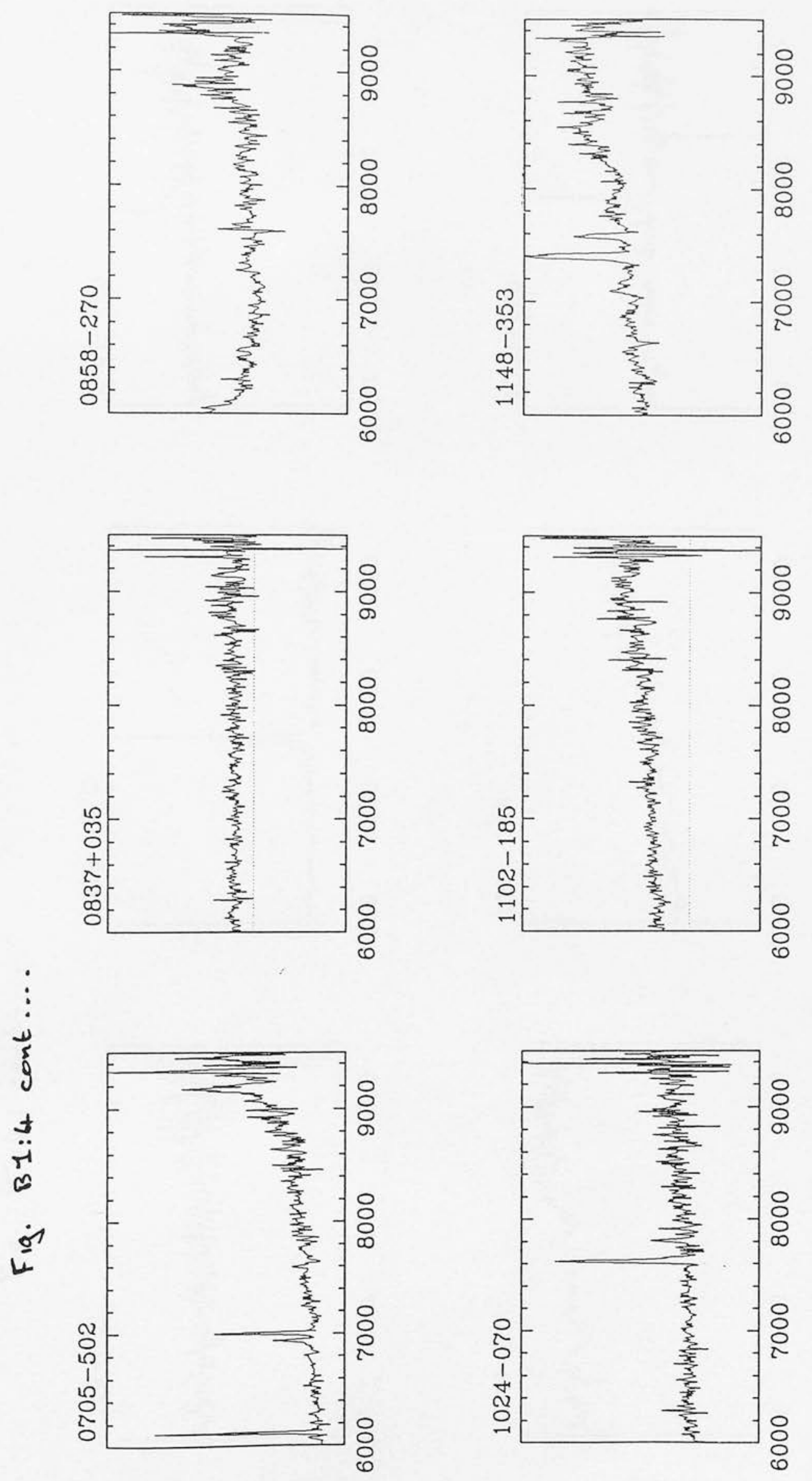

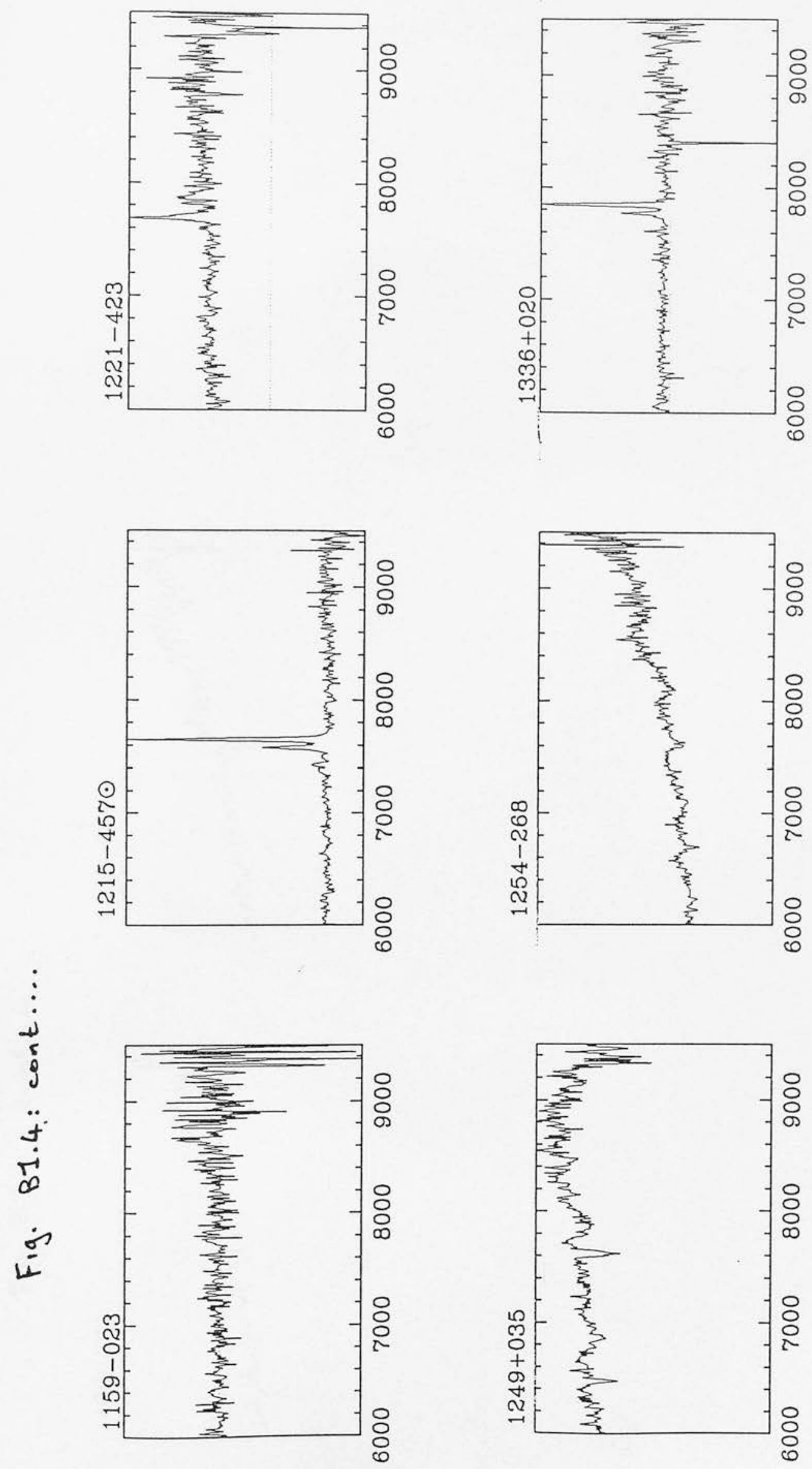

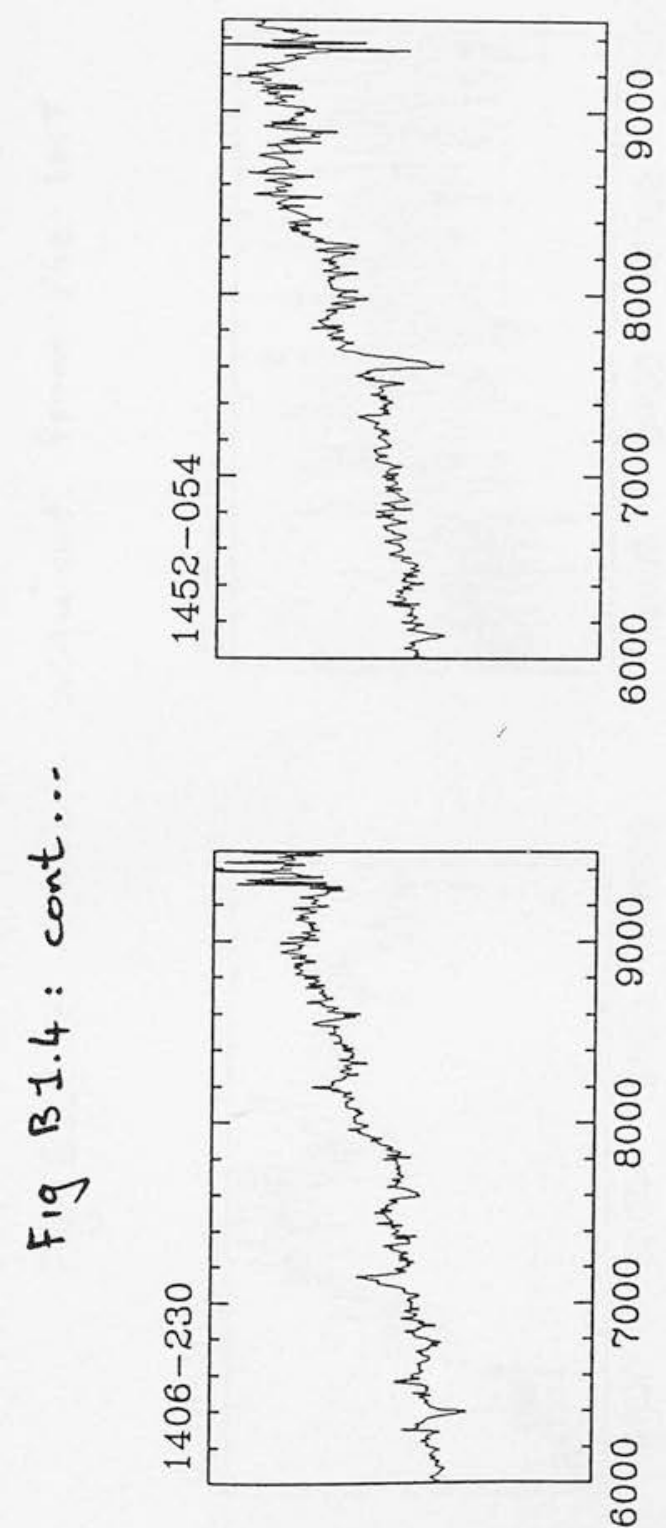

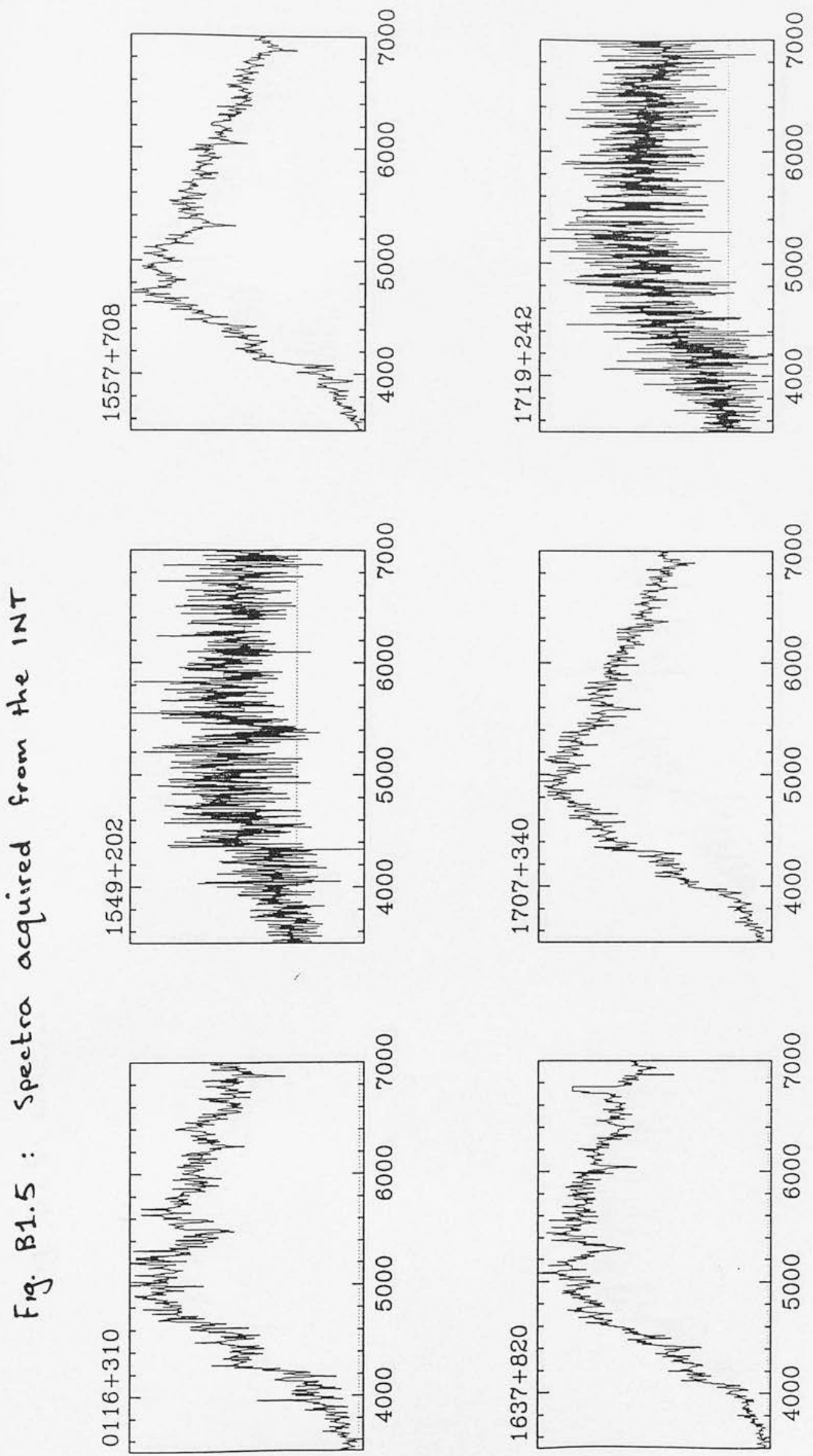

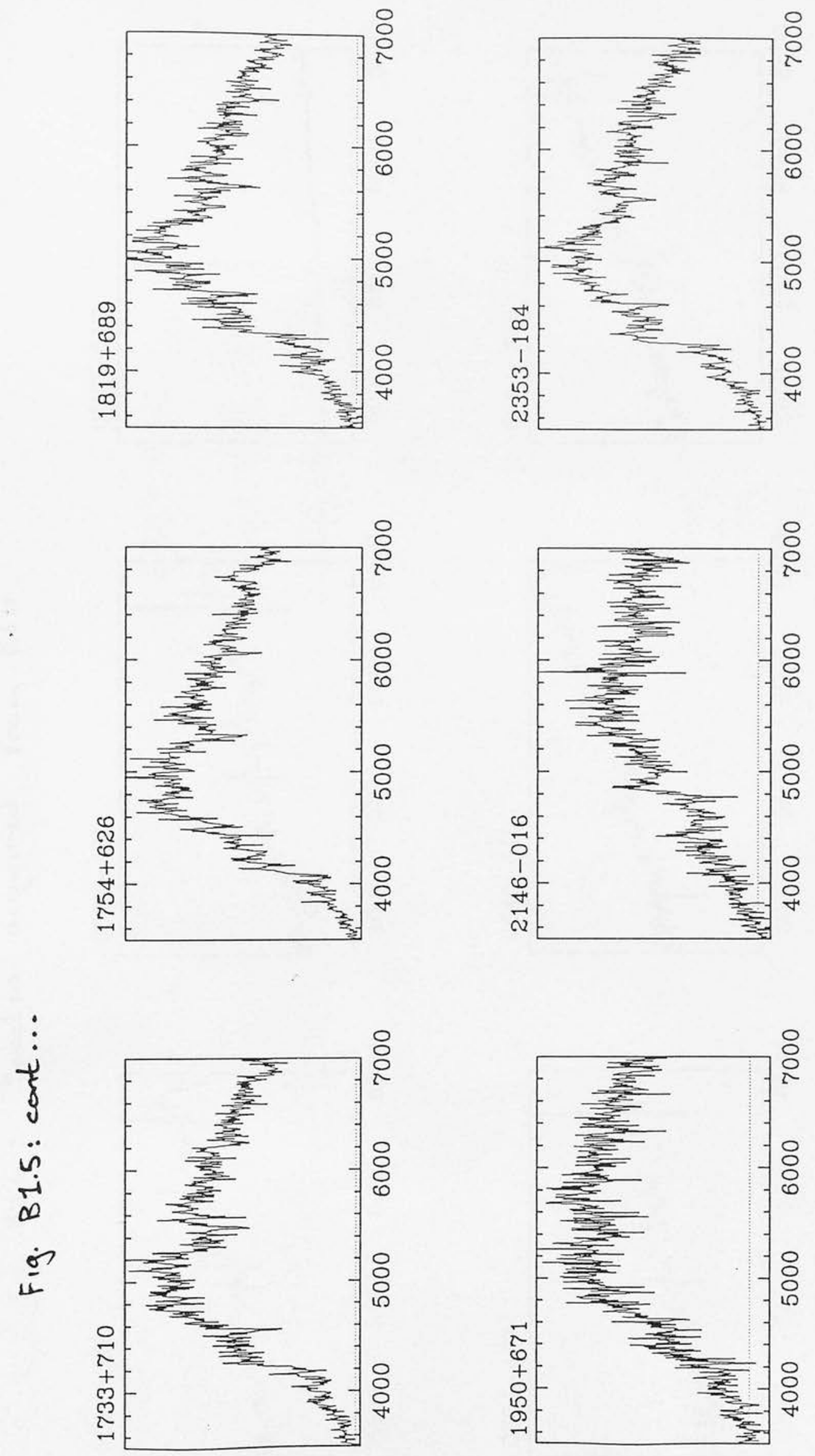

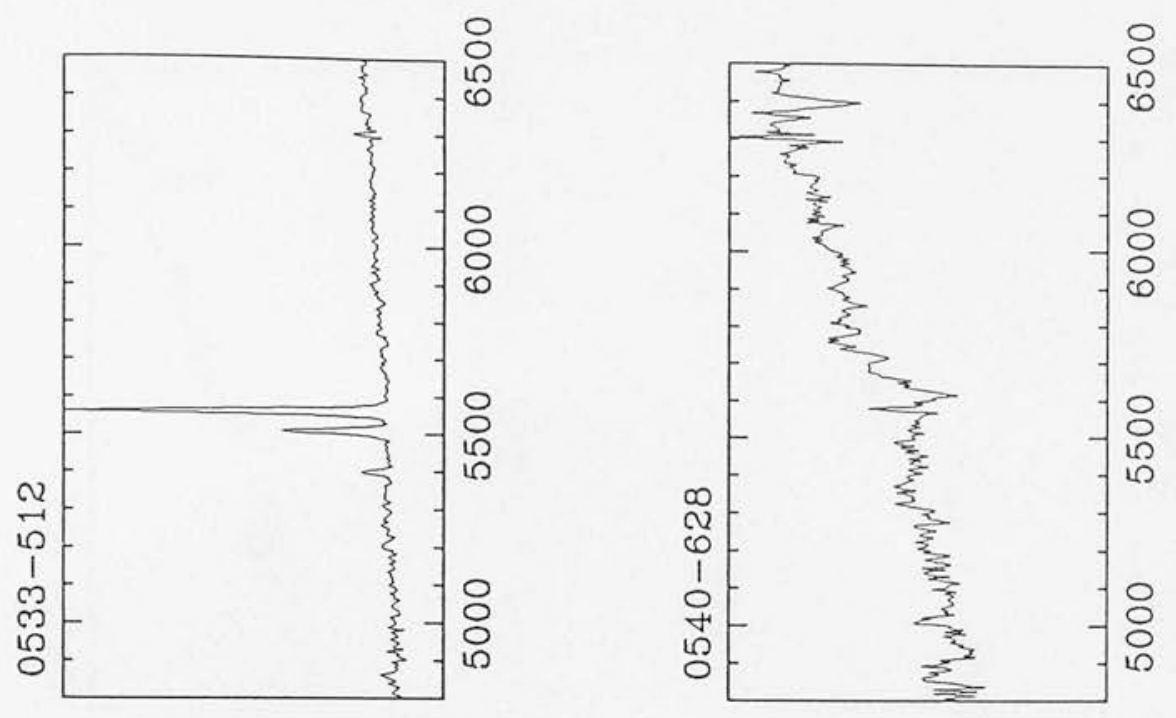

0
4
4
5
5
5
5
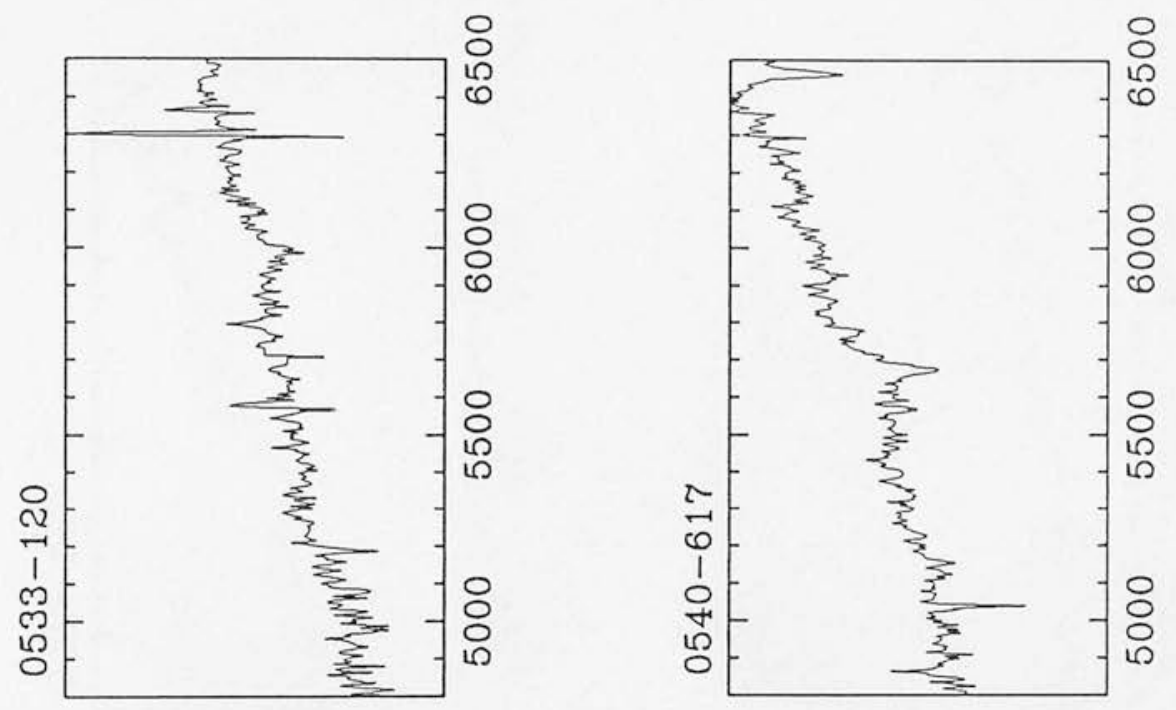

$\delta$
u
ڤ్

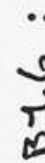
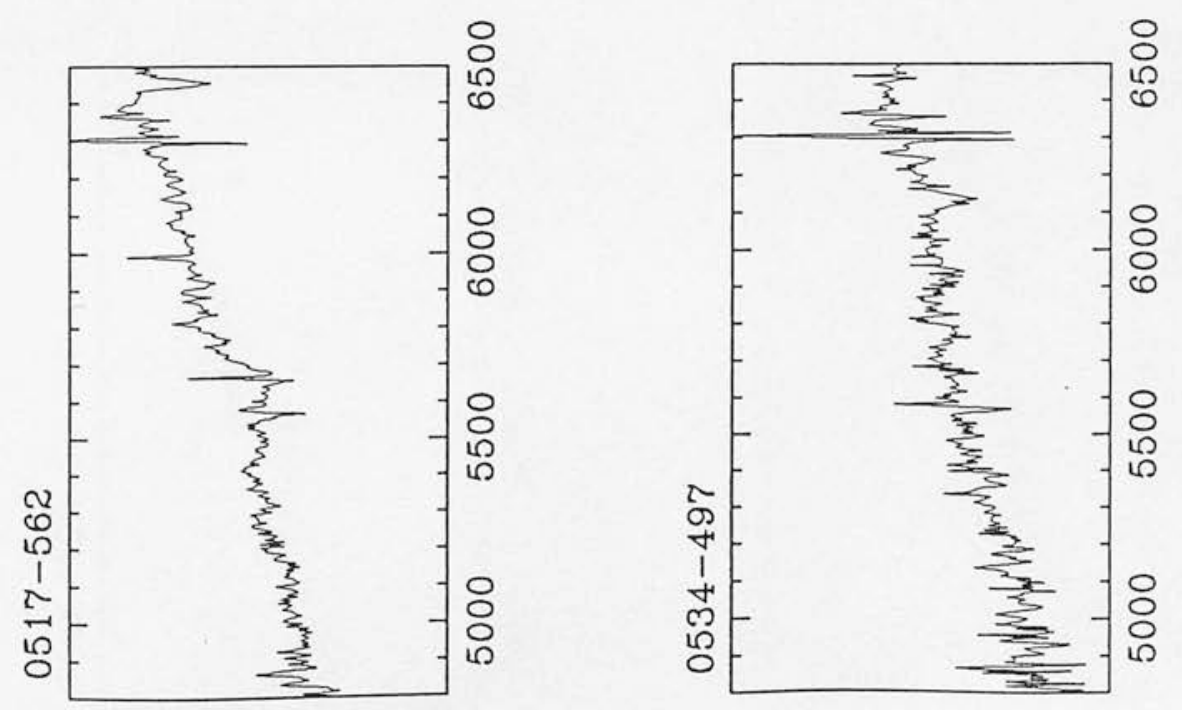

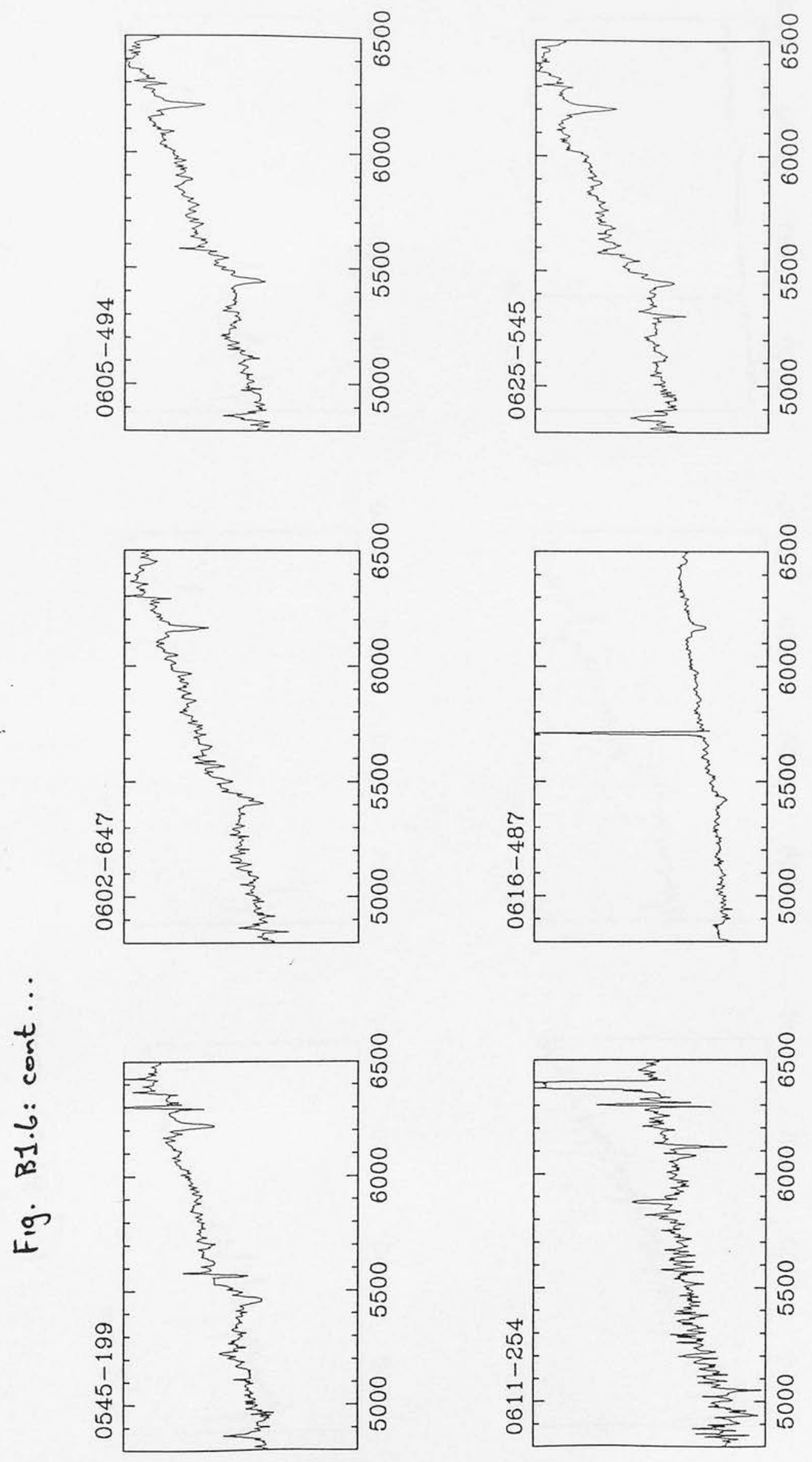

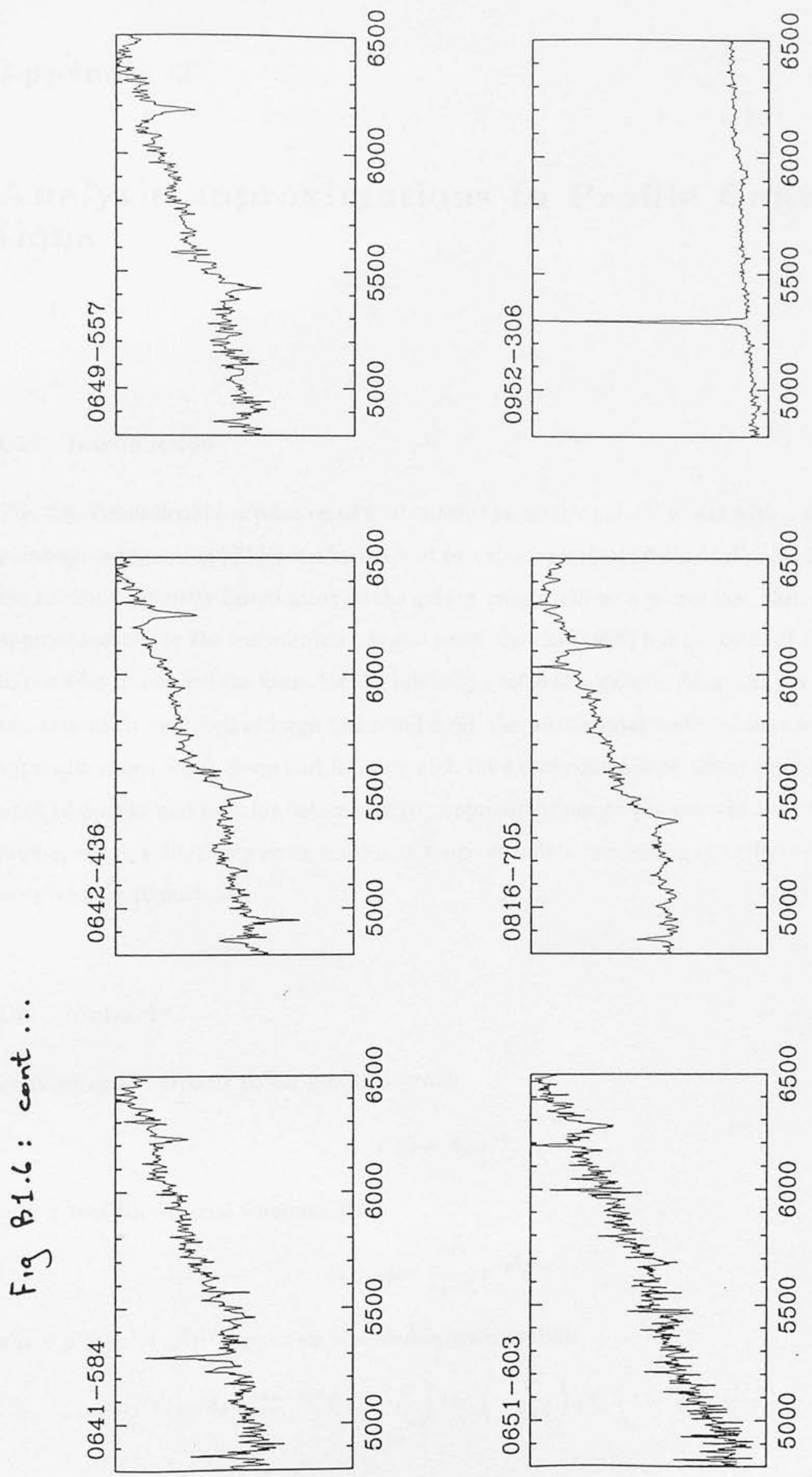


\section{Appendix C}

\section{Analytic approximations to Profile Convolu- tions}

\section{C1 Introduction}

The two-dimensional convolution of a circularly symmetric galaxy model with a Gaussian point-spread function (PSF) can be reduced to a single integral (Bailey \& Sparks 1983). If the intrinsic intensity distribution of the galaxy model follows a power law, then analytic approximations to the convolution integral exist. Sparks (1988) has generalised this work to consider more realistic forms for the intensity profile of a galaxy. Although his analytic expressions fit very well at large and small radii, there is an intermediate range where the approximations break down and fail to match the exact convolution. Here we extend the work of Sparks and look for better analytic approximations to the convolution of an $r^{1 / 4}$ profile, which collectively cover the entire range of radius over which the effects of seeing are probably important.

\section{C2 Method}

Convolving an intrinsic power-law light profile

$$
i(\rho)=A_{k} \rho^{-k}
$$

with a two-dimensional Gaussian PSF

$$
S(x, y)=\frac{1}{2 \pi \sigma^{2}} e^{-\rho^{2} / 2 \sigma^{2}}
$$

where $\rho=\left(x^{2}+y^{2}\right)^{1 / 2}$, gives an observed intensity profile

$$
i_{\text {obs }}(r)=A_{k} \sigma^{-k} 2^{-k / 2} \Gamma\left(1-\frac{k}{2}\right) \exp \left(-\frac{r^{2}}{2 \sigma^{2}}\right){ }_{1} F_{1}\left(1-\frac{k}{2}, 1 ; \frac{r^{2}}{2 \sigma^{2}}\right)
$$


where $\Gamma(z)$ is the usual gamma-function and ${ }_{1} F_{1}(\alpha, \beta ; z)$ is the confluent hypergeometric function. A more flexible representation of the intensity distribution of a galaxy is

$$
I(s)=I_{e} e^{-\alpha\left(s^{\gamma}\right)-1}=I_{0} e^{-\alpha s^{\gamma}}
$$

where $s$ is the ratio of radius to characteristic size $r_{0}$ and $I_{0}$ is a characteristic or effective intensity. This expression accommodates the empirical relations which have been derived for ellipticals $(\alpha \simeq 7.67, \gamma=0.25)$ and the exponential disc of spirals $(\alpha \simeq 1.68, \gamma=1)$. To make use of the analytic solution to the convolution integral given by equation $(i)$ the intensity distribution in equation ()$^{C}$ must be recast as a power-law. Thus, adopting the usual expansion for an exponential series one obtains

$$
I(s)=\sum_{n=0}^{\infty} I_{0} \frac{\left(-\alpha s^{\gamma}\right)^{n}}{n !} \equiv \sum_{n=0}^{\infty} i^{(n)}
$$

and the $n$th term of the series is

$$
i^{(n)}=I_{0} \frac{\left(-\alpha r_{0}^{-\gamma}\right)^{n}}{n !} r^{n \gamma}
$$

which is of the desired power-law form with $k=-\gamma n$ and $A_{k}=I_{0} \frac{\left(-\alpha r_{0}^{-\gamma} \gamma\right)^{n}}{n !}$.

These are completely general formulae for galaxies with power-law light profiles. Here we shall only consider $r^{1 / 4}$ law galaxies since this empirical fir to the data in Chapter 4 was found to be sufficiently adequate for most of the radio galaxies.

\section{C2.1 ANALYTIC APPROXIMATION FOR SMALL RADII}

This can be derived by noting that the confluent hypergeometric function can be expanded in a power series as follows:

$$
{ }_{1} F_{1}(a ; b ; z)=1+\frac{a}{b} \frac{z}{1 !}+\frac{a(a+1)}{b(b+1)} \frac{z^{2}}{2 !}+\cdots
$$

valid for all $z$. Substituting this into equation ()$_{C \cdot 3}$ and expanding the exponential term gives, for a power law $i(\rho)$

$$
i_{\mathrm{obs}} \sim A_{k} 2^{-k / 2} \sigma^{-k} \Gamma\left(1-\frac{k}{2}\right)\left(1-\frac{k}{2} z\right)
$$

for small $z$ and where, as before, $z=r^{2} / 2 \sigma^{2}$. For the model galaxy profile we set $k=-\gamma n$ and perform an infinite sum.

$$
I(s)=\sum_{0}^{\infty} A_{k} 2^{n \gamma / 2} \sigma^{n \gamma} \Gamma\left(1+\frac{n \gamma}{2}\right)\left(1+\frac{n \gamma}{2} z\right)
$$

where the appropriate form for $A_{k}$ has been given above. 


\section{C2.2 ANALYTIC APPROXIMATION FOR LARGE RADII}

To derive this, use is made of the asymptotic expansion of the confluent hypergeometric function ${ }_{1} F_{1}(a, b ; z)$ valid for large $z$. This is

$$
{ }_{1} F_{1}(a, b ; z) \sim \frac{\Gamma(b)}{\Gamma(a)} z^{a-b} e^{z}\left(\sum_{n=0}^{S-1} \frac{(1-a) n(b-a) n}{n ! z^{n}}+O\left(z^{-S}\right)\right)
$$

where $(\theta)_{n}$ denotes $\theta(\theta+1) \cdots(\theta+n-1)$. Upon substitution into equation $\hat{d}^{C}$, the leading terms cancel and so at large radii a power-law intensity profile produces an observed profile of the form

$$
i_{\mathrm{obs}} \sim A_{k} r^{-k} \sum_{n=0}^{S-1} \frac{(k / 2)_{n}^{2}}{n ! z^{n}}
$$

For $k=-\gamma n$ and the expression for $A_{k}$, a general term for $i^{(n)}$ can be found and the observed intensity calculated by summing up from $n=0 \rightarrow \infty$. After some considerable algebra, the results is

$$
I_{\text {obs }}(r)=I_{0} e^{\left(-r^{\gamma}\right)}\left[1+\frac{\gamma^{2} \sigma^{2}}{2} r^{\gamma-2}\left(r^{\gamma}-1\right)\right]
$$

\section{C3 Results}

In fact it turns out that the analytic formulae derived by Sparks and written down in thre preceding Section can be made to fit a large range of radius provided one is prepared to take enough terms in the series expansion for the confluent hypergeometric function. Figure C.1 shows the result of applying these expressions to an $r^{1 / 4}$ profile convolved with a PSF of $\sigma=5 r_{0}$. An excellent match to the true convolution, computed by numerical integration, is found over all radii for which the effects of seeing on the light profile are important. This fit required 8 terms in the summation over ${ }_{1} F_{1}(a, b ; z)$ for the long-range approximation.

\section{C4 Conclusion}

Numerical integration for the effects of seeing on power-law galaxy light profiles are computationally intensive. Analytic expressions to the integral have been found by Sparks but 


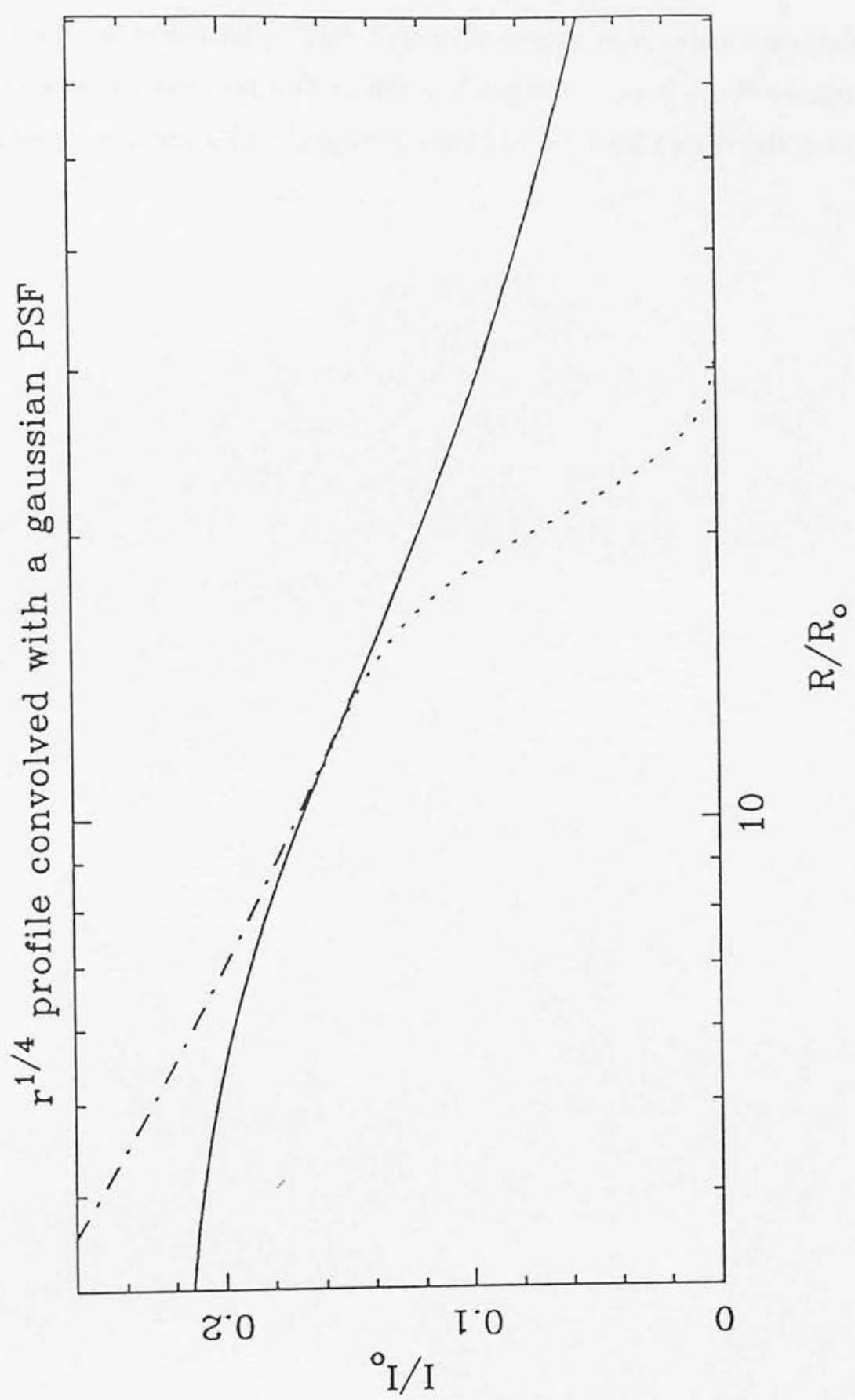

ig. C.1: Analytic approximate fit to the convolution of an $r^{1 / 4}$ profile with a gaussian $\mathrm{SF}$ equal to $5 r_{0}$. The bold line is for the numerical solution to the 2 -d convolution tegral. The short- and long-range approximations are shown by the dotted and dashedot lines respectively. 
these were not tested fully. This Appendix demonstrates that the approximations work very well for an assumed $r^{1 / 4}$ profile and require many fewer floating-point operations, and hence much less CPU time, than would be required by the full 2-d integral. 


\section{Appendix D}

\section{Environment Parameters for The G7 Ellipti- cals}

This Appendix provides listings of the cluster environment parameters, $A_{g e}$ and $B_{g e}$ defined in Chapter 4, for the G7 elliptical galaxies. For galaxies north of $\delta=-23^{\circ}$, both the Abell cluster catalogue and Lick galaxy counts were used to derive estimates. The Lick estimate is preferable however and this was used in preference for the biased peculiar velocities analysis presented in Chapter 7 . 
Table D.1: Local galaxian environment parameters $A_{g e}$ and $B_{g e}$ for the G7 ellipticals.

\begin{tabular}{|c|c|c|c|c|c|c|}
\hline ID\# & GP\# & IAU & $A_{g e}^{\mathrm{Abell}}$ & $A_{g e}^{\text {Lick }} \pm \delta A_{g e}$ & $B_{g e}^{\mathrm{Abell}}$ & $B_{g e}^{\text {Lick }} \pm \delta B_{g e}$ \\
\hline 1 & 0 & $0002-307$ & 0.139 & & 64.0 & \\
\hline 4 & 0 & $0035-181$ & -0.015 & $-0.084 \pm 0.151$ & -6.5 & $-50.3 \pm 90.4$ \\
\hline 23 & 0 & $0105-471$ & 0.987 & & 439.8 & \\
\hline 45 & 0 & $0136-427$ & -0.017 & & -7.3 & \\
\hline 56 & 0 & $0150-139$ & -0.001 & $-0.119 \pm 0.122$ & -0.5 & $-114.1 \pm 116.9$ \\
\hline 66 & 0 & $0204-413$ & -0.012 & & -5.1 & \\
\hline 67 & 0 & $0205+107$ & -0.001 & $-0.085 \pm 0.210$ & -0.5 & $-81.5 \pm 201.3$ \\
\hline 76 & 0 & $0240+322$ & -0.011 & $0.066 \pm 0.147$ & -4.7 & $42.4 \pm 94.2$ \\
\hline 101 & 0 & $0336-225$ & -0.008 & $-0.101 \pm 0.121$ & -3.2 & $-70.5 \pm 84.5$ \\
\hline 112 & 0 & $0406-211$ & -0.007 & $0.004 \pm 0.126$ & -3.0 & $2.7 \pm 88.3$ \\
\hline 117 & 0 & $0427-269$ & -0.007 & & -2.8 & \\
\hline 120 & 0 & $0429+731$ & -0.007 & $-0.018 \pm 0.119$ & -3.1 & $-12.7 \pm 82.9$ \\
\hline 128 & 0 & $0456+005$ & -0.008 & $-0.118 \pm 0.242$ & -3.6 & $-79.3 \pm 162.6$ \\
\hline 129 & 0 & $0457-078$ & -0.007 & $0.776 \pm 0.257$ & -2.9 & $545.3 \pm 180.6$ \\
\hline 130 & 0 & $0459-212$ & -0.009 & $0.042 \pm 0.183$ & -3.7 & $28.3 \pm 122.1$ \\
\hline 131 & 0 & $0504-176$ & -0.009 & $-0.154 \pm 0.334$ & -4.0 & $-100.6 \pm 218.2$ \\
\hline 132 & 0 & $0524-467$ & -0.007 & & -3.1 & \\
\hline 137 & 0 & $0645-266$ & -0.002 & & -0.7 & \\
\hline 138 & 0 & $0648-642$ & -0.005 & & -2.2 & \\
\hline 139 & 0 & $0700-286$ & -0.002 & & -0.9 & \\
\hline 141 & 0 & $0703+754$ & -0.006 & $0.100 \pm 0.172$ & -2.6 & $71.7 \pm 123.1$ \\
\hline 149 & 0 & $0721-274$ & -0.001 & & -0.5 & \\
\hline 150 & 0 & $0732-503$ & 0.000 & & -0.2 & \\
\hline 152 & 0 & $0759+095$ & -0.009 & $0.185 \pm 0.184$ & -3.8 & $119.2 \pm 118.7$ \\
\hline 153 & 0 & $0817+212$ & -0.009 & $0.368 \pm 0.204$ & -3.8 & $237.9 \pm 131.9$ \\
\hline 157 & 0 & $0843-336$ & -0.002 & & -0.7 & \\
\hline 163 & 0 & $0902+185$ & -0.007 & $0.223 \pm 0.062$ & -3.0 & $150.0 \pm 41.7$ \\
\hline 167 & 0 & $0917+720$ & -0.005 & $-0.182 \pm 0.053$ & -2.2 & $-133.6 \pm 39.0$ \\
\hline 168 & 0 & $0917+012$ & -0.011 & $0.132 \pm 0.066$ & -4.7 & $80.9 \pm 40.4$ \\
\hline 172 & 0 & $0922-635$ & -0.003 & & -1.4 & \\
\hline 173 & 0 & $0922+116$ & -0.004 & $-0.089 \pm 0.116$ & -1.8 & $-66.2 \pm 86.1$ \\
\hline 175 & 0 & $0940-034$ & -0.001 & $-0.021 \pm 0.097$ & -0.6 & $-18.5 \pm 83.7$ \\
\hline 178 & 0 & $0955+105$ & -0.012 & $-0.052 \pm 0.145$ & -5.2 & $-31.2 \pm 86.9$ \\
\hline 182 & 0 & $0958-027$ & -0.015 & $0.203 \pm 0.092$ & -6.6 & $115.0 \pm 52.2$ \\
\hline 183 & 0 & $1002-074$ & 0.000 & $0.274 \pm 0.104$ & -0.1 & $276.4 \pm 104.9$ \\
\hline 188 & 0 & $1010+390$ & -0.019 & $0.159 \pm 0.077$ & -8.5 & $86.1 \pm 41.7$ \\
\hline 190 & 0 & $1017-212$ & 0.143 & $-0.062 \pm 0.049$ & 60.2 & $-43.6 \pm 34.5$ \\
\hline 202 & 0 & $1038-459$ & 0.270 & & 121.9 & \\
\hline 206 & 0 & $1045-098$ & -0.002 & $-0.065 \pm 0.090$ & -1.0 & $-53.2 \pm 73.3$ \\
\hline 209 & 0 & $1048-127$ & -0.008 & $-0.061 \pm 0.080$ & -3.4 & $-39.8 \pm 52.2$ \\
\hline 210 & 0 & $1050-400$ & -0.010 & & -4.1 & \\
\hline 211 & 0 & $1101-198$ & -0.006 & $-0.169 \pm 0.005$ & -2.5 & $-118.0 \pm 3.5$ \\
\hline 212 & 0 & $1107-372$ & -0.001 & & -0.5 & \\
\hline 213 & 0 & $1109+731$ & -0.018 & $0.118 \pm 0.113$ & -8.2 & $65.0 \pm 62.3$ \\
\hline 224 & 0 & $1139-058$ & -0.001 & $-0.236 \pm 0.182$ & -0.4 & $-214.0 \pm 165.1$ \\
\hline 231 & 0 & $1143+140$ & -0.004 & $-0.021 \pm 0.065$ & -1.8 & $-15.6 \pm 48.2$ \\
\hline 240 & 0 & $1201+021$ & -0.014 & $0.137 \pm 0.097$ & -6.4 & $78.4 \pm 55.4$ \\
\hline 244 & 0 & $1209+134$ & -0.002 & $0.200 \pm 0.202$ & -0.9 & $164.4 \pm 166.3$ \\
\hline 246 & 0 & $1216+060$ & -0.002 & $0.161 \pm 0.026$ & -0.9 & $134.0 \pm 21.7$ \\
\hline 278 & 0 & $1235+017$ & -0.001 & $-0.135 \pm 0.253$ & -0.6 & $-118.1 \pm 221.1$ \\
\hline 289 & 0 & $1246-055$ & -0.001 & $0.054 \pm 0.085$ & -0.2 & $50.9 \pm 80.3$ \\
\hline
\end{tabular}




\begin{tabular}{|c|c|c|c|c|c|c|}
\hline ID \# & GP\# & IAU & $A_{g e}^{\mathrm{Abell}}$ & $A_{g e}^{\text {Lick }} \pm \delta A_{g e}$ & $B_{g e}^{\mathrm{Abell}}$ & $B_{g e}^{\text {Lick }} \pm \delta B_{g e}$ \\
\hline 301 & 0 & $1249-215$ & -0.019 & $0.132 \pm 0.054$ & -8.6 & $70.8 \pm 29.1$ \\
\hline 306 & 0 & $1251-065$ & -0.009 & $-0.042 \pm 0.079$ & -3.8 & $-26.9 \pm 50.6$ \\
\hline 312 & 0 & $1254-194$ & -0.005 & $0.070 \pm 0.048$ & -1.9 & $51.2 \pm 35.2$ \\
\hline 320 & 0 & $1257-219$ & -0.019 & $-0.025 \pm 0.114$ & -8.7 & $-13.4 \pm 61.1$ \\
\hline 329 & 0 & $1258-042$ & -0.004 & $-0.350 \pm 0.054$ & -1.7 & $-261.7 \pm 40.4$ \\
\hline 334 & 0 & $1305-492$ & -0.001 & & -0.3 & \\
\hline 347 & 0 & $1328+469$ & -0.003 & $-0.116 \pm 0.165$ & -1.1 & $-95.2 \pm 135.5$ \\
\hline 359 & 0 & $1403-336$ & 1.232 & & 523.9 & \\
\hline 360 & 0 & $1405-477$ & -0.001 & & -0.3 & \\
\hline 365 & 0 & $1418+399$ & -0.001 & $-0.344 \pm 0.108$ & -0.3 & $-327.6 \pm 102.9$ \\
\hline 366 & 0 & $1425-373$ & 0.053 & & 22.2 & \\
\hline 367 & 0 & $1426+260$ & -0.008 & $-0.369 \pm 0.078$ & -3.6 & $-243.0 \pm 51.3$ \\
\hline 374 & 0 & $1458-072$ & -0.002 & $-0.106 \pm 0.107$ & -0.7 & $-91.8 \pm 92.3$ \\
\hline 385 & 0 & $1554+225$ & -0.008 & $-0.085 \pm 0.174$ & -3.4 & $-57.1 \pm 116.8$ \\
\hline 386 & 0 & $1602+240$ & 0.265 & $0.264 \pm 0.127$ & 129.6 & $127.1 \pm 61.1$ \\
\hline 390 & 0 & $1618+580$ & -0.009 & $0.437 \pm 0.102$ & -3.7 & $289.4 \pm 67.6$ \\
\hline 399 & 0 & $1734+608$ & -0.006 & $-0.306 \pm 0.152$ & -2.4 & $-224.8 \pm 111.7$ \\
\hline 405 & 0 & $1845+456$ & -0.009 & $0.248 \pm 0.079$ & -3.9 & $165.2 \pm 52.5$ \\
\hline 406 & 0 & $1845+454$ & -0.002 & $0.315 \pm 0.149$ & -1.0 & $273.1 \pm 129.0$ \\
\hline 409 & 0 & $1856-578$ & 0.019 & & 8.2 & \\
\hline 417 & 0 & $2002-403$ & 0.253 & & 111.1 & \\
\hline 424 & 0 & $2020-278$ & -0.014 & & -6.0 & \\
\hline 430 & 0 & $2100-492$ & 0.359 & & 163.6 & \\
\hline 435 & 0 & $2105-436$ & 1.857 & & 887.9 & \\
\hline 451 & 0 & $2203-645$ & -0.004 & & -1.4 & \\
\hline 456 & 0 & $2222-315$ & -0.027 & & -12.6 & \\
\hline 458 & 0 & $2247+113$ & 0.424 & $0.136 \pm 0.083$ & 194.3 & $71.9 \pm 43.7$ \\
\hline 467 & 0 & $2330-453$ & -0.004 & & -1.7 & \\
\hline 474 & 0 & $2352+056$ & -0.006 & $-0.486 \pm 0.135$ & -2.5 & $-364.6 \pm 101.3$ \\
\hline 481 & 0 & $0645-266$ & -0.002 & & -0.9 & \\
\hline 482 & 0 & $0816-252$ & -0.001 & & -0.6 & \\
\hline 483 & 0 & $0928-301$ & -0.002 & & -1.0 & \\
\hline 484 & 0 & $1000-314$ & 0.000 & & 0.1 & \\
\hline 488 & 0 & $1113-335$ & -0.001 & & -0.6 & \\
\hline 494 & 0 & $1248-261$ & -0.005 & & -1.9 & \\
\hline 553 & 0 & $2248-017$ & -0.004 & $-0.229 \pm 0.141$ & -1.6 & $-187.2 \pm 115.3$ \\
\hline 2 & 21 & $0018+220$ & -0.013 & $0.271 \pm 0.157$ & -5.7 & $168.1 \pm 97.3$ \\
\hline 3 & 21 & $0018+221$ & -0.013 & $0.270 \pm 0.126$ & -5.7 & $167.7 \pm 78.1$ \\
\hline 12 & 23 & $0054-530$ & 0.075 & & 34.8 & \\
\hline 13 & 23 & $0054-532$ & -0.025 & & -11.4 & \\
\hline 17 & 24 & $0104+322$ & -0.010 & $0.343 \pm 0.161$ & -4.2 & $228.2 \pm 107.1$ \\
\hline 18 & 24 & $0104+322$ & -0.010 & $0.301 \pm 0.144$ & -4.2 & $200.0 \pm 95.8$ \\
\hline 19 & 24 & $0104+321$ & -0.010 & $0.310 \pm 0.145$ & -4.2 & $206.4 \pm 96.5$ \\
\hline 20 & 24 & $0104+321$ & -0.010 & $0.308 \pm 0.145$ & -4.2 & $205.1 \pm 96.5$ \\
\hline 21 & 24 & $0104+320$ & -0.010 & $0.295 \pm 0.145$ & -4.2 & $196.5 \pm 96.5$ \\
\hline 24 & 24 & $0105+328$ & -0.010 & $0.230 \pm 0.080$ & -4.2 & $152.8 \pm 53.2$ \\
\hline 27 & 24 & $0108+328$ & -0.010 & $0.288 \pm 0.126$ & -4.2 & $191.2 \pm 83.8$ \\
\hline 34 & 25 & $0122+014$ & 0.181 & $-0.521 \pm 0.128$ & 77.9 & $-327.9 \pm 80.5$ \\
\hline 35 & 25 & $0123-016$ & 0.757 & $0.665 \pm 0.146$ & 326.1 & $418.1 \pm 91.8$ \\
\hline 36 & 25 & $0123-015$ & 0.757 & $0.602 \pm 0.133$ & 326.2 & $378.5 \pm 83.6$ \\
\hline 37 & 25 & $0123-016$ & 0.758 & $0.626 \pm 0.137$ & 326.4 & $393.6 \pm 86.1$ \\
\hline
\end{tabular}




\begin{tabular}{|c|c|c|c|c|c|c|}
\hline ID\# & GP\# & IAU & $A_{g e}^{\mathrm{Abell}}$ & $A_{g e}^{\text {Lick }} \pm \delta A_{g e}$ & $B_{g e}^{\mathrm{Abell}}$ & $B_{g e}^{\text {Lick }} \pm \delta B_{g e}$ \\
\hline 38 & 25 & $0123-014$ & 0.743 & $0.550 \pm 0.139$ & 320.2 & $345.5 \pm 87.4$ \\
\hline 41 & 25 & $0125-021$ & 0.667 & $0.472 \pm 0.121$ & 287.6 & $296.8 \pm 76.1$ \\
\hline 42 & 26 & $0128-071$ & -0.002 & $-0.109 \pm 0.250$ & -0.6 & $-102.9 \pm 236.0$ \\
\hline 43 & 26 & $0130-072$ & -0.002 & $-0.111 \pm 0.071$ & -0.6 & $-104.8 \pm 67.0$ \\
\hline 46 & 26 & $0136-077$ & -0.002 & $-0.126 \pm 0.155$ & -0.6 & $-118.8 \pm 146.2$ \\
\hline 49 & 27 & $0146+355$ & 0.723 & $-0.064 \pm 0.121$ & 306.2 & $-43.4 \pm 81.4$ \\
\hline 52 & 27 & $0147+361$ & 0.785 & $0.171 \pm 0.279$ & 332.4 & $114.8 \pm 187.7$ \\
\hline 55 & 27 & $0149+359$ & 0.843 & $0.406 \pm 0.292$ & 357.0 & $272.9 \pm 196.4$ \\
\hline 60 & 27 & $0153+328$ & 0.206 & $-1.013 \pm 0.274$ & 87.2 & $-681.4 \pm 184.4$ \\
\hline 61 & 27 & $0154+329$ & 0.212 & $-1.003 \pm 0.105$ & 89.7 & $-674.7 \pm 70.6$ \\
\hline 64 & 27 & $0157+311$ & 0.143 & $-0.371 \pm 0.111$ & 60.8 & $-249.6 \pm 74.7$ \\
\hline 80 & 29 & $0259-150$ & -0.002 & $0.031 \pm 0.002$ & -0.9 & $27.4 \pm 1.8$ \\
\hline 81 & 29 & $0301-158$ & -0.002 & $0.098 \pm 0.116$ & -0.9 & $86.2 \pm 101.6$ \\
\hline 83 & 29 & $0303-158$ & -0.002 & $0.166 \pm 0.136$ & -0.9 & $145.2 \pm 119.1$ \\
\hline 84 & 30 & $0313+41.1$ & 1.717 & $1.341 \pm 0.139$ & 740.0 & $843.0 \pm 87.4$ \\
\hline 85 & 30 & $0316+413$ & 1.811 & $2.147 \pm 0.201$ & 780.2 & $1349.4 \pm 126.3$ \\
\hline 86 & 30 & $0316+413$ & 1.796 & $2.097 \pm 0.176$ & 773.7 & $1318.2 \pm 110.6$ \\
\hline 87 & 30 & $0316+414$ & 1.808 & $2.118 \pm 0.201$ & 779.3 & $1331.4 \pm 126.3$ \\
\hline 88 & 30 & $0316+413$ & 1.789 & $2.048 \pm 0.165$ & 770.6 & $1287.3 \pm 103.7$ \\
\hline 89 & 30 & $0316+411$ & 1.762 & $2.070 \pm 0.178$ & 759.2 & $1301.2 \pm 111.9$ \\
\hline 90 & 30 & $0316+412$ & 1.764 & $2.098 \pm 0.192$ & 760.0 & $1318.7 \pm 120.7$ \\
\hline 91 & 30 & $0318+412$ & 1.632 & $1.748 \pm 0.186$ & 703.4 & $1098.4 \pm 116.9$ \\
\hline 567 & 30 & $0314+412$ & 1.789 & $1.784 \pm 0.138$ & 770.7 & $1121.3 \pm 86.8$ \\
\hline 568 & 30 & $0315+412$ & 1.817 & $2.060 \pm 0.196$ & 782.9 & $1295.1 \pm 123.2$ \\
\hline 569 & 30 & $0316+413$ & 1.803 & $2.118 \pm 0.188$ & 776.7 & $1331.5 \pm 118.2$ \\
\hline 570 & 30 & $0316+414$ & 1.808 & $2.118 \pm 0.201$ & 779.3 & $1331.4 \pm 126.3$ \\
\hline 572 & 30 & $0316+412$ & 1.791 & $2.095 \pm 0.197$ & 771.7 & $1316.5 \pm 123.8$ \\
\hline 573 & 30 & $0316+412$ & 1.791 & $2.095 \pm 0.197$ & 771.7 & $1316.5 \pm 123.8$ \\
\hline 574 & 30 & $0316+411$ & 1.763 & $2.097 \pm 0.177$ & 759.6 & $1318.3 \pm 111.3$ \\
\hline 575 & 30 & $0316+411$ & 1.734 & $2.103 \pm 0.180$ & 747.1 & $1322.0 \pm 113.1$ \\
\hline 576 & 30 & $0315+409$ & 1.719 & $2.091 \pm 0.198$ & 740.8 & $1314.5 \pm 124.5$ \\
\hline 577 & 30 & $0316+409$ & 1.721 & $2.104 \pm 0.202$ & 741.6 & $1322.7 \pm 127.0$ \\
\hline 94 & 31 & $0326-324$ & -0.091 & & -36.1 & \\
\hline 95 & 31 & $0326-312$ & -0.076 & & -30.0 & \\
\hline 96 & 31 & $0328-350$ & -0.171 & & -67.9 & \\
\hline 97 & 31 & $0333-354$ & -0.360 & & -142.9 & \\
\hline 98 & 31 & $0334-356$ & -0.388 & & -153.8 & \\
\hline 100 & 31 & $0336-356$ & -0.413 & & -163.9 & \\
\hline 102 & 31 & $0336-357$ & -0.405 & & -160.8 & \\
\hline 105 & 31 & $0338-376$ & -0.146 & & -58.0 & \\
\hline 106 & 31 & $0340-355$ & -0.332 & & -131.8 & \\
\hline 111 & 31 & $0352-361$ & -0.105 & & -41.6 & \\
\hline 99 & 32 & $0336-231$ & -0.037 & & -14.9 & \\
\hline 103 & 32 & $0337-188$ & -0.030 & $0.214 \pm 0.092$ & -11.9 & $205.2 \pm 88.1$ \\
\hline 104 & 32 & $0337-187$ & -0.030 & $0.190 \pm 0.091$ & -11.9 & $182.1 \pm 87.1$ \\
\hline 107 & 32 & $0340-222$ & -0.035 & $-0.233 \pm 0.092$ & -14.1 & $-222.8 \pm 88.0$ \\
\hline 108 & 32 & $0342-220$ & -0.035 & $-0.250 \pm 0.115$ & -13.9 & $-238.9 \pm 109.9$ \\
\hline 118 & 33 & $0428+005$ & -0.005 & $-0.056 \pm 0.119$ & -2.2 & $-41.8 \pm 88.4$ \\
\hline 119 & 33 & $0428+005$ & -0.005 & $-0.062 \pm 0.112$ & -2.2 & $-45.7 \pm 83.2$ \\
\hline 121 & 34 & $0429-051$ & -0.010 & $0.178 \pm 0.124$ & -4.3 & $115.3 \pm 80.3$ \\
\hline 122 & 34 & $0429-052$ & -0.010 & $0.180 \pm 0.124$ & -4.3 & $116.5 \pm 80.3$ \\
\hline
\end{tabular}




\begin{tabular}{|c|c|c|c|c|c|c|}
\hline ID\# & GP\# & IAU & $A_{g e}^{\mathrm{Abell}}$ & $A_{g e}^{\text {Lick }} \pm \delta A_{g e}$ & $B_{g e}^{\mathrm{Abell}}$ & $B_{g e}^{\text {Lick }} \pm \delta B_{g e}$ \\
\hline 123 & 34 & $0429-052$ & -0.010 & $0.154 \pm 0.116$ & -4.3 & $99.5 \pm 75.1$ \\
\hline 256 & 35 & $1222-394$ & 0.131 & & 54.3 & \\
\hline 259 & 35 & $1224-390$ & 0.136 & & 56.5 & \\
\hline 140 & 36 & $0701+506$ & 0.255 & $0.052 \pm 0.134$ & 111.8 & $31.1 \pm 79.6$ \\
\hline 142 & 36 & $0705+487$ & 0.732 & $1.135 \pm 0.162$ & 320.6 & $673.8 \pm 96.1$ \\
\hline 143 & 36 & $0705+487$ & 0.728 & $1.091 \pm 0.130$ & 318.7 & $647.6 \pm 77.1$ \\
\hline 144 & 36 & $0705+487$ & 0.726 & $1.130 \pm 0.137$ & 318.0 & $670.8 \pm 81.3$ \\
\hline 145 & 36 & $0707+502$ & 0.320 & $0.739 \pm 0.190$ & 140.3 & $438.6 \pm 112.8$ \\
\hline 158 & 39 & $0846+192$ & -0.006 & $0.076 \pm 0.103$ & -2.6 & $52.5 \pm 71.5$ \\
\hline 159 & 39 & $0846+192$ & -0.006 & $0.099 \pm 0.105$ & -2.6 & $69.0 \pm 72.9$ \\
\hline 166 & 41 & $0916+339$ & 0.625 & $0.374 \pm 0.088$ & 283.1 & $202.7 \pm 47.7$ \\
\hline 169 & 41 & $0918+336$ & 0.573 & $0.059 \pm 0.063$ & 259.2 & $32.2 \pm 34.1$ \\
\hline 170 & 41 & $0919+340$ & 0.589 & $0.054 \pm 0.069$ & 266.6 & $29.2 \pm 37.4$ \\
\hline 184 & 44 & $1004-671$ & -0.001 & & -0.5 & \\
\hline 186 & 44 & $1009-667$ & -0.001 & & -0.5 & \\
\hline 189 & 45 & $1015+221$ & -0.001 & $0.102 \pm 0.082$ & -0.3 & $96.2 \pm 77.0$ \\
\hline 192 & 45 & $1020+201$ & -0.001 & $-0.153 \pm 0.006$ & -0.3 & $-143.1 \pm 5.6$ \\
\hline 193 & 46 & $1024-396$ & 0.000 & & 0.2 & \\
\hline 194 & 46 & $1026-354$ & 0.150 & & 61.7 & \\
\hline 195 & 46 & $1026-353$ & 0.150 & & 62.0 & \\
\hline 196 & 46 & $1026-353$ & 0.151 & & 62.3 & \\
\hline 197 & 46 & $1027-350$ & 0.156 & & 64.2 & \\
\hline 203 & 46 & $1038-368$ & 0.002 & & 1.0 & \\
\hline 199 & 47 & $1034-271$ & 1.083 & & 454.4 & \\
\hline 200 & 47 & $1034-272$ & 1.095 & & 459.3 & \\
\hline 201 & 47 & $1034-272$ & 1.089 & & 456.5 & \\
\hline 216 & 48 & $1114+182$ & 0.000 & $-0.099 \pm 0.251$ & -0.2 & $-94.5 \pm 239.7$ \\
\hline 217 & 48 & $1114+183$ & 0.000 & $-0.094 \pm 0.252$ & -0.2 & $-89.8 \pm 240.7$ \\
\hline 218 & 48 & $1114+184$ & 0.000 & $-0.063 \pm 0.247$ & -0.2 & $-60.2 \pm 235.9$ \\
\hline 221 & 50 & $1118+035$ & -0.001 & $0.003 \pm 0.060$ & -0.4 & $2.6 \pm 54.2$ \\
\hline 222 & 50 & $1118+034$ & -0.001 & $0.009 \pm 0.049$ & -0.4 & $8.2 \pm 44.3$ \\
\hline 225 & 51 & $1141+201$ & 2.333 & $1.029 \pm 0.150$ & 1044.1 & $573.3 \pm 83.6$ \\
\hline 227 & 51 & $1141+202$ & 2.331 & $1.032 \pm 0.094$ & 1043.1 & $575.0 \pm 52.4$ \\
\hline 228 & 51 & $1142+198$ & 2.325 & $0.860 \pm 0.057$ & 1040.5 & $479.0 \pm 31.8$ \\
\hline 230 & 51 & $1143+200$ & 2.331 & $0.792 \pm 0.091$ & 1043.2 & $441.2 \pm 50.7$ \\
\hline 234 & 51 & $1150+212$ & 0.783 & $0.274 \pm 0.068$ & 350.6 & $152.7 \pm 37.9$ \\
\hline 232 & 52 & $1146-289$ & -0.002 & & -0.6 & \\
\hline 233 & 52 & $1148-285$ & -0.002 & & -0.6 & \\
\hline 237 & 53 & $1155-180$ & -0.001 & $-0.045 \pm 0.251$ & -0.5 & $-40.0 \pm 223.3$ \\
\hline 239 & 53 & $1158-175$ & -0.001 & $-0.101 \pm 0.109$ & -0.5 & $-89.9 \pm 97.0$ \\
\hline 242 & 54 & $1205+653$ & -0.001 & $-0.059 \pm 0.001$ & -0.5 & $-53.4 \pm 0.9$ \\
\hline 243 & 54 & $1205+654$ & -0.001 & $-0.048 \pm 0.001$ & -0.5 & $-43.4 \pm 0.9$ \\
\hline 247 & 55 & $1217+295$ & 0.000 & $0.308 \pm 0.142$ & -0.1 & $310.1 \pm 143.2$ \\
\hline 248 & 55 & $1217+295$ & 0.000 & $0.316 \pm 0.171$ & -0.1 & $318.7 \pm 172.5$ \\
\hline 254 & 56 & $1221+075$ & -0.001 & $0.089 \pm 0.223$ & -0.2 & $84.6 \pm 212.1$ \\
\hline 255 & 56 & $1222+131$ & -0.001 & $0.028 \pm 0.063$ & -0.2 & $26.3 \pm 60.0$ \\
\hline 258 & 56 & $1223+132$ & -0.001 & $0.011 \pm 0.072$ & -0.2 & $10.8 \pm 68.6$ \\
\hline 260 & 56 & $1224+084$ & -0.001 & $-0.429 \pm 0.109$ & -0.2 & $-408.7 \pm 103.7$ \\
\hline 261 & 56 & $1226+135$ & -0.001 & $-0.030 \pm 0.189$ & -0.2 & $-28.6 \pm 180.1$ \\
\hline 265 & 56 & $1227+082$ & -0.001 & $-0.335 \pm 0.130$ & -0.2 & $-318.8 \pm 123.7$ \\
\hline 266 & 56 & $1227+137$ & -0.001 & $-0.072 \pm 0.207$ & -0.2 & $-68.6 \pm 197.2$ \\
\hline
\end{tabular}


Table D.1 cont....

\begin{tabular}{|c|c|c|c|c|c|c|}
\hline ID\# & GP\# & IAU & $A_{g e}^{\mathrm{Abell}}$ & $A_{g e}^{\text {Lick }} \pm \delta A_{g e}$ & $B_{g e}^{\mathrm{Abell}}$ & $B_{g e}^{\text {Lick }} \pm \delta B_{g e}$ \\
\hline 268 & 56 & $1227+126$ & -0.001 & $-0.536 \pm 0.162$ & -0.2 & $-510.6 \pm 154.3$ \\
\hline 270 & 56 & $1228+126$ & -0.001 & $-0.543 \pm 0.159$ & -0.2 & $-517.6 \pm 151.5$ \\
\hline 274 & 56 & $1233+128$ & -0.001 & $-0.007 \pm 0.281$ & -0.2 & $-6.7 \pm 267.8$ \\
\hline 276 & 56 & $1233+117$ & -0.001 & $0.172 \pm 0.139$ & -0.2 & $164.3 \pm 132.4$ \\
\hline 279 & 56 & $1239+119$ & -0.001 & $0.029 \pm 0.068$ & -0.2 & $27.3 \pm 64.8$ \\
\hline 283 & 56 & $1240+029$ & -0.001 & $-0.073 \pm 0.151$ & -0.2 & $-69.5 \pm 143.7$ \\
\hline 284 & 56 & $1241+118$ & -0.001 & $-0.074 \pm 0.072$ & -0.2 & $-70.8 \pm 68.6$ \\
\hline 286 & 56 & $1242+114$ & -0.001 & $-0.056 \pm 0.040$ & -0.2 & $-53.4 \pm 38.1$ \\
\hline 207 & 57 & $1045+142$ & 0.000 & $-0.153 \pm 0.098$ & -0.1 & $-152.5 \pm 97.7$ \\
\hline 208 & 57 & $1045+128$ & 0.000 & $-0.099 \pm 0.182$ & -0.1 & $-98.7 \pm 181.4$ \\
\hline 285 & 58 & $1241-414$ & 0.518 & & 213.8 & \\
\hline 290 & 58 & $1246-410$ & 0.723 & & 298.5 & \\
\hline 291 & 58 & $1246-407$ & 0.710 & & 293.2 & \\
\hline 299 & 58 & $1248-408$ & 0.657 & & 271.1 & \\
\hline 304 & 58 & $1251-394$ & 0.281 & & 115.8 & \\
\hline 280 & 59 & $1239-403$ & 0.348 & & 148.7 & \\
\hline 293 & 59 & $1247-411$ & 0.702 & & 299.7 & \\
\hline 294 & 59 & $1247-411$ & 0.702 & & 299.9 & \\
\hline 303 & 59 & $1250-409$ & 0.565 & & 241.5 & \\
\hline 302 & 60 & $1250-102$ & -0.008 & $-0.093 \pm 0.075$ & -3.4 & $-60.9 \pm 49.1$ \\
\hline 288 & 61 & $1245+274$ & 0.590 & $-0.309 \pm 0.079$ & 267.9 & $-166.1 \pm 42.4$ \\
\hline 305 & 61 & $1251+273$ & 0.963 & $0.006 \pm 0.062$ & 437.3 & $3.3 \pm 33.3$ \\
\hline 309 & 61 & $1253+277$ & 1.373 & $0.584 \pm 0.080$ & 623.7 & $313.6 \pm 43.0$ \\
\hline 311 & 61 & $1253+280$ & 1.692 & $0.809 \pm 0.077$ & 768.5 & $435.0 \pm 41.4$ \\
\hline 313 & 61 & $1255+277$ & 1.849 & $1.046 \pm 0.079$ & 839.9 & $562.3 \pm 42.5$ \\
\hline 315 & 61 & $1255+287$ & 1.837 & $0.175 \pm 0.068$ & 834.5 & $94.0 \pm 36.6$ \\
\hline 316 & 61 & $1256+279$ & 1.991 & $1.717 \pm 0.088$ & 904.2 & $923.0 \pm 47.3$ \\
\hline 317 & 61 & $1256+283$ & 2.075 & $1.613 \pm 0.086$ & 942.3 & $867.3 \pm 46.2$ \\
\hline 318 & 61 & $1256+282$ & 2.094 & $1.869 \pm 0.089$ & 951.2 & $1004.7 \pm 47.8$ \\
\hline 319 & 61 & $1256+281$ & 2.111 & $1.916 \pm 0.071$ & 958.8 & $1029.9 \pm 38.2$ \\
\hline 321 & 61 & $1257+282$ & 2.120 & $1.854 \pm 0.087$ & 962.8 & $996.6 \pm 46.8$ \\
\hline 323 & 61 & $1257+281$ & 2.103 & $1.940 \pm 0.086$ & 955.4 & $1042.9 \pm 46.2$ \\
\hline 324 & 61 & $1257+285$ & 2.070 & $1.237 \pm 0.079$ & 940.3 & $664.7 \pm 42.5$ \\
\hline 325 & 61 & $1257+282$ & 2.116 & $1.753 \pm 0.085$ & 961.2 & $942.3 \pm 45.7$ \\
\hline 326 & 61 & $1257+282$ & 2.106 & $1.801 \pm 0.088$ & 956.7 & $968.1 \pm 47.3$ \\
\hline 327 & 61 & $1258+281$ & 2.060 & $1.729 \pm 0.084$ & 935.7 & $929.6 \pm 45.2$ \\
\hline 328 & 61 & $1258+282$ & 2.067 & $1.526 \pm 0.082$ & 939.0 & $820.2 \pm 44.1$ \\
\hline 330 & 61 & $1259+281$ & 1.985 & $1.241 \pm 0.081$ & 901.4 & $667.1 \pm 43.5$ \\
\hline 331 & 61 & $1259+278$ & 1.894 & $0.485 \pm 0.083$ & 860.3 & $260.6 \pm 44.6$ \\
\hline 333 & 61 & $1302+278$ & 1.142 & $-0.005 \pm 0.067$ & 518.5 & $-2.5 \pm 36.0$ \\
\hline 497 & 61 & $1256+282$ & 2.094 & $1.865 \pm 0.088$ & 951.1 & $1002.5 \pm 47.3$ \\
\hline 498 & 61 & $1256+280$ & 2.075 & $1.960 \pm 0.090$ & 942.4 & $1053.4 \pm 48.4$ \\
\hline 499 & 61 & $1256+280$ & 2.068 & $1.959 \pm 0.087$ & 939.2 & $1052.9 \pm 46.8$ \\
\hline 500 & 61 & $1256+282$ & 2.097 & $1.889 \pm 0.089$ & 952.5 & $1015.4 \pm 47.8$ \\
\hline 503 & 61 & $1257+282$ & 2.110 & $1.894 \pm 0.082$ & 958.4 & $1018.0 \pm 44.1$ \\
\hline 504 & 61 & $1257+281$ & 2.119 & $1.925 \pm 0.085$ & 962.3 & $1034.9 \pm 45.7$ \\
\hline 505 & 61 & $1257+282$ & 2.112 & $1.828 \pm 0.087$ & 959.3 & $982.4 \pm 46.8$ \\
\hline 507 & 61 & $1257+283$ & 2.077 & $1.585 \pm 0.089$ & 943.5 & $851.9 \pm 47.8$ \\
\hline 508 & 61 & $1257+282$ & 2.105 & $1.721 \pm 0.086$ & 956.0 & $924.9 \pm 46.2$ \\
\hline 509 & 61 & $1257+283$ & 2.214 & $1.662 \pm 0.079$ & 1005.6 & $893.5 \pm 42.5$ \\
\hline 510 & 61 & $1257+284$ & 2.091 & $1.490 \pm 0.091$ & 949.8 & $801.1 \pm 48.9$ \\
\hline
\end{tabular}




\begin{tabular}{|c|c|c|c|c|c|c|}
\hline ID\# & GP\# & IAU & $A_{g e}^{\mathrm{Abell}}$ & $A_{g e}^{\text {Lick }} \pm \delta A_{g e}$ & $B_{g e}^{\text {Abell }}$ & $B_{g e}^{\text {Lick }} \pm \delta B_{g e}$ \\
\hline 511 & 61 & $1257+283$ & 2.122 & $1.683 \pm 0.092$ & 963.8 & $904.8 \pm 49.5$ \\
\hline 515 & 61 & $1258+283$ & 2.068 & $1.440 \pm 0.082$ & 939.2 & $773.9 \pm 44.1$ \\
\hline 332 & 62 & $1302-433$ & 0.155 & & 64.3 & \\
\hline 335 & 62 & $1309-428$ & 0.130 & & 53.7 & \\
\hline 336 & 63 & $1310-164$ & -0.003 & $-0.146 \pm 0.158$ & -1.3 & $-114.6 \pm 124.0$ \\
\hline 337 & 63 & $1310-192$ & -0.003 & $-0.031 \pm 0.025$ & -1.3 & $-24.0 \pm 19.6$ \\
\hline 338 & 63 & $1312-161$ & -0.003 & $-0.148 \pm 0.165$ & -1.3 & $-116.2 \pm 129.6$ \\
\hline 340 & 63 & $1316-123$ & -0.003 & $-0.108 \pm 0.095$ & -1.3 & $-84.7 \pm 74.6$ \\
\hline 343 & 65 & $1318-434$ & 0.101 & & 42.3 & \\
\hline 346 & 65 & $1325-439$ & 0.086 & & 36.0 & \\
\hline 354 & 66 & $1350-282$ & 1.093 & & 466.3 & \\
\hline 356 & 66 & $1353-300$ & 1.262 & & 538.4 & \\
\hline 523 & 66 & $1400-337$ & 1.313 & & 560.0 & \\
\hline 364 & 68 & $1418+035$ & -0.001 & $-0.102 \pm 0.030$ & -0.4 & $-92.5 \pm 27.2$ \\
\hline 368 & 68 & $1427+034$ & -0.001 & $0.012 \pm 0.100$ & -0.4 & $11.1 \pm 90.8$ \\
\hline 371 & 69 & $1446-201$ & -0.005 & $-0.016 \pm 0.063$ & -2.0 & $-12.1 \pm 46.2$ \\
\hline 372 & 69 & $1455-190$ & -0.005 & $-0.072 \pm 0.118$ & -2.1 & $-52.8 \pm 86.6$ \\
\hline 373 & 69 & $1456-164$ & -0.005 & $-0.092 \pm 0.192$ & -2.1 & $-67.7 \pm 140.8$ \\
\hline 375 & 70 & $1458+018$ & -0.001 & $-0.211 \pm 0.088$ & -0.5 & $-191.2 \pm 79.8$ \\
\hline 377 & 70 & $1501+014$ & -0.001 & $-0.156 \pm 0.017$ & -0.5 & $-141.4 \pm 15.4$ \\
\hline 380 & 70 & $1503+017$ & -0.001 & $-0.142 \pm 0.054$ & -0.5 & $-128.8 \pm 49.0$ \\
\hline 381 & 71 & $1515-239$ & -0.002 & & -0.9 & \\
\hline 383 & 71 & $1515-238$ & -0.002 & & -0.9 & \\
\hline 556 & 72 & $2345-285$ & 2.835 & & 1339.3 & \\
\hline 557 & 72 & $2345-284$ & 2.828 & & 1336.2 & \\
\hline 558 & 72 & $2345-284$ & 2.830 & & 1336.8 & \\
\hline 559 & 72 & $2345-284$ & 2.820 & & 1332.1 & \\
\hline 561 & 72 & $2344-283$ & 2.796 & & 1321.2 & \\
\hline 562 & 72 & $2344-283$ & 2.801 & & 1323.3 & \\
\hline 563 & 72 & $2344-283$ & 2.809 & & 1327.1 & \\
\hline 564 & 72 & $2344-284$ & 2.796 & & 1321.1 & \\
\hline 565 & 72 & $2344-283$ & 2.887 & & 1363.9 & \\
\hline 566 & 72 & $2344-283$ & 2.660 & & 1256.6 & \\
\hline 410 & 77 & $1941-544$ & 0.016 & & 6.4 & \\
\hline 411 & 77 & $1952-570$ & 0.013 & & 5.2 & \\
\hline 414 & 80 & $1959-484$ & 0.082 & & 33.6 & \\
\hline 416 & 80 & $2002-485$ & 0.118 & & 47.9 & \\
\hline 420 & 80 & $2006-485$ & 0.130 & & 52.8 & \\
\hline 425 & 80 & $2024-471$ & 0.030 & & 12.0 & \\
\hline 428 & 82 & $2054-488$ & 0.351 & & 149.9 & \\
\hline 431 & 82 & $2101-483$ & 0.619 & & 264.6 & \\
\hline 434 & 82 & $2104-473$ & 1.001 & & 427.6 & \\
\hline 436 & 83 & $2108-494$ & -0.002 & & -0.9 & \\
\hline 437 & 83 & $2114-487$ & -0.002 & & -0.9 & \\
\hline 442 & 84 & $2149-484$ & -0.002 & & -0.6 & \\
\hline 443 & 84 & $2150-481$ & -0.002 & & -0.6 & \\
\hline 445 & 85 & $2159-322$ & -0.003 & & -1.2 & \\
\hline 454 & 86 & $2212+135$ & -0.025 & $0.811 \pm 0.117$ & -11.2 & $428.4 \pm 61.8$ \\
\hline 455 & 86 & $2212+135$ & -0.025 & $0.802 \pm 0.116$ & -11.2 & $423.7 \pm 61.3$ \\
\hline 462 & 87 & $2313+064$ & -0.005 & $0.149 \pm 0.095$ & -2.1 & $116.0 \pm 74.1$ \\
\hline 463 & 87 & $2317+078$ & -0.005 & $0.266 \pm 0.110$ & -2.1 & $207.7 \pm 85.8$ \\
\hline
\end{tabular}


Table D.1 cont....

\begin{tabular}{|c|c|c|c|c|c|c|}
\hline ID\# & GP\# & $\mathrm{IAU}$ & $A_{g e}^{\mathrm{Abell}}$ & $A_{g e}^{\text {Lick }} \pm \delta A_{g e}$ & $B_{g e}^{\text {Abell }}$ & $B_{g e}^{\text {Lick }} \pm \delta B_{g e}$ \\
\hline 464 & 87 & $2317+079$ & -0.005 & $0.229 \pm 0.100$ & -2.1 & $178.2 \pm 78.0$ \\
\hline 465 & 87 & $2318+079$ & -0.005 & $0.206 \pm 0.095$ & -2.1 & $160.5 \pm 74.1$ \\
\hline 469 & 89 & $2344-282$ & 2.712 & & 1258.4 & \\
\hline 473 & 89 & $2349-286$ & 2.555 & & 1185.6 & \\
\hline 249 & 98 & $1218+756$ & -0.001 & $0.166 \pm 0.126$ & -0.4 & $153.5 \pm 116.8$ \\
\hline 277 & 98 & $1235+744$ & -0.001 & $0.159 \pm 0.066$ & -0.5 & $147.5 \pm 61.2$ \\
\hline 282 & 98 & $1239+746$ & -0.001 & $0.178 \pm 0.067$ & -0.5 & $164.7 \pm 62.1$ \\
\hline 124 & 100 & $0443-024$ & -0.007 & $0.029 \pm 0.115$ & -2.9 & $20.1 \pm 81.0$ \\
\hline 127 & 100 & $0454-049$ & -0.007 & $-0.003 \pm 0.090$ & -2.9 & $-1.9 \pm 63.2$ \\
\hline 450 & 102 & $2202-503$ & -0.003 & & -1.4 & \\
\hline 452 & 102 & $2203-502$ & -0.003 & & -1.4 & \\
\hline 29 & 103 & $0120+332$ & -0.009 & $0.544 \pm 0.144$ & -3.9 & $366.1 \pm 96.9$ \\
\hline 31 & 103 & $0120+329$ & -0.009 & $0.582 \pm 0.146$ & -3.9 & $391.9 \pm 98.2$ \\
\hline 388 & 104 & $1615+350$ & -0.032 & $0.973 \pm 0.075$ & -15.5 & $476.4 \pm 36.7$ \\
\hline 389 & 104 & $1615+351$ & -0.032 & $1.062 \pm 0.088$ & -15.5 & $519.8 \pm 43.1$ \\
\hline 440 & 105 & $2142-486$ & -0.012 & & -5.1 & \\
\hline 441 & 105 & $2142-485$ & -0.012 & & -5.1 & \\
\hline 6 & 201 & $0036+027$ & -0.010 & $0.181 \pm 0.087$ & -4.2 & $121.4 \pm 58.2$ \\
\hline 9 & 202 & $0040-018$ & -0.008 & $-0.094 \pm 0.213$ & -3.4 & $-66.0 \pm 149.6$ \\
\hline 48 & 203 & $0141+284$ & -0.006 & $-0.007 \pm 0.125$ & -2.3 & $-5.3 \pm 94.7$ \\
\hline 50 & 204 & $0147+217$ & -0.004 & $-0.213 \pm 0.334$ & -1.4 & $-176.1 \pm 276.2$ \\
\hline 59 & 205 & $0153+053$ & -0.011 & $0.118 \pm 0.093$ & -4.8 & $75.8 \pm 59.6$ \\
\hline 74 & 206 & $0235+019$ & -0.016 & $0.391 \pm 0.107$ & -7.0 & $228.2 \pm 62.4$ \\
\hline 75 & 207 & $0238-084$ & -0.001 & $0.002 \pm 0.063$ & -0.3 & $1.8 \pm 62.6$ \\
\hline 110 & 208 & $0343-041$ & -0.007 & $0.046 \pm 0.184$ & -2.8 & $32.8 \pm 131.1$ \\
\hline 114 & 210 & $0411-317$ & -0.050 & & -20.0 & \\
\hline 115 & 211 & $0414-557$ & -0.001 & & -0.2 & \\
\hline 151 & 212 & $0735-691$ & -0.001 & & -0.3 & \\
\hline 160 & 214 & $0853+515$ & -0.008 & $-0.008 \pm 0.121$ & -3.4 & $-5.5 \pm 80.9$ \\
\hline 164 & 215 & $0907+602$ & -0.001 & $-0.315 \pm 0.008$ & -0.3 & $-304.0 \pm 7.7$ \\
\hline 165 & 216 & $0909+352$ & -0.001 & $-0.171 \pm 0.030$ & -0.6 & $-150.6 \pm 26.4$ \\
\hline 180 & 218 & $0956-339$ & 0.000 & & 0.0 & \\
\hline 179 & 219 & $0956-266$ & 0.000 & & -0.2 & \\
\hline 181 & 220 & $0957-193$ & -0.005 & $0.425 \pm 0.111$ & -2.3 & $302.0 \pm 78.8$ \\
\hline 205 & 221 & $1043+731$ & -0.003 & $0.476 \pm 0.172$ & -1.3 & $386.3 \pm 139.4$ \\
\hline 345 & 224 & $1321+142$ & -0.020 & $0.341 \pm 0.055$ & -9.2 & $181.1 \pm 29.2$ \\
\hline 349 & 225 & $1333-337$ & 4.124 & & 1727.4 & \\
\hline 357 & 227 & $1400+096$ & -0.014 & $0.139 \pm 0.136$ & -6.0 & $80.8 \pm 79.3$ \\
\hline 363 & 228 & $1416+367$ & -0.005 & $0.102 \pm 0.154$ & -2.1 & $74.3 \pm 112.6$ \\
\hline 384 & 229 & $1537+595$ & -0.003 & $-0.064 \pm 0.287$ & -1.3 & $-51.6 \pm 231.3$ \\
\hline 459 & 231 & $2254-367$ & -0.001 & & -0.4 & \\
\hline 460 & 232 & $2258+161$ & -0.002 & $0.166 \pm 0.065$ & -0.8 & $153.1 \pm 60.0$ \\
\hline 387 & 233 & $1610+296$ & 0.654 & $0.485 \pm 0.065$ & 319.4 & $233.8 \pm 31.3$ \\
\hline 472 & 234 & $2348+268$ & 0.915 & $0.466 \pm 0.283$ & 420.2 & $244.5 \pm 148.5$ \\
\hline 272 & 235 & $1228+260$ & -0.001 & $-0.025 \pm 0.131$ & -0.2 & $-23.8 \pm 125.0$ \\
\hline 238 & 236 & $1156+253$ & -0.007 & $0.266 \pm 0.091$ & -3.1 & $178.5 \pm 61.0$ \\
\hline 361 & 237 & $1405+552$ & -0.001 & $0.289 \pm 0.306$ & -0.6 & $260.6 \pm 275.8$ \\
\hline 362 & 238 & $1407+177$ & -0.011 & $-0.035 \pm 0.104$ & -4.8 & $-21.5 \pm 63.9$ \\
\hline 236 & 240 & $1155+284$ & -0.005 & $-0.135 \pm 0.110$ & -2.1 & $-97.4 \pm 79.4$ \\
\hline 492 & 242 & $1127-361$ & -0.003 & & -1.4 & \\
\hline 461 & 243 & $2309-288$ & -0.001 & & -0.4 & \\
\hline
\end{tabular}


Table D.1 cont....

\begin{tabular}{|c|c|c|c|c|c|c|}
\hline ID\# & GP\# & IAU & $A_{g e}^{\mathrm{Abell}}$ & $A_{g e}^{\text {Lick }} \pm \delta A_{g e}$ & $B_{g e}^{\mathrm{Abell}}$ & $B_{g e}^{\text {Lick }} \pm \delta B_{g e}$ \\
\hline 235 & 244 & $1152-136$ & -0.001 & $-0.008 \pm 0.095$ & -0.4 & $-7.1 \pm 84.9$ \\
\hline 241 & 245 & $1204-294$ & -0.002 & & -0.7 & \\
\hline 147 & 246 & $0715+858$ & -0.002 & $0.136 \pm 0.327$ & -0.7 & $120.8 \pm 290.8$ \\
\hline 177 & 247 & $0941-210$ & -0.002 & $-0.071 \pm 0.115$ & -0.8 & $-59.8 \pm 96.2$ \\
\hline 468 & 251 & $2335+267$ & 1.122 & $1.478 \pm 0.209$ & 538.1 & $724.9 \pm 102.5$ \\
\hline 187 & 252 & $1010+033$ & -0.001 & $-0.337 \pm 0.127$ & -0.3 & $-313.5 \pm 118.2$ \\
\hline 348 & 253 & $1330+629$ & -0.004 & $-0.008 \pm 0.124$ & -1.5 & $-6.3 \pm 97.1$ \\
\hline 353 & 254 & $1347+604$ & -0.002 & $-0.119 \pm 0.081$ & -0.6 & $-105.2 \pm 71.6$ \\
\hline 358 & 255 & $1401+353$ & -0.006 & $-0.072 \pm 0.241$ & -2.6 & $-50.5 \pm 169.0$ \\
\hline 439 & 266 & $2128-388$ & -0.012 & & -5.2 & \\
\hline 427 & 268 & $2045-381$ & -0.003 & & -1.2 & \\
\hline 422 & 269 & $2013-710$ & -0.007 & & -2.9 & \\
\hline 14 & 271 & $0055+300$ & -0.011 & $-0.083 \pm 0.106$ & -4.5 & $-53.9 \pm 69.2$ \\
\hline 73 & 272 & $0222-586$ & -0.085 & & -40.8 & \\
\hline 161 & 273 & $0851-028$ & -0.001 & $-0.055 \pm 0.117$ & -0.6 & $-48.3 \pm 102.7$ \\
\hline 339 & 274 & $1315-265$ & -0.001 & & -0.6 & \\
\hline 518 & 275 & $1301-302$ & 1.290 & & 535.4 & \\
\hline 369 & 277 & $1439-363$ & 0.000 & & 0.0 & \\
\hline 401 & 278 & $1754-636$ & 0.020 & & 8.4 & \\
\hline 408 & 279 & $1852-546$ & -0.003 & & -1.2 & \\
\hline 220 & 281 & $1115+582$ & -0.001 & $0.053 \pm 0.109$ & -0.6 & $47.0 \pm 97.1$ \\
\hline 155 & 283 & $0842+741$ & -0.002 & $-0.011 \pm 0.194$ & -0.8 & $-9.7 \pm 168.9$ \\
\hline 171 & 284 & $0921-229$ & -0.003 & $-0.106 \pm 0.192$ & -1.1 & $-85.9 \pm 155.6$ \\
\hline 214 & 285 & $1110-264$ & -0.001 & & -0.4 & \\
\hline
\end{tabular}

Table D.2: Group identification code for the 5 most populous clusters in the sample.

\begin{tabular}{ccc}
\hline GP\# & $\begin{array}{c}\text { No. } \\
\text { Galaxies }\end{array}$ & Cluster \\
\hline & & \\
30 & 18 & Perseus \\
31 & 11 & Fornax \\
56 & 16 & Virgo \\
61 & 33 & Coma \\
72 & 10 & DC2345-28
\end{tabular}




\section{References}

Aaronson, M., Bothun, G., Mould, J., Schommer, R.A. \& Cornell, M.E., 1986. Astrophys. $J ., 302,536$.

Abell, G.O., 1958. Astrophys. J. Suppl. Ser., 3, 211.

Abell, G.O., Corwin, H.G. \& Olowin, R.P, 1989. Astrophys. J. Suppl. Ser., 70, 1.

Auriemma, C., Perola, G.C., Ekers, R., Fanti, R., Lari, C., Jaffe, W.J. \& Ulrich, M.H., 1977. Astron. Astrophys., 57, 41.

Bahcall, N.A. \& Burgett, W.S., 1986. Astrophys. J. Lett., 300, L35.

Bahcall, N.A. \& Soneira, R.M., 1983. Astrophys. J., 270, 20.

Bahcall, N.A., 1979. Astrophys. J., 232, 689.

Bahcall, N.A., 1988. Ann. Rev. Astron. Astrophys., 26, 631.

Bailey, M.E. \& Sparks, W.B., 1983. Mon. Not. R. Astr. Soc., 204, 53P.

Baldwin, J.E. \& Stone, R.P.S, 1983. Mon. Not. R. astr. Soc., 204, 347.

Bardeen, J.M., Bond, J.R., Kaiser, N. \& Szalay, A.S., 1986. Astrophys. J., 304, 15.

Bardeen, J.M., Steinhardt, P.J. \& Turner, M.S., 1983. Phys. Rev. D., 28, 679.

Barnes, J., 1983. Mon. Not. R. Astr. Soc., 203, 223.

Bean, A.J., Efstathiou, G., Ellis, R.S., Peterson, B.A. \& Shanks, T., 1983. Mon. Not. R. astr. Soc., 205, 605.

Bertschinger, E. \& Juszkiewicz, R., 1988. Astrophys. J. Lett., 334, L59.

Blumenthal, G.R., Faber, S.M., Primack, J.R. \& Rees, M.J., 1984. Nature, 311, 517.

Bolton, J.G., Wright, A.E. \& Savage, A., 1979. Aust. J. Phys. Astrophys. Suppl., 46, 1.

Bond, J.R. \& Efstathiou, 1984. Astrophys. J., 285, L45.

Bond, J.R. \& Efstathiou, 1987. Mon. Not. R. astr. Soc., 226, 655.

Bond, J.R. \& Szalay, A.S., 1983. Astrophys. J., 274, 443.

Bond, J.R., 1988. in "The Early Universe", eds. Unruh, W.G. \& Semenoff, G.W., Reidel, Dordrecht.

Bond, J.R., 1989. in "Large-Scale Motions in the Universe" proc. Vatican Study Week, eds. V.C. Rubin \& G. Coyne.

Braccesi, A. et al. 1969. Nuovo Cimento, 62B, 13.

Broadhurst, T.J., Ellis, R.S., Koo, D.C. \& Szalay, A.S., 1990. Nature, 343, 726.

Bruzual, G.A., 1983. Rev. Mex. Astr. Astrofis., 8, 63. 
Burstein, D. \& Heiles, C., 1982. Astron. J., 87, 1165.

Burstein, D., 1990. Reports on Progress in Physics, 53, 421.

Burstein, D., Davies, R.L., Dressler, A., Faber, S.M., Stone, R.P.S, Lynden-Bell, D., Terlevich, R., Wegner, G., 1987. Astrophys. J. Suppl. Ser. 64, 601.

Burstein, D., Rubin, V.C., Thonnard, N. \& Ford, W.K., 1982. Astrophys. J., 253, 70.

Carlberg, R.G. \& Couchman, H.M.P., 1989. Astrophys. J., 340, 47.

Casali, M.M., Aspin, C.A. \& Maclean, I.S., 1987. IRCAM Users Guide.

Chambers, K.C., Miley, G.K. \& van Breugel, W.J.M., 1988. Astrophys. J. Lett., 327, 247.

Clutton-Brock, M. \& Peebles, P.J.E., 1981. Astron. J., 86, 1115.

Clutton-Brock, M., 1982. Astron. J, 87, 1127

Cohen, A.M., Porcas, R.W., Browne, I.W.A., Daintree, E.J. \& Walsh, D., 1977. Mem. R. astr. Soc., $84,1$.

Cole, S. \& Kaiser, N., 1989. Mon. Not. R. astr. Soc., 237, 1127.

Collins, C.A., Joseph, R.D. \& Robertson, N.A., 1986. Nature, 320, 506

Corwin, H.G. \& Emerson, D., 1982. Mon. Not. R. astr. Soc., 200, 621.

Costero, R. \& Osterbrock, D.E., 1977. Astrophys. J., 211, 675.

Davies, R.D., Lasenby, A.N., Watson, R.A., Daintree, E.J., Hopkins, J., Beckman, J., Sanchez-Almeida, J. \& Rebolo, R., 1987. Nature, 326, 462.

Davis, M. \& Djorgovski, 1985. Astrophys. J., 299, 15.

Davis, M. \& Geller, M.J., 1976. Astrophys. J., 208, 13.

Davis, M. \& Peebles, P.J.E. 1983a. Ann. Rev. Astron. Astrophys., 21, 109

Davis, M. \& Peebles, P.J.E., 1983b. Astrophys. J., 267, 465.

Davis, M., Efstathiou, G., Frenk, C.S. \& White, S.D.M., 1985. Astrophys. J., 292, 371.

Davis, M., Huchra, J.P., Latham, D.W. \& Tonry, J., 1982. Astrophys. J., 253, 423.

Day, G.A., Shimmins, A.J., Ekers, R.D. \& Cole, D.J., 1964. Austr. J. Phys., 17, 340.

Dekel, A. \& Rees, M.J. 1987, Nature, 326, 455.

Dekel, A., Blumenthal, G.R., Primack, J.R. \& Oliver, S., 1989. Astrophys. J., 338, L5.

Dekel, A., \& Silk, J., 1986. Astrophys. J., 303, 39.

Djorgovski, S. \& Davis, M., 1987. Astrophys. J., 313, 59.

Djorgovski, S., de Carvalho, R. \& Han, M.S., 1988. in "The Extragalactic Distance Scale", ed. S. van den Bergh. 
Dressler, A. 1980, Astrophys. J., 236, 351

Dressler, A. \& Sandage, A., 1983. Astrophys. J., 265, 644.

Dressler, A., 1987. Astrophys. J., 317, 1.

Dressler, A., Faber, S.M, Burtein, D., Davies, R.L., Lynden-Bell, D., Terlevich, R., Wegner, G. 1987, Astrophys. J., 313, L37.

Dressler, A., Lynden-Bell, D., Burstein, D., Davies, R.L., Faber, S.M., Terlevich, R.J. \& Wegner, G., 1987. Astrophys. J., 313, 42.

Dunlop, J.S. \& Peacock, J.A., 1990. Mon. Not. R. astr. Soc, 247, 19.

Efstathiou, G. \& Silk, J., 1983. Fund. Cosm. Phys., 9, 1.

Efstathiou, G., 1990. in Physics of the Early Universe, proc $36^{\text {th }}$ Scottish Universities Summer School in Physics, eds Peacock, J.A., Heavens, A.F. \& Davies, A.T. (Adam Hilger), p361.

Efstathiou, G., Ellis, R.S. \& Peterson, B.A., 1988. Mon. Not. R. astr. Soc., 232, 431.

Eisenhardt, P.P.M. \& Lebofsky, M.J., 1987. Astrophys. J., 316, 70.

Evans, D.S., Menzies, A. \& Stoy, R.H., 1959. Mon. Not. R. astr. Soc., 119, 638.

Evrard, A.E., 1987. Astrophys. J., 316, 36.

Faber, S.M. \& Burstein, D., 1988. In "Large-Scale Motions in the Universe", Proc. of Vatican Study Week XXVII, eds. V.C. Rubin \& G. Coyne.

Faber, S.M. \& Gallagher, J.S., 1979. Ann. Rev. Astron. Astrophys., 17, 135

Faber, S.M. \& Jackson, R.E., 1976. Astrophys. J., 204, 668.

Faber, S.M., Wegner, G., Burstein, D., Davies, R.L., Dressler, A. \& Lynden-Bell, D., 1989. Astrophys. J. Suppl. Ser., 69, 361.

Faber, S.M., 1982. in "Astrophysical Cosmology", proc. Vatican study week, eds H.A. Brück, G.V.Coyne \& M.S. Longair, p191.

Fairall, A.P. \& Jones, A., 1988. Publ. Dept. Astr. Univ. Cape Town, No. 10.

Fall, S.M. \& Jones, B.J.T., 1976. Nature, 262, 457

Fanaroff, B.L. \& Riley, J.M., 1974. Mon. Not. R. astr. Soc. 167, 31P

Fehrenbach, C.H. \& Duflot, M., 1980. Astr. Astrophys. Suppl., 39, 311.

Fixsen, D.J., Cheng, E.S. \& Wilkinson, D.T., 1983. Phys. Rev. Lett., 277, L23.

Frenk, C.S., White, S.D.M. \& Davis, M., 1983. Astrophys. J., 271, 417.

Giovanelli, R., Haynes, M.P. \& Chincarini, G.L. 1986. Astrophys. J., 300, 77

Groth, E.J. \& Peebles, P.J.E., 1977. Astrophys. J., 217, 385 
Groth, E.J., Juskiewicz, R. \& Ostriker, J.P., 1989. Astrophys. J., 345, 558.

Grueff, G. \& Vigotti, M., 1972. Astr. Astrophys. Suppl. Ser., 6, 1.

Grueff, G. \& Vigotti, M., 1973. Astr. Astrophys. Suppl. Ser., 11, 41.

Grueff, G. \& Vigotti, M., 1979. Astr. Astrophys. Suppl. Ser., 35, 371.

Gunn, J.E. In. "The Extragalactic Distance Scale", 1988. ASP Workshop, eds. S. Van den Bergh \& C. Pritchett.

Gunn, J.E. \& Oke, J.B., 1975. Astrophys. J., 195, 255

Gunn, J.E. \& Tinsley, B.M., 1976. Astrophys. J., 210, 1.

Gunn, J.E., 1978. in "The 8'th Advanced Course of Swiss. Soc. of Ast. Astr.", p1.

Gunn, J.E., Hoessel, J.G. \& Oke, J.B., 1986. Astrophys. J., 306, 30.

Guth, A.H., 1981. Phys. Rev. D., 23, 347.

Hamabe, M. \& Kormendy, J., 1987. in "Structure and Dynamics of Elliptical Galaxies", ed. T. de Zeeuw.

Hamilton, A.J.S., 1988. Astrophys. J. Lett., 331, L59.

Hart, L. \& Davies, R.D., 1982. Nature, 297, 191.

Hausman, M.A. \& Ostriker, J.P., 1978. Astrophys. J., 224, 320.

Heavens, A.F. \& Peacock, J.A., 1988. Mon. Not. R. astr. Soc., 232, 339.

Heckman, T.M., Smith, E.P., Baum, S.A., van Breugel, W.J.M., Miley, G.K., Illingworth, G.D., Bothun, G.D. \& Balick, B., 1986. Astrophys. J., 311, 526.

Heydon-Dumbleton, N.H., Collins, C.A. \& MacGillivray, H.T., 1989. Mon. Not. R. astr. Soc., 238,378 .

Hine, R.G. \& Longair, M.S., 1979. Mon. Not. R. astr. Soc, 188, 111.

Hoessel, J.G., 1987. in "Structure and Dynamics of Elliptical Galaxies", ed. T. de Zeeuw.

Hoessel, J.G., 1980. Astrophys. J., 241, 493.

Hoffman, Y., Shahmim, J. \& Shaviv, G., 1982. Astrophys. J., 262, 413.

Iovino, A., Melnick, J. \& Shaver, P.A. in "Large-Scale Structure and Motions in the Universe", ed. M. Mezzetti et al.

Jedrzejewski, R.I., 1987. in "Structure and Dynamics of Elliptical Galaxies", IAU No. 127, ed. de Zeeuw, T.

Jenkins, C.R., 1984. Mon. Not. R. astr. Soc., 207, 361.

Kühr, H., Johnston, K.J., Odenwald, S. \& Adlhoch, J., 1987. Astr. Astrophys. Suppl. Ser., 71, 493 . 
Kühr, H., Pauliny-Toth, I.I.K., Witzel, A. \& Schmidt, J., 1981. Astr. J., 86, 854.

Kaiser, N. 1984, Astrophys. J, 284, L9

Kaiser, N. \& Lahav, O., 1989. Mon. Not. R. astr. Soc., 237, 129.

Kaiser, N., 1984. Astrophys. J., 284, L9.

Kaiser, N., 1986. Mon. Not. R. astr. Soc., 222, 323.

Kaiser, N., 1988a. in "Large-Scale Structures of the Universe", proc. IAU symp. no. 130, eds J. Audouze, M.-C. Pelletan \& A. Szalay (D. Reidel), p43.

Kaiser, N., 1988b. in "Large-Scale Structure and Motions in the Universe", eds. M. Mezzetti et al.

Kaiser, N., 1988c. Mon. Not. R. astr. Soc., 231, 149.

Kidger, M., 1898. preprint.

Klypin, A.A. \& Shandarin, S.F., 1983. Mon. Not. R. astro. Soc., 204, 891

Kolb, E.W. \& Turner, M.S., 1990. "The Early Universe", Addison-Wesley Publishing Company.

Kormendy, J. \& Ilingworth, G., 1983. Astrophys. J., 265, 632.

Kormendy, J., 1977. Astrophys. J, 218, 33

Laing, R.A., Riley, J.M. \& Longair, M.S., 1983. Mon. Not. R. astr. Soc., 204, 151.

Landau, L.D. \& Lifschitz, E.M., 1975, "Classical Theory of Fields", Pergamon Press, 4th Edition.

Landolt, A.U. 1983. Astron. J., 88, 439.

Langston, G., 1988. in "Large-Scale Structure and Motions in the Universe", eds. M.Mezzetti et al., Kluwer Acad. Publ., p387

de Lapparent, V. \& Geller, M.J. \& Huchra, J.P., 1986. Astrophys. J. Lett., 302, L1.

Larson, R.B., 1974. Mon. Not. R. astr. Soc., 169, 229.

Layzer, D., 1985. in "Stars and Stellar Systems", eds. A. Sandage, M. Sandage, J. Kristian.

Lilje, P.B. \& Efstathiou, G., 1988. Mon. Non. R. astr. Soc., 231, 635.

Lilje, P.B., Yahil, A., \& Jones, B.J.T., 1986. Astrophys. J., 307, 91.

Lilly, S.J. \& Longair, M.S., 1984. Mon. Not. R. astr. Soc., 211, 833.

Lilly, S.J. \& Prestage, R.M., 1987. Mon. Not. R. astr. Soc., 225, 531

Lilly, S.J., 1988. Astrophys. J., 333, 161.

Lilly, S.J., Mclean, I.S. \& Longair, M.S., 1984. Mon. Not. R. astr. Soc., 209, 401. 
Ling, E.N., Frenk, C.S. \& Barrow, J., 1986. Mon. Not. R. astr. Soc., 223, 21 P.

Longair, M.S. \& Scheuer, P.A.G., 1970. Mon. Not. R. Astron. Soc 151, 45

Longair, M.S. \& Seldner, M., 1979. Mon. Not. R. astr. Soc., 189, 433.

Longair, M.S., 1978. In. "The 8'th Advance Course of Swiss. Soc. of Ast. Astr.", p125.

Lonsdale, C.J. \& Hacking, P.B., 1989. Astrophys. J., 339, 712.

Lubin, P.M., Villela, T., Epstein, G. \& Smoot, G., 1985, Astrophys. J. Lett., 298, L1.

Lucey, J.R., 1983. Mon. Not. R. astr. Soc, 204, 33.

Lynden-Bell, D., Faber, S.M., Burstein, D., Davies, R.L., Dressler, A., Terlevich, R., Wegner, G. 1988. Astrophys. J. 326, 19

Mackay, C.D., 1986, Ann. Revs. Astron. Astrophys. 24, 256.

Maddox, S.J., Efstathiou, G., Sutherland, W.J. \& Loveday, J., 1990. Mon. Not. R. astro. Soc., 242, 43P.

Malmquist, K.G., 1920. Medd. Lunds Astr. Obs., II, 22

Malumuth, E.M. \& Kirshner, R.P., 1985. Astrophys. J., 291, 8.

Masson, C., 1979. Mon. Not. R. astr. Soc., 188, 261.

Masson, C.R., 1979. Mon. Not. R. astr. Soc., 187, 253.

Mayer, C.J., 1979. Mon. Not. R. astr. Soc., 186, 99.

Melchiorri, F., Melchiorri, B.O., Ceccarelli, C. \& Pietranara, L., 1981, Astrophys. J., 250, L1.

Merritt, D., 1984. Astrophys. J., 276, 26

Merritt, D., 1987. Astrophys. J., 313, 121.

Narayan, R. \& White, S.D.M., 1988. Mon. Not. R. astr. Soc., 231, 97P

Oke, J.B. \& Gunn, J.E., 1983. Astrophys. J., 266, 713.

Owen, F.N., White, R.A. \& Thronson, H.A., 1988. Astron. J., 95, 1

Parker, Q.A., MacGillivaray, H.T., Hill, P.W. \& Dodd, R.J., 1986. Mon. Not. r. Astr. Soc., 220, 901.

Peacock, J.A. \& Heavens, A.F., 1985. Mon. Not. R. astr. Soc., 217, 805.

Peacock, J.A. \& Nicholson, D., 1991. in preparation.

Peacock, J.A., Miller, L., Collins, C.A., Nicholson, D. \& Lilly, S.J., 1988. in Large-Scale Structures in the Universe, eds. Audouze, J., Pelletan, M.C. \& Szalay, A.S, p579.

Peacock, J.A., 1990. Mon. Not. R. astr. Soc., 243, 517

Peebles, P.J.E. 1986, Nature, 321, 27 
Peebles, P.J.E., 1973. Astrophys. J., 185, 413.

Peebles, P.J.E., 1980, The Large Scale Structure of the Universe, Princeton University Press.

Peebles, P.J.E., 1987a. Astrophys. J., 315, L51.

Peebles, P.J.E., 1987b. Nature, 327, 210.

Peebles, P.J.E., 1989. in "The Epoch Of Galaxy Formation", eds. C. Frenk et al.

Pence, W., 1976. Astrophys. J., 203, 39

Pierce, M.J., 1986., in. "Galaxy Distances and Deviations from Universal Expansion", NATO ASI Conference Proceedings.

Porcas, R.W., Urry, C.M., Browne, I.W.A., Cohen, A.M., Daintree, E.J. \& Walsh, D., 1980. Mon. Not. R. astr. Soc., 191, 607.

Postman, M., \& Geller, M.J. 1984, Astrophys. J., 281, 95

Press, W.H. \& Schechter, P., 1974. Astrophys. J., 187, 425. (PS)

Prestage, R.M. \& Peacock, J.A., 1988. Mon. Not. R. astr. Soc., 230, 131.

Prestage, R.M. \& Peacock, J.A., 1989. Mon. Not. R. astr. Soc., 236, 959

Primack, J.R. \& Blumenthal, G.R., 1984. in "Clusters and Groups of Galaxies", eds. Giurcin, G. \& Mezzetti, M., Reidel.

Readhead, A.C.S., Lawrence, C.R., Myers, S.T., Sargent, W.L.W, Hardebeck, H.E. \& Moffet, A.T., 1989, Astrophys. J., 346, 566.

Rice, S.O., 1954. in Selected papers on Noise and Stochastic Processes, p. 133, ed. Wax, N., Dover.

Rubin, V.C., Ford, W.K., Thonnard, N. \& Burstein, D. 1982, Ap. J., 261, 439

Rubin, V.C., Thonnard, N., Ford, W.K. \& Roberts, M.S., 1976. Astron. J., 81, 719.

Sansom, A.E. et al., 1987. Mon. Not. R. Astr. Soc., 229, 15.

Sansom, A.E. 1987. in "Structure and Dynamics of Elliptical Galaxies", ed. T. de Zeeuw.

Saslaw, W.C. \& Hamilton, A.J.S., 1984. Astrophys. J., 276, 13.

Savage, A. \& Bolton, J.G., 1979. Aust. J. Phys. Astrophys. Suppl., 1979. 46, 19.

Savage, A. \& Wall, J.V., 1976. Aust. J. Phys. Astrophys. Suppl., 39, 39

Savage, A., 1976. Mon. Not. R. astr. Soc, 174, 259.

Schaeffer, R. \& Silk, J. 1985, Astrophys. J., 292, 315

Schaeffer, R., 1987. Astr. Astrophys., 180, L5.

Schechter, P,L, 1977. Astron. J, 82, 569. 
Schectman, S.A., 1985, Astrophys. J., 57, 77.

Schombert, J.M. 1986, Astrophys. J. Suppl., 60, 603.

Schweizer, F., 1979 Astrophys. J, 233, 23.

Seldner, M. \& Peebles, P.J.E., 1977. Astrophys. J., 215, 703.

Seldner, M. \& Peebles, P.J.E., 1978. Astrophys. J., 225, 7.

Shane, C.D. \& Wirtanen, C.A., 1967. Publ. Lick. Obs., No. 22, part 1.

Shanks, T., Stevenson, P.R.F, Fong, R., Macgillivray, H.T., 1984. Mon. Not. R. astr. Soc., 206, 767.

Shaver, P.A. \& Pierre, M., 1989. Astr. Astrophys., 220, 35.

Shimmins, A.J., Day, G.A., 1968. Austr. J. Phys., 21, 377.

Silk, J. \& Norman, C., 1981. Astrophys. J., 247, 59.

Silk, J., 1968, Astrophys. J., 151, 459.

Silk, J., 1987. in Cosmology and Particle Physics. Proceedings of the XVII GIFT International Seminar on Theoretical Physics.

Silk, J., 1988. in Comets to Cosmology. Proceedings of the Third IRAS Conference.

Silk, J., 1989. Astrophys. J. Lett., 345, L1.

Smith, E.P. \& Heckman, T.M., 1989. Astrophys. J., 341, 658.

Smoot, G.F., Gorenstein, M.V. \& Muller, R.A., 1977. Phys. Rev. Lett., 39, 898

Sparks, W.B., 1988. Astron. J., 95, 1569.

Staveley-Smith, L. \& Davies, R.D., 1989. Mon. Not. R. astr. Soc., 241, 787.

Staveley-Smith, L., 1985. University of Manchester, PhD Thesis.

Strauss, M.A. \& Davis, M., 1988. in "Large-Scale Structure and Motions in the Universe".

A Vatican study week, eds. V.C. Rubin \& G.V. Coyne, p116.

Subrahmanya, C.R. \& Hunstead, R.W., 1986. Astr. Astrophys., 170, 27.

Sunyaev, R.A. \& Zeldovich, Ya. B., 1972. Astron. Astrophys, 20, 189.

Sutherland, W.J., 1988. Mon. Not. R. astr. Soc., 234, 159.

Sutherland, W.J., Maddox, S.J., Efstathiou, G.P. \& Loveday, J., 1988. Bull. Am. Astron. Soc., 20, 1035.

Tammann, G. in Physical Cosmology, Les Houches 1979, eds. Balian, R., Audouze, J. \& Schramm, D.

Tammann, G.A. \& Sandage, A. 1985, Astrophys. J., 294, 81.

Thuan, T.X., Gott, J.R. \& Schneider, S.E, 1987. Astrophys. J. Lett., 315, L93. 
Tonry, J. \& Davis, M., 1979. Astron. J., 84, 1511.

Tully, R.B., 1986. Astrophys. J., 303, 25.

Tully, R.B. \& Fisher, J.R., 1977. Astr. Astrophys., 54, 661.

Umemura, M. \& Ikeuchi, S., 1985. Ap. J., 294, 583.

Uson, J.M. \& Wilkinson, D.T., 1984. Nature, 312, 427.

de Vaucouleurs, G., 1948. Ann. d'Astrophys., 11, 267.

de Vaucouleurs, G., de Vaucouleurs, A. \& Corwin, H.G., 1976, Second Reference Catalogue of Galaxies.

Véron-Cetty, M.P. \& Véron, P., 1983. Astr. Astrophys. Suppl., 53, 219.

Waggett, P.C., Warner, P.J. \& Baldwin, J.E., 1977. Mon. Not. R. astr. Soc., 181, 465.

Wall, J.V. \& Laing, R.A., 1986. Users' Guide to the Prime Focus CCD Camera on the INT.

Wall, J.V. \& Peacock, J.A., 1985. Mon. Not. R. astr. Soc., 216, 173.

Webster, A.S., 1976. Mon. Not. R. astr. Soc., 175, 61.

Webster, A.S., 1977. in Radio astronomy and cosmology, proc. IAU symp no. 74, ed D.L. Jauncey (Dordrecht; D. Reidel), p75.

Weinberg, S., 1972. "Gravitation and Cosmology", J. Wiley \& Sons, New York.

West, M.J. \& Richstone, D.O., 1988 Astrophys. J., 335, 532.

West, R.M. \& Barbier, B., 1982. Astr. Astrophys., 106, 53.

White, S.D.M., Davis, M., Efstathiou, G. \& Frenk, C.S., 1987. Nature, 330, 451.

Whitmore, B.C. \& Kirshner, R.P., 1981. Astrophys. J., 250, 43.

Whittet, D.C.B., Bode, M.F., Murdin, P. 1987 Vistas Astron., 30, 135.

Willis, A.G., Strom, R.G. \& Wilson, A.S., 1974. Nature, 250, 625.

Wills, D. \& Bolton, J.G., 1969. Austr. J. Phys., 22, 775.

Windhorst, R.A., van Heerde, G.M. \& Katgert, P., 1984. Astron. Astrophys. Suppl. Ser., 58,1 .

Yahil, A., 1988. in "Large-Scale Structure and Motions in the Universe", eds. V.C. Rubin \& G.V. Coyne.

Yang, J., Turner, M.S, Steigman, G., Schramm, D.N. \& Olive, K.A., 1984. Astrophys. J., $281,493$.

Yates, M.G., Miller, L. \& Peacock, J.A., 1989. Mon. Not. R. astr. Soc., 240, 129.

Yee, H.K.C. \& Green, R.F., 1987. Astrophys. J., 319, 28. 
Young, P., 1976. Astron. J., 81, 807.

Zeldovich, Ya. B., 1970. Astron. Astrophys., 5, 84. 217, 805.

Zwicky, F. 1933. Helv. Phys. Acta, 6, 110.

Zwicky, F., Herzog, E., Wild, P., Karpowicz, M \& Kowal ,C.T., 1961-68. Catalog of Glaxies and Clusters of Galaxies. 